\title{
INVENTORY OF SPRINGS IN THE STATE OF NEW MEXICO
}

By W.E. White and G.E. Kues

\section{U.S. GEOLOGICAL SURVEY \\ Open-File Report 92-118}

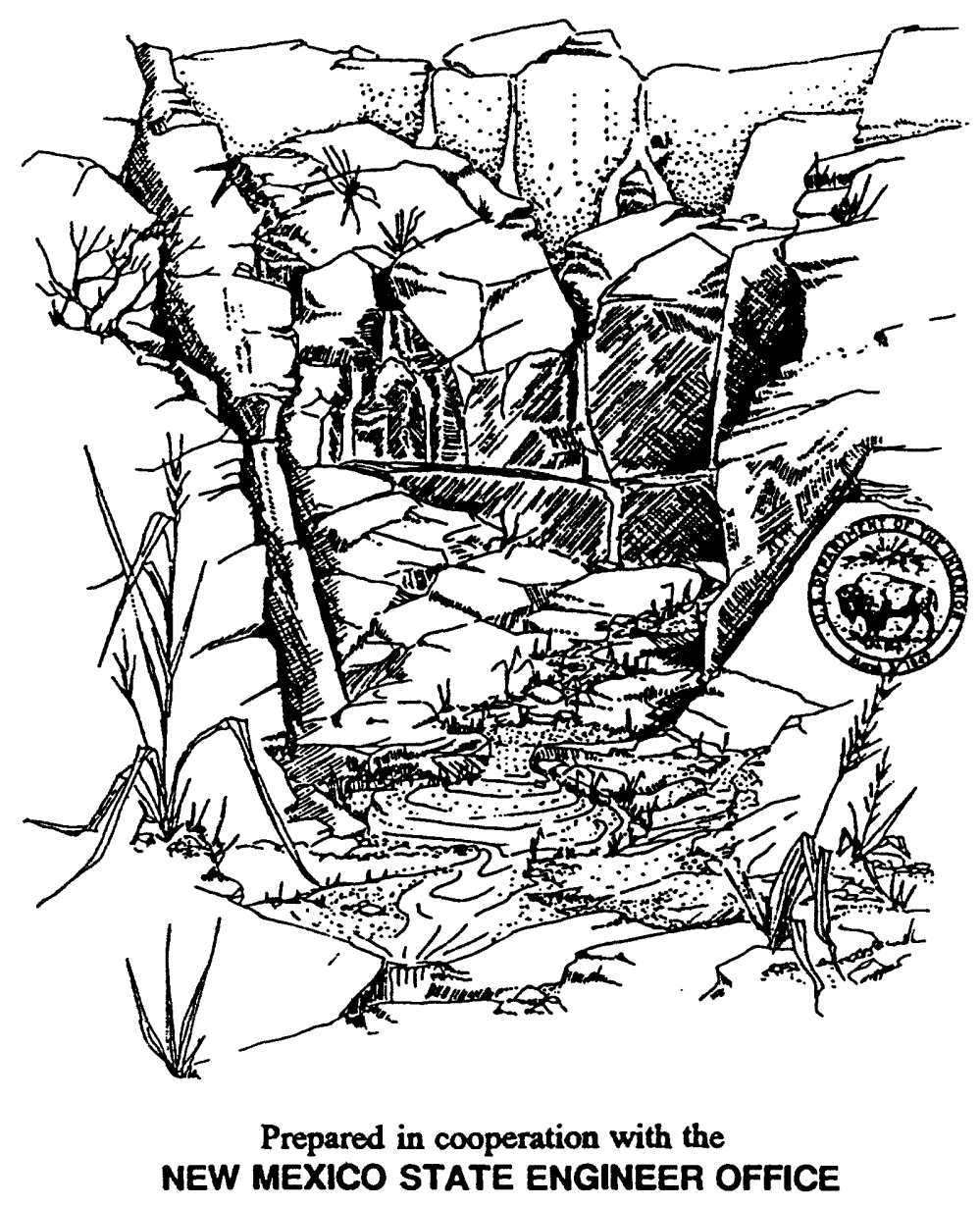

Albuquerque, New Mexico

1992 


$$
\begin{array}{r|l}
\text { U.S. DEPARTMENT OF THE INTERIOR } \\
\text { MANUEL LUJAN, JR., Secretary } \\
\text { U.S. GEOLOGICAL SURVEY } \\
\text { Dallas L. Peck, Director }
\end{array}
$$

For additional information write to:

District Chief U.S. Geological Survey Water Resources Division Pinetree Corporate Centre 4501 Indian School Rd. NE, Suite 200 Albuquerque, New Mexico 87110
Copies of this report can be purchased from:

U.S. Geological Survey Books and Open-File Reports Federal Center Box 25425

Denver, Colorado 80225 


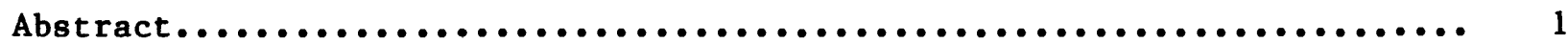

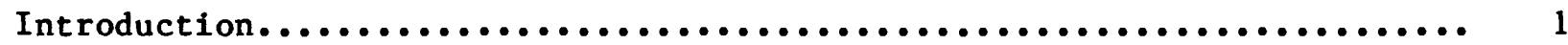

Approach ...............................................

Format of the report.................................... 1

Spring-numbering systems...............................

Standard spring-numbering system........................ 3

Navajo Reservation spring-numbering system................ 4

References ..............................................

\section{PIGURES}

Figure $\quad 1$. Location of counties in New Mexico.................

2. Diagram showing system of numbering springs on lands other than the Navajo Reservation................

3. Diagram showing system of numbering springs on the Navajo Reservation..........................

4-25. Maps showing location of inventoried springs in:

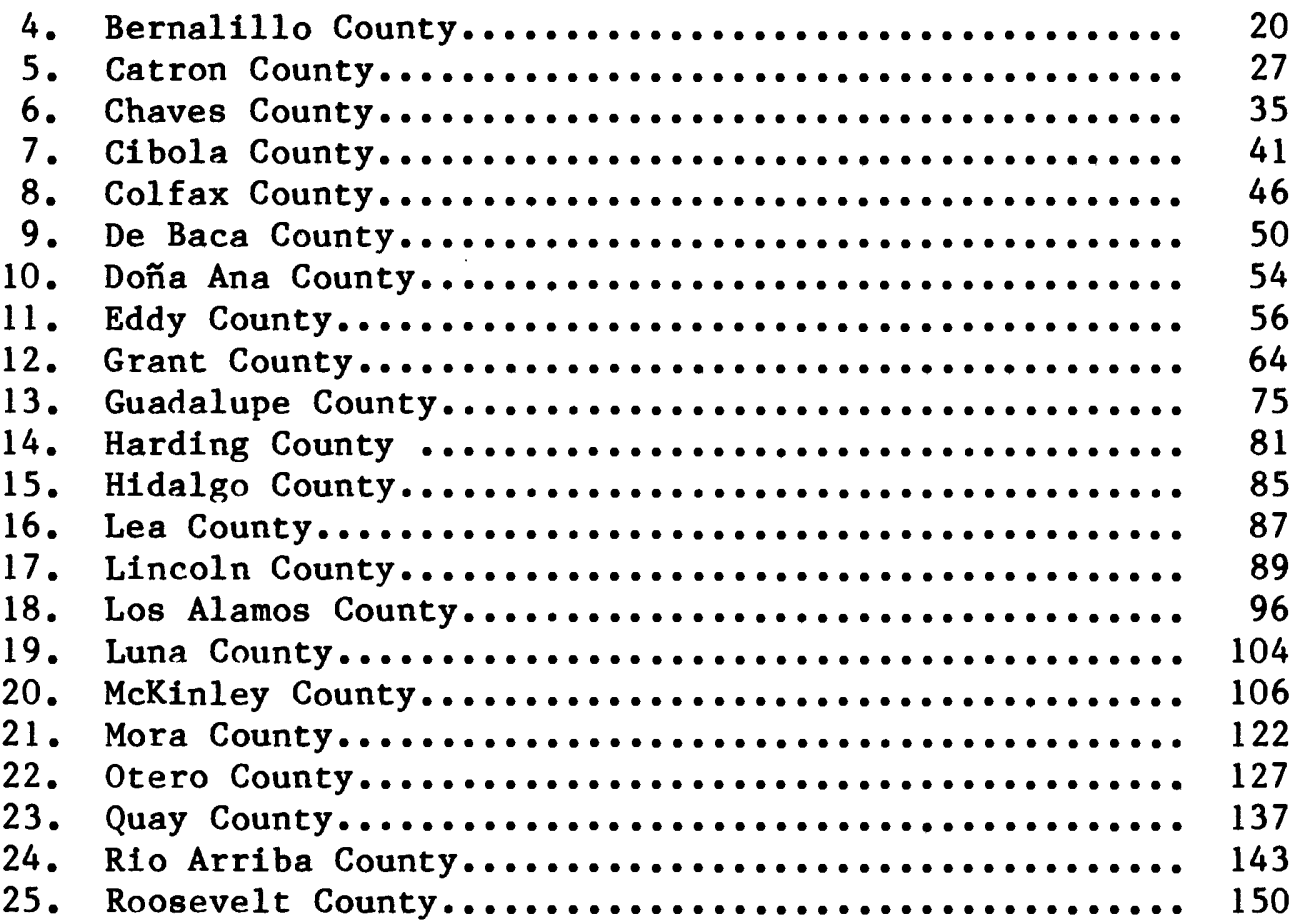




\section{FIGURES-Concluded}

Figures 26-35. Maps showing location of inventoried springs in:

26. Sandoval County .......................... 152

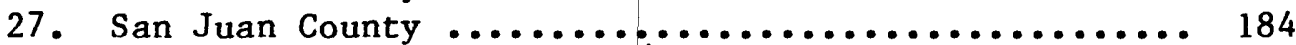

28. San Miguel County .......................... 205

29. Santa Fe County ............................. 209

30. Sierra County ............................. 220

31. Socorro County ............................ 223

32. Taos County ............................. 236

33. Torrance County ............................ 239

34. Union County ............................. 243

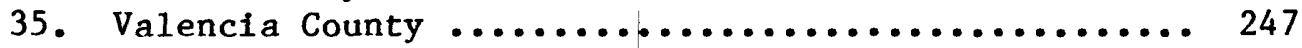

\section{TABLES}

Table 1. Geologic unit explanation table.................... 15

2-22. Physical characteristics of springs in:

2. Bernalillo County.......................... 21

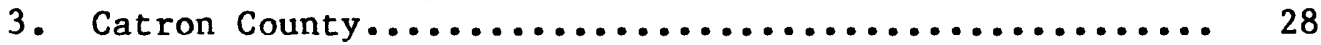

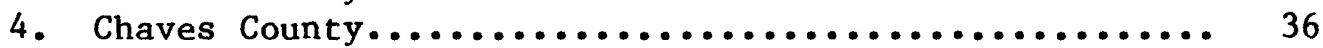

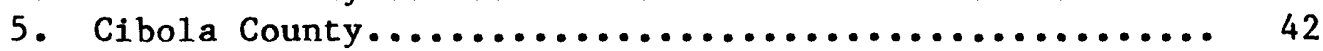

6. Colfax County............................. 47

7. De Baca County............................. 51

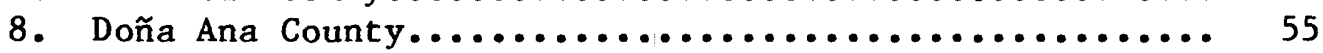

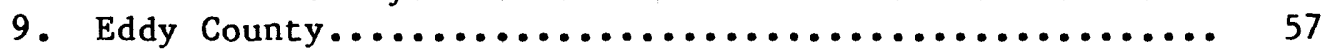

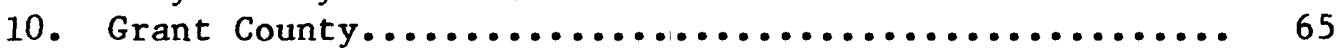

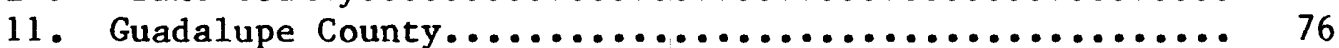

12. Harding County.............................. 82

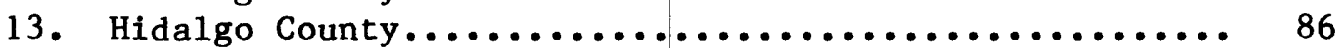

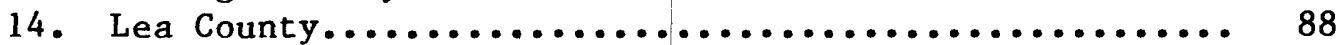

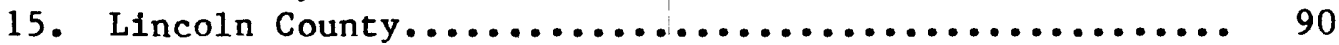

16. Los Alamos County............................ 97

17. Luna County................................. 105

18. McKinley County............................ 107

19. Mora County................................ 123

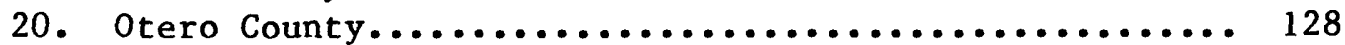

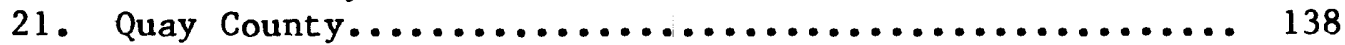

22. Rio Arriba County.............................. 144

23. Roosevelt County............................ 151

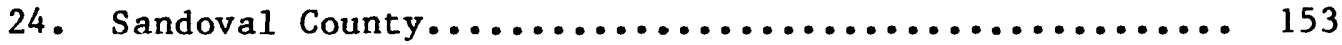

25. San Juan County............................ 185

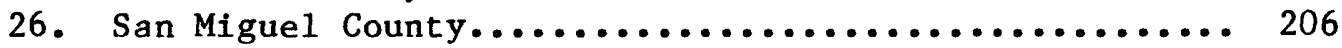

27. Santa Fe County............................. 210

28. Sierra County............................. 221

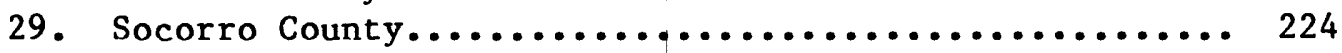

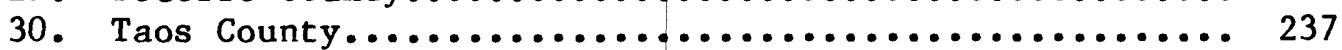

31. Torrence County............................. 240

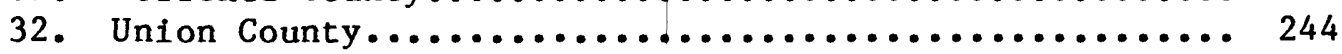

33. Valencia County............................. 248 


\section{CONVERSION FACTORS AND VERTICAL DATUM}

\section{Multiply}

inch

foot

mile

acre

gallon

gallon per minute

cubic foot per second
By

$$
\begin{gathered}
25.40 \\
0.3048 \\
1.609 \\
4.047 \\
3.785 \\
0.06309 \\
28.32
\end{gathered}
$$

To obtain

millimeter

meter

kilometer

square meter

liter

liter per second

liter per second

Degrees Celsius $\left({ }^{\circ} \mathrm{C}\right)$ can be converted to degrees Fahrenheit $\left({ }^{\circ} \mathrm{F}\right)$ by the following equation:

$$
{ }^{\circ} \mathrm{F}=9 / 5\left({ }^{\circ} \mathrm{C}\right)+32
$$

Sea level: In this report sea level refers to the National Geodetic Vertical Datum of 1929--a geodetic datum derived from a general adjustment of the first-order level nets of the United States and Canada, formerly called Sea Level Datum of 1929. 


\title{
INVENTORY OF SPRINGS IN THE STATE OF NEW MEXICO
}

\author{
By W.E. White and G.E. Kues
}

\begin{abstract}
Data on 1,425 springs, collected as of 1978, were compiled and tabulated. The purpose of the tabulation was to condense information published in a variety of references and residing in U.S. Geological Survey computer files into a single source.

The data include spring location and name, owner's name, topographic situation, geologic source, altitude, and yield of selected springs. Also included are spring water temperature, specific conductance, and spring wateruse information. Information is provided for all counties in the State except Curry County, for which no information on springs was found.
\end{abstract}

\section{INTRODUCTION}

This report is a compilation and tabulation of data up to and including 1978 on springs of New Mexico contained in published reports and in U.S. Geological Survey computer files. The purpose of this report, prepared in cooperation with the New Mexico State Engineer office, is to present information on the occurrence and quality of spring water in a single source.

The spring sites described in this report have not been field checked. This report demonstrates the need for the collection of quantitative data in areas where spring sites have not been intensively surveyed.

\section{Approach}

Sources of information for this report include many of the published geologic and hydrologic studies of the New Mexico Bureau of Mines and Mineral Resources, New Mexico State Engineer Office, and U.S. Geological Survey. The location and topographic situation of each reported spring were verified, when

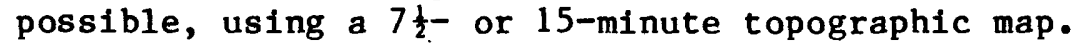

\section{Format of the Report}

The data are grouped by county (fig. 1). County tables and maps are arranged in alphabetical order by county name. Available physical characteristics of springs in all but Curry County have been reported. Explanations for tables in the report precede the county sections. 


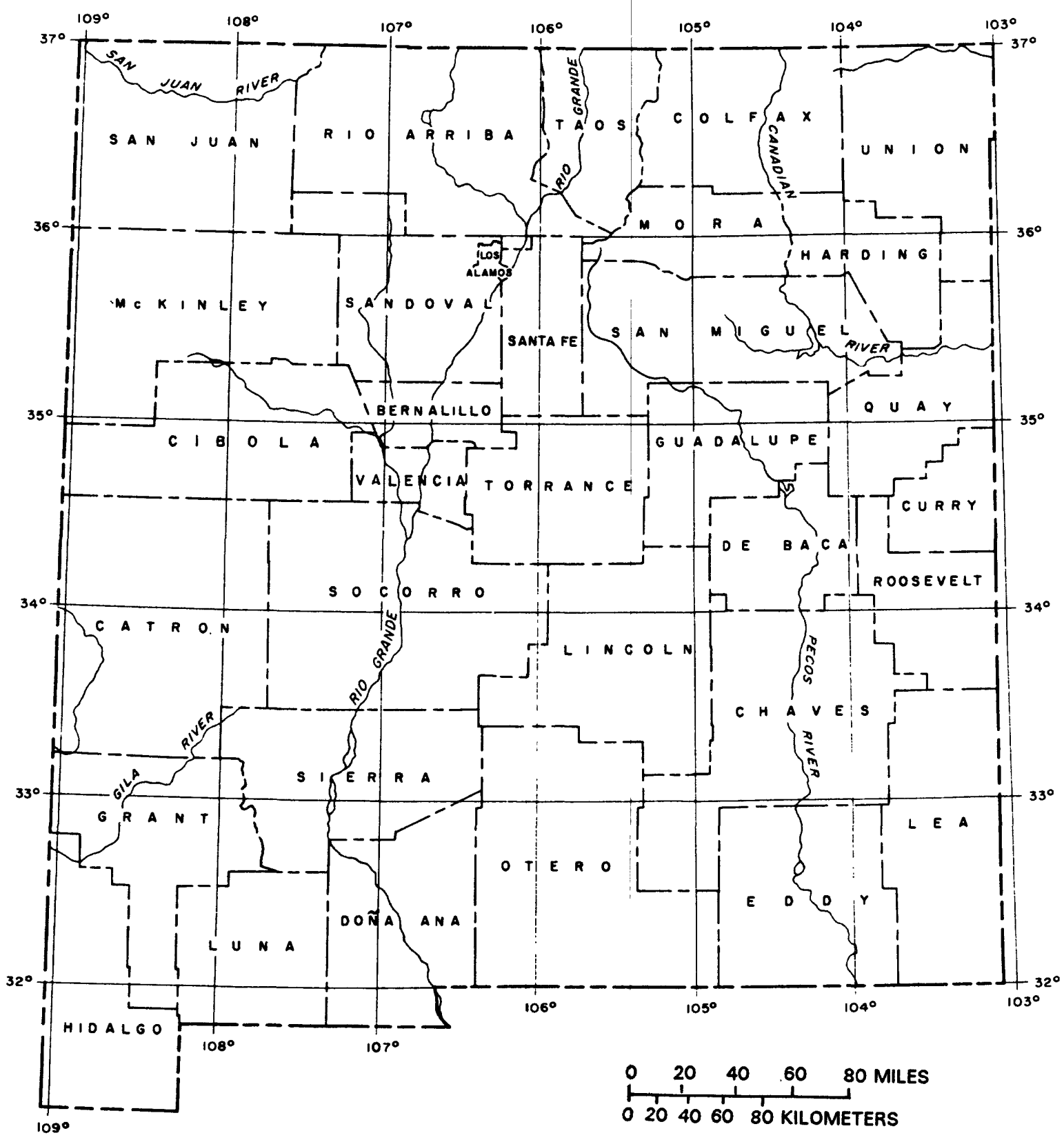

Figure 1.--Location of counties in New Mexico. 


\section{Spring-Numbering Systems}

\section{Standard Spring-Numbering System}

The standard system of numbering springs in New Mexico is based on the common subdivision of public lands into sections. The spring number, in addition to designating the spring, locates its position to the nearest 10acre tract in the land network. This number is divided into four segments. The first segment denotes the township north or south of the New Mexico base line; the second denotes the range east or west of the New Mexico principal meridian, and the third denotes the section. The fourth segment of the number, which consists of three digits, denotes the 160-, 40-, and 10-acre tracts, respectively, in which the spring is situated. For this purpose, the section is divided into four quarters, numbered $1,2,3$, and 4 , in the normal reading order, for the northwest, northeast, southwest, and southeast quarters, respectively. The first digit of the fourth segment gives the quarter section, which is a tract of 160 acres. Similarly, the quarter section is divided into four 40-acre tracts numbered in the same manner, and the second digit denotes the 40-acre tract. Finally, the 40-acre tract is divided into four 10-acre tracts, and the third digit denotes the 10-acre

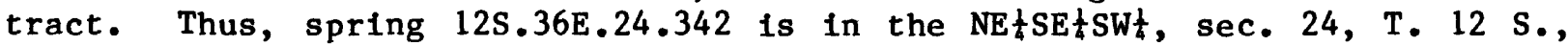
R. $36 \mathrm{E}$. If a spring is not located accurately within a 10-acre tract, a zero is used as the third digit, and if it is not located within a 40-acre tract, zeros are used for both the second and third digits.

The letters a, b, $c$, etc. that sometimes appear at the end of a location number indicate the first, second, third, and succeeding springs located within the same 10-acre tract. An example of this method of site location is shown in figure 2 .

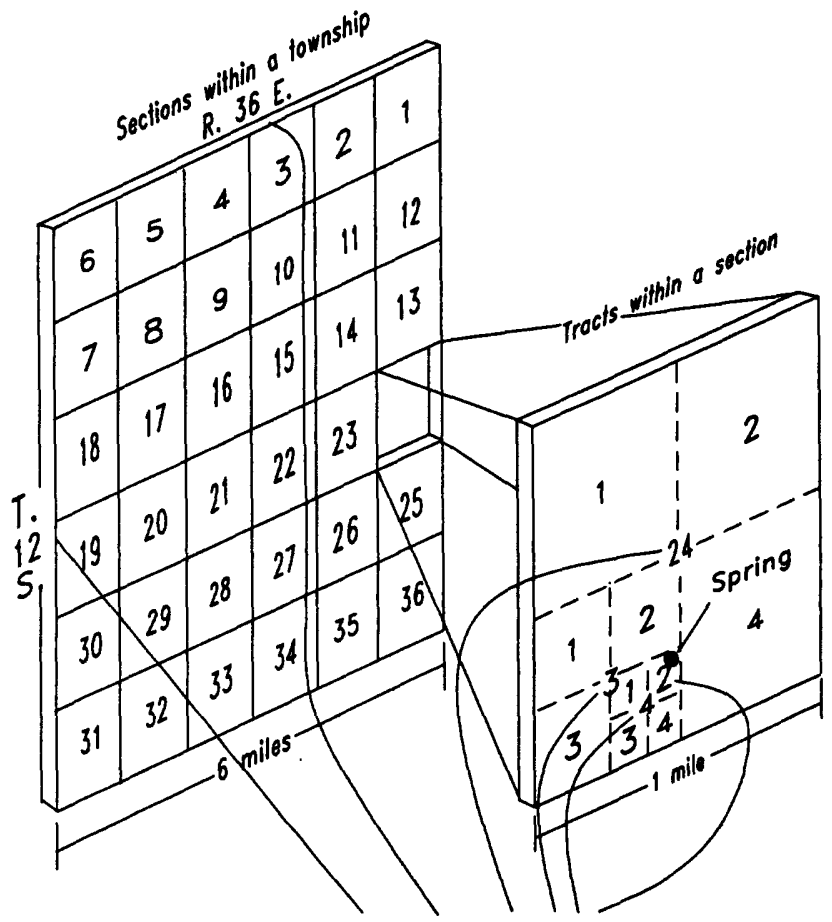

Spring 12S.36E.24.342

Figure 2.--System of numbering springs on lands other than the Navajo Reservation. 
Spanish land grants, Indian reservations, and national forests have not been divided into townships and sections. When such lands share boundaries with land that has been sectionalized, irregular-shaped townships, sections, and tracts result. If a township, section, or tract is not square, the boundaries of the township were informally extended, and the area was treated as if it were square for the purpose of locating a spring.

In the tables, springs located on lands that have not been divided into sections and do not have chemical analyses available will not have a detailed location number or latitude and longitude reported. To locate these springs, refer to the spring-location maps at the beginning of each county section. All springs having a chemical analysis will have a latitude and longitude reported and may have an extended location number reported in the remarks column.

\section{Navajo Reservation Spring-Numbering System}

Public land surveys have not been made for the Navajo Reservation. Springs located on the reservation have a local identifier based on a system of letters and numbers that begins with the letters NR followed by three segments. The first segment is a three-digit map number. The number is assigned to one of a series of U.S. Geological Survey 15-minute quadrangle maps or a group of four contiguous $7 \frac{1}{2}$-minute quadrangle maps (fig. 3 ). The second segment, which is separated from the first by a decimal point, consists of two numbers separated by an " $x$ ". The other numbers are the spring's coordinates in hundredths of a mile from the east and north boundaries of the map area, respectively. For example, spring number 049.1181 x 1074 is located on map-area number $49,11.81$ miles west of the area's east boundary and 10.74 miles south of the area's north boundary (fig. 3 ). 


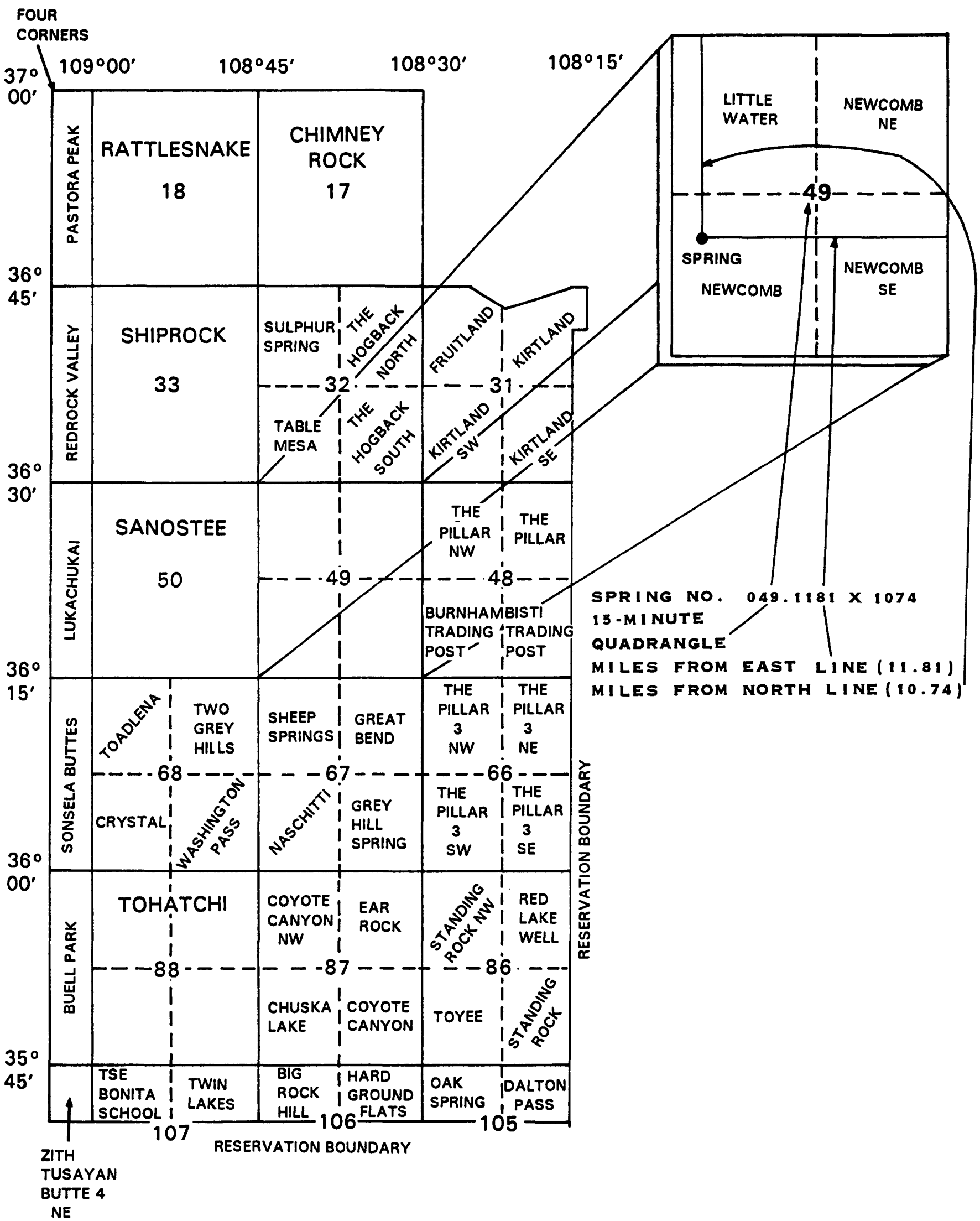

Figure 3.--System of numbering springs on the Navajo Reservation. 


\section{REFERENCES}

Akers, J.P., Shorty, J.C., and Stevens, P.R., 1971, Hydrogeology of the Cenozoic igneous rocks, Navajo and Hopi Indian Reservations, Arizona, New Mexico, and Utah: U.S. Geological Survey Professional Paper 521-D, 18 p.

Baltz, E.H., and West, S.W., 1967, Ground-water resources of the southern part of Jicarilla Apache Indian Reservation and adjacent areas, New Mexico: U.S. Geologica1 Survey Water-Supp1y Paper 1576-H, 86 p.

Berkstresser, C.F., Jr., and Mourant, W.A., 1966, Ground-water resources and geology of Quay County, New Mexico: Socorro, New Mexico Bureau of Mines and Mineral Resources Ground-Water Report 9, 115 p.

Bjorklund, L.J., and Maxwe11, B.W., 1961, Availability of ground water in the Albuquerque area, Bernalillo and Sandoval Counties, New Mexico: New Mexico State Engineer Technical Report 21, 117 p., 13 figs.

Blodgett, D.D., and Titus, F.B., 1973, Hydrogeology of the San Augustin Plains, New Mexico: Socorro, New Mexico Bureau of Mines and Mineral Resources Open-File Report 51, 54 p.

Borton, R.L., 1974, General geology and ground-water conditions in the Truchas-Espańola-Velarde area of Rio Arriba County, New Mexico, in Guidebook to Ghost Ranch, central-northern New Mexico: New Mexico Geological Society, 25th Field Conference, p. 351-354.

compiler, 1978, Bibliography of ground-water studies in New Mexico, 1873-1977: New Mexico State Engineer Special Pub1ication, 121 p.

Brown, D.R., and Stone, W.J., 1978, Hydrogeology of the Aztec quadrangle (San Juan County, New Mexico): Socorro, New Mexico Bureau of Mines and Minera1 Resources Hydrologic Sheet 1.

Bushman, F.X., 1955, Ground-water data for Dwyer quadrangle, Grant and Luna Counties, New Mexico: Socorro, New Mexico Bureau of Mines and Mineral Resources Circular 37, 18 p.

Bushman, F.X., and Valentine, C.P., 1954, Water we11 records and we11 water quality in southwestern San Agustin Plains, Catron County, New Mexico: Socorro, New Mexico Bureau of Mines and Mineral Resources Circular 26, $19 \mathrm{p}$.

California State Water Quality Control Board, 1963, Water quality criteria, 2d ed.: California State Water Quality Contro1 Board Publication 3, 548 p., 4 figs.

Clark, F.W., 1893, Miscellaneous analyses--Report on work done in the division of chemistry during the fiscal years 1881-92 and 1892-93: U.S. Geological Survey Bulletin 113, p. 114 . 


\section{REFERENCES-Continued}

Conover, C.S., 1953, Letter to John H. Bliss, State Engineer of New Mexico, describing ground-water conditions in the upper Black River valley, New Mexico: U.S. Geological Survey open-file report, 5 p.

Conover, C.S., Theis, C.V., and Griggs, R.L., 1963, Geology and hydrology of Valle Grande and Valle Toledo, Sandoval County, New Mexico: U.S. Geological Survey Water-Supply Paper 1619-Y, 37 p.

Cooper, J.B., 1958, Ground water in the vicinity of Carrizozo, Lincoln County, New Mexico: U.S. Geological Survey open-file report, 45 p.

Cooper, J.B., and Davis, L.V., 1967, General occurrence and quality of ground water in Union County, New Mexico: Socorro, New Mexico Bureau of Mines and Mineral Resources Ground-Water Report 8, 168 p.

Cooper, J.B., and John, E.C., 1968, Geology and ground-water occurrence in southeastern McKinley County, New Mexico: New Mexico State Engineer Technica1 Report 35, 118 p.

Davis, L.V., and Busch, F.E., 1965, Summary of hydrologic investigations by the United States Geological Survey at White Sands Missile Range, New Mexico: U.S. Geological Survey open-file report, 146 p.

Davis, G.E., Hardt, W.F., Thompson, L.K., and Cooley, M.E., 1963, Geohydrologic data in the Navajo and Hopi Indian Reservations, Arizona, New Mexico, and Utah--Part I, Records of ground-water supplies: Arizona State Land Department Water Resources Report 12-A, 59 p.

Davis, P., Wilcox, R.W., and Gross, G.W., 1980, Spring characteristics of the western Roswell artesian basin: Las Cruces, New Mexico Water Resources Research Institute Report 116, 93 p.

Dinwiddie, G.A., 1963, Municipal water supply and uses, southeastern New Mexico: New Mexico State Engineer Technical Report 29A, 140 p.

1964, Municipal water supplies and uses, northeastern New Mexico: New Mexico State Engineer Technical Report 29B, 64 p.

Dinwiddie, G.A., and Clebsch, Alfred, Jr., 1973, Water resources of Guadalupe County, New Mexico: Socorro, New Mexico Bureau of Mines and Mineral Resources Hydrologic Report 3, 43 p.

Dinwiddie, G.A., Mourant, W.A., and Basler, J.A., 1966a, Municipal water supplies and uses, northwestern New Mexico: New Mexico State Engineer Technical Report 29C, 197 p.

1966b, Municipal water supplies and uses, southeastern New Mexico: New Mexico State Engineer Technical Report 29D, 98 p. 


\section{REFERENCES-Continued}

Doty, G.C., 1960, Reconnaissance of ground water in Playas Valley, Hidalgo County, New Mexico: New Mexico State Engineer Technical Report 15, 40 p.

1967, Potential sources of water supply for Cañoncito Navajo Day School, Bernalillo County, New Mexico: U.S. Geological Survey open-file report, 29 p.

Erickson, J.R., 1955, Ground-water conditions in the vicinity of Rattlesnake Springs, Eddy County, New Mexico: New Mexico State Engineer Technical Report 3, 54 p.

Fisher, C.A., 1906, Geology and underground waters of the Roswell artesian area, New Mexico: U.S. Geological Survey Water-Supply Paper 158, 29 p.

Garza, Sergio, and McLean, J.S., 1977, Fresh-water resources in the southeastern part of the Tularosa basin: New Mexico State Engineer Technical Report 40,67 p.

Gordon, E.D., 1961, Geology and ground-water resources of the Grants-Bluewater area, Valencia County, New Mexico: New Mexico State Engineer Technical Report 20, 109 p.

Griggs, R.L., 1948, Geology and ground-water resources of the eastern part of Colfax County, New Mexico: Socorro, New Mexico Bureau of Mines and Mineral Resources Ground-Water Report 1, 180 p.

1964, Geology and ground-water resources of the Los Alamos area, New Mexico: U.S. Geological Survey Water-Supply Paper 1753, 105 p.

Griggs, R.L., and Hendrickson, G.E., 1951, Geology and ground-water resources of San Miguel County, New Mexico: Socorro, New Mexico Bureau of Mines and Mineral Resources Ground-Water Report 2, 121 p.

Gross, W.G., Davis, P., and Rehfeldt, K.R., 1980, Paul Spring--An investigation of recharge in the Roswell (NM) artesian basin: Las Cruces, New Mexico Water Resources Research Institute, $135 \mathrm{p}$.

Hale, W.E., 1945, Ground-water conditions in the vicinity of Carlsbad, New Mexico: New Mexico State Engineer 16th and 17th Biennial Report, p. 195-260.

Hall, F.R., 1963, Springs in the vicinity of Socorro, New Mexico, in Guidebook to Socorro Region: Socorro, New Mexicol Geological Society, 14th Field Conference, p. 160-179.

Halpenny, L.C., and Whitcomb, H.A., 1949, Water-supply investigation at Thoreau, McKinley County, New Mexico: U.S. Geological Survey open-file report, $16 \mathrm{p}$. 


\section{REFERENCES-Continued}

Hart, D.L., Jr., and Smith, Christian, 1979, Ground water in the vicinity of Capulin, Colfax, and Union Counties, New Mexico: U.S. Geological Survey Water-Resources Investigations 79-79, $58 \mathrm{p}$.

Hem, J.D., 1959, Study and interpretation of the chemical characteristics of natural water: U.S. Geological Survey Water-Supp1y Paper 1473, lst ed., 269 p.

Hendrickson, G.E., and Jones, R.S., 1952, Geology and ground-water resources of Eddy County, New Mexico: Socorro, New Mexico Bureau of Mines and Mineral Resources Ground-Water Report 3, 169 p.

Holmes, C.R., 1963, Tritium studies, Socorro Spring, in Guidebook to Socorro Region: New Mexico Geological Society, 14th Field Conference, p. 152-154.

Hood, J.W., 1958, Ground-water resources and related geology in the vicinity of Holloman Air Force Base, Otero County, New Mexico: U.S. Geologica1 Survey open-file report, $261 \mathrm{p}$.

$1960 \mathrm{a}$, Ground water in the vicinity of the Atlas site, Holloman Air Force Base, Otero County, New Mexico: U.S. Geological Survey open-file report, $38 \mathrm{p}$.

$1960 \mathrm{~b}$, Availability of ground water in the vicinity of Cloudcroft, Otero County, New Mexico: U.S. Geological Survey open-file report, 27 p.

Hood, J.W., and Kister, L.R., 1962, Saline-water resources of New Mexico: U.S. Geological Survey Water-Supply Paper 1601, 70 p.

Hudson, J.D., 1978, A compilation of hydrologic data before and during highway contruction in parts of Tijeras Canyon, New Mexico, 1972-1977: U.S. Geological Survey open-file report, $82 \mathrm{p}$.

Iorns, W.V., Hembree, C.H., Phoenix, D.A., and Oakland, G.L., 1964, Water resources of the upper Colorado River basin - basic data: U.S. Geological Survey Professional Paper 442, p. 742-754.

John, E.C., Enyart, Eugene, and Purtyman, W.D., 1967, Records of we11s, test holes, springs, and surface-water stations in the Los Alamos area, New Mexico: U.S. Geological Survey open-file report, 129 p.

Kelly, Clyde, and Anspach, E.V., 1913, A preliminary study of waters of the Jemez Plateau, New Mexico: Albuquerque, University of New Mexico, Chemical Series Bulletin, v. 1, no. 1, 72 p.

Kister, L.R., and Hatchett, J.L., 1963, Geohydrologic data in the Navajo and Hopi Indian Reservations, Arizona, New Mexico, and Utah--Part II, Selected chemical analyses of the ground water: Arizona State Land Department Water Resources Report 12-B, 58 p. 


\section{REFERENCES--Continued}

Lovering, T.G., 1956, Contributions to the geology of uranium: U.S. Geological Survey Bulletin 1009-L, p. 315-390.

McLean, J.S., 1970, Saline ground-water resources of the Tularosa basin, New Mexico: U.S. Geological Survey Research and Development Progress Report 561, 128 p.

1977, Hydrologic maps and data in the Mimbres basin, New Mexico: U.S. Geological Survey Open-File Report 77-314, 531 p.

Meinzer, O.E., 1927, Large springs in the United States: U.S. Geological Survey Water-Supp1y Paper 557, p. 89-91.

Mercer, J.W., 1967, Inventory of wells and springs within a 10-mile radius of Project Gasbuggy, Rio Arriba County, New Mexico: U.S. Geological Survey Technical Letter, Gasbuggy-3, $12 \mathrm{p}$.

Mercer, J.W., and Lappala, E.G., 1972, Ground-water resources of the Mora River drainage basin, western Mora County, New Mexico: New Mexico State Engineer Technical Report 37, 91 p.

Mourant, W.A., 1957, Reconnaissance of ground-water resources in the upper part of Sacramento River Canyon, Otero County, New Mexico: U.S. Geological Survey open-file report, 22 p.

1963, Water resources and geology of the Rio Hondo drainage basin, Chaves, Lincoln, and Otero Counties, New Mexico: New Mexico State Engineer Technical Report 28, 85 p.

Mourant, W.A., and Shomaker, J.W., 1970, Reconnaissance of water resources of De Baca County, New Mexico: Socorro, New Mexico Bureau of Mines and Mineral Resources Ground-Water Report 10, 87 p.

Murray, C.E., 1942, Report on the investigations of water resources east of Albuquerque, New Mexico: U.S. Geological Survey open-file report, 5 p.

1959, Ground-water conditions in the nonthermal artesian-water basin south of Hot Springs, Sierra County, New Mexico: New Mexico State Engineer Technical Report 10, $33 \mathrm{p}$.

Peale, A.C., 1886, New Mexico, in List and analysis of the mineral springs of the United States (a preliminary study): U.S. Geological Survey Bulletin 32 , p. 193-195.

Purtyman, W.A., 1966, Geology and hydrology of White Rock Canyon from Otowi to the confluence of Frijoles Canyon, Los Alamos and Santa Fe Counties, New Mexico: U.S. Geological Survey open-file report, 27 p. 


\section{REFERENCES-Continued}

Purtyman, W.D., Adams, W.H., and Stoker, A.K., 1978, Water quality in vicinity of Fenton Hill site, 1976: Los Alamos Scientific Laboratory Informal Report LA-7307-MS, $17 \mathrm{p}$.

Purtyman, W.D., Adams, W.H., Stoker, A.K., and West, F.G., 1976, Water quality in vicinity of Fenton Hill site, 1975: Los Alamos Scientific Laboratory Informa1 Report LA-6511-MS, $21 \mathrm{p}$.

Purtyman, W.D., Stoker, A.K., Adams, W.H., and Owens, J.W., 1978, Water quality in vicinity of Fenton Hill site, 1977: Los Alamos Scientific Laboratory Progress Report LA-7468-PR, 16 p.

Purtyman, W.D., West, F.G., and Adams, W.H., 1974, Preliminary study of the quality of water in the drainage area of the Jemez River and Rio Guadalupe: Los Alamos Scientific Laboratory Informal Report LA-5595-MS, 26 p.

Reagan, A.B., 1903, Geology of the Jemez-Albuquerque region, New Mexico: The American Geologist, v. 31, no. 2, p. 98-103.

Renick, B.C., 1926, Geology and ground-water resources of the drainage basin of the Rio Peñasco above Hope, New Mexico: New Mexico State Engineer Seventh Biennial Report, p. 109-138.

1931, Geology and ground-water resources of western Sandoval County, New Mexico: U.S. Geological Survey Water-Supply Paper 620, 117 p.

Scott, R.C., and Barker, F.B., 1962, Data on uranium and radium in ground water in the United States, 1954 to 1957: U.S. Geological Survey Professional Paper 426, p. 77-79.

Shomaker, J.W., 1968, Availability of ground water in and near the mouth of Dulce Canyon, Rio Arriba County, New Mexico: Unpublished data on file in Albuquerque office of the U.S. Geological Survey.

1971, Water resources of Fort Wingate Army Depot and adjacent areas, McKinley County, New Mexico: U.S. Geological Survey open-file report, 228 p.

1976, Summary of well and spring records near Star Lake mine area (McKinley County, New Mexico): Consultant's report to Genge Environmental Consultants, $14 \mathrm{p}$.

smith, R.E., 1957, Geology and ground-water resources of Torrance County, New Mexico: Socorro, New Mexico Bureau of Mines and Mineral Resources GroundWater Report 5, 186 p.

Spiegel, Zane, 1955, Geology and ground-water resources of northeastern Socorro County, New Mexico: Socorro, New Mexico Bureau of Mines and Mineral Resources Ground-Water Report 4, 99 p. 


\section{REFERENCES-Continued}

Spiegel, Zane, and Baldwin, Brewster, 1963, Geology and water resources of the Santa Fe area, New Mexico: U.S. Geological Survey Water-Supply Paper 1525, 258 p.

Stearns, N.D., Stearns, H.T., and Waring, G.A., 1937, Thermal springs in the United States: U.S. Geological Survey Water-Supply Paper 679-B, p. $87,107,166-169$.

Summers, W.K., 1965, Chemical characteristics of New Mexico's thermal waters-A critique: Socorro, New Mexico Bureau of Mines and Mineral Resources Circular 83, $27 \mathrm{p}$.

1969, Ground-water resources of the San Cristoval Grant, Santa Fe County, New Mexico: New Mexico Technical Research Foundation Report, 50 p.

1976, Catalog of thermal waters in New Mexico: Socorro, New Mexico Bureau of Mines and Mineral Resources Hydrologic Report 4, 80 p.

Summers, W.K., Schwab, G.E., and Brandvold, L.A., 1972, Ground-water characteristics in a recharge area, Magdalena Mountains, Socorro County, New Mexico: Socorro, New Mexico Bureau of Mines and Mineral Resources Circular 124,18 p.

Theis, C.V., Taylor, G.C., Jr., and Murray, C.R., 1942, Thermal waters of the Hot Springs artesian basin, Sierra County, New Mexico: New Mexico State Engineer 14th and 15th Biennia1 Report, 1938-42, p. 419-492.

Titus, F.B., 1963, Geology and ground-water conditions in eastern Valencia County, New Mexico: Socorro, New Mexico Bureau of Mines and Minera1 Resources Ground-Water Report 7, 113 p.

Trainer, F.W., 1974, Ground water in the southwestern part of the Jemez Mountains volcanic region, New Mexico, in Guidebook to Ghost Ranch, central-northern New Mexico: New Mexico Geological Society, 25th Field Conference, p. 337-345.

1978, Geohydrologic data from the Jemez Mountains and vicinity, northcentral New Mexico: U.S. Geological Survey Water-Resources Investigations 77-131, $147 \mathrm{p}$.

Trauger, F.D., 1953, Memorandum of ground water for public supply at the Cañoncito Reservation Day School, Bernali11o County, New Mexico: U.S. Geological Survey open-file report, 6 p.

1963, Geology and availability of ground water in the vicinity of Gila Cliff Dwellings National Monument, Catron County, New Mexico: U.S. Geologica1 Survey open-file report, $24 \mathrm{p}$. 


\section{REFERENCES-Continued}

Trauger, F.D., 1972, Water resources and general geology of Grant County, New Mexico: Socorro, New Mexico Bureau of Mines and Mineral Resources Hydrologic Report 2, 211 p.

Trauger, F.D., and Bushman, F.X., 1964, Geology and ground water in the vicinity of Tucumcari, Quay County, New Mexico: New Mexico State Engineer Technical Report 30,178 p.

U.S. Environmental Protection Agency, 1975, National interim primary drinking water regulations: Federal Register, v. 40, no. 248, Wednesday, December 24, 1975, pt. IV, p. 59566-59587.

1976, Interim primary drinking water regulations - promulgation of regulations on radionuclides: Federal Register, v. 41, no. 133, Friday, July 9, 1976, pt. III, p. 28402-29409.

1977, National secondary drinking water regulations: Federal Register, v. 42, no. 62, Thursday, March 31, 1977, pt. I, p. 17143-17147.

U.S. Geological Survey, 1965-70, Water resources data for New Mexico, 1965-69, Part 1--Surface-water records: U.S. Geological Survey Water-Data Reports NM-65-1 to NM-69-1 (published annually).

U.S. National Resources Planning Board, 1942a, Regional planning--Part X, The Pecos River Joint Investigation in the Pecos River basin in New Mexico and Texas--Summary, analyses, findings: Washington, D.C., U.S. Government Printing Office, 407 p.

1942b, The Pecos River Joint Investigation--Atlas to accompany the reports of the Pecos River Joint Investigations: Washington, D.C., U.S. Government Printing Office, $34 \mathrm{p}$.

Waring, G.A., 1965, Thermal springs of the United States and other countries of the world--A summary: U.S. Geological Survey Professional Paper 492, p. $37-38,270$.

Weir, J.E., Jr., 1965, Geology and availability of ground water in the northern part of the White Sands Missile Range and vicinity of New Mexico: U.S. Geological Survey Water-Supply Paper 1801, 78 p.

West, S.W., 1959, Availability of ground water in the Gallup area, New Mexico: U.S. Geological Survey open-file report, $146 \mathrm{p}$.

Whitcomb, H.A., Halpenny, L.C., and Harshbarger, J.W., 1951, An investigation of the water supply and ground-water resources at Zuni Day School, Zuni Indian Reservation, McKinley County, New Mexico: U.S. Geological Survey open-file report, $14 \mathrm{p}$. 


\section{REFERENCES-Concluded}

White, D.E., Hem, J.D., and Waring, G.A., 1963, Data of geochemistry, in Chemical composition of subsurface waters: U.S. Geological Survey Professional Paper 440-F, p. 13, 14, 22, 24, 50-51, 56-57.

Winograd, I.J., 1959, Ground-water conditions and geology of Sunshine Valley and western Taos County, New Mexico: New Mexico State Engineer Technical Report 12, $70 \mathrm{p}$. 
Table 1.-Geologic unit explanation table

\begin{tabular}{|c|c|c|c|c|}
\hline \multirow[b]{2}{*}{ Erathem } & \multirow[b]{2}{*}{ System } & \multirow{2}{*}{$\begin{array}{l}\text { Time before } \\
\text { present (in } \\
\text { millions of years) }\end{array}$} & \multicolumn{2}{|r|}{ Geologic unit } \\
\hline & & & $\begin{array}{l}\text { Abbre- } \\
\text { viation }\end{array}$ & Stratigraphic description \\
\hline \multirow[t]{30}{*}{ Cenozoic } & Quaternary & Holocene to 2 & Qab & $\begin{array}{l}\text { Alluvium, bolson, and other } \\
\text { superficial deposits }\end{array}$ \\
\hline & & & $\begin{array}{l}\text { Qal } \\
Q b\end{array}$ & $\begin{array}{l}\text { Alluvium, mainly in flood plains } \\
\text { Basalt flows }\end{array}$ \\
\hline & & & Qbc & Bolson fill \\
\hline & & & Qbu & Extrusive rocks, unconsolidated \\
\hline & & & $Q_{c}$ & Colluvial deposits \\
\hline & & & Qcg & Terrace gravels \\
\hline & & & Qcl & Conglomerate \\
\hline & & & Q1 & Landslide debris \\
\hline & & & Qr & Bandelier Tuff \\
\hline & & & $Q t$ & Terrace deposits \\
\hline & & & Qtsv & $\begin{array}{l}\text { Basalt flows associated with } \\
\text { the Santa Fe Group }\end{array}$ \\
\hline & & & Qv & $\begin{array}{l}\text { Products of diatremic } \\
\text { explosions and other volcanic } \\
\text { rocks }\end{array}$ \\
\hline & Quaternary & 2 to 63 & QTa & Ancha Formation \\
\hline & and Tertiary & & QTb & Basalt and basalt flows \\
\hline & & & QTg & Gila Conglomerate \\
\hline & & & QTp & $\begin{array}{l}\text { Pediment, terrace, and other } \\
\text { deposits }\end{array}$ \\
\hline & & & QTs & Santa Fe Group, undivided \\
\hline & & & QTsf & Santa Fe Group, upper part \\
\hline & & & QTt, QTu & Terrace deposits \\
\hline & Tertiary & & $\mathrm{Tb}$ & $\begin{array}{l}\text { Andesite and basalt flows, } \\
\text { breccias, and tuffs }\end{array}$ \\
\hline & & & Tc & Chuska Sandstone \\
\hline & & & $\mathrm{Td}$ & Datil Formation \\
\hline & & & $\mathrm{Te}$ & $\begin{array}{l}\text { Basalt, andesites, and } \\
\text { extrusives }\end{array}$ \\
\hline & & & $\mathrm{Tg}$ & Galisteo Formation \\
\hline & & & $\mathrm{Ti}$ & Dikes, sills, and plugs \\
\hline & & & Tn & Nacimiento Formation \\
\hline & & & To & Ogallala Formation \\
\hline & & & $\mathrm{Tp}$ & Popotosa Formation \\
\hline & & & $\operatorname{Trp}$ & Rubio Peak Formation \\
\hline & & & Tsf & Santa Fe Group \\
\hline
\end{tabular}


Table 1.-Geologic unit explanation table-Continued

\begin{tabular}{|c|c|c|c|c|}
\hline \multirow[b]{2}{*}{ Erathem } & \multirow[b]{2}{*}{ System } & \multirow{2}{*}{$\begin{array}{c}\text { Time before } \\
\text { present (in } \\
\text { miliions of years) }\end{array}$} & \multicolumn{2}{|r|}{ Geologic unit } \\
\hline & & & $\begin{array}{l}\text { Abbre- } \\
\text { viation }\end{array}$ & Stratigraphic description \\
\hline Cenozoic & Tertiary & 2 to 63 & $\begin{array}{l}\text { Tsj, Tsje } \\
\text { Tsr } \\
\text { Tv } \\
\text { Toa }\end{array}$ & $\begin{array}{l}\text { San Jose Formation } \\
\text { Regina Member of San Jose } \\
\text { Formation } \\
\text { Extrusive rocks of varied } \\
\text { composition and age } \\
\text { Ojo Alamo Sandstone }\end{array}$ \\
\hline $\begin{array}{l}\text { Cenozoic and } \\
\text { Mesozoic }\end{array}$ & $\begin{array}{l}\text { Tertiary and } \\
\text { Cretaceous }\end{array}$ & & $\begin{array}{l}\text { TKi } \\
\text { IKr }\end{array}$ & $\begin{array}{l}\text { Dikes, sills, and plugs } \\
\text { Raton Formation }\end{array}$ \\
\hline Mesozoic & Cretaceous & 63 to 138 & $\begin{array}{l}\mathrm{Kd} \\
\mathrm{Kdp} \\
\mathrm{Kg} \\
\mathrm{Kgg} \\
\mathrm{Kk} \\
\mathrm{Kkm} \\
\mathrm{Kkf} \\
\mathrm{K} 1 \\
\mathrm{Km} \\
\mathrm{Kmf} \\
\mathrm{Kmn} \\
\mathrm{Knf} \\
\mathrm{Kpc}\end{array}$ & $\begin{array}{l}\text { Cretaceous System, undivided } \\
\text { Carlile Shale } \\
\text { Bartlett Barren and Dilco Coal } \\
\text { Members of Crevasse Canyon } \\
\text { Formation } \\
\text { Crevasse Canyon Formation } \\
\text { Dalton Sandstone Member of } \\
\text { Crevasse Canyon Formation } \\
\text { Cliff House Sandstone } \\
\text { Cleary Coal Member of Menefee } \\
\text { Formation and Gibson Coal Member } \\
\text { of Crevasse Canyon Formation, } \\
\text { undivided } \\
\text { Dakota Sandstone } \\
\text { Paguate Tongue of Dakota Sandstone } \\
\text { Gallup Sandstone } \\
\text { Greenhorn and Graneros Formations, } \\
\text { undivided } \\
\text { Kirtland Shale } \\
\text { Kirtland Shale and Mancos Shale, } \\
\text { undivided } \\
\text { Kirtland Shale and Fruitland } \\
\text { Formation, undivided } \\
\text { Lewis Shale } \\
\text { Mancos Shale } \\
\text { Menefee Formation } \\
\text { Mesaverde Group } \\
\text { Fort Hays Limestone Member of } \\
\text { Niobrara Formation } \\
\text { Pictured Cliffs Sandstone }\end{array}$ \\
\hline
\end{tabular}


Table 1.-Geologic unit explanation table-Concluded

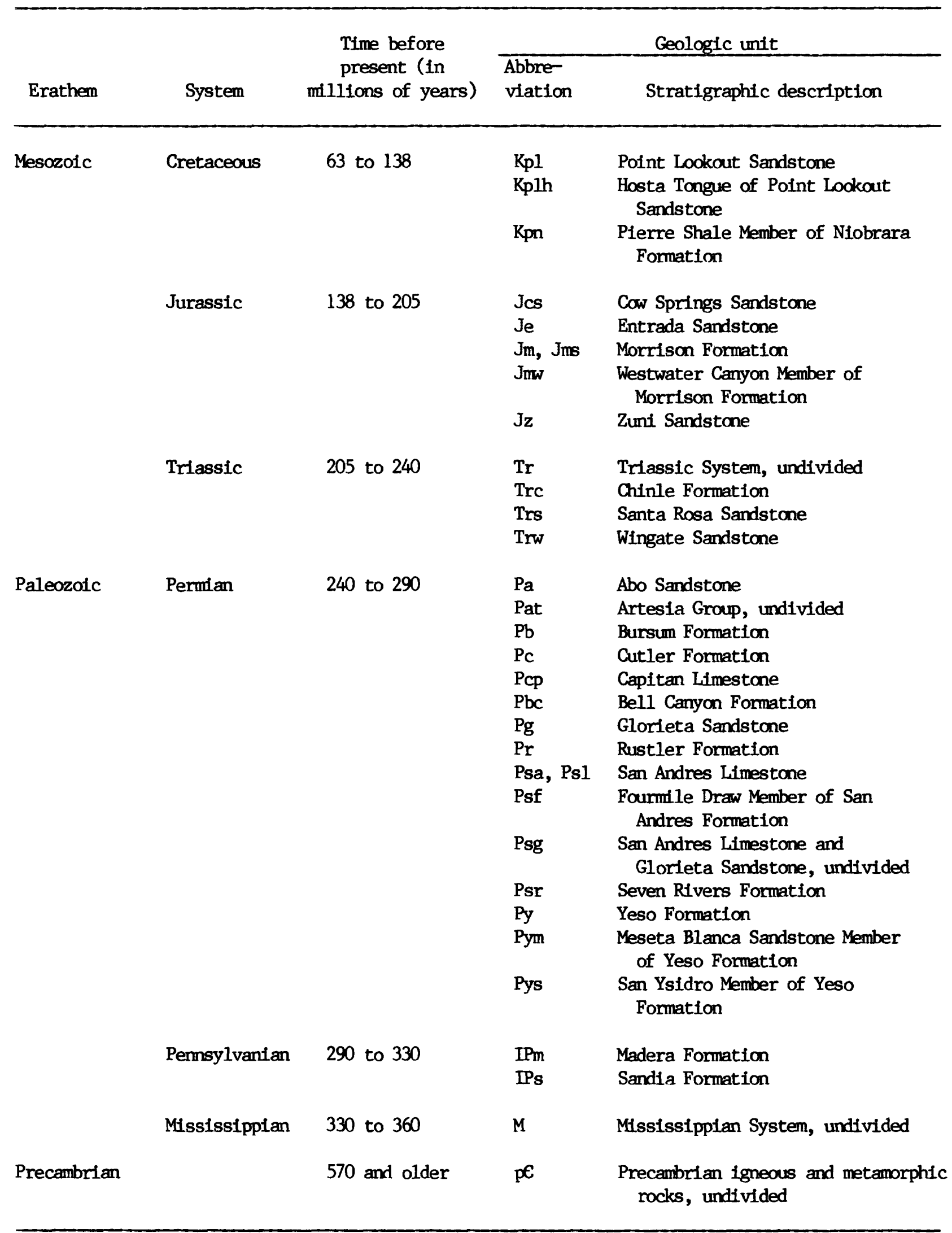




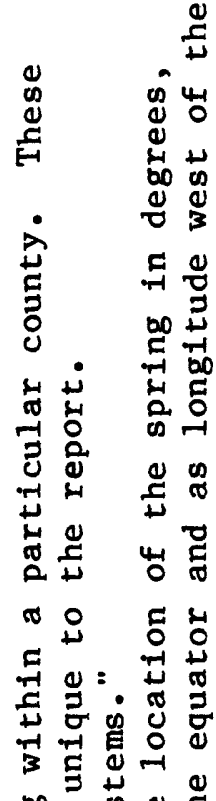

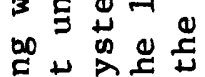

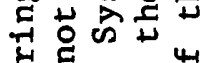

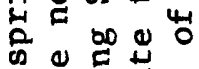

㐘

บ

औ

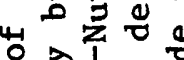

०

EE

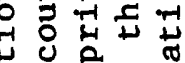

记 क त

ن

( ) 百。

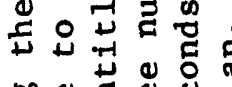

oo

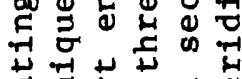

Ш का $\Rightarrow$ 엉 है के ช 0 \& - 告至 क岂

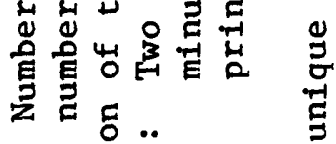

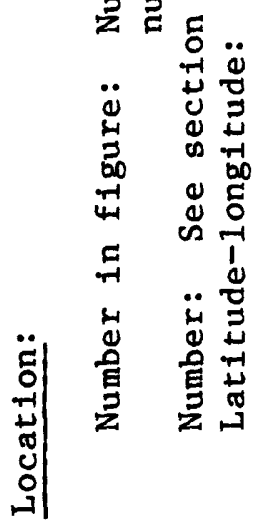

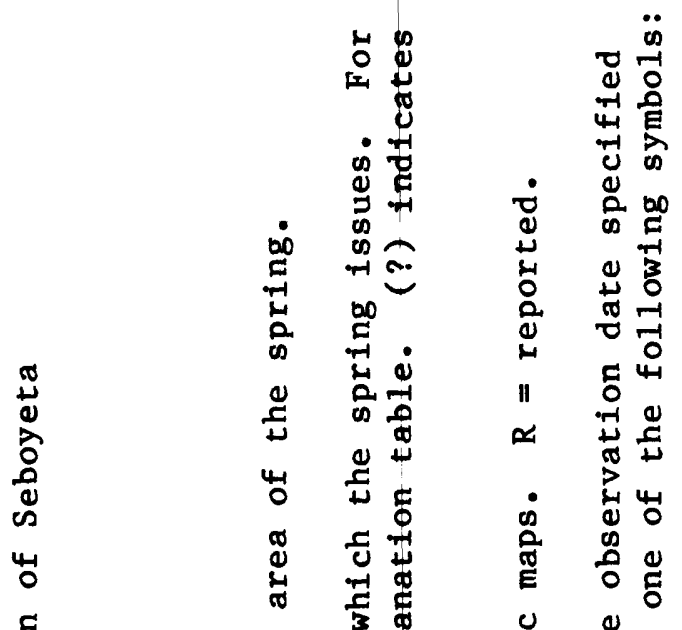

ำ

ه

足先 告

$\forall$ 出 00 0

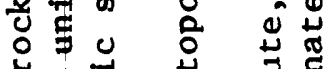

$\omega$ 出

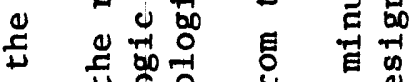

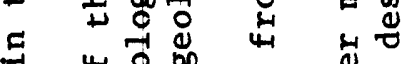

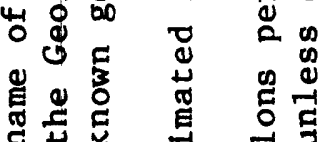

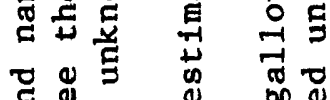

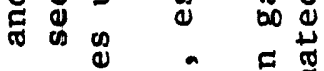

0

क 口

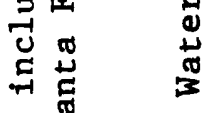

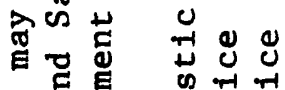

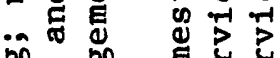

อ 0 क

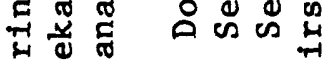

命造 స

क स्ते

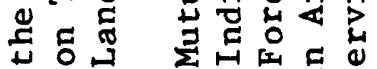
的

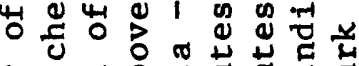

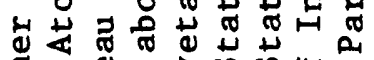

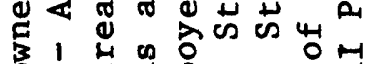

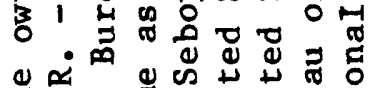

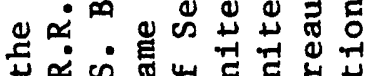
山幽 : 0 为 111

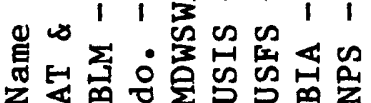

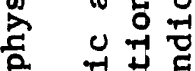

岁

엉 1

$\infty$ 色

导 ब

4 o

는 造

क 4 ह

동 4ㅓㅓㅇ 웅

年

Th

(2) कू

苛

대엉

की 0 D

บ

60 든

긍 웡

गे

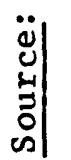

통

岌

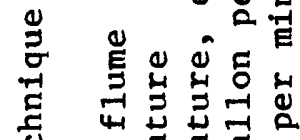
4 거

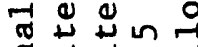
क्न न्न สิ वृ A

ए ह

के क्ष屯 ? उ 5 Tे ह

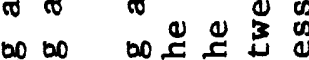

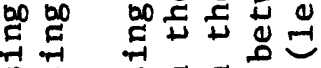

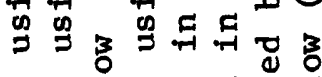

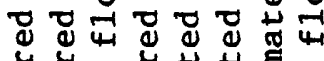

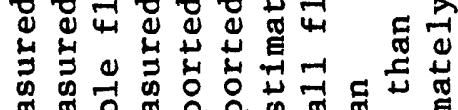
\%

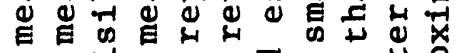

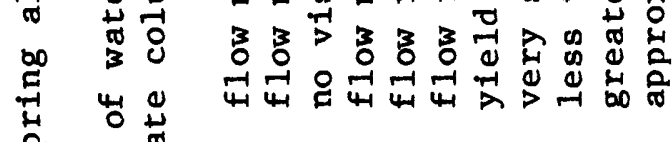

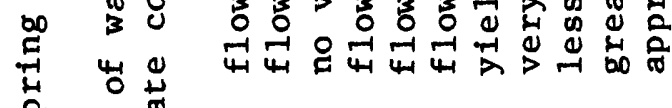
की \& 苛 

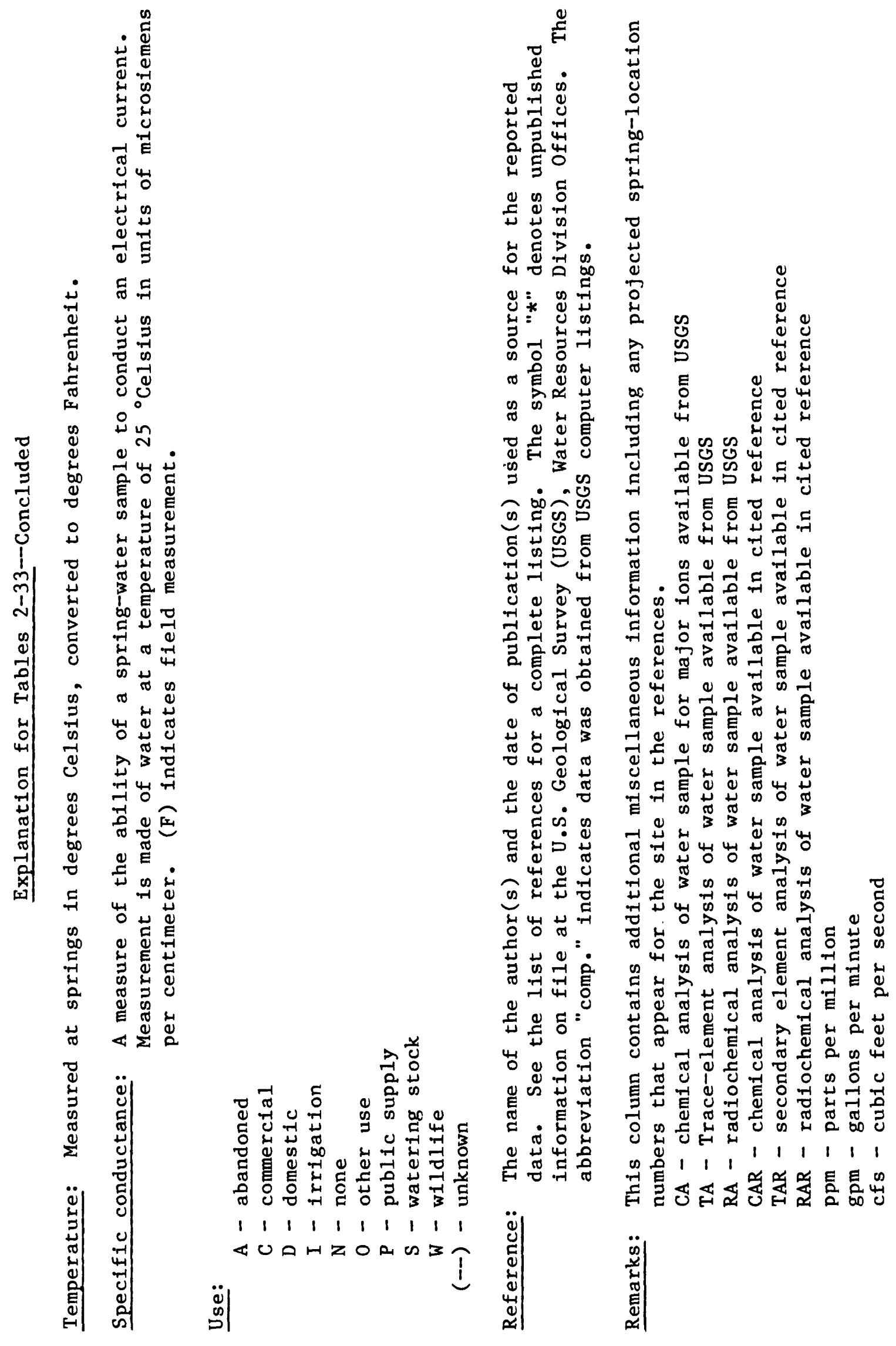


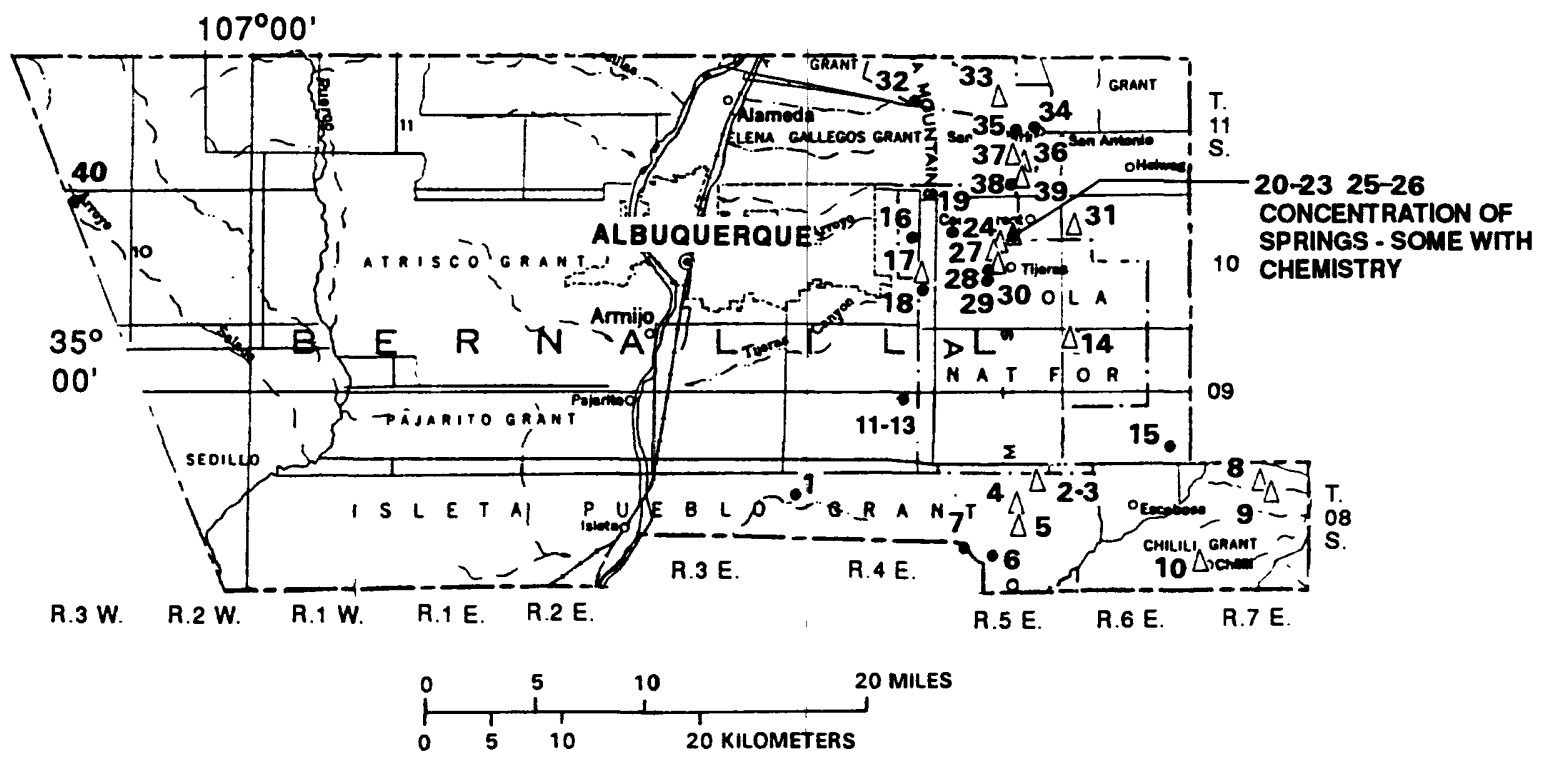

\section{EXPLANATION}

- SPRING WITH CHEMISTRY

$\triangle$ SPRING WITHOUT CHEMISTRY

10 SPRING NUMBER REFERS TO TABLE 2

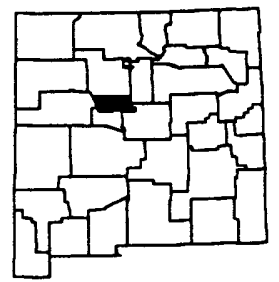

Figure 4.---Location of inventoried springs in Bernalillo County. 


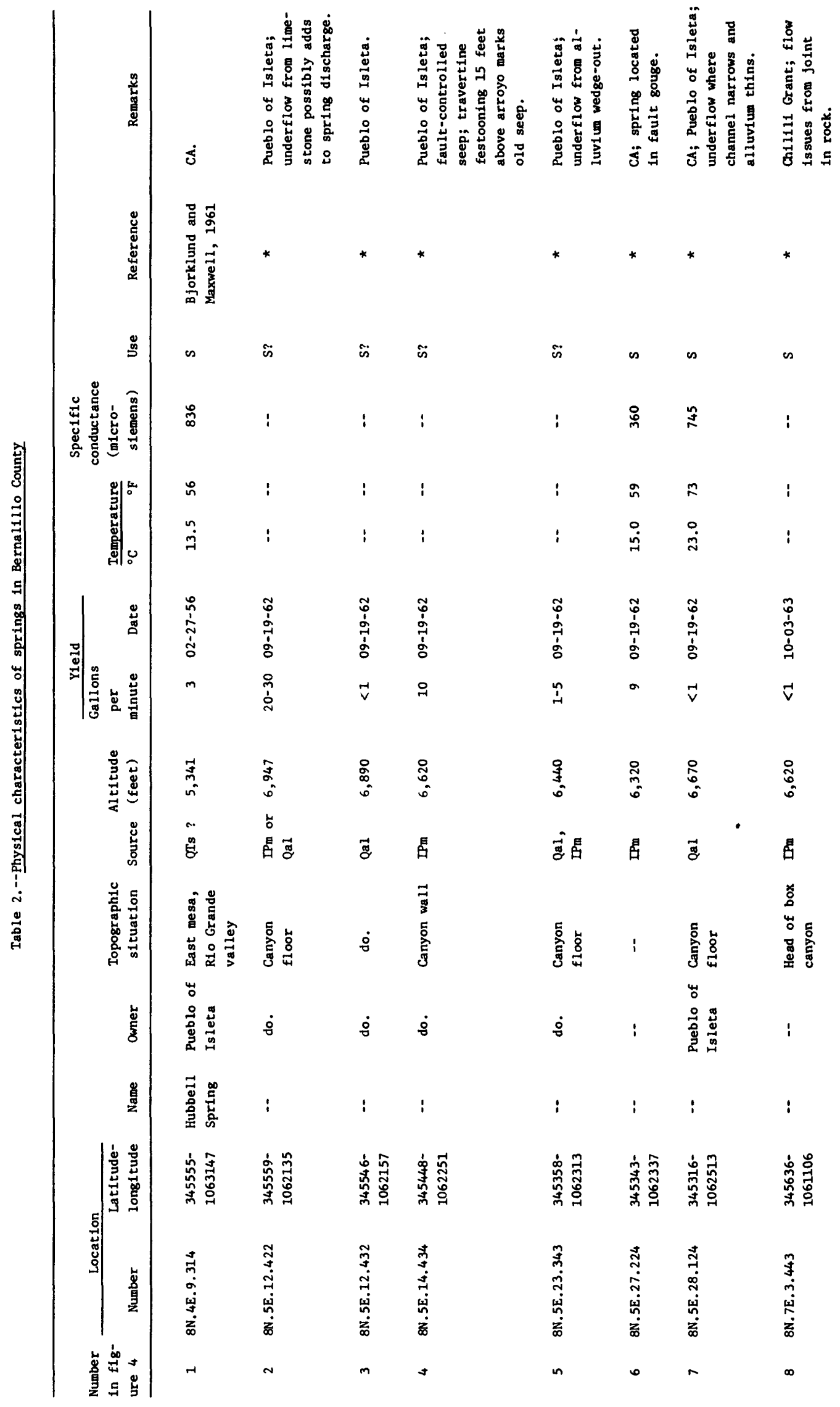




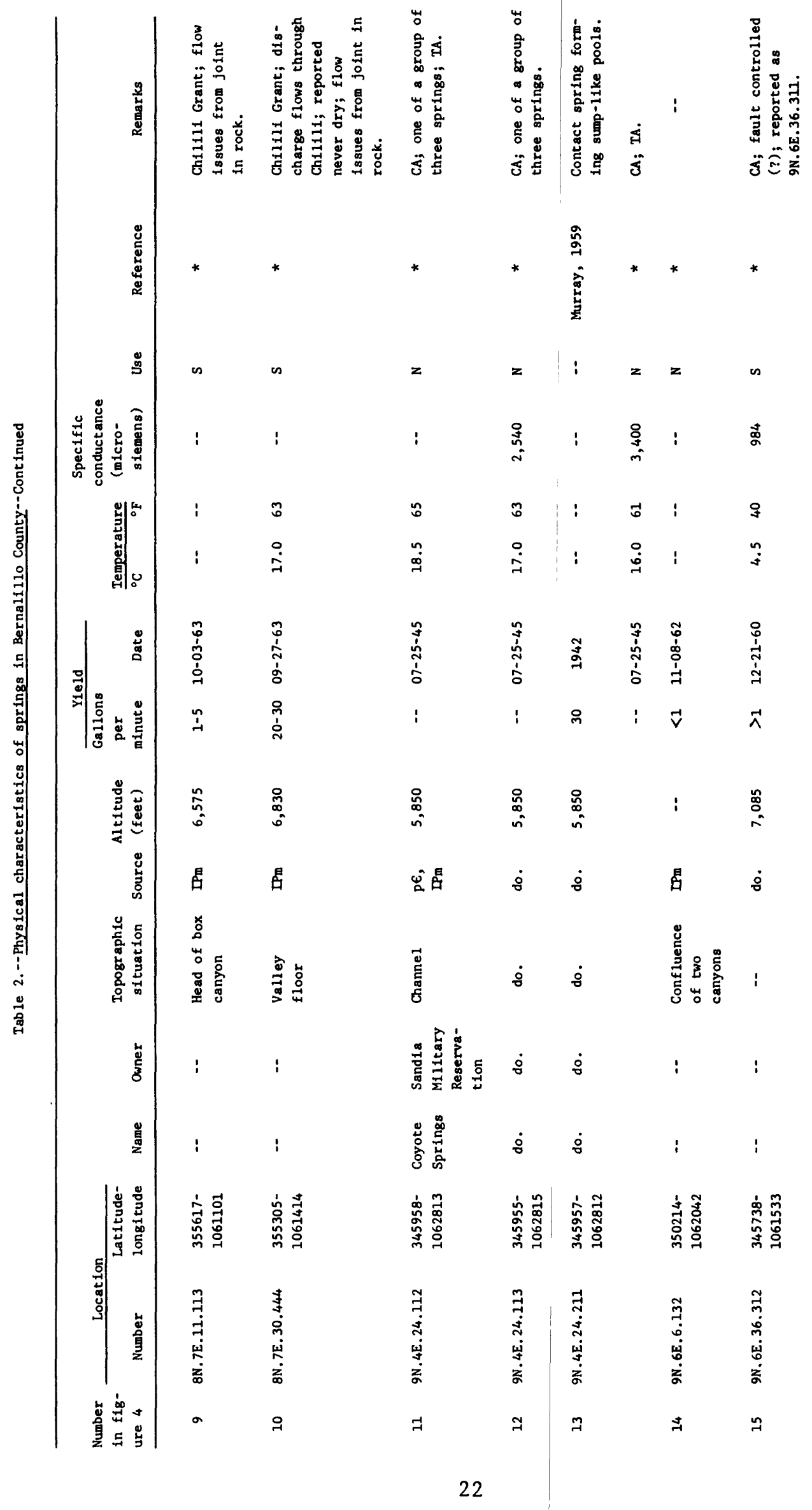




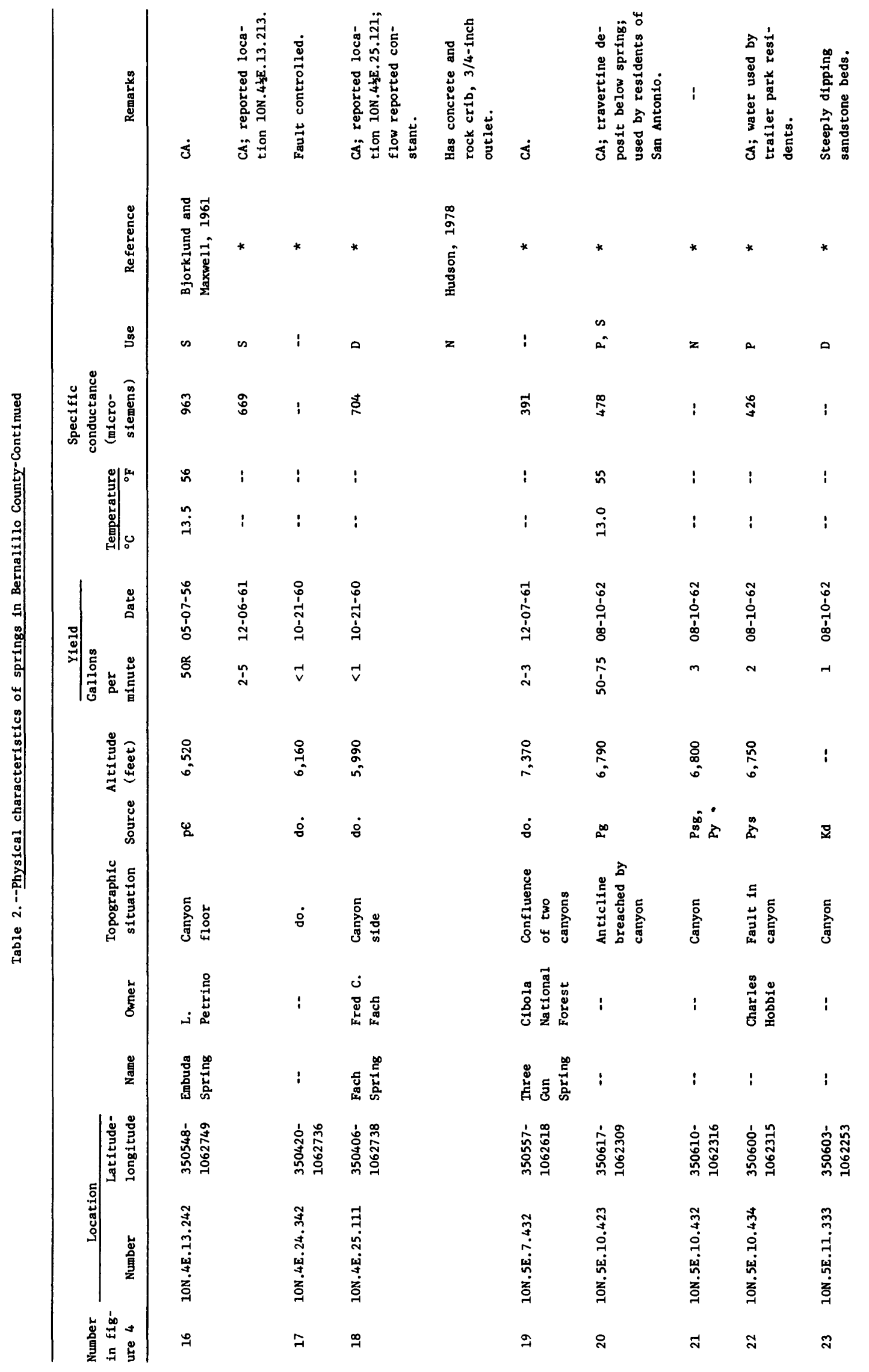




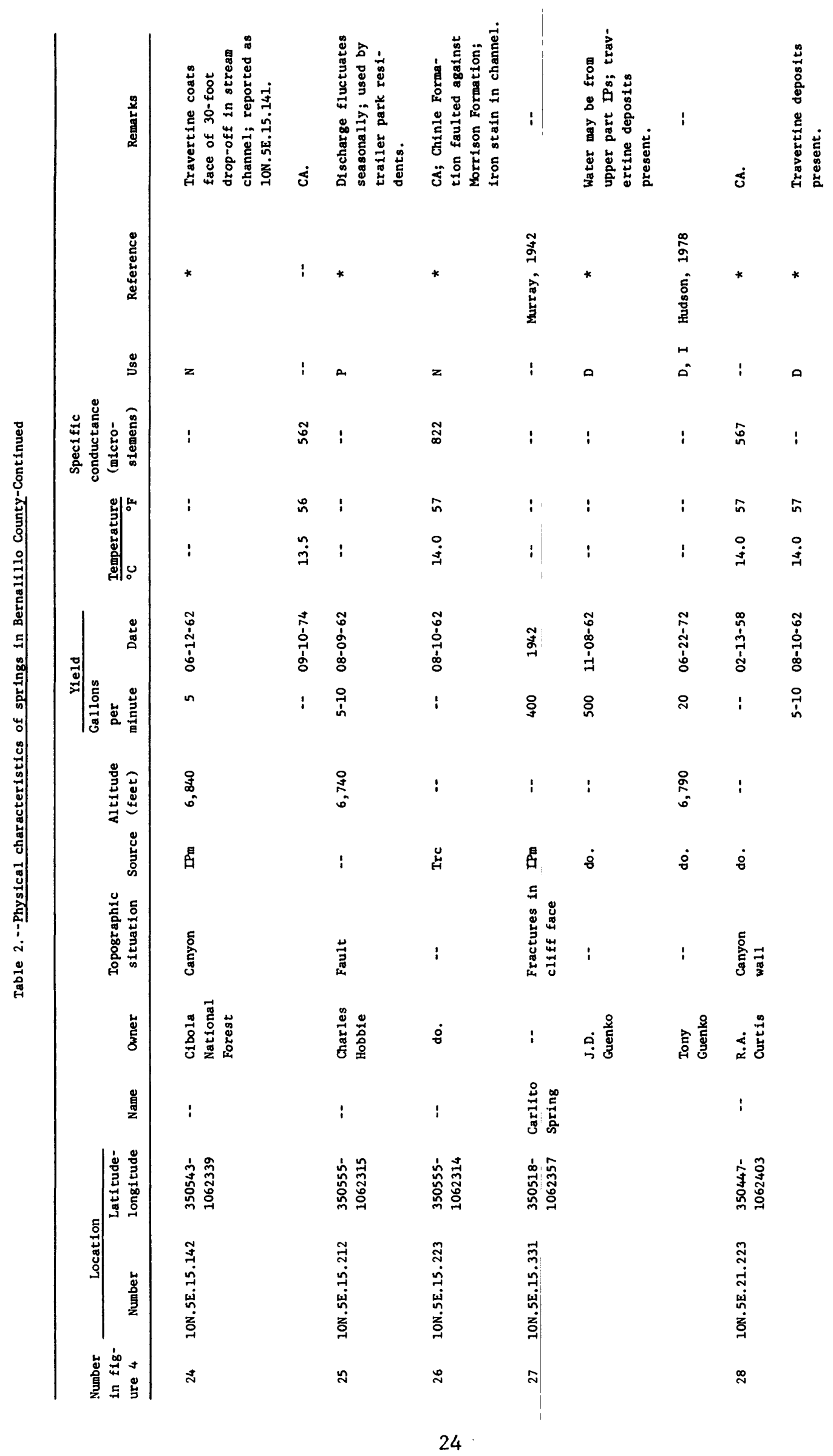




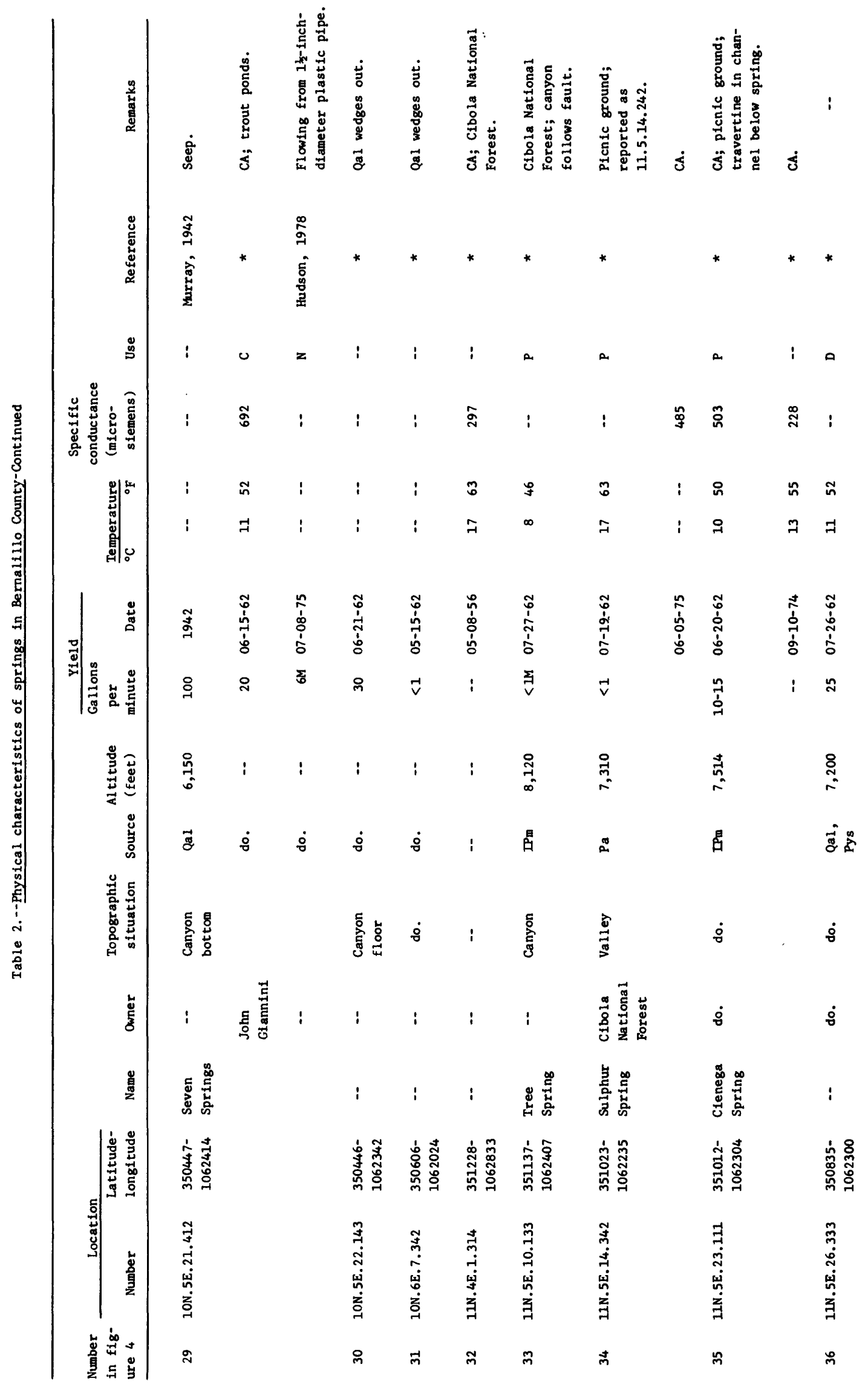




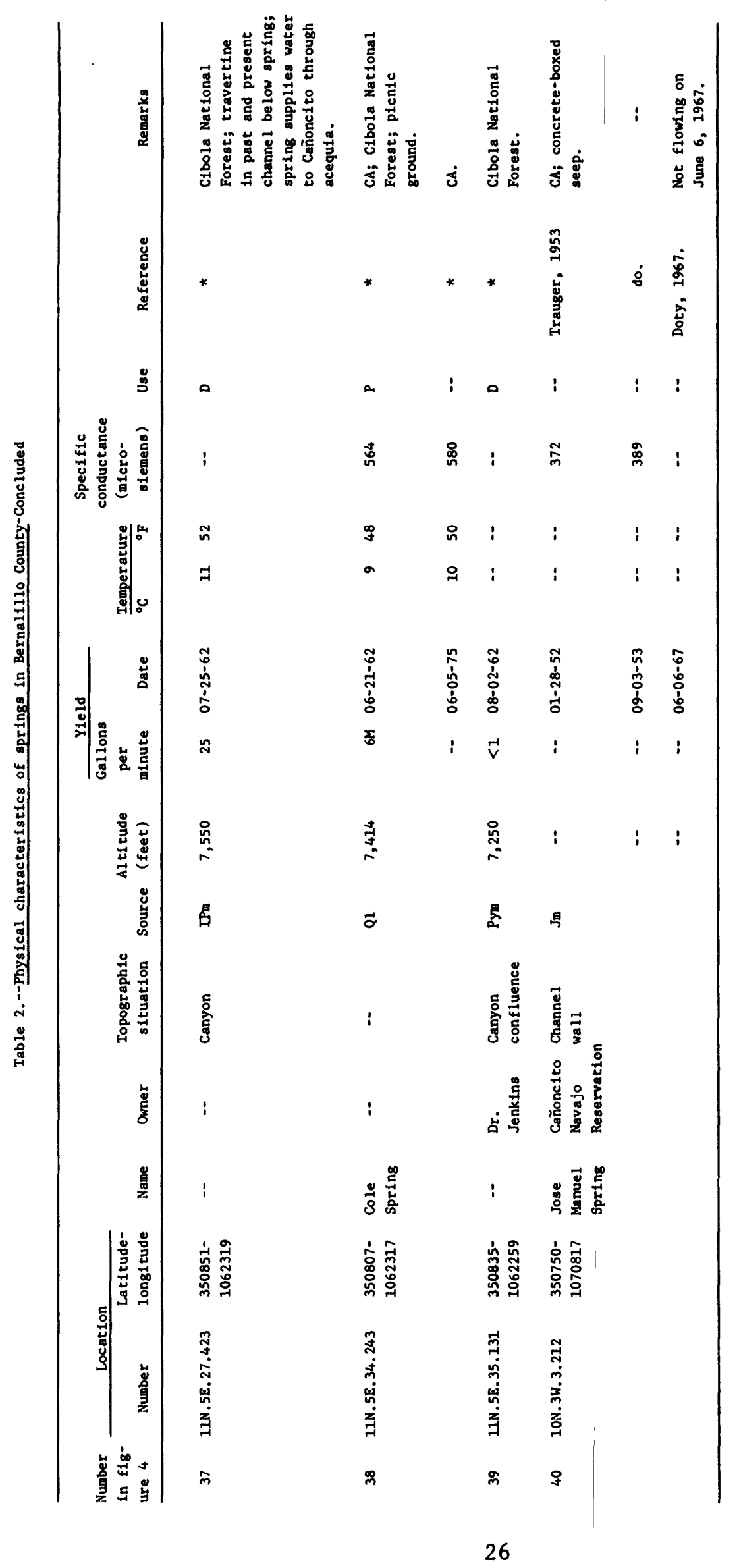




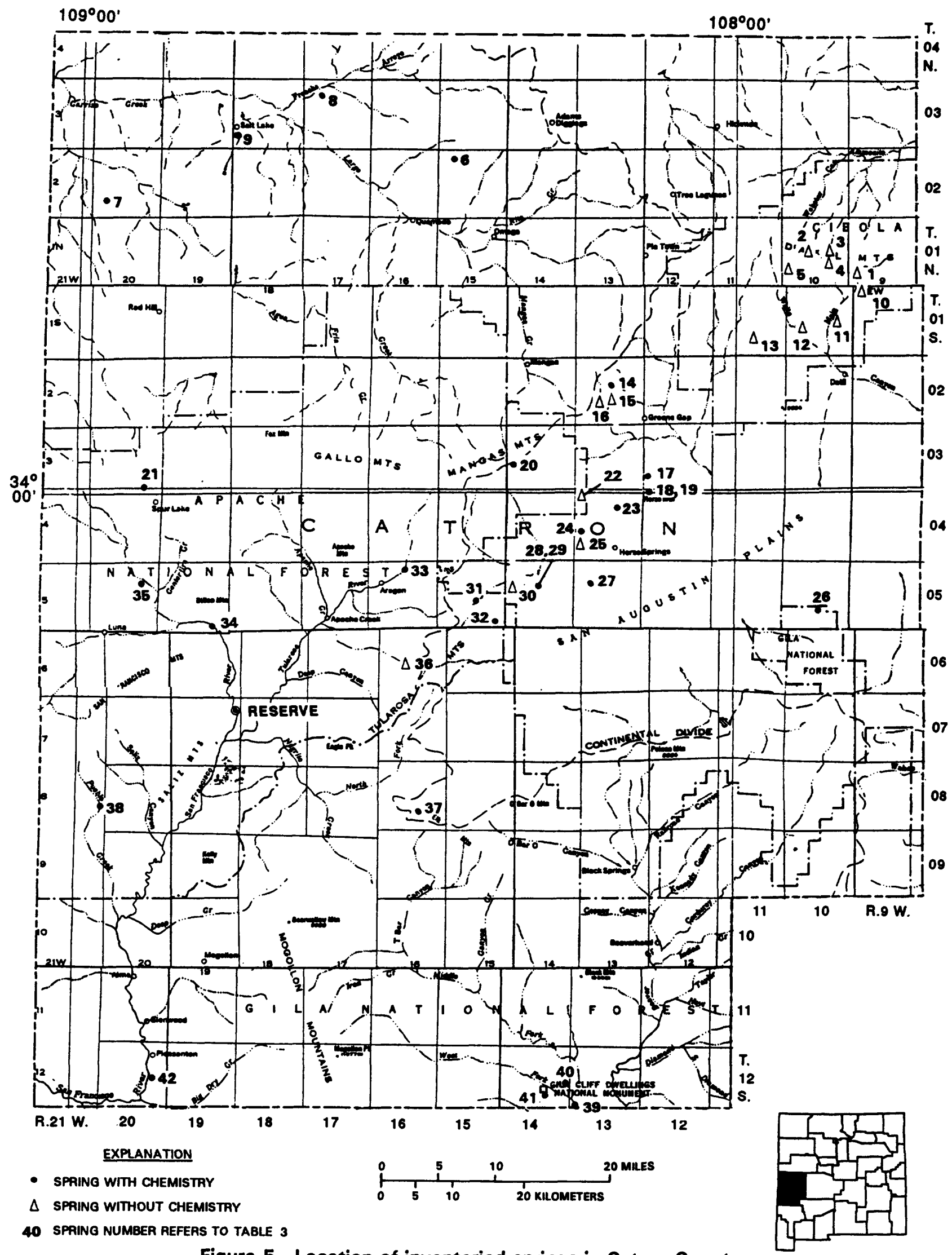

Figure 5.--Location of inventoried springs in Catron County. 


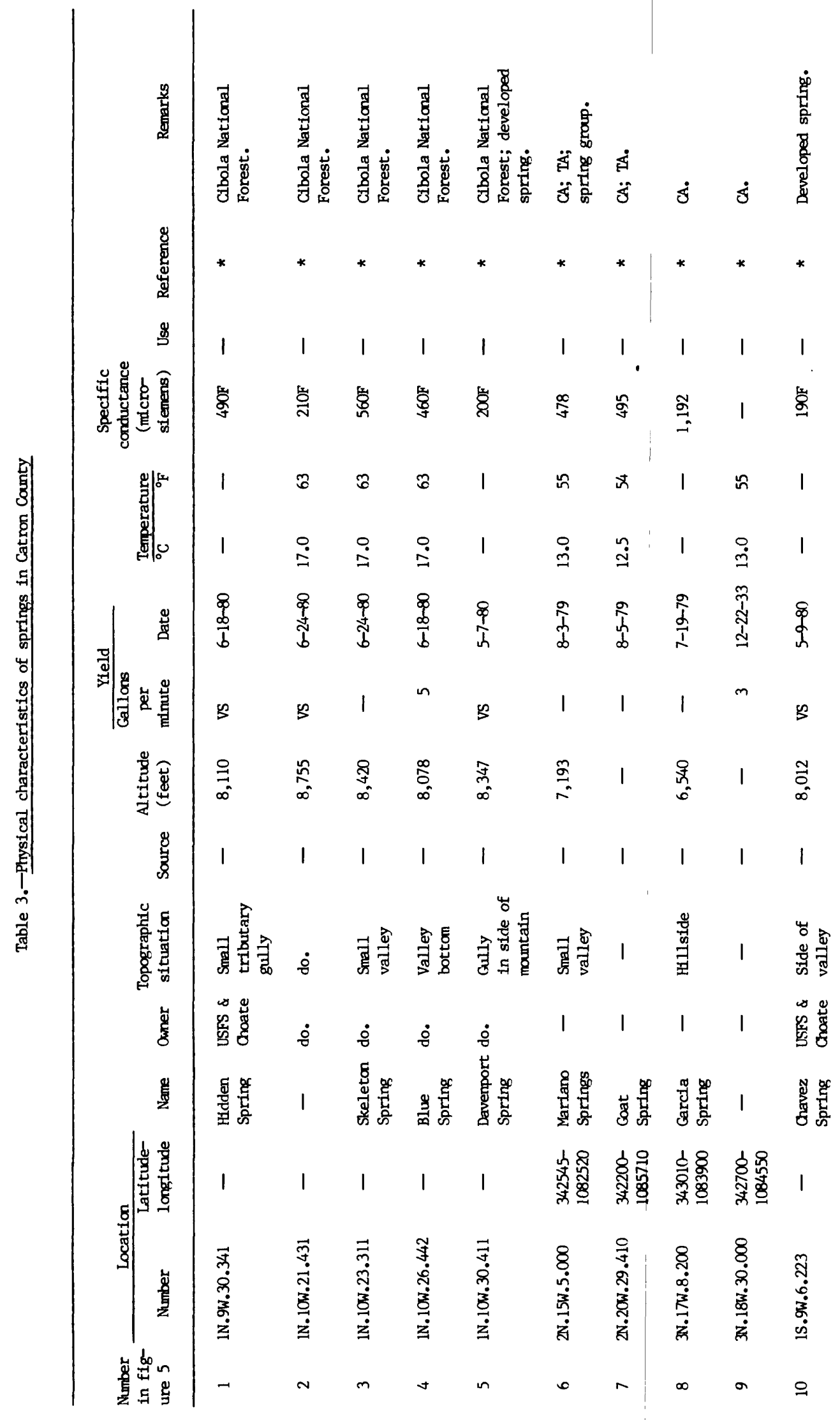




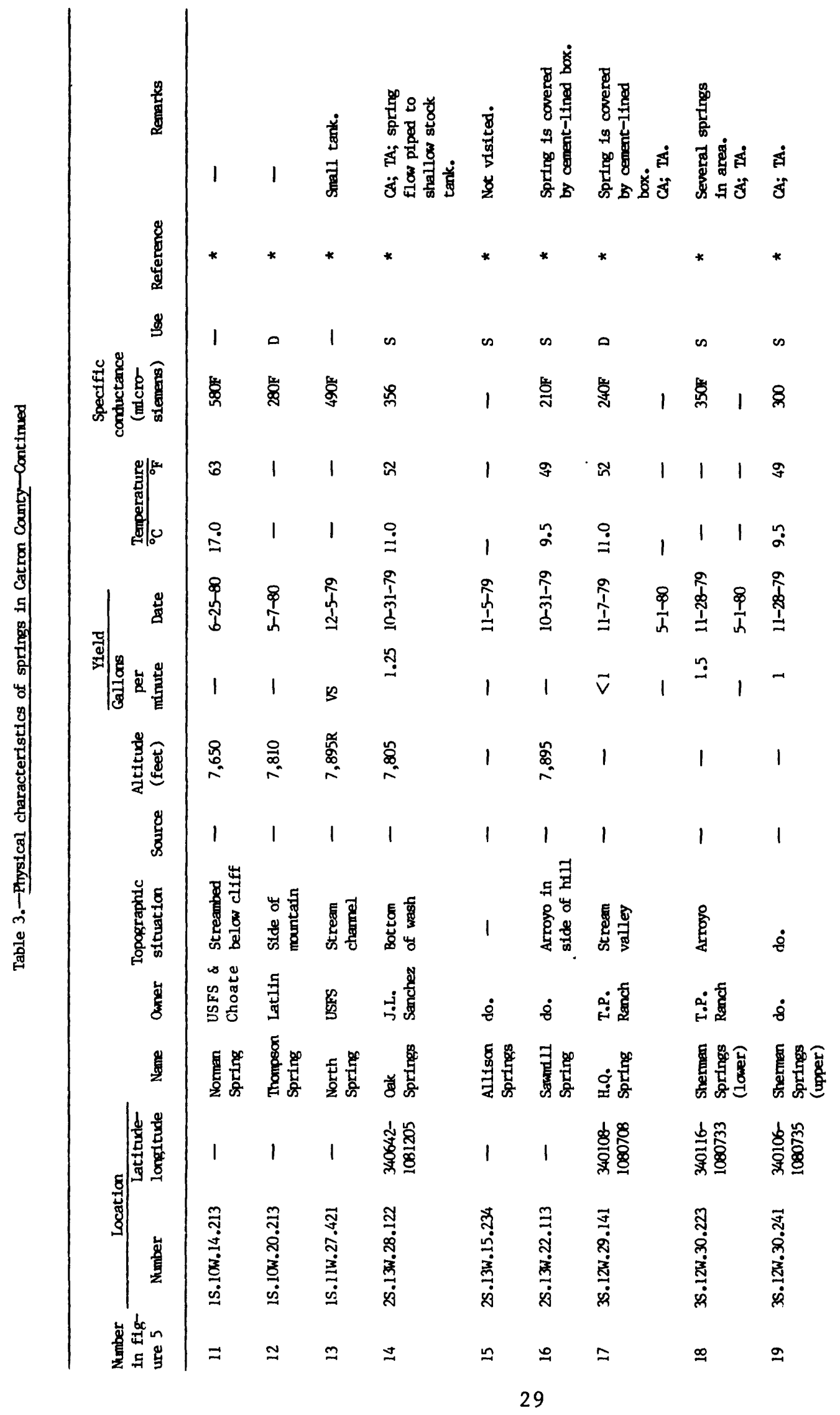




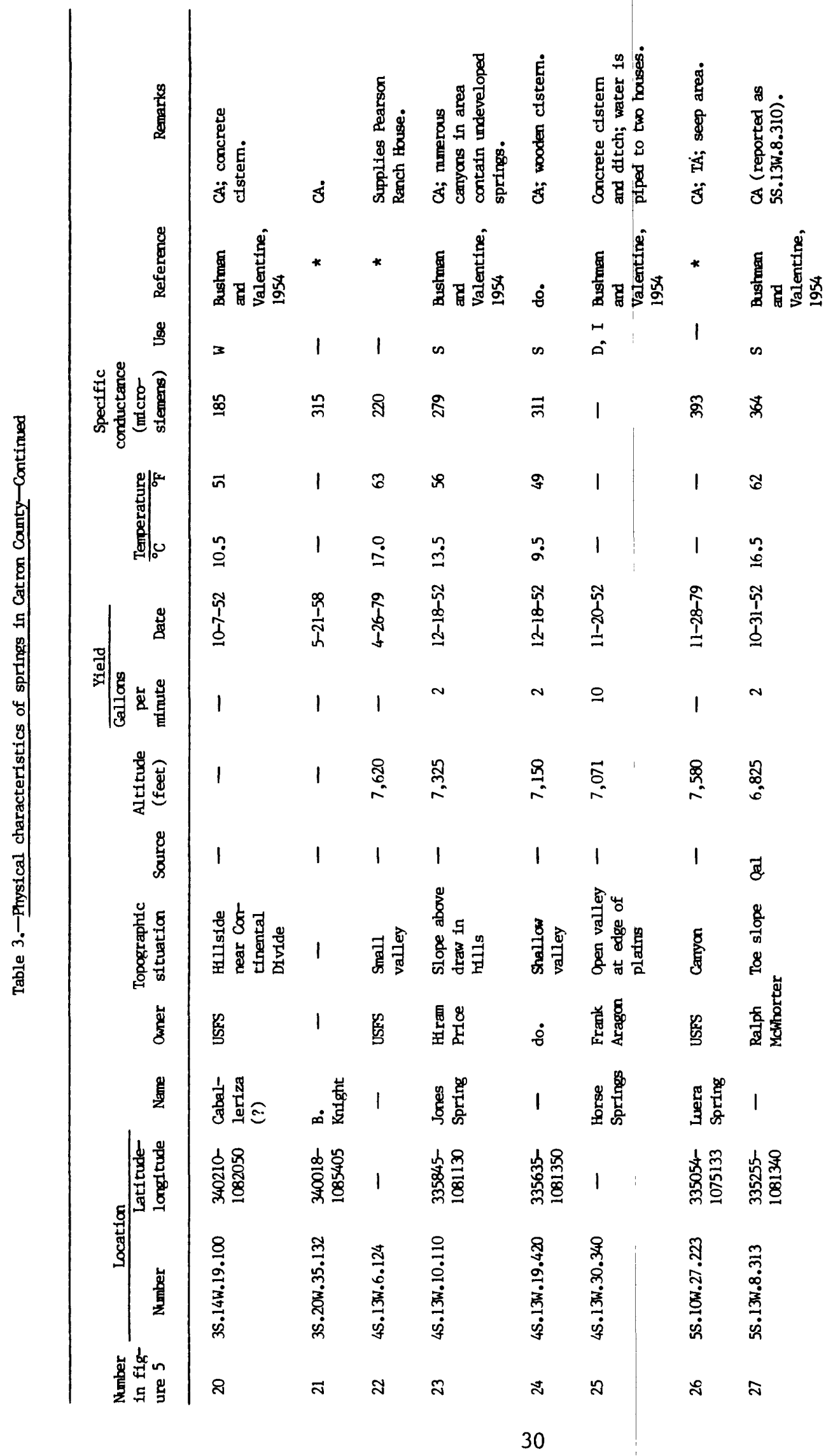




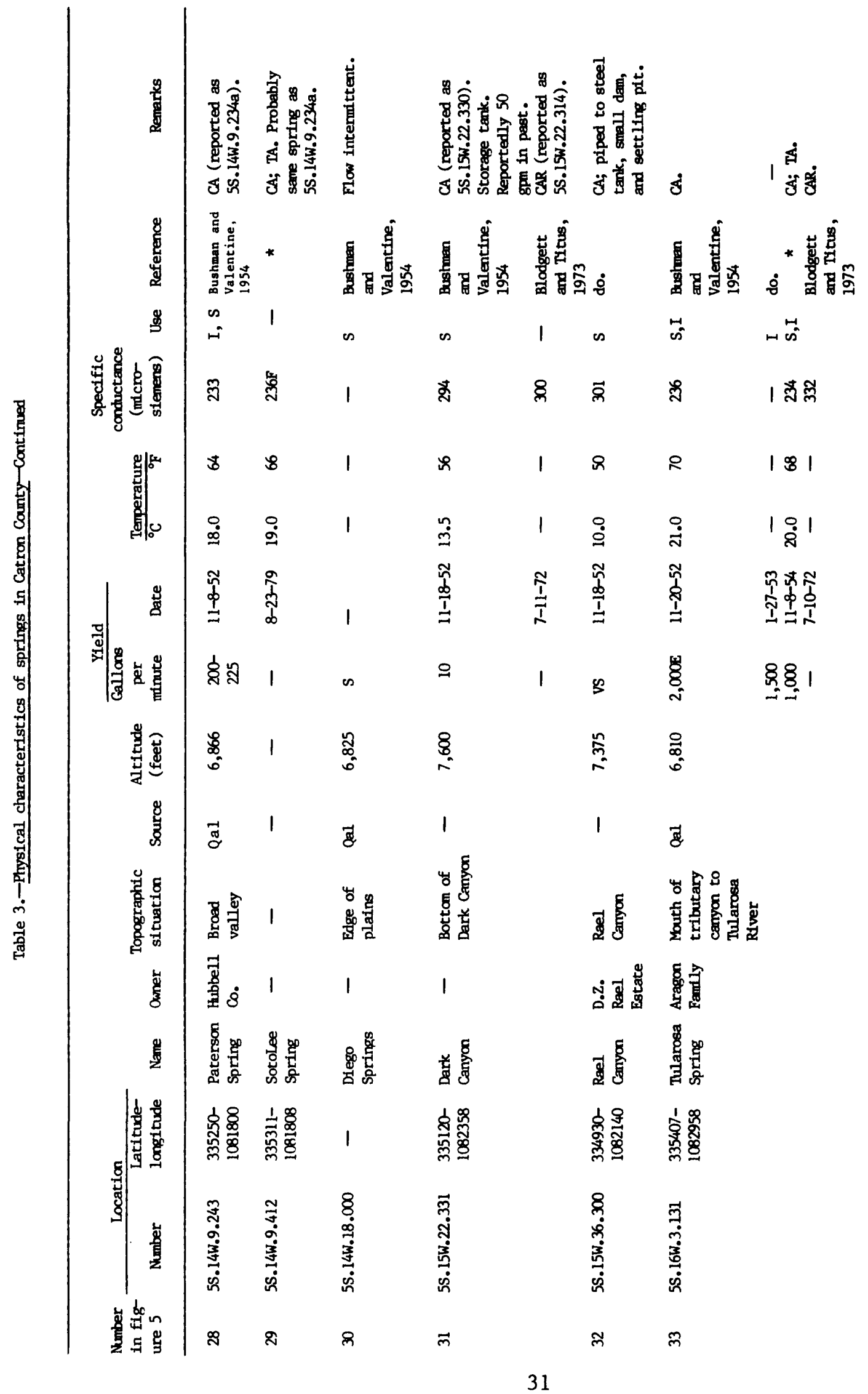




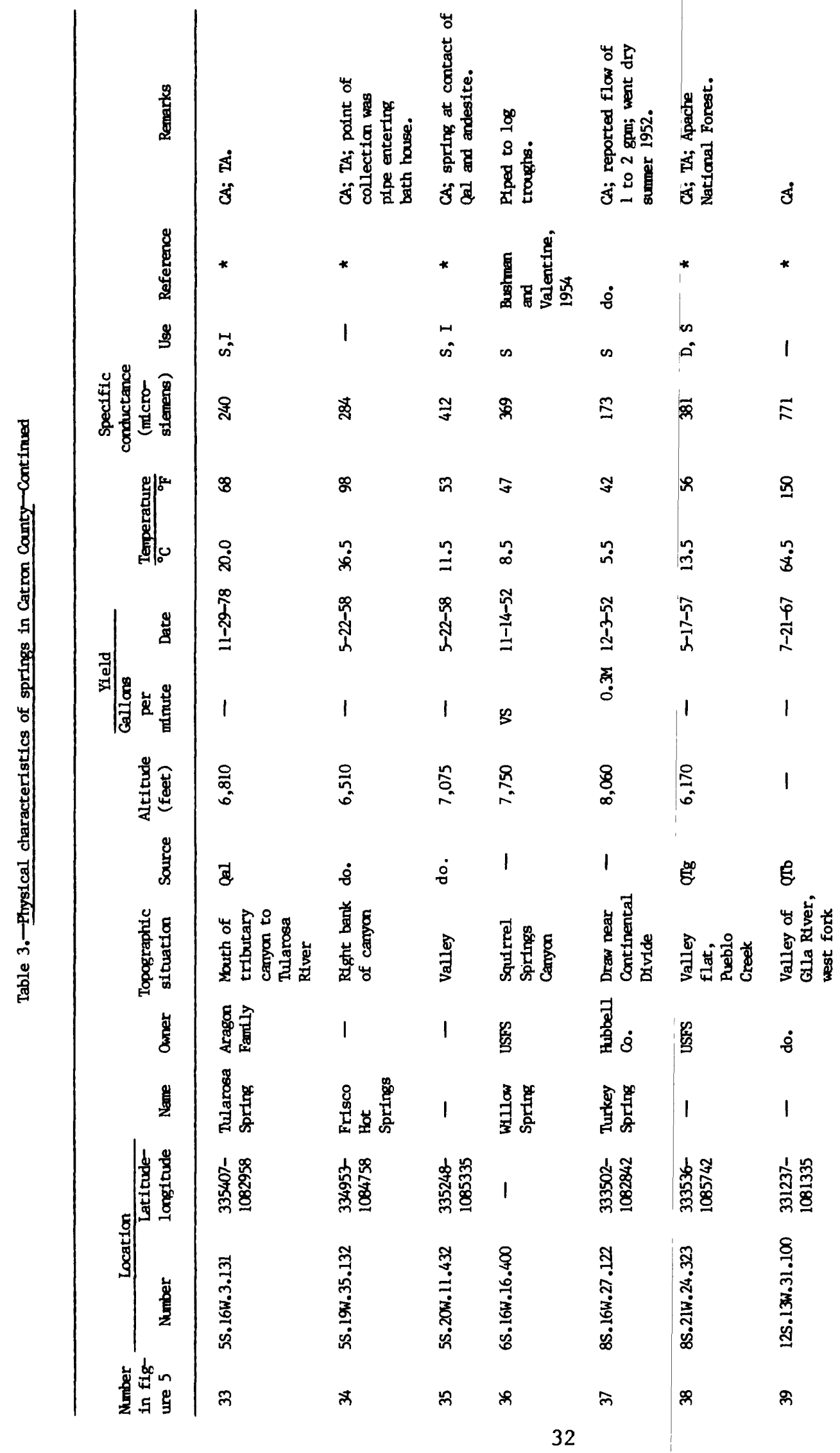




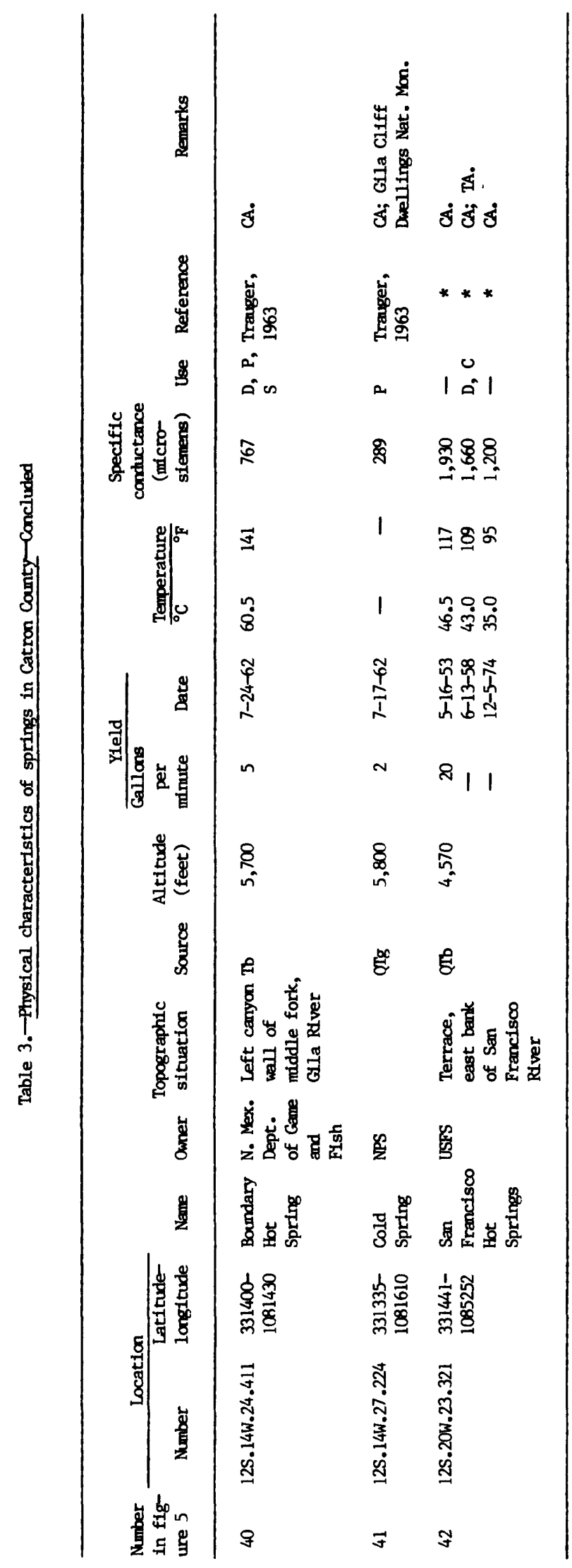




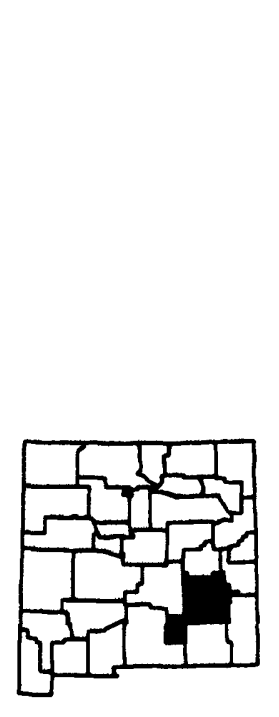

EXPLANATION

- SPRING WITH CHEMISTRY

$\triangle$ SPAING WITHOUT CHEMISTRY

22 SPRING NUMBER REFERS TO TABLE 4
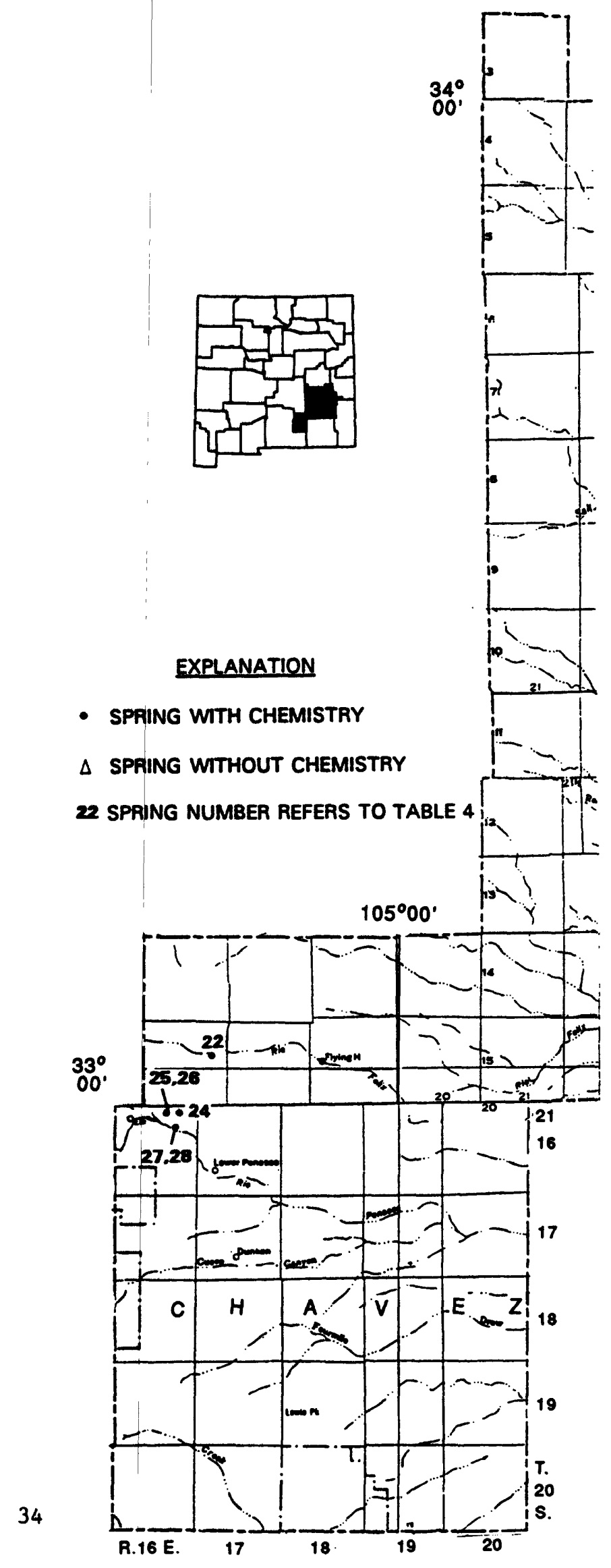


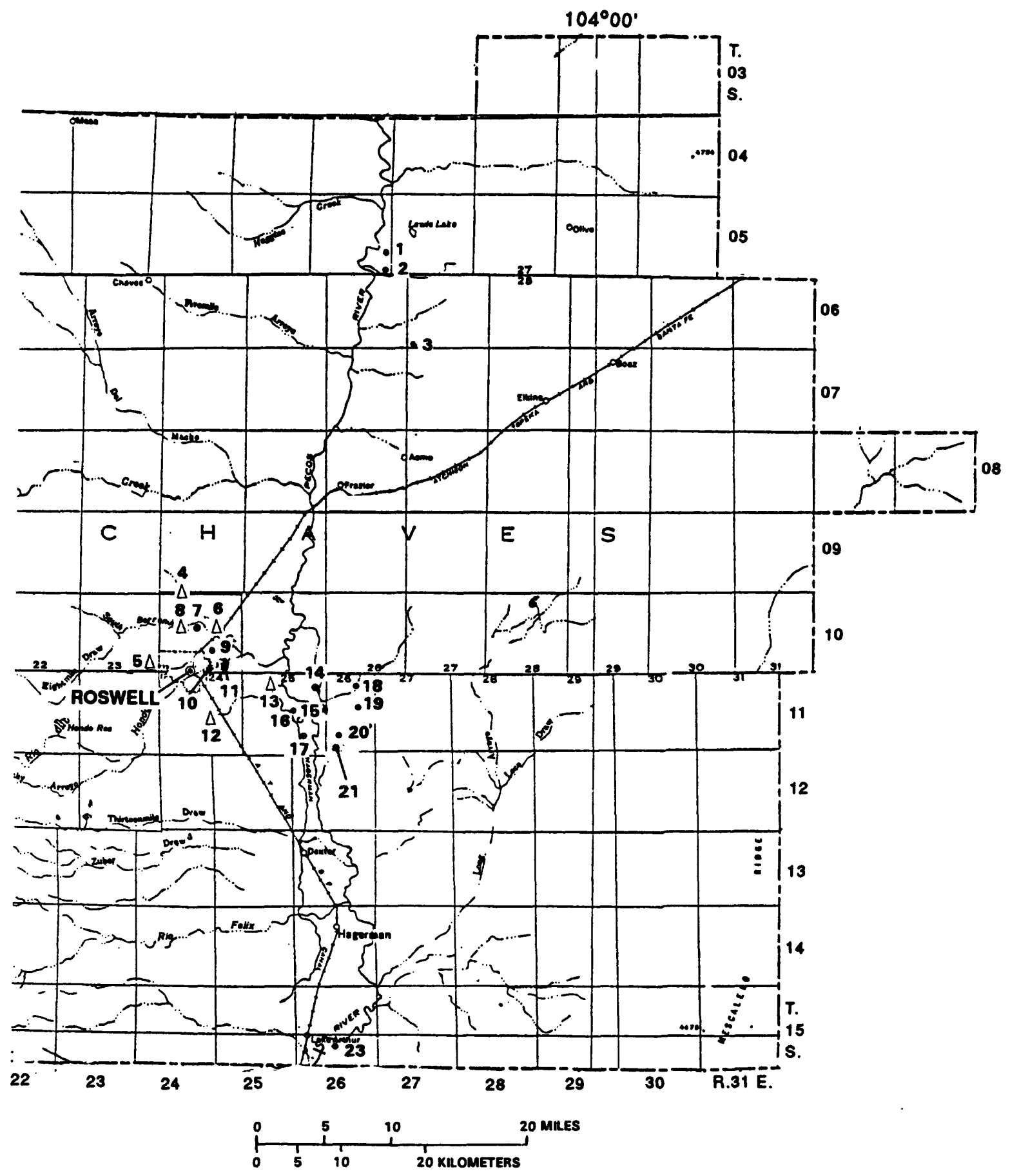

Figure 6.--Location of inventoried springs in Chaves County; 


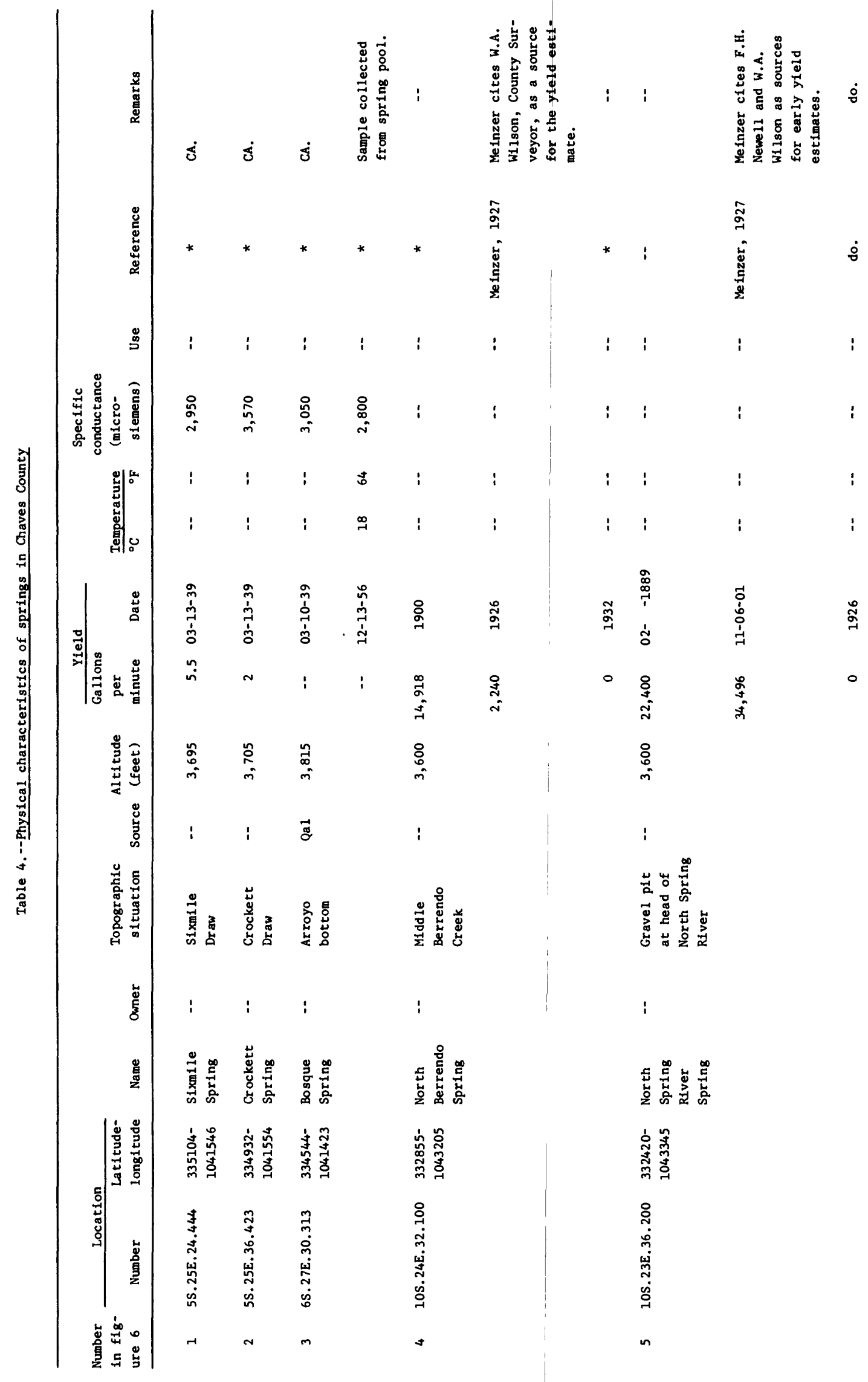




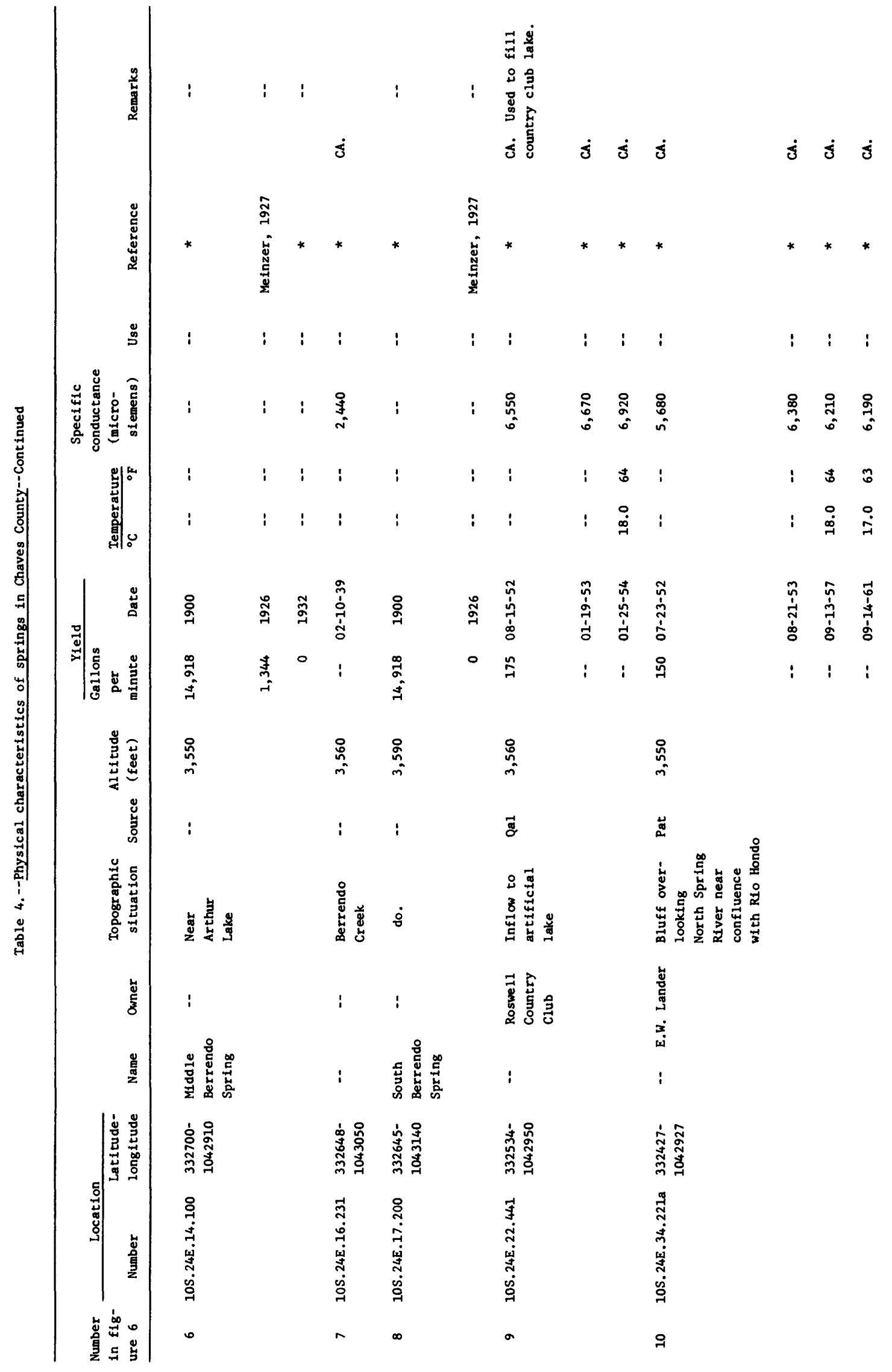




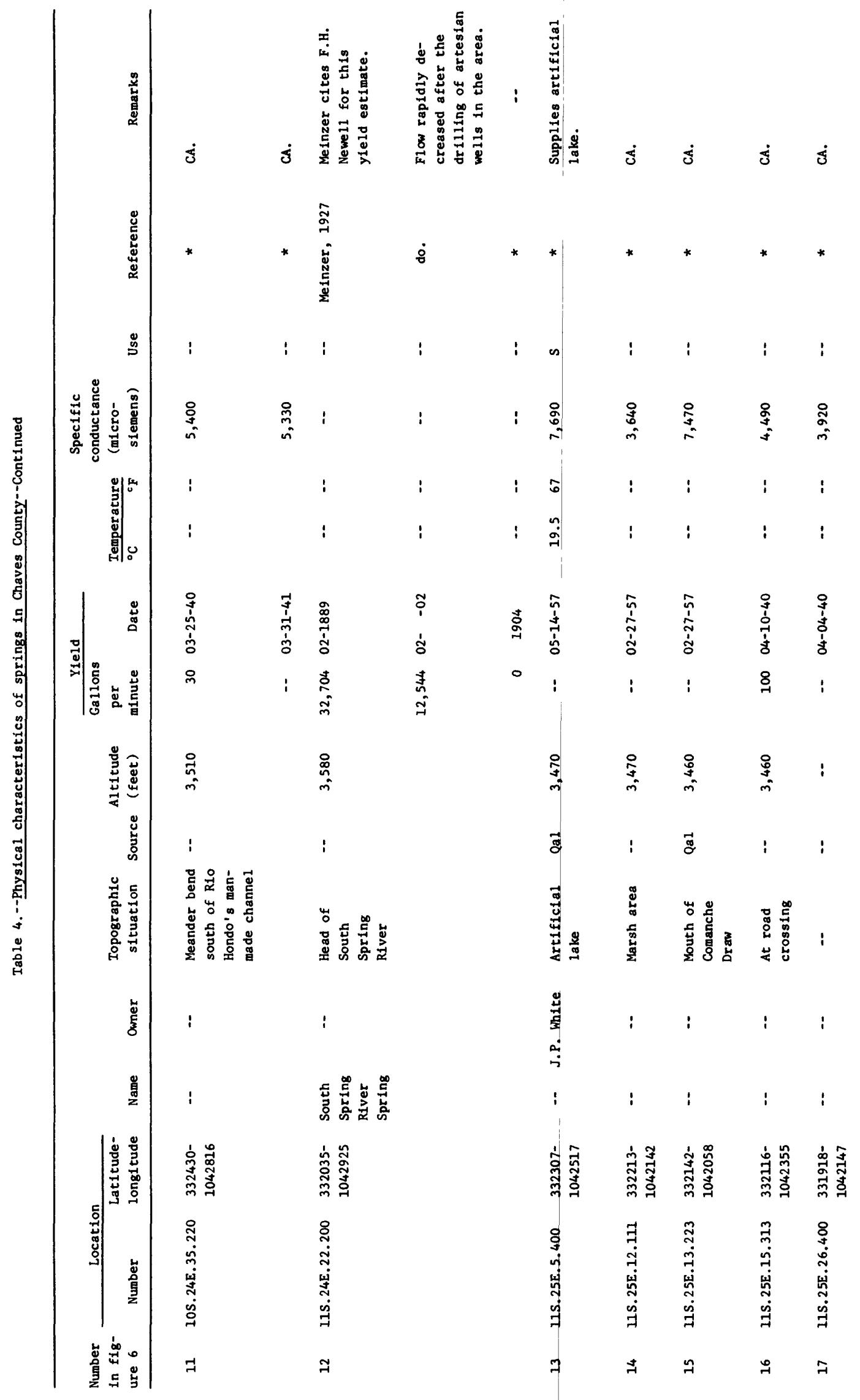




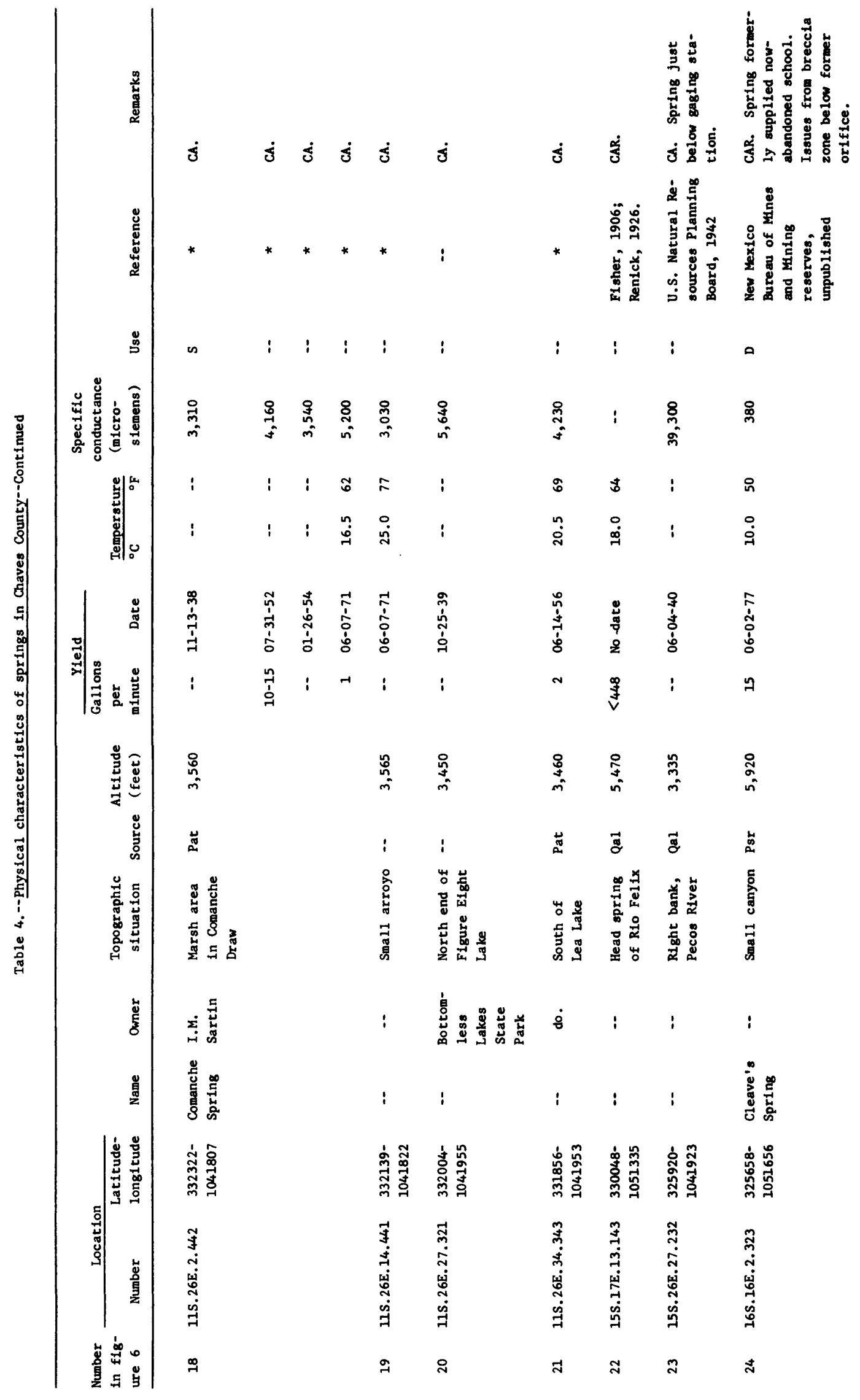




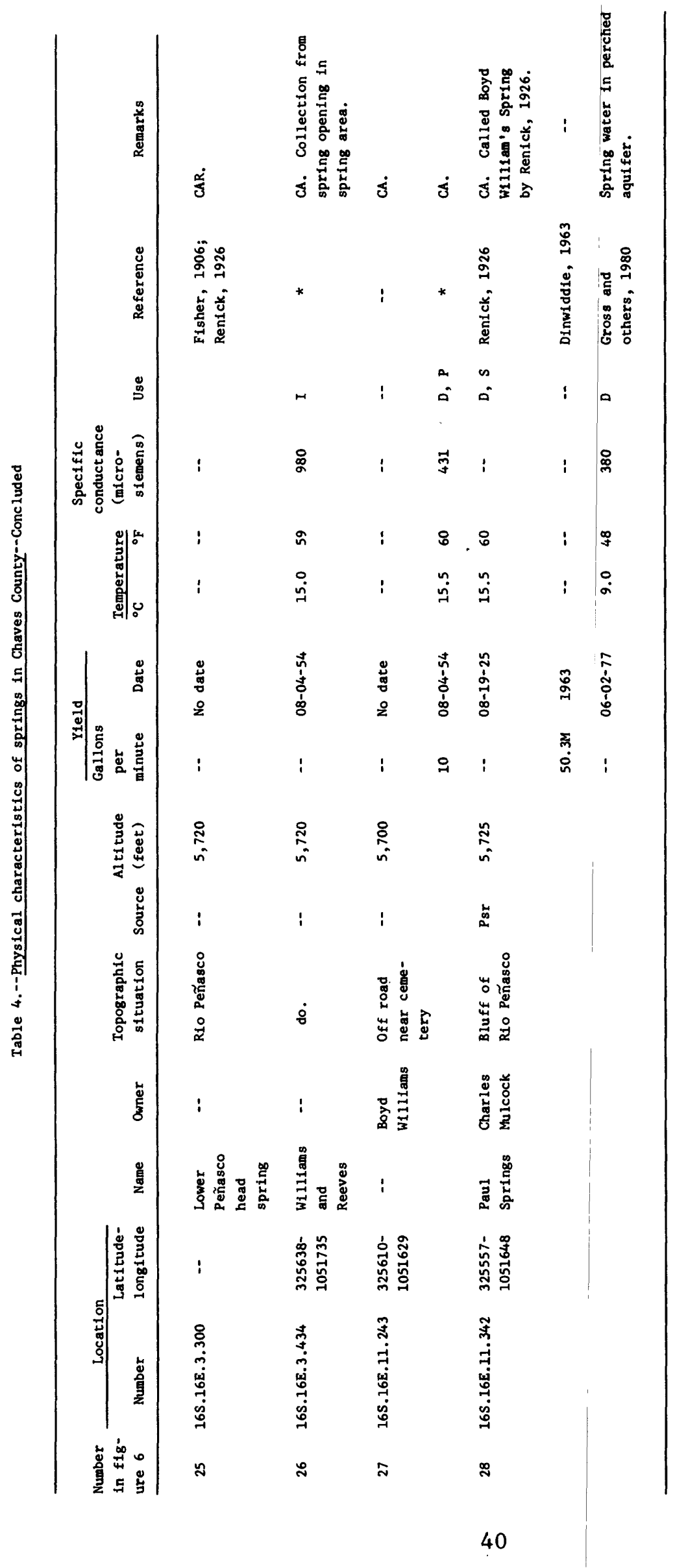




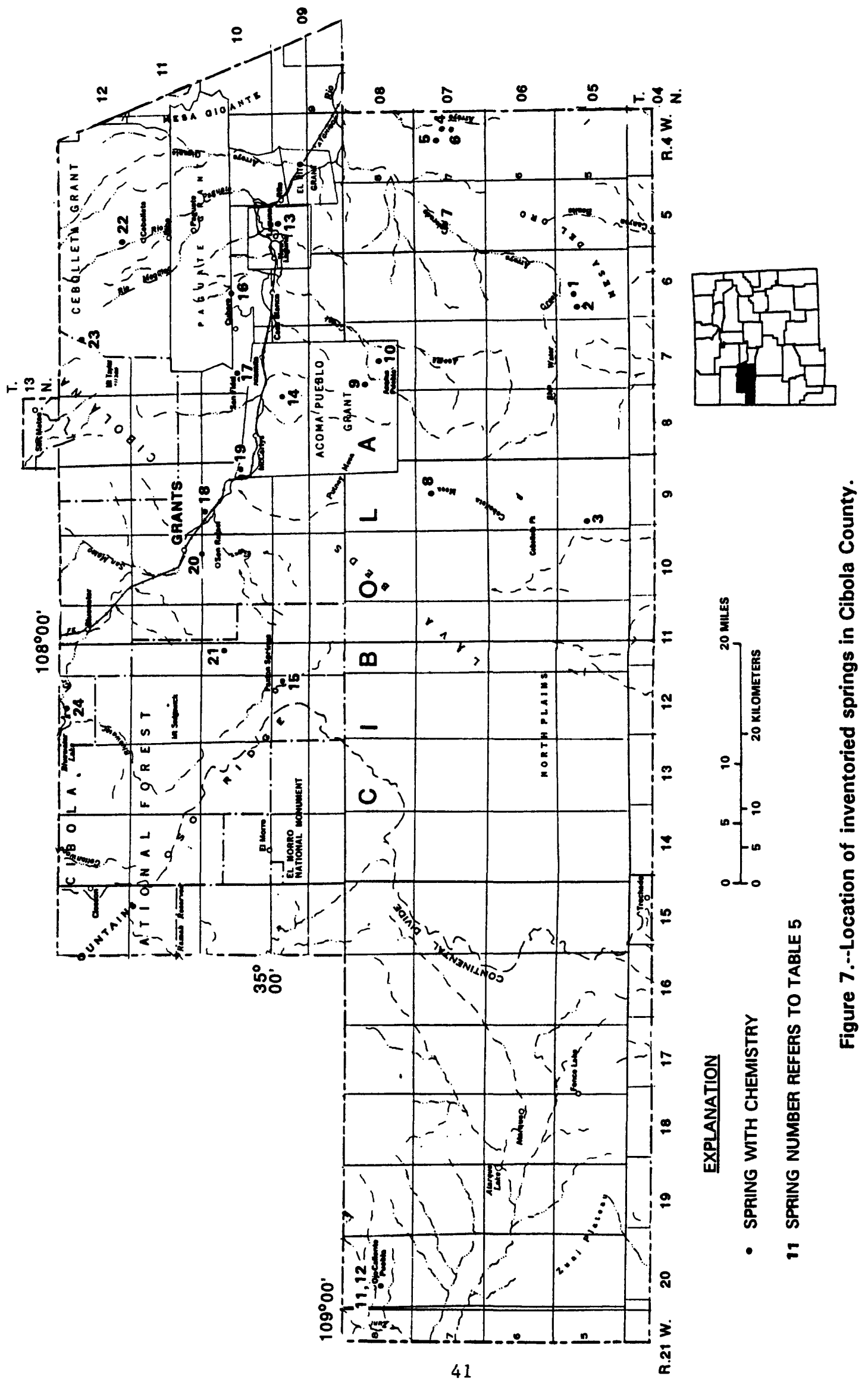




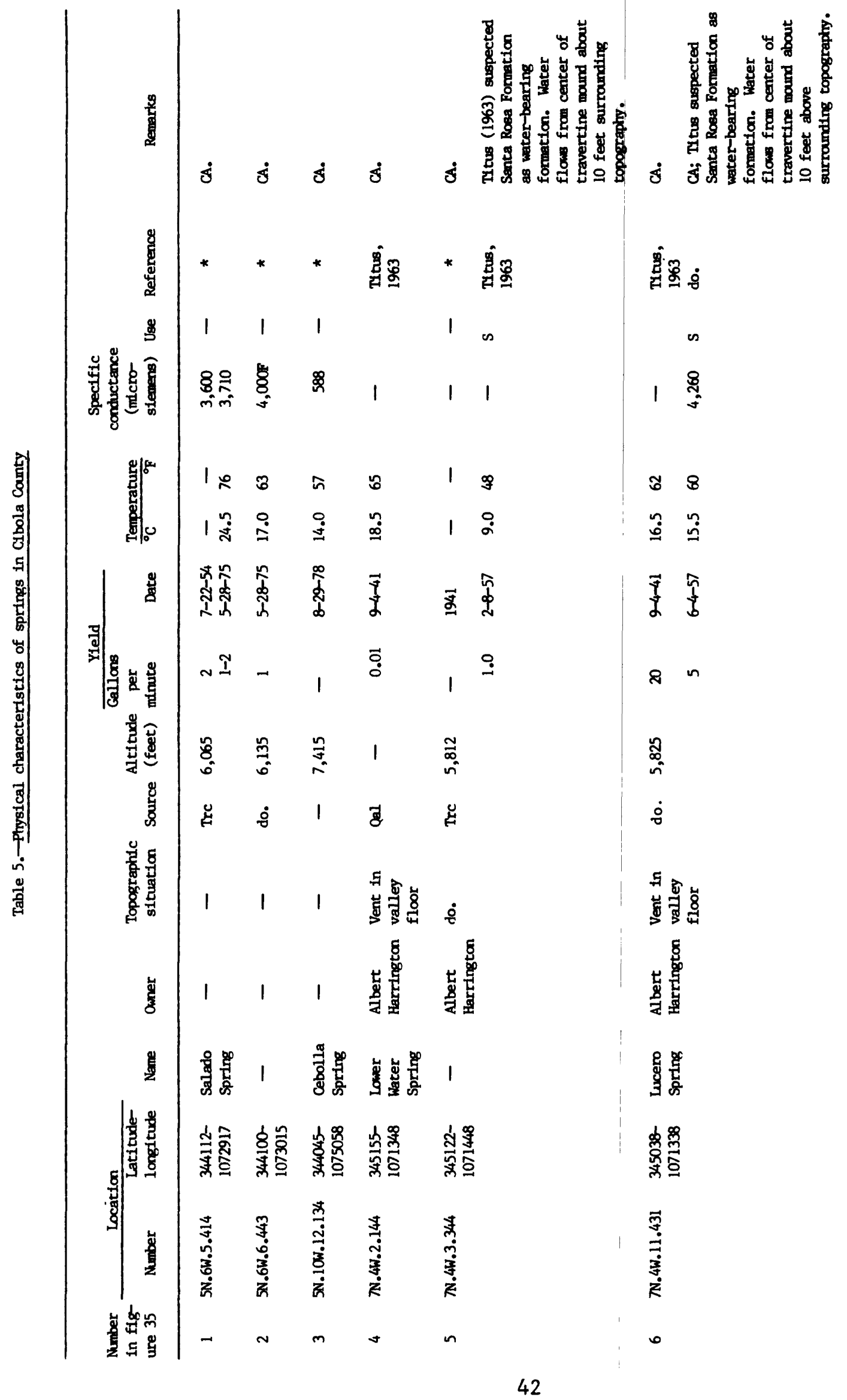




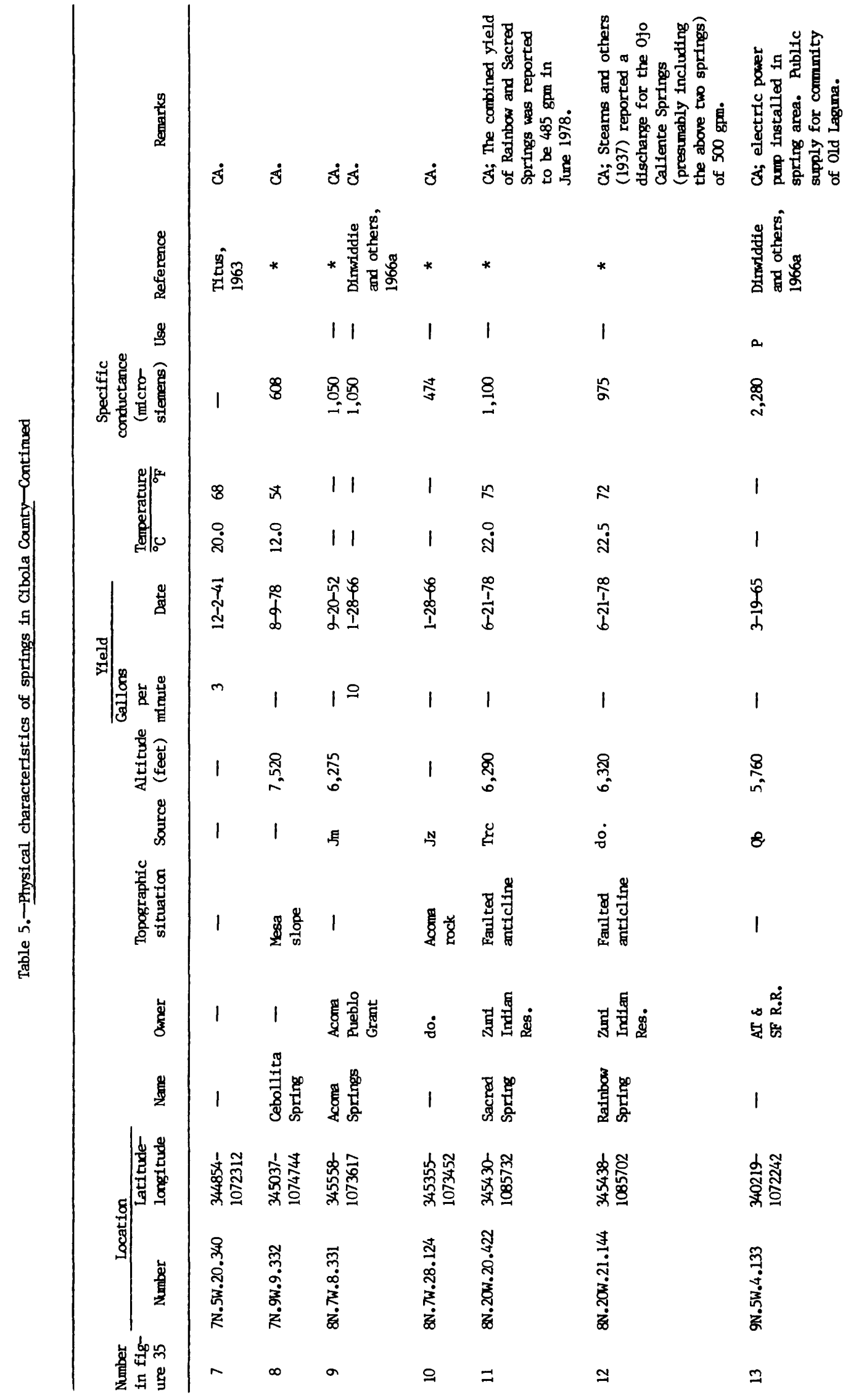




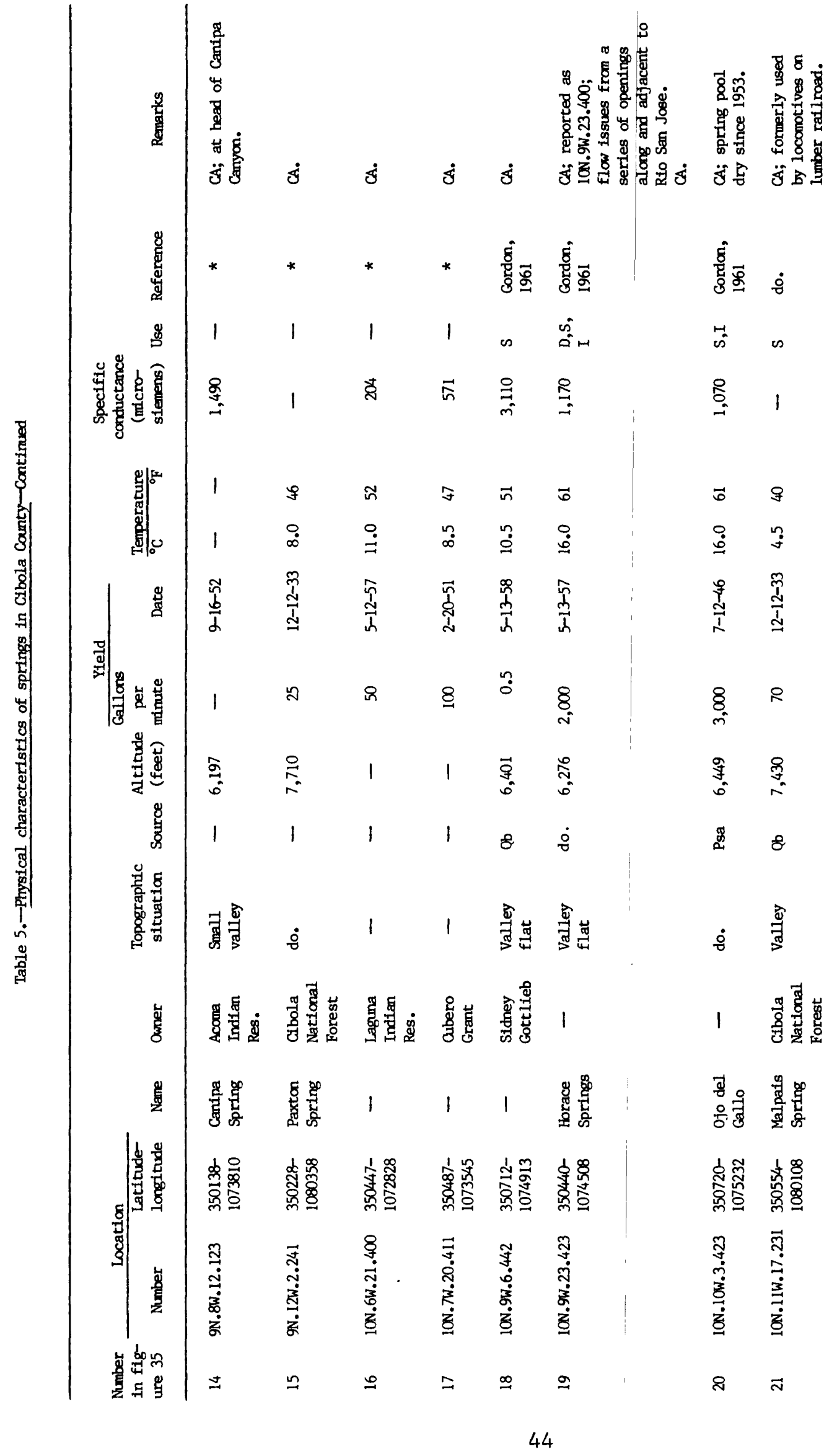




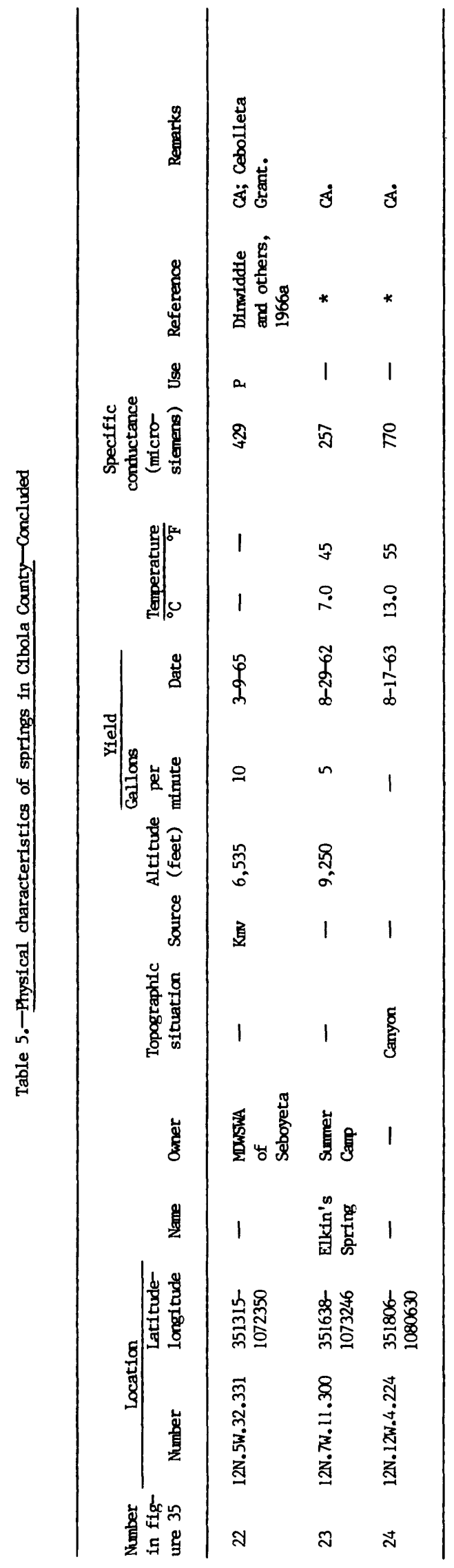




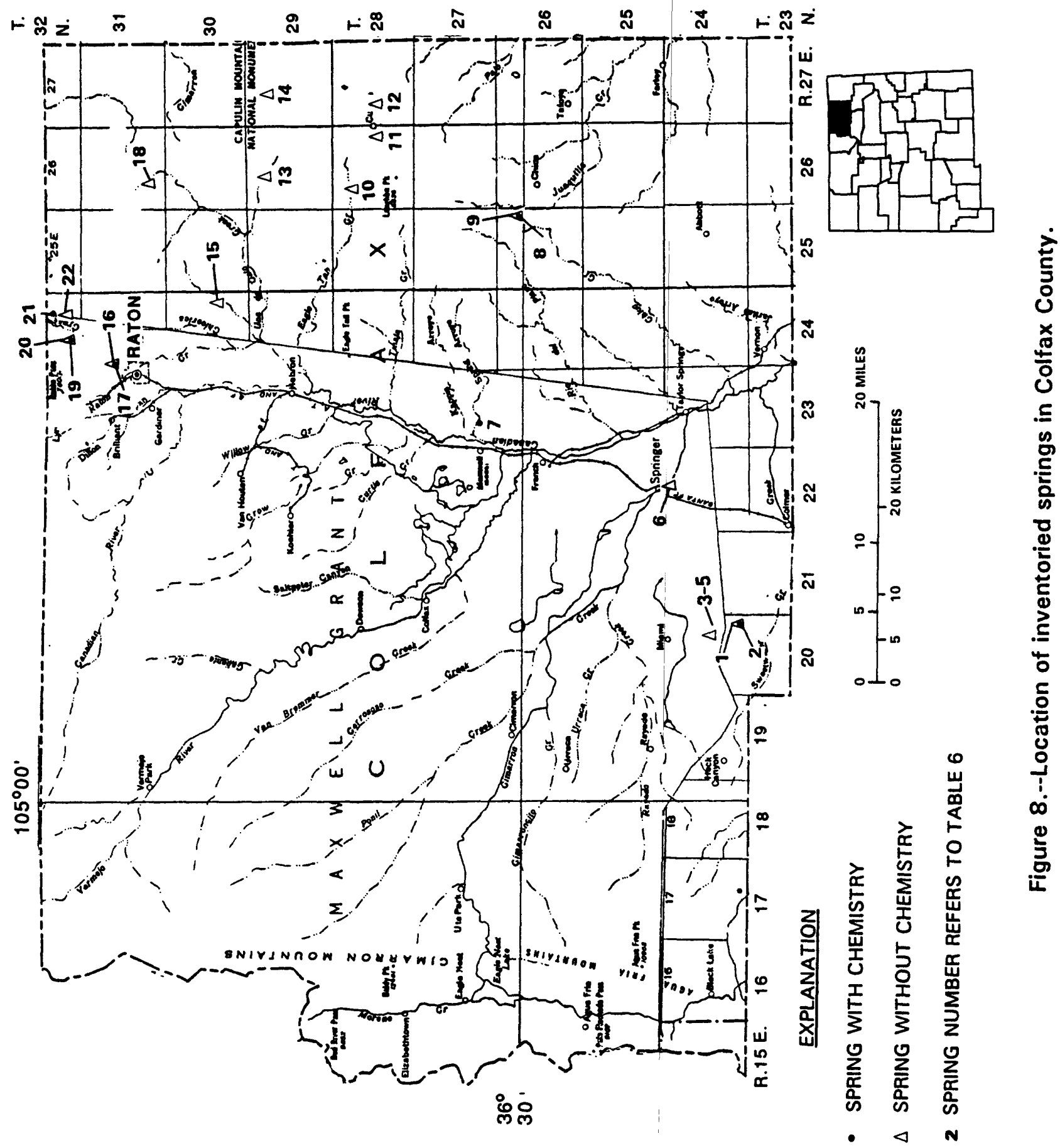




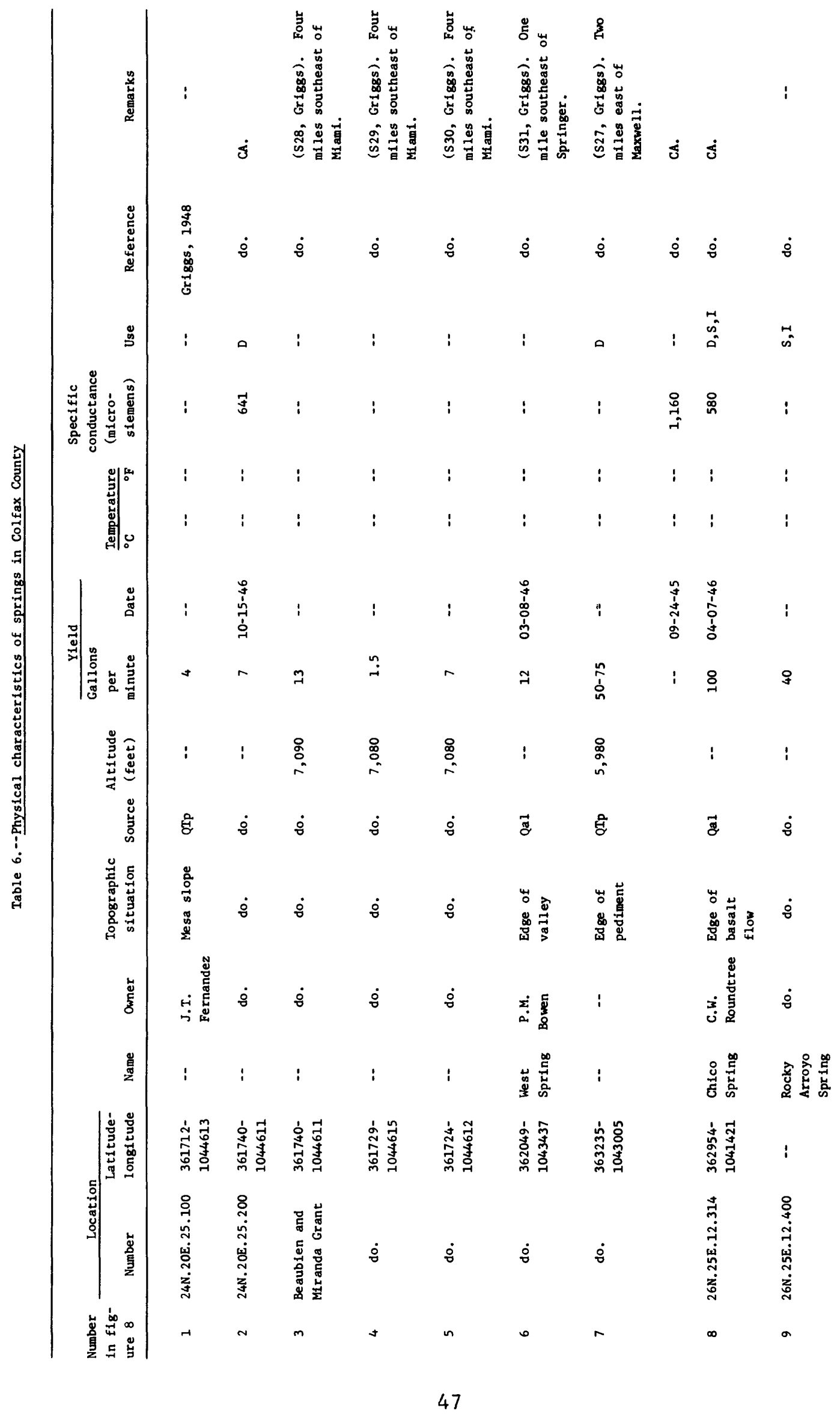




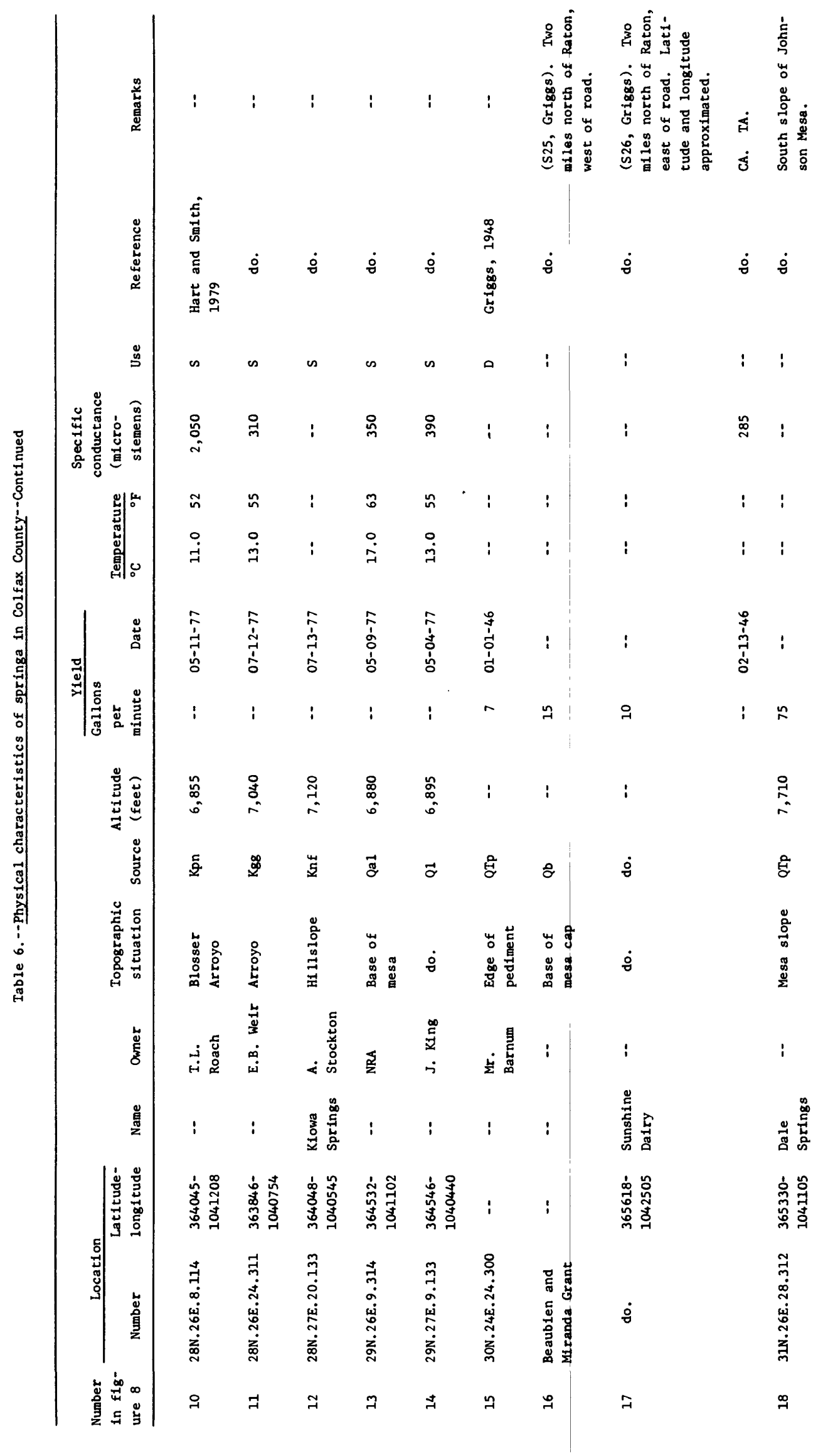




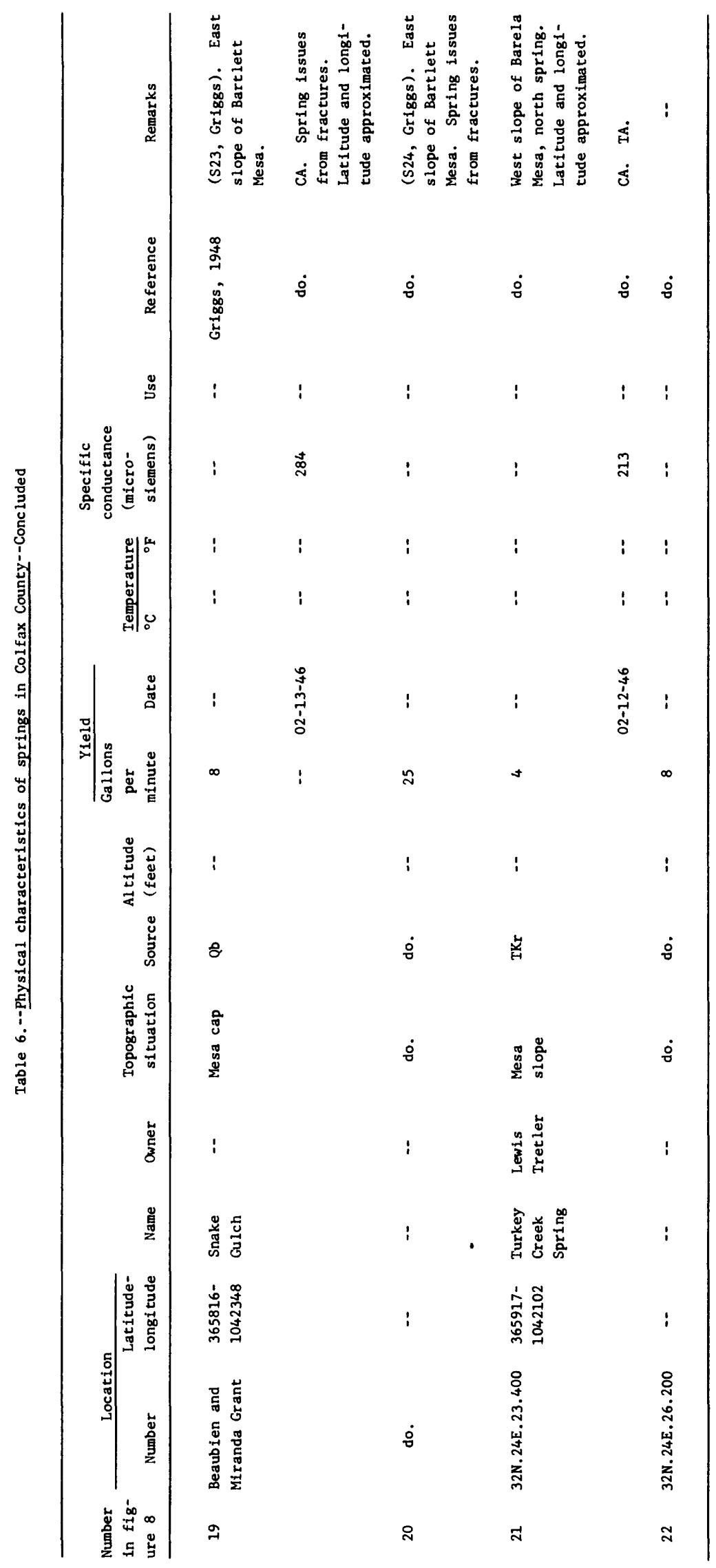




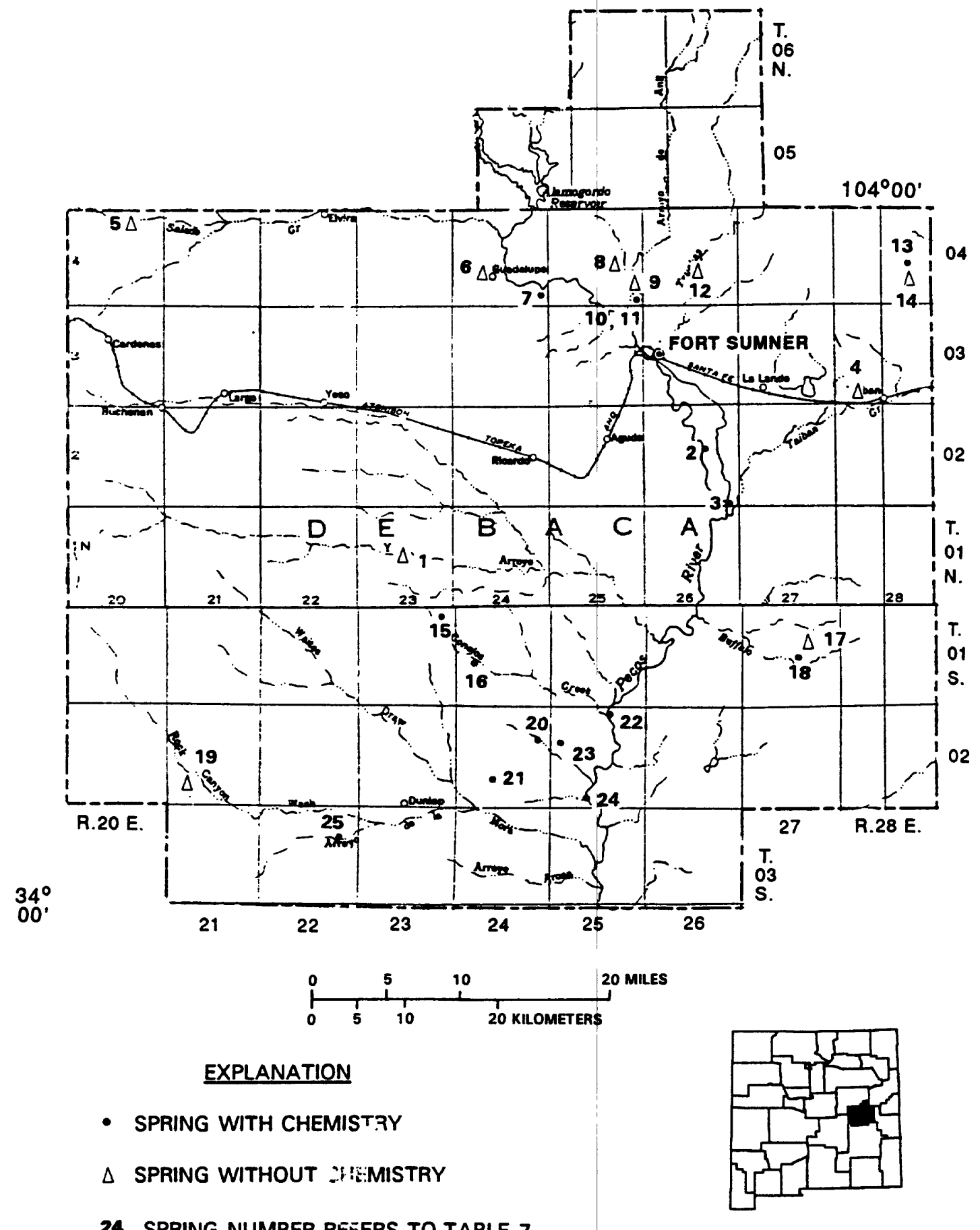

Figure 9.--Location of inventoried springs in De Baca County. 


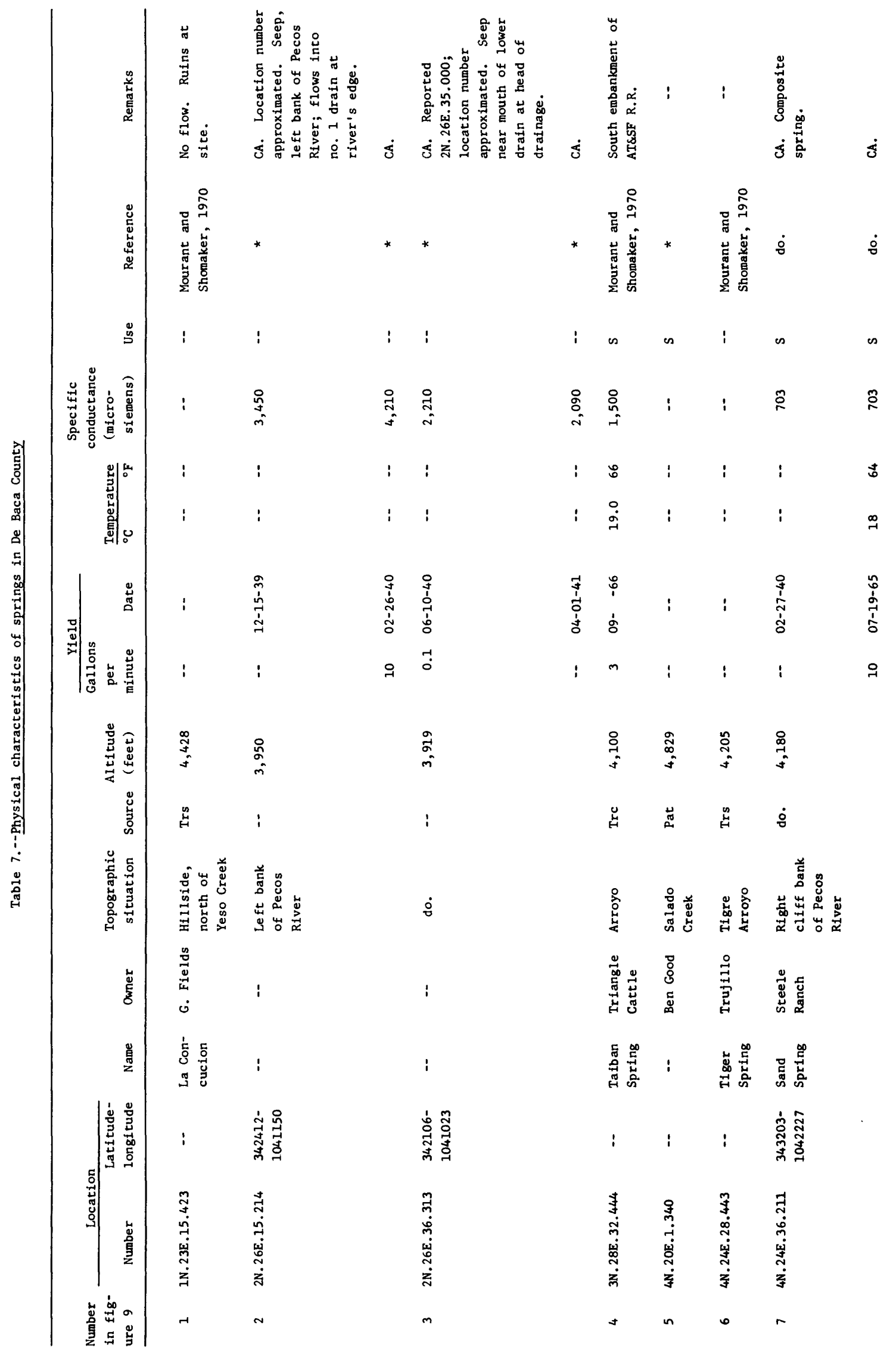




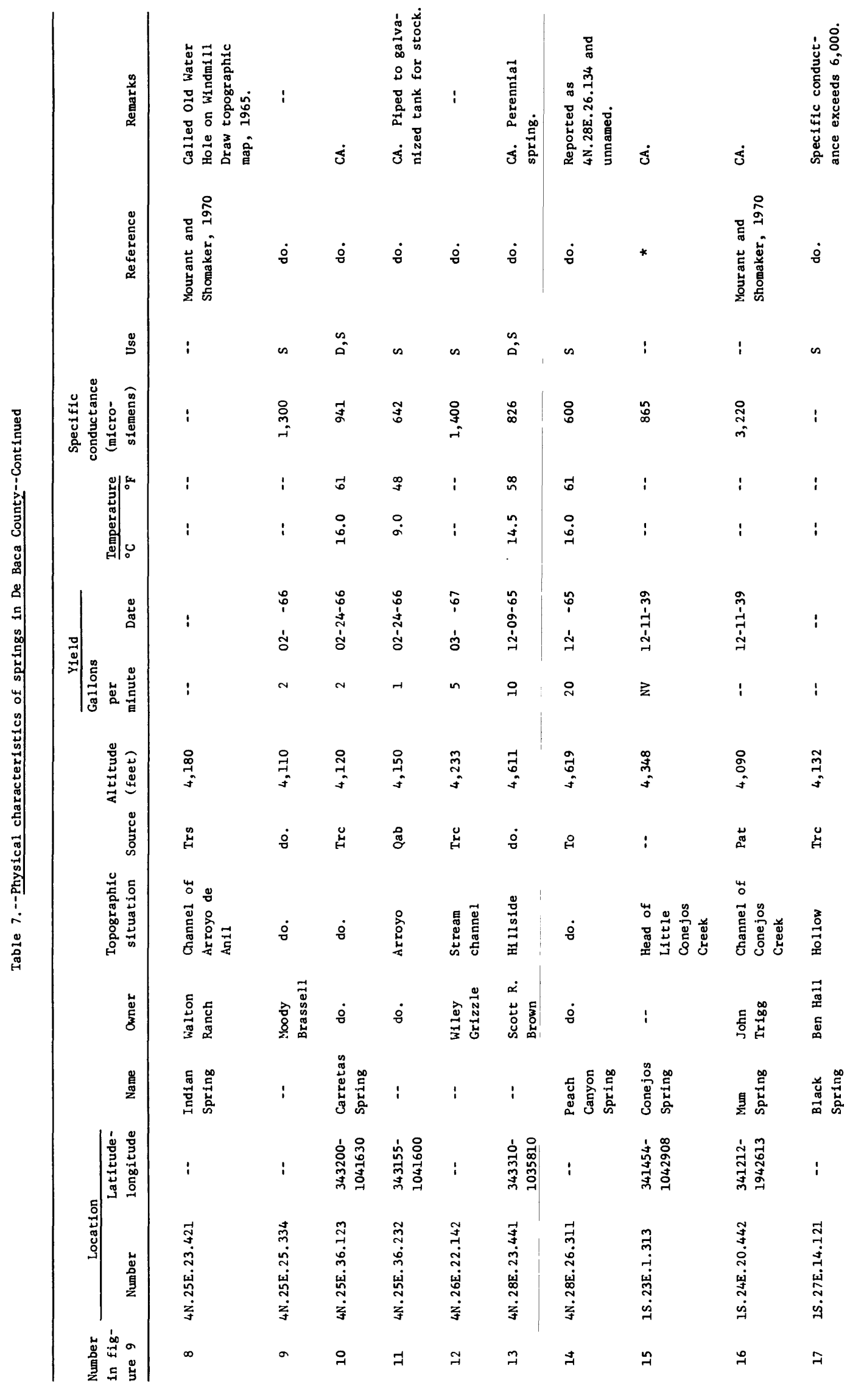




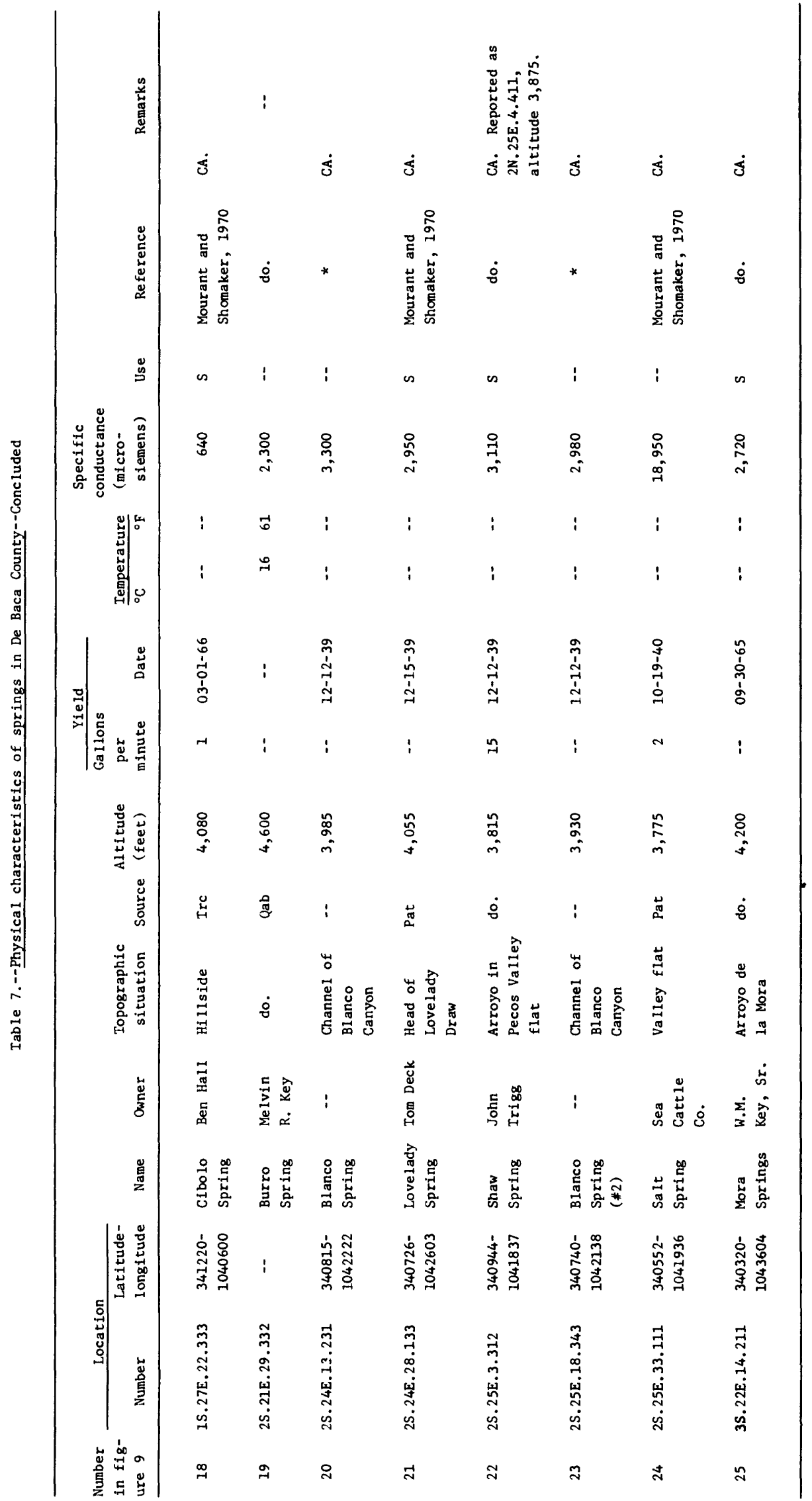


EXPLANATION

- SPRING WITH CHEMISTRY

7 SPRING NUMBER REFERS

TO TABLE 8

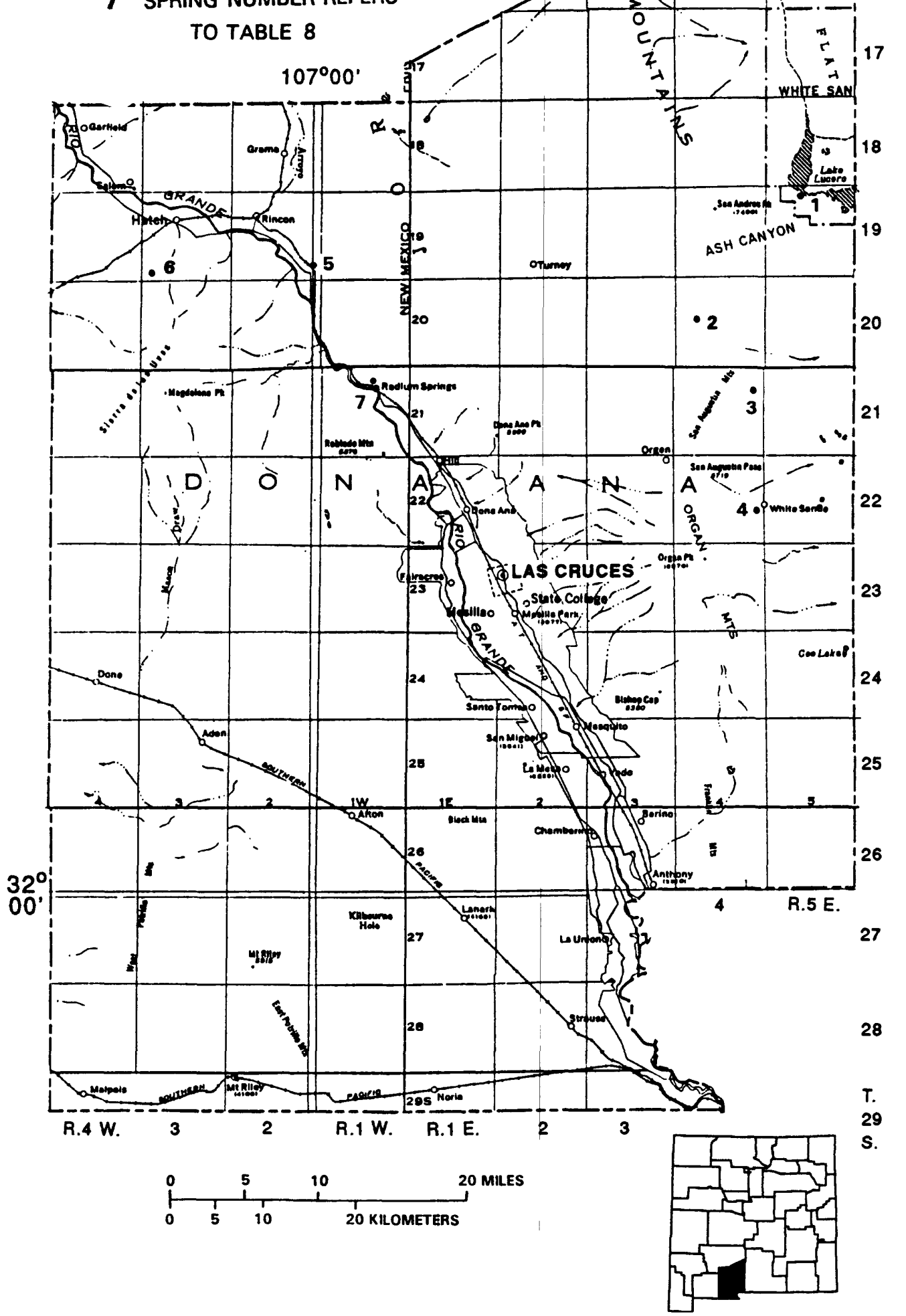

Figure 10.--Location of inventoried springs in Doña Ana County. 


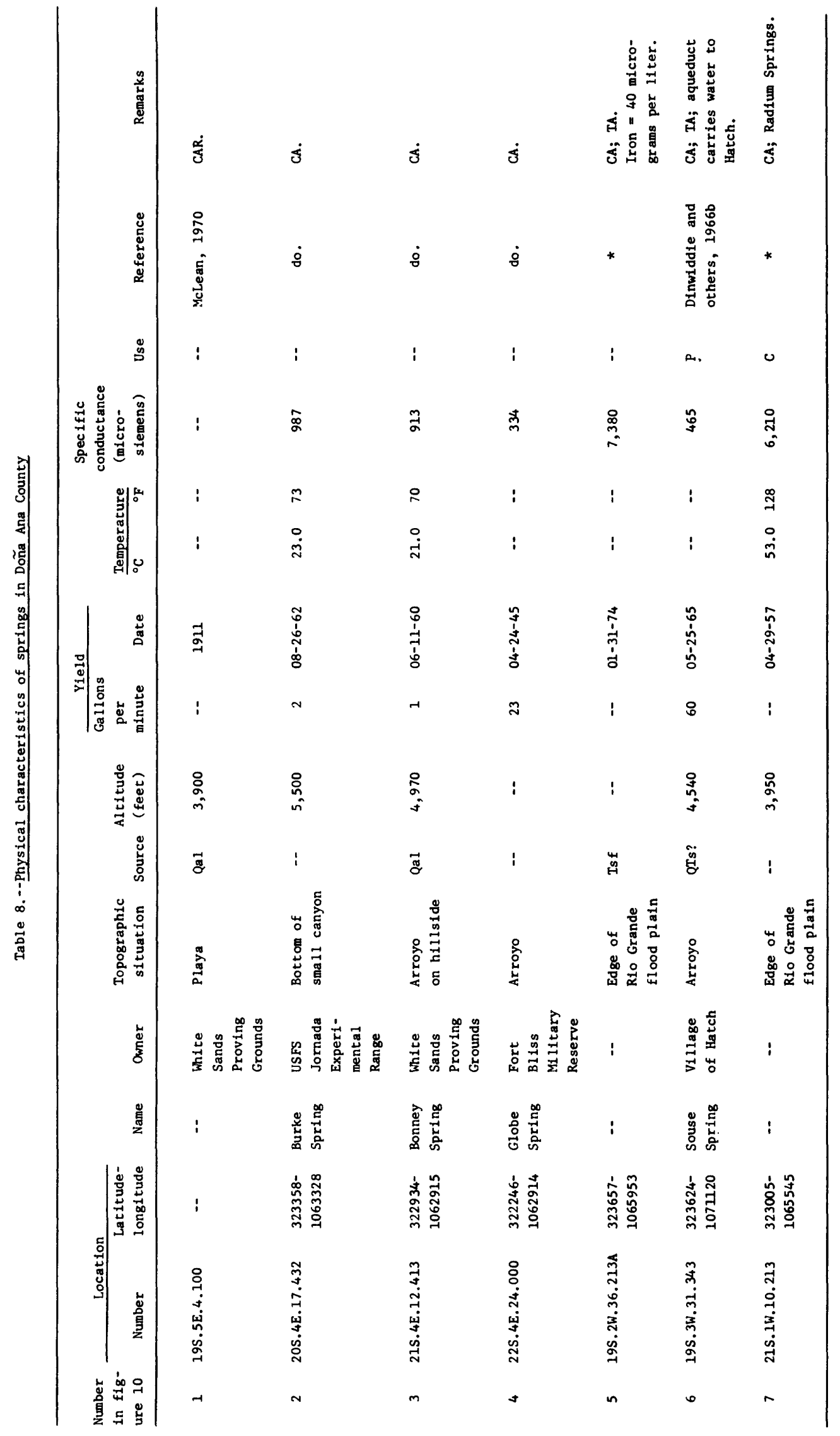




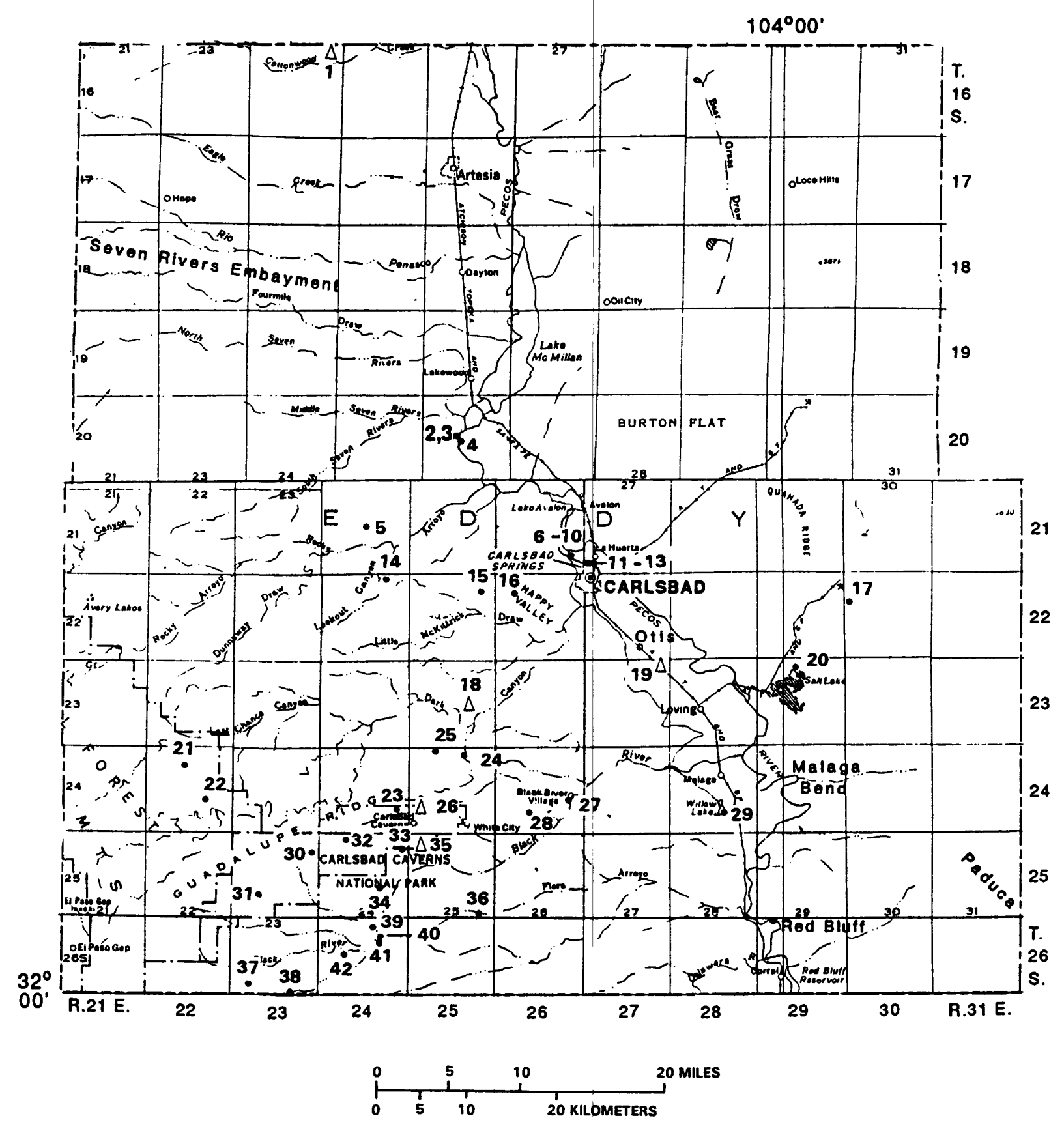

EXPLANATION

- SPRING WITH CHEMISTRY

$\triangle$ SPRING WITHOUT CHEMISTRY

38 SPRING NUMBER REFERS TO TABLE 9

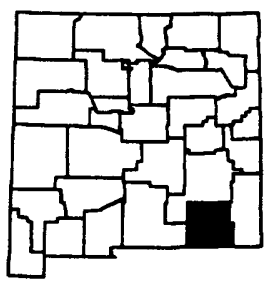

Figure 11.--Location of inventoried springs in Eddy County. 


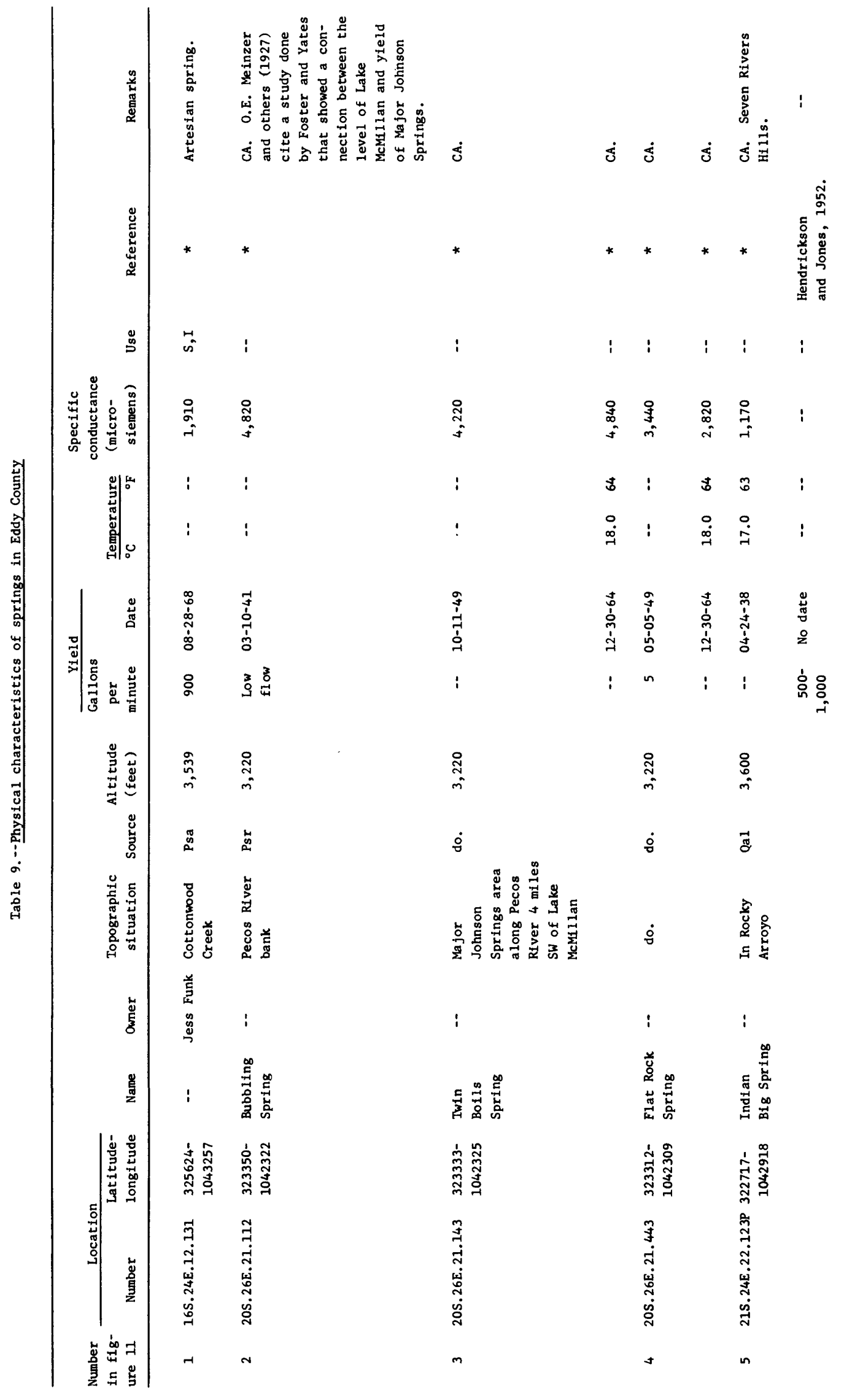




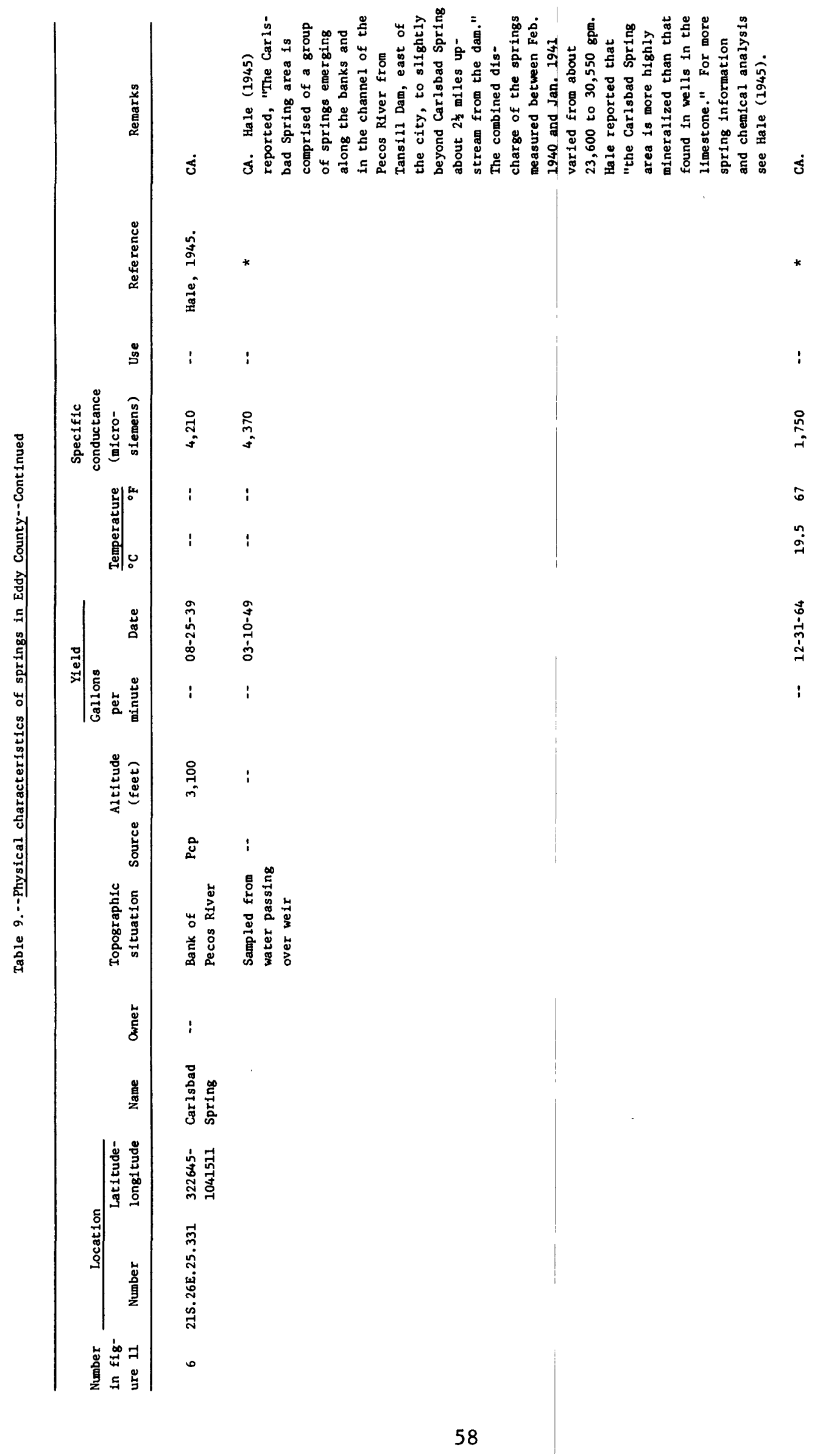




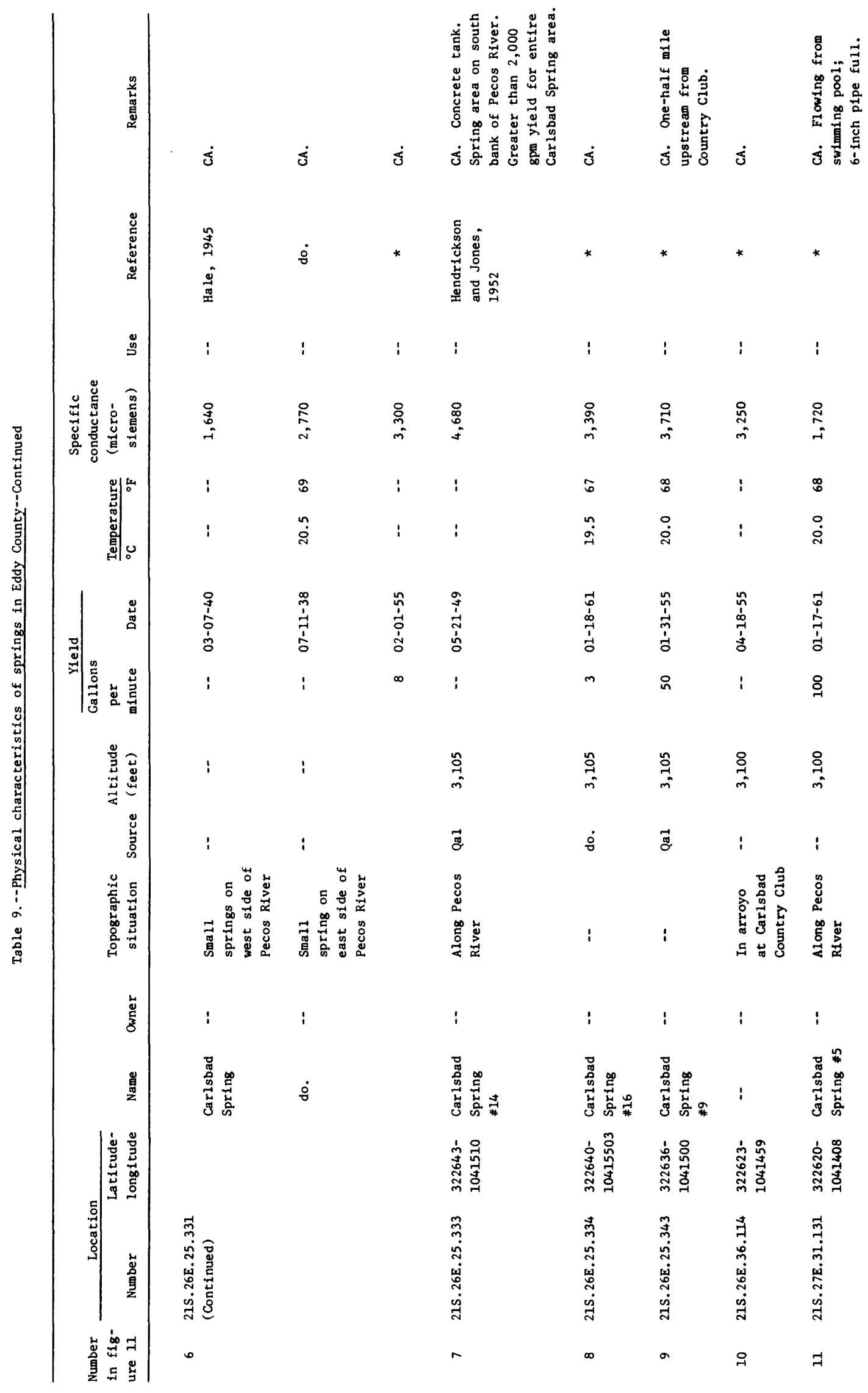




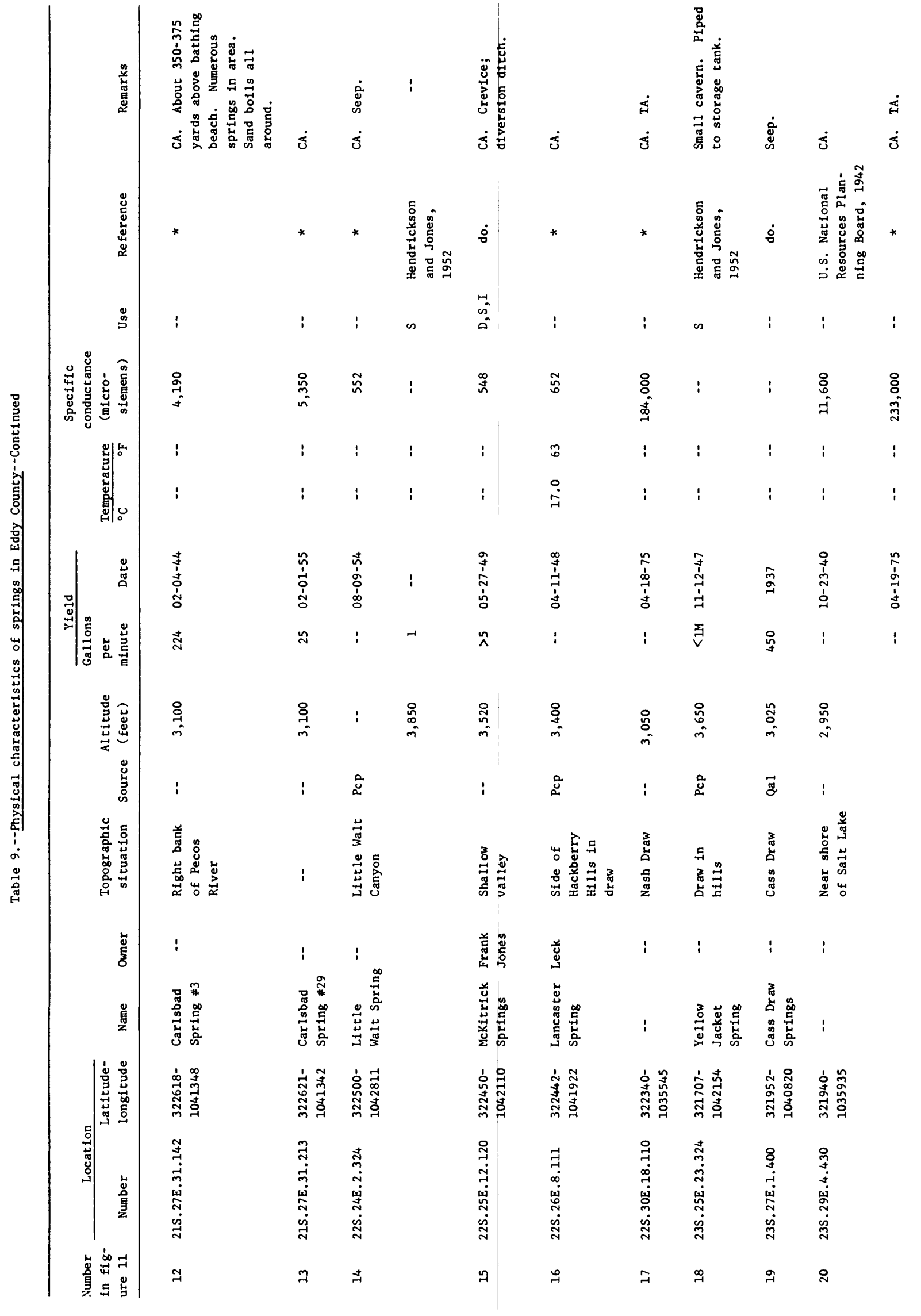




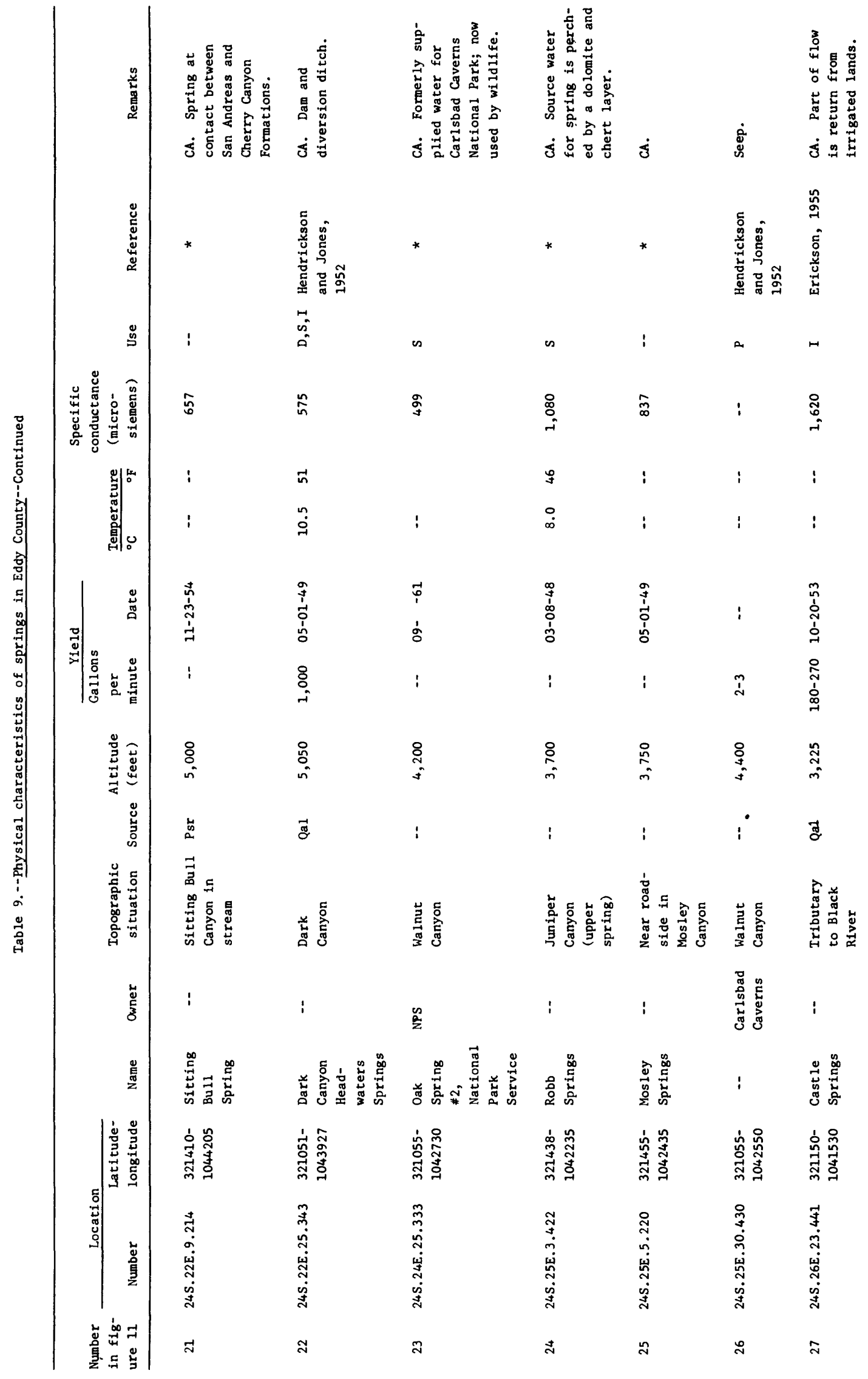




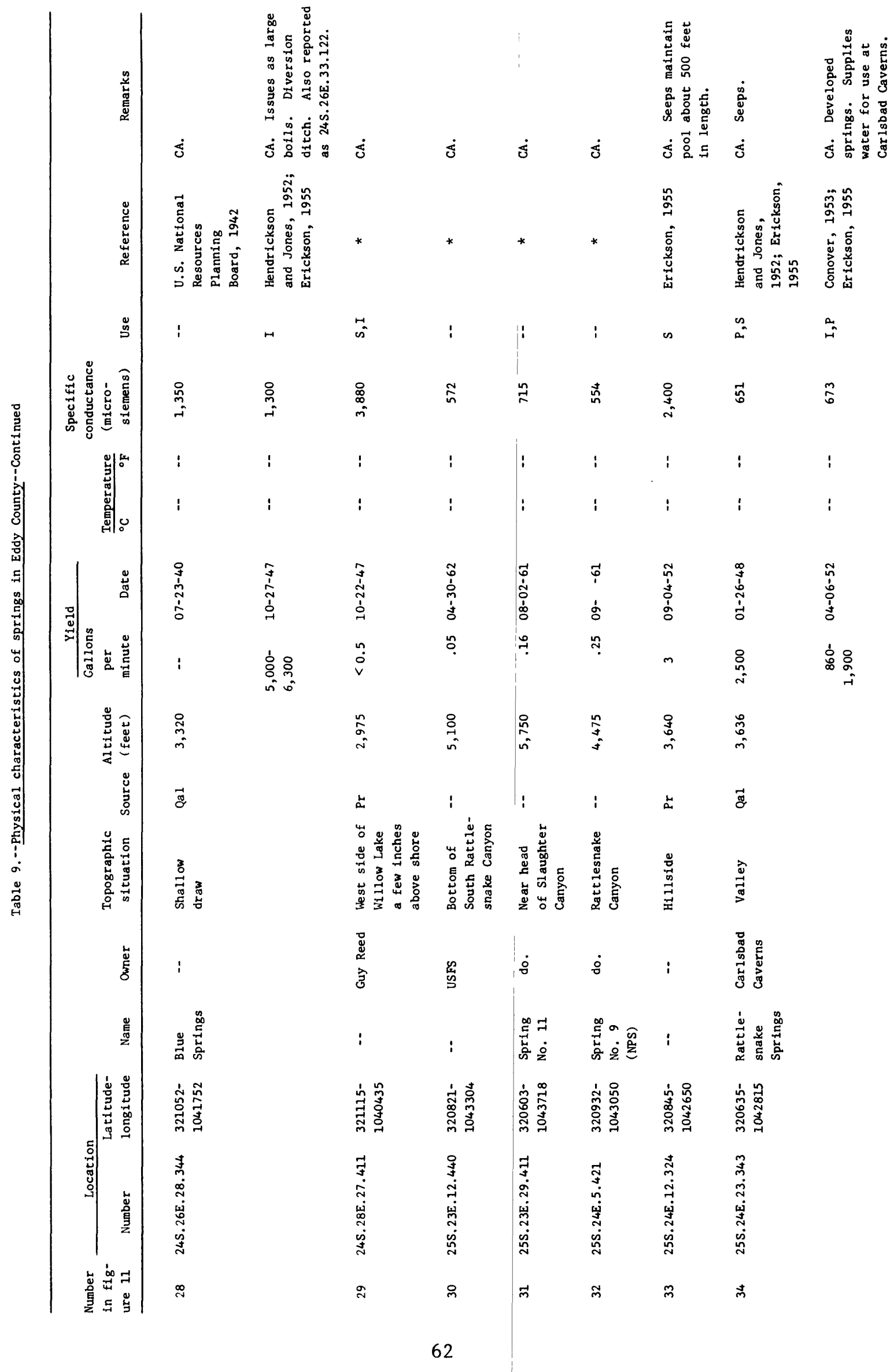




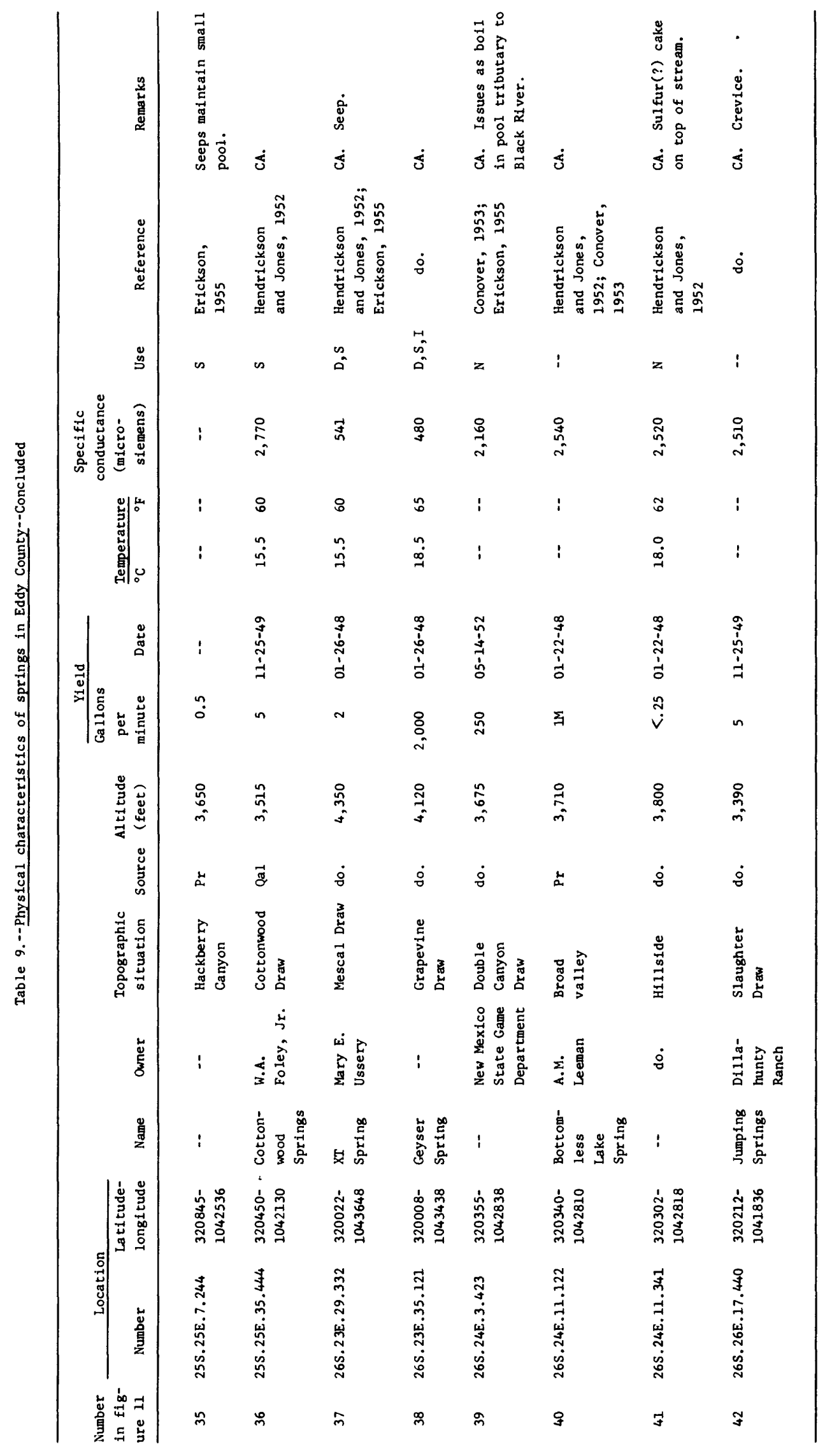




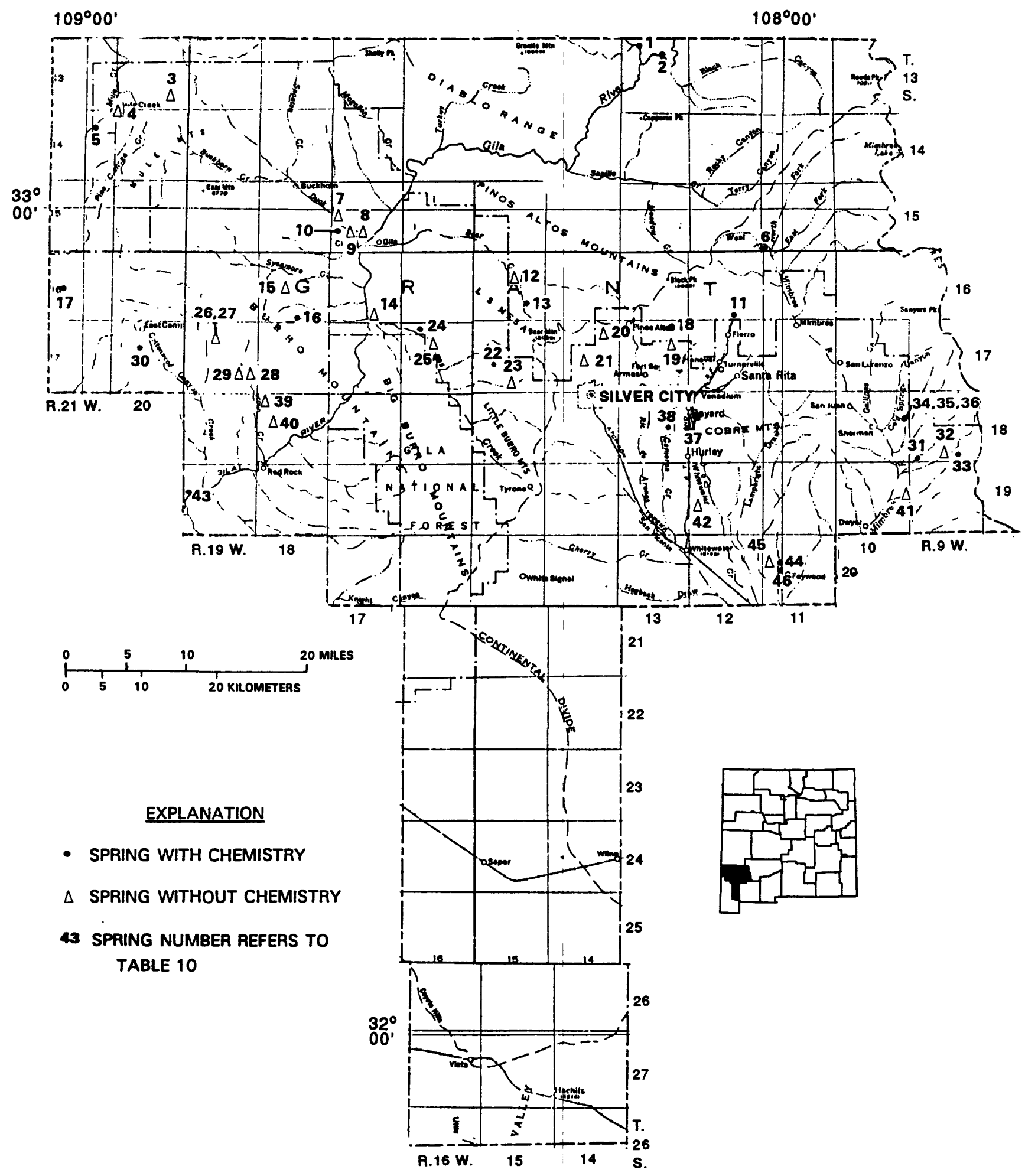

Figure 12.--Location of inventoried springs in Grant County. 


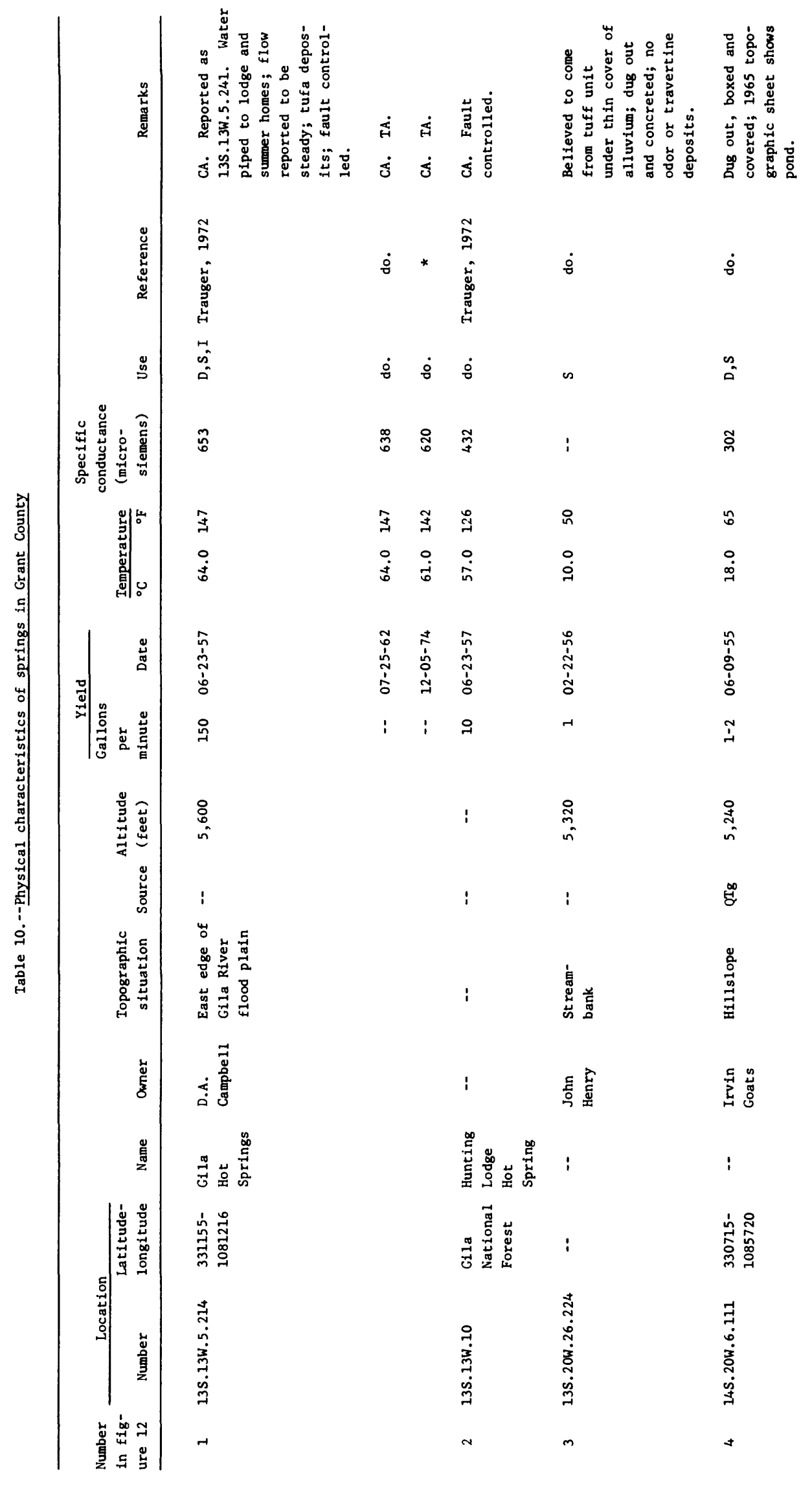




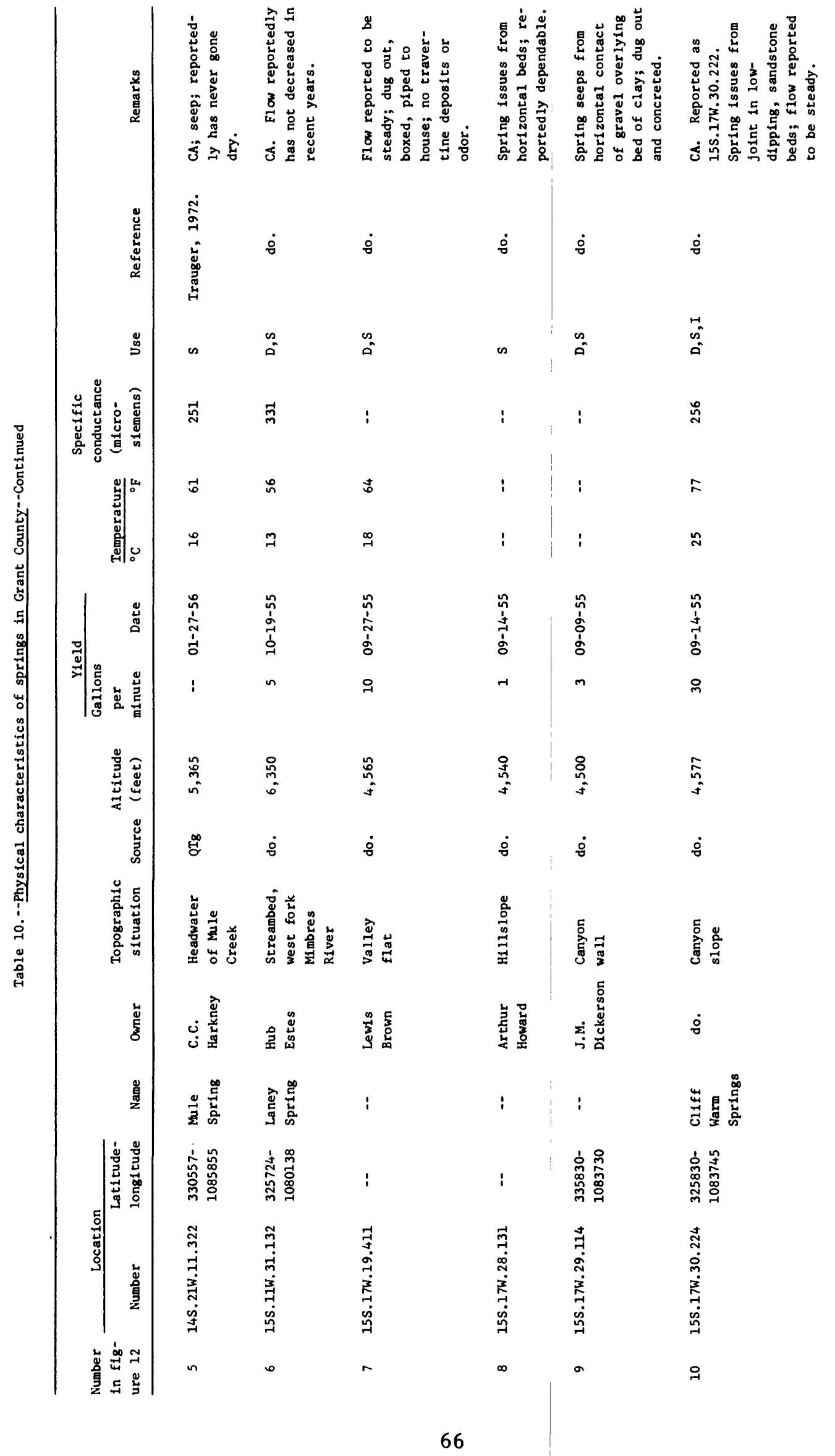




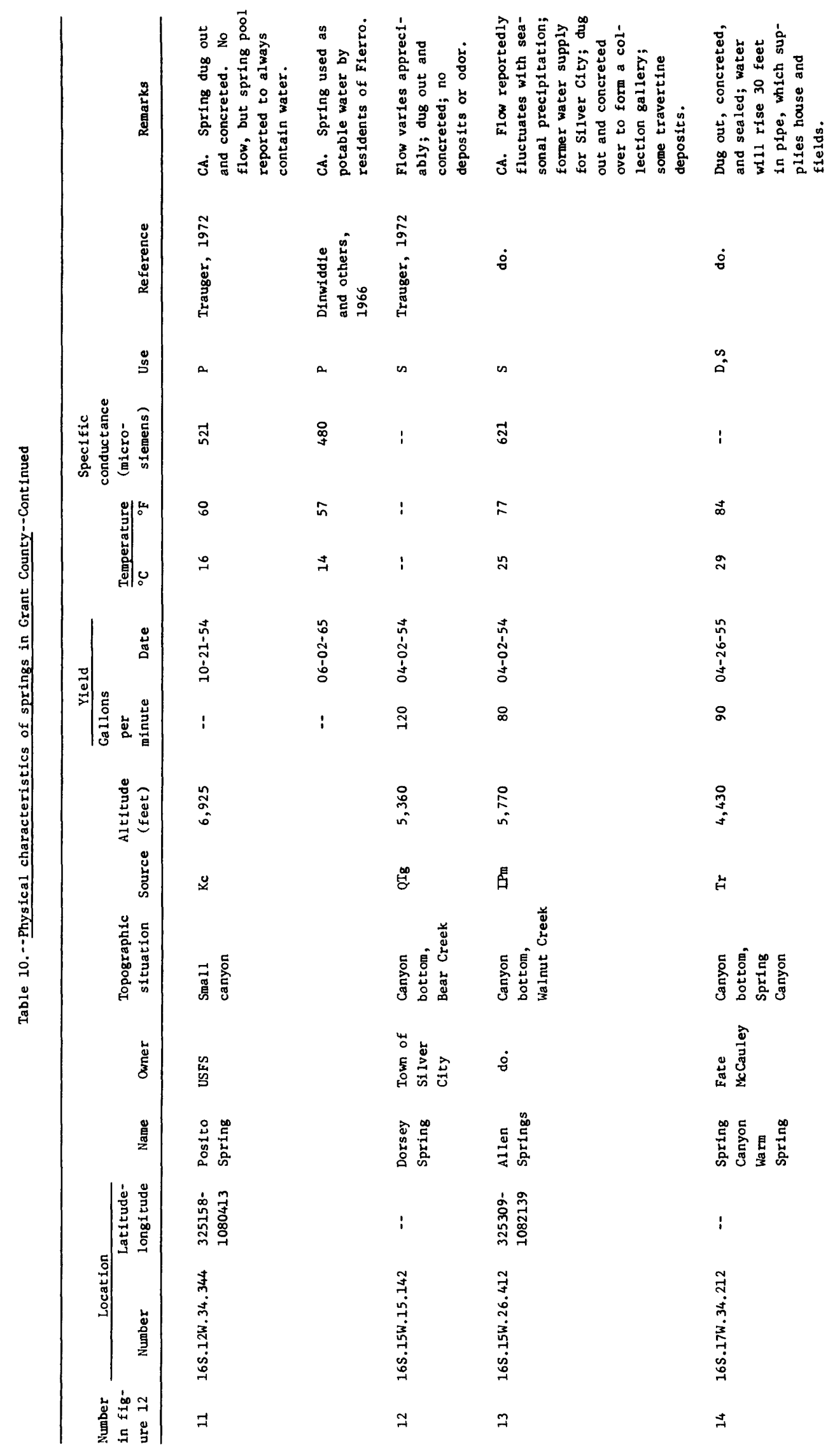




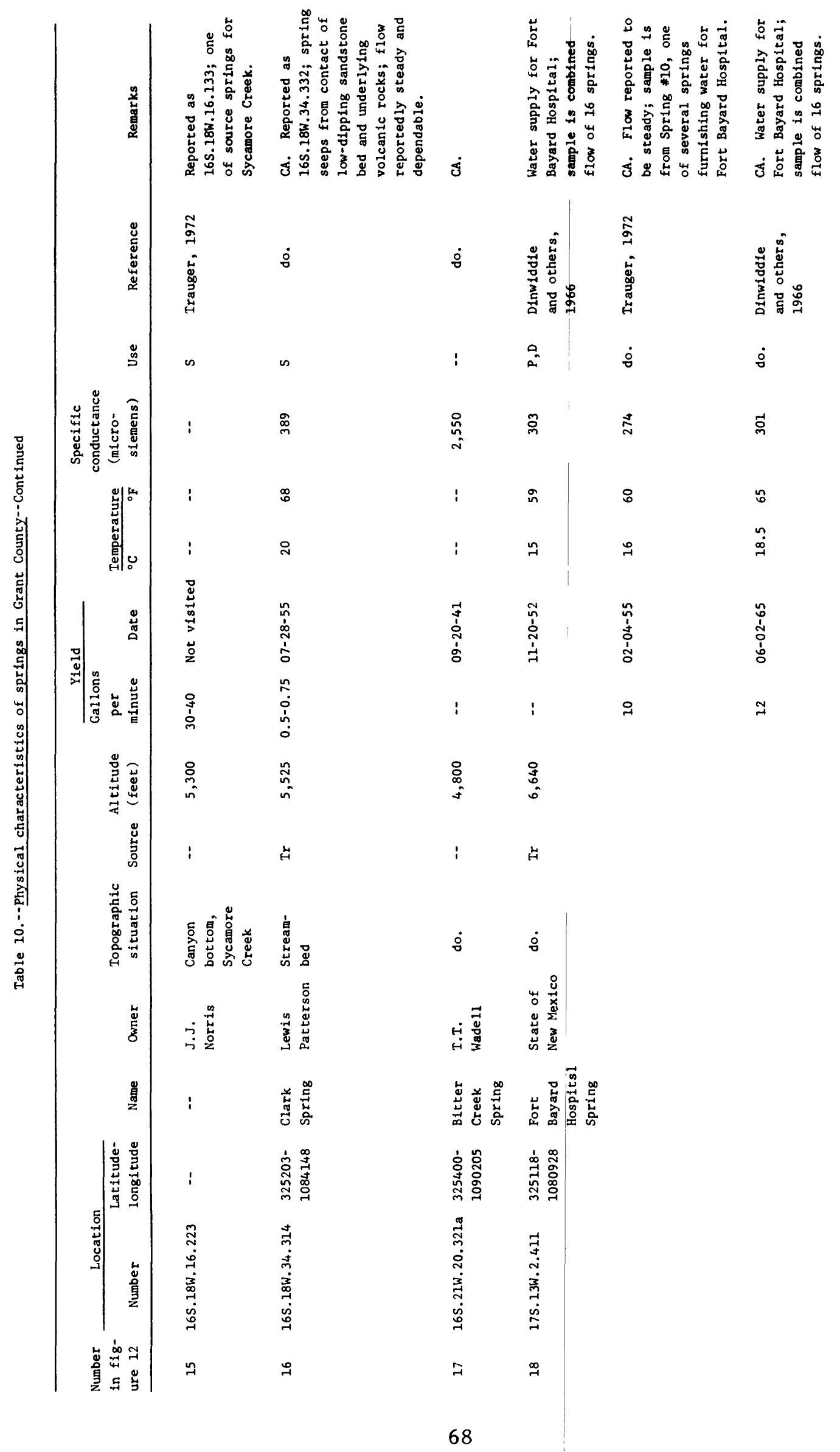




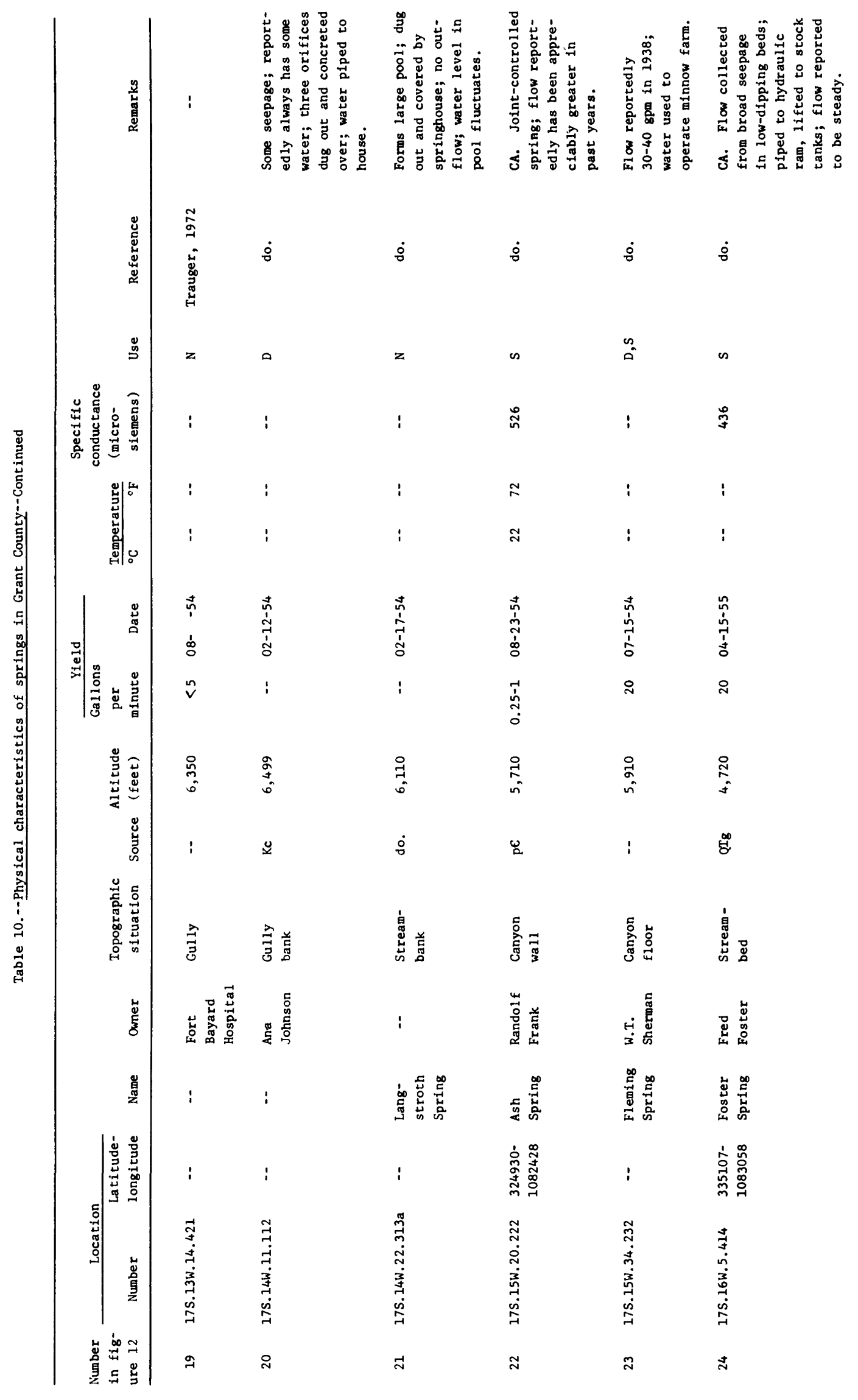




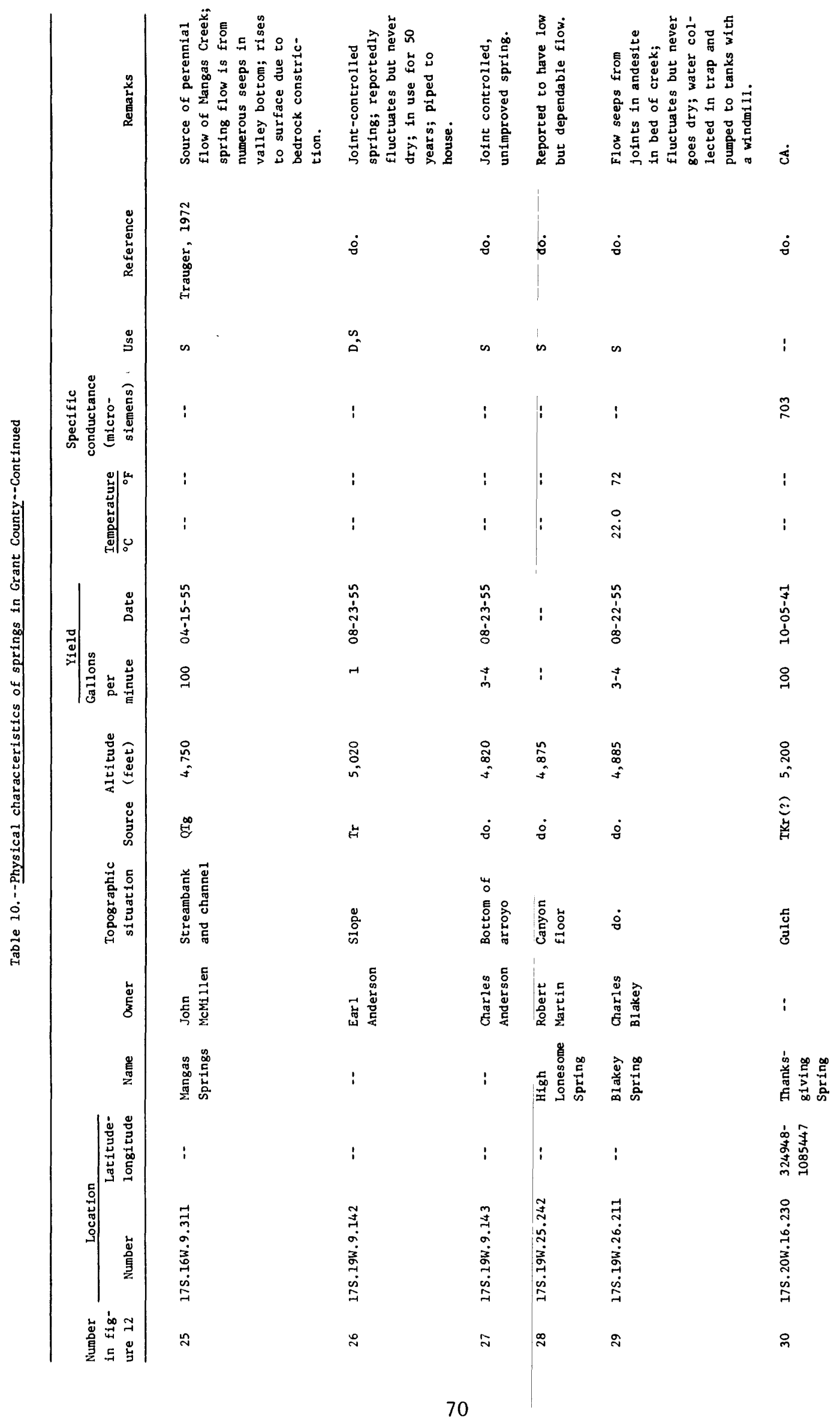




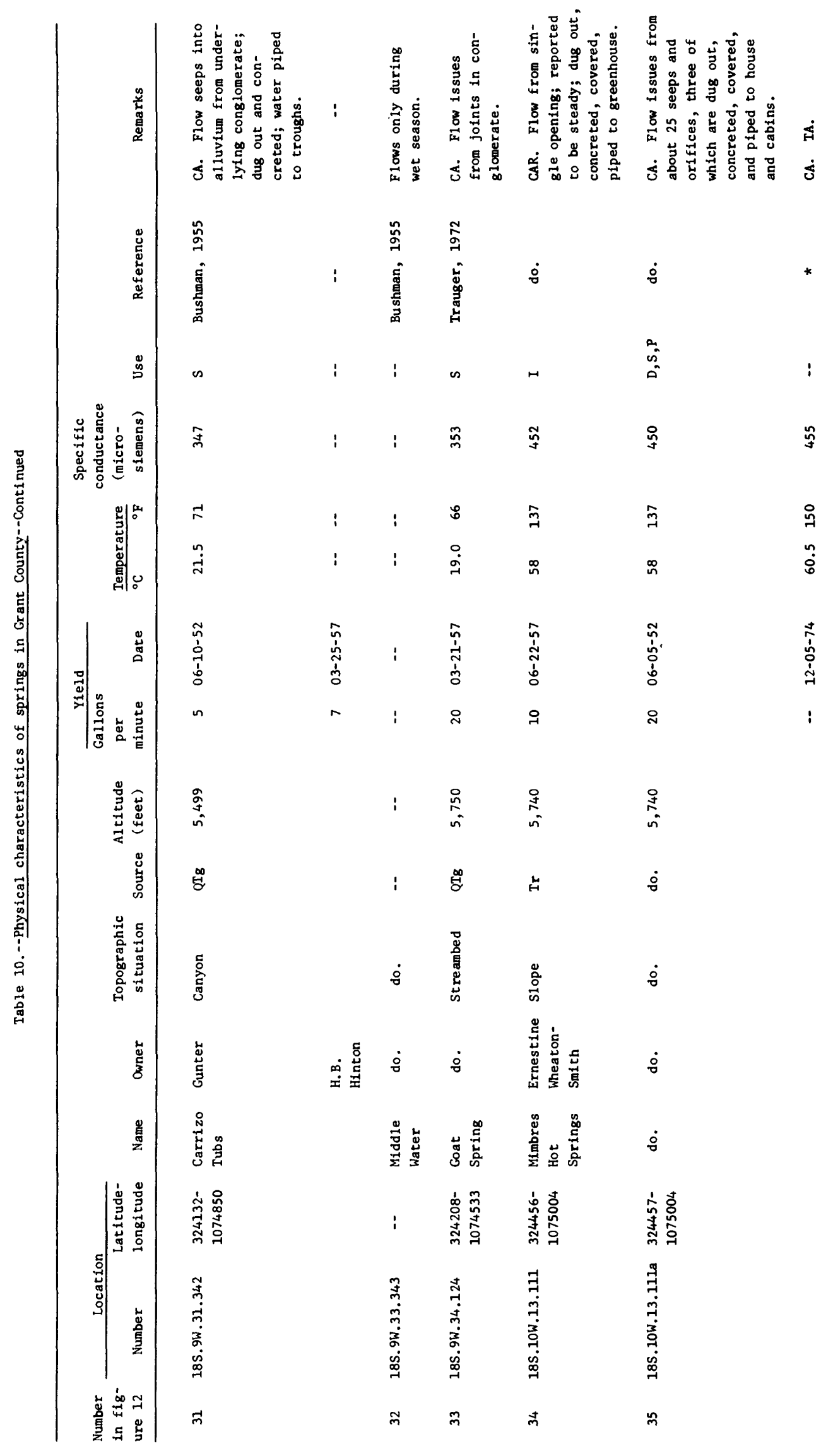




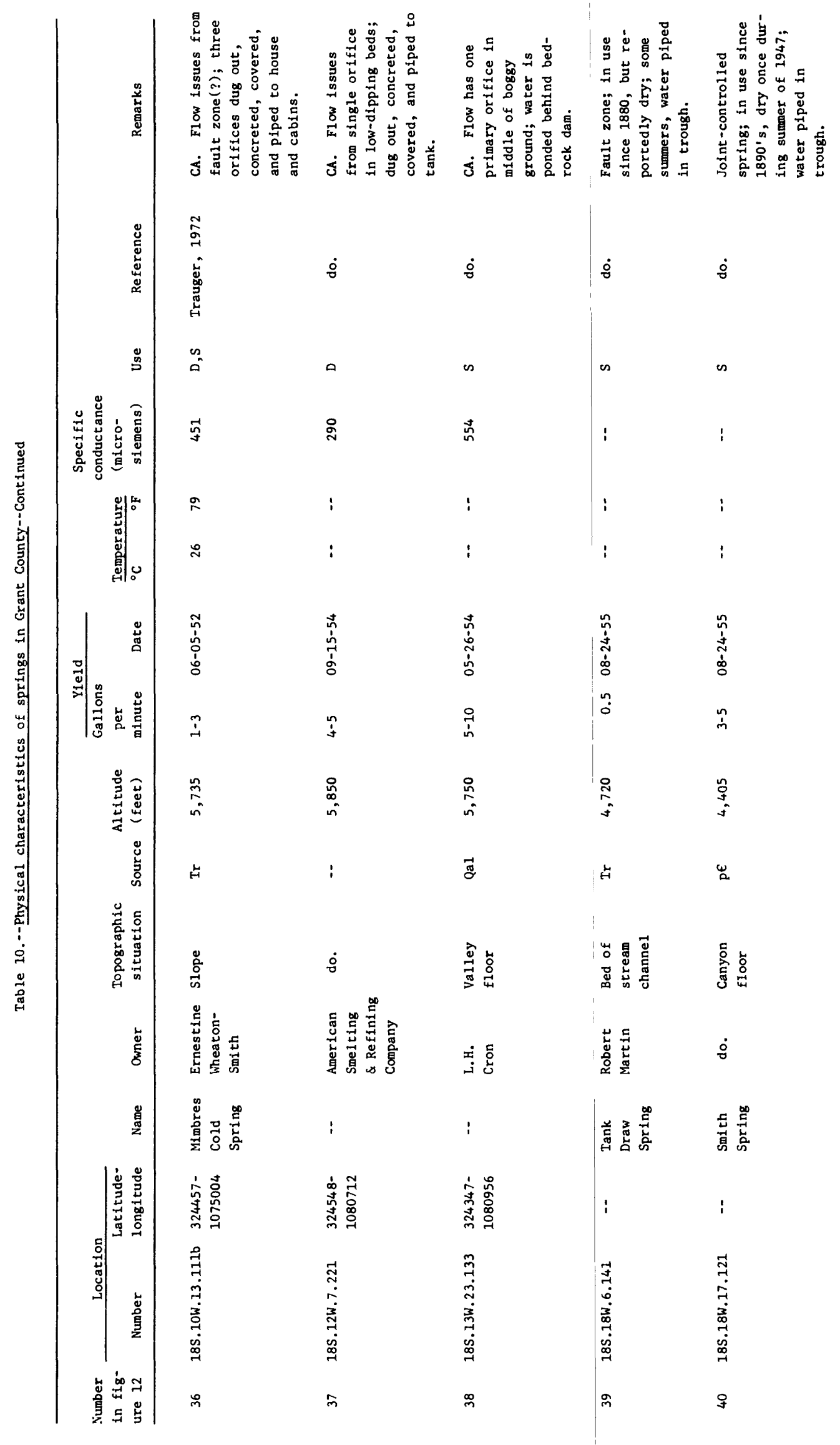




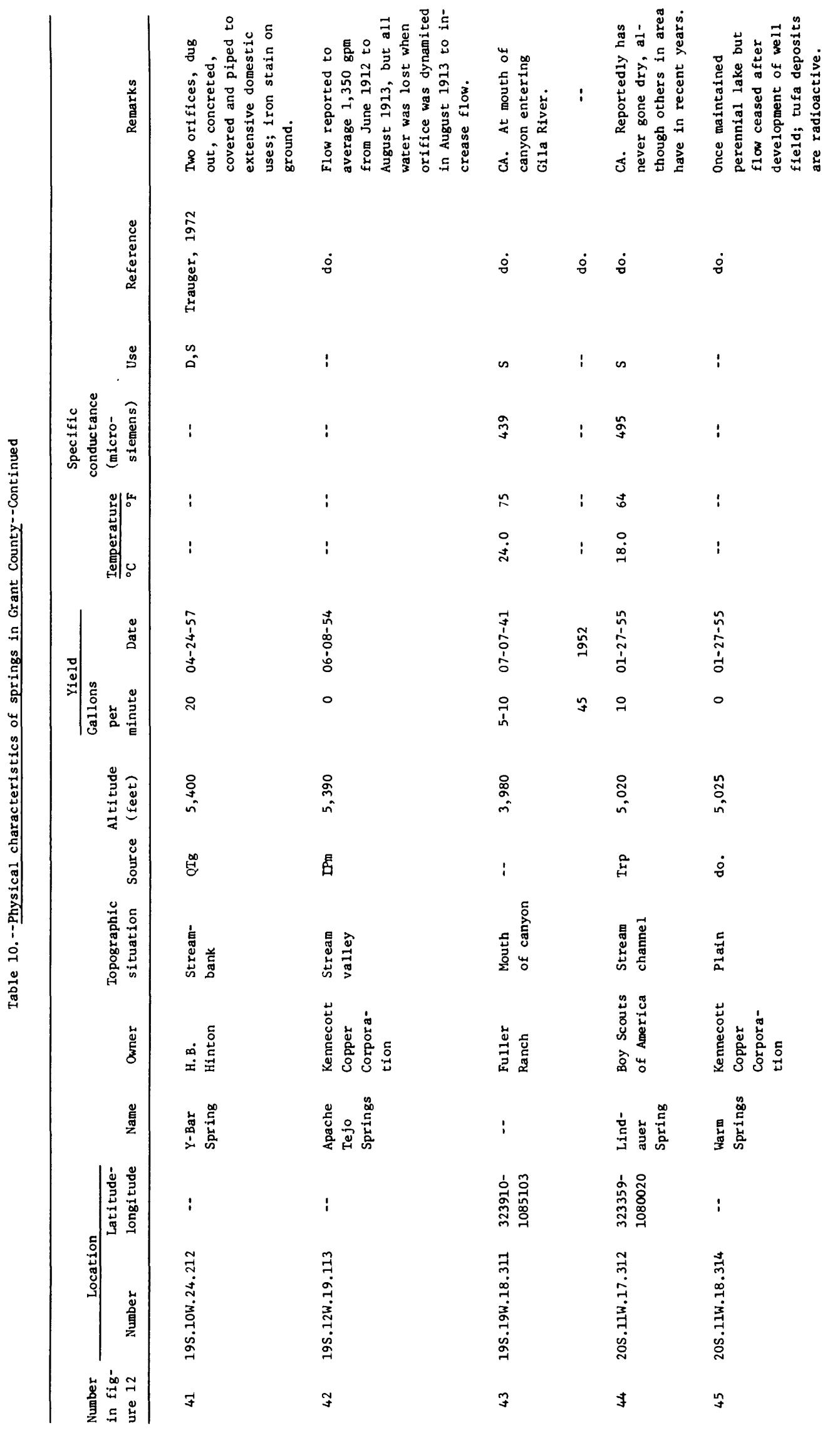




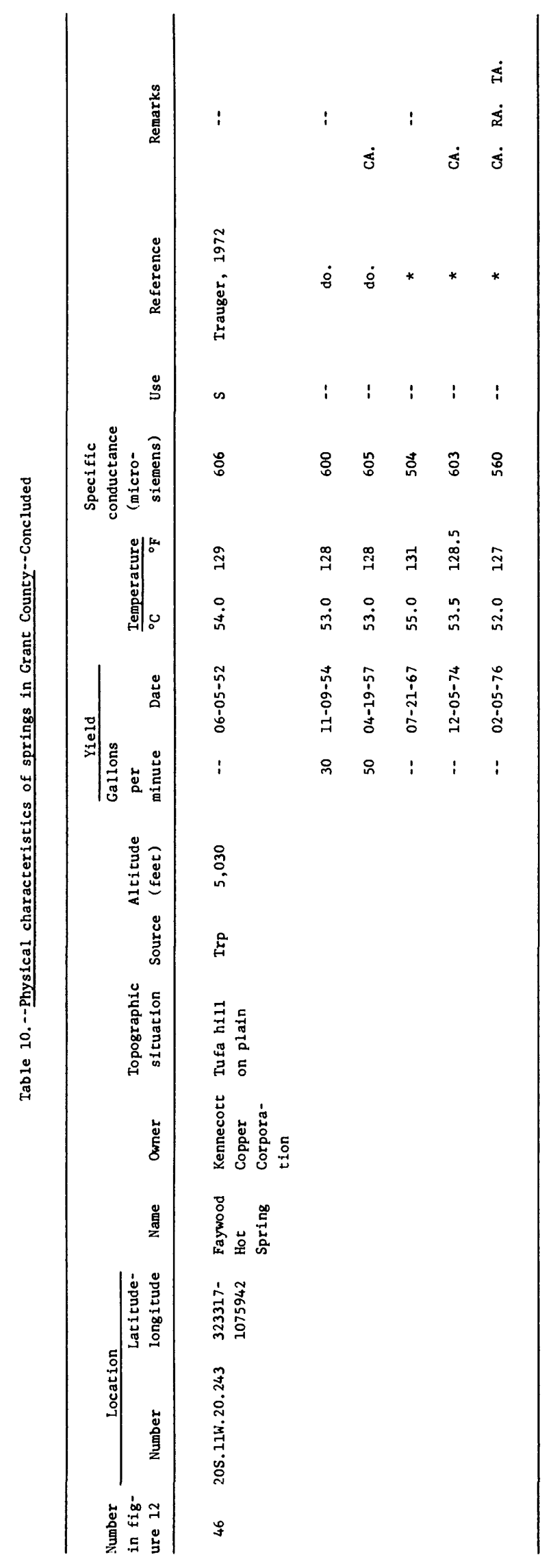




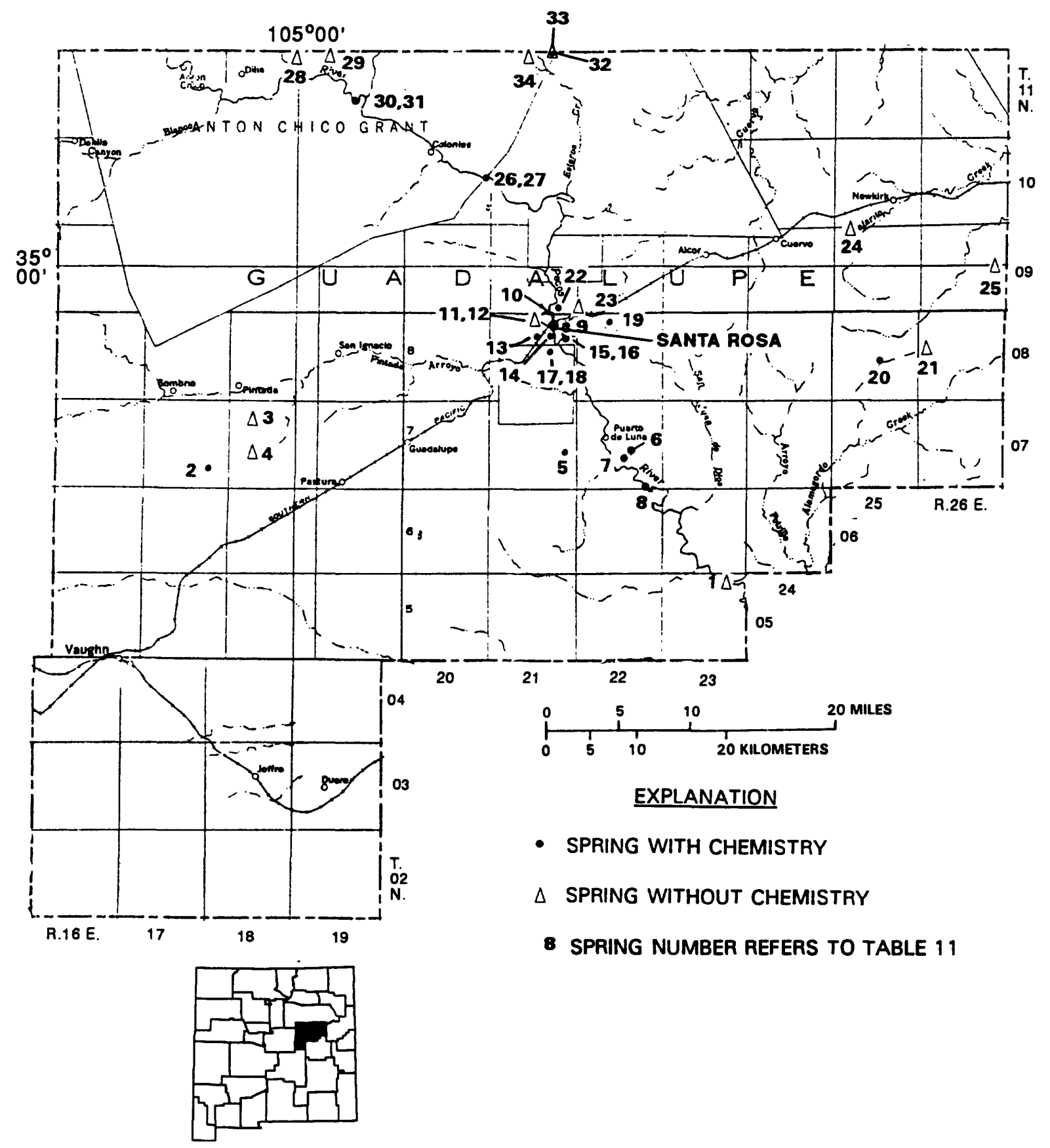

Figure 13.--Location of inventoried springs in Guadalupe County. 


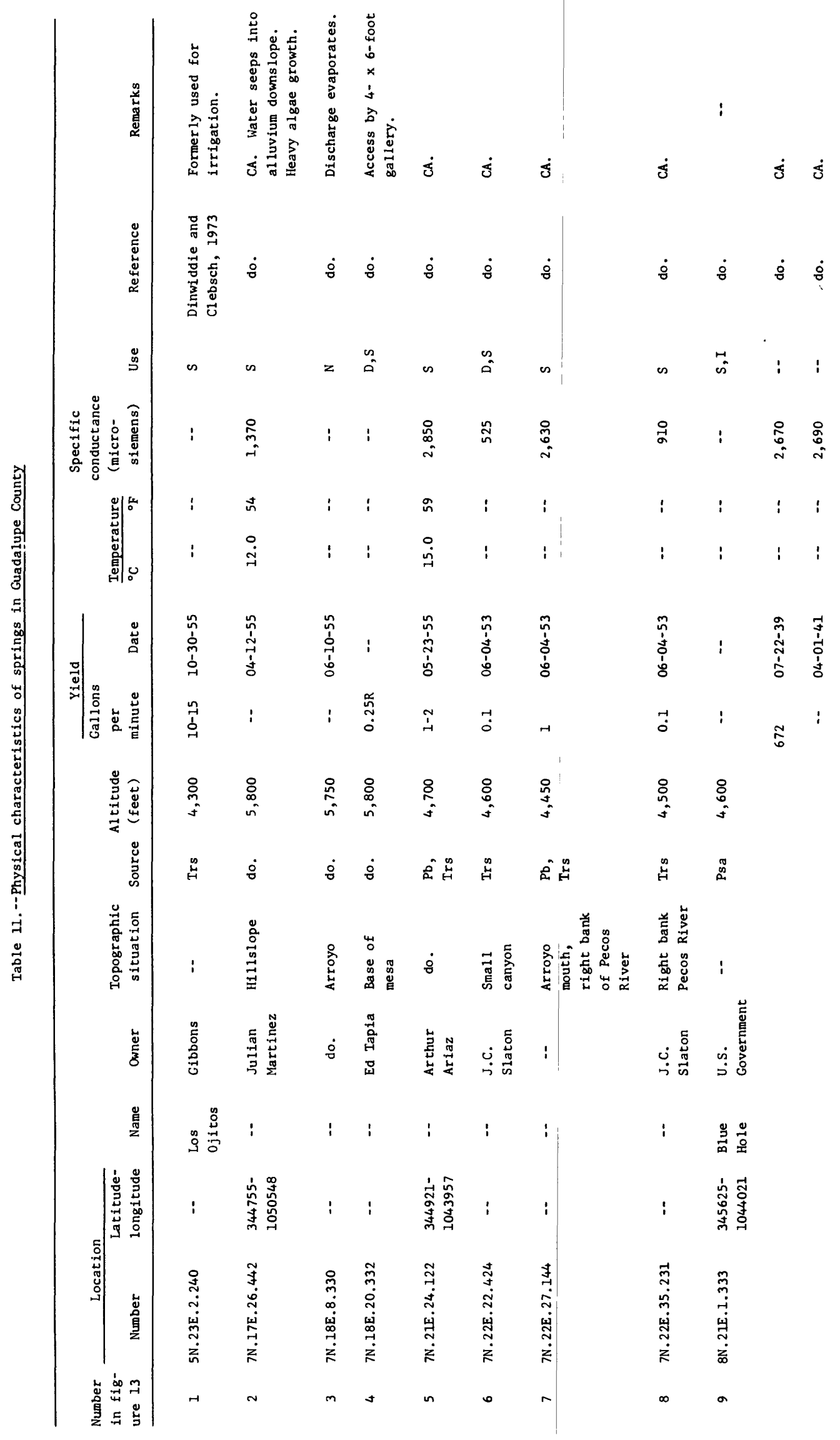




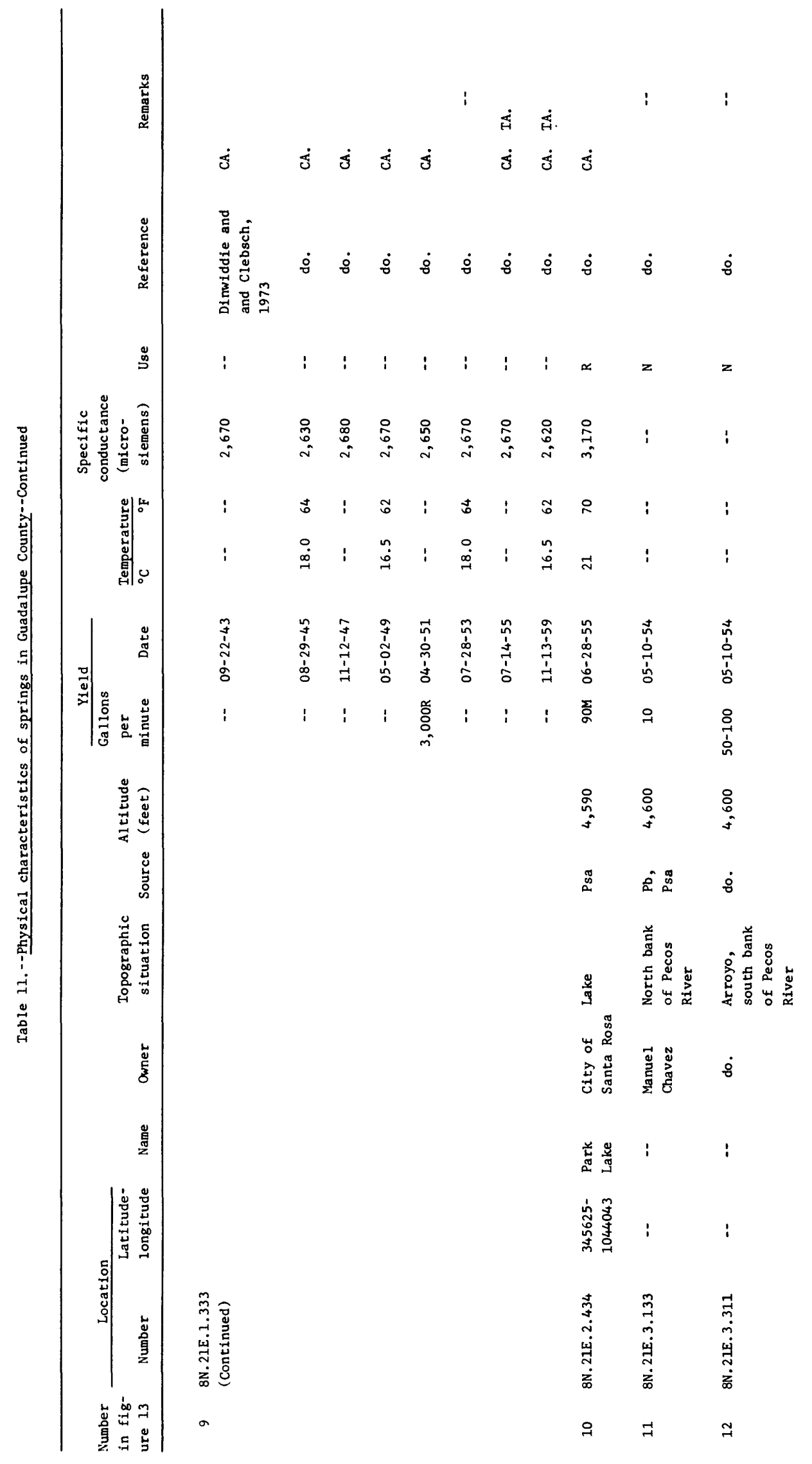




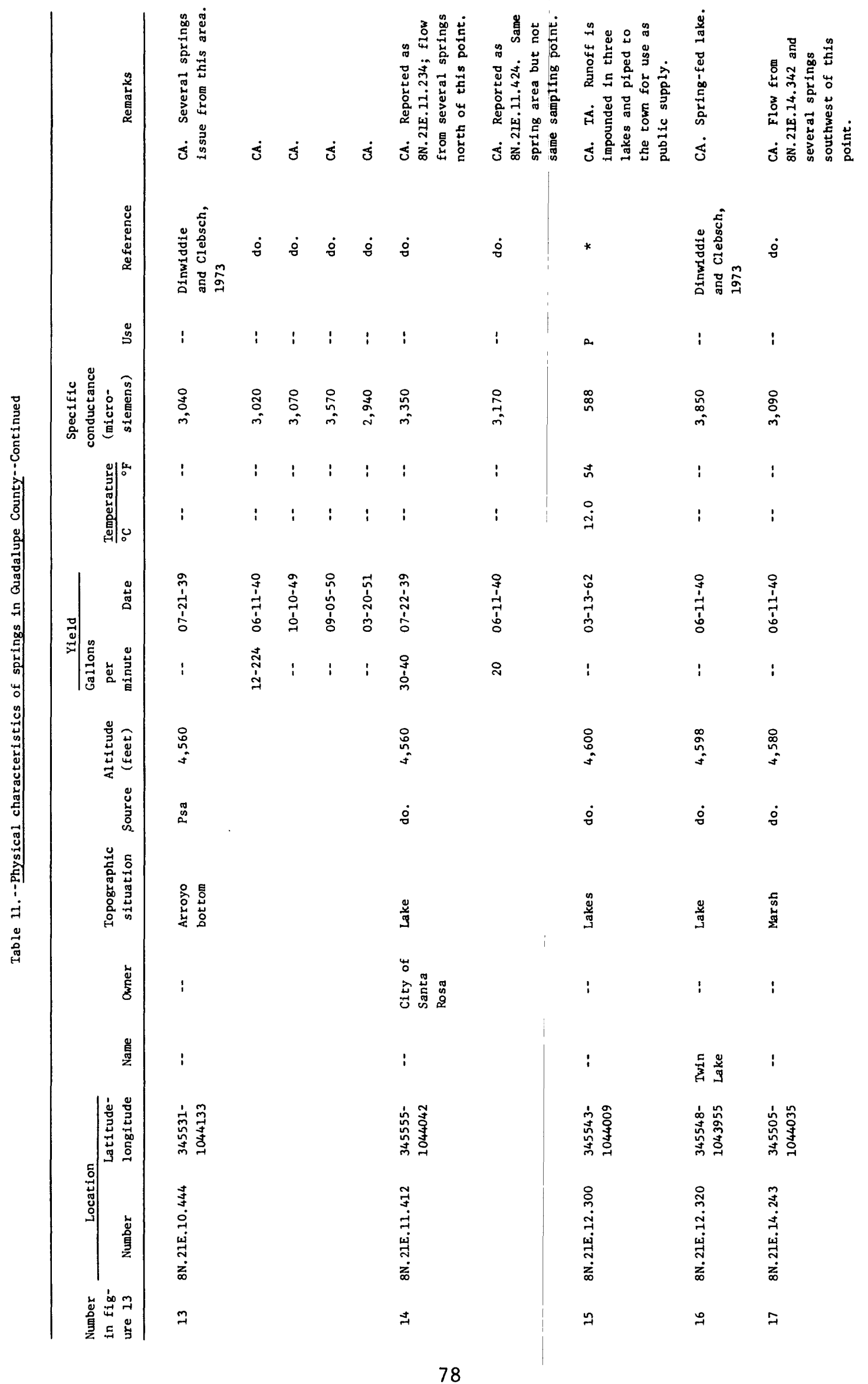




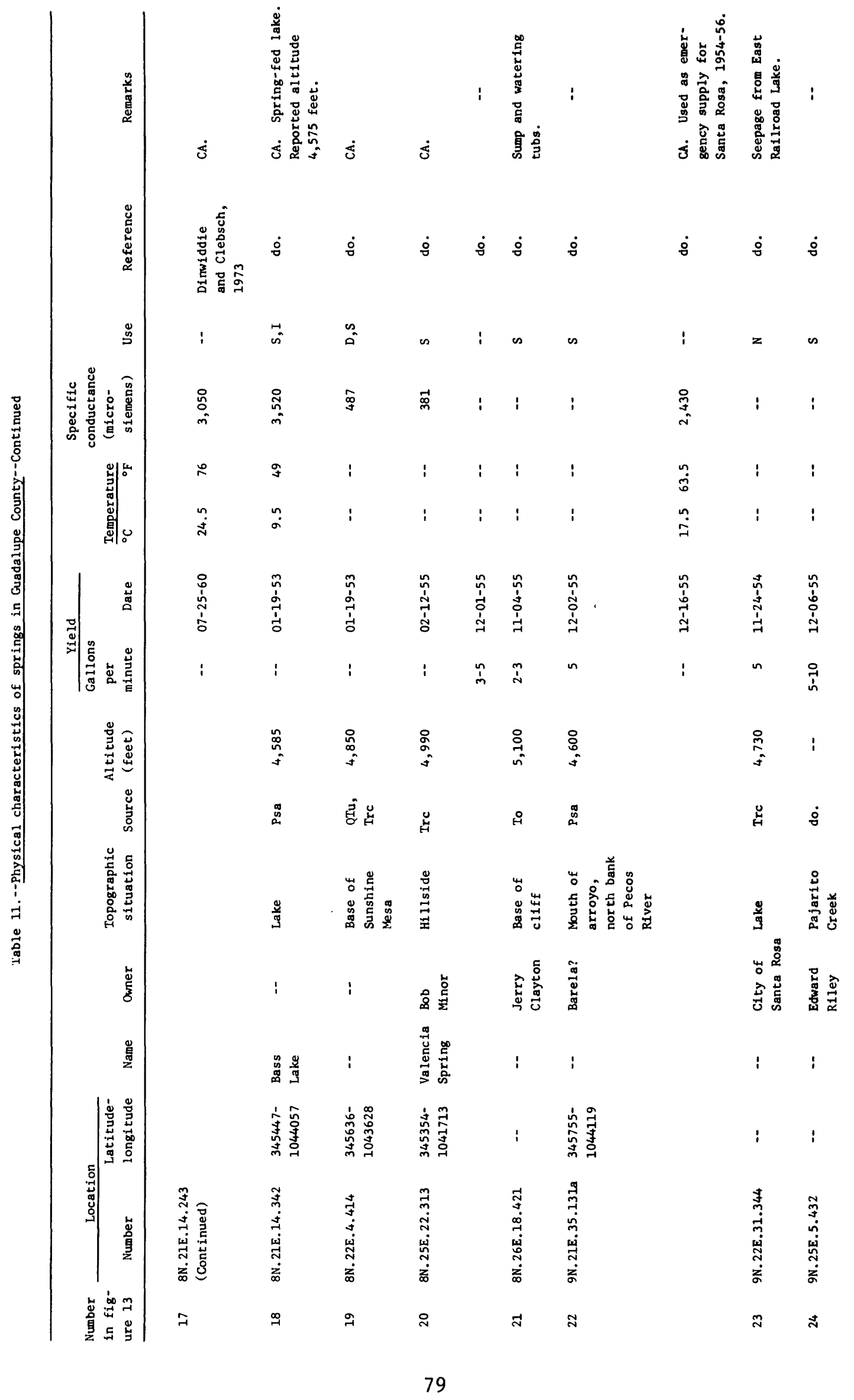




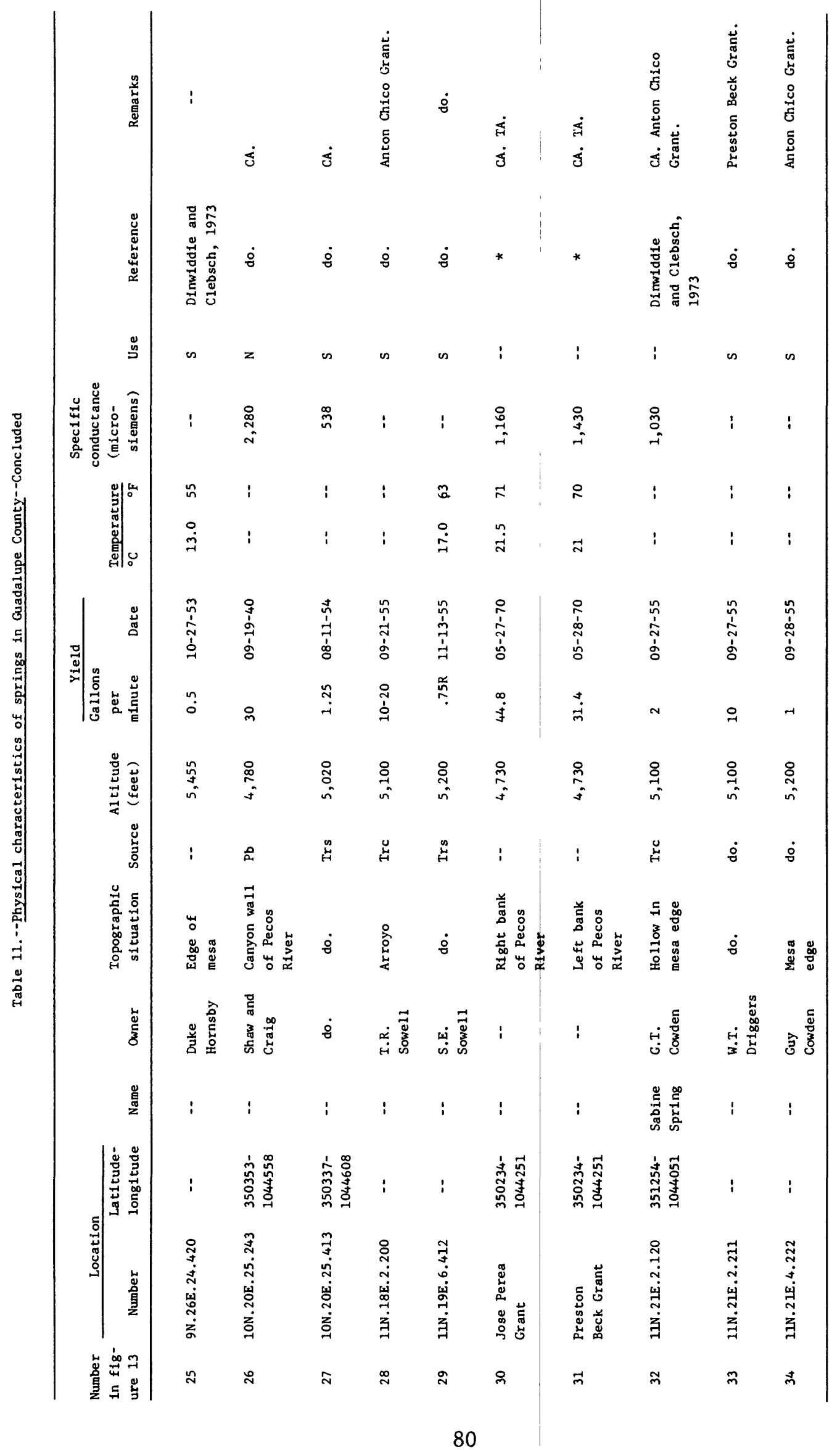




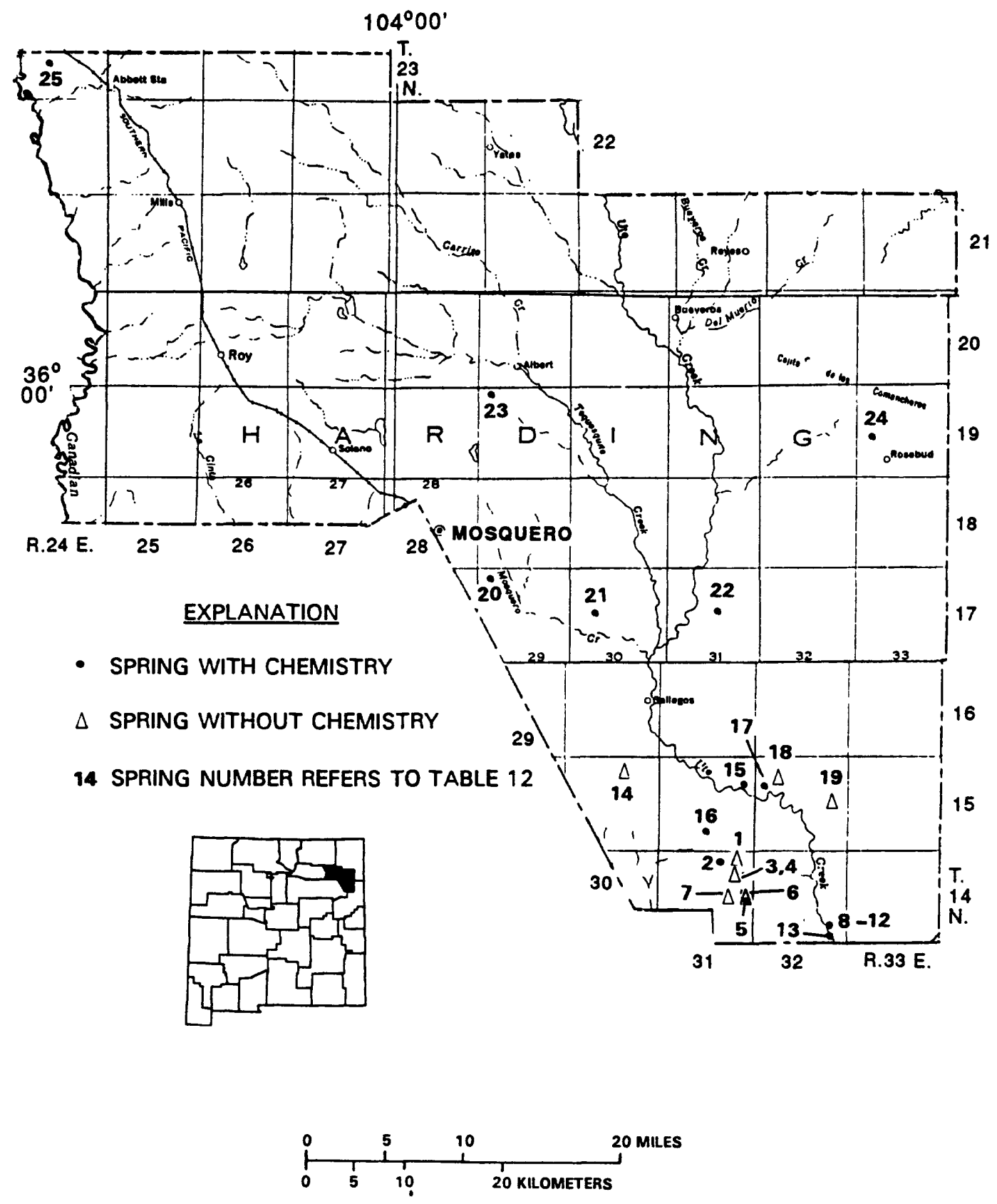

Figure 14.--Location of inventoried springs in Harding County. 


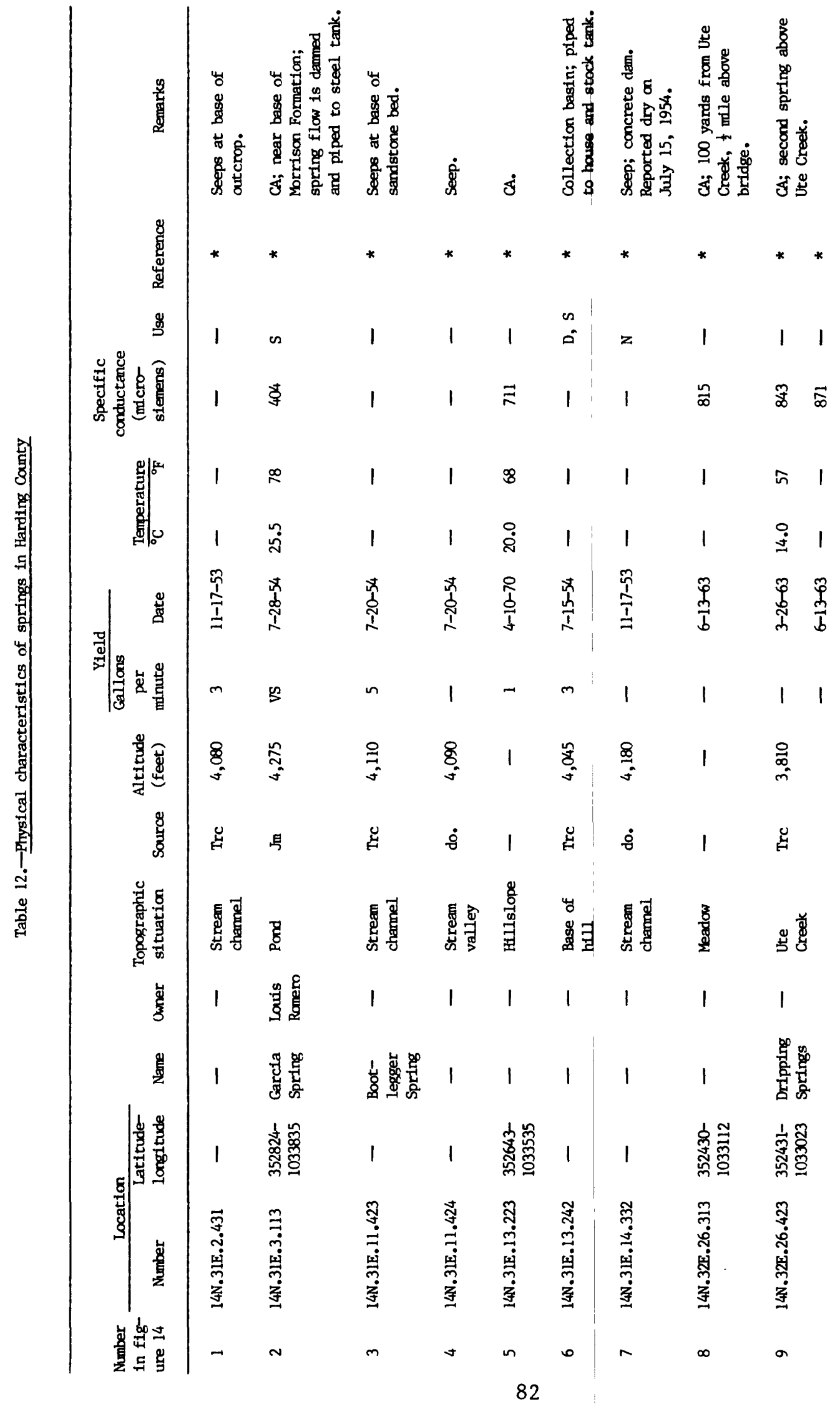




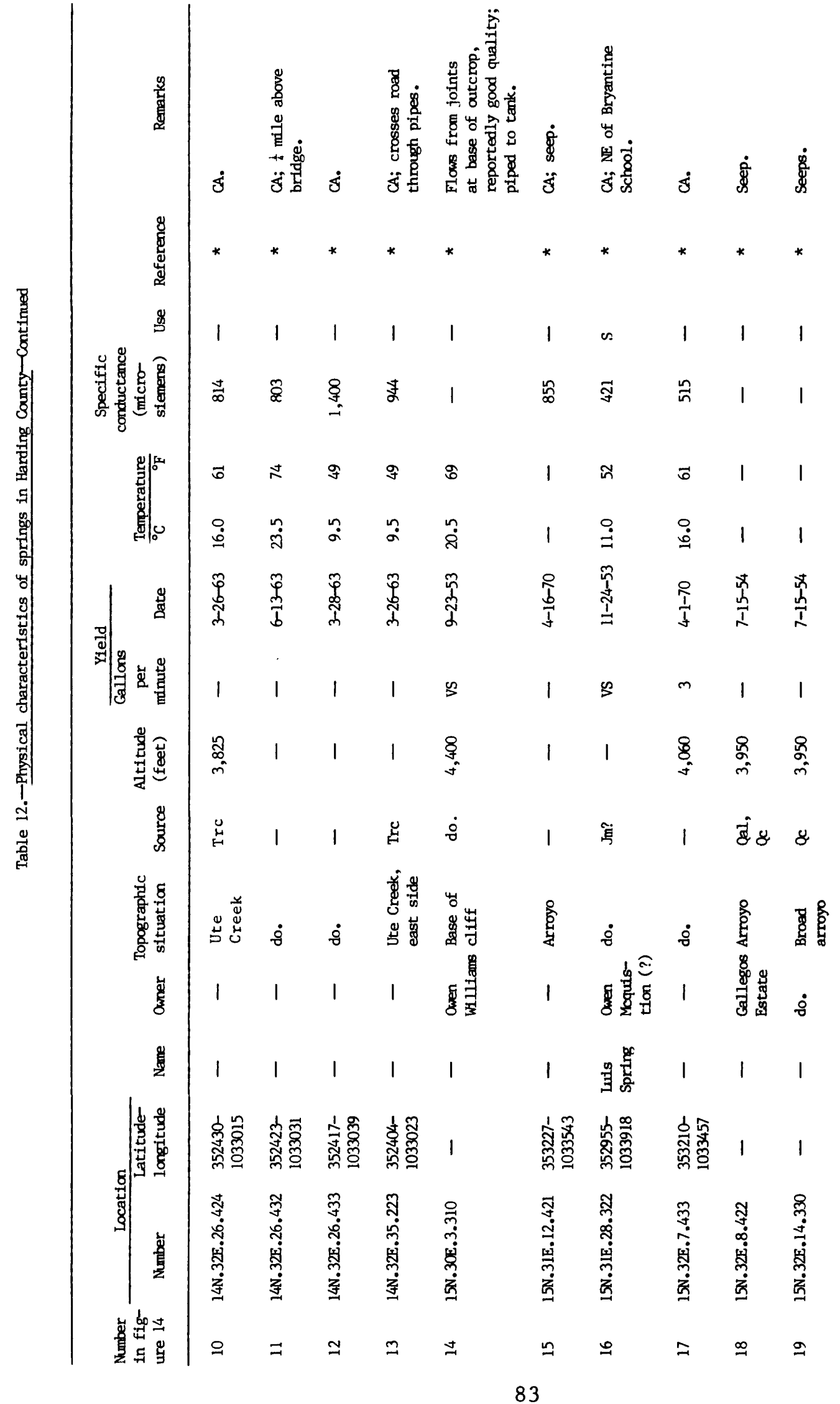




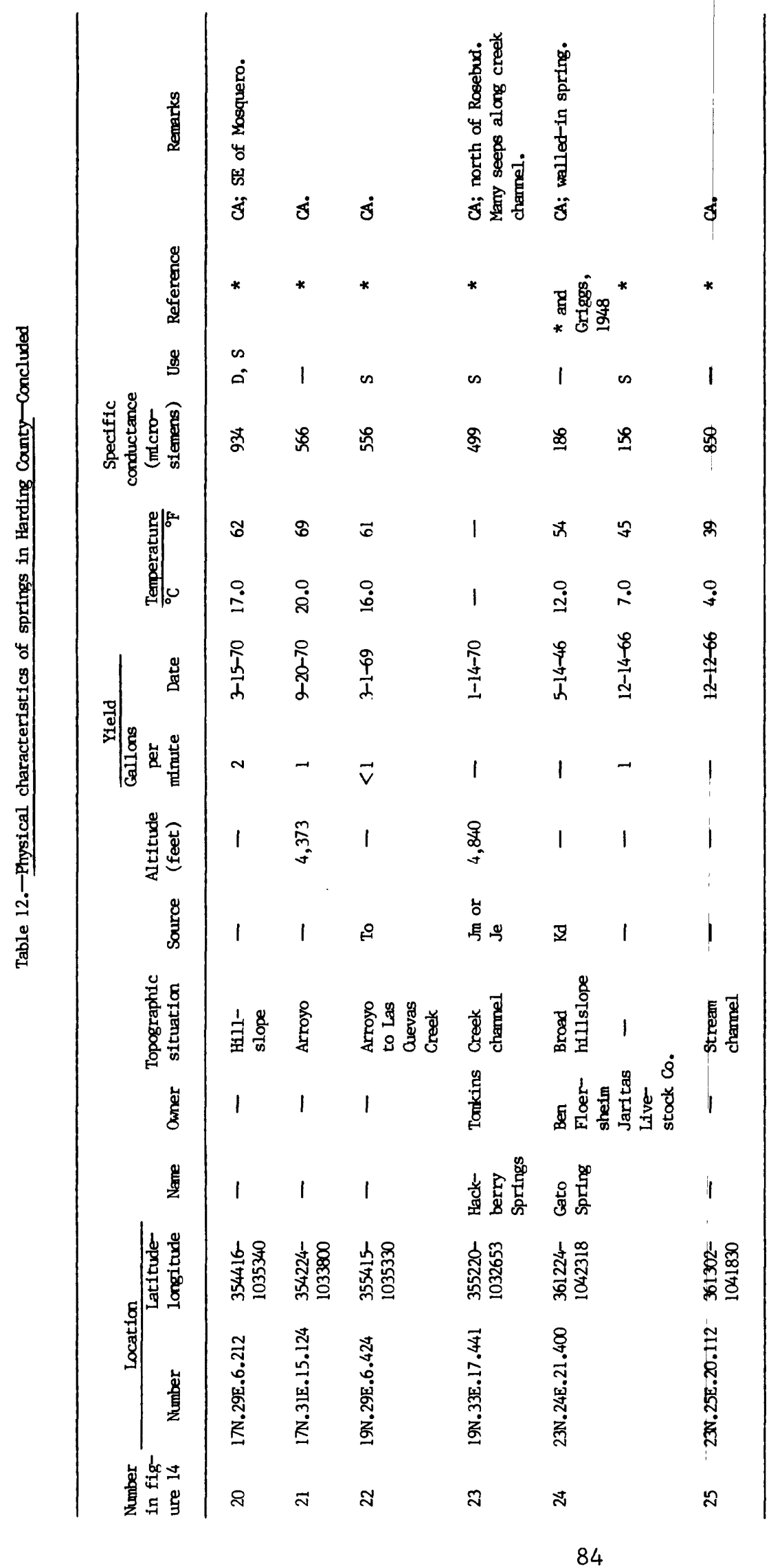




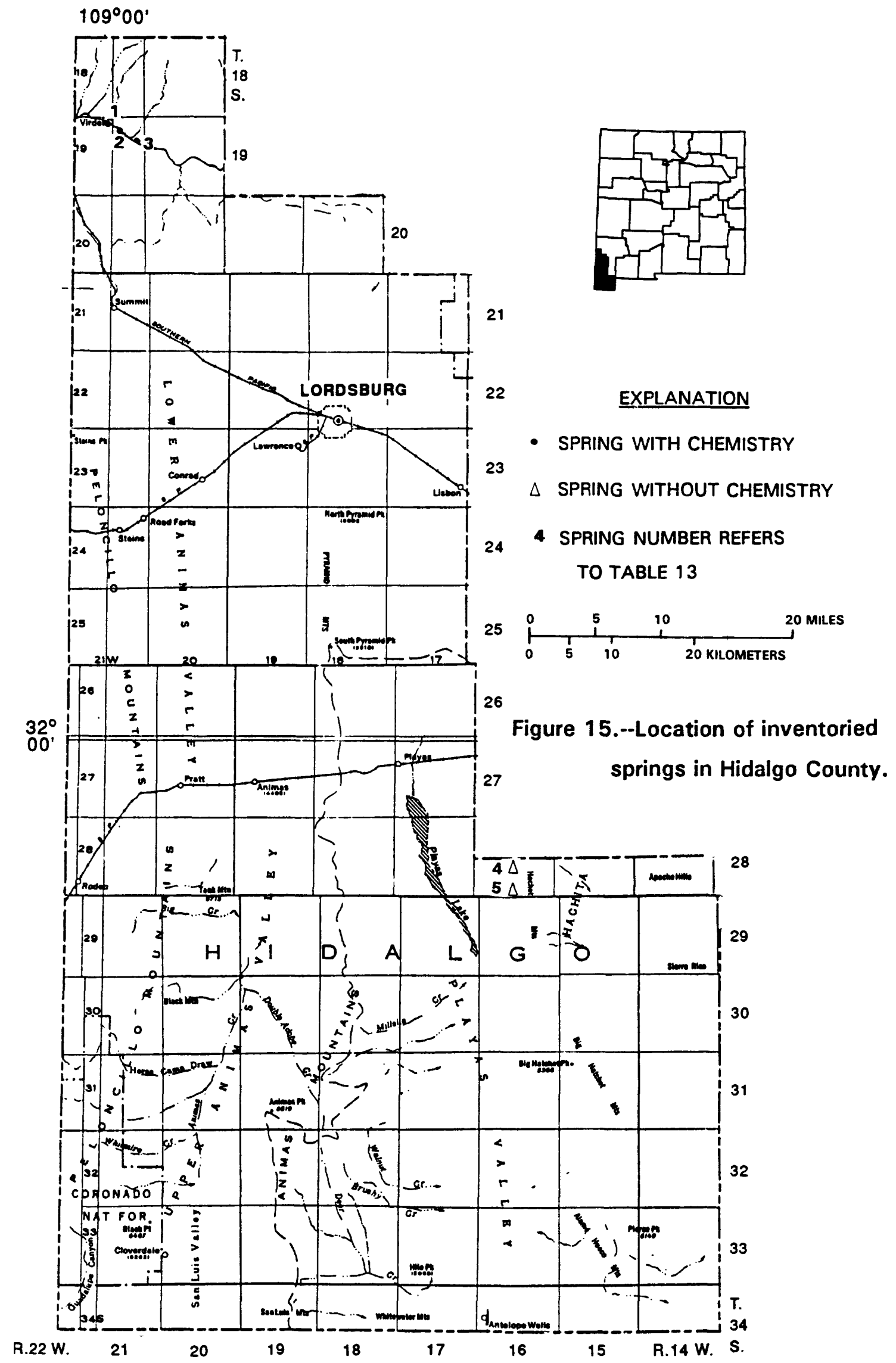




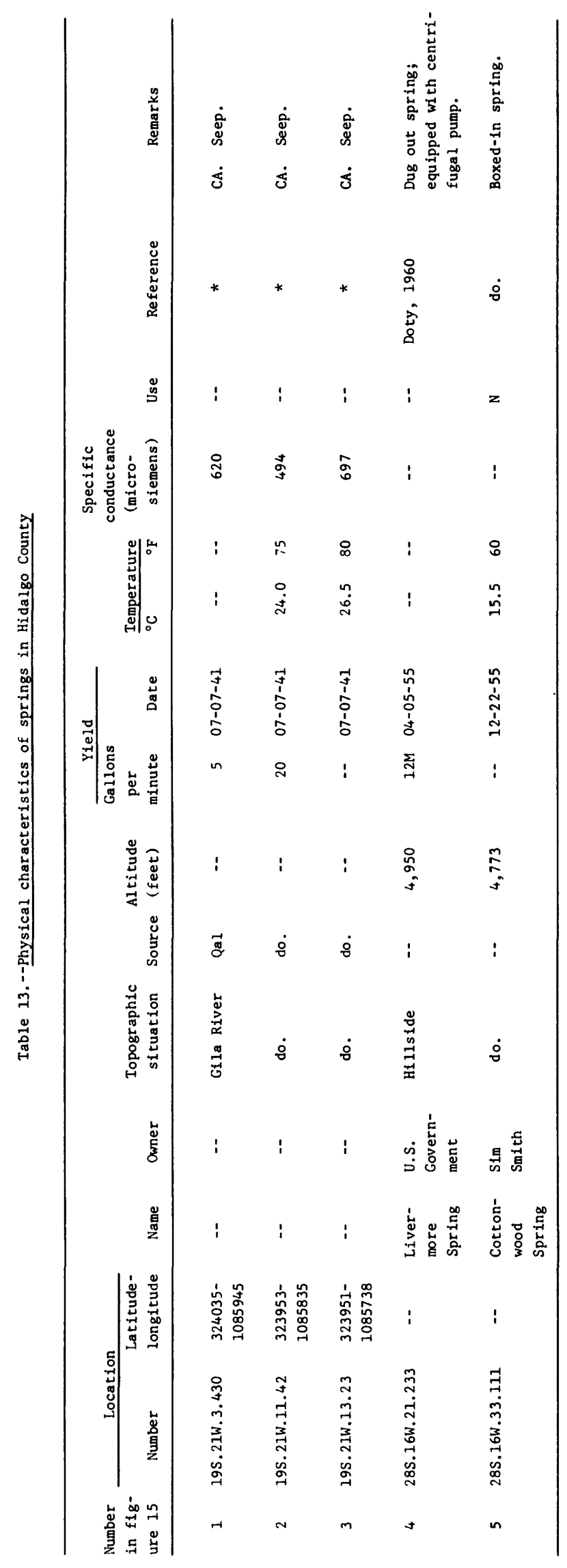




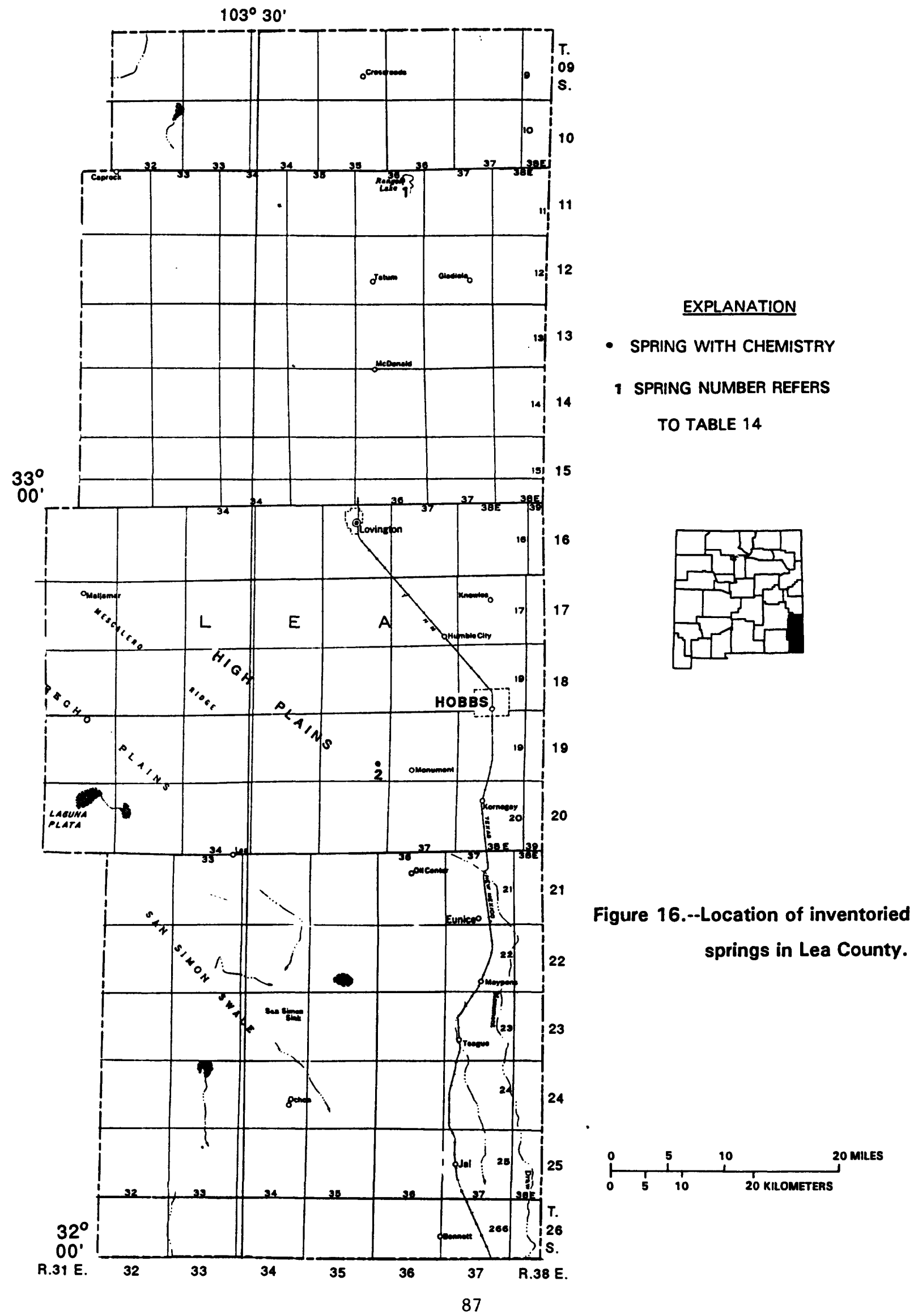




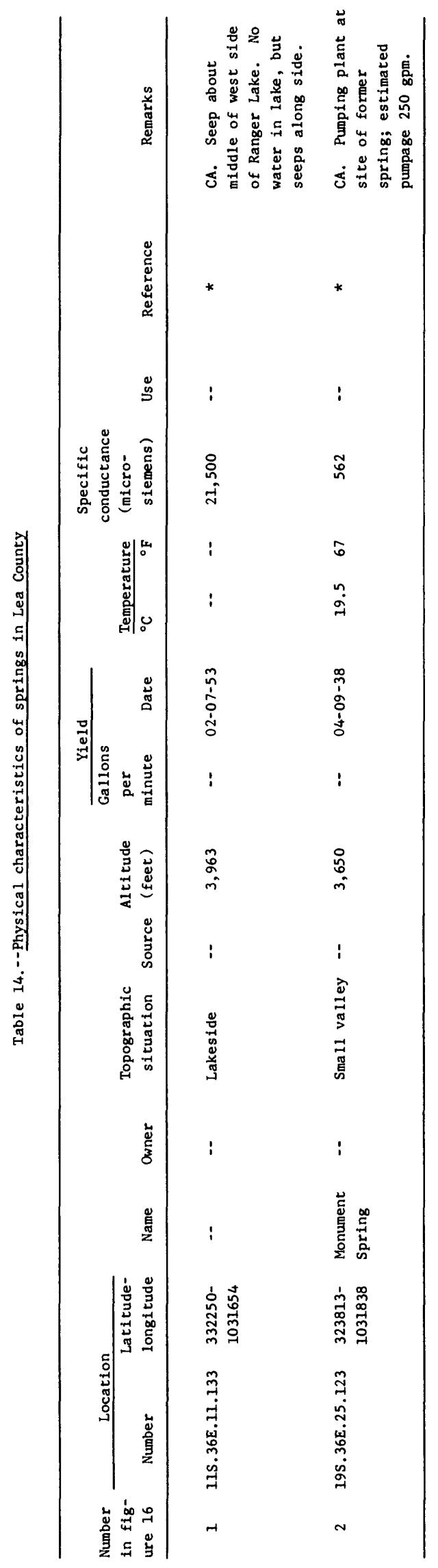




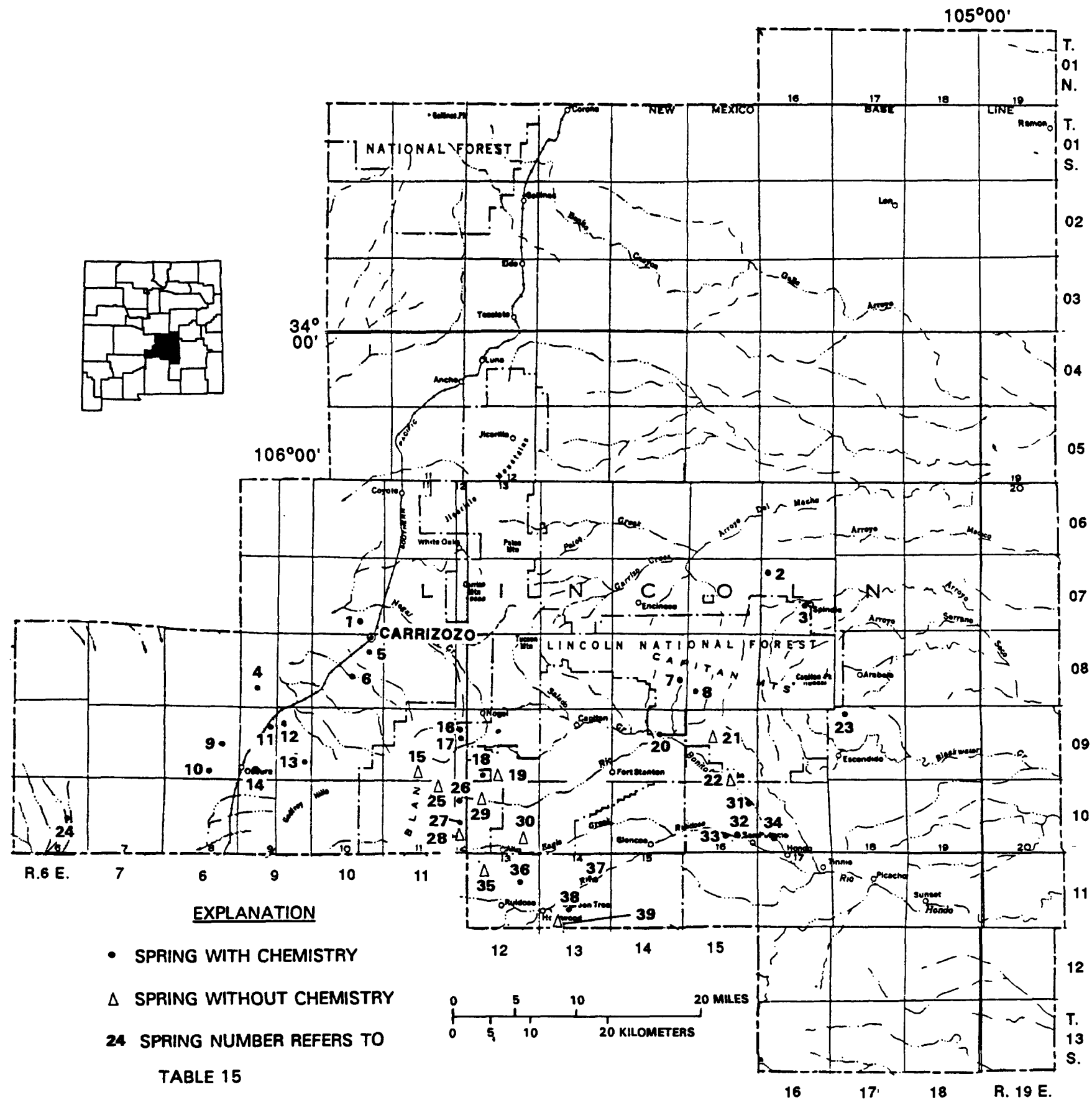

Figure 17.--Location of inventoried springs in Lincoln County. 


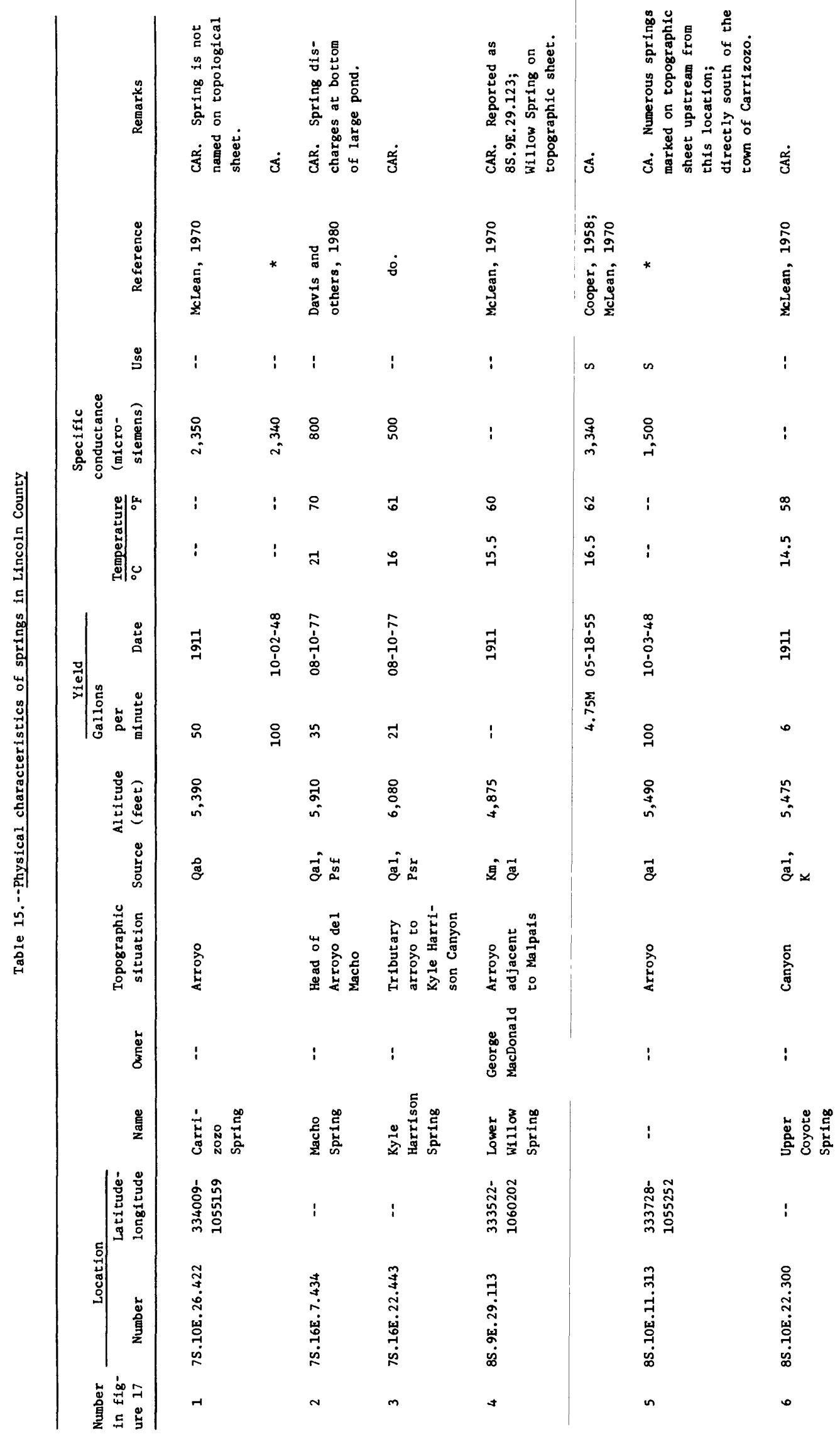




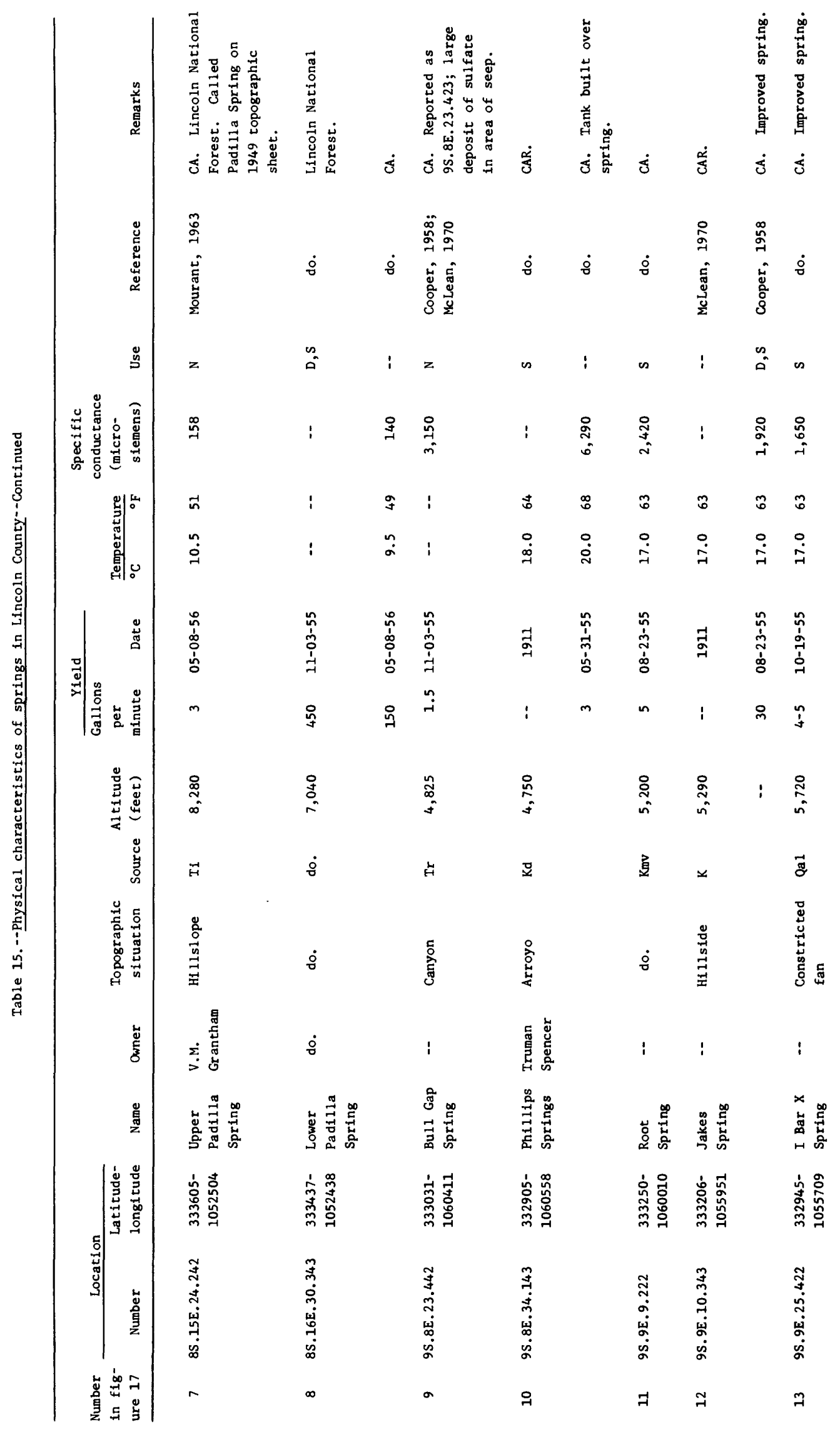




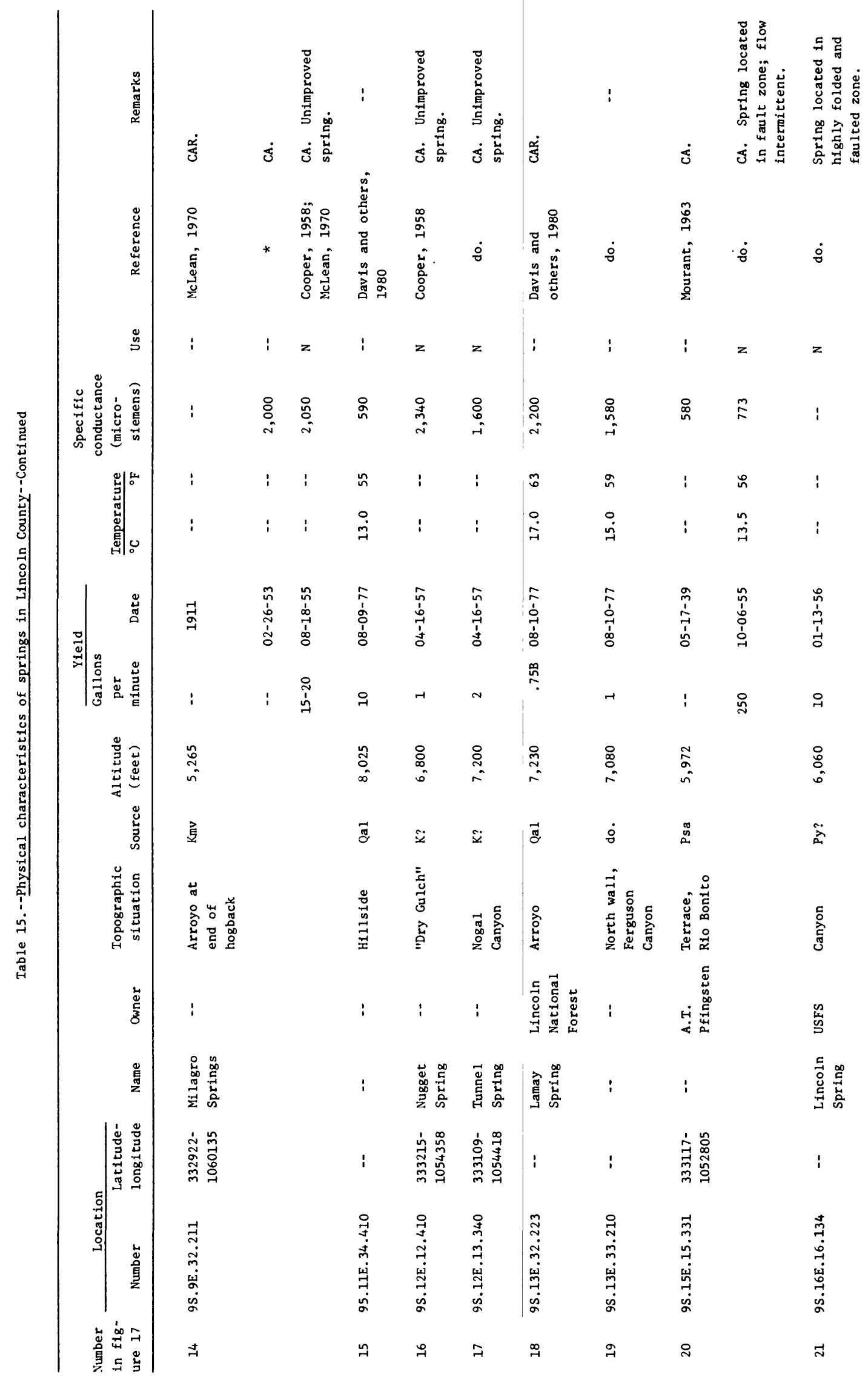




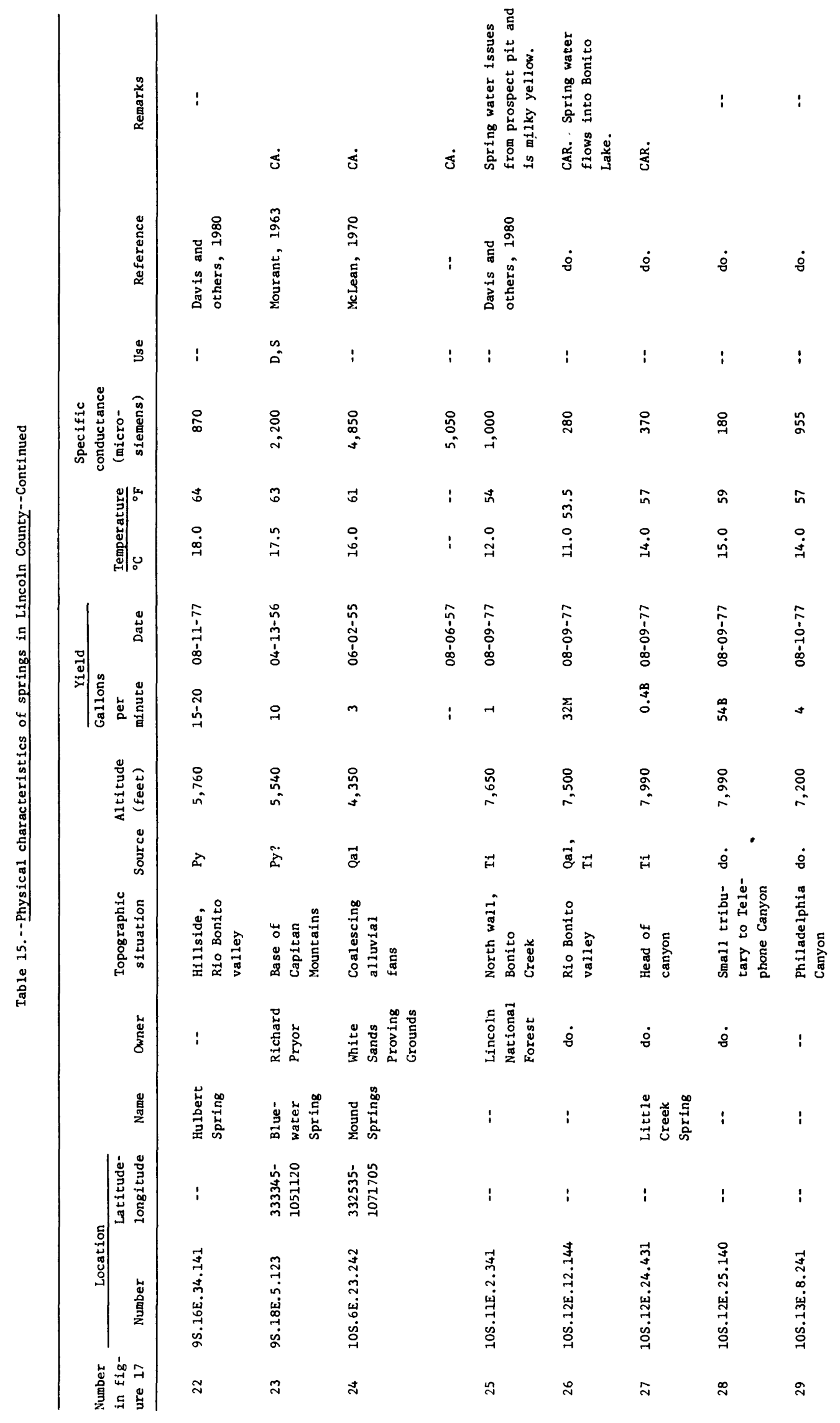




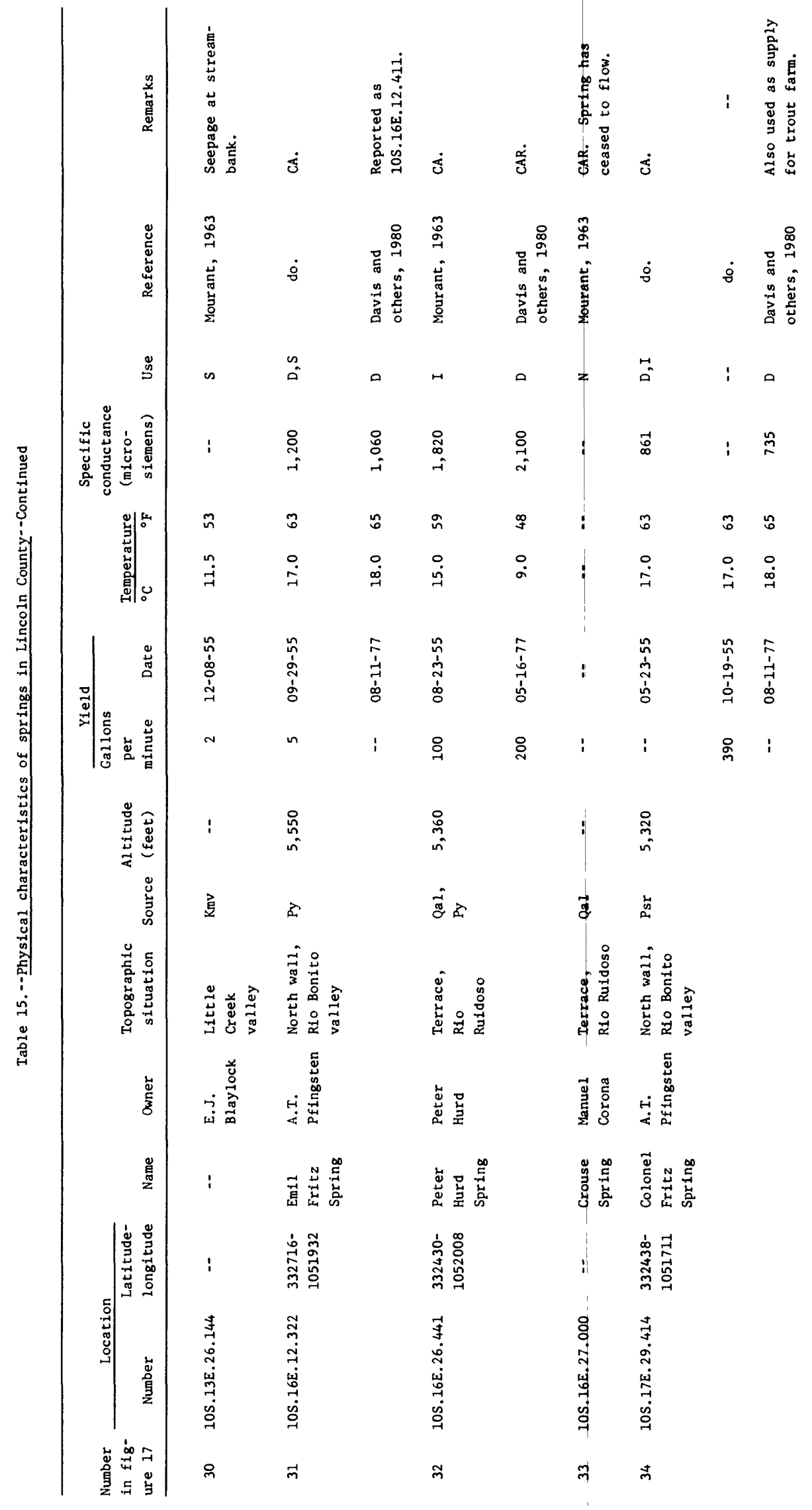




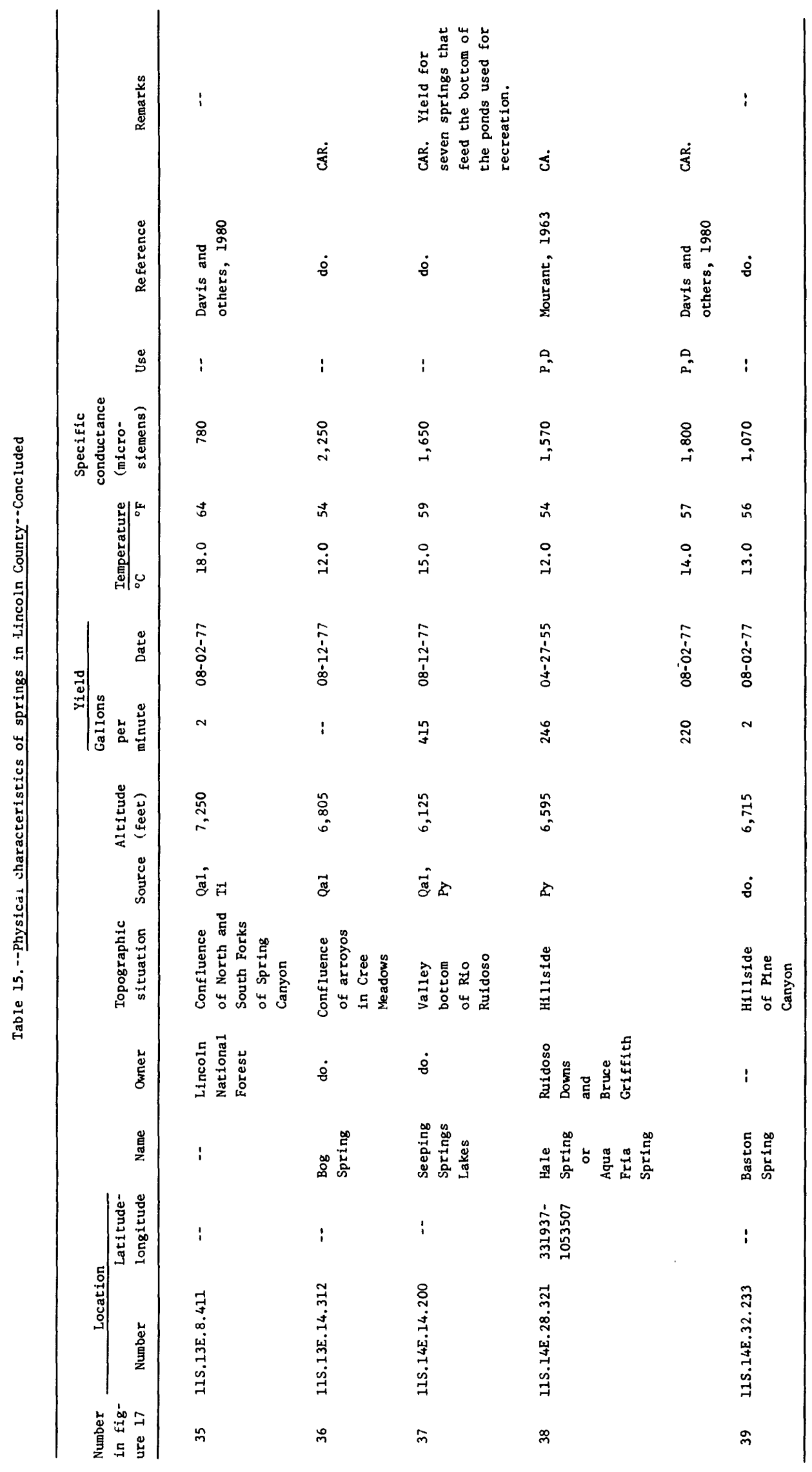




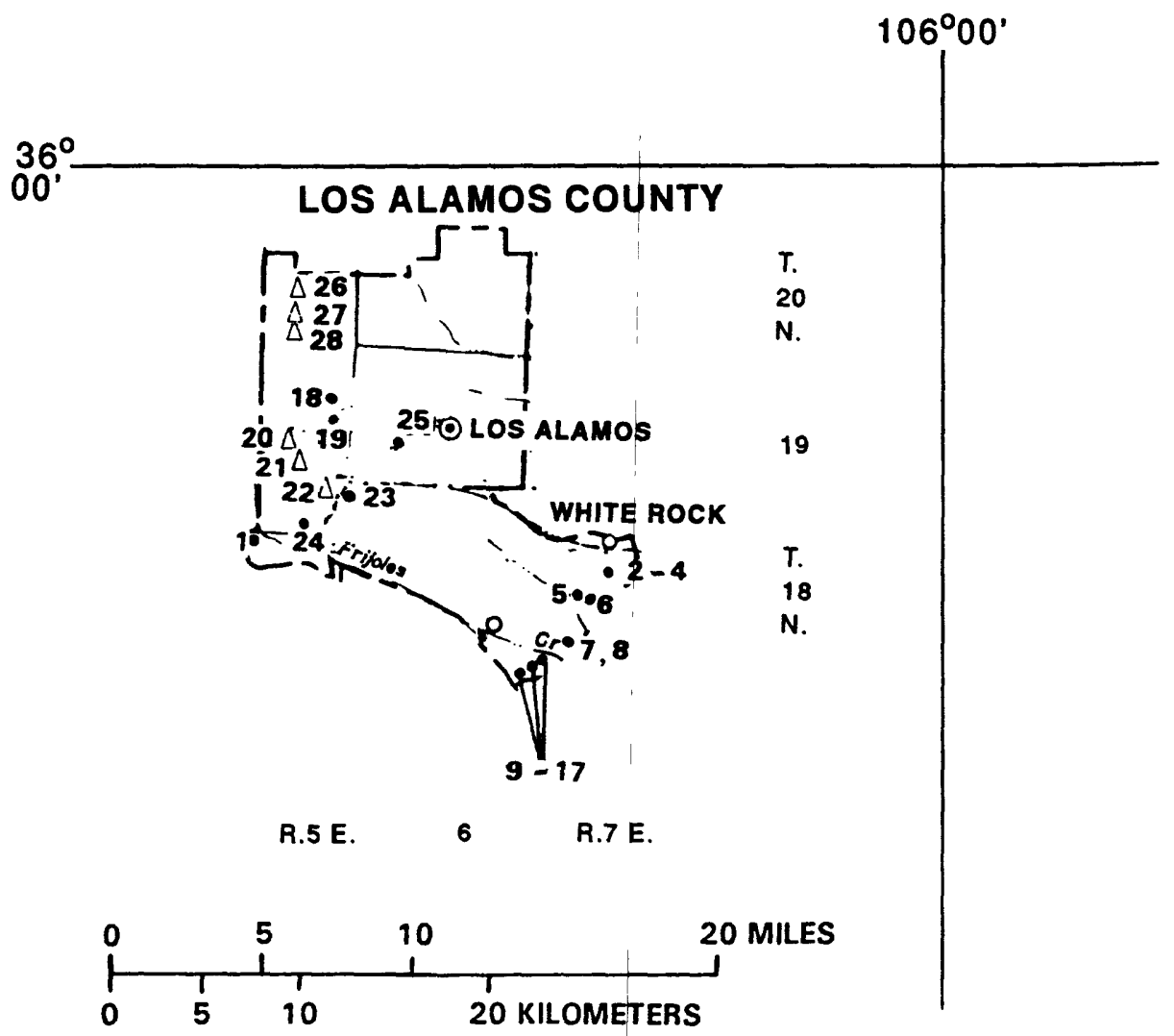

\section{EXPLANATION}

- SPRING WITH CHEMISTRY

$\triangle$ SPRING WITHOUT CHEMISTRY

9 SPRING NUMBER REFERS TO

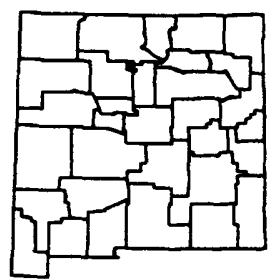

TABLE 16

Figure 18.--Location of inventoried springs in Los Alamos County. 


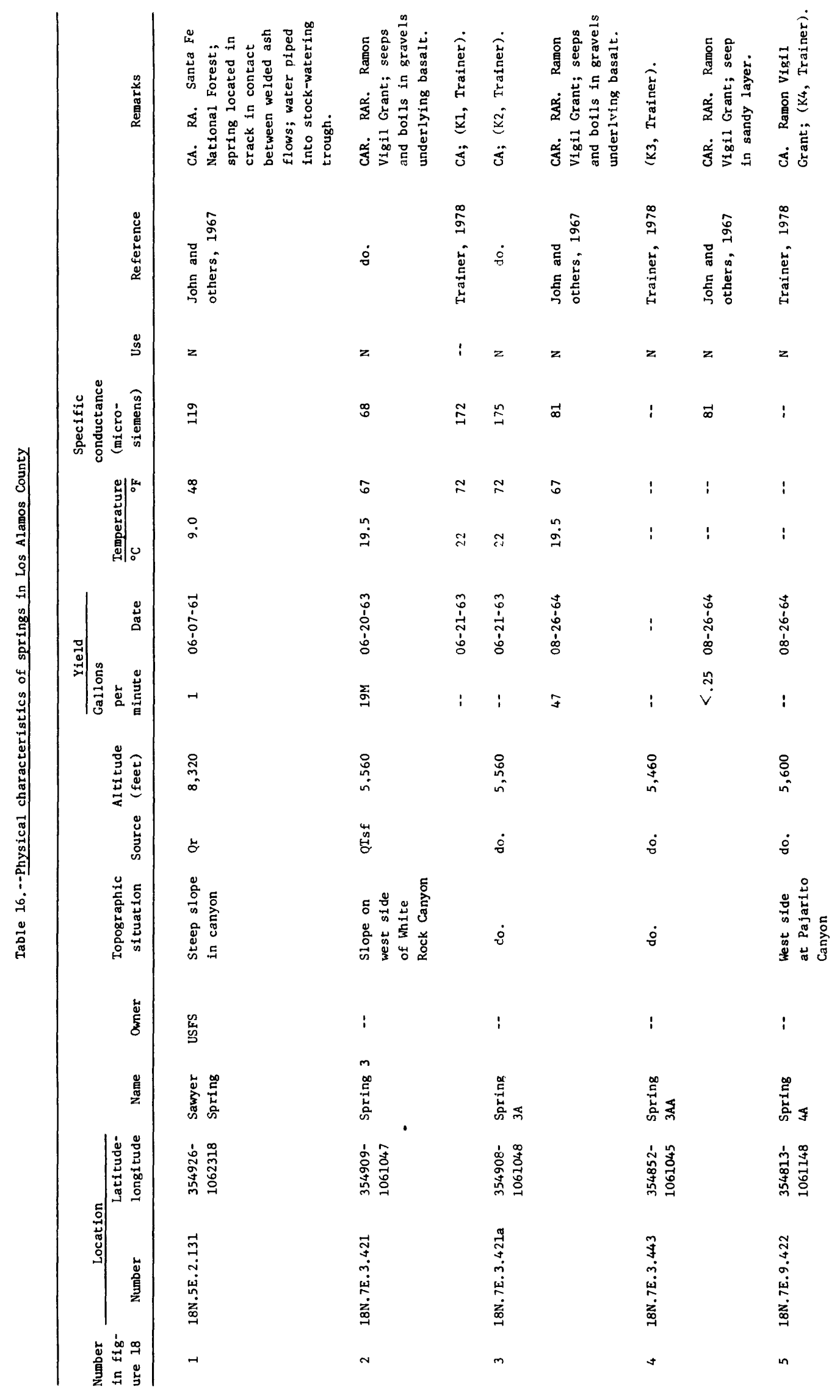




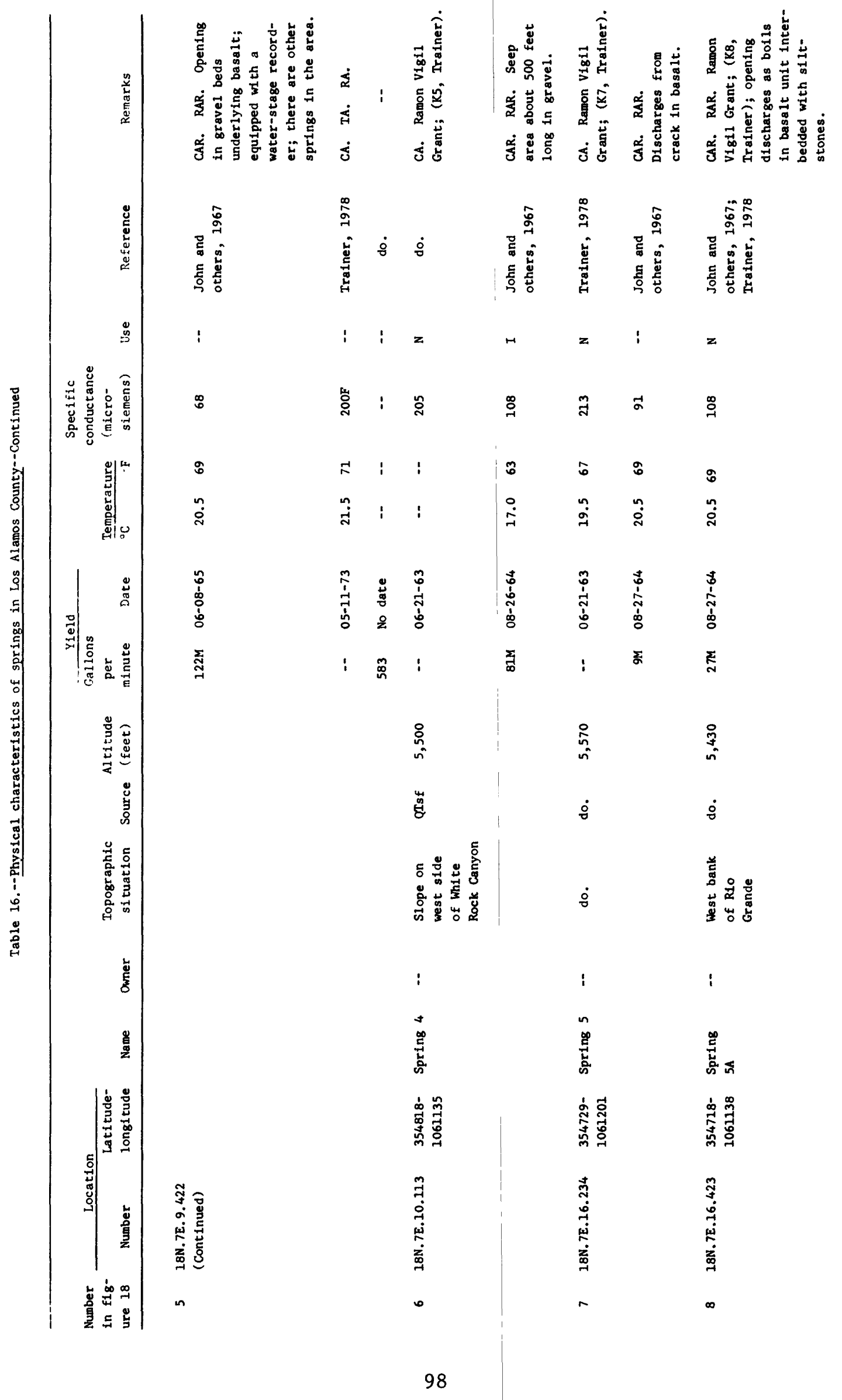




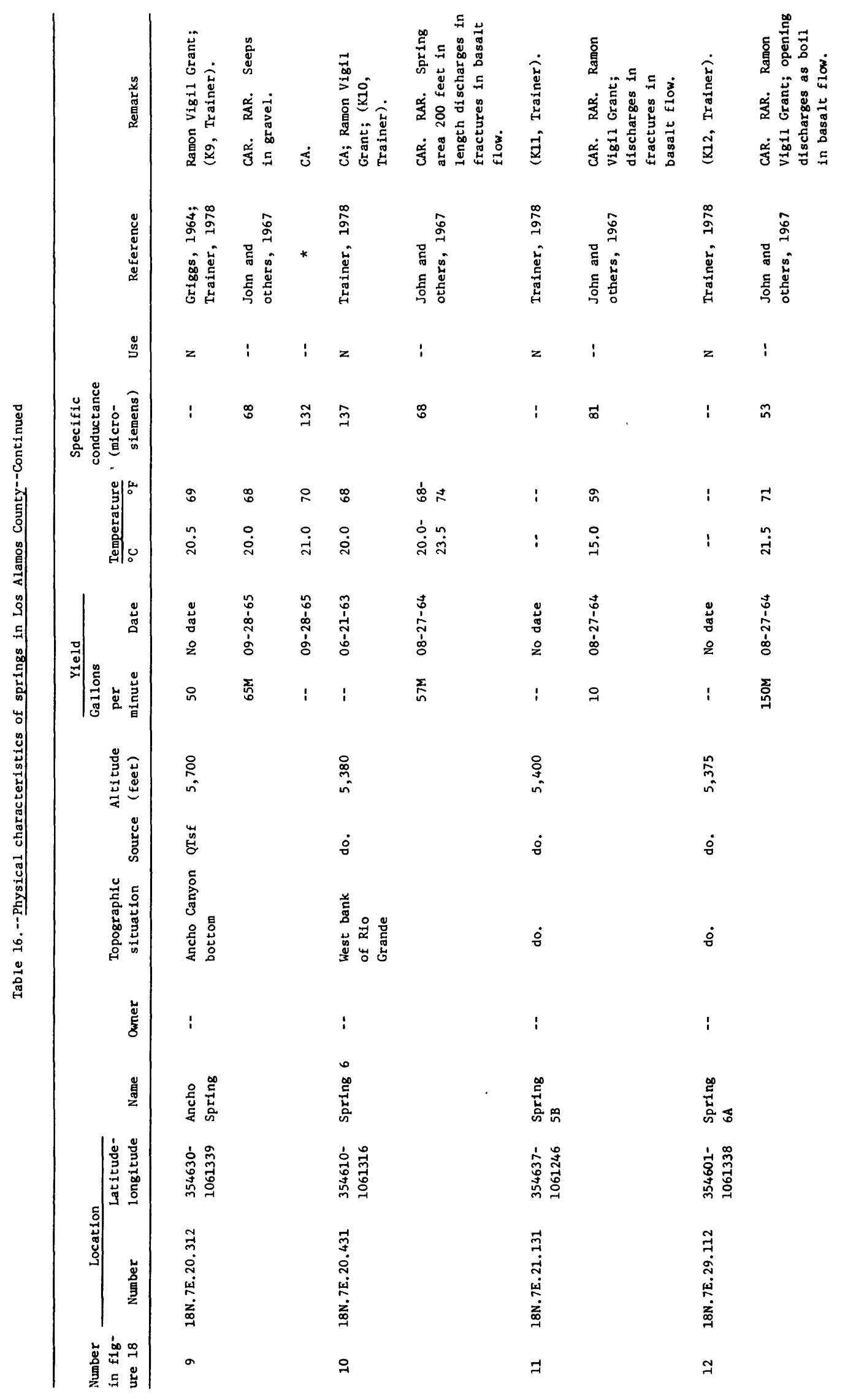




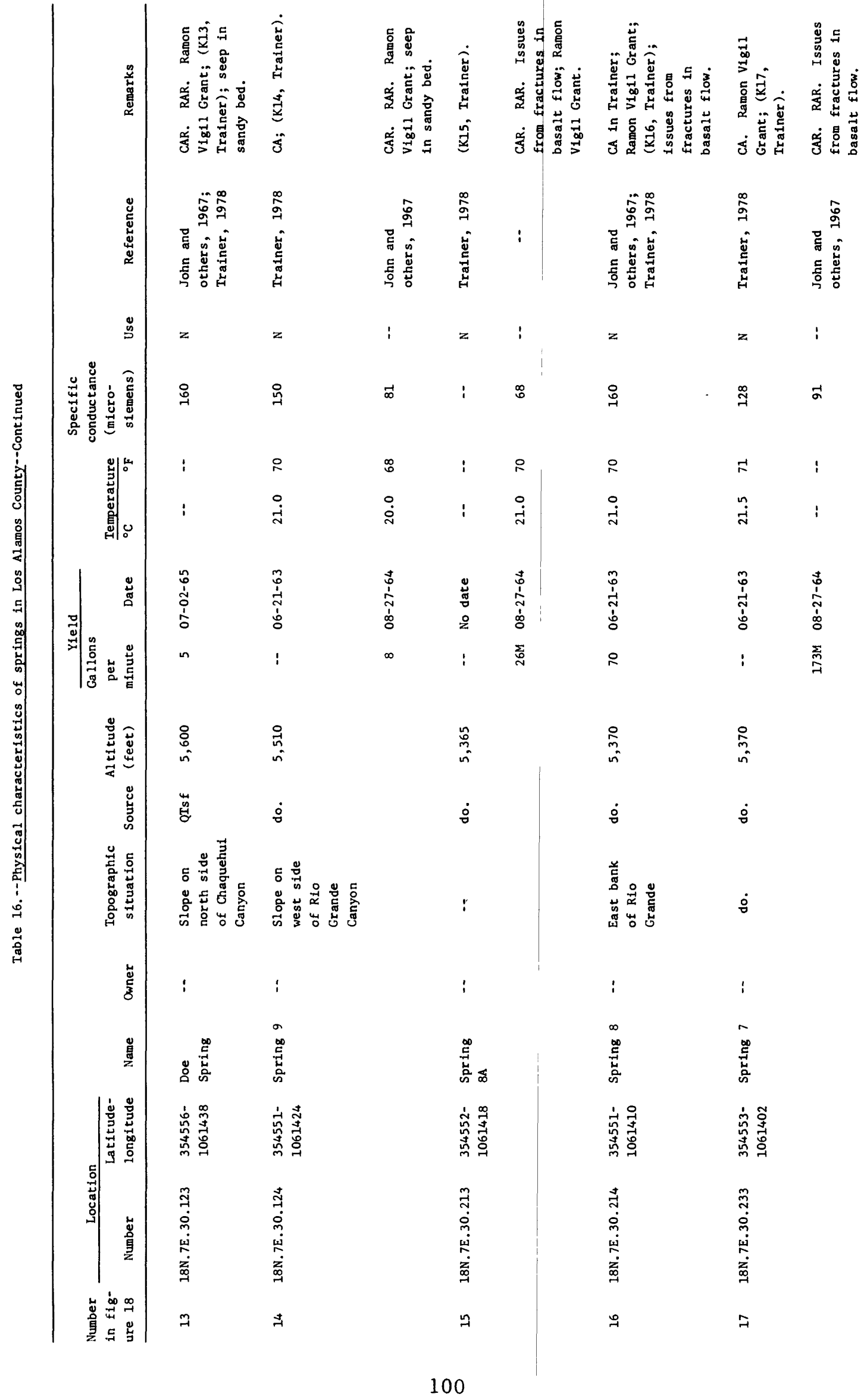




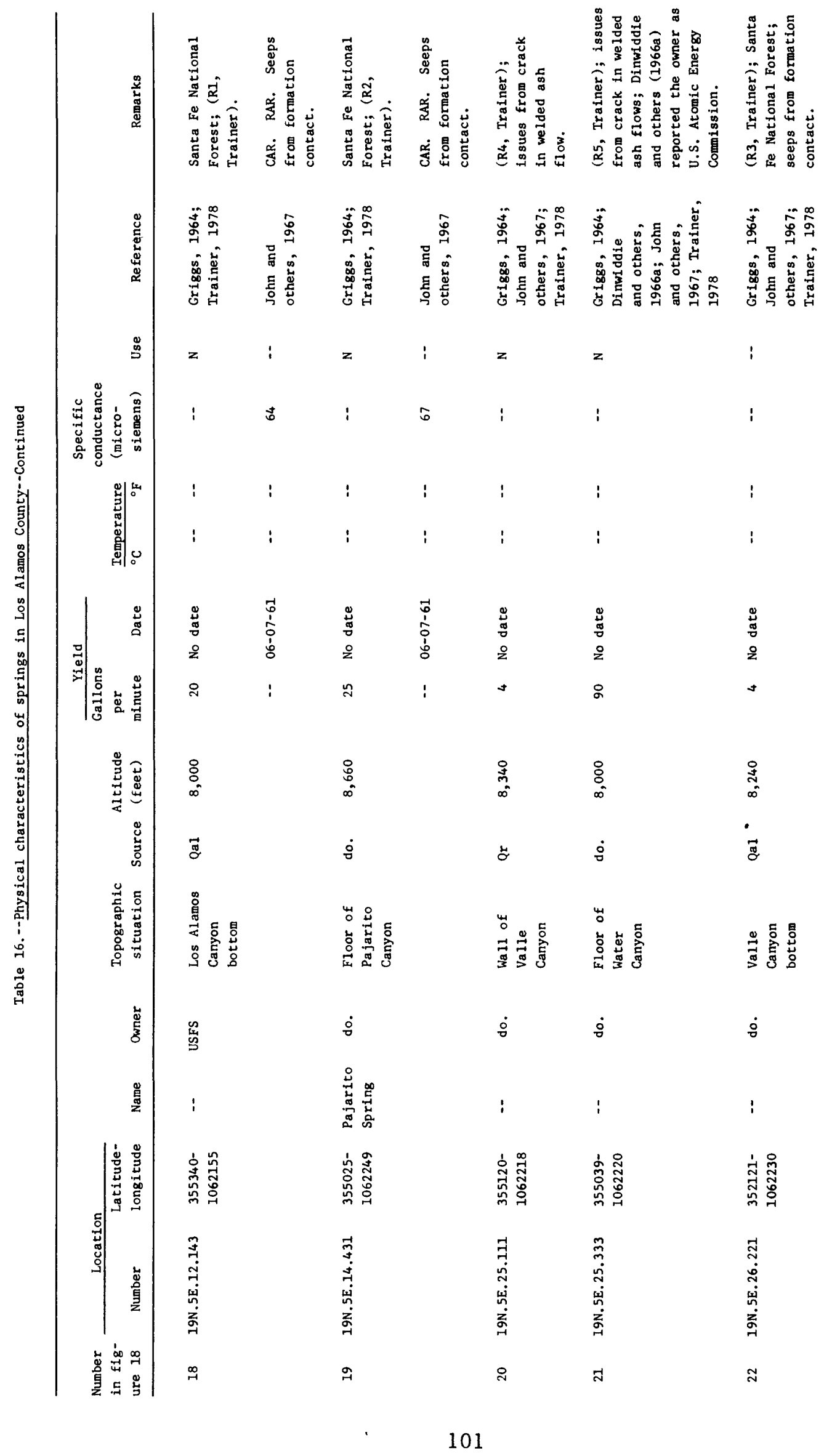




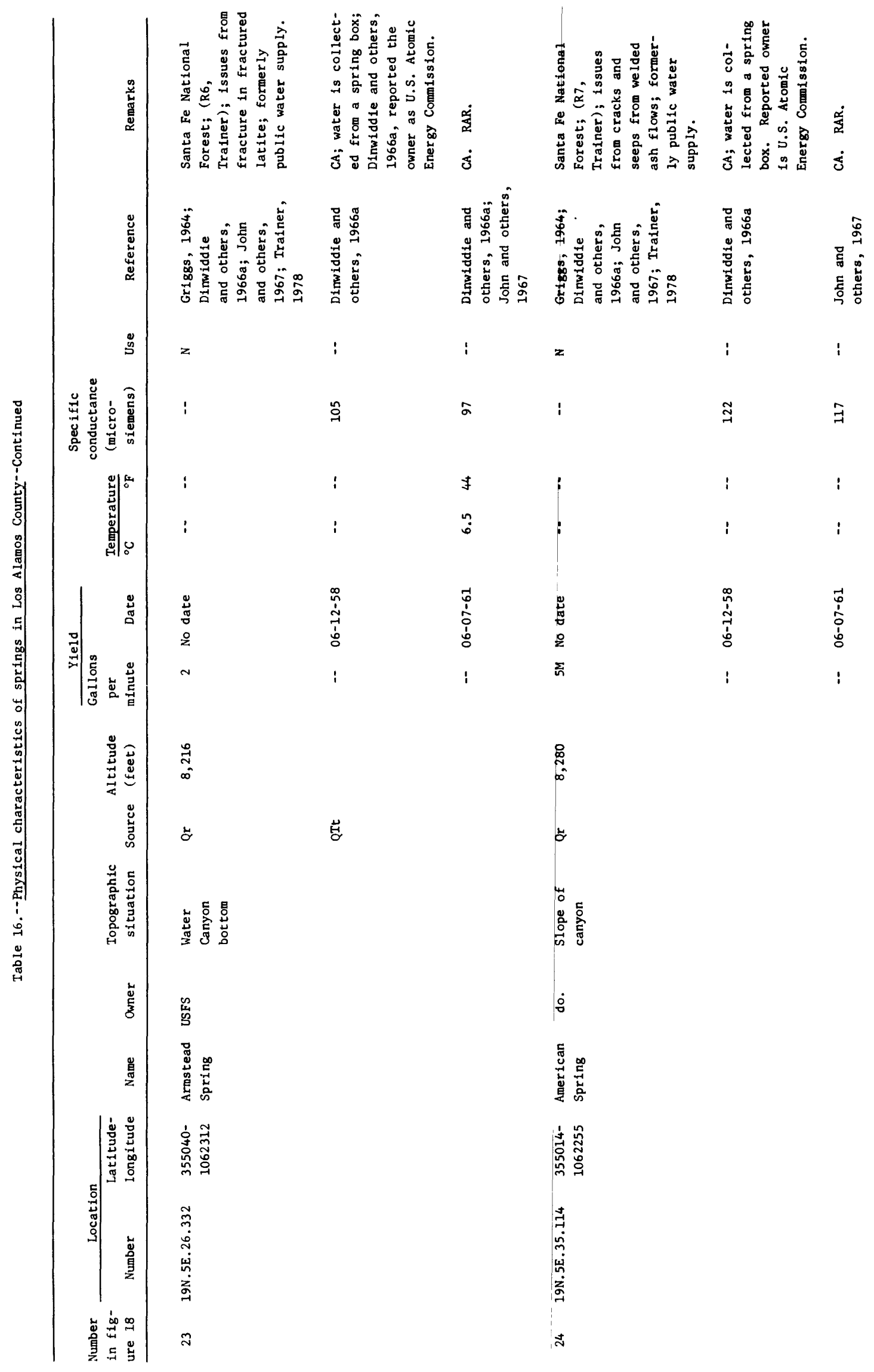




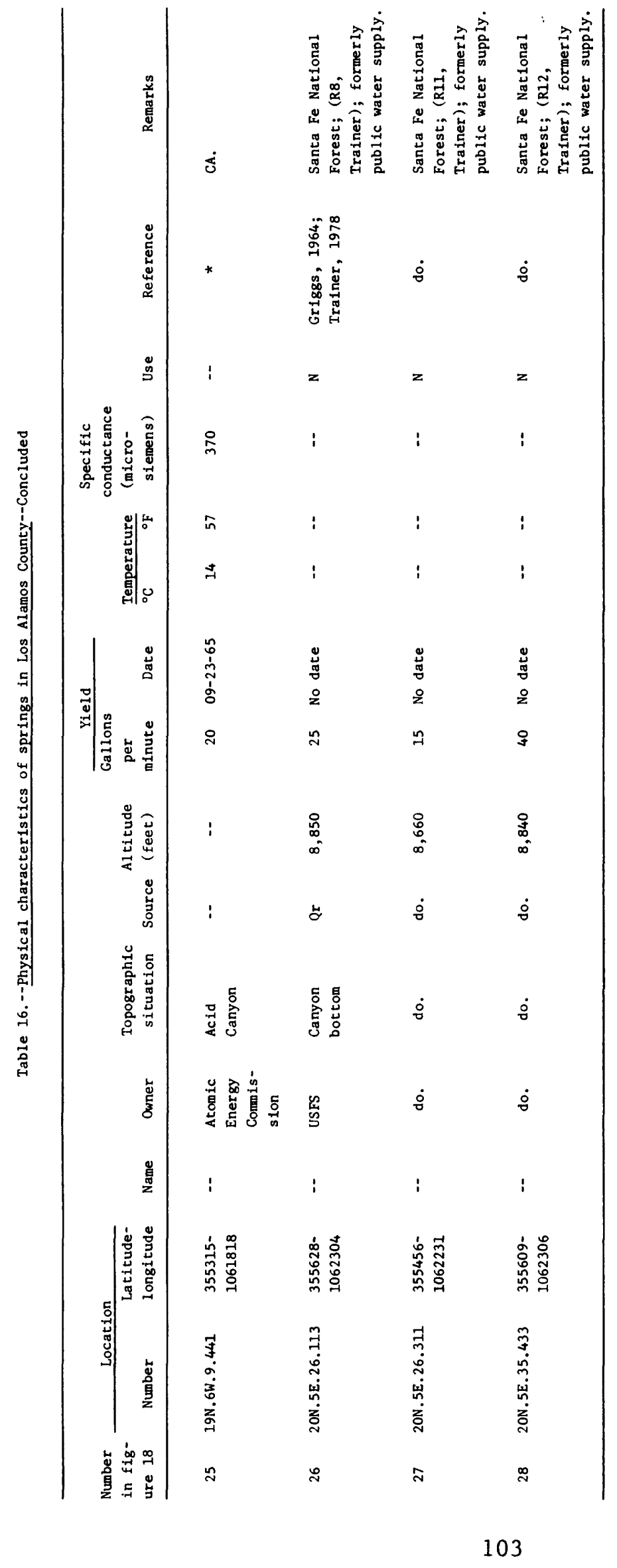




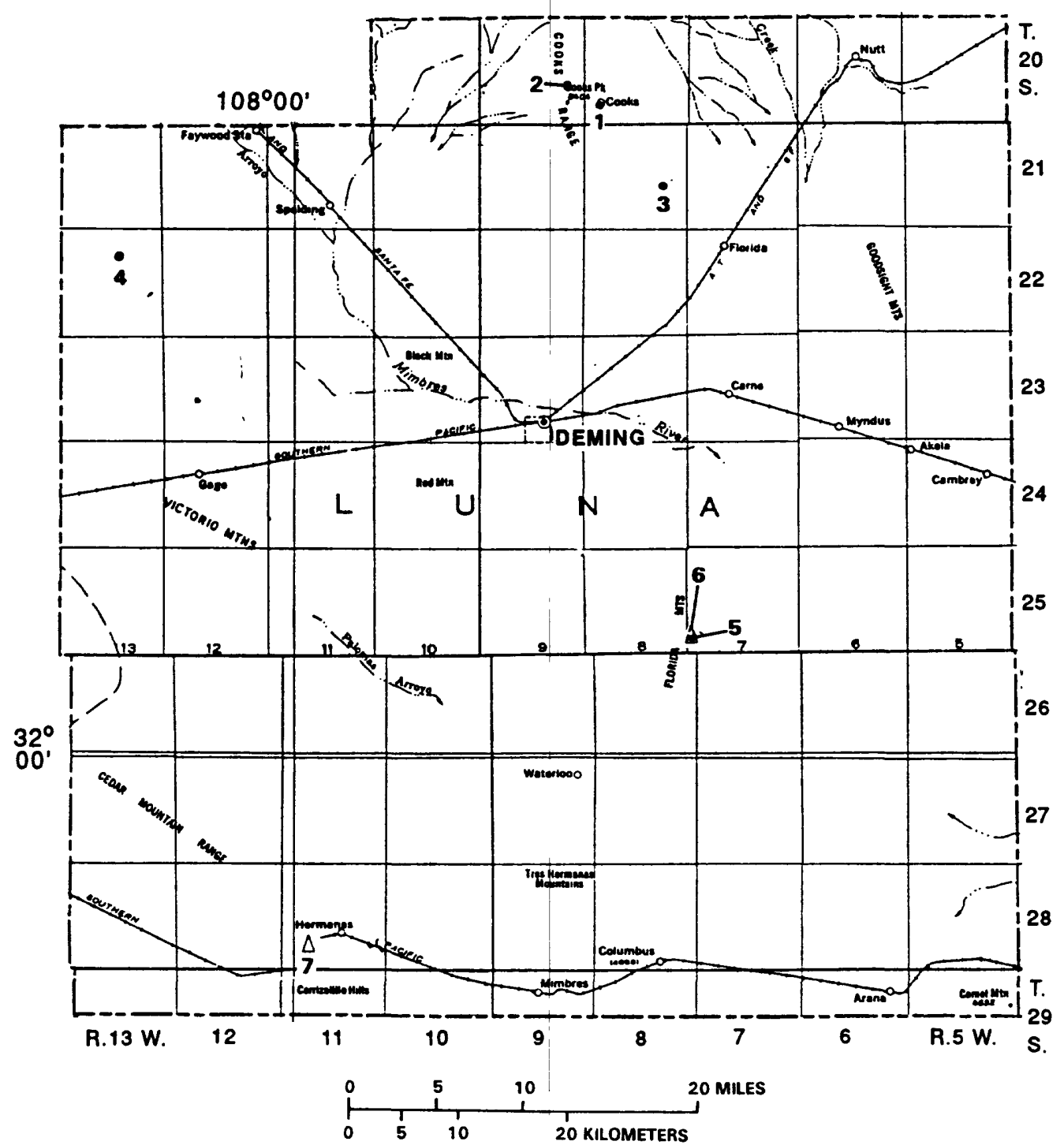

EXPLANATION

- SPRING WITH CHEMISTRY

$\triangle$ SPRING WITHOUT CHEMISTRY

5 SPRING NUMBER REFERS TO TABLE 17

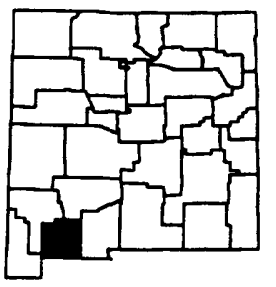

Figure 19.--Location of inventoried springs in Luna County. 


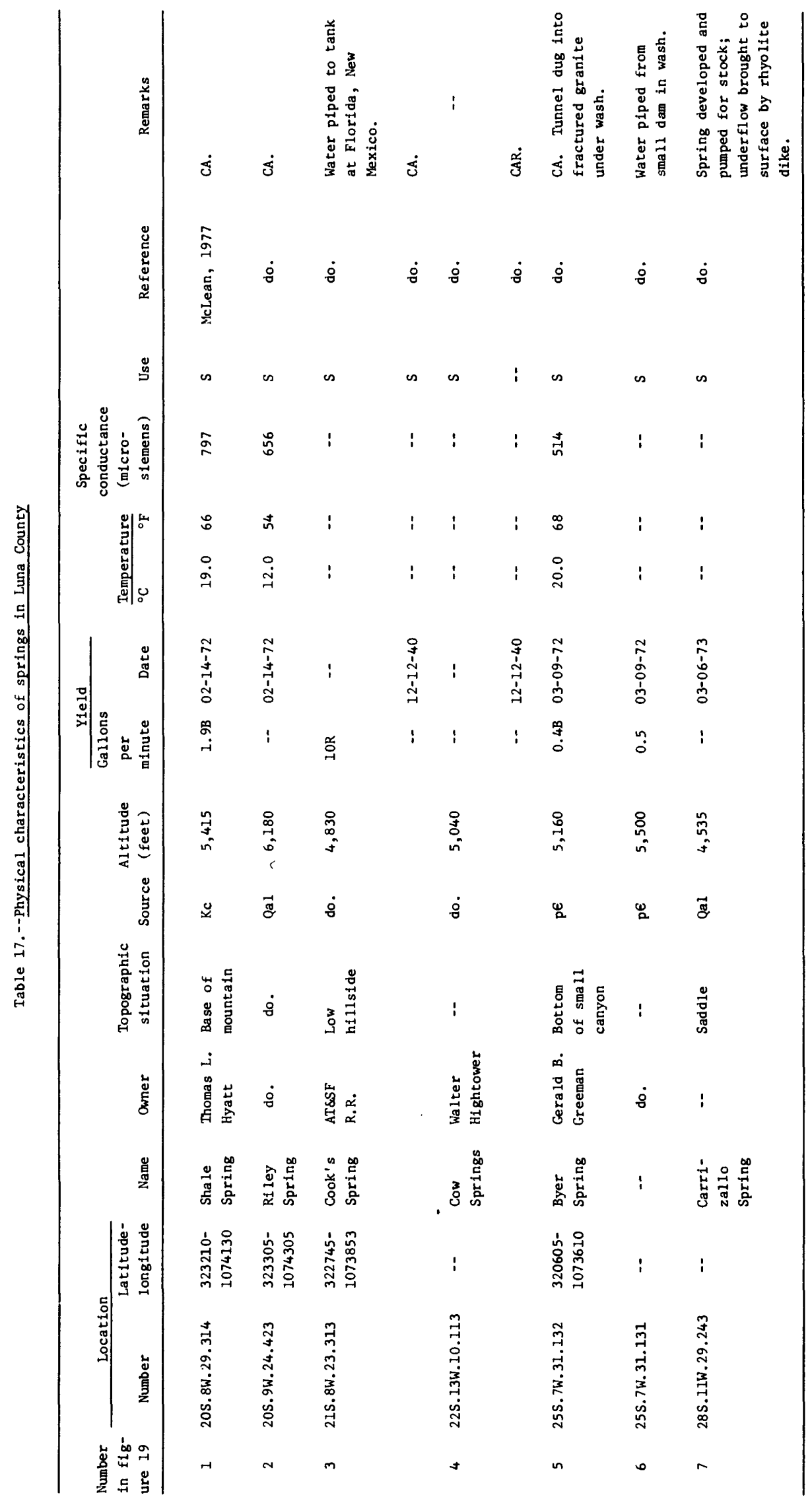




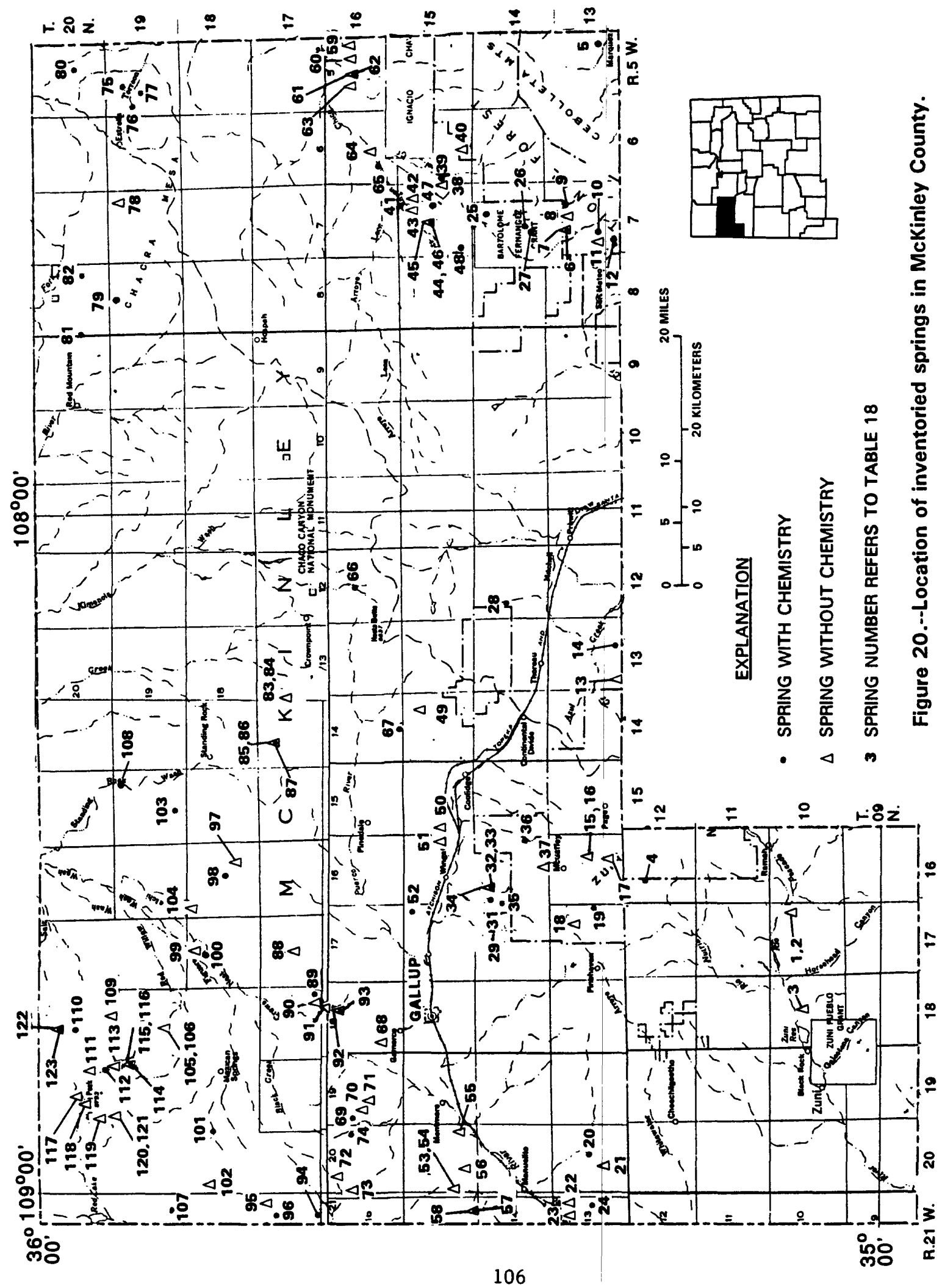




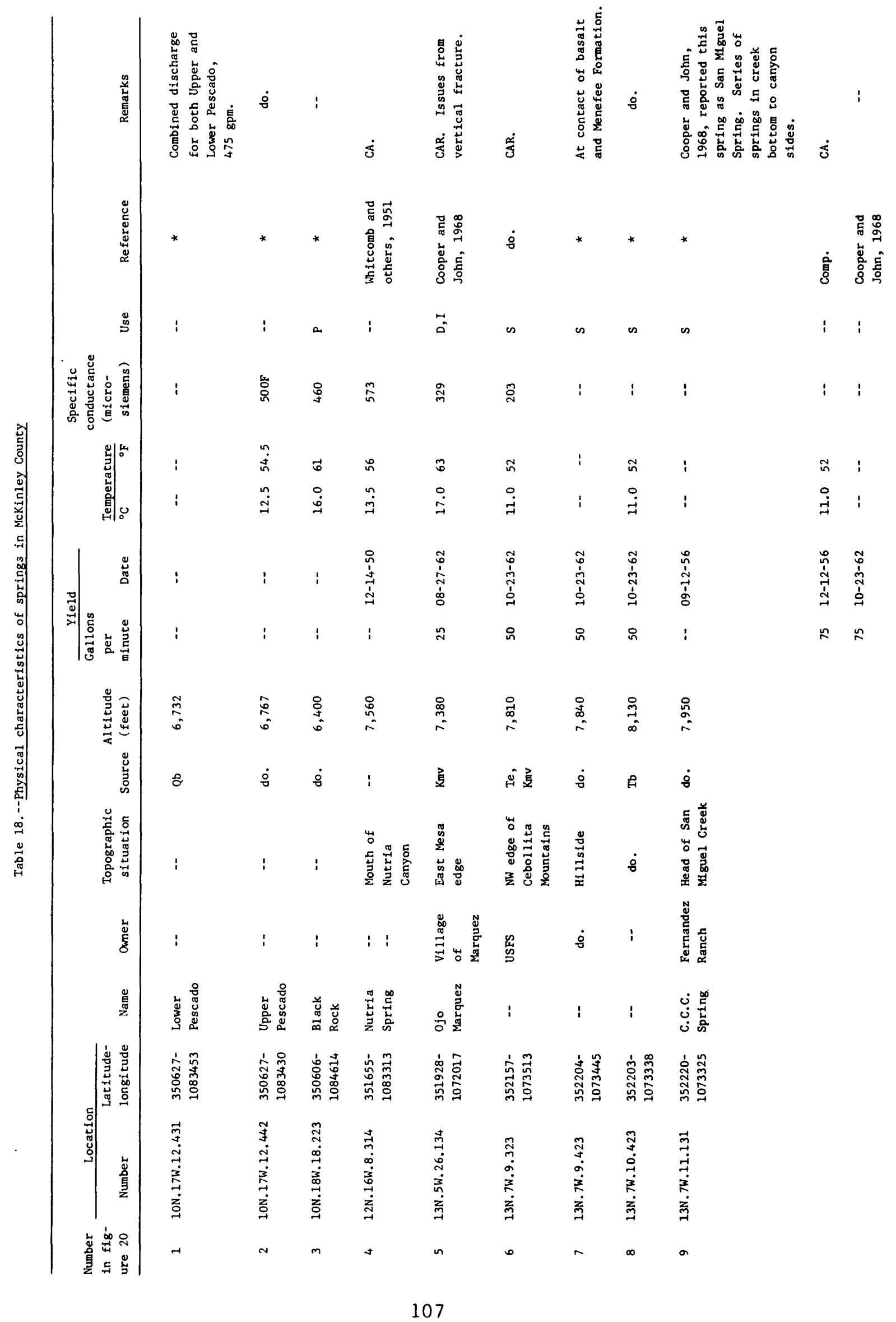




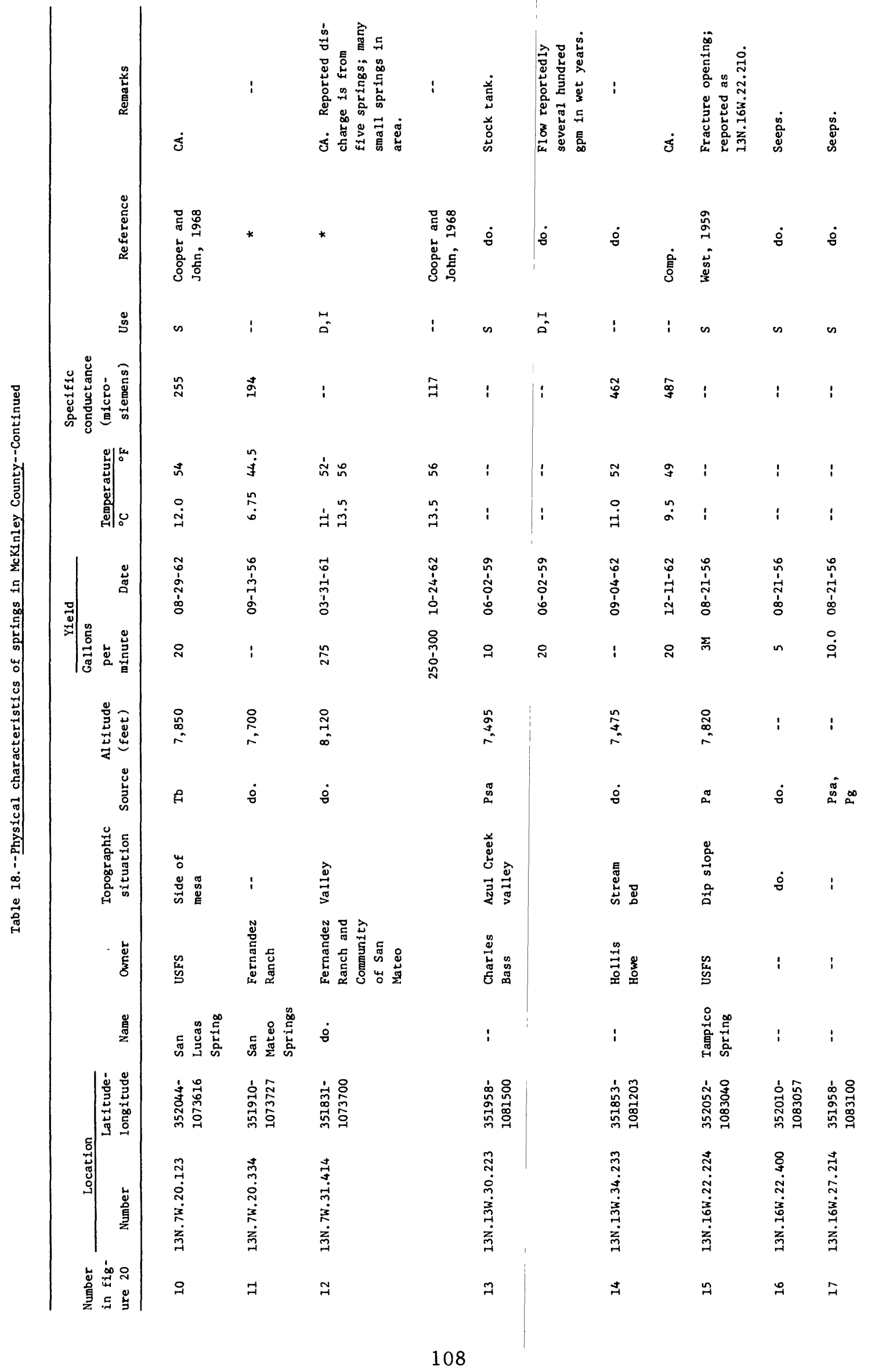




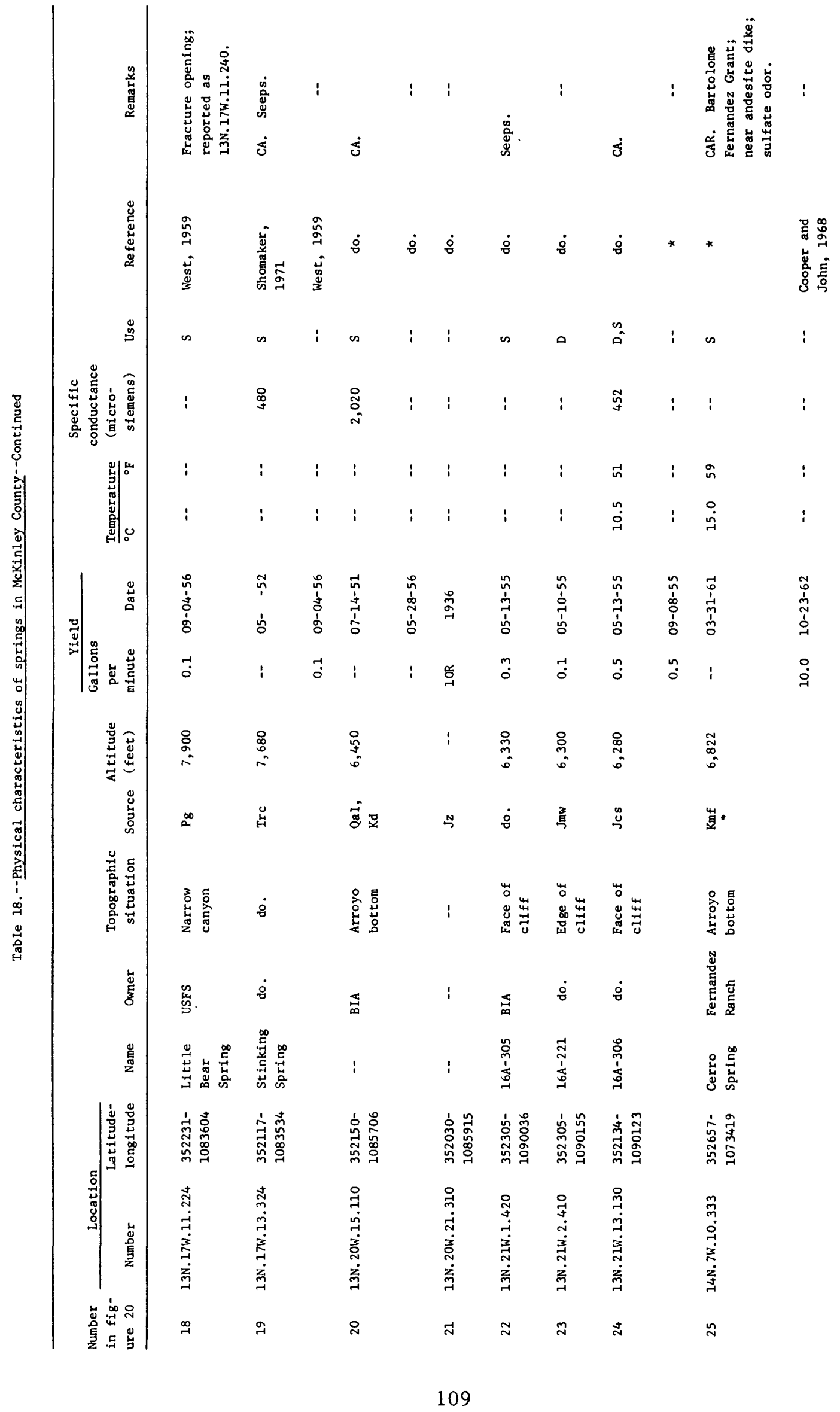




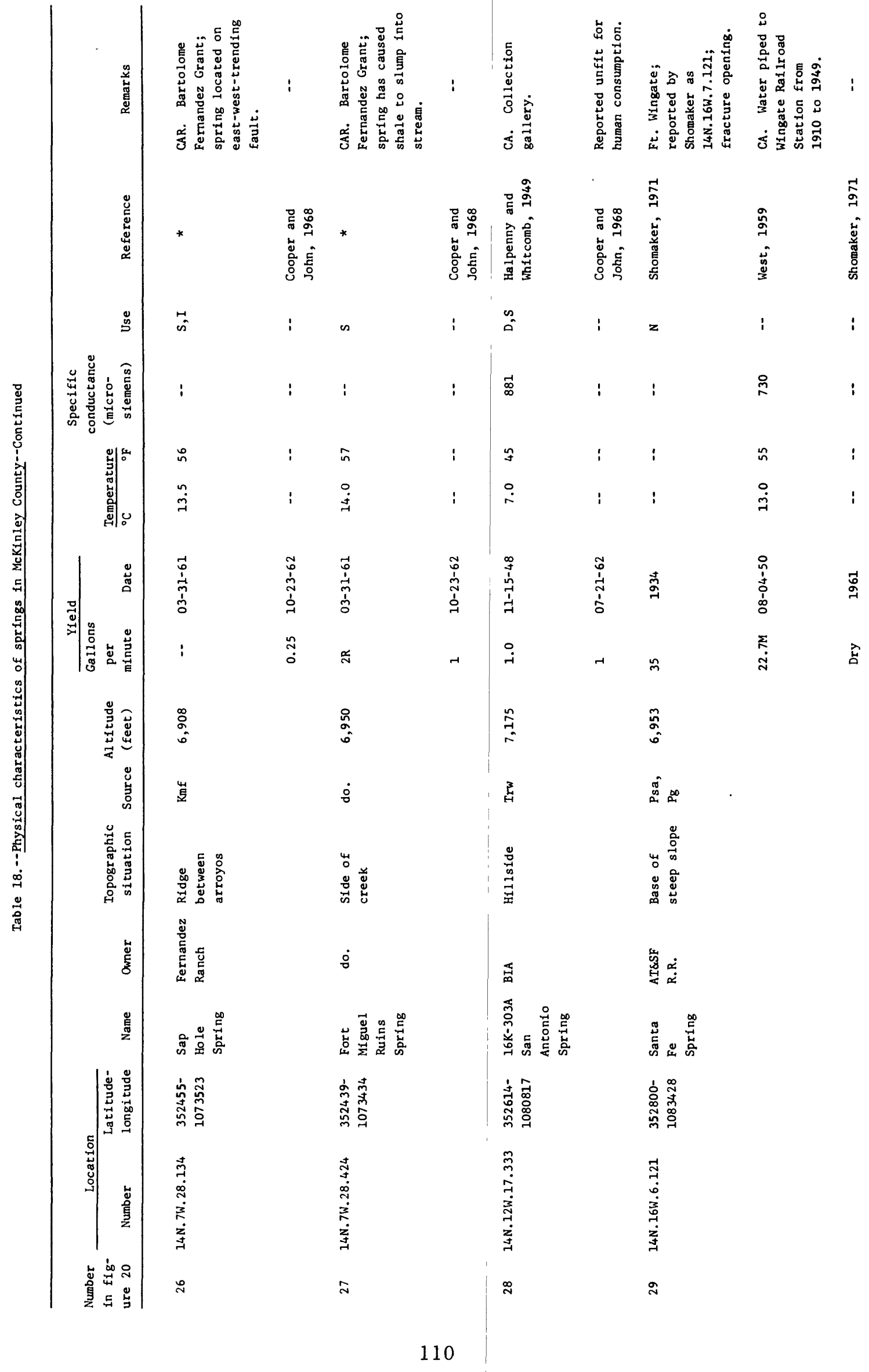




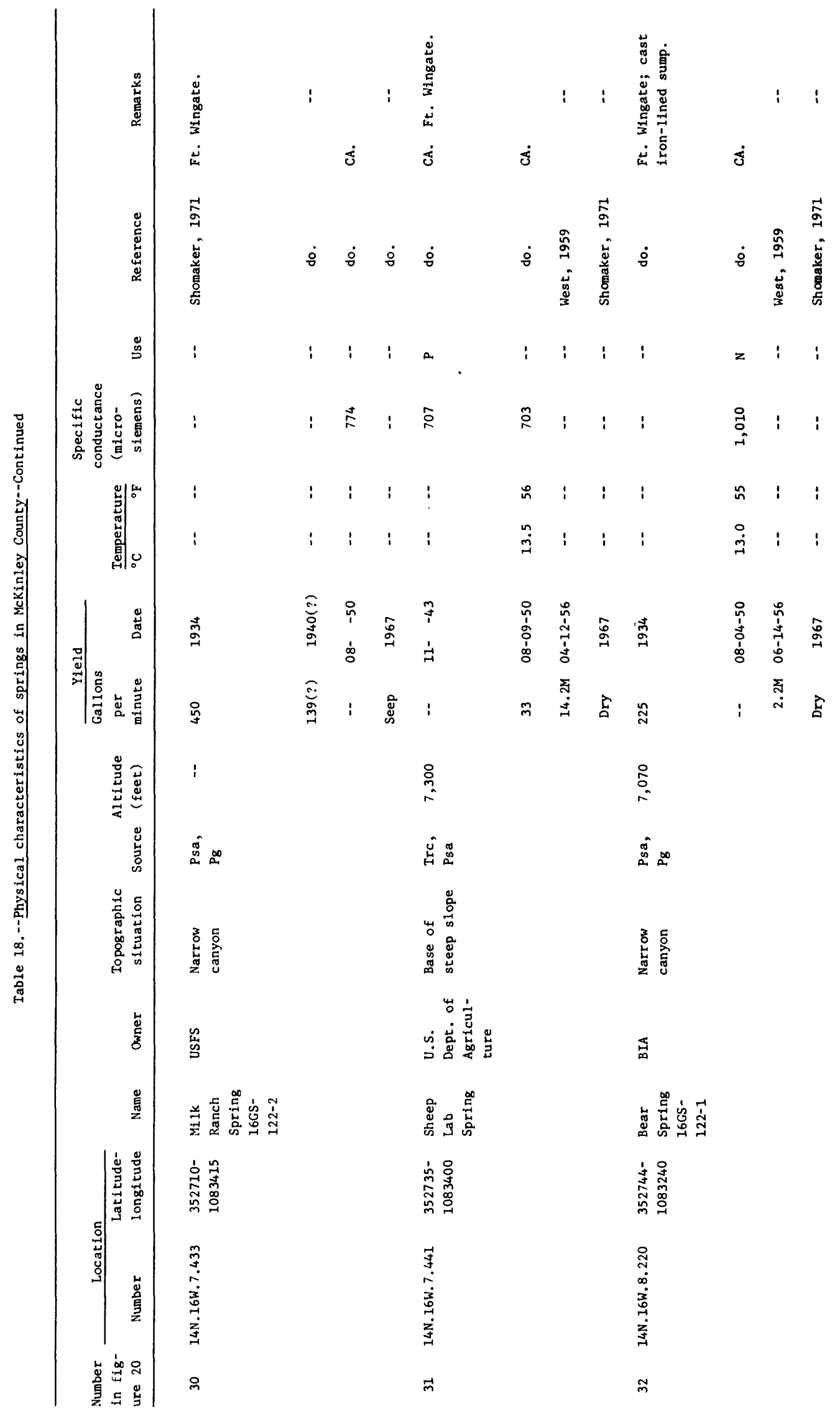




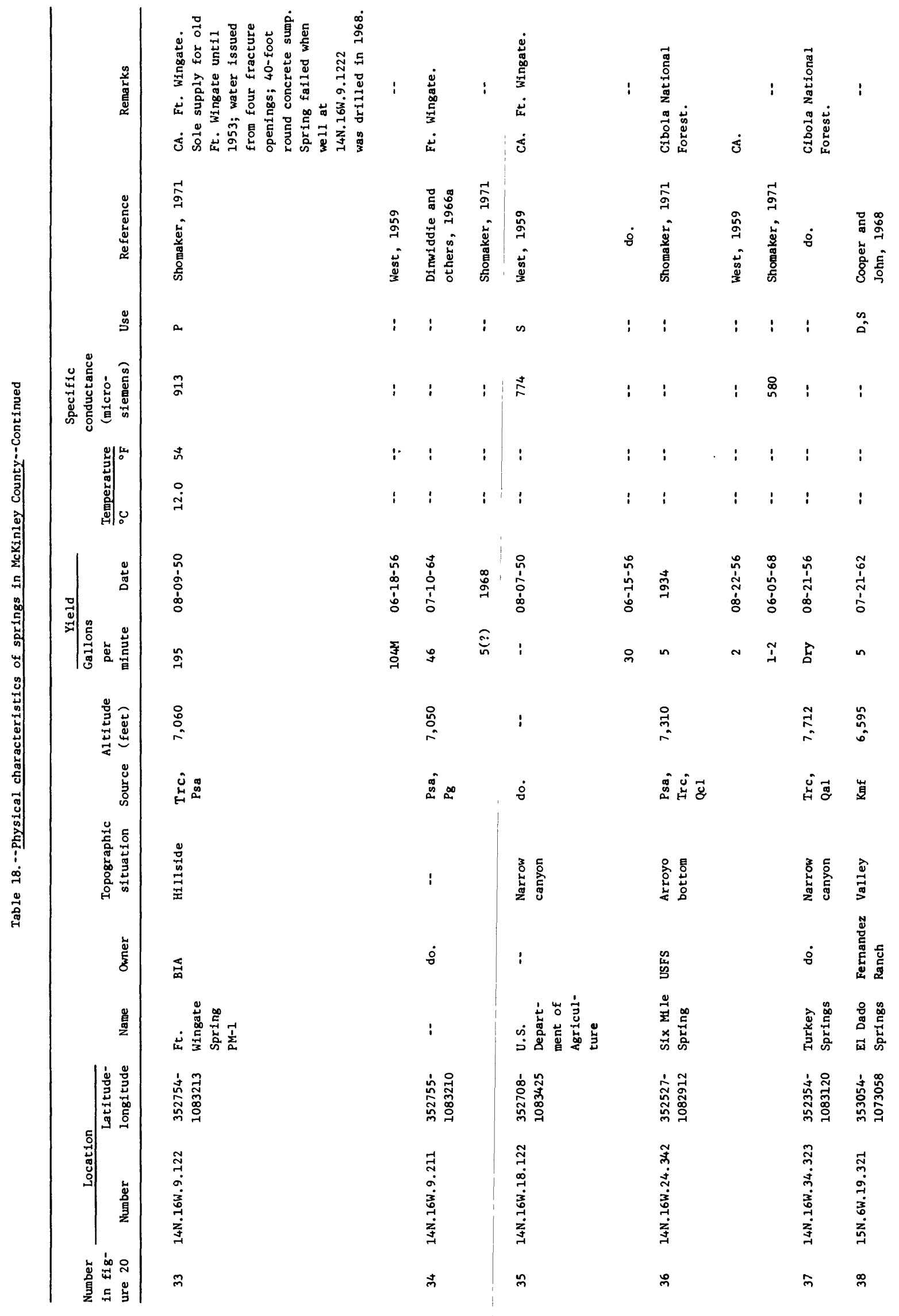




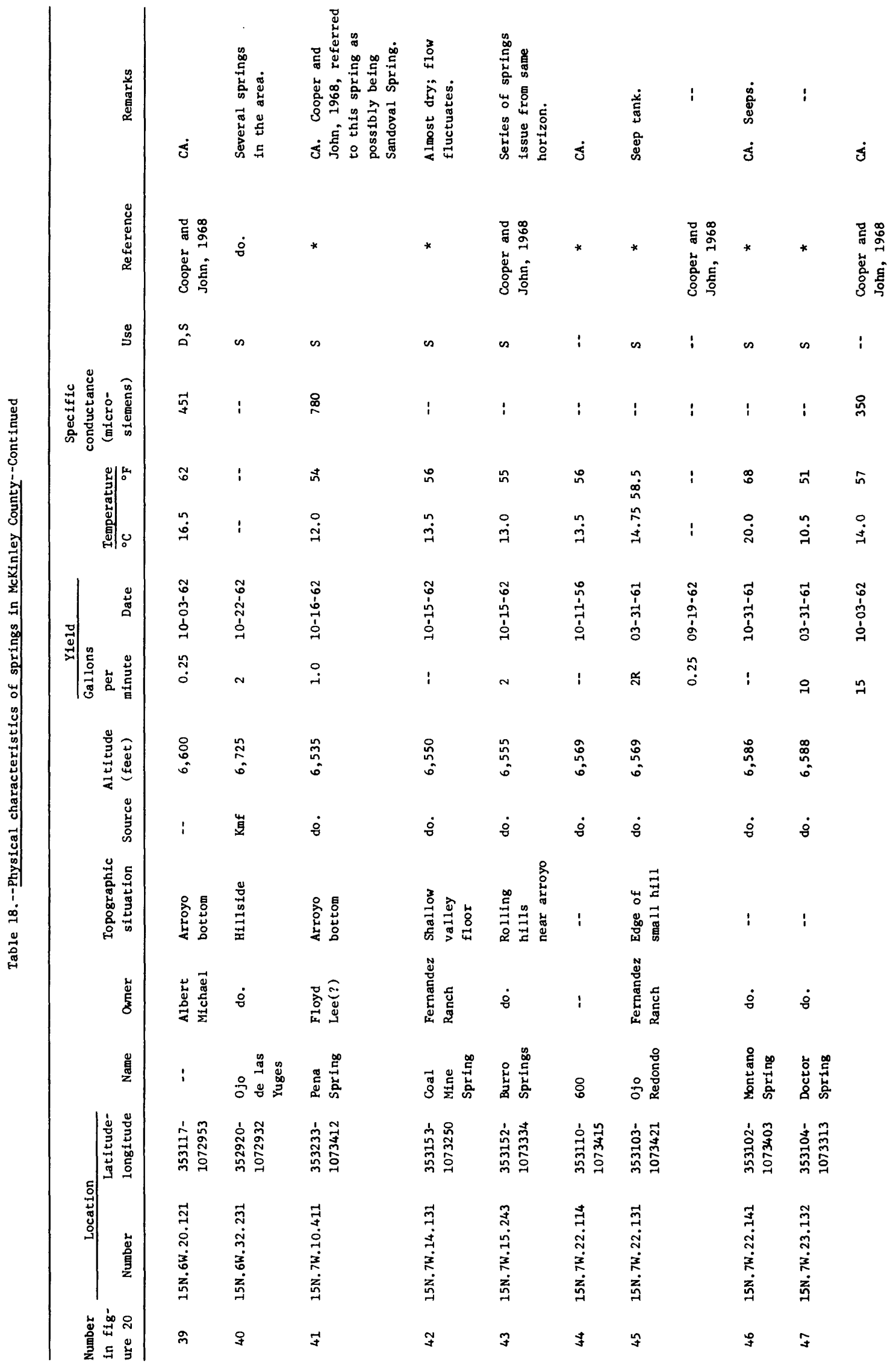




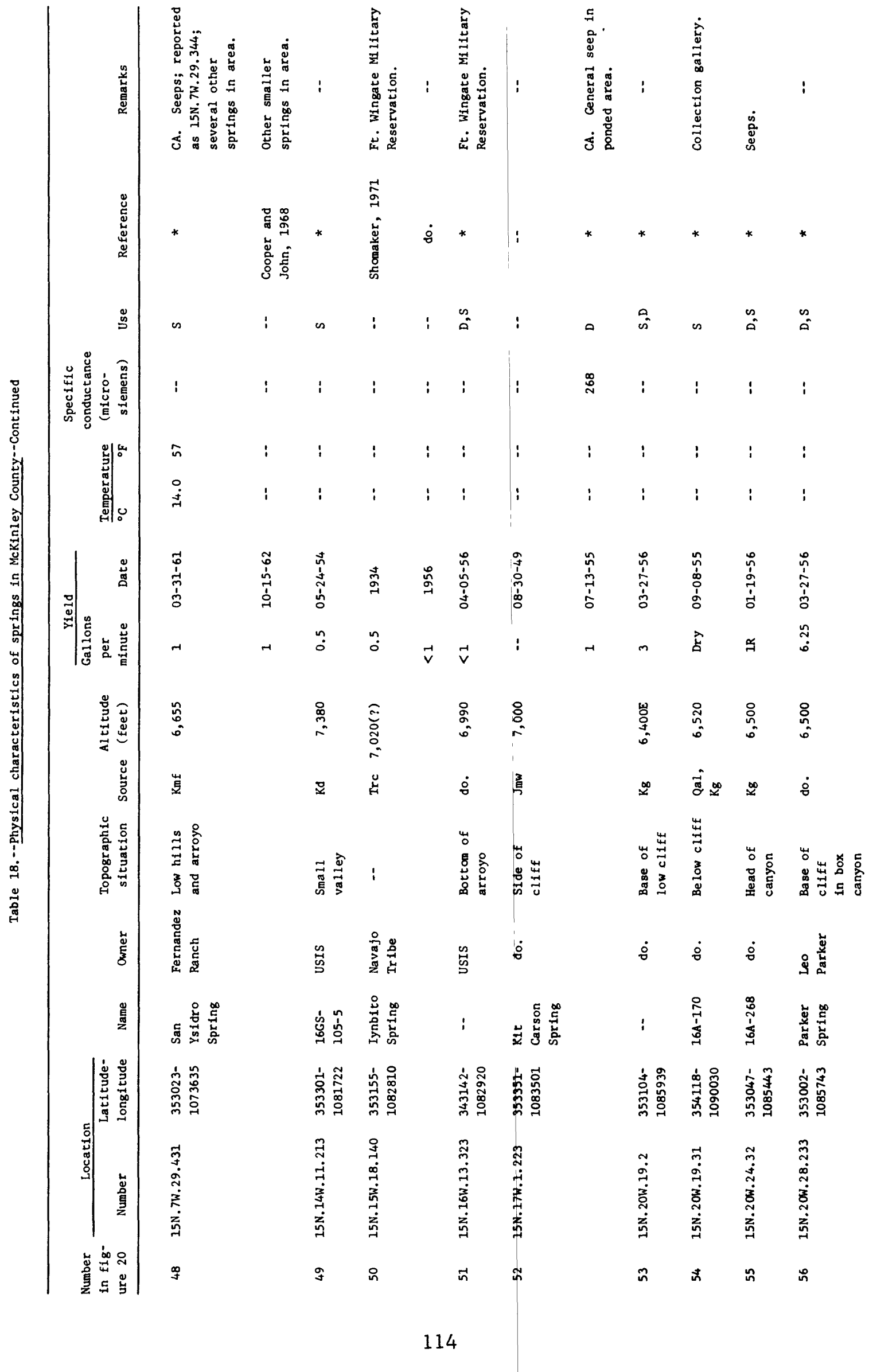




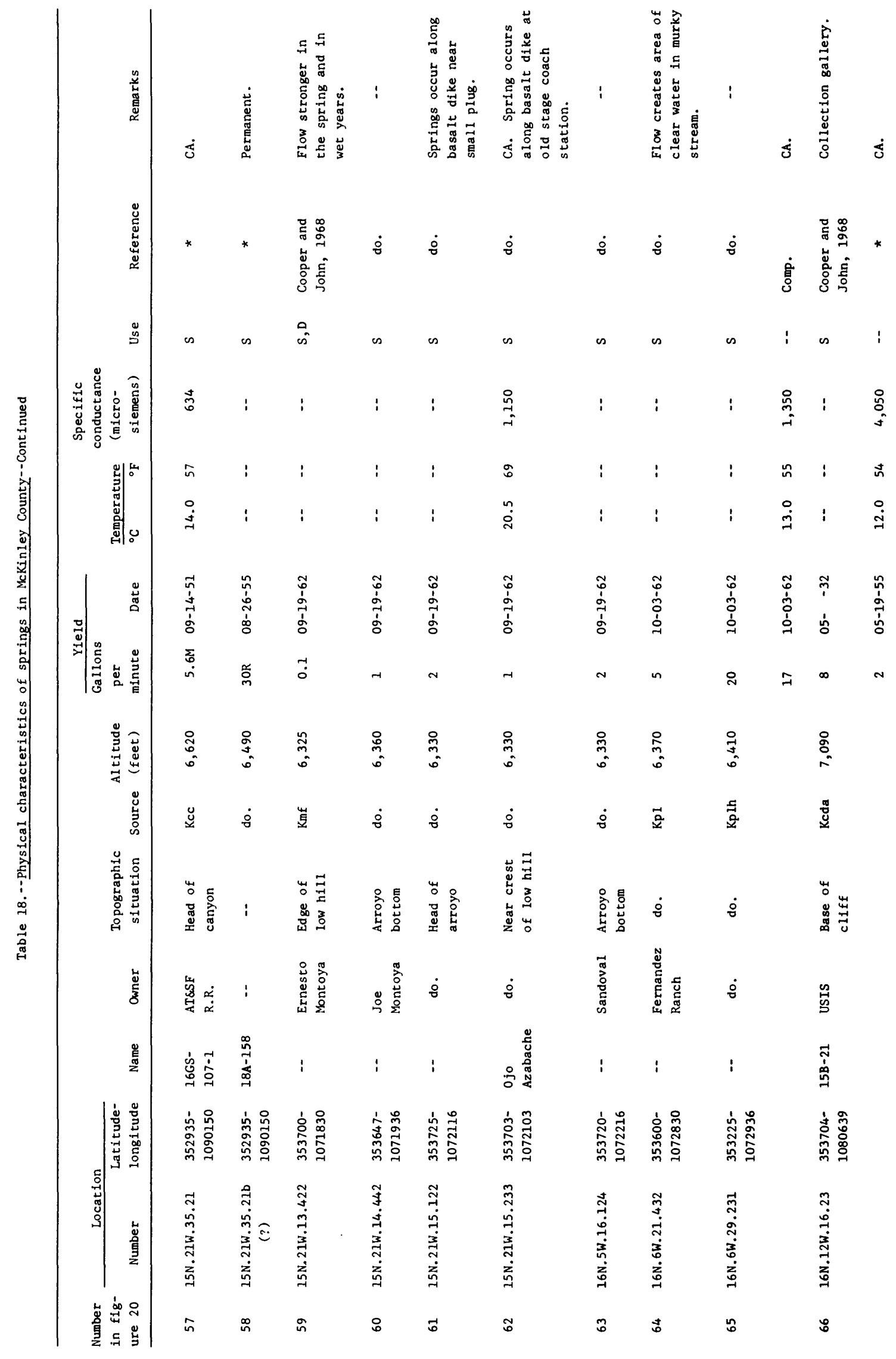




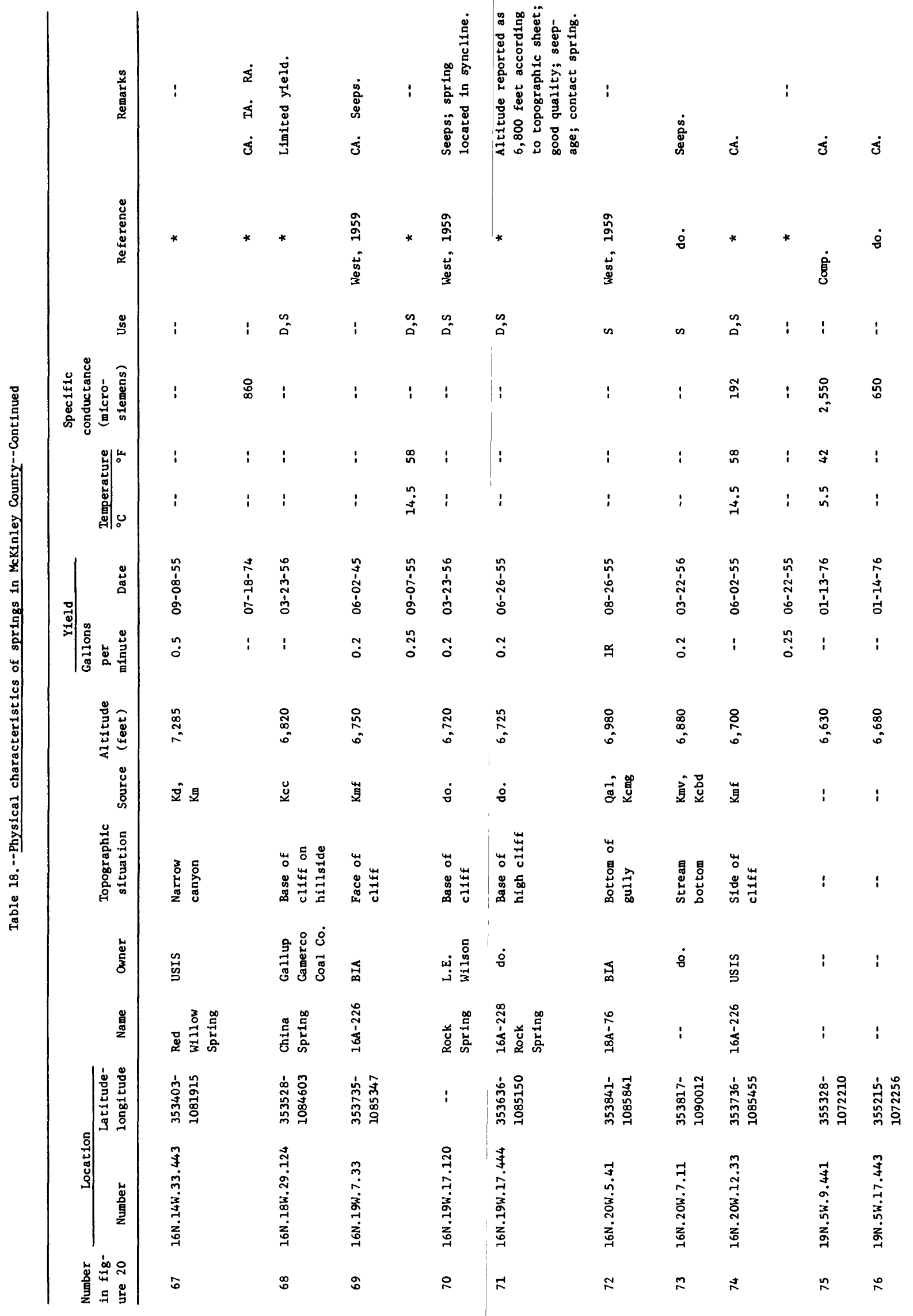




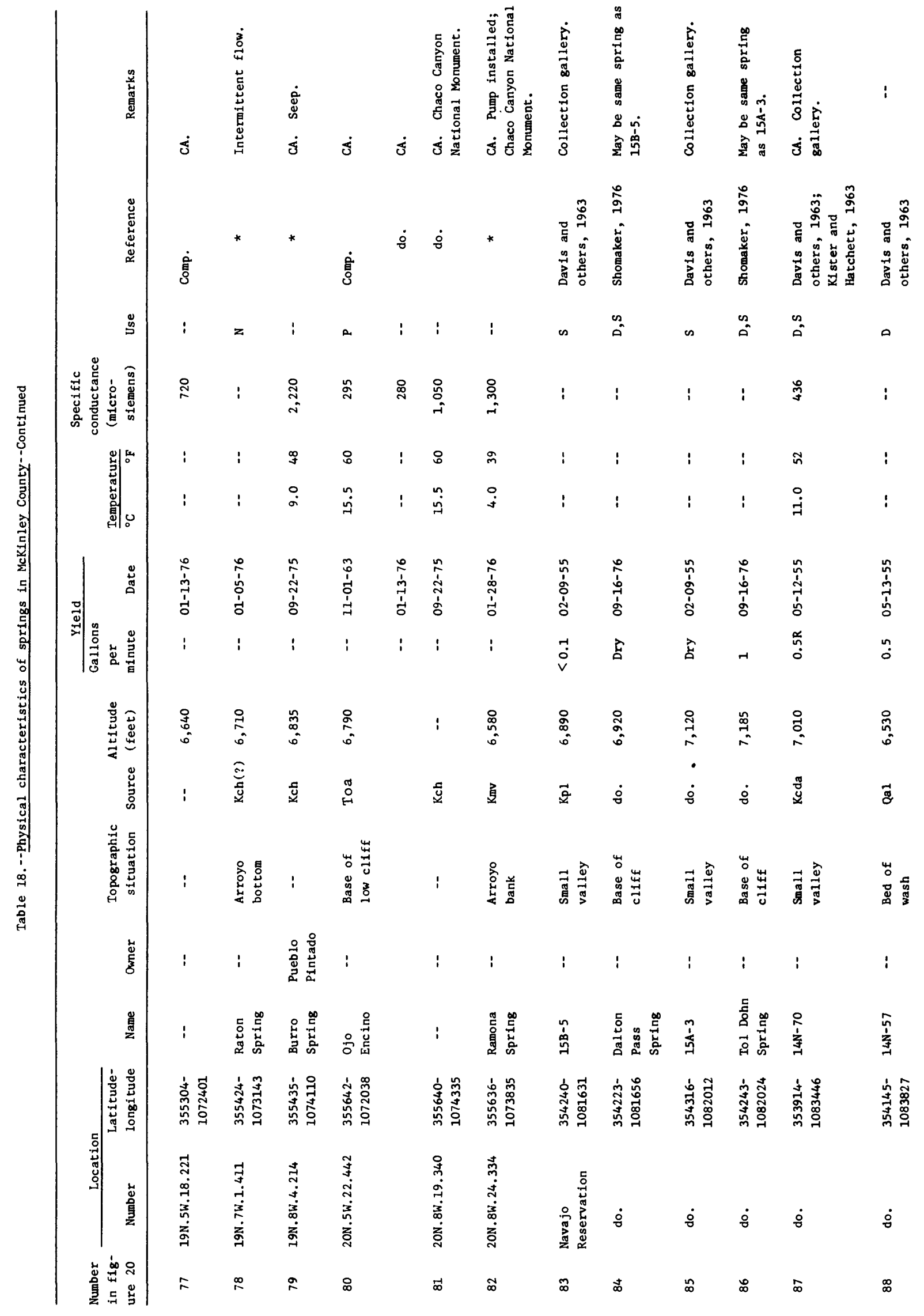




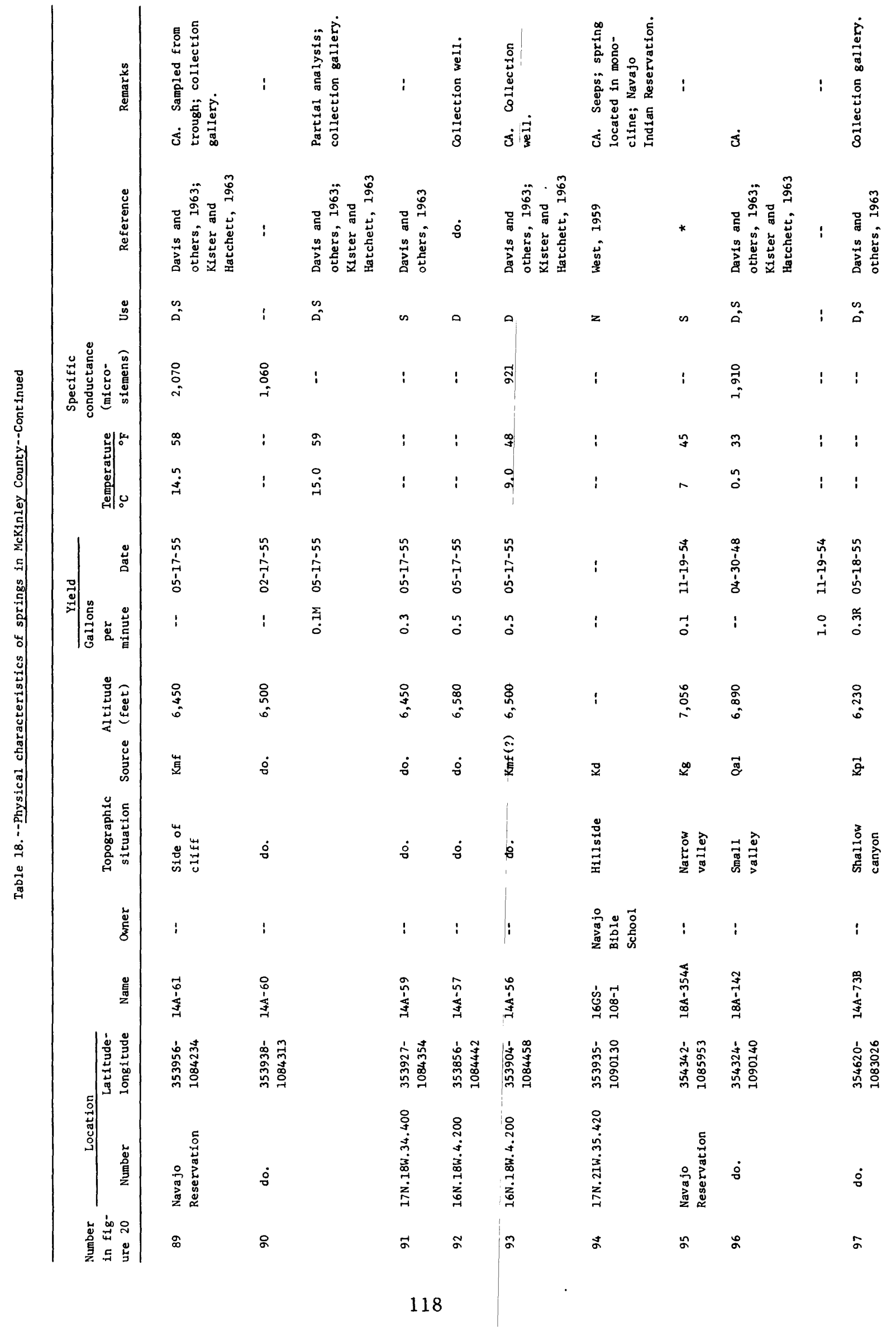




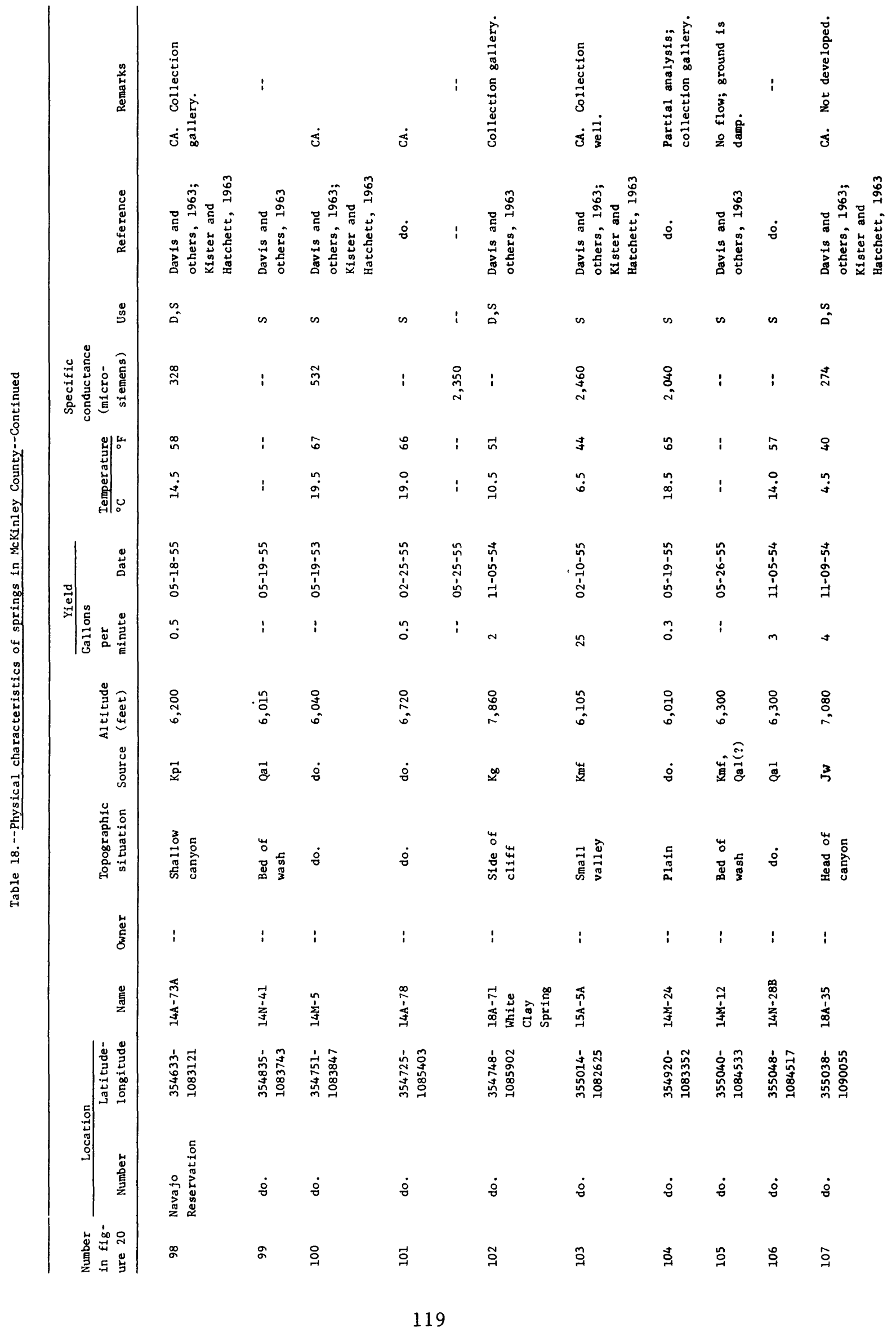




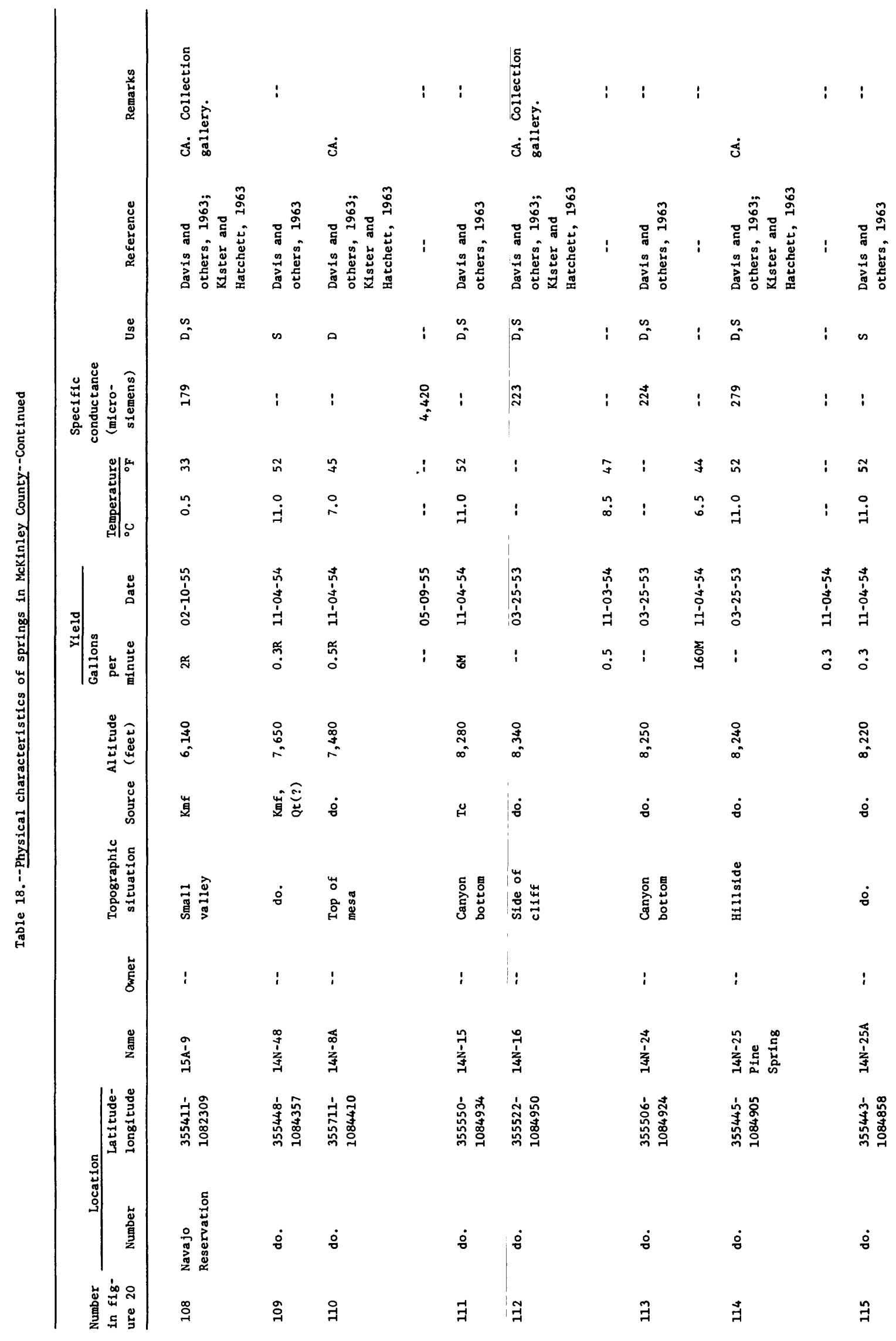




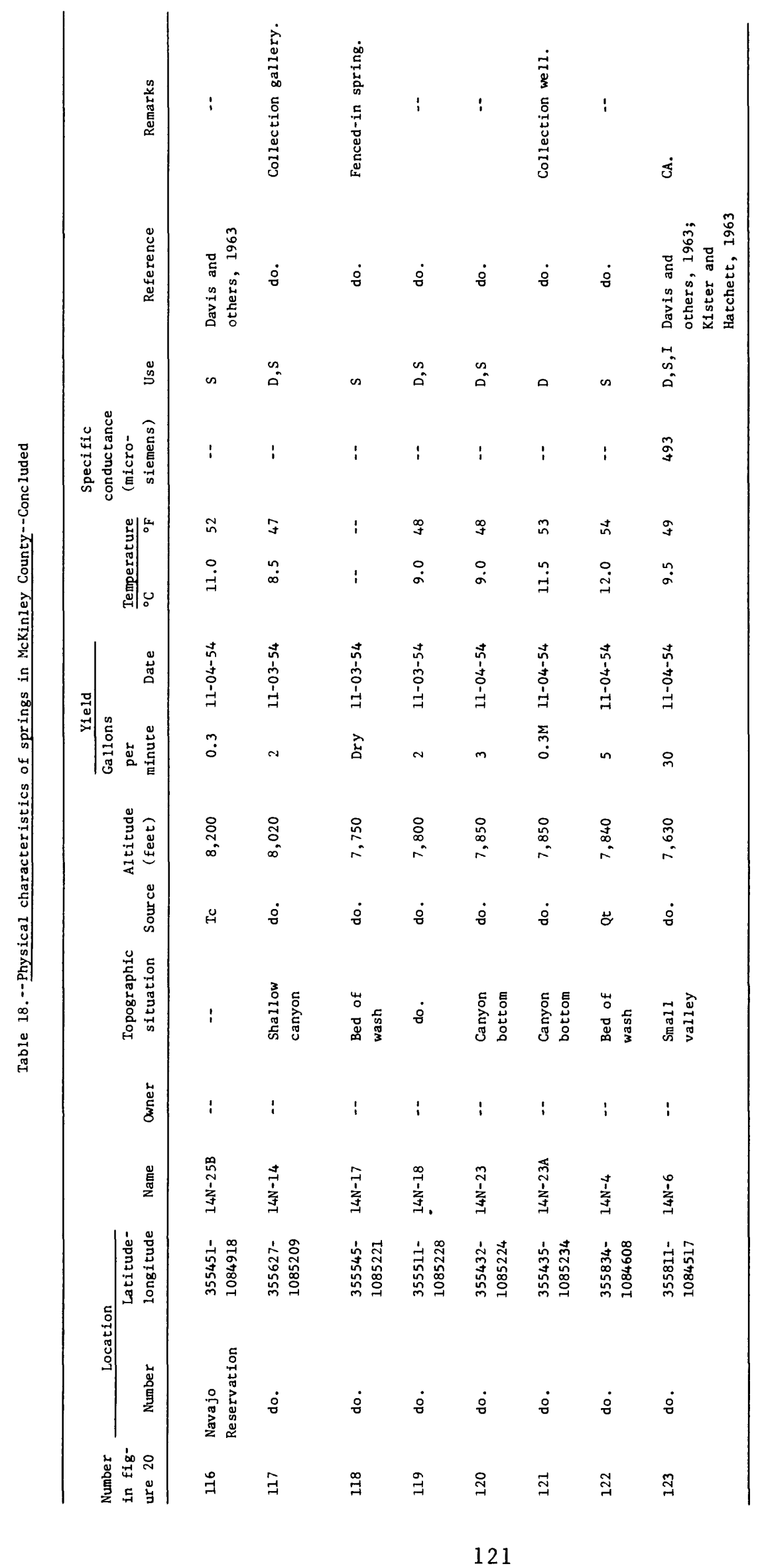




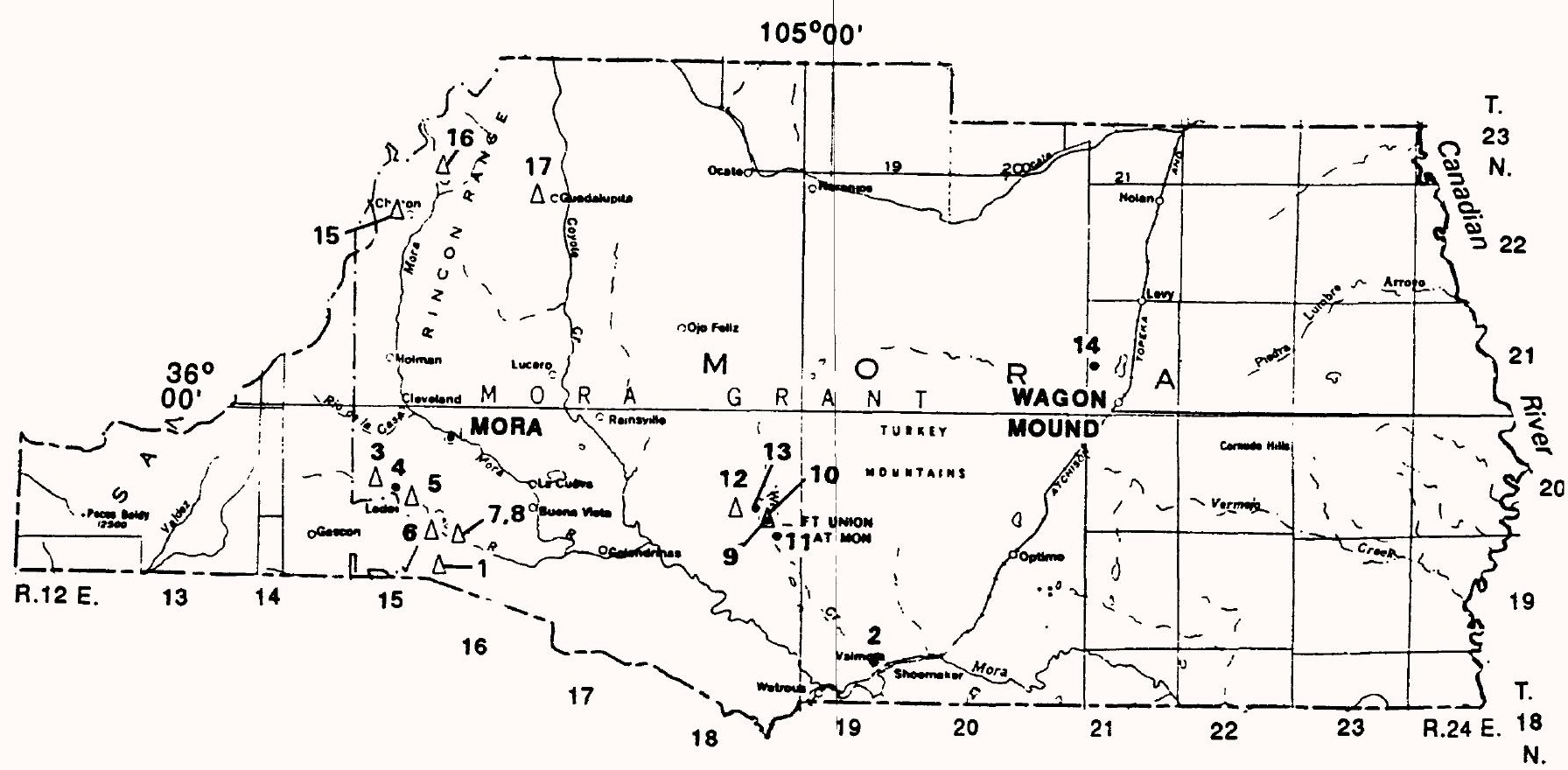

\section{EXPLANATION}

- SPRING WITH CHEMISTRY

$\triangle$ SPRING WITHOUT CHEMISTRY

2 SPRING NUMBER REFERS TO TABLE 19

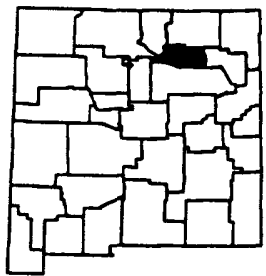

Figure 21.--Location of inventoried springs in Mora County. 


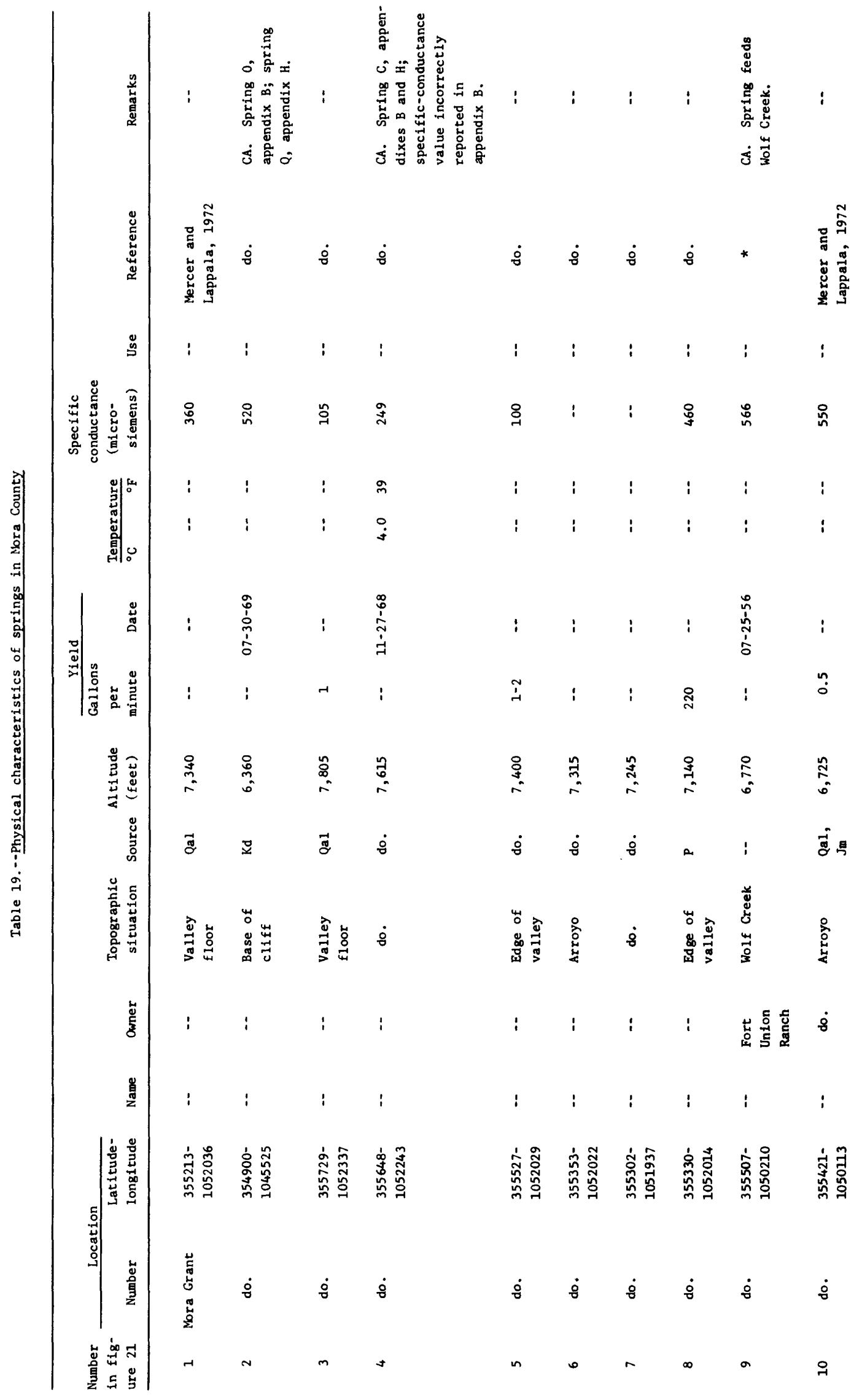




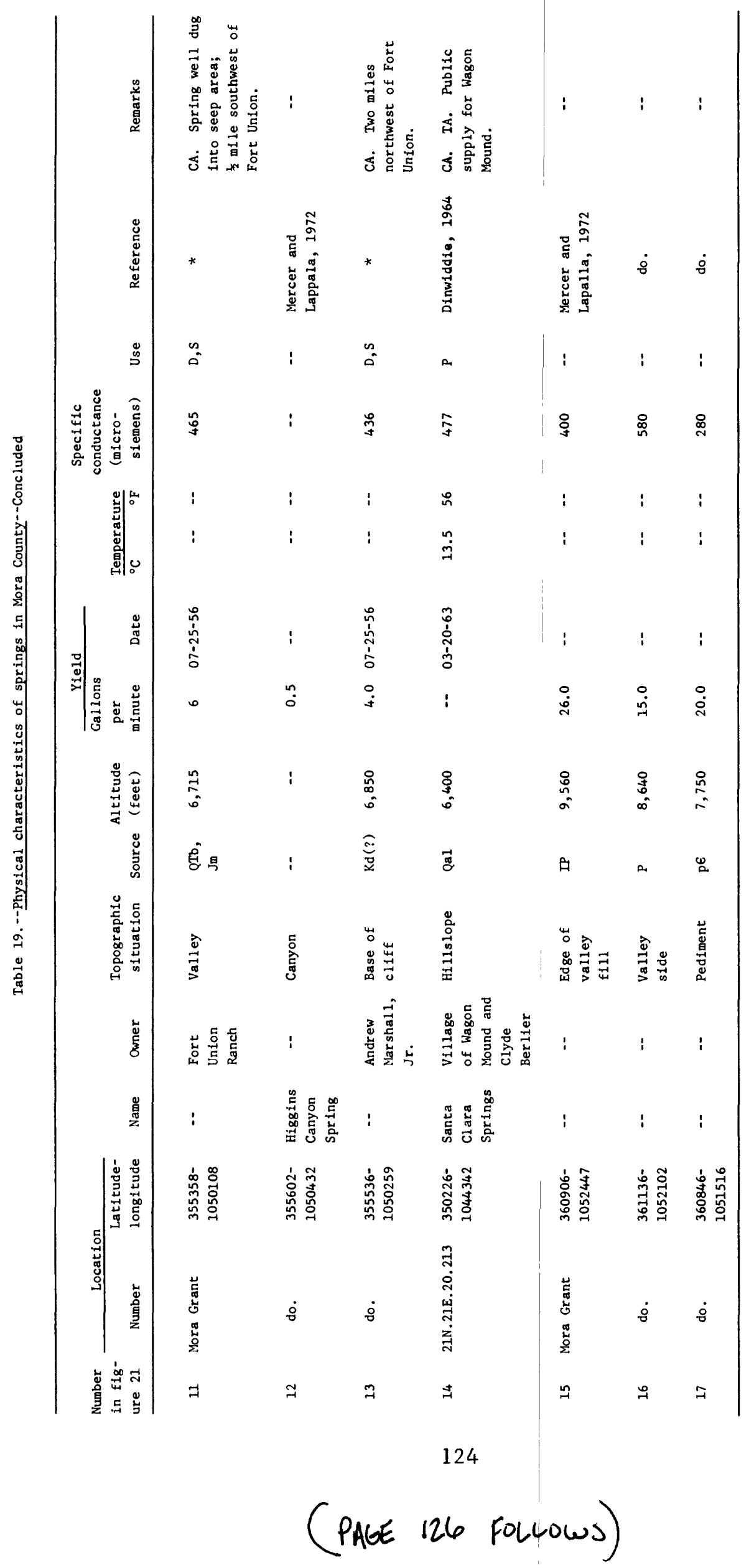




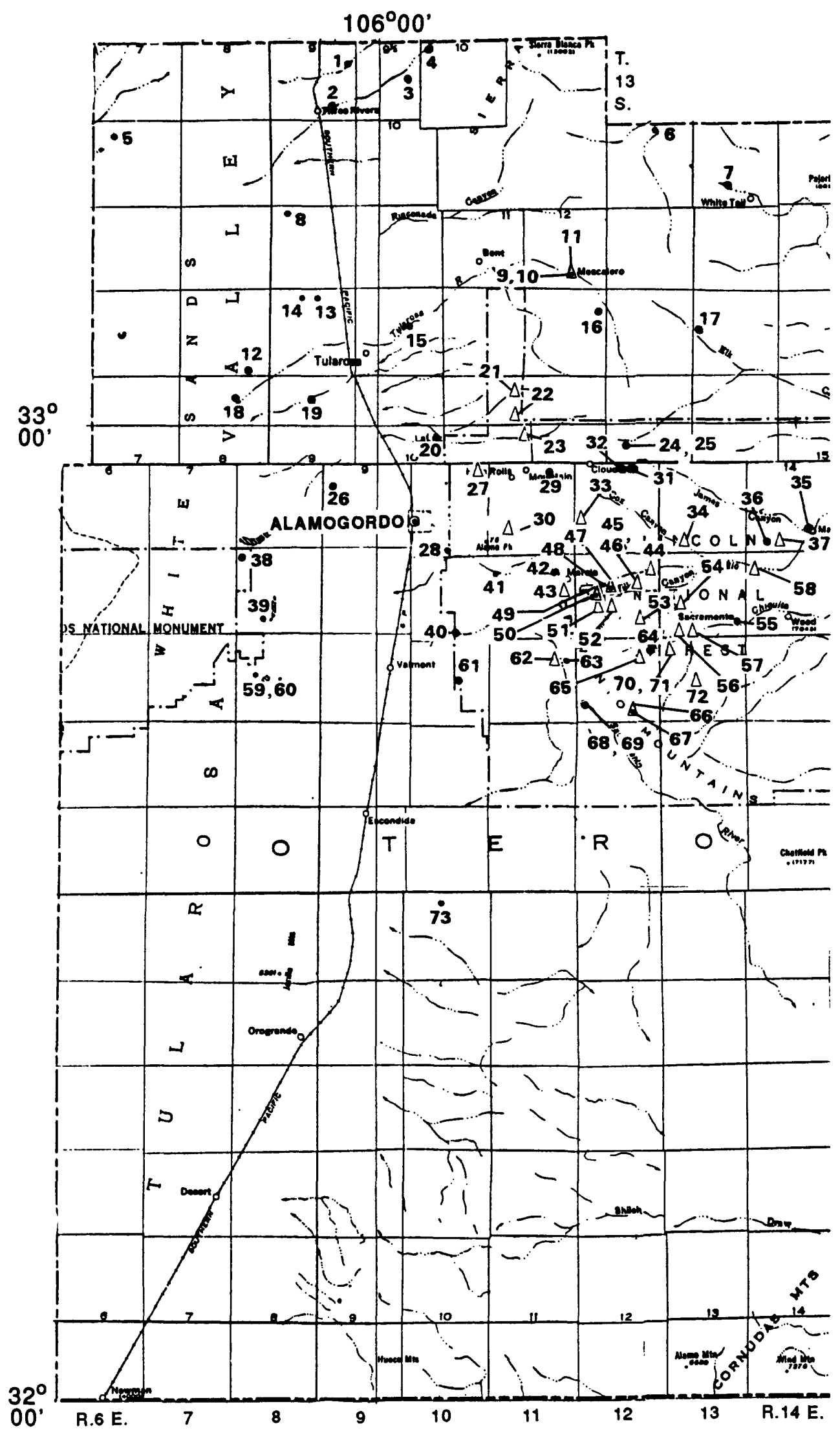




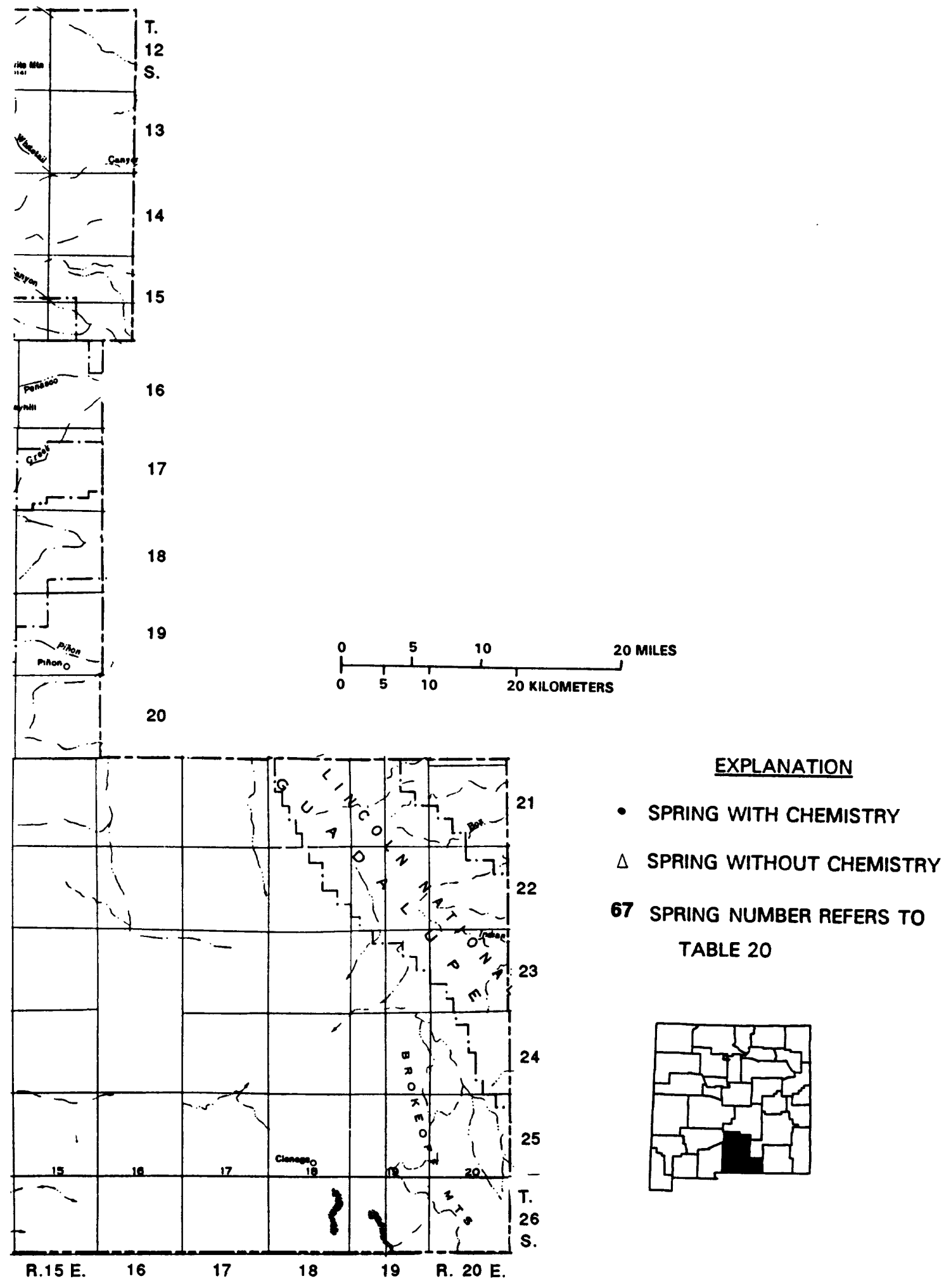

Figure 22.--Location of inventoried springs in Otero County. 


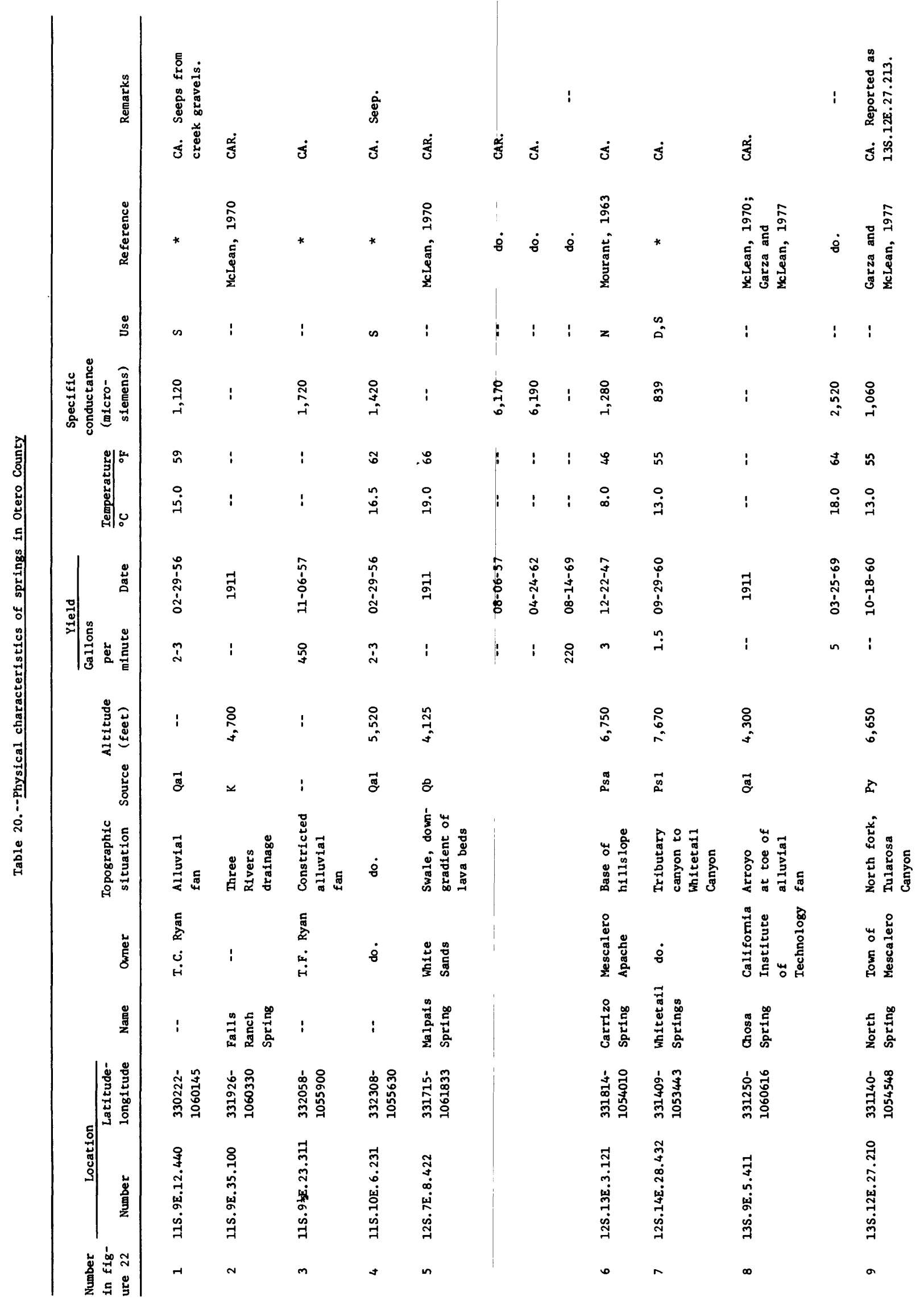




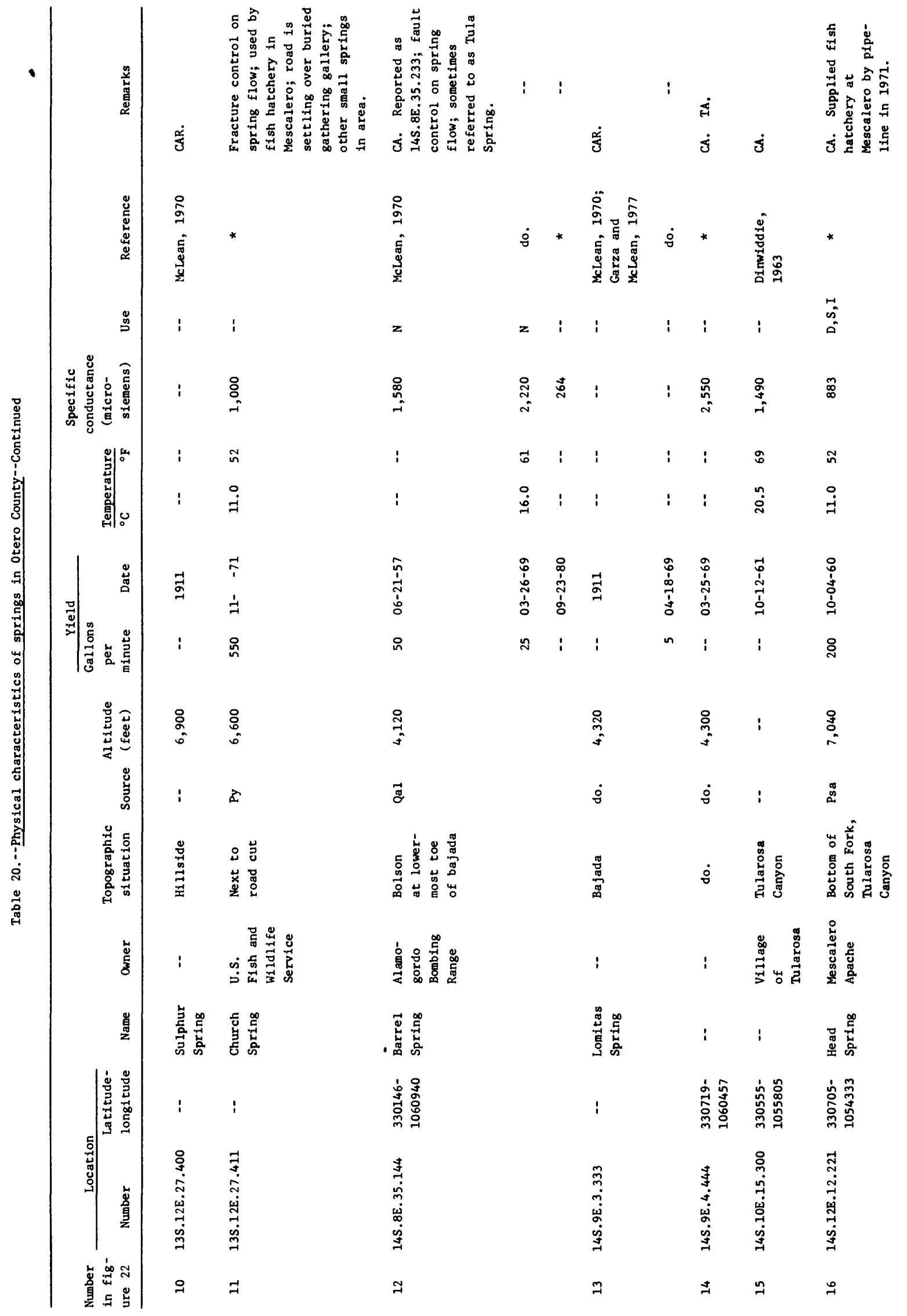




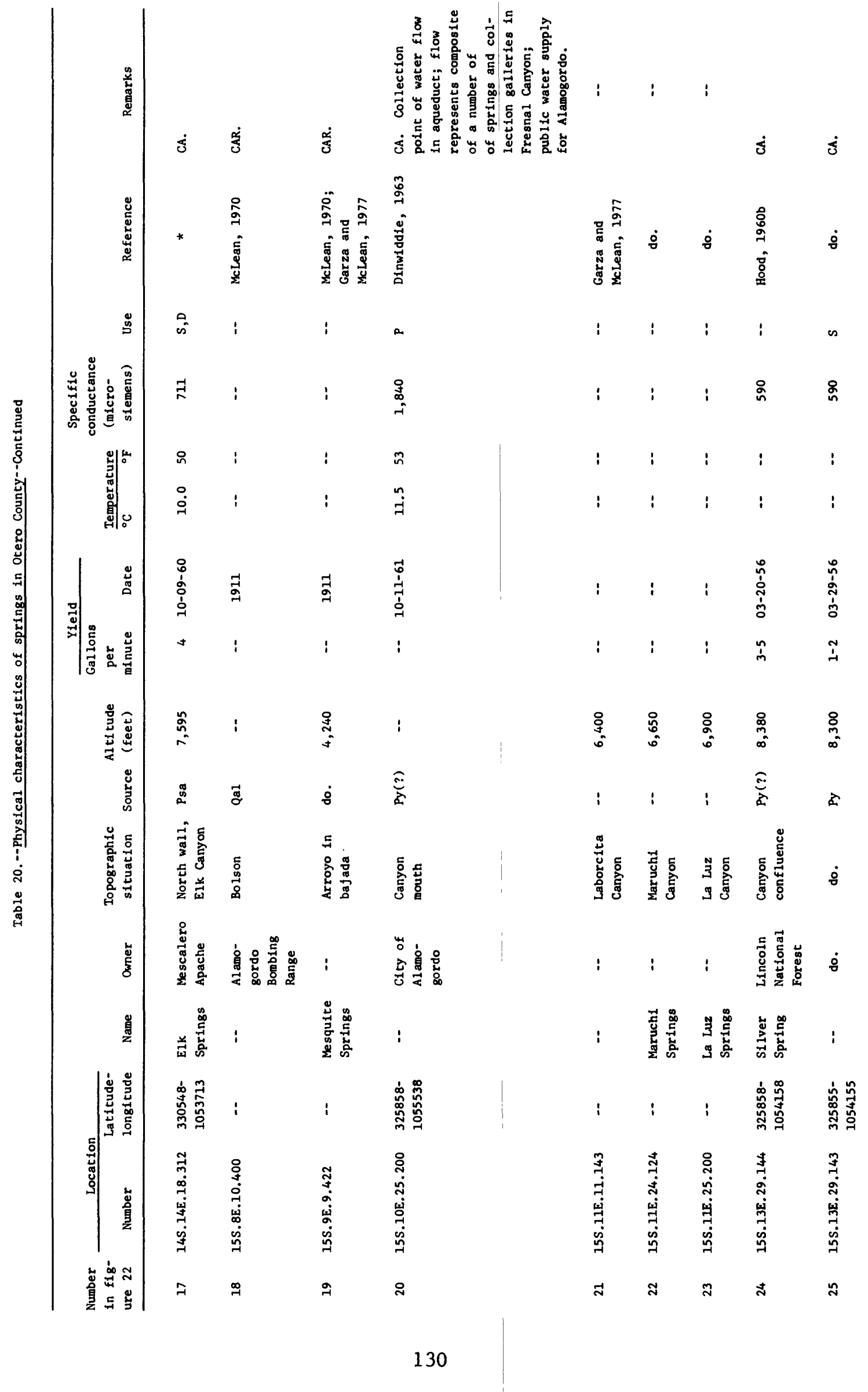




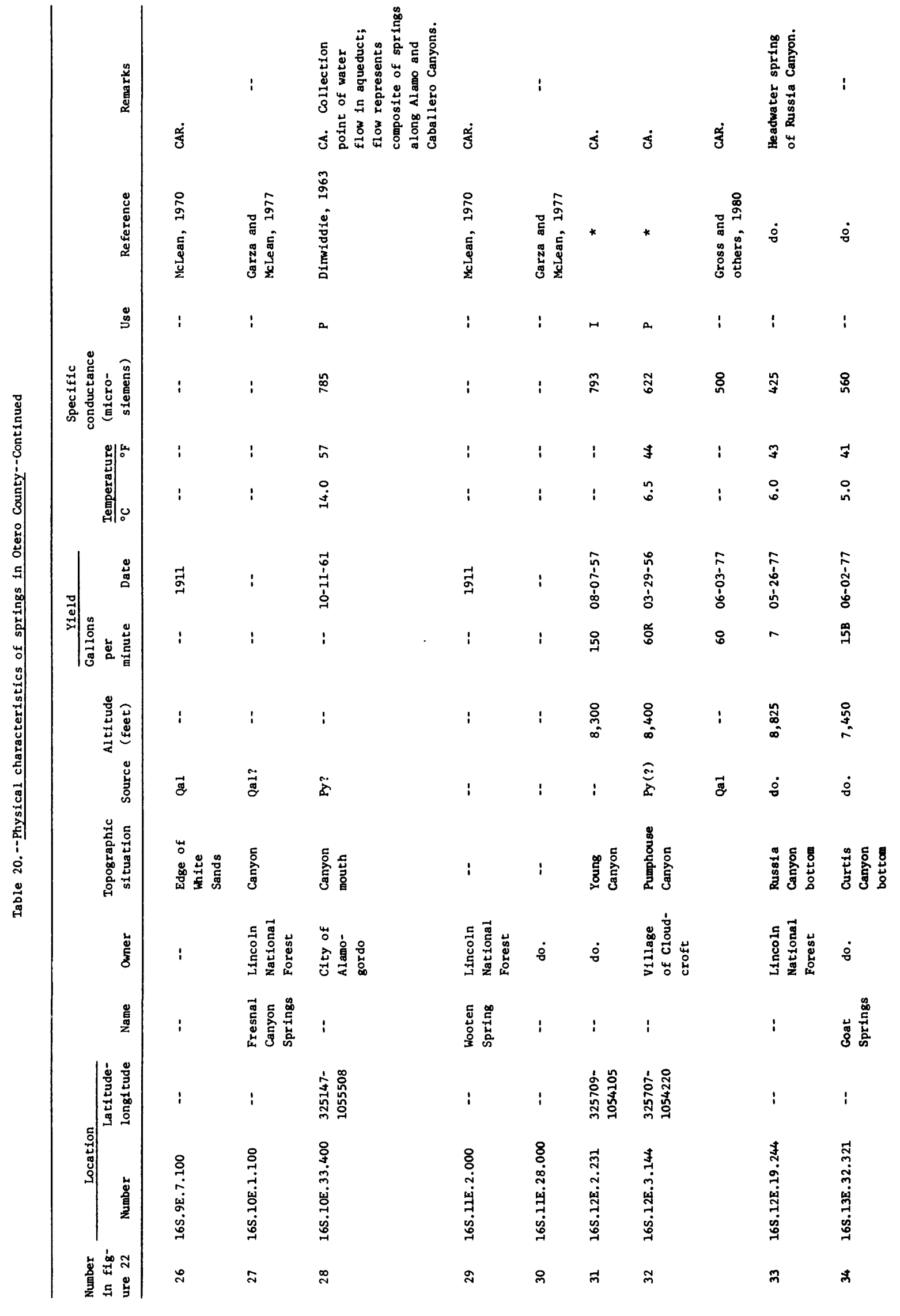




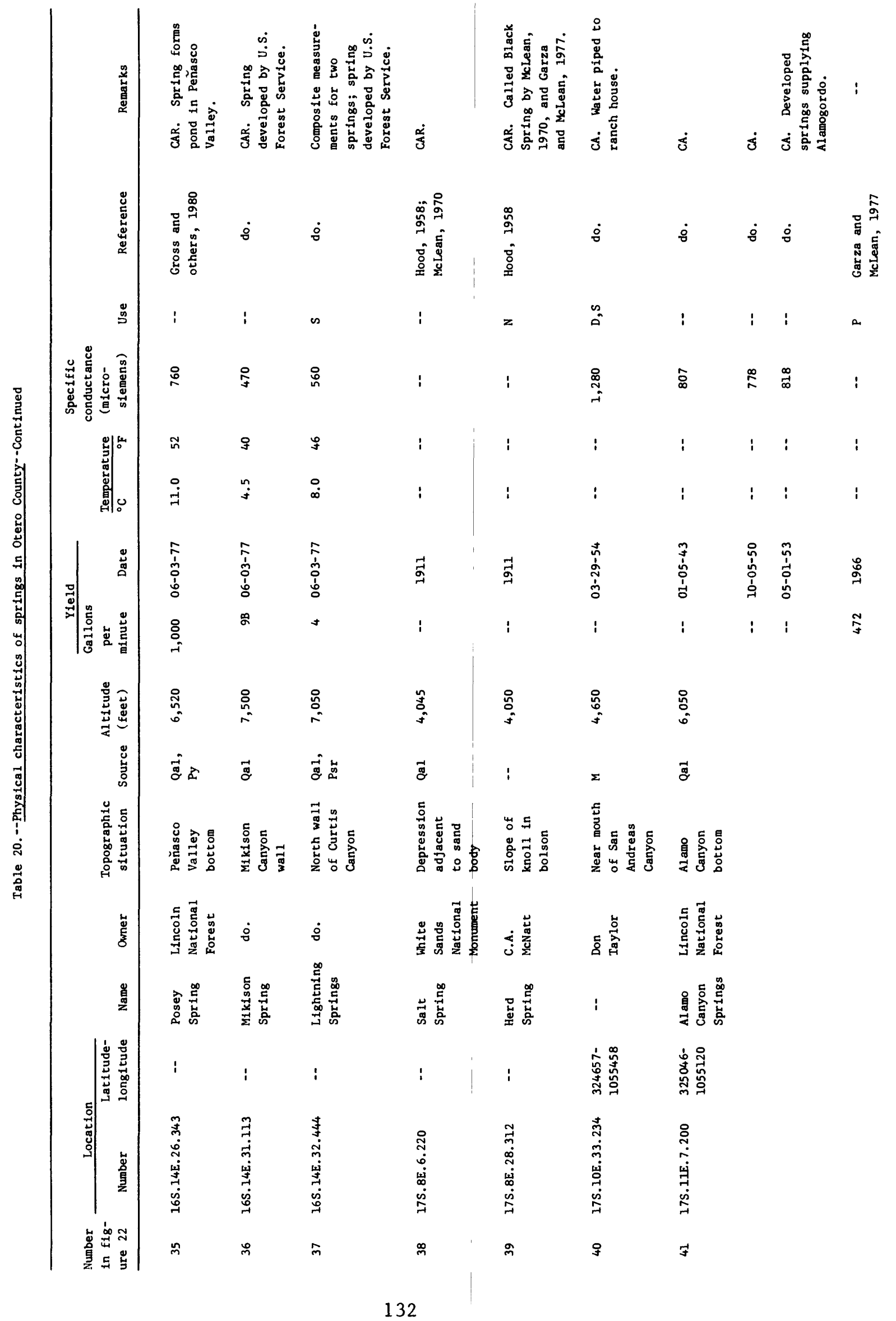




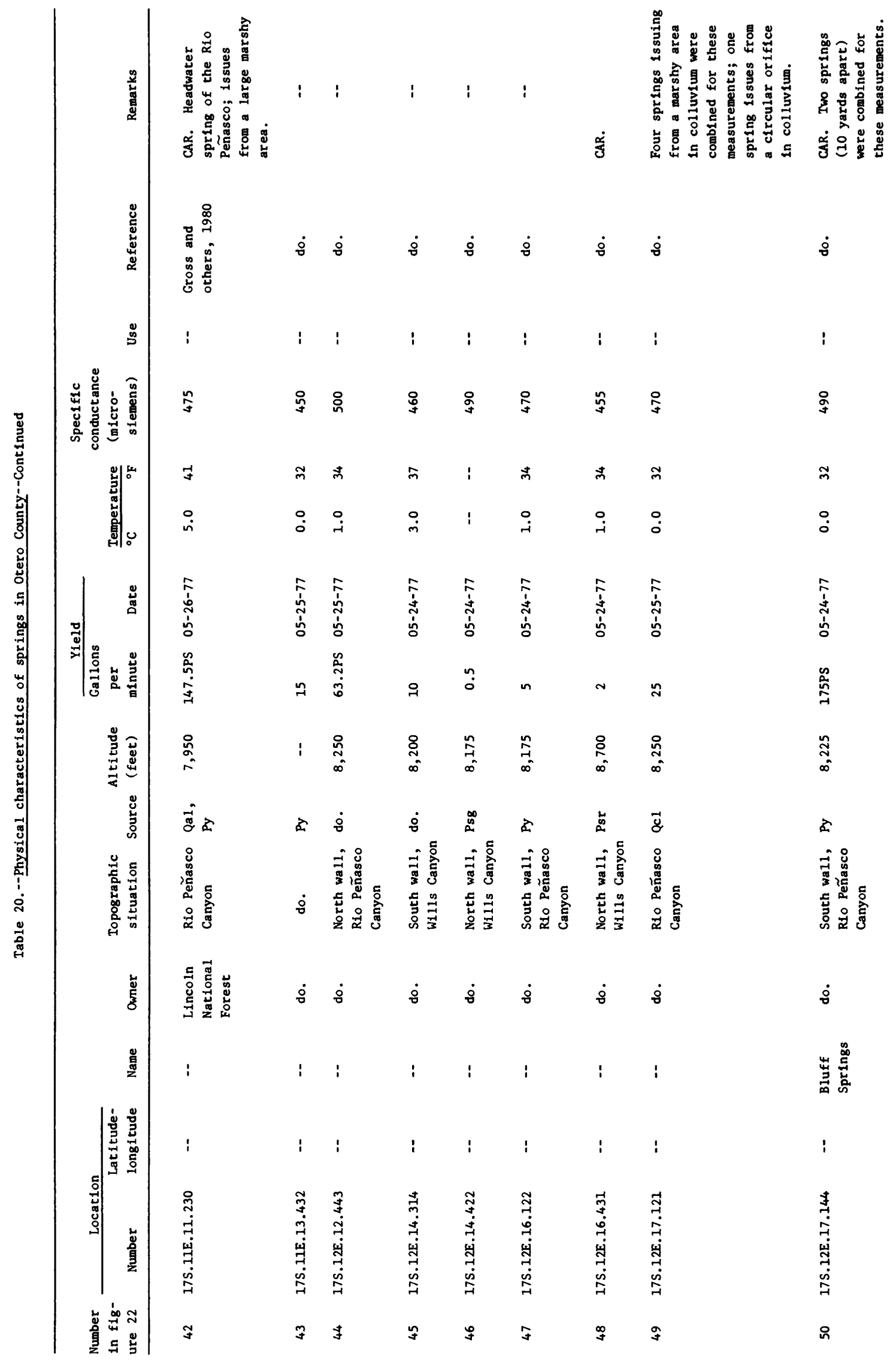




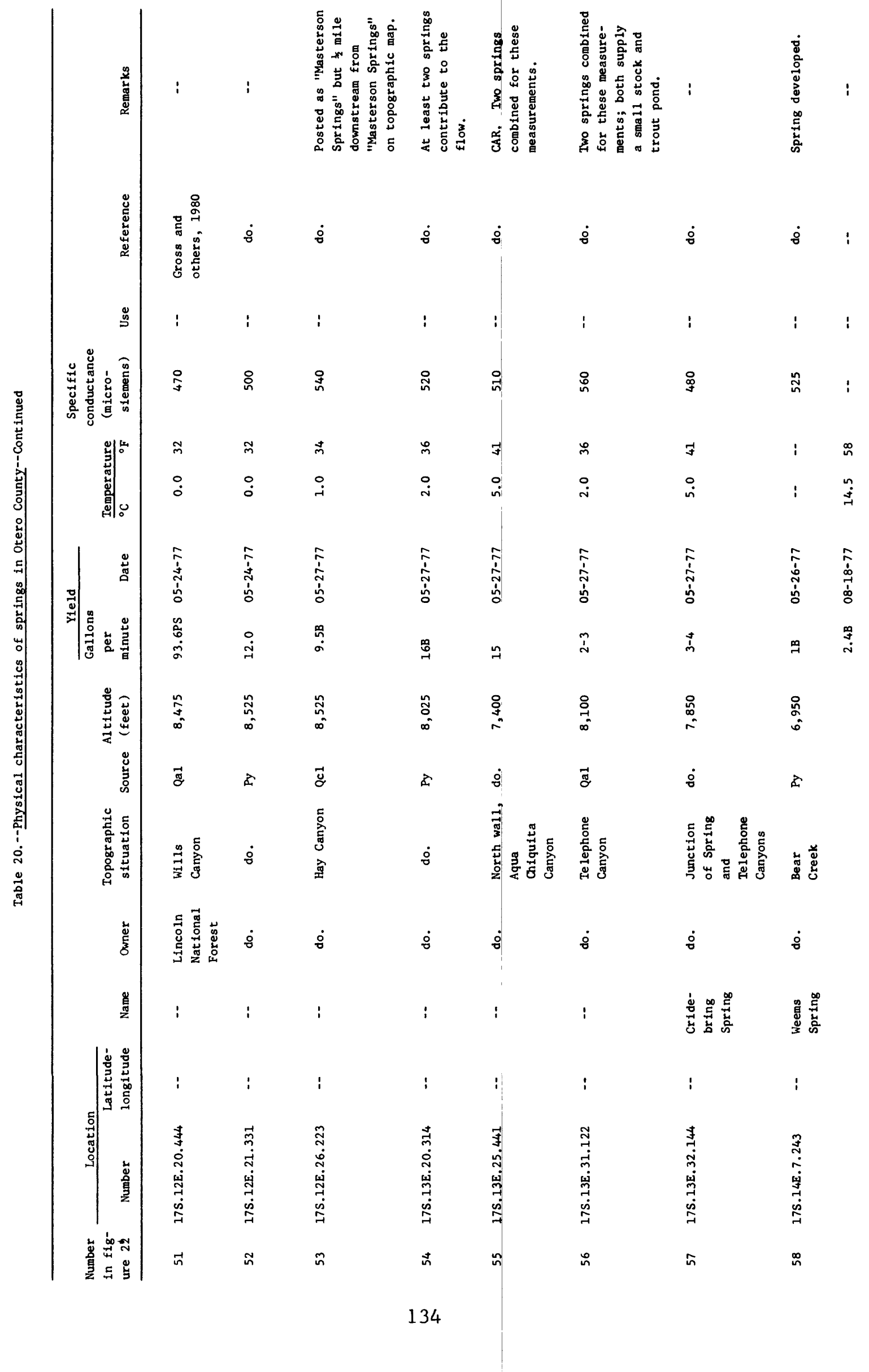




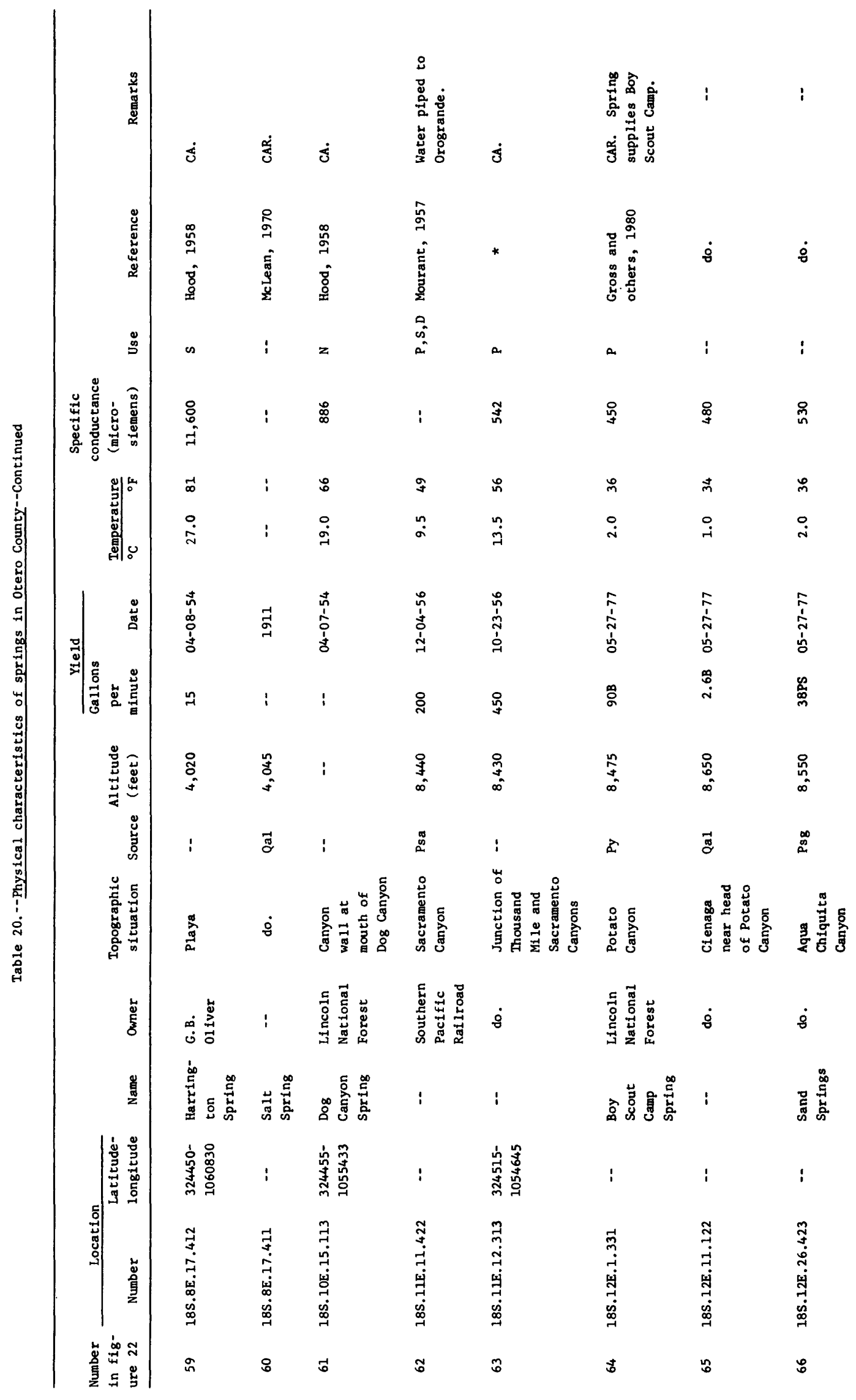




\begin{tabular}{|c|c|c|c|c|c|c|c|c|}
\hline 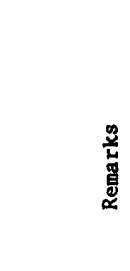 & 总 & 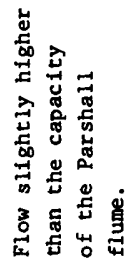 & 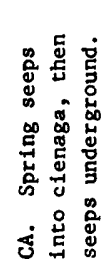 & 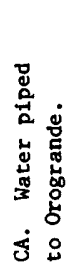 & 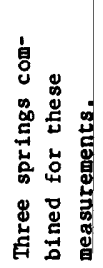 & 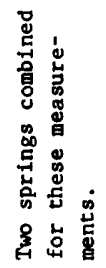 & ! & $\dot{3}$ \\
\hline 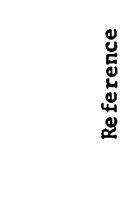 & 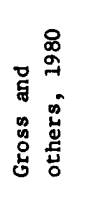 & $\dot{8}$ & 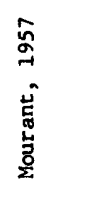 & $\dot{8}$ & 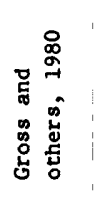 & $\dot{8}$ & $\dot{8}$ & * \\
\hline$\stackrel{:}{:}$ & $:$ & $i$ & $z$ & a & : & i & i & : \\
\hline & nึ & 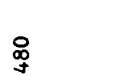 & $\tilde{\mathrm{G}}$ & $\underset{\mathscr{\infty}}{\stackrel{8}{0}}$ & nั & 굴 & : & ৪ి \\
\hline$y_{0}^{2}$ & $\stackrel{m}{\circ}$ & 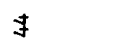 & $q$ & : & $\stackrel{m}{m}$ & $\stackrel{\circ}{m}$ & $\stackrel{\infty}{\infty}$ & t \\
\hline 氤。 & $\stackrel{\stackrel{i}{i}}{ }$ & $\stackrel{n}{6}$ & $\stackrel{n}{a}$ & $:$ & $\stackrel{\circ}{i}$ & $\stackrel{\circ}{i}$ & $\stackrel{\circ}{\dot{m}}$ & $\begin{array}{l}0 \\
\infty \\
\end{array}$ \\
\hline 미 & $\begin{array}{l}\hat{i} \\
\dot{\tilde{n}} \\
\dot{a}\end{array}$ & $\begin{array}{l}\hat{\hat{d}} \\
\vec{\phi} \\
\overrightarrow{0} \\
\dot{0}\end{array}$ & 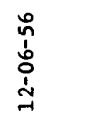 & 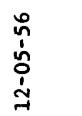 & $\begin{array}{l}\hat{i} \\
\hat{i} \\
\dot{n} \\
0\end{array}$ & $\begin{array}{l}\hat{i} \\
\hat{a} \\
\dot{n} \\
0\end{array}$ & $\begin{array}{l}\hat{n} \\
\hat{a} \\
\dot{n} \\
o\end{array}$ & $\begin{array}{l}\hat{n} \\
\hat{n} \\
\dot{j}\end{array}$ \\
\hline " & 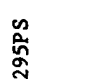 & 离 & 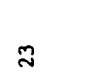 & $\approx$ & n & $a$ & 京 & $:$ \\
\hline 苛 & $\begin{array}{c}0 \\
i n \\
\infty \\
\infty\end{array}$ & & $\underset{\substack{\infty \\
\text { D. }}}{0}$ & 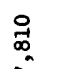 & $\stackrel{8}{-1}$ & 욤 & $\hat{\sigma}$ & : \\
\hline 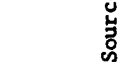 & 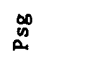 & & 恕 & i & $z$ & $\dot{g}$ & $\overrightarrow{\mathbb{g}}$ & $\stackrel{\mathscr{2}}{\infty}$ \\
\hline 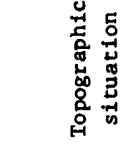 & 营营 & & 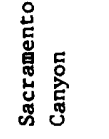 & $\dot{8}$ & 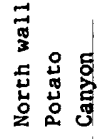 & 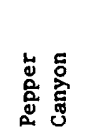 & 总 & 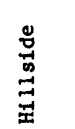 \\
\hline$\frac{\breve{v}}{3}$ & 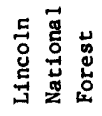 & & 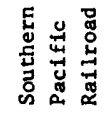 & $\dot{8}$ & 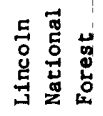 & $\dot{8}$ & $\dot{8}$ & 它会 \\
\hline$\frac{\text { 基 }}{2}$ & 预 & & i & i & : & : & 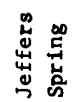 & i \\
\hline 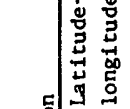 & $i$ & & 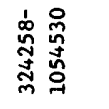 & 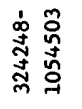 & $i$ & i & i & 㝘 \\
\hline 兽 & 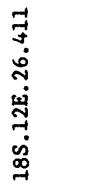 & & 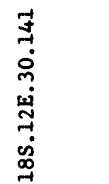 & 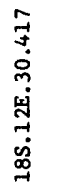 & 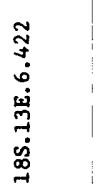 & 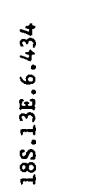 & 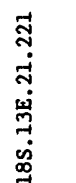 & 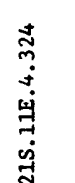 \\
\hline 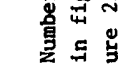 & $\hat{6}$ & & ๑ & o & $?$ & $\vec{F}$ & $\approx$ & $\stackrel{n}{\sim}$ \\
\hline
\end{tabular}




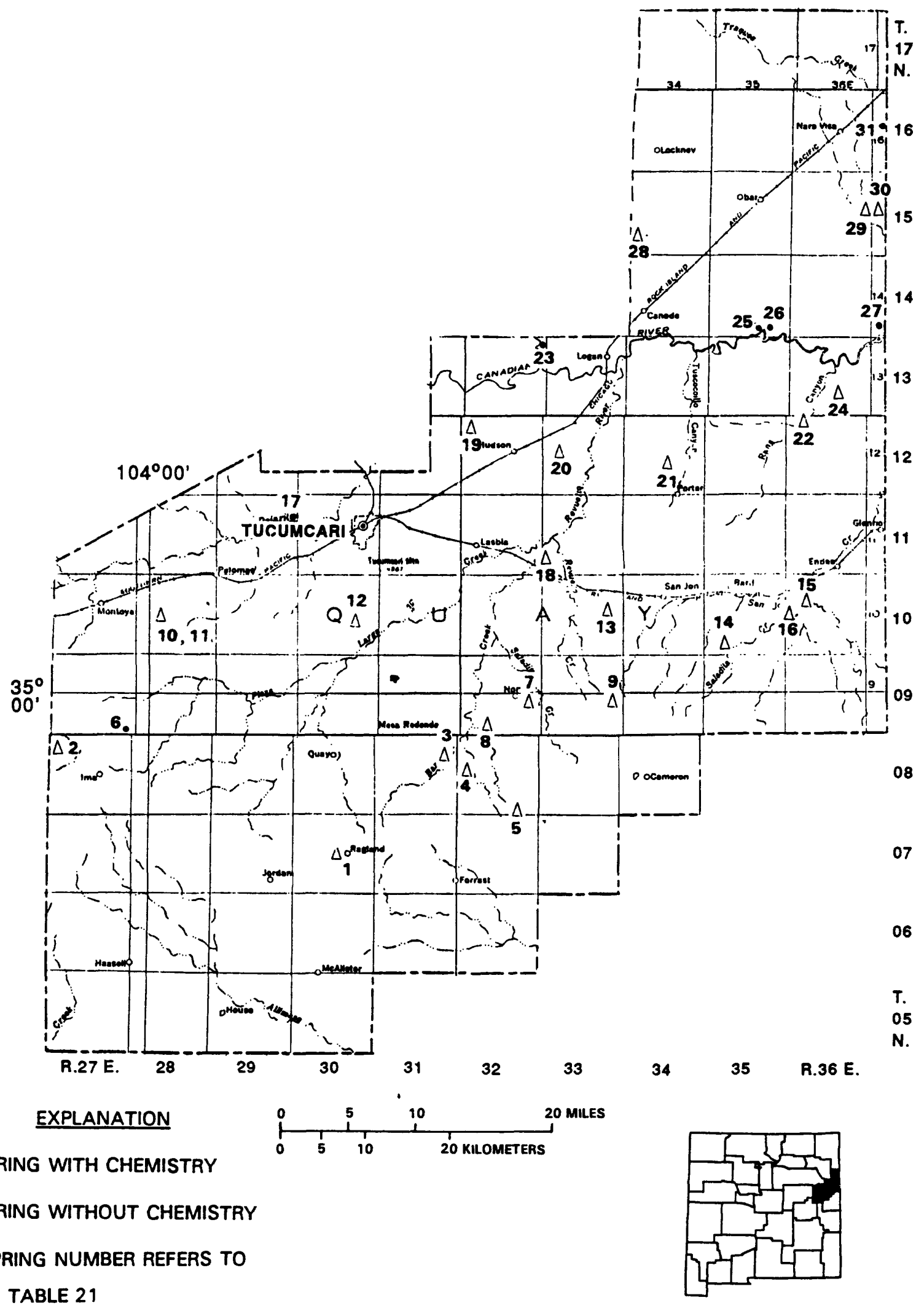

Figure 23.--Location of inventoried springs in Quay County. 


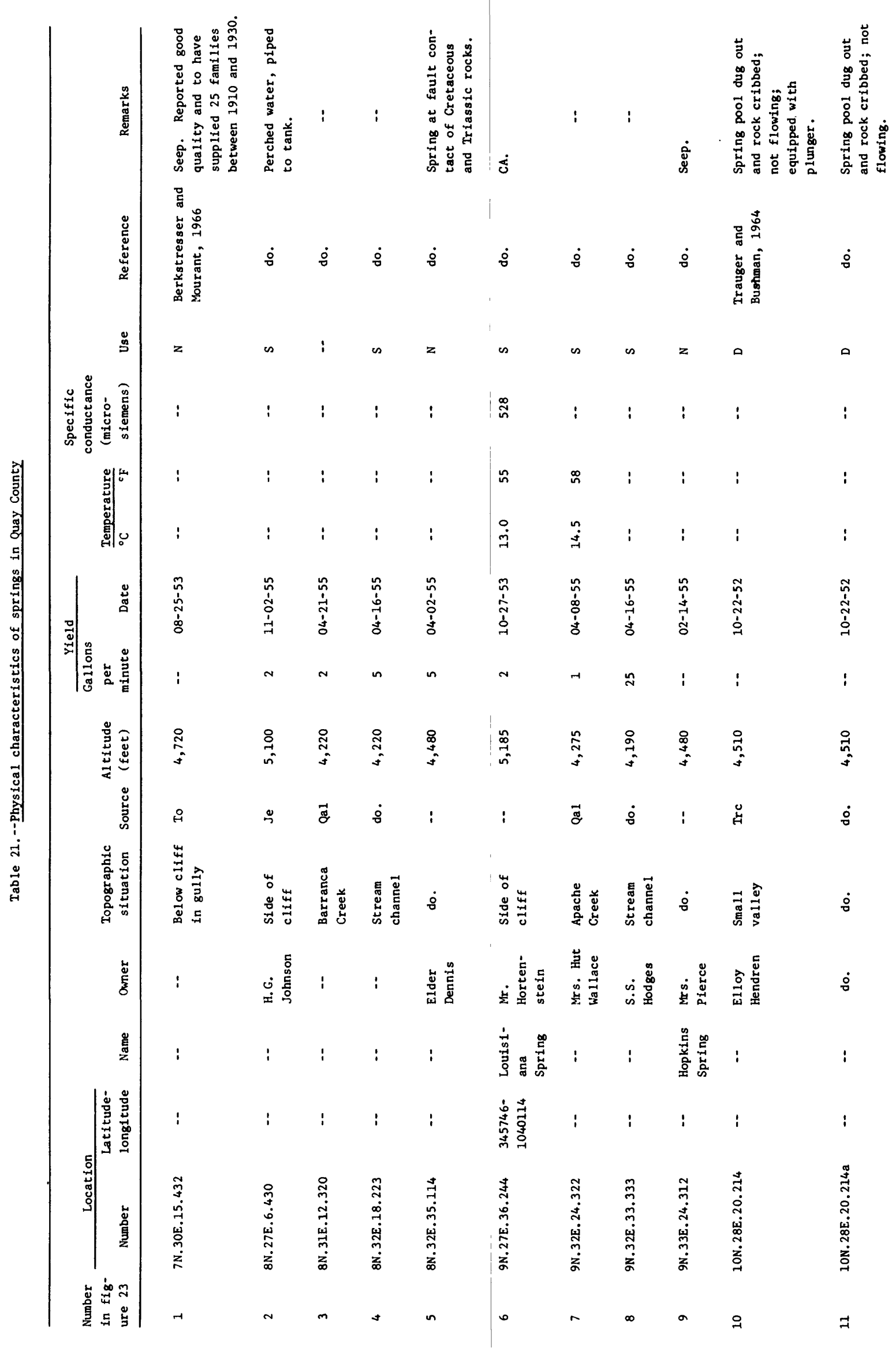




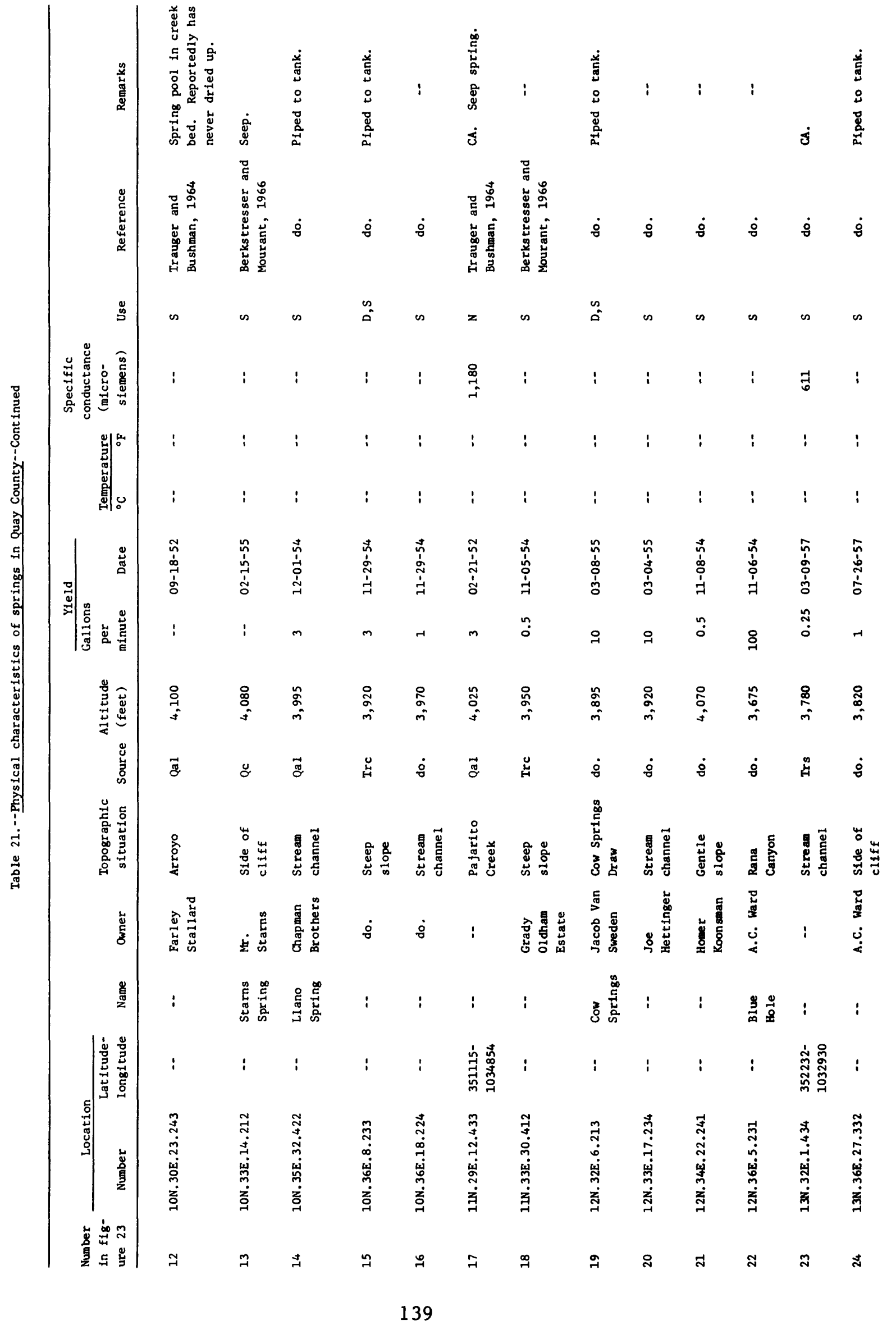




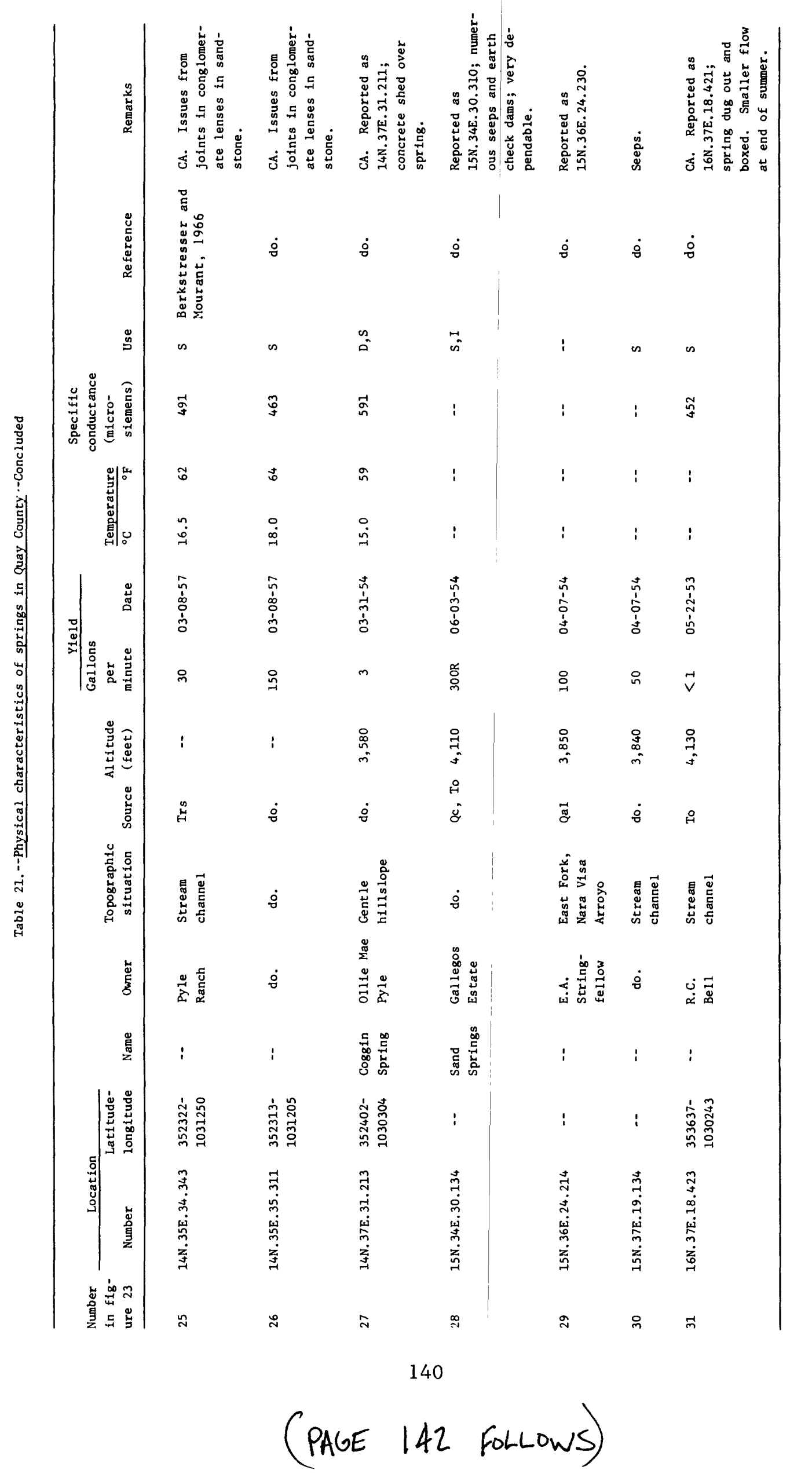




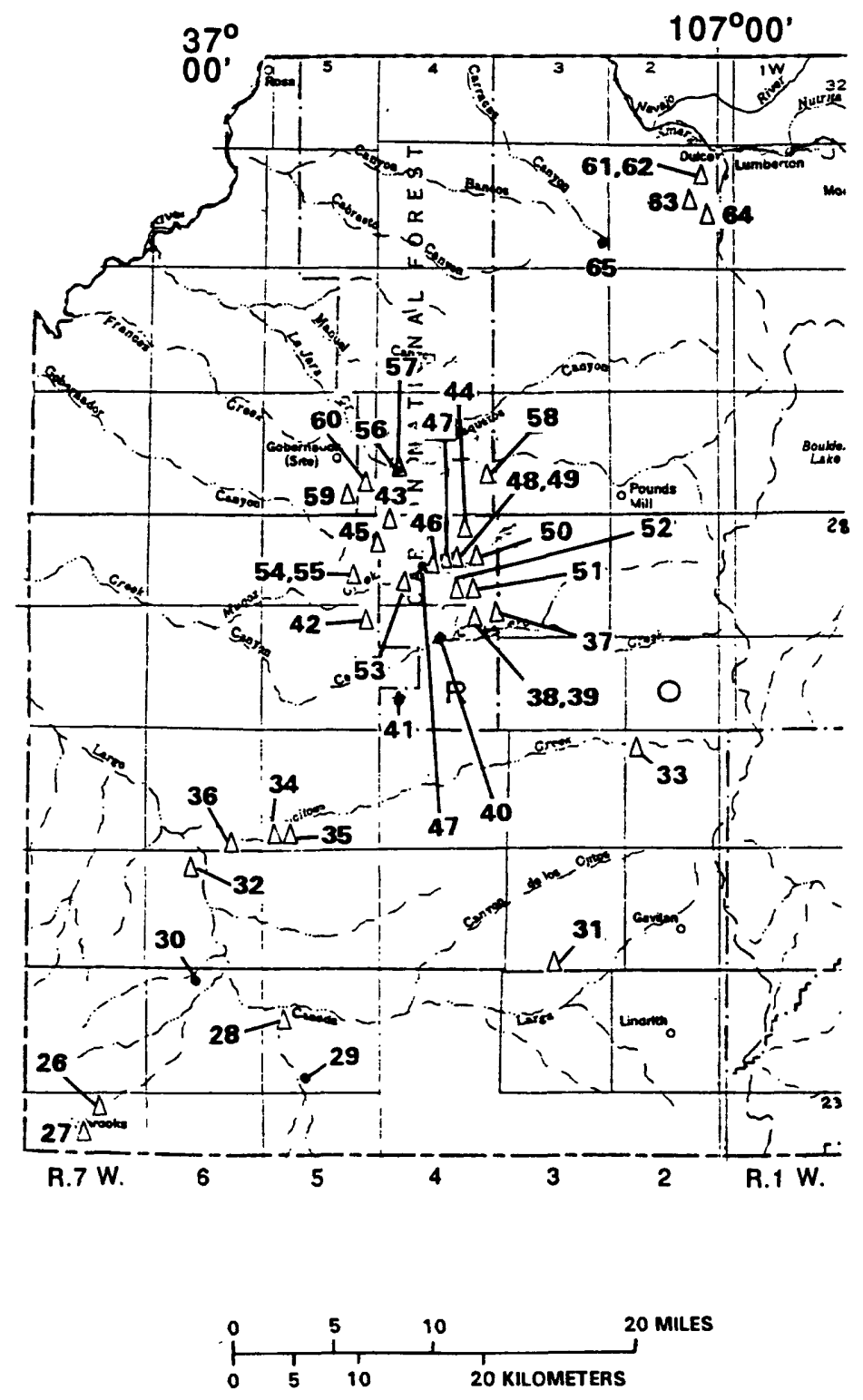

EXPLANATION

- SPRING WITH CHEMISTRY

$\triangle$ SPRING WITHOUT CHEMISTRY

29 SPRING NUMBER REFERS TO TABLE 22 


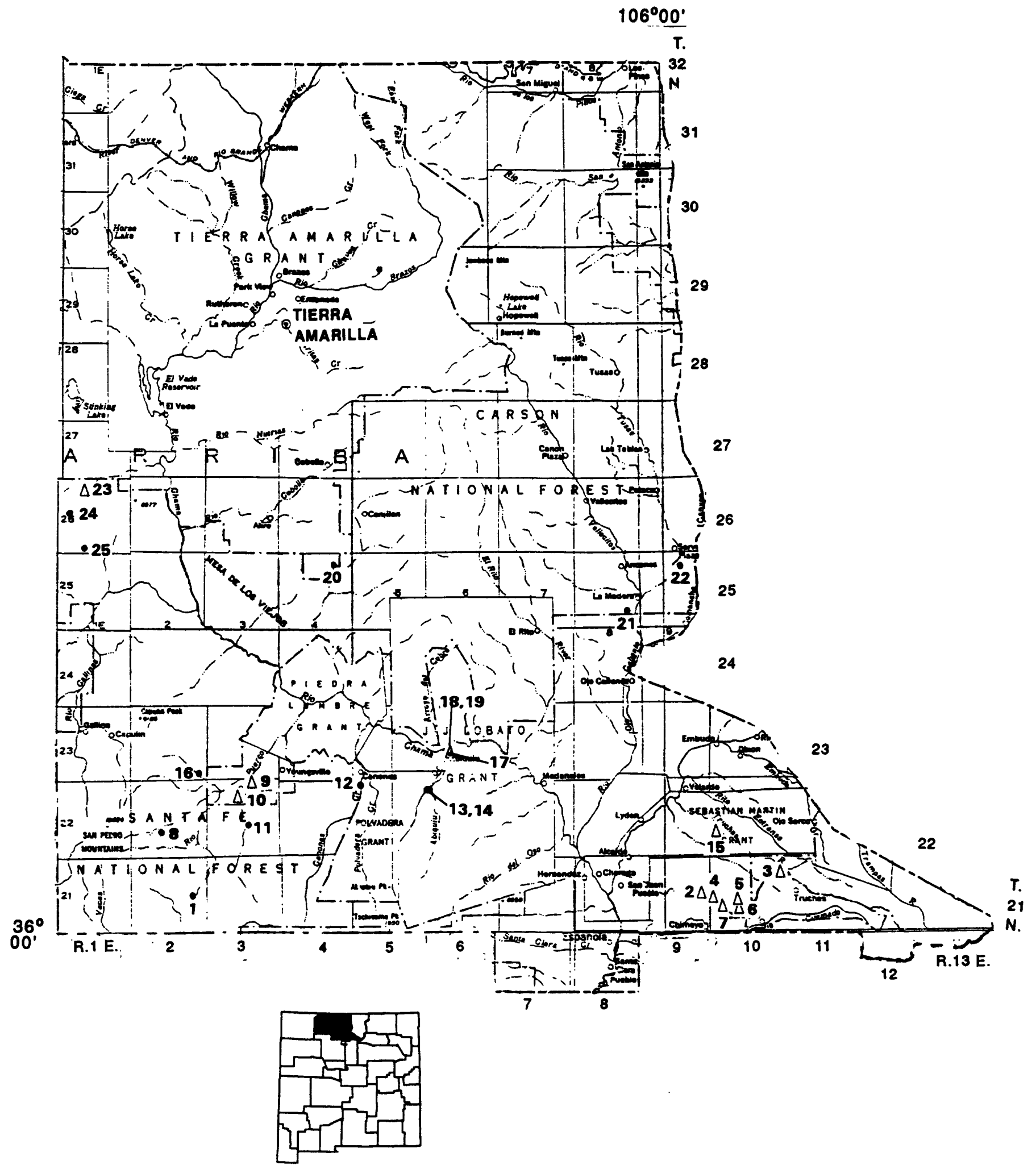

Figure 24.--Location of inventoried springs in Rio Arriba County. 


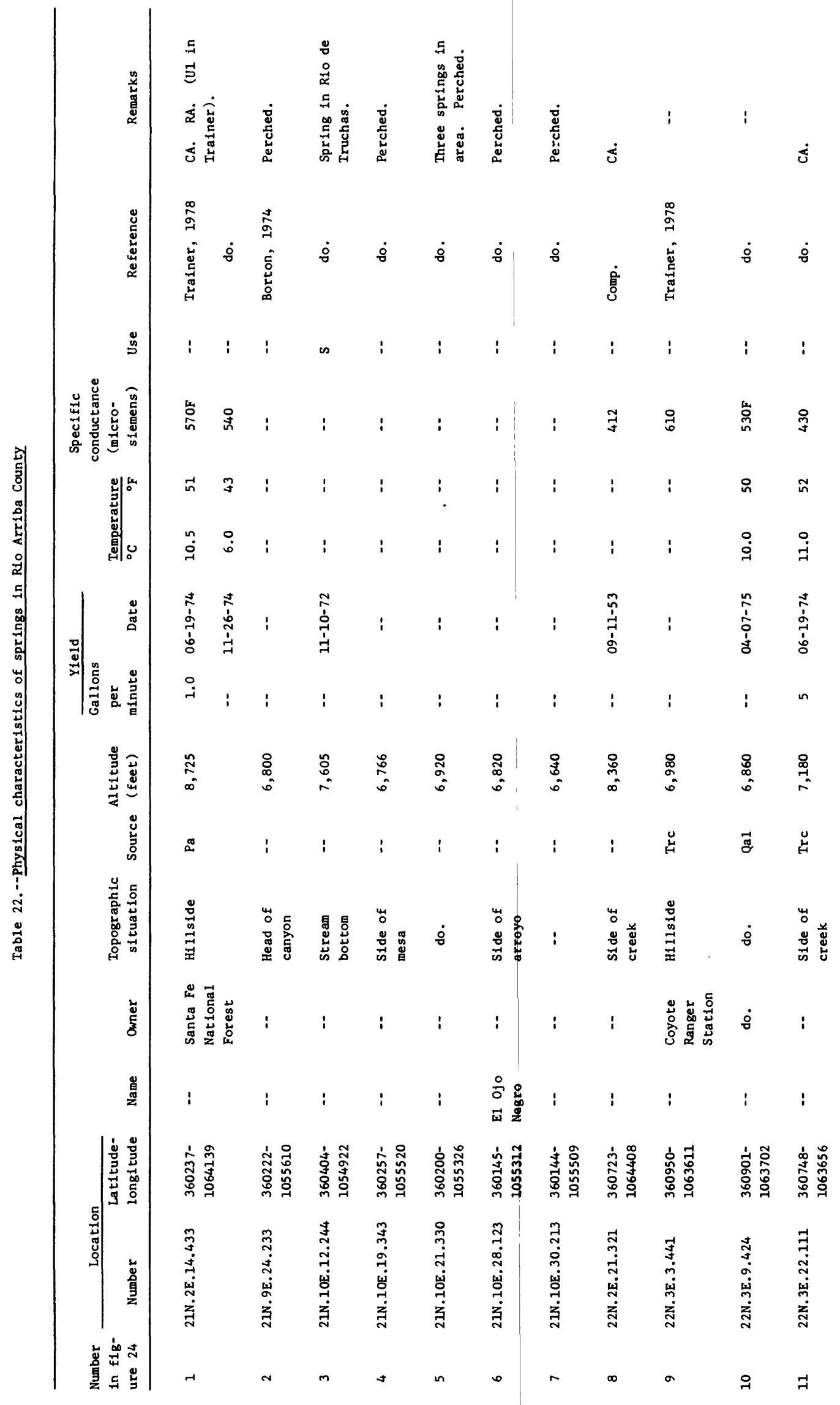




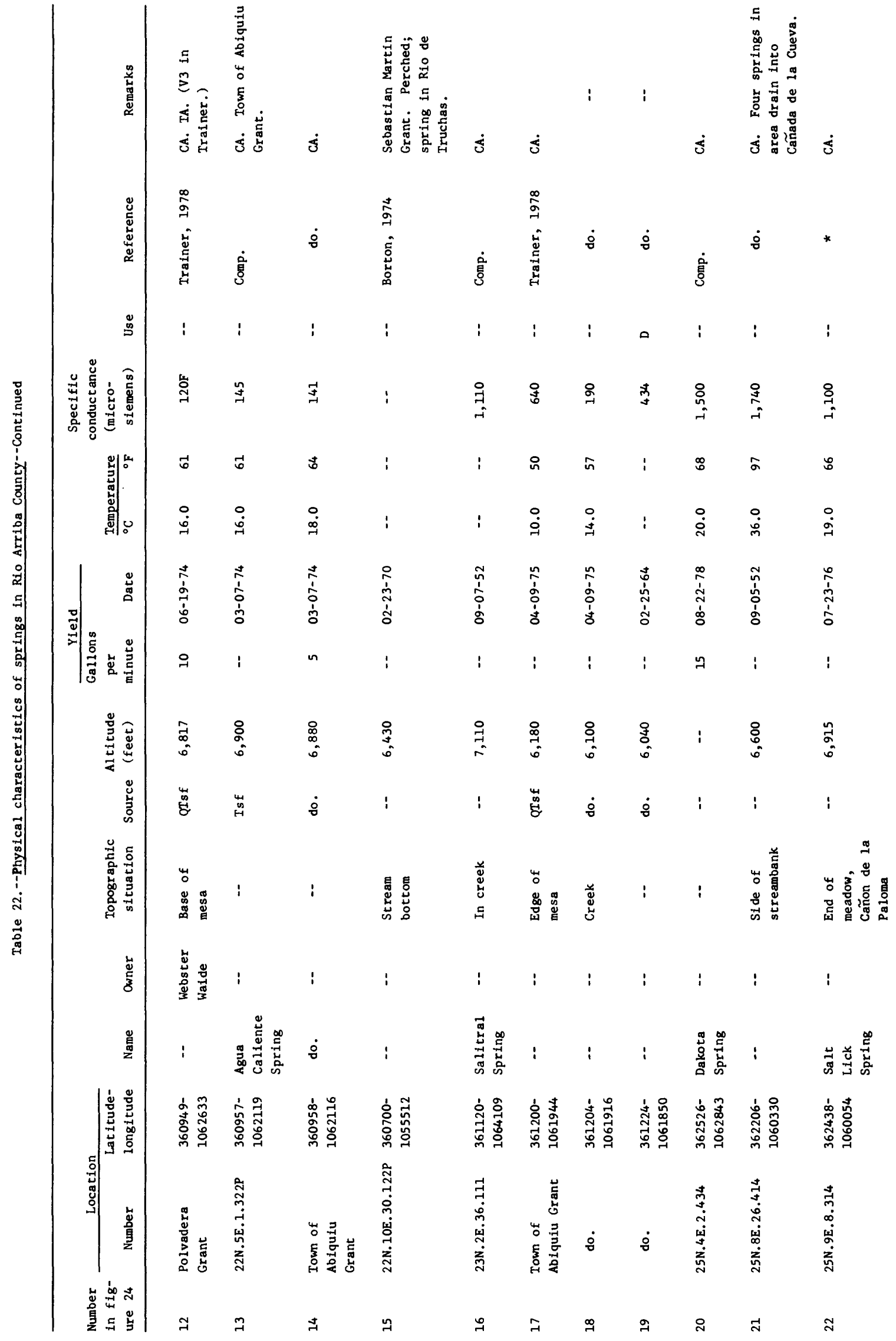




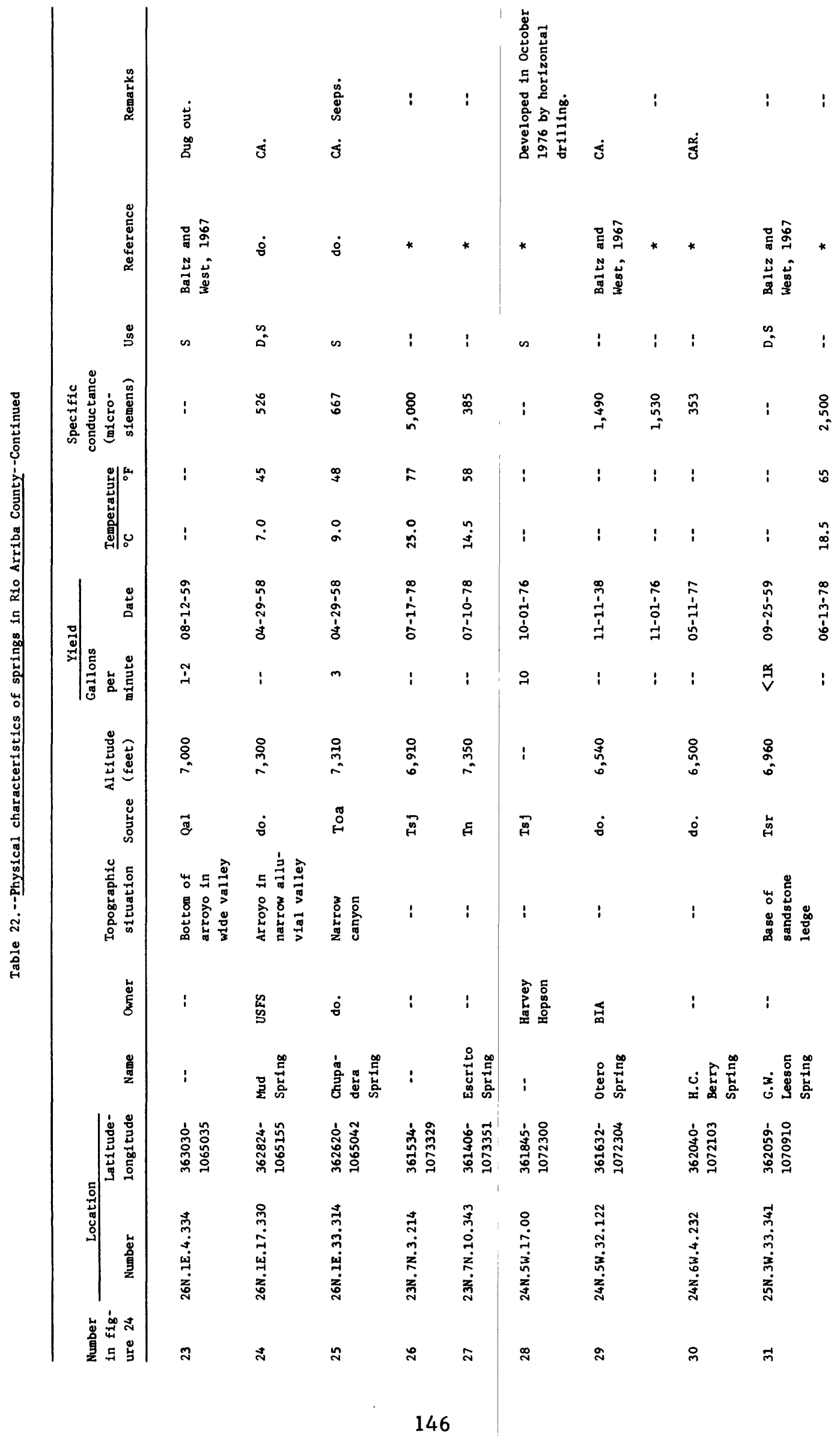




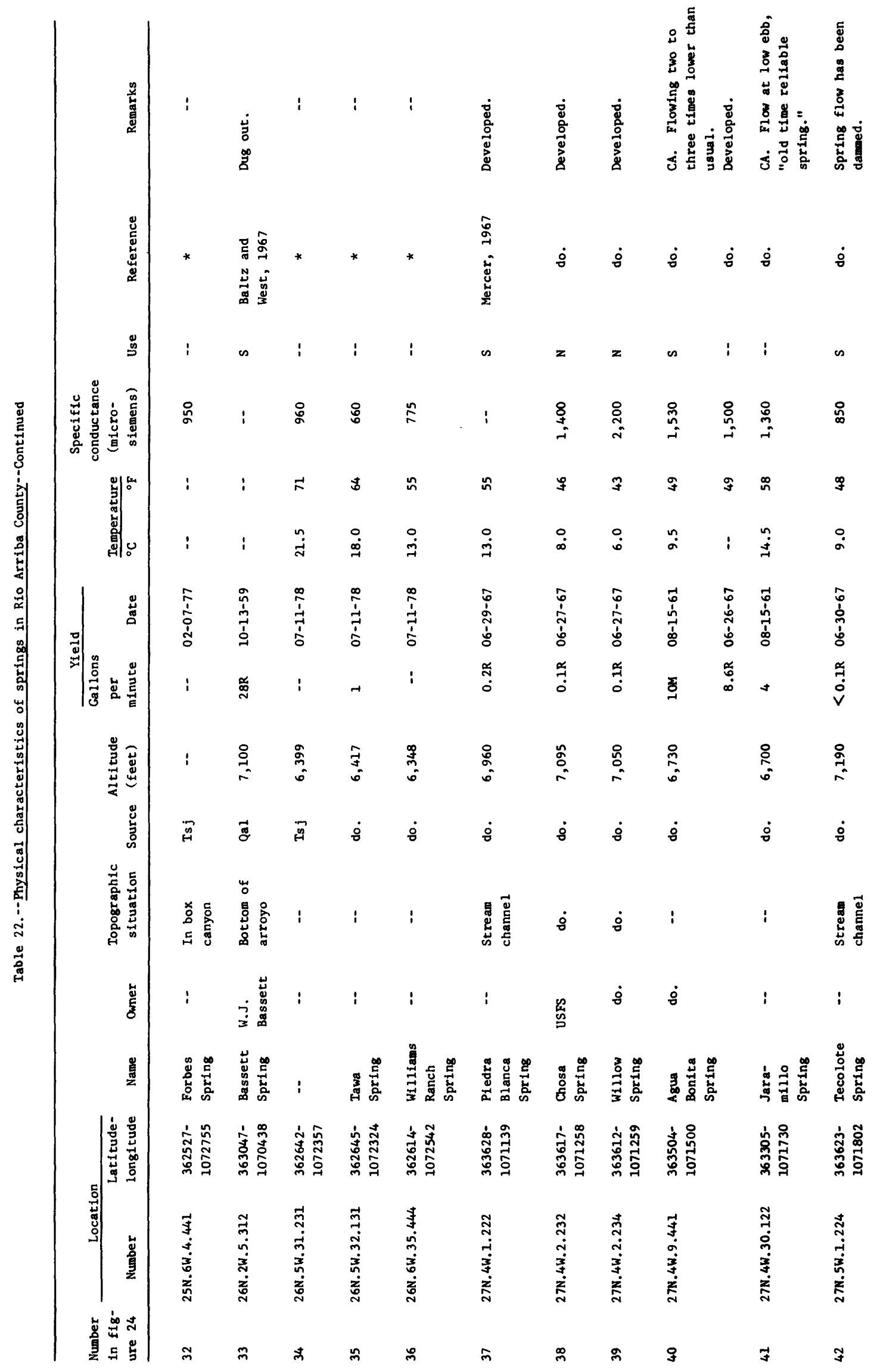




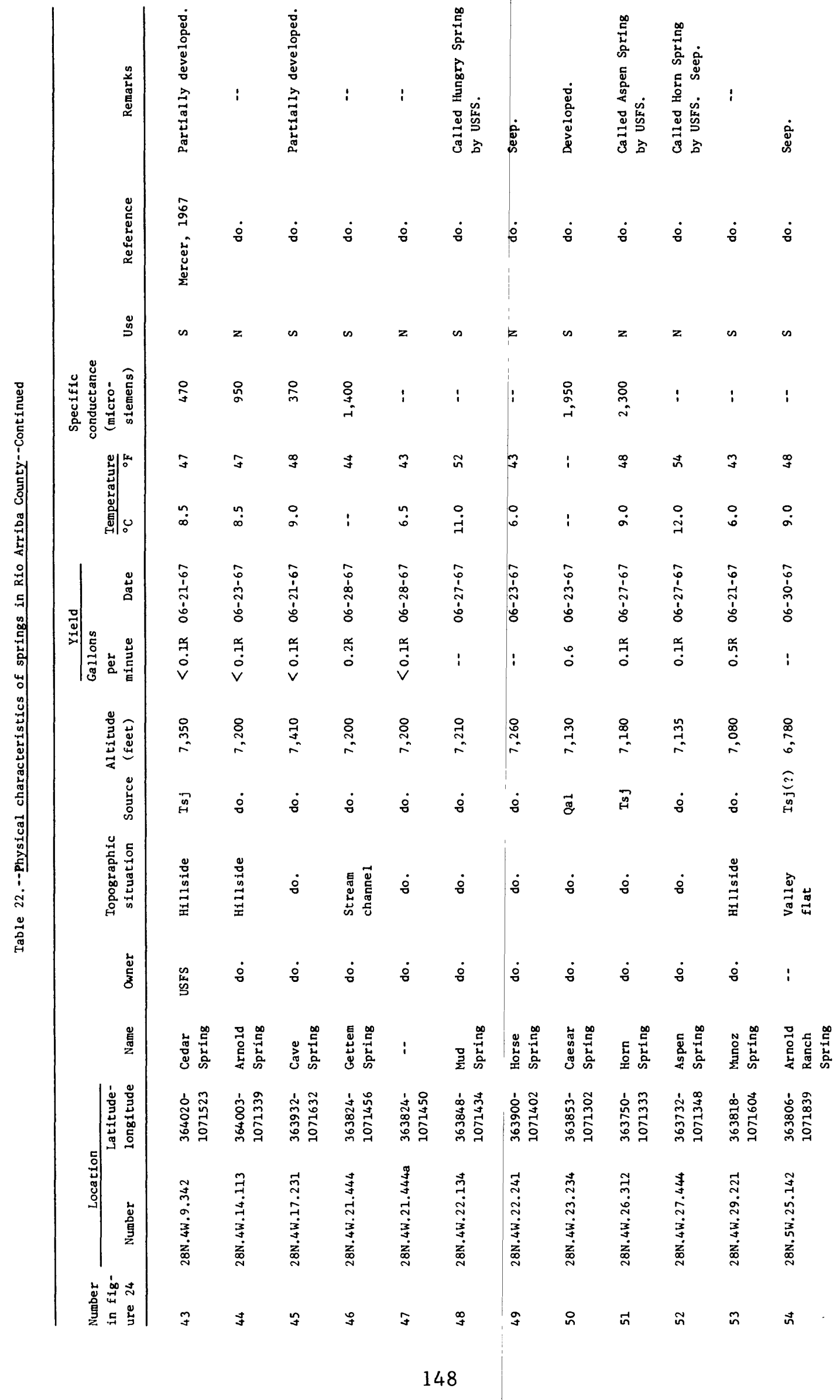




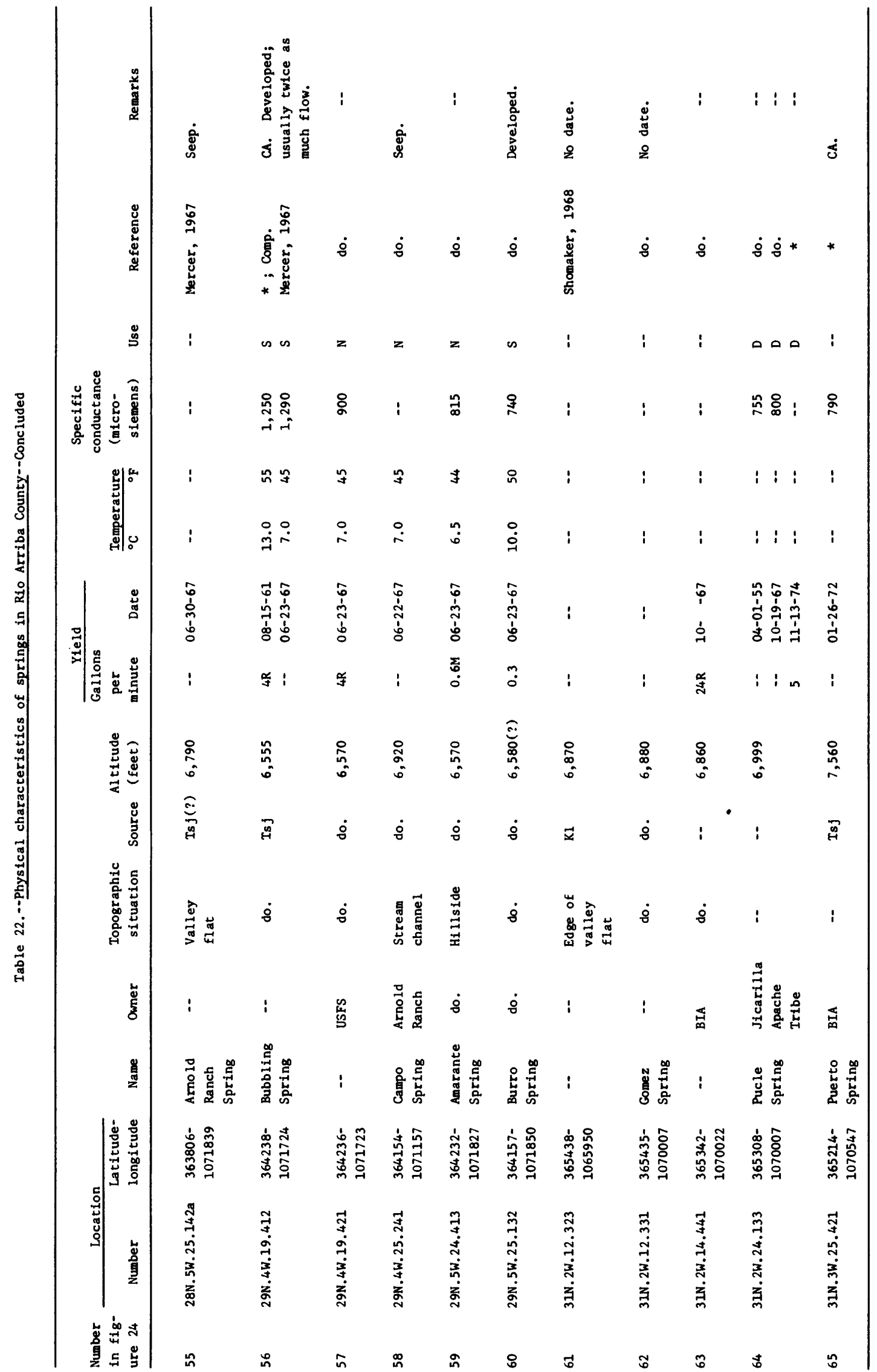




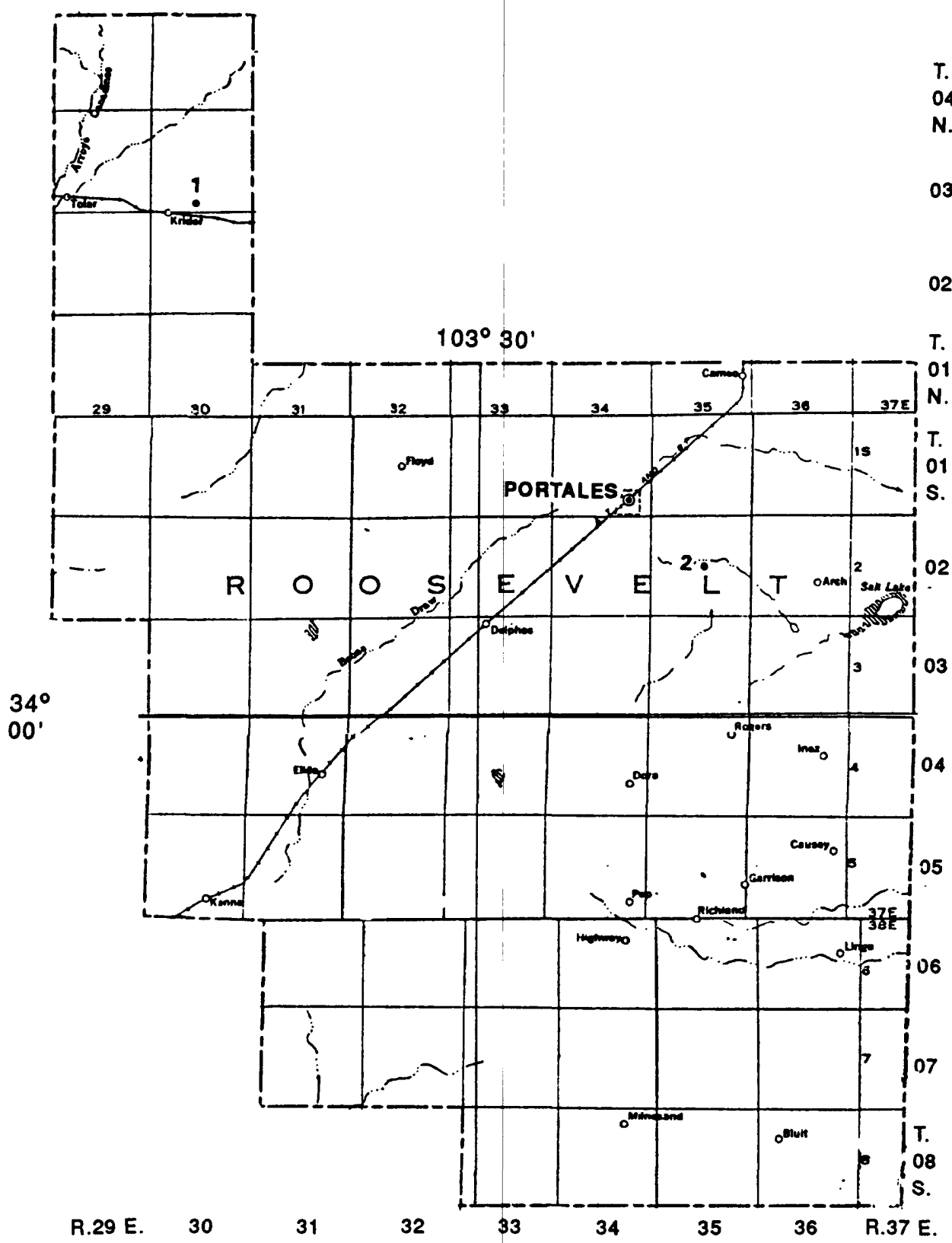

02

T. $\mathrm{N}$. 01 


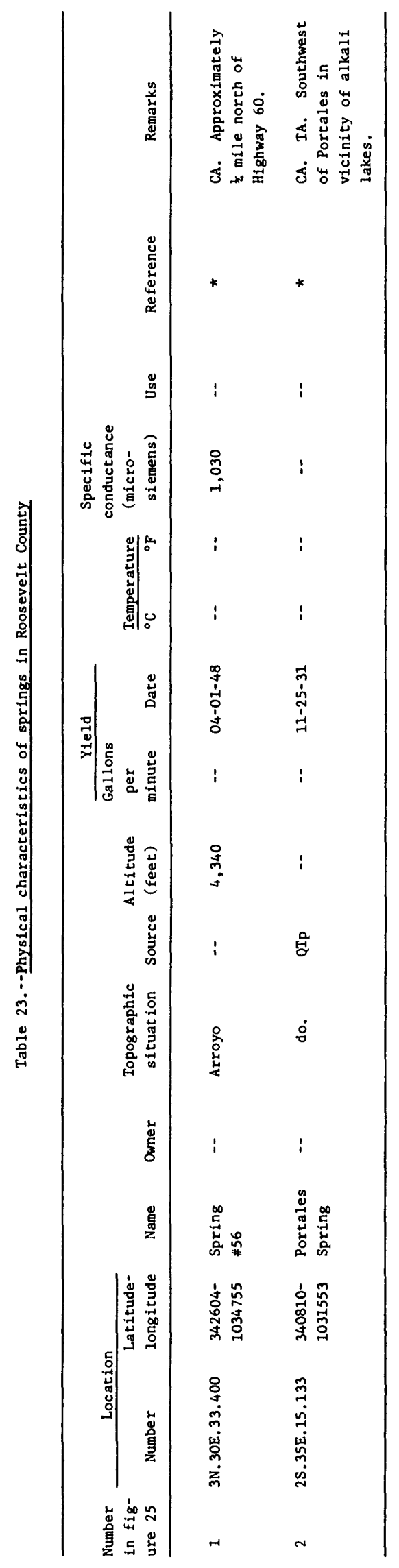




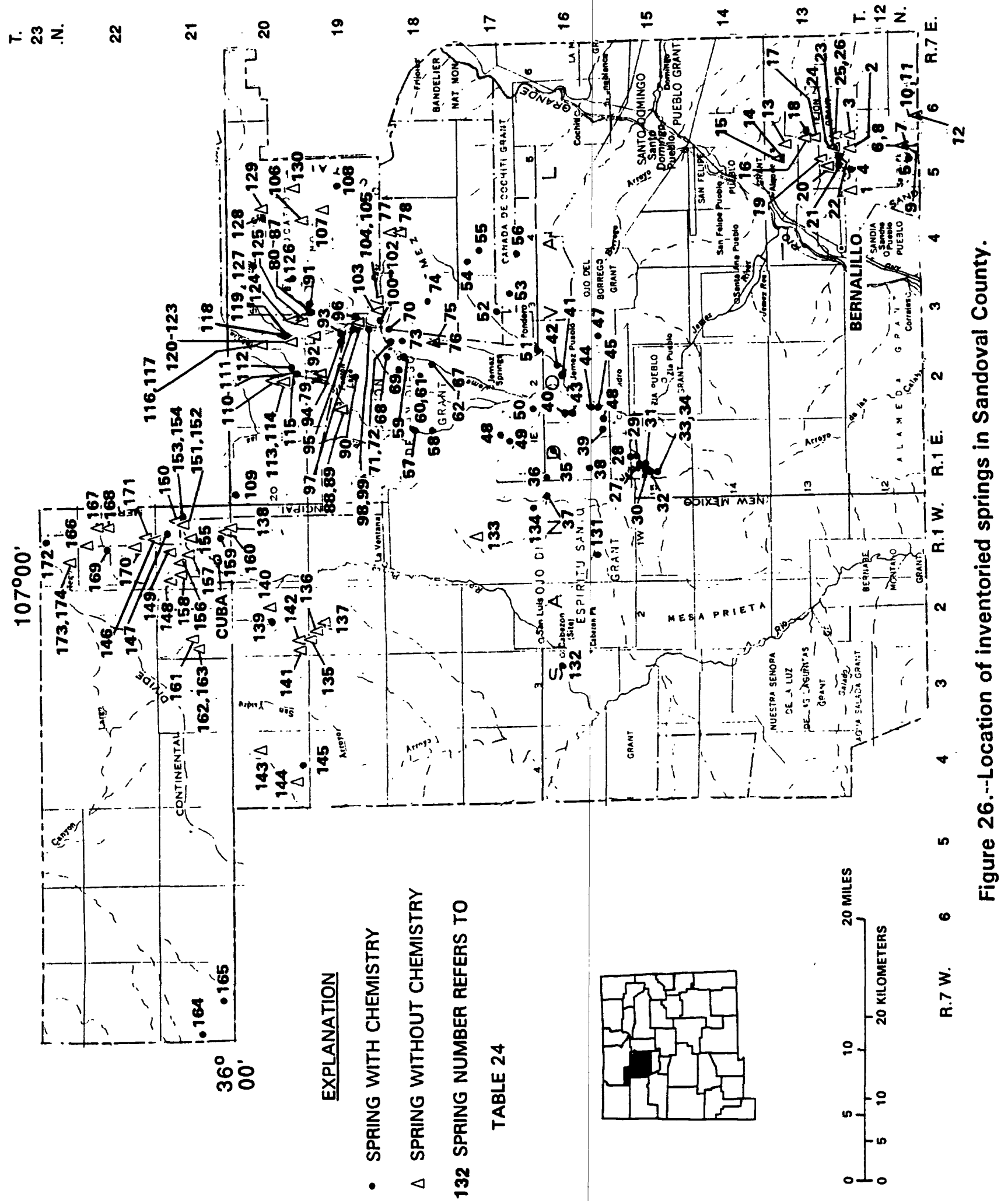




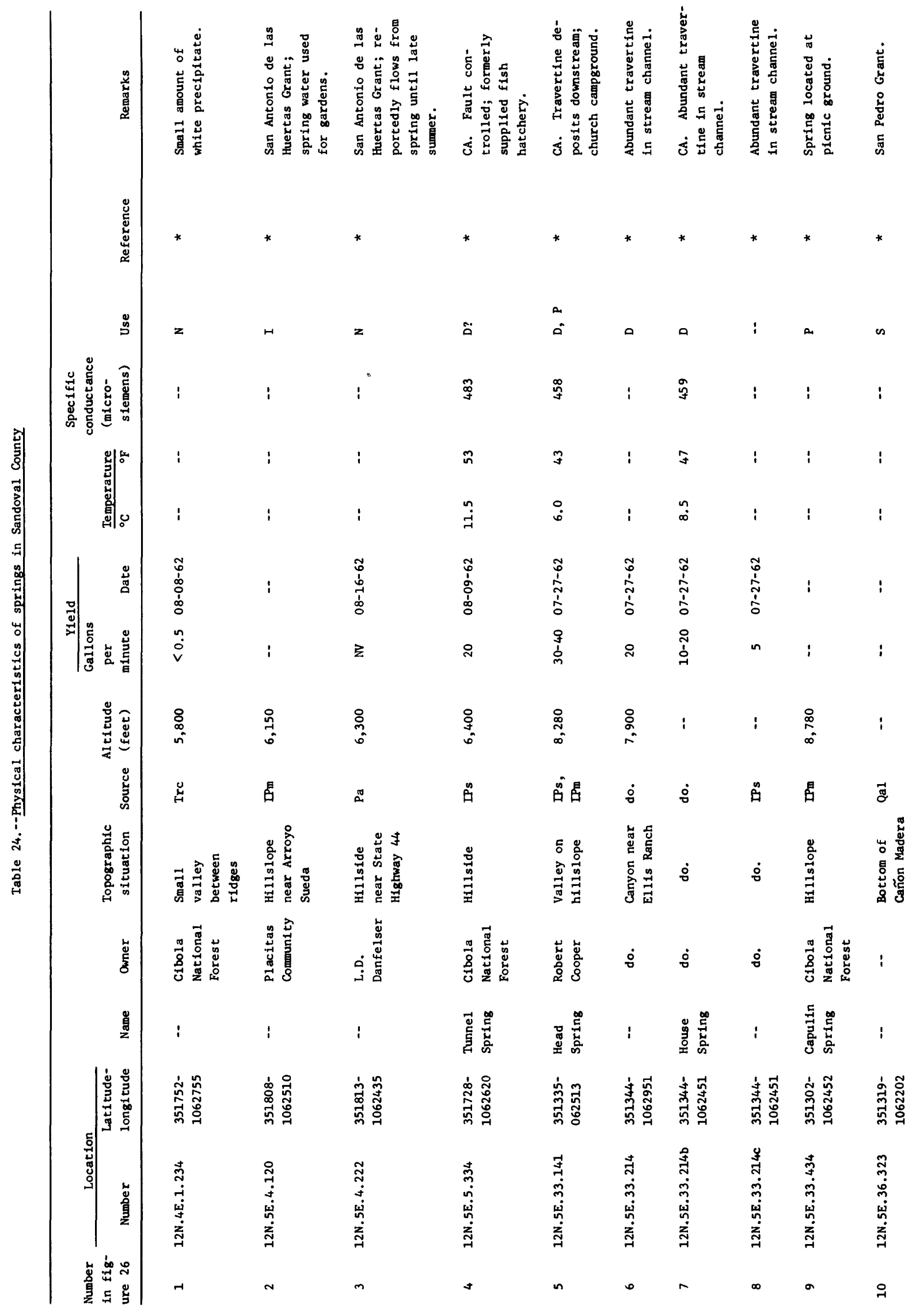




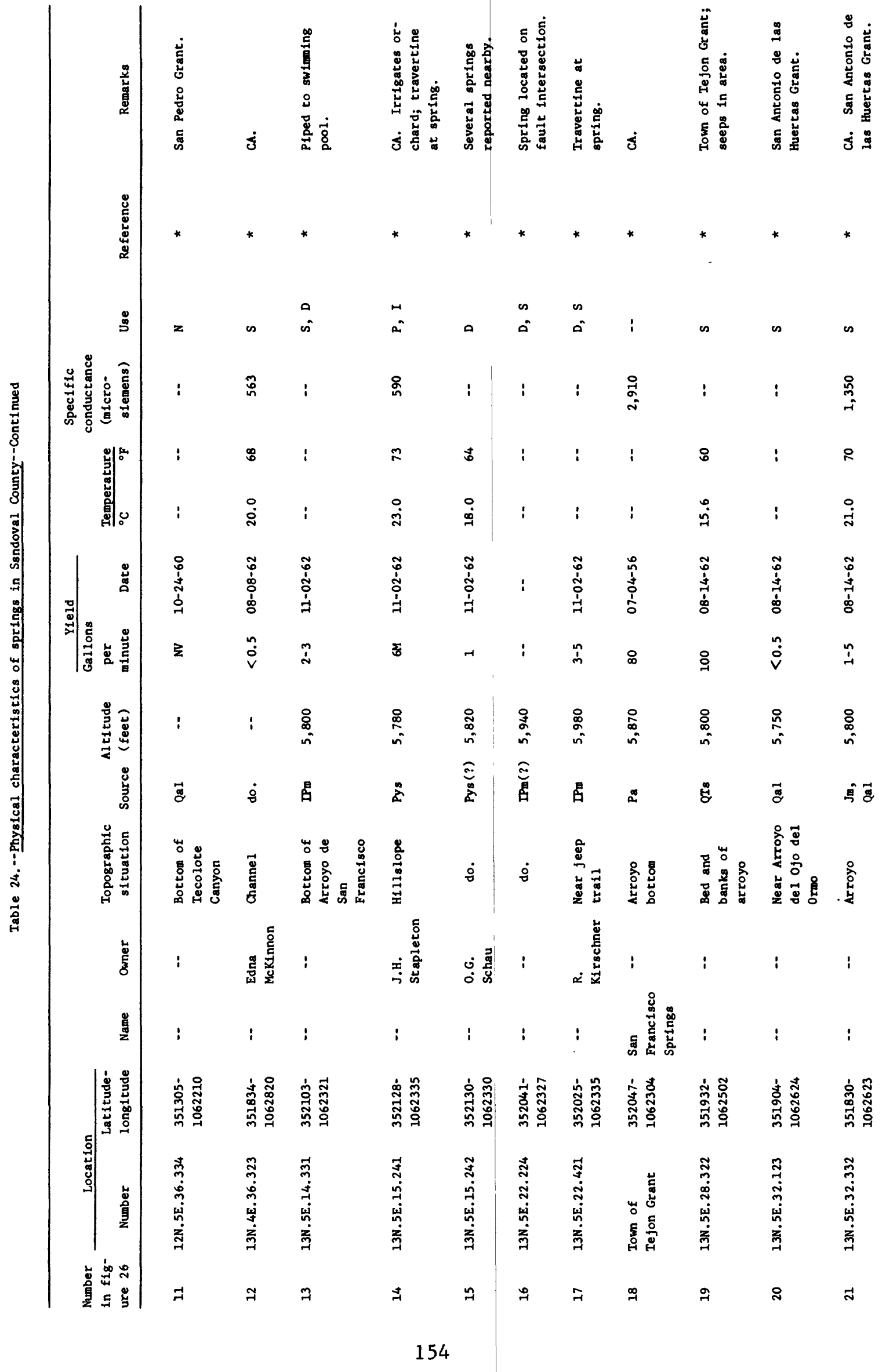




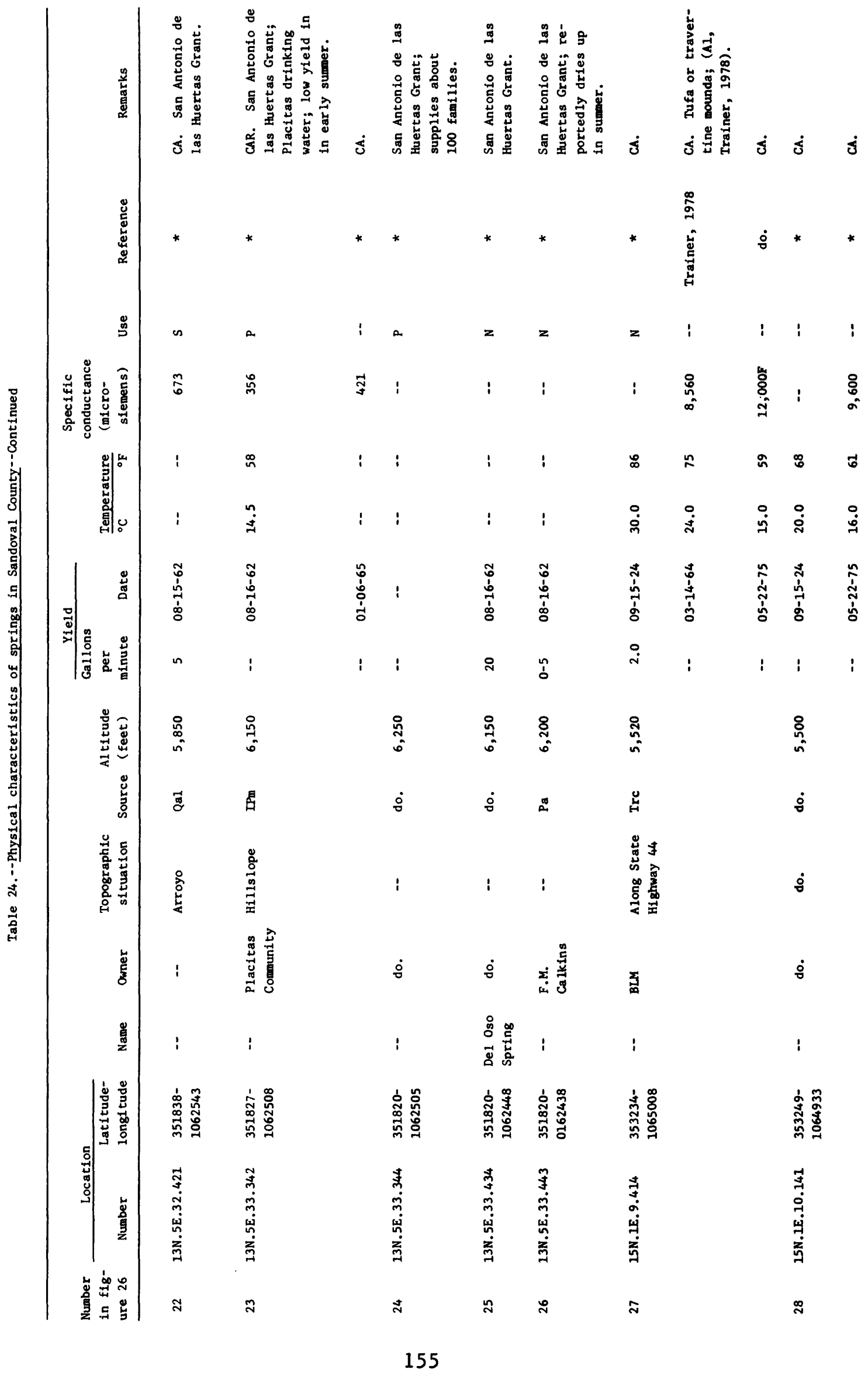




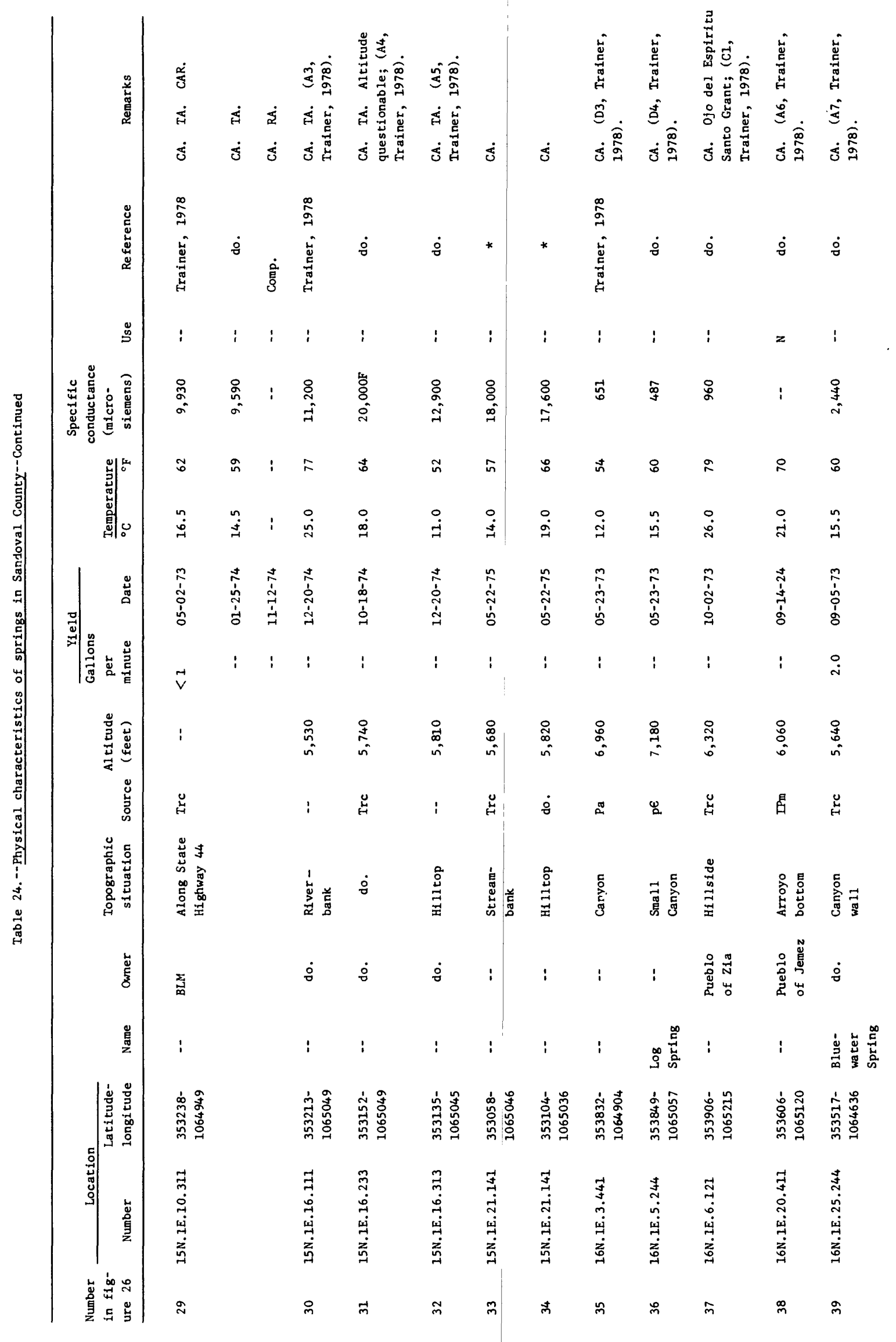




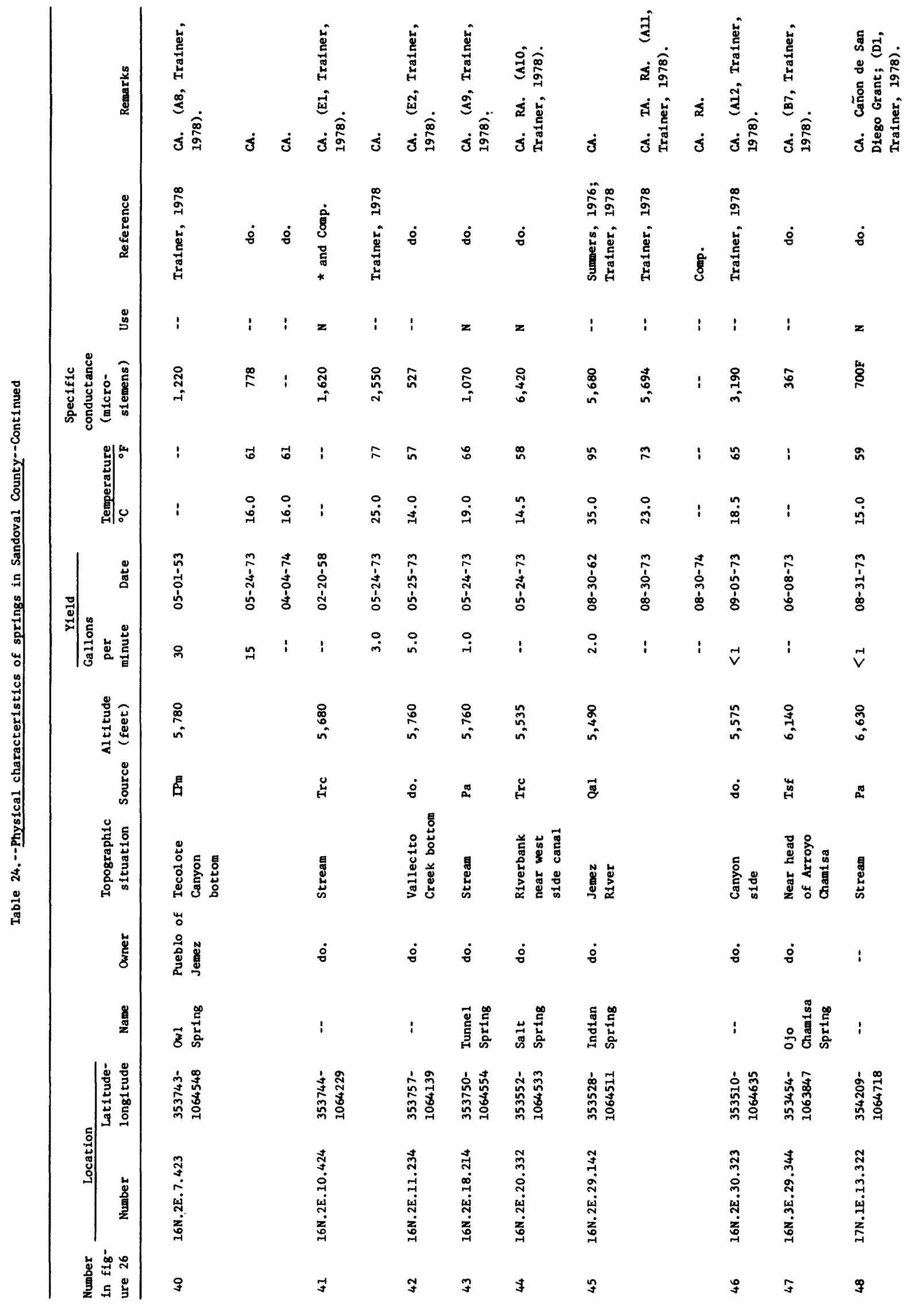




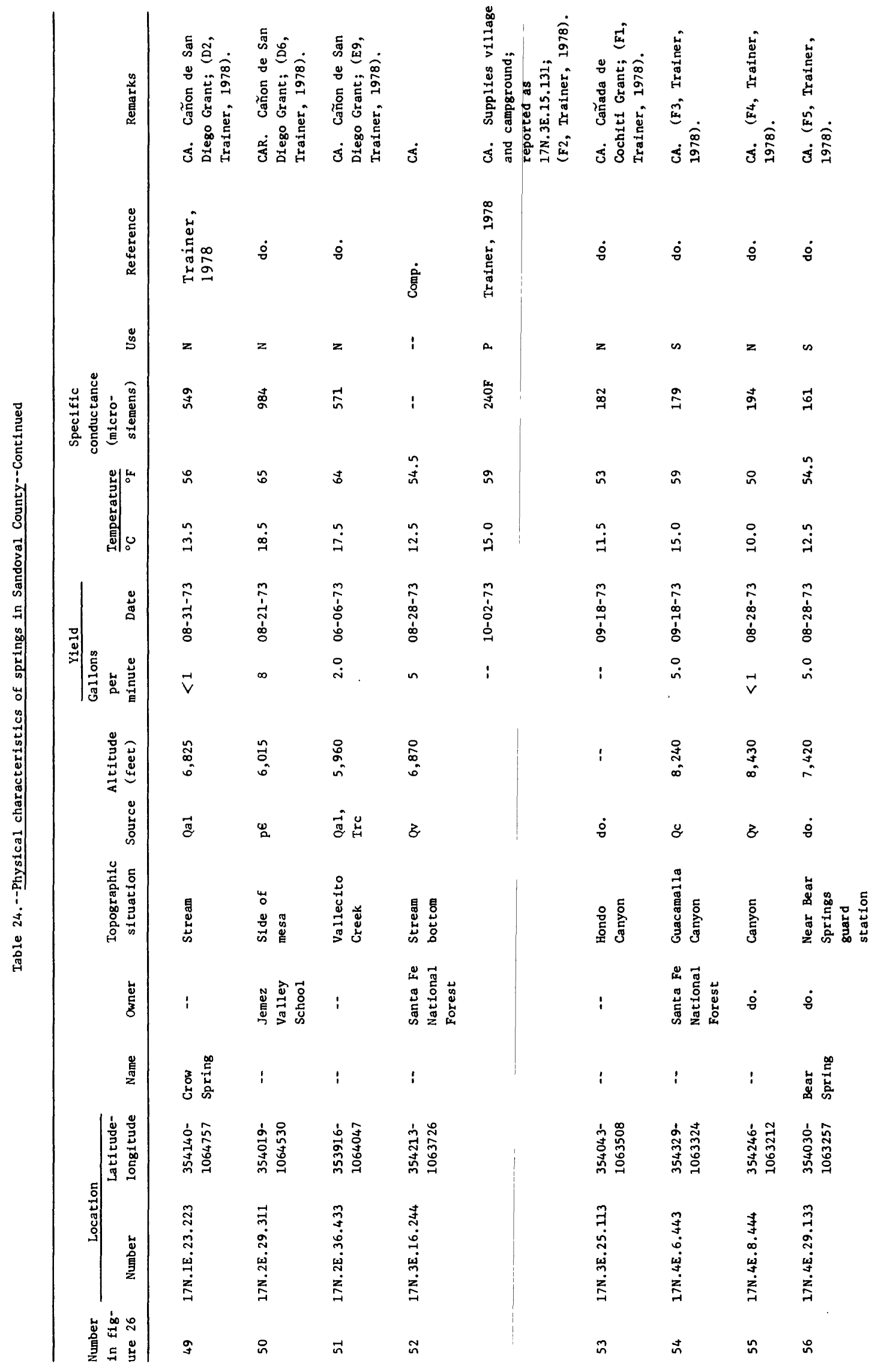




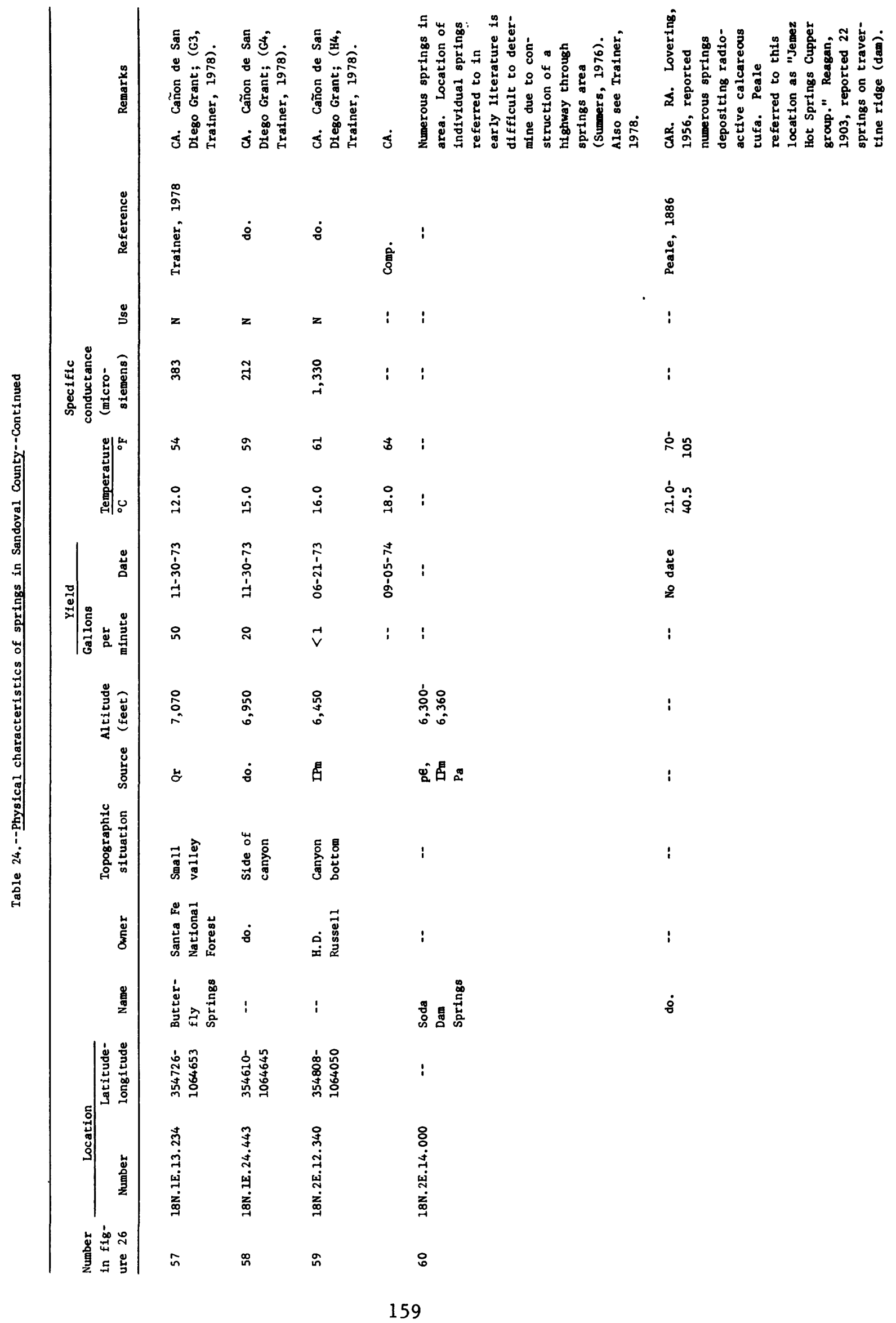




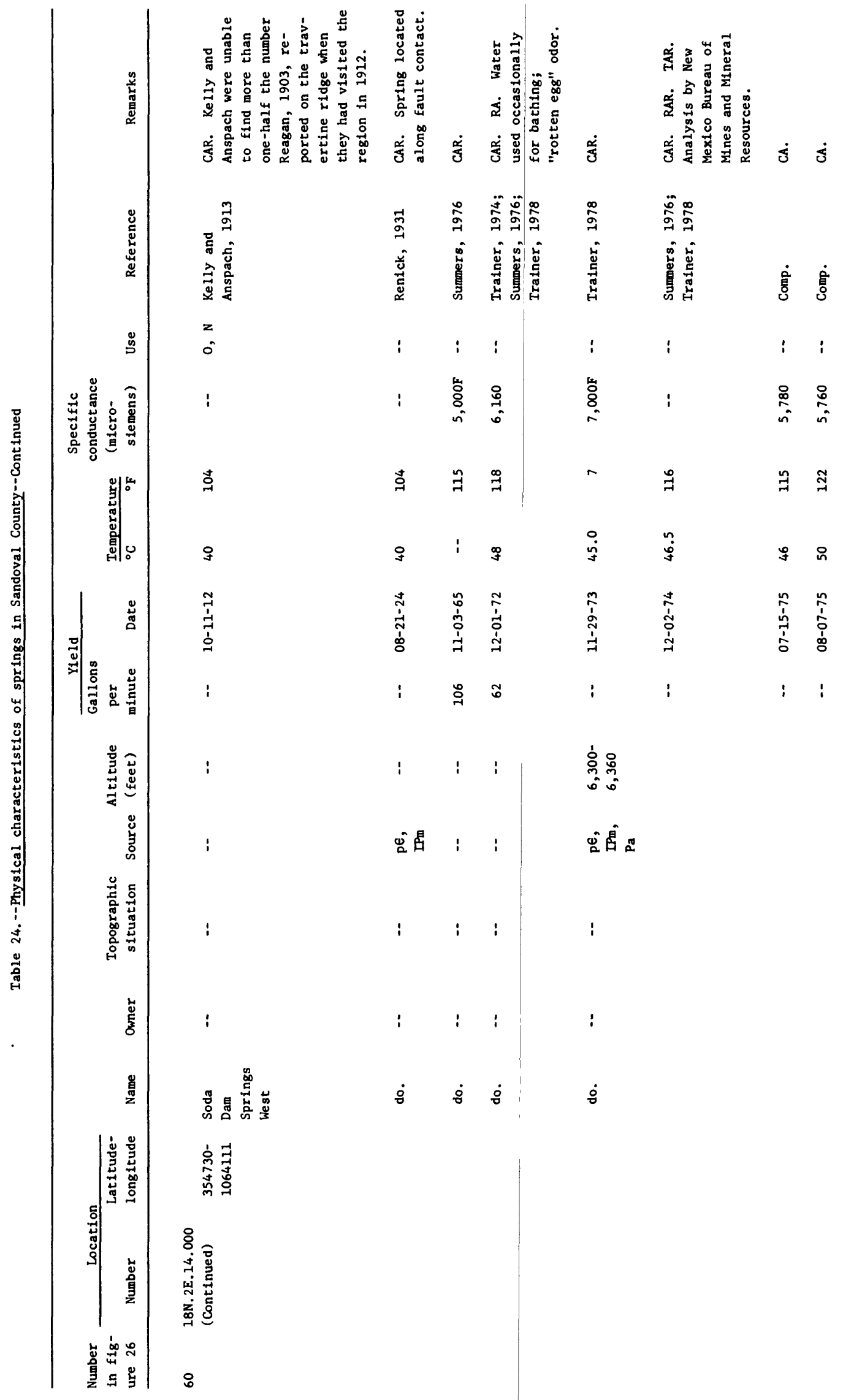




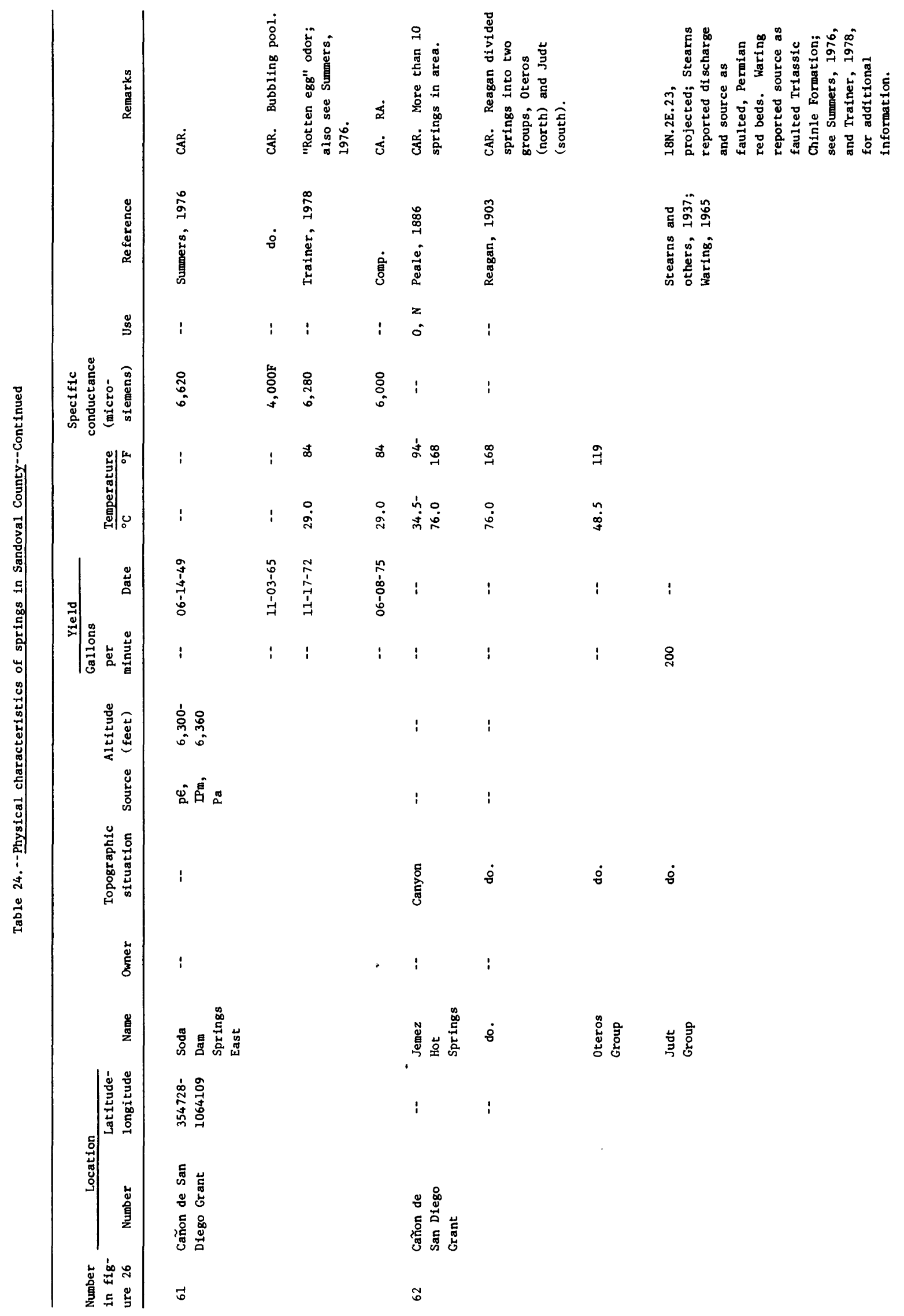




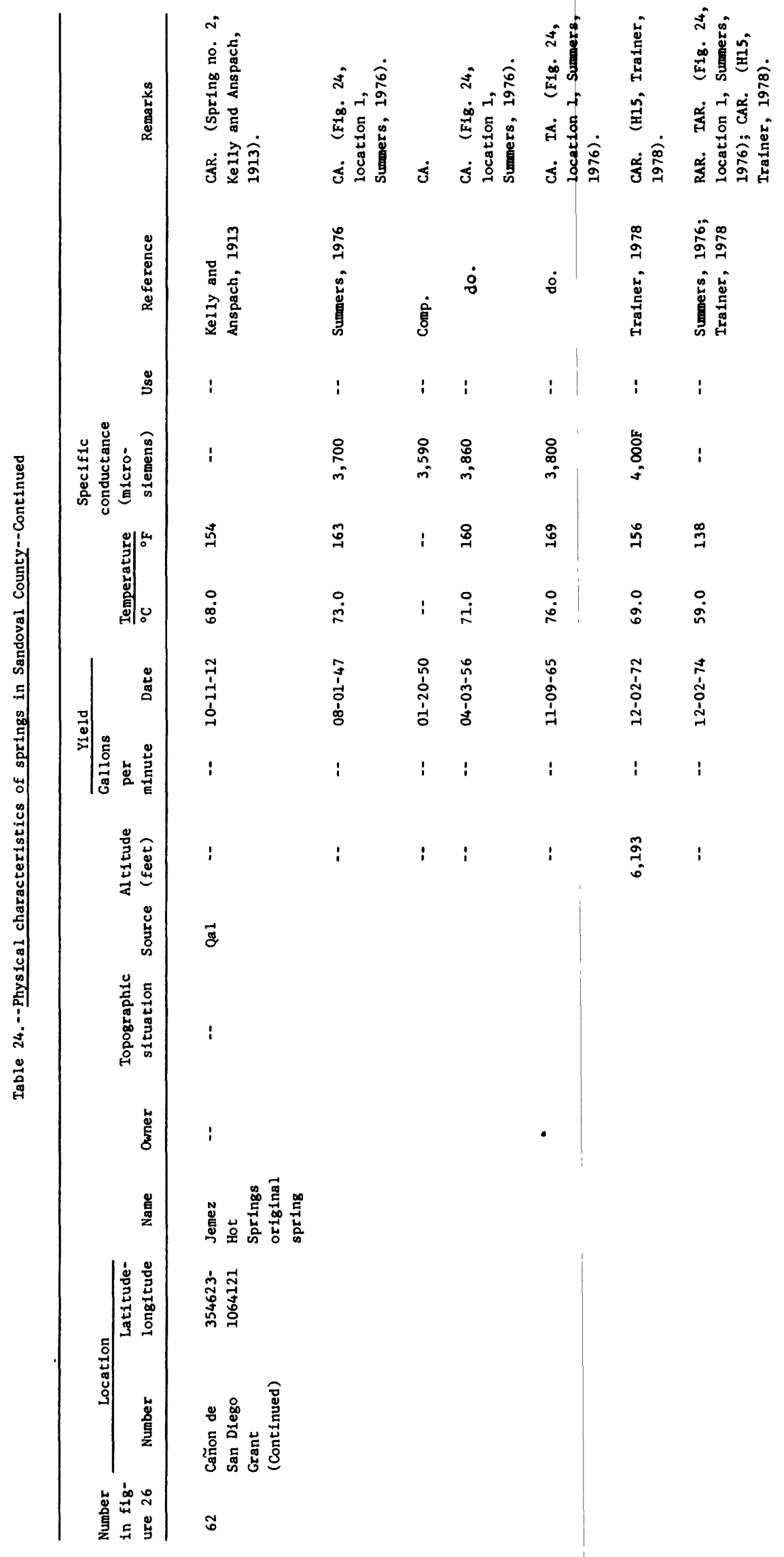




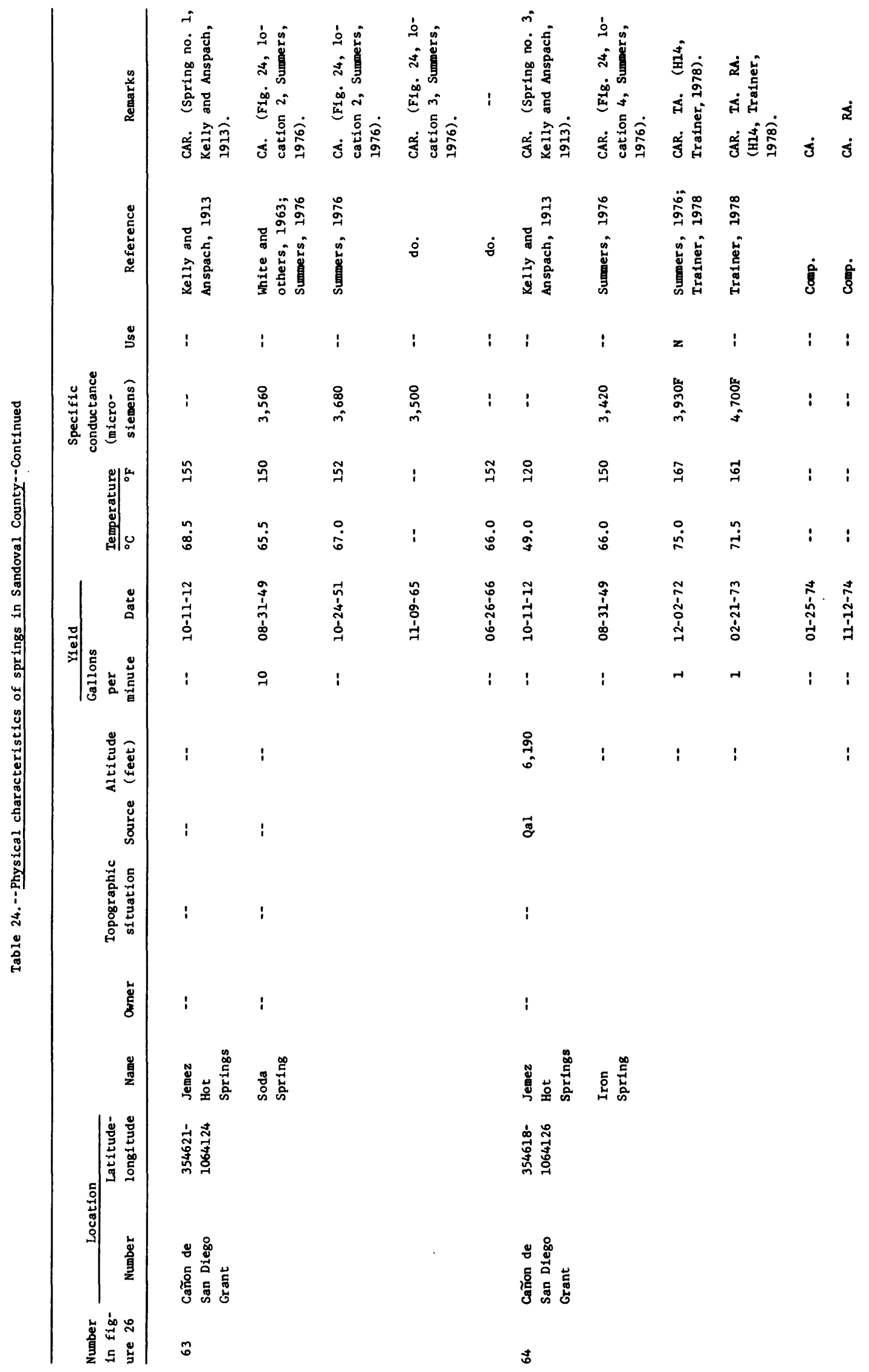




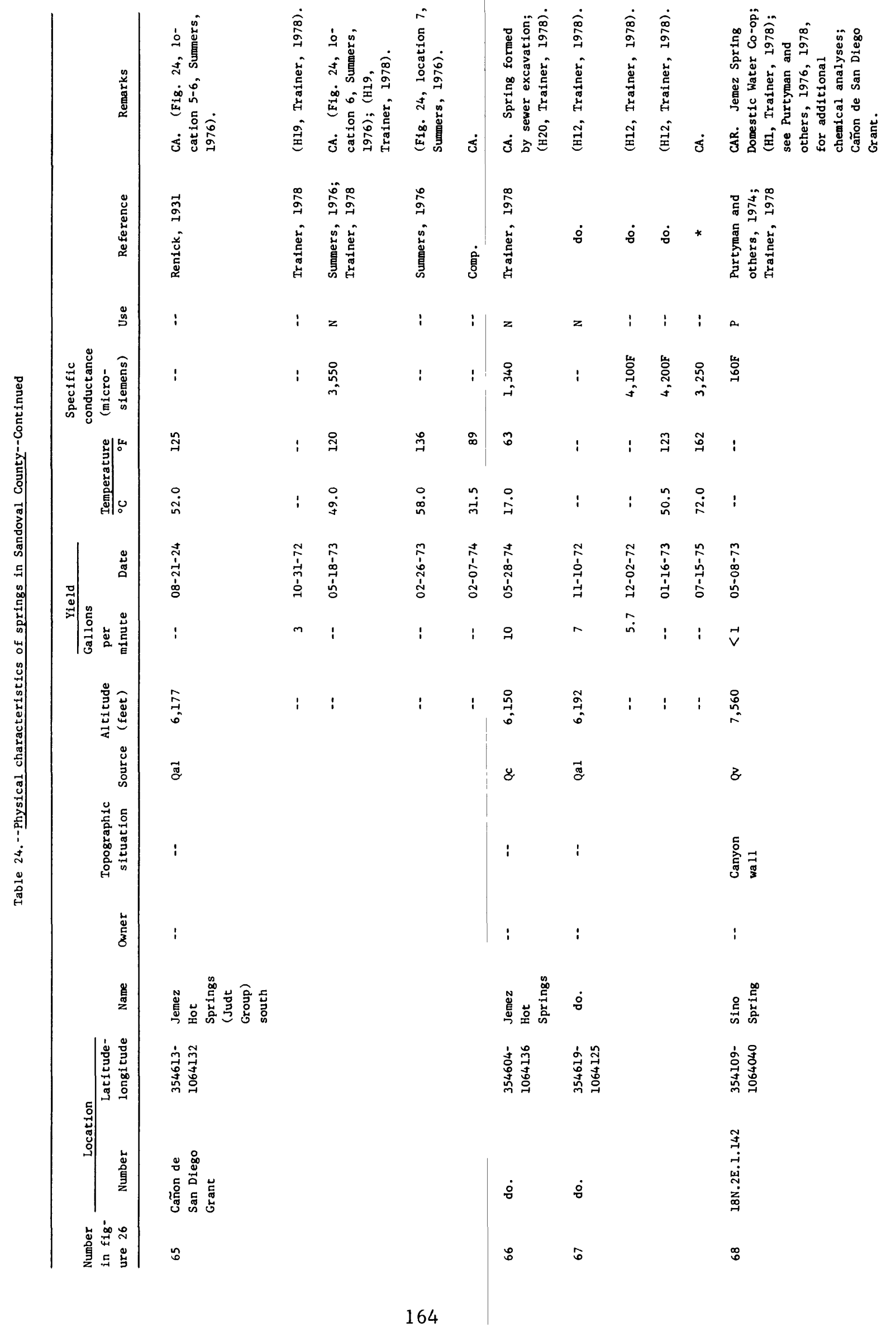




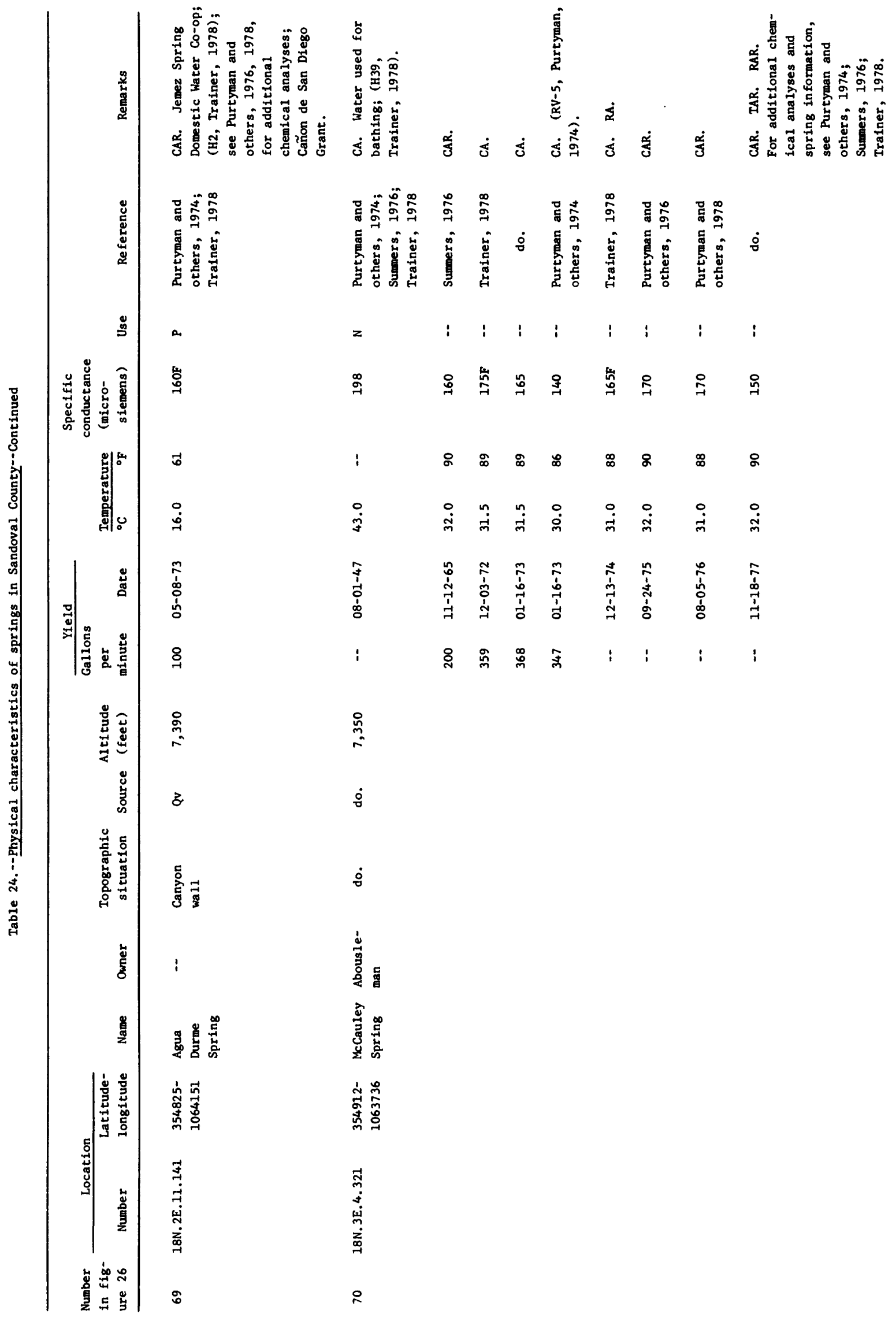




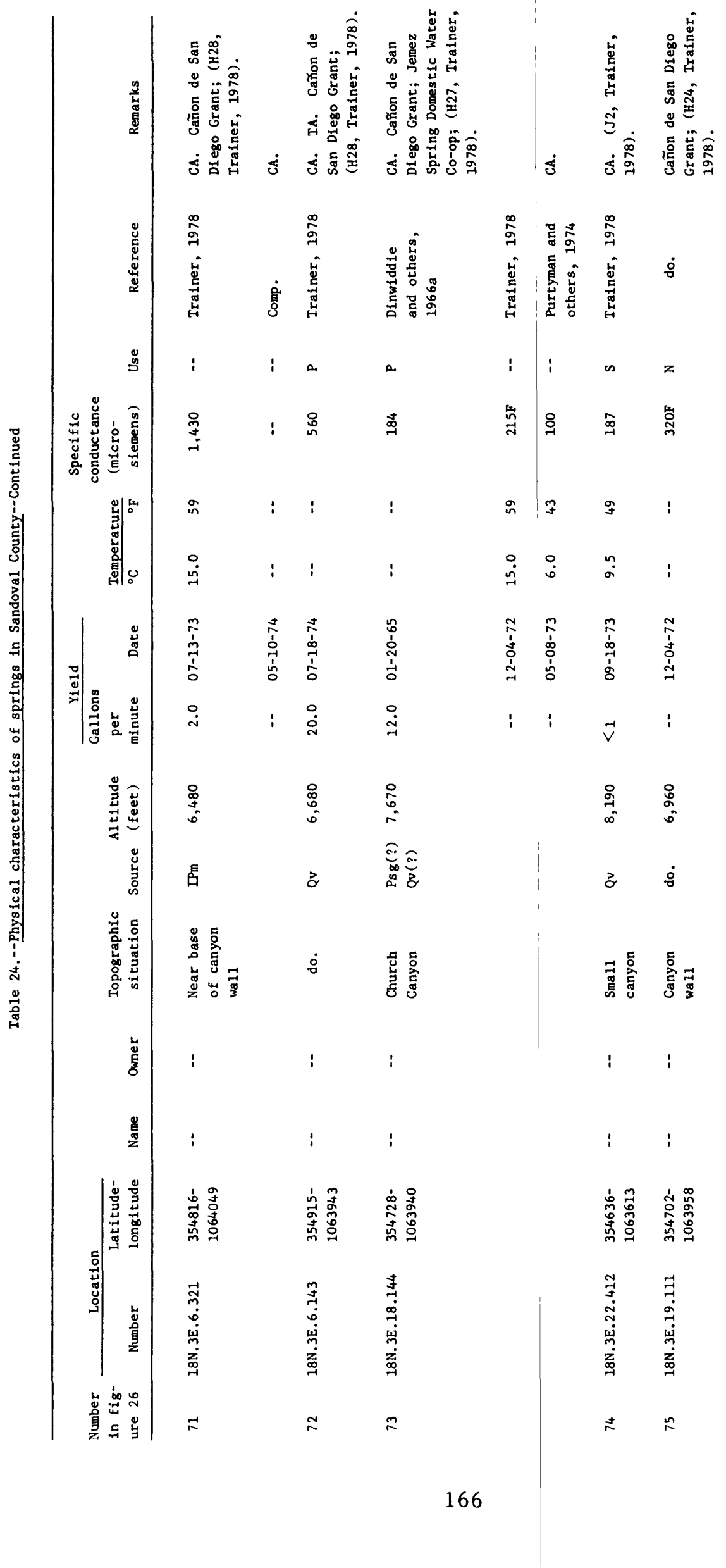




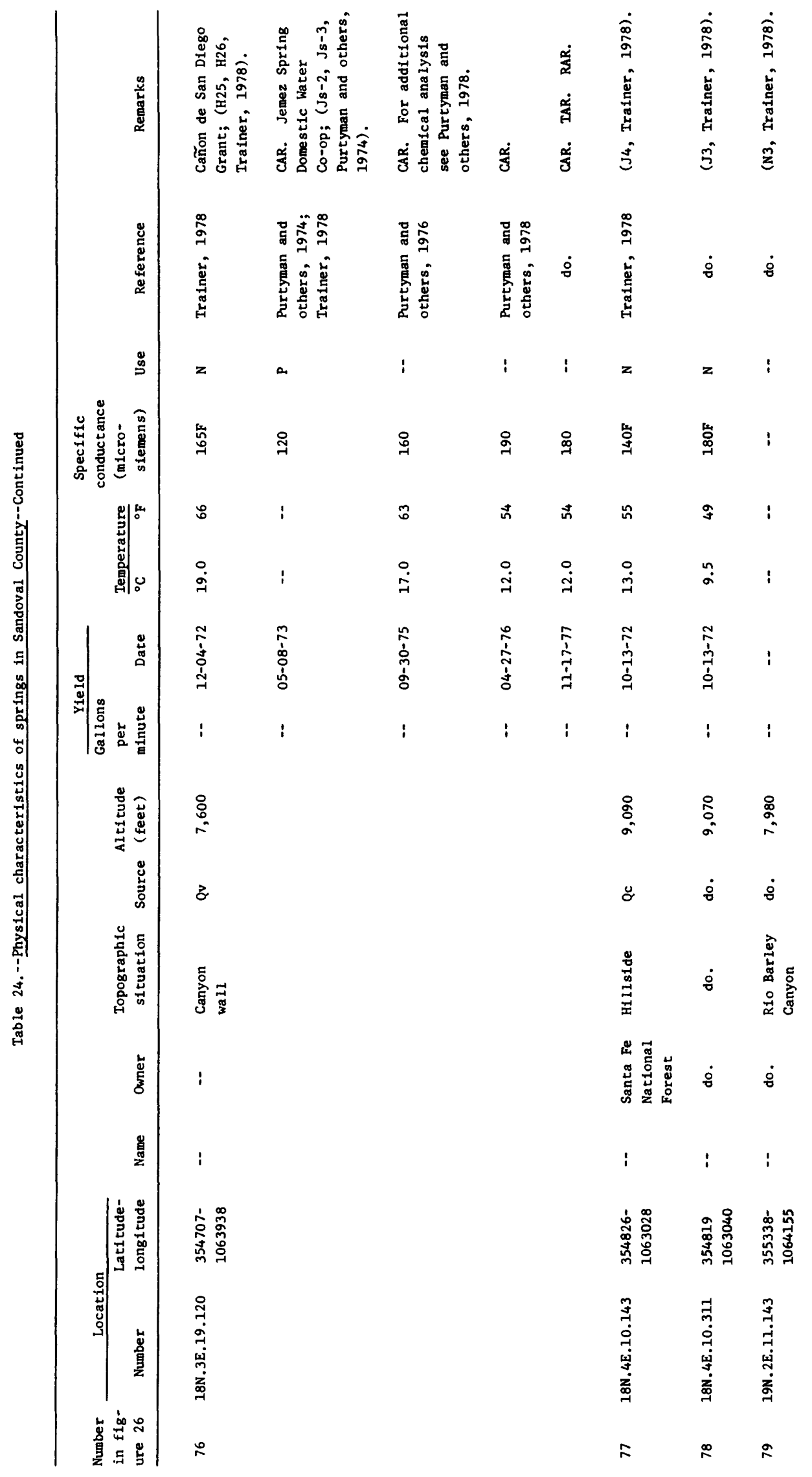




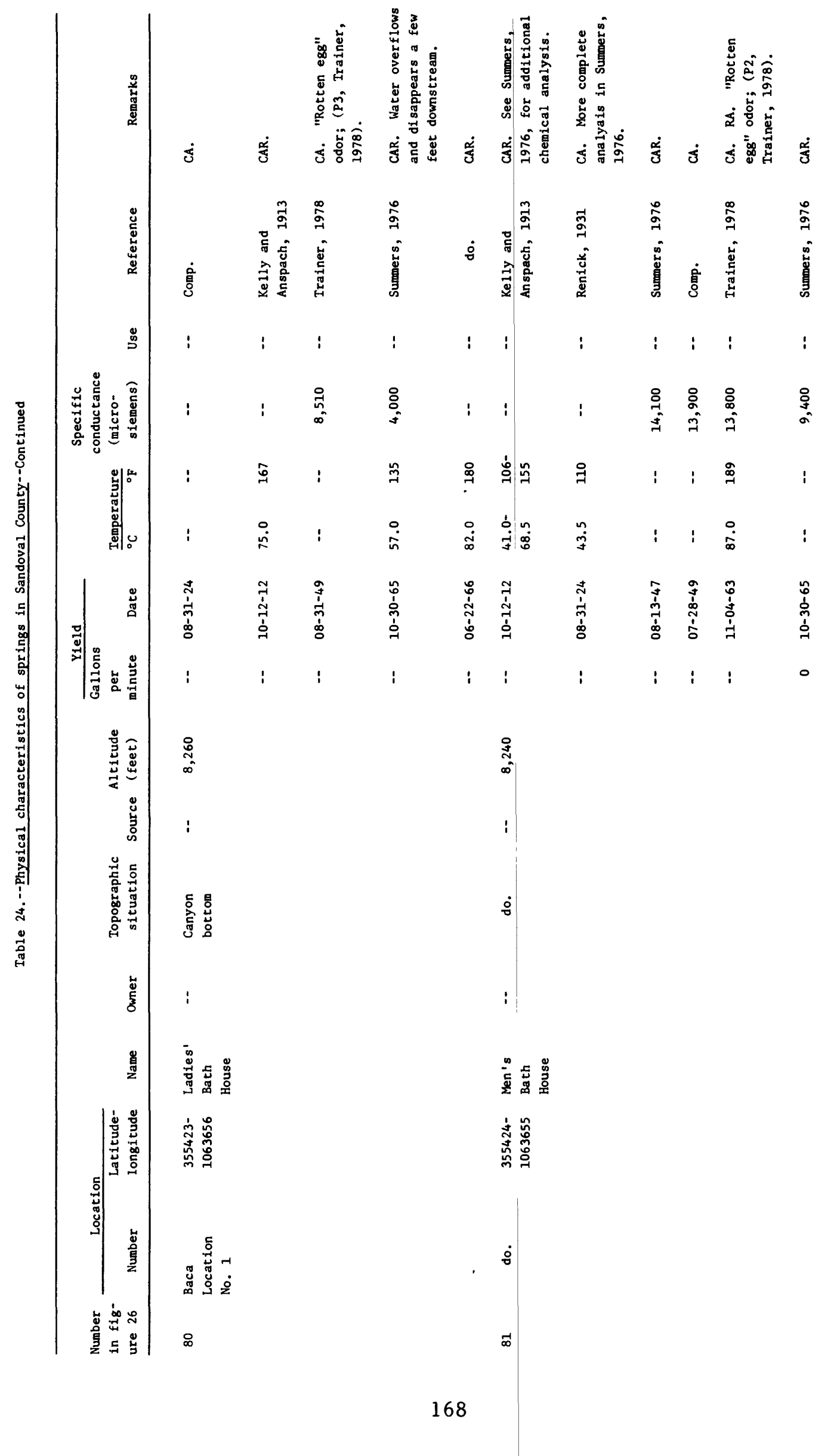




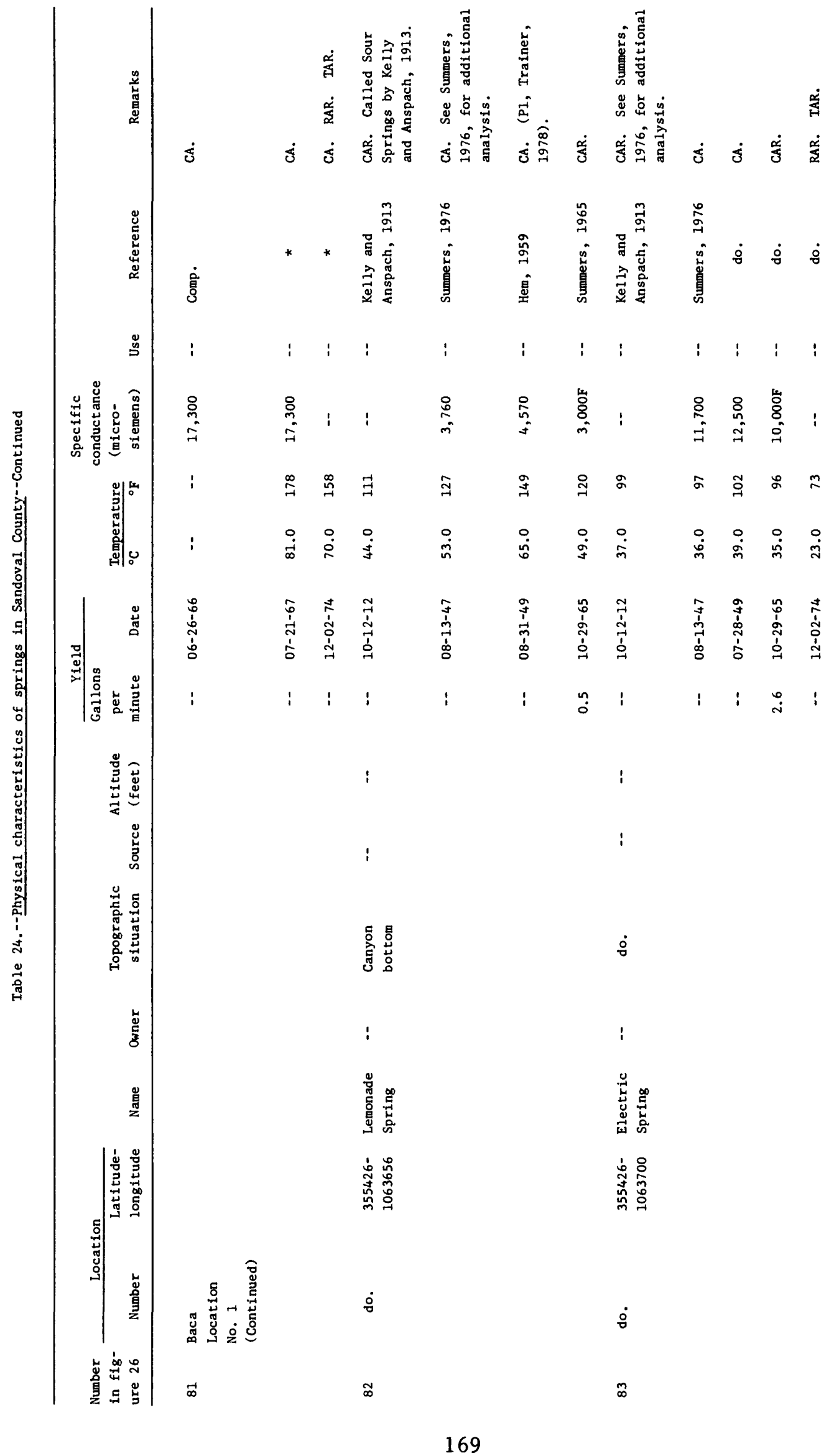




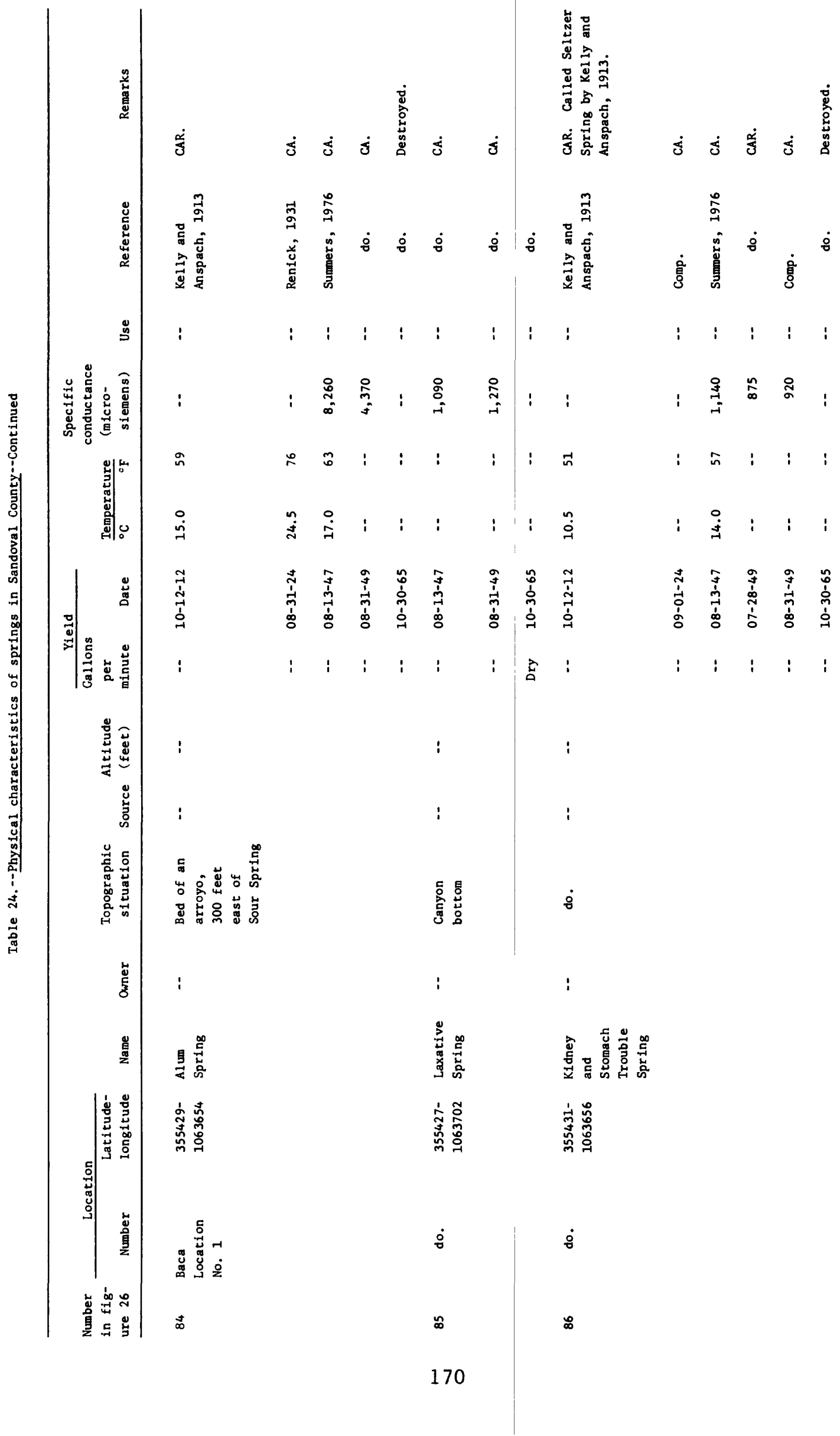




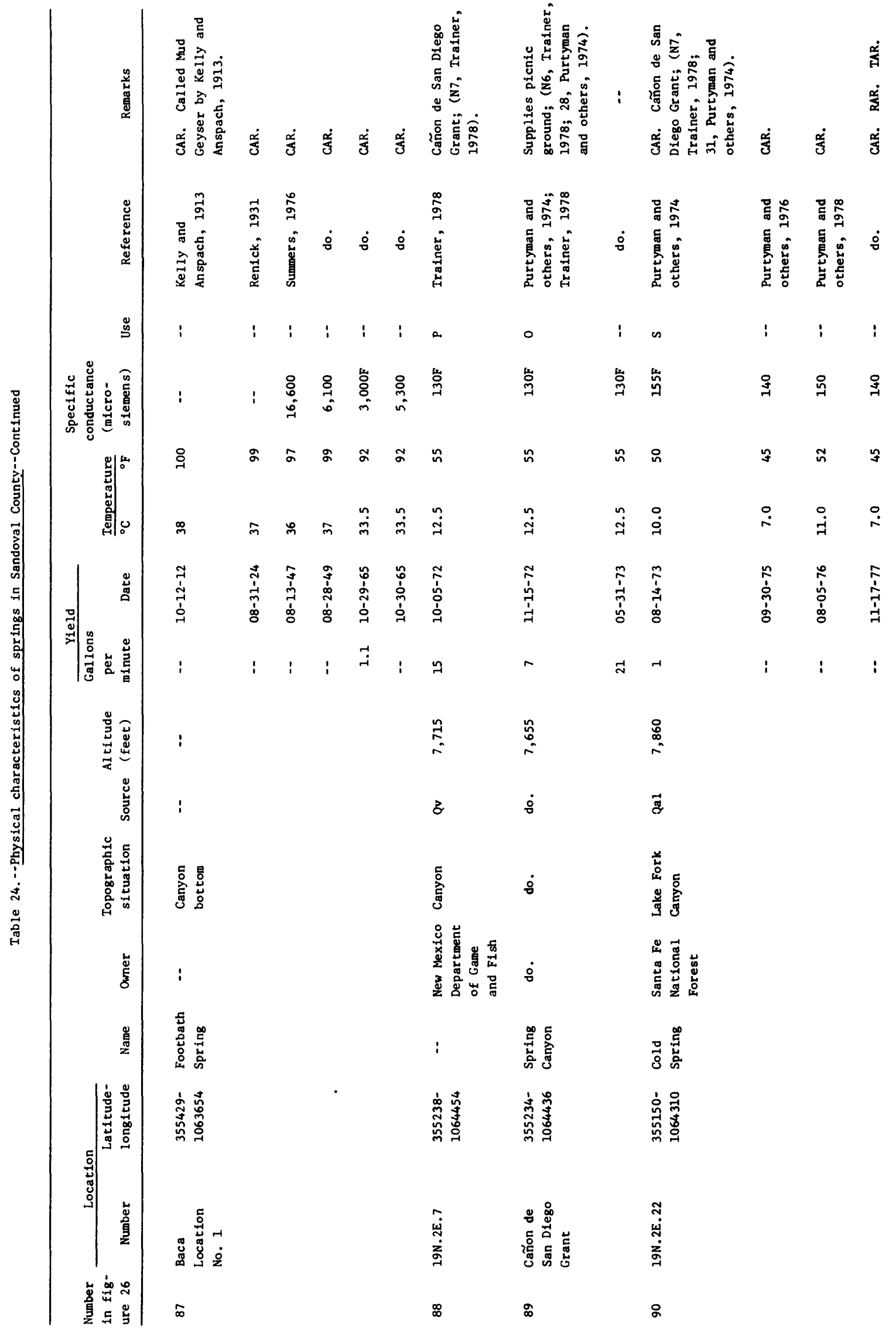




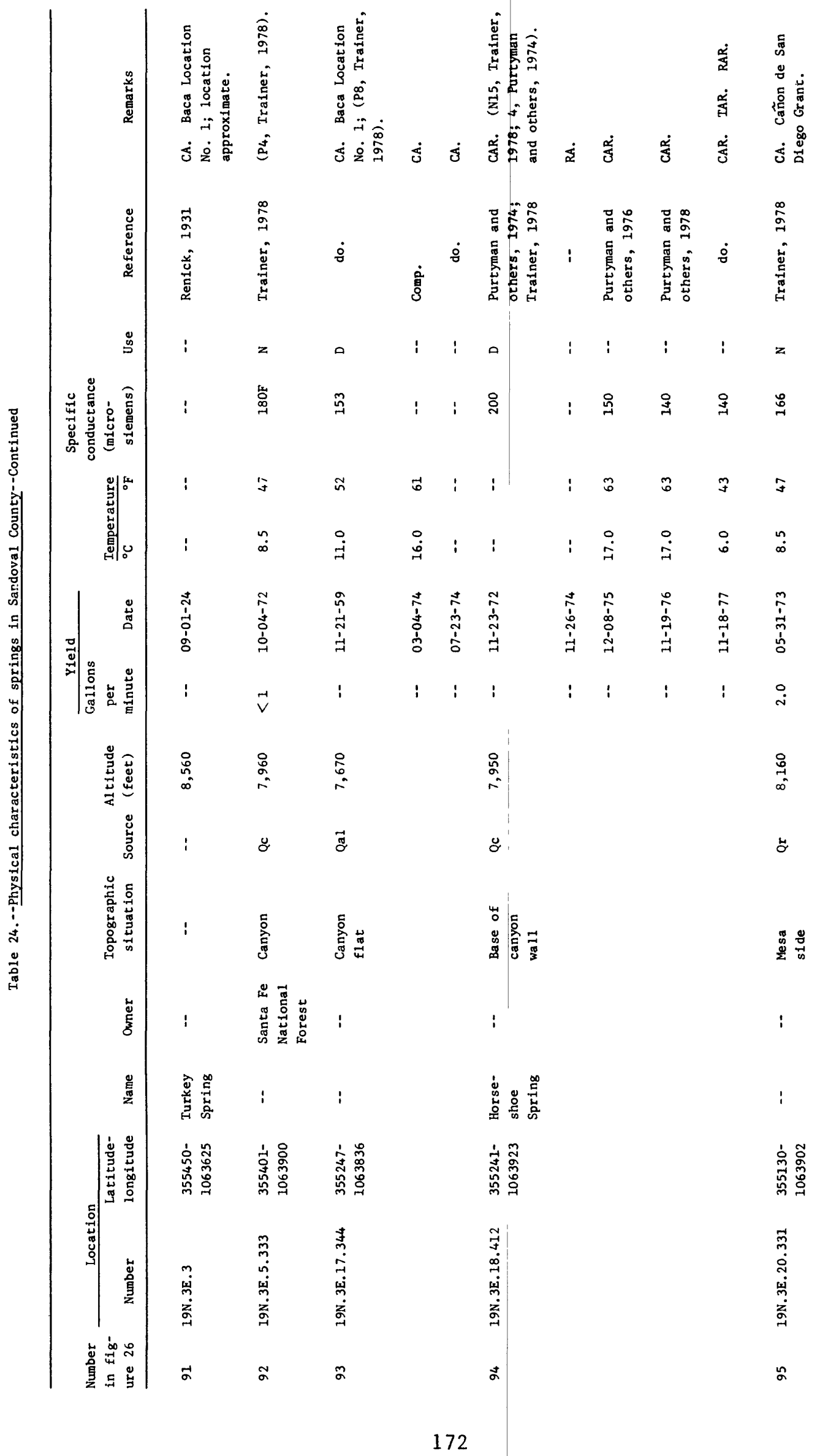




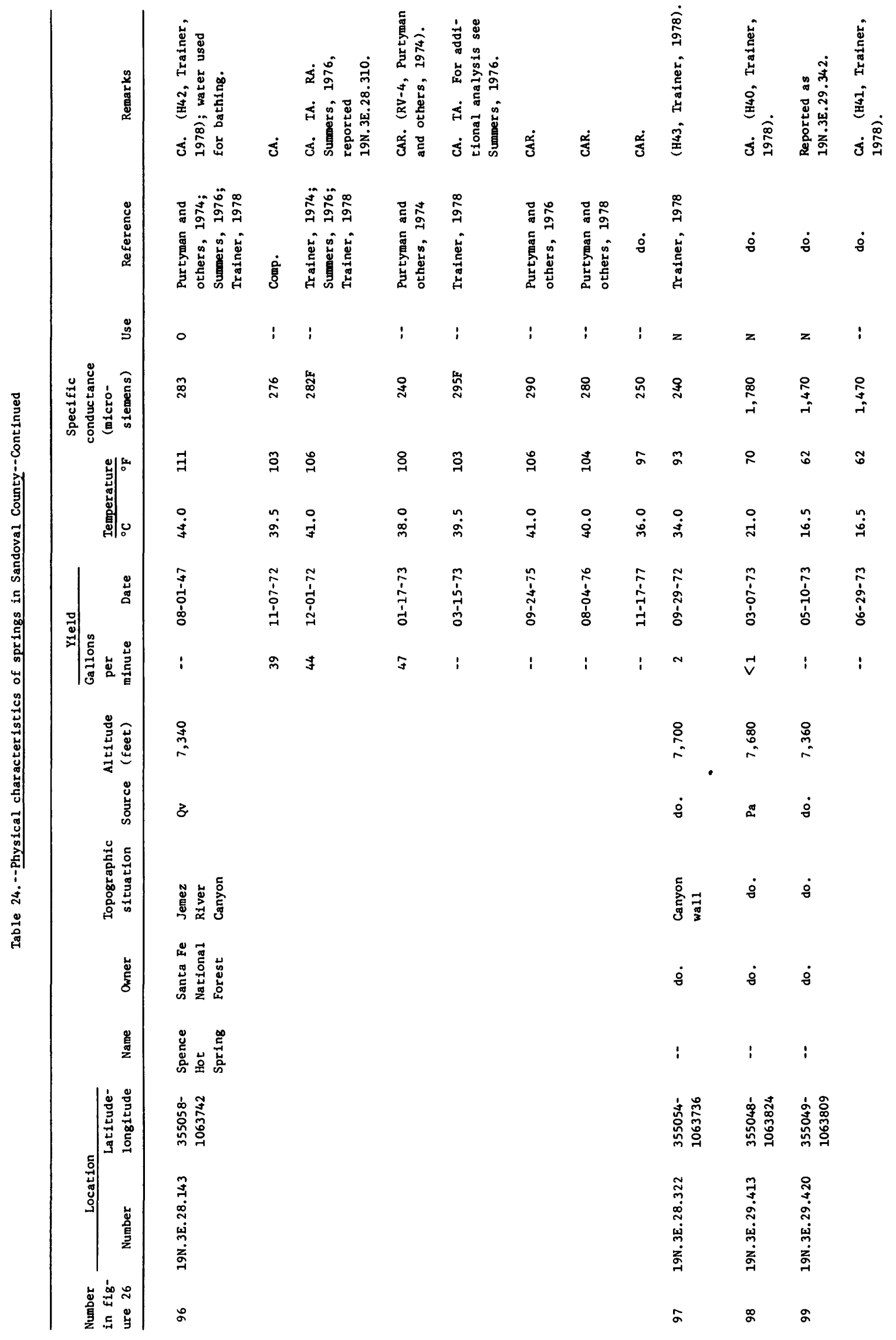




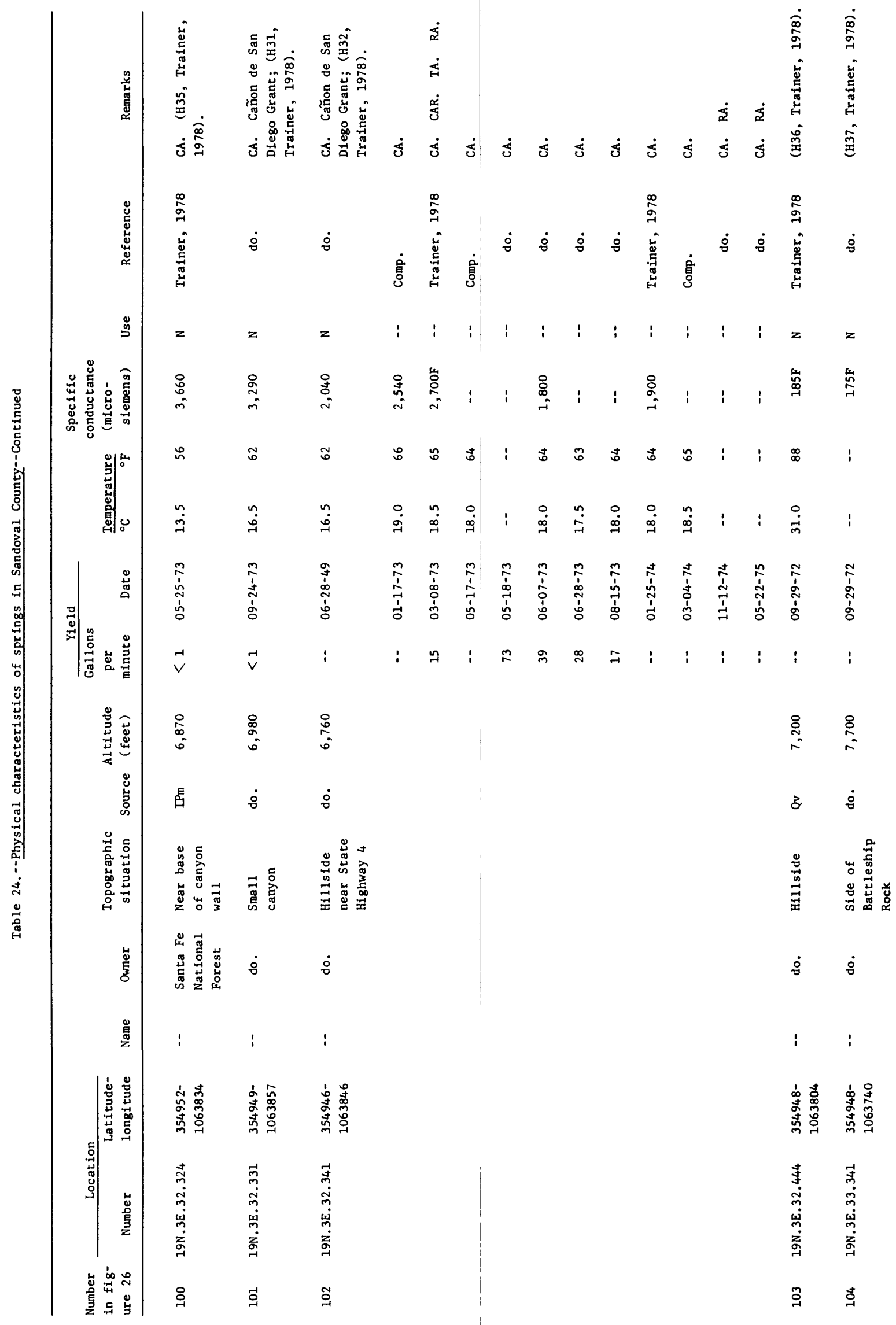




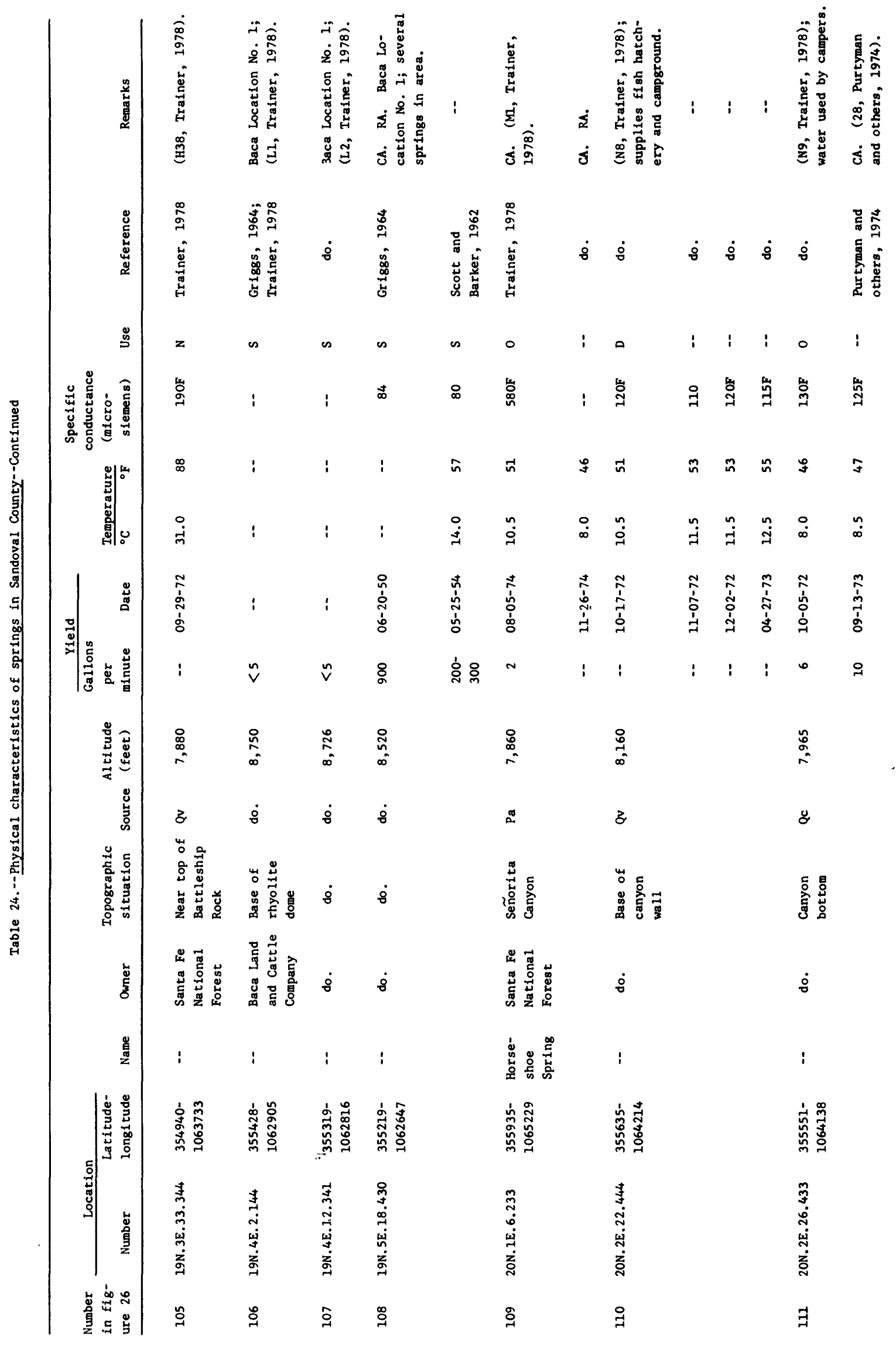




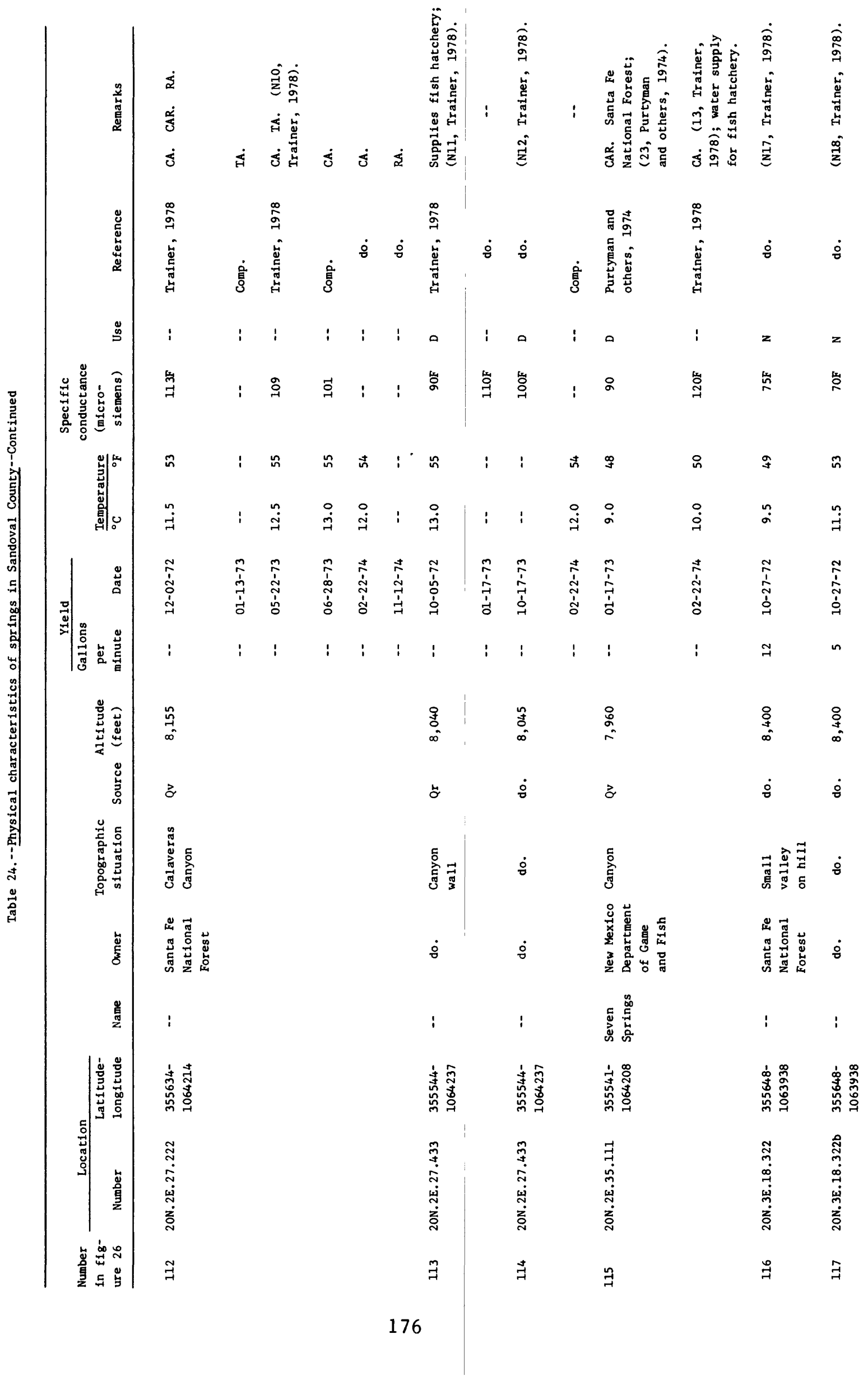




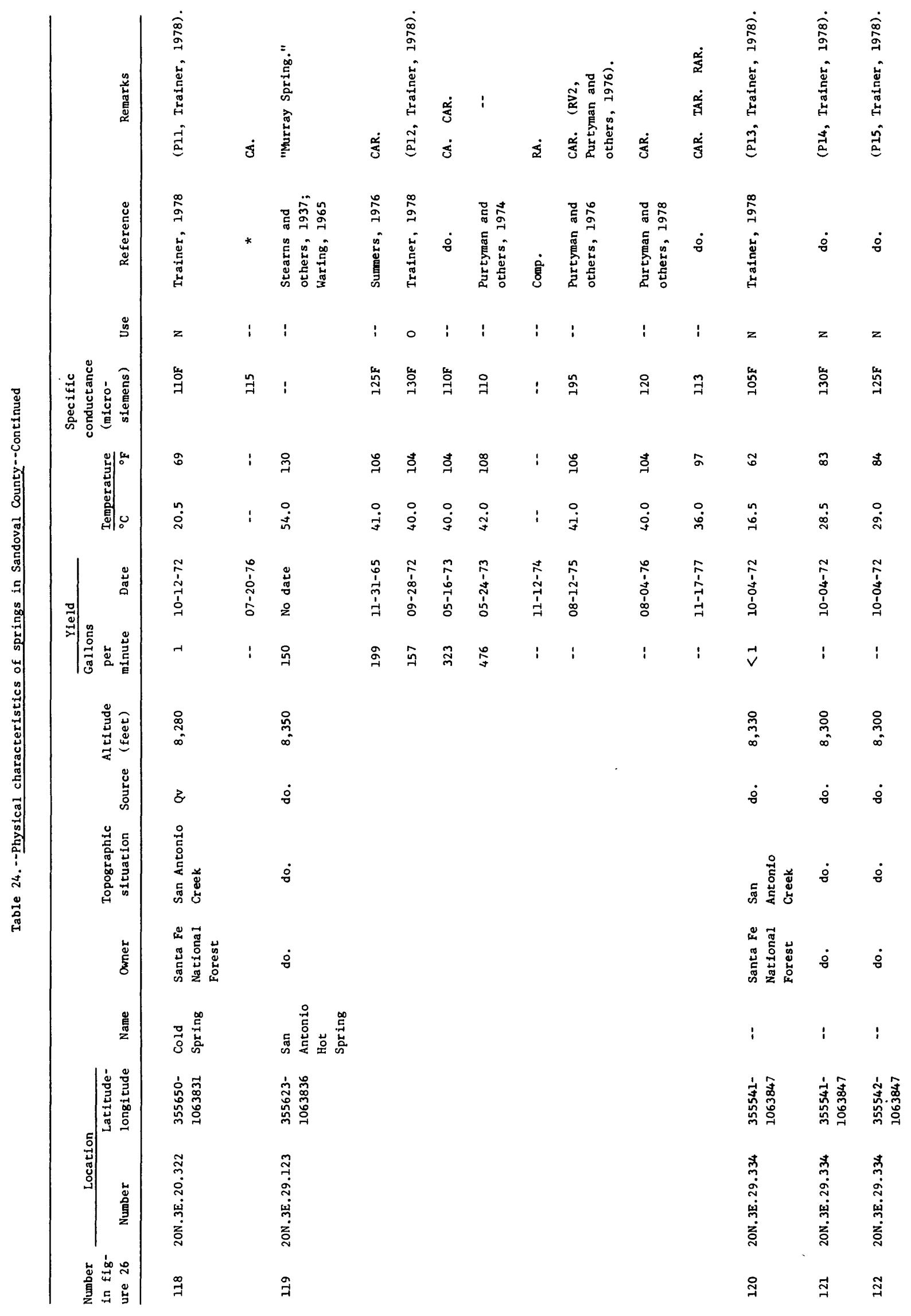




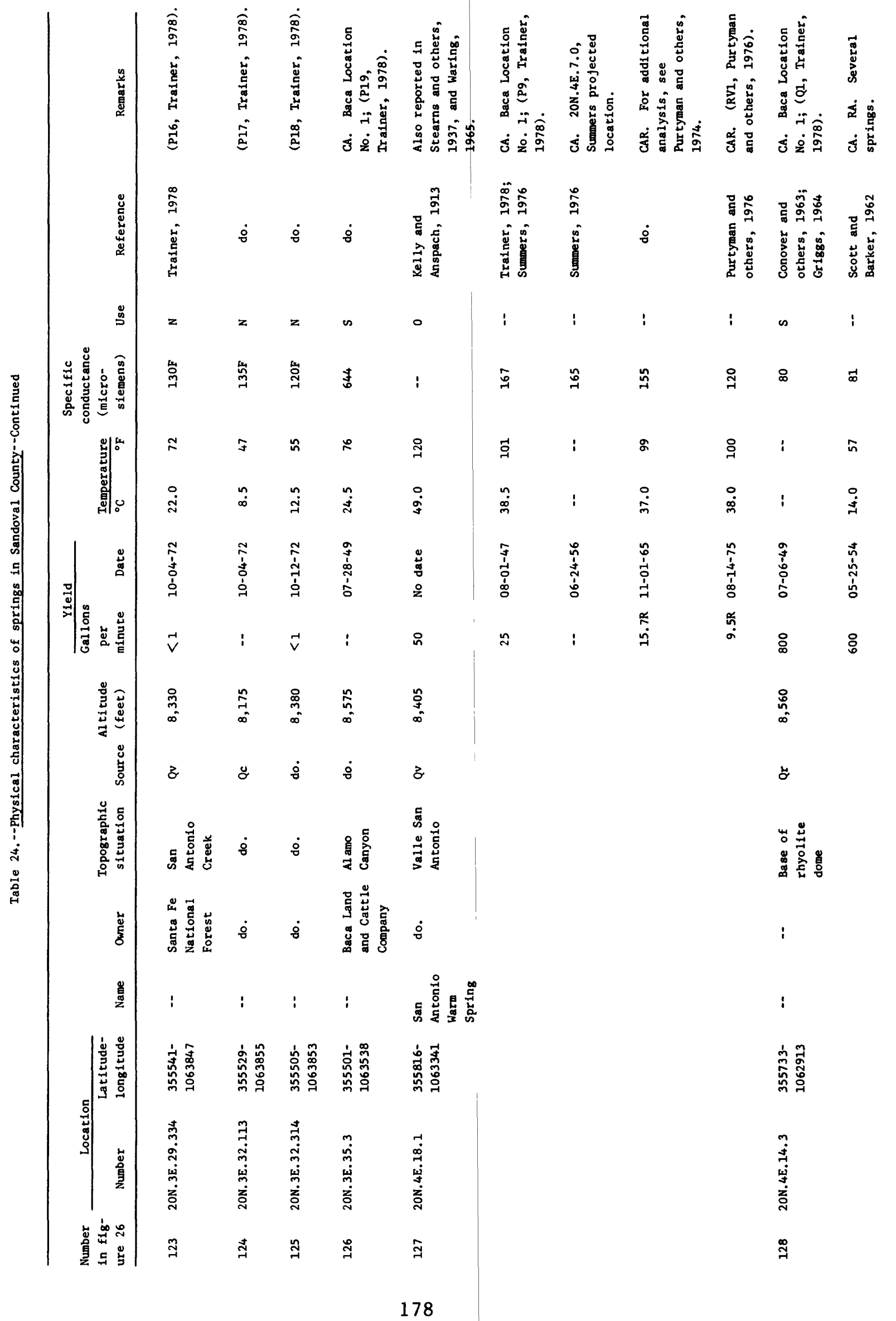




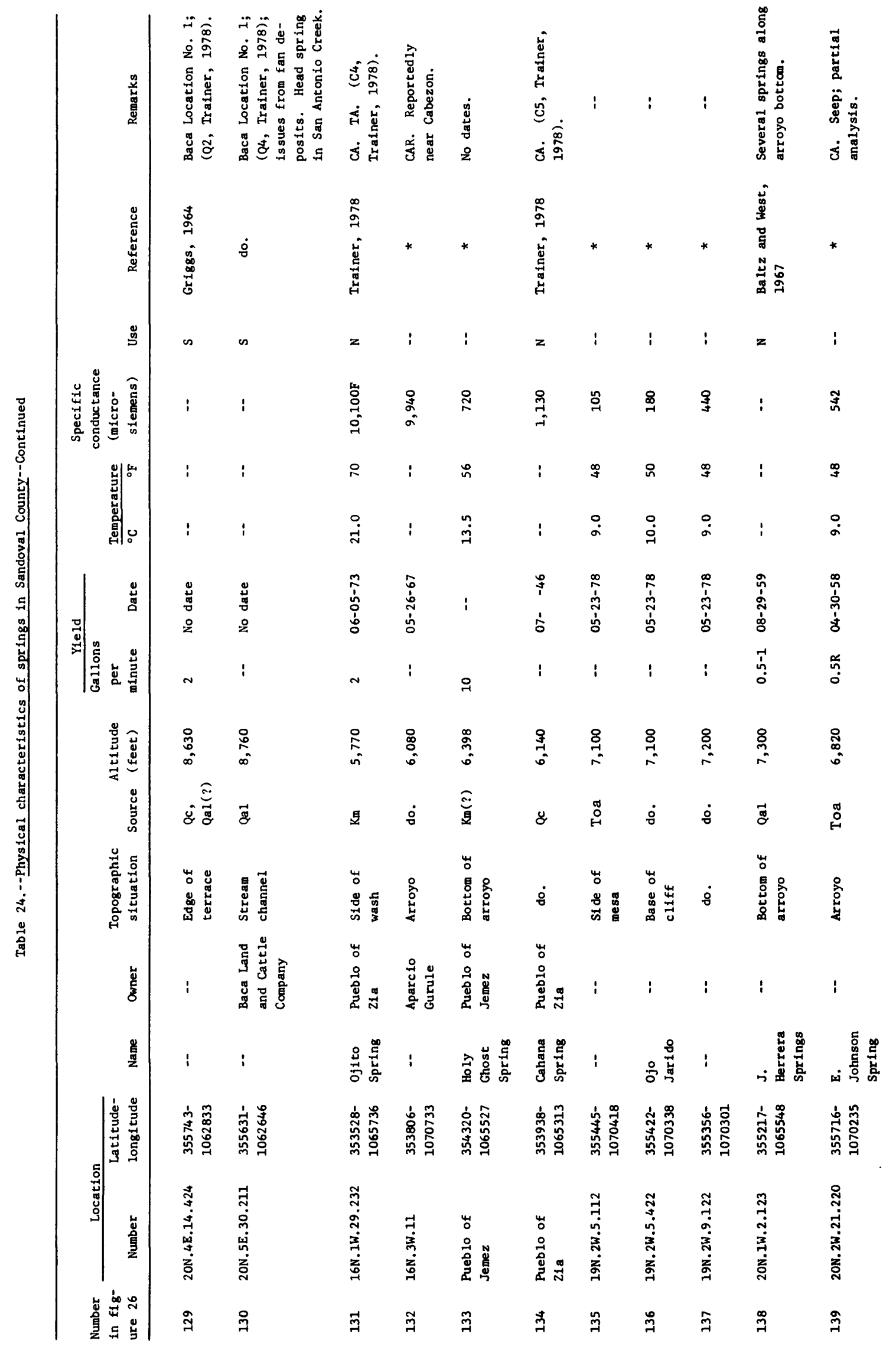




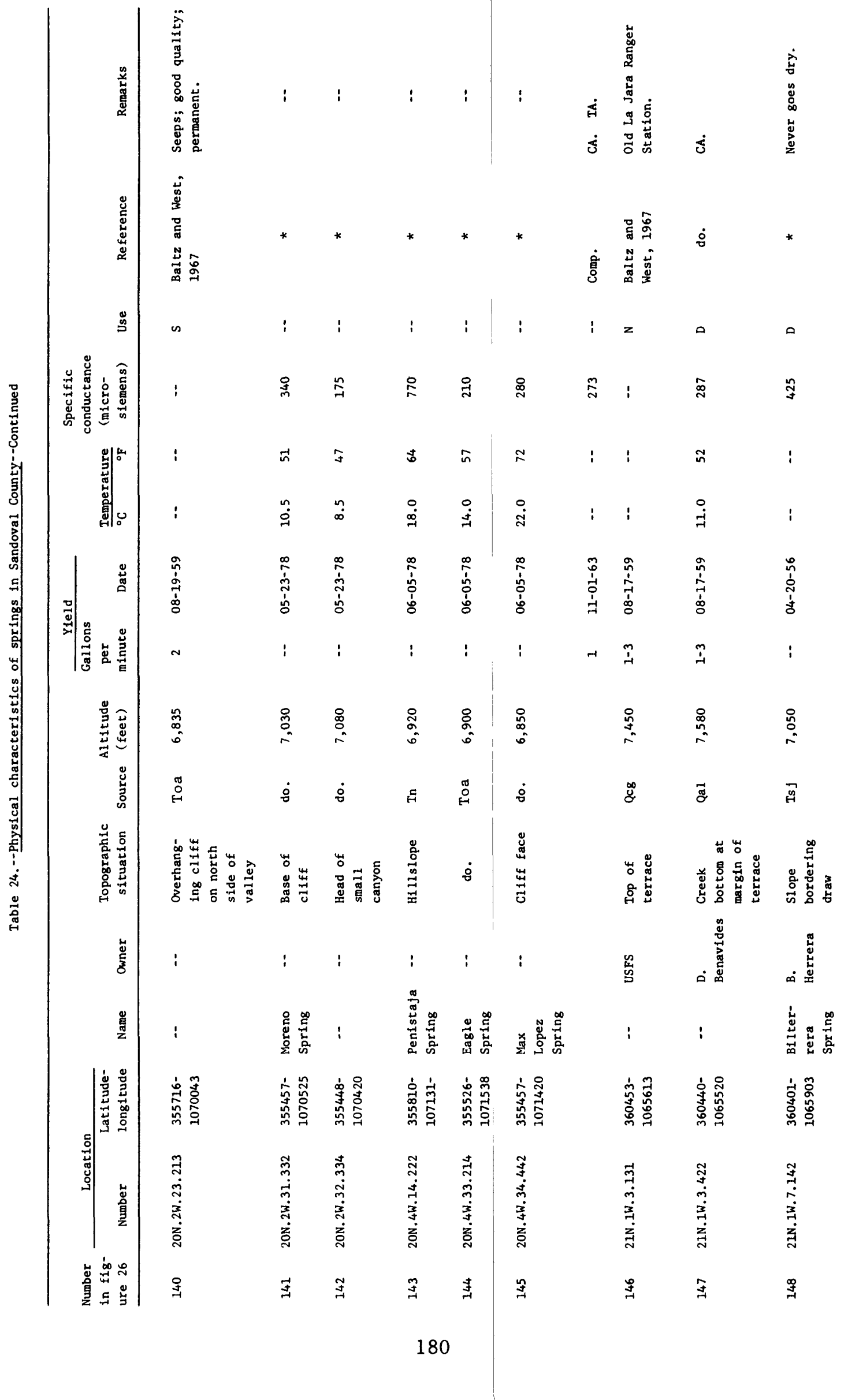




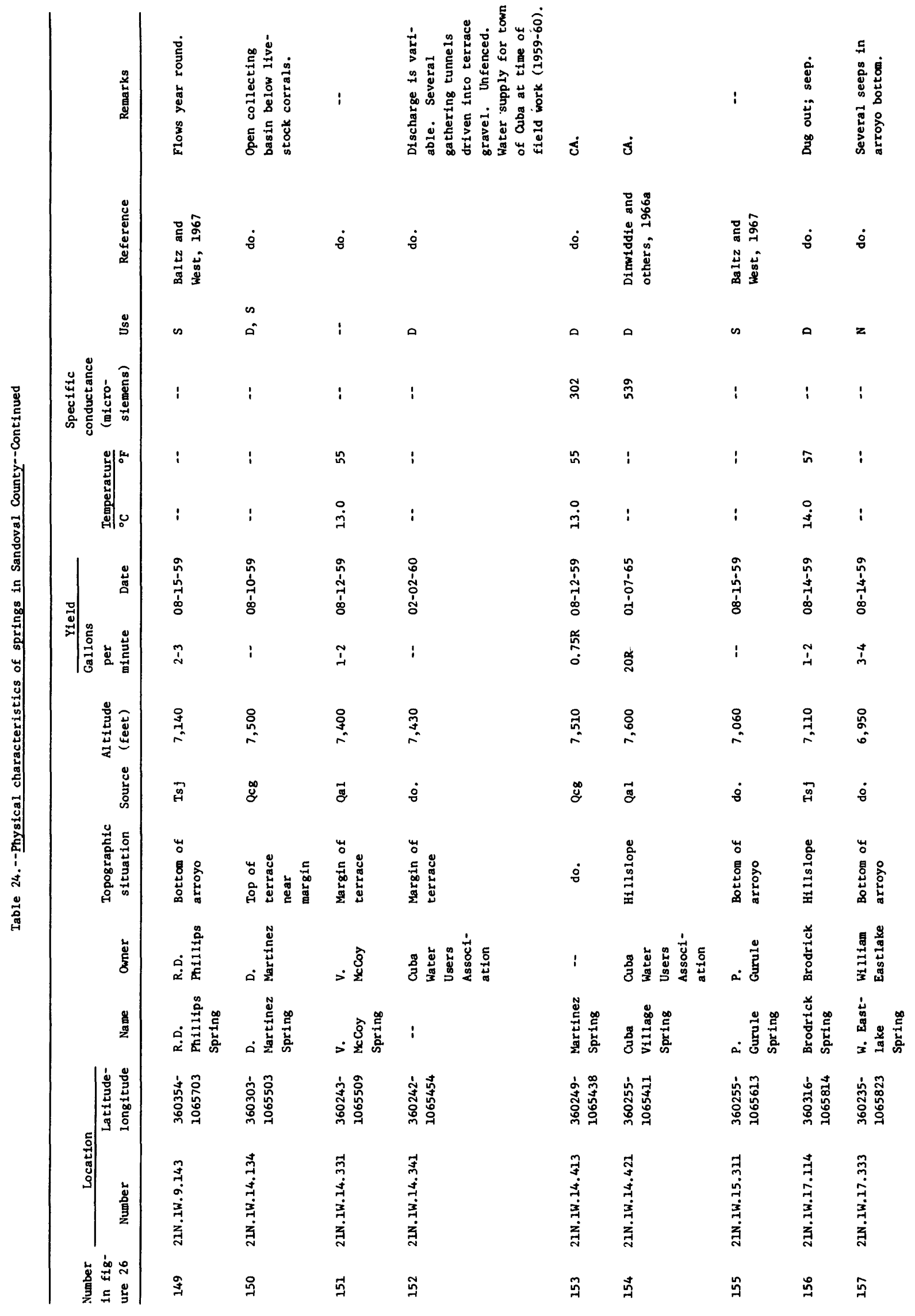




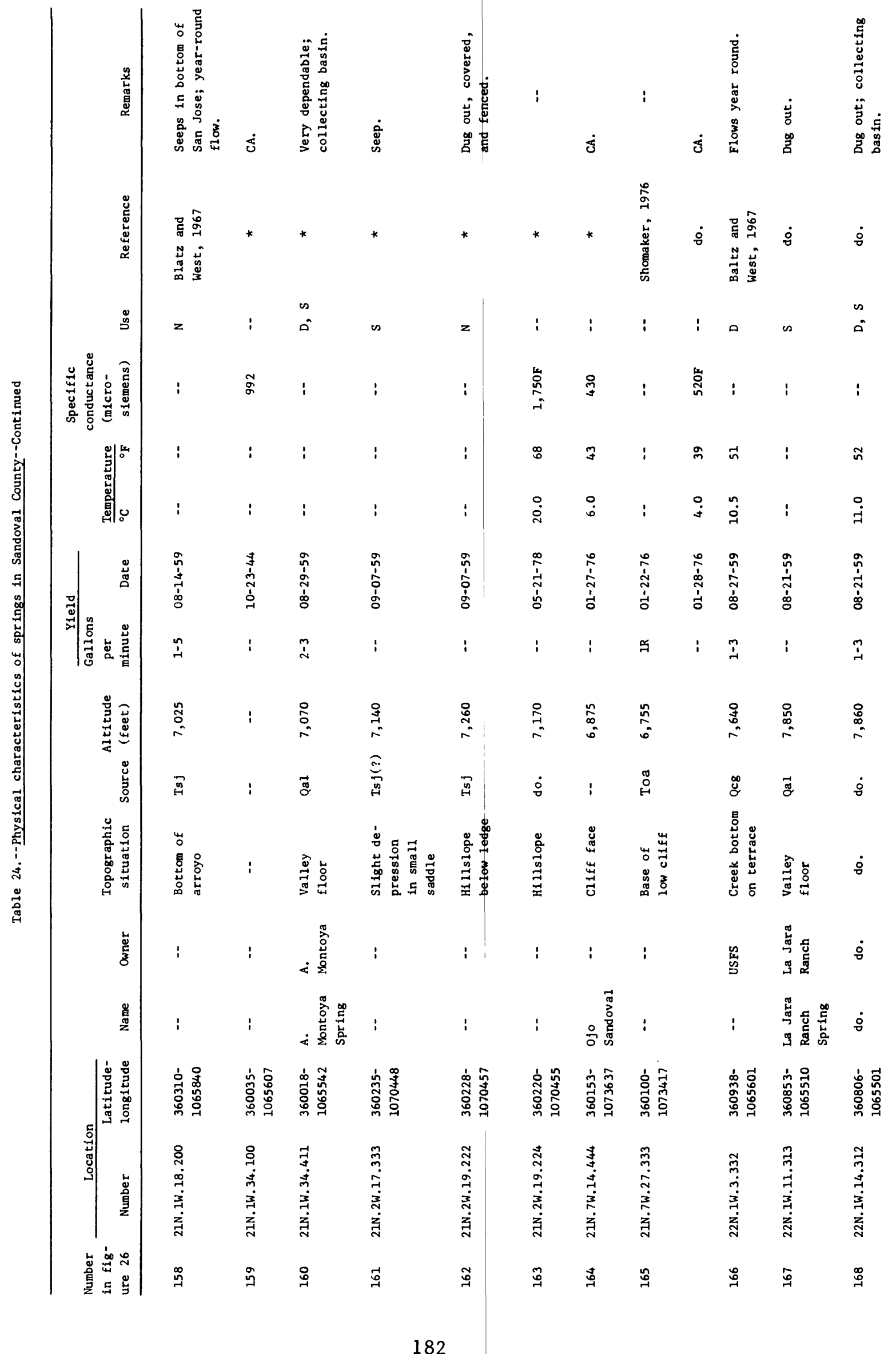




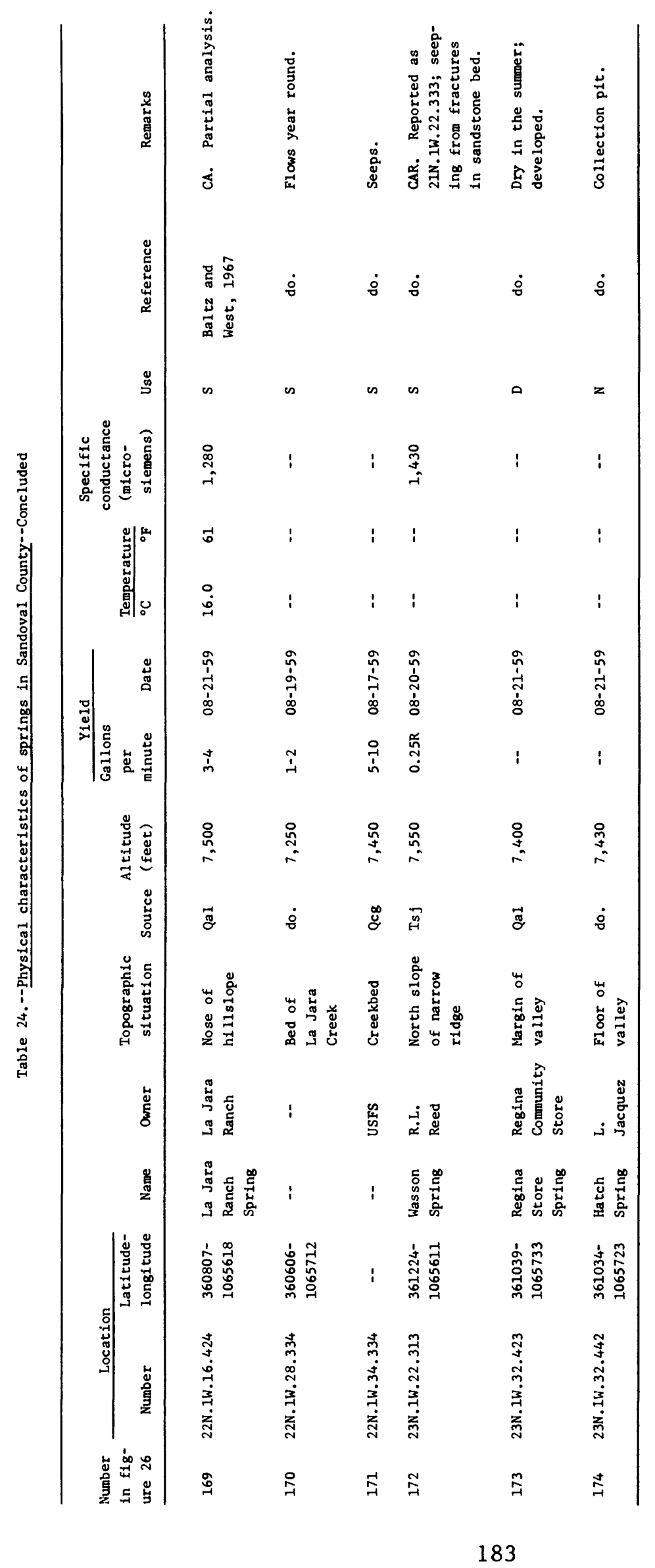




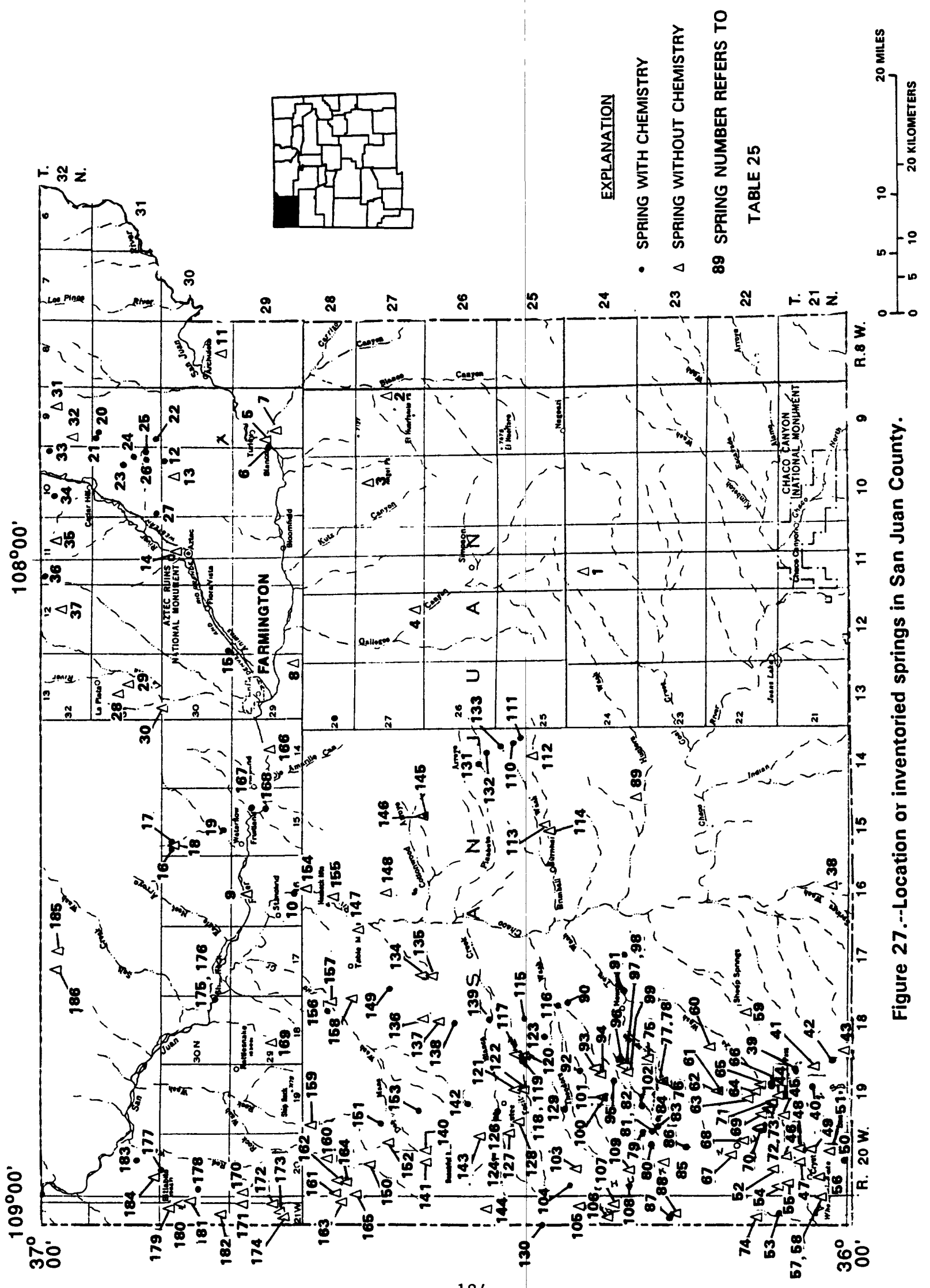




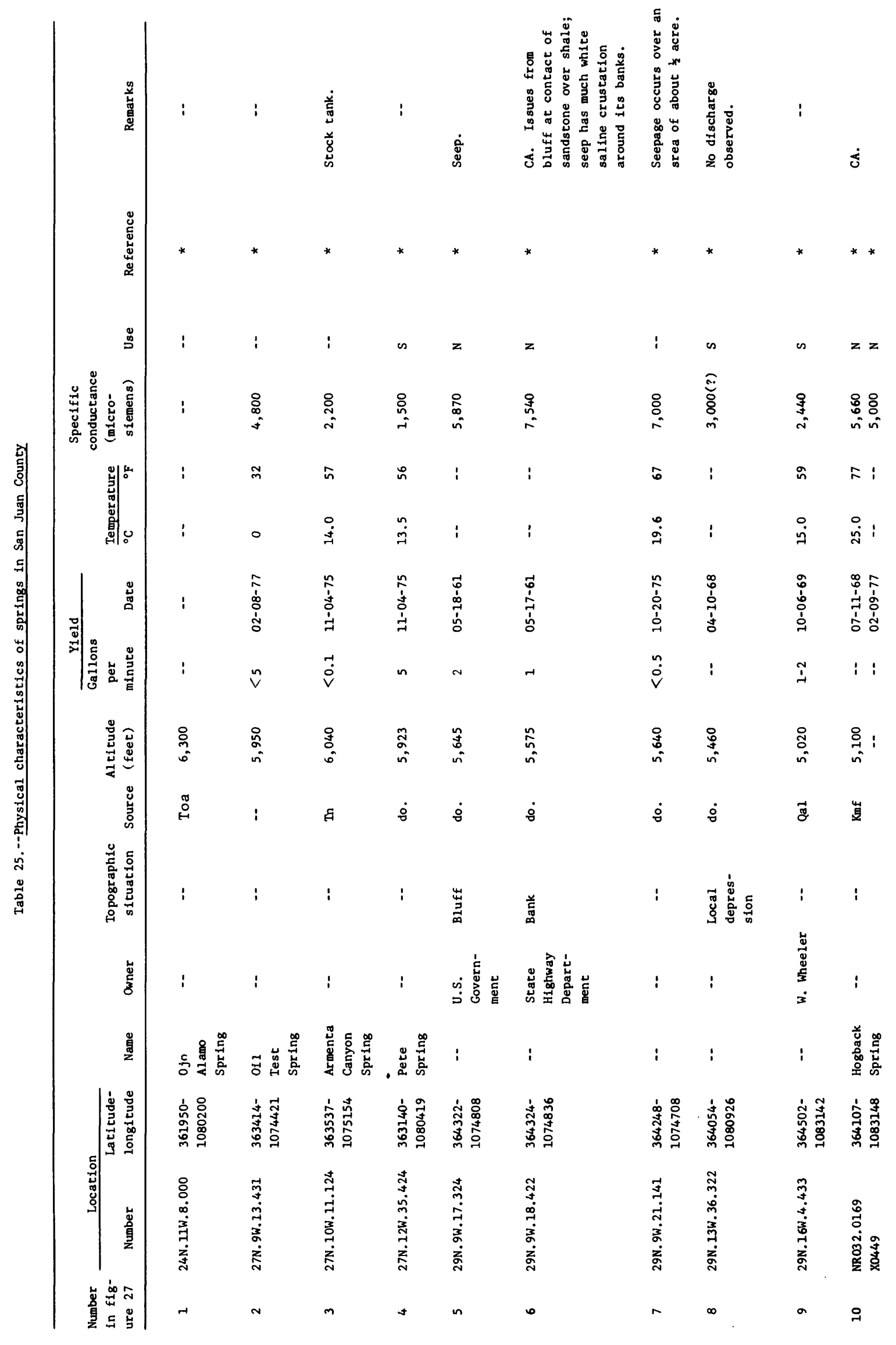




\begin{tabular}{|c|c|c|c|c|c|c|c|c|c|c|c|}
\hline 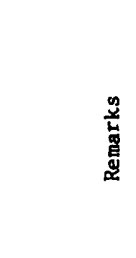 & $i$ & 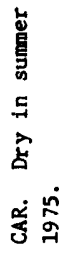 & 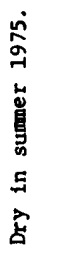 & $i$ & $\begin{array}{l}\dot{\forall} \\
\dot{\sigma}\end{array}$ & $\begin{array}{c}1 \\
\text { d }\end{array}$ & $\begin{array}{l}\dot{\leftrightarrow} \\
\dot{\Delta}\end{array}$ & 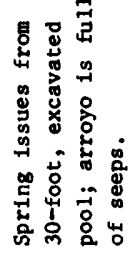 & ذ̇ & కัّ & 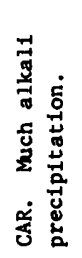 \\
\hline 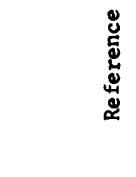 & * & 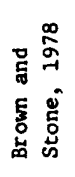 & $\dot{8}$ & * & * & * & $\dot{8}$ & * & 㻤 & 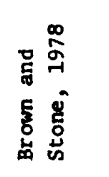 & $\dot{8}$ \\
\hline 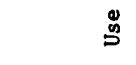 & : & $\infty$ & i & $\begin{array}{l}n \\
a \\
a\end{array}$ & a & : : & $:$ & $:$ & ! & $\infty$ & $\infty$ \\
\hline 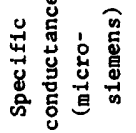 & $\begin{array}{c}o_{0} \\
m \\
-1\end{array}$ & $\stackrel{8}{0}$ & : & : & $\begin{array}{l}\text { 离 } \\
\text { on } \\
-1\end{array}$ & 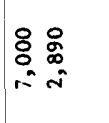 & $\begin{array}{l}\stackrel{8}{0} \\
\stackrel{-}{9}\end{array}$ & $\begin{array}{l}0 \\
0 \\
0 \\
0\end{array}$ & 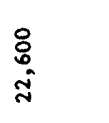 & $\stackrel{\infty}{\oplus}$ & 惫 \\
\hline$\left.\mathbf{y}^{\infty}\right|^{\infty}$ & $!$ & i & : & $:$ & $:$ & $q$ : & i & ก & $m$ & : & $:$ \\
\hline 氤。 & $i$ & $!$ & $i$ & $i$ & $:$ & $\stackrel{0}{i}:$ & $:$ & $\stackrel{n}{\infty}$ & $\stackrel{\circ}{\dot{n}}$ & $i$ & $i$ \\
\hline 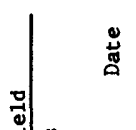 & 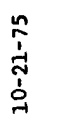 & $\begin{array}{l}n \\
1 \\
\vdots \\
0\end{array}$ & : & 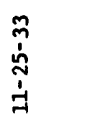 & \begin{tabular}{l}
0 \\
\multirow{1}{1}{} \\
$\hat{i}$ \\
$\dot{\alpha}$ \\
0
\end{tabular} & 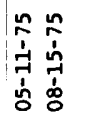 & 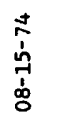 & $\begin{array}{l}n \\
\hat{\omega} \\
\grave{a} \\
\end{array}$ & $\begin{array}{l}\overrightarrow{0} \\
\hat{n} \\
\vec{n} \\
\dot{0}\end{array}$ & $\begin{array}{l}n \\
\hat{1} \\
\overline{1} \\
\dot{8}\end{array}$ & $\begin{array}{l}n \\
\hat{1} \\
\hat{7} \\
\dot{8} \\
8\end{array}$ \\
\hline $\mid$ & ฆั & $:$ & $i$ & $:$ & ' & :! : & 芯 & $!$ & i & $i$ & i \\
\hline 总 & 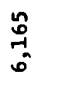 & 员 & $\overbrace{0}^{8}$ & $i$ & 疍 & 离 & in & స్ల్ & : & in & $\begin{array}{l}\stackrel{0}{n} \\
\hat{n}^{\circ}\end{array}$ \\
\hline 苞 & i & $\bar{n}$ & $\dot{8}$ & i & $\widehat{\widehat{\mathscr{I}}}$ & 岁 & $:$ & $\bar{d}$ & i & $\overrightarrow{\tilde{H}}$ & $\dot{8}$ \\
\hline 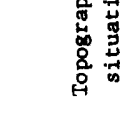 & 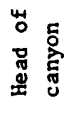 & i & 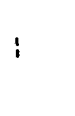 & $:$ & 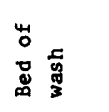 & i & $i$ & 产 & $:$ & : & : \\
\hline 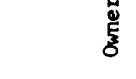 & i & $!$ & $i$ & i & 煦 & : & $i$ & $i$ & $:$ & ' & : \\
\hline 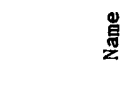 & $i$ & 总总 & 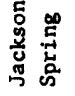 & 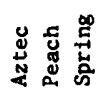 & 的总总营 & 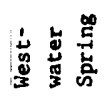 & $\dot{8}$ & $i$ & 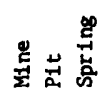 & 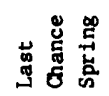 & 点品 \\
\hline 总 & 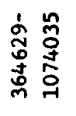 & 㝘总 & 话总 & 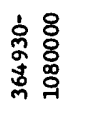 & 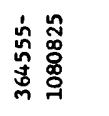 & 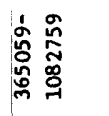 & 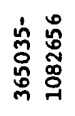 & 容商 & 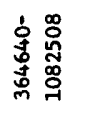 & 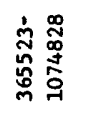 & 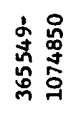 \\
\hline 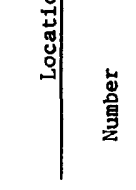 & 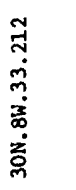 & 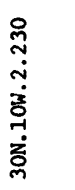 & 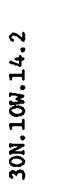 & $\begin{array}{l}8 \\
\vdots \\
\vdots \\
\vdots \\
\vdots \\
7 \\
\dot{z} \\
\dot{z}\end{array}$ & 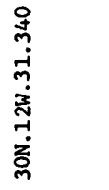 & 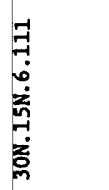 & 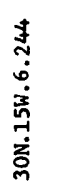 & 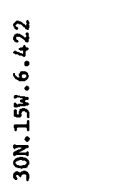 & 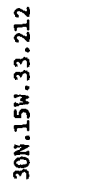 & 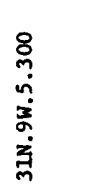 & $\begin{array}{l}\stackrel{8}{8} \\
\vdots \\
\vdots \\
\vdots \\
\vdots \\
\dot{j} \\
\dot{m}\end{array}$ \\
\hline 圎至苛 & $\vec{F}$ & 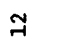 & $\eta$ & \pm & $\cong$ & $\stackrel{1}{1}$ & 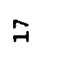 & $\stackrel{\infty}{-1}$ & 9 & $\stackrel{\sim}{\circ}$ & $\pi$ \\
\hline
\end{tabular}




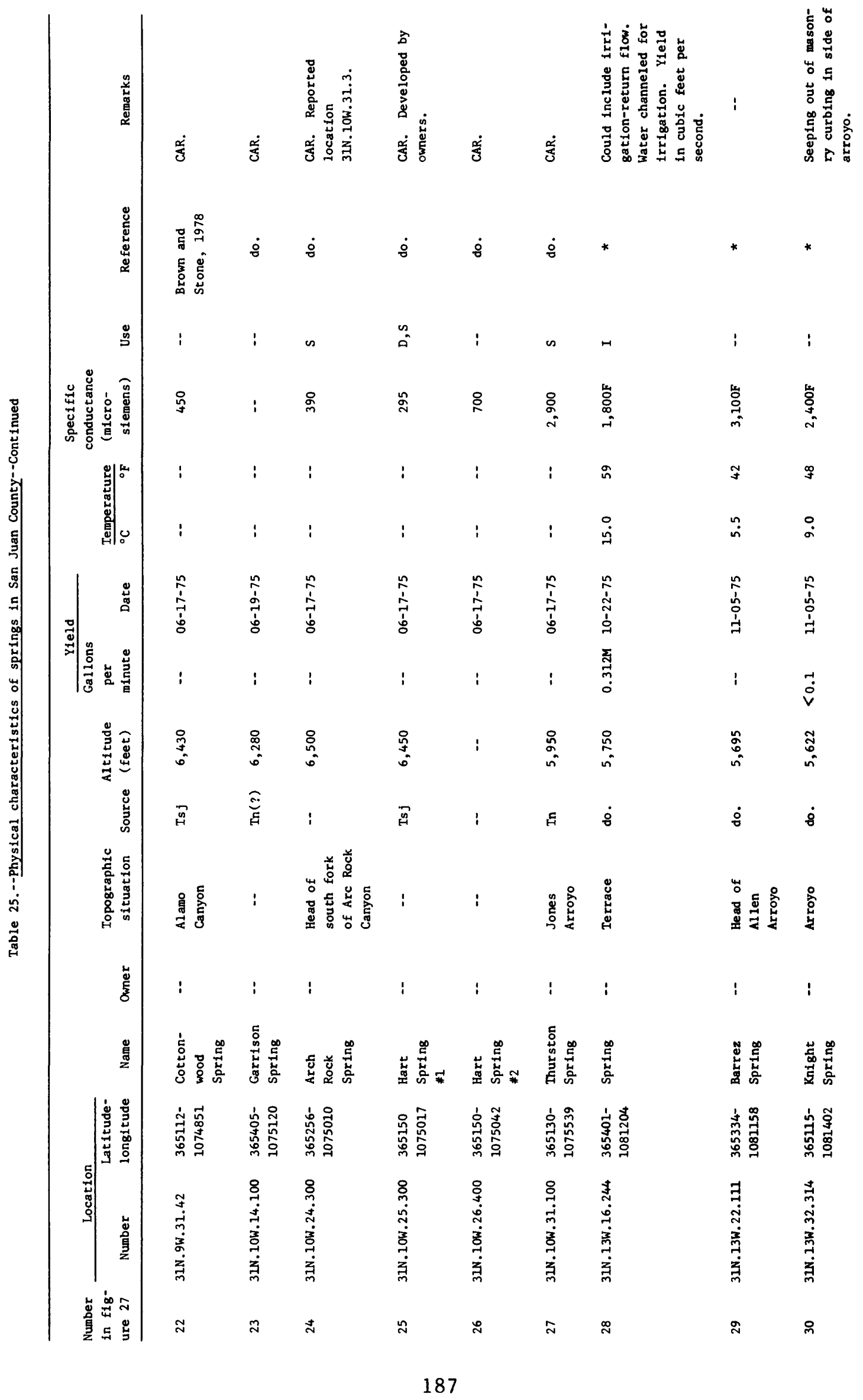




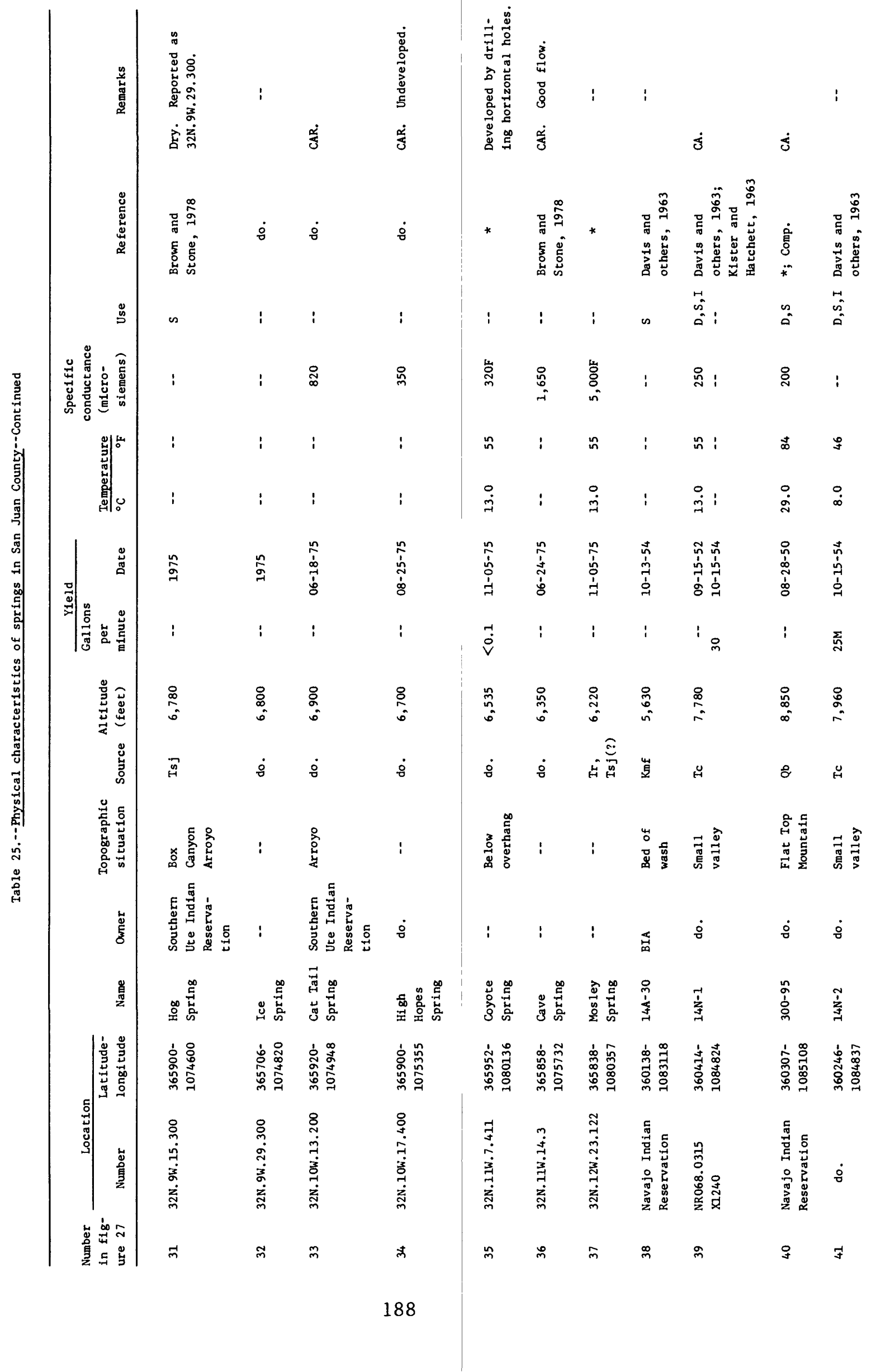




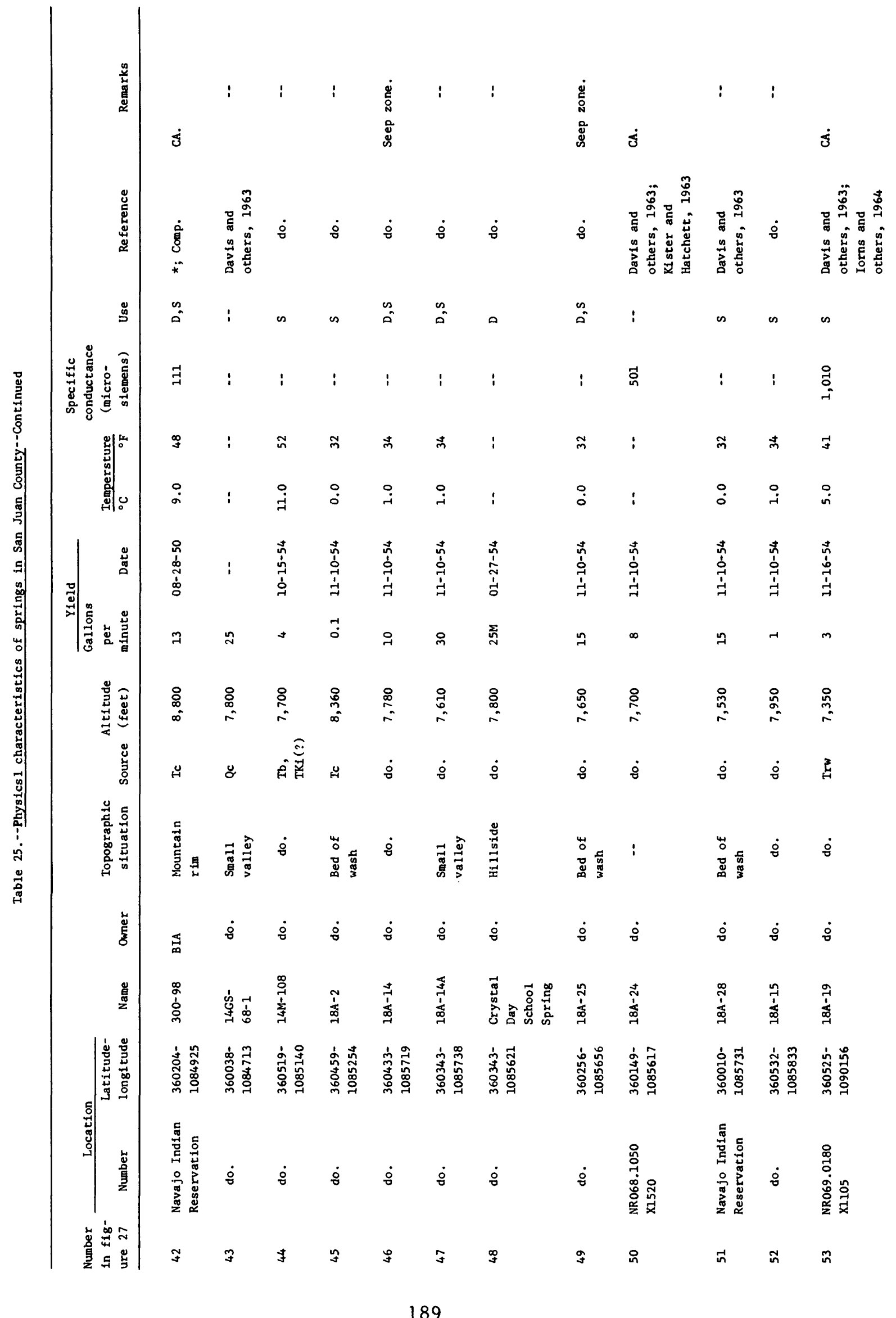




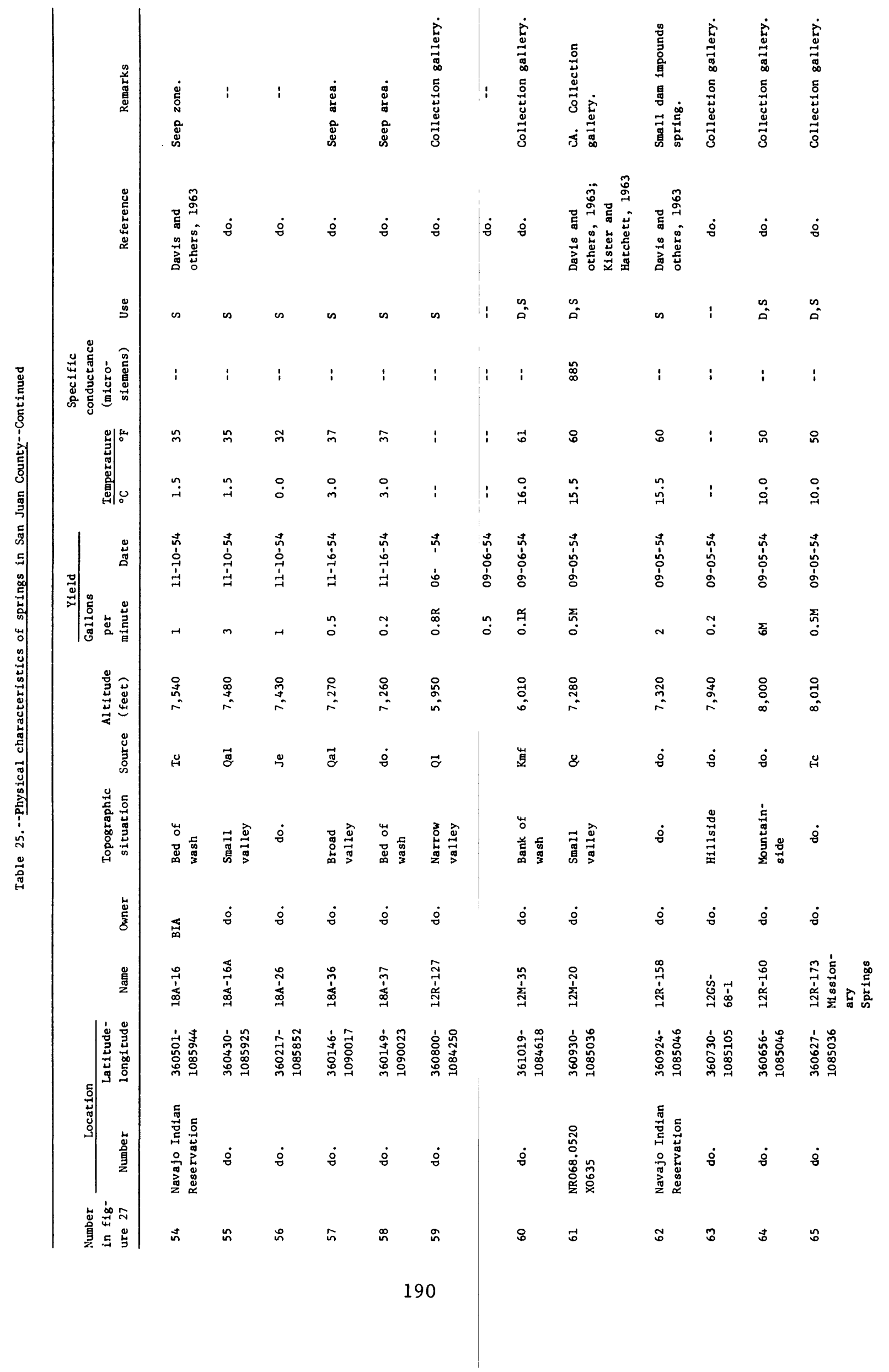




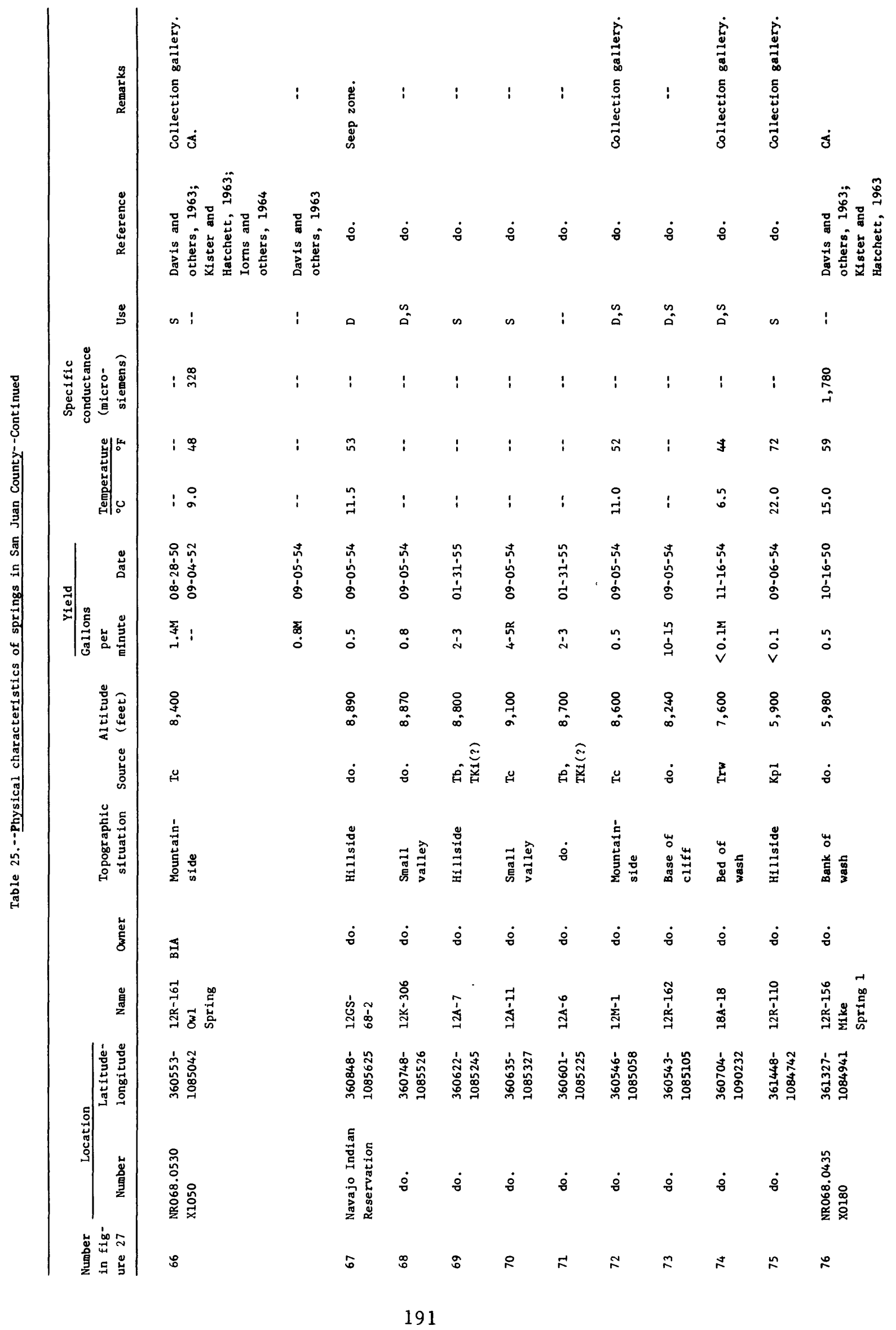




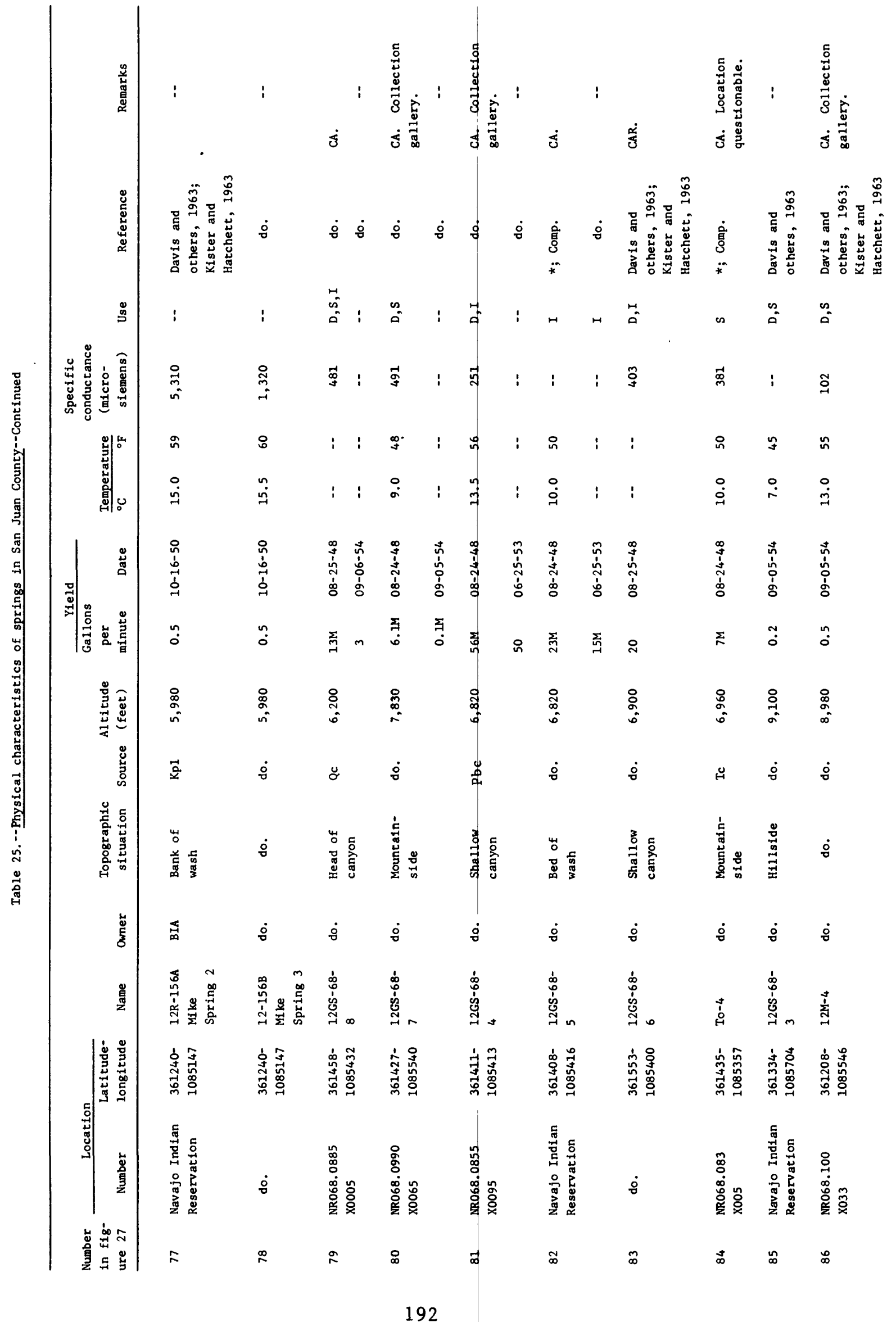




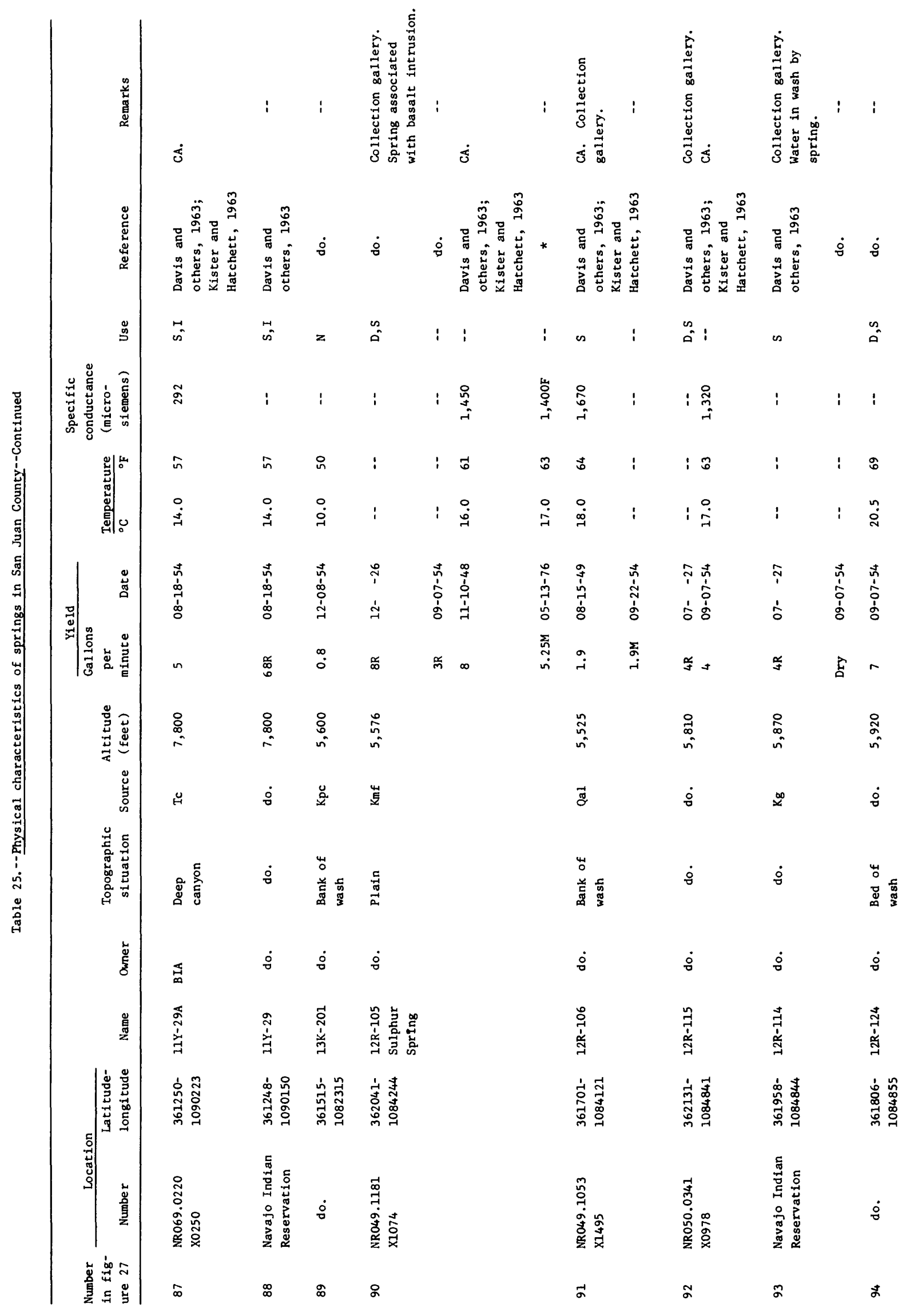




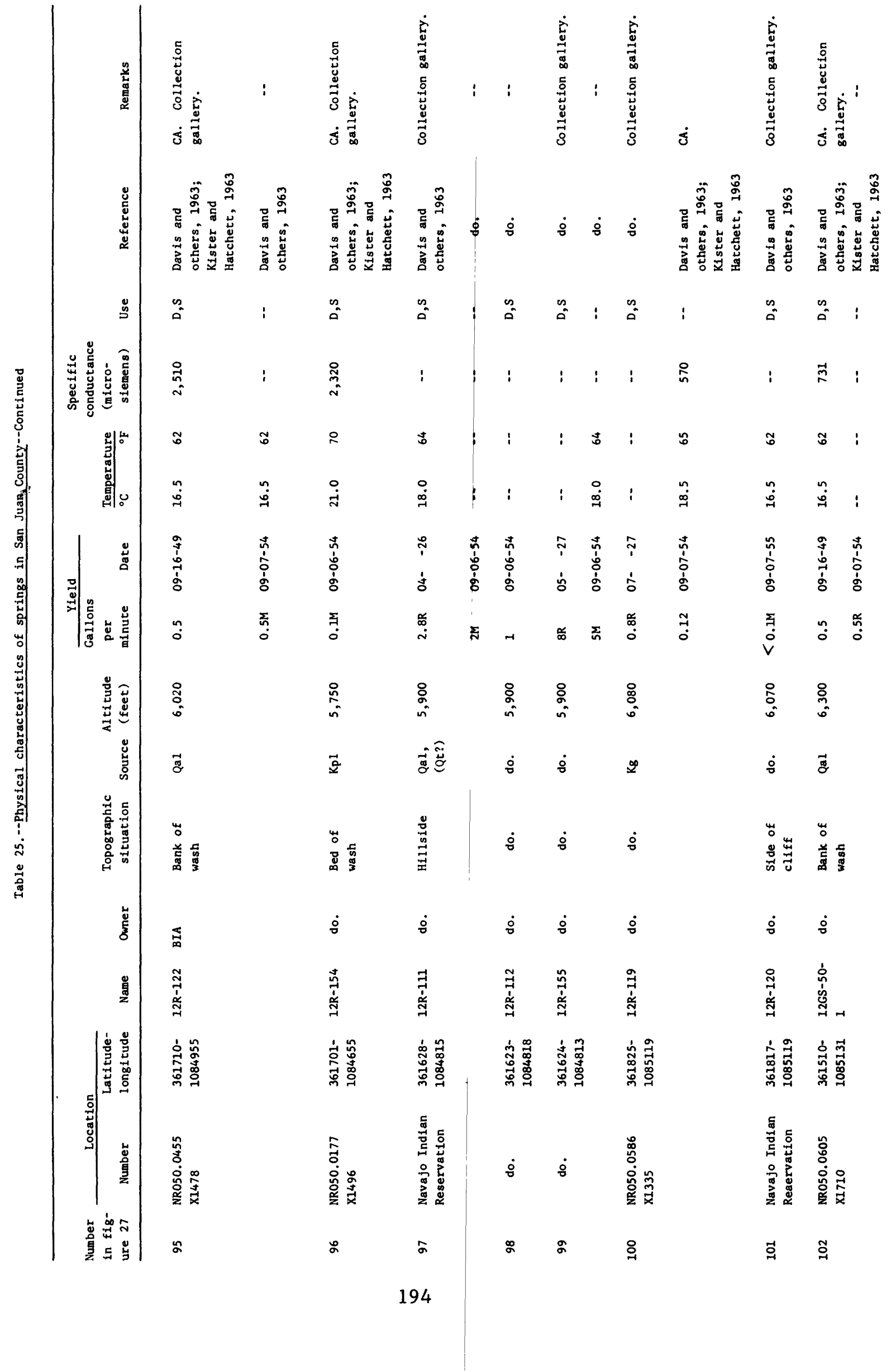




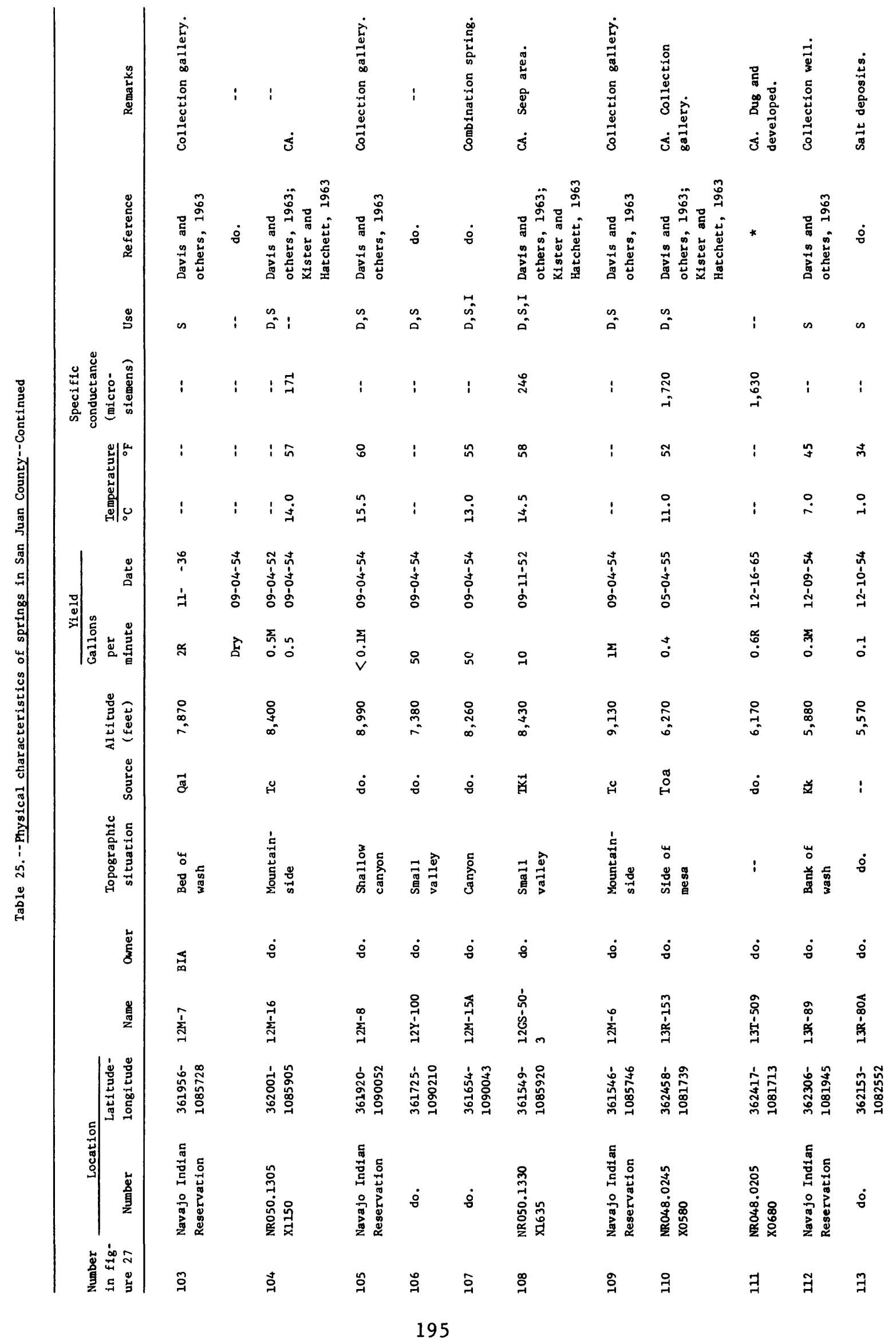




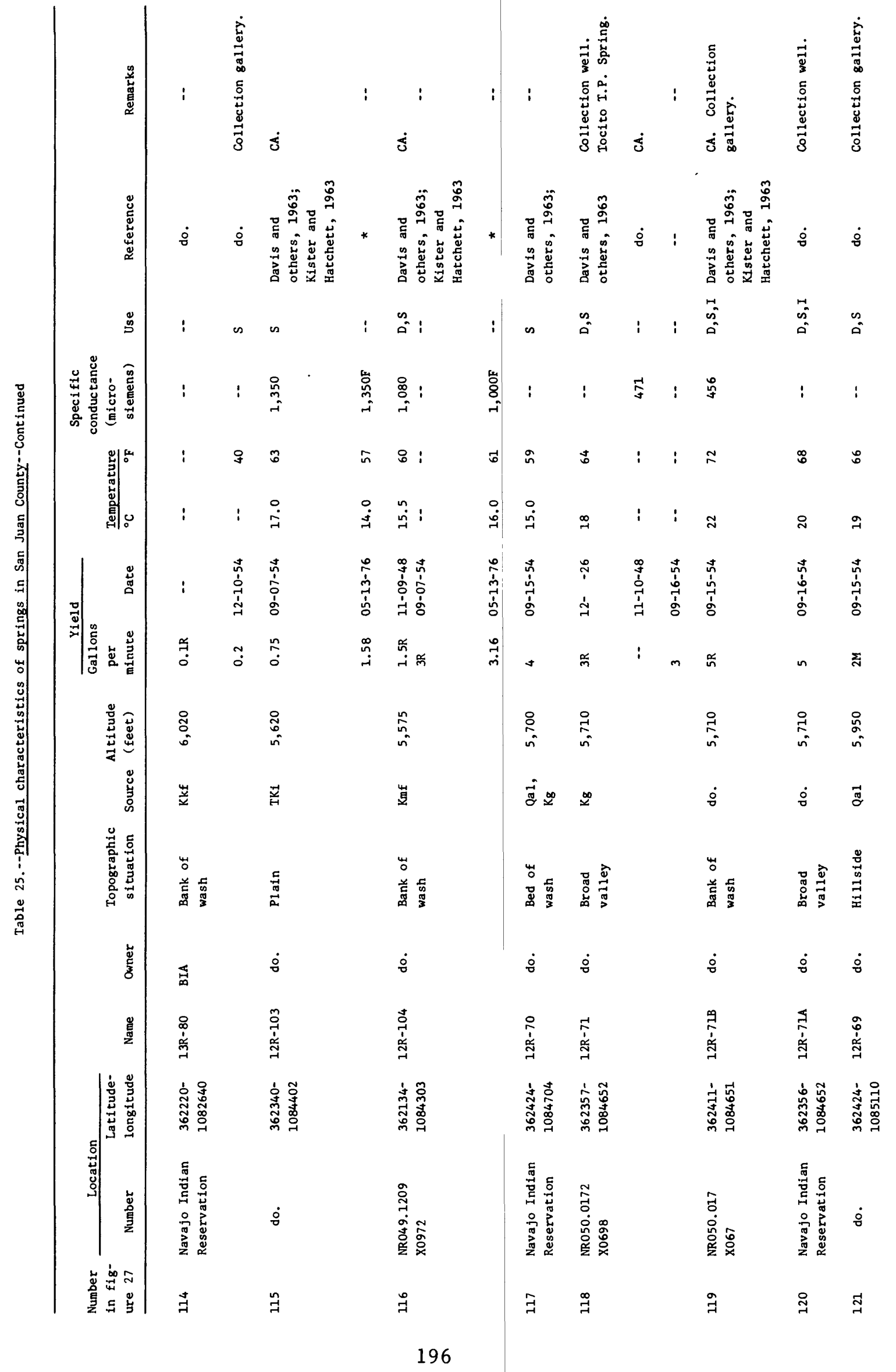




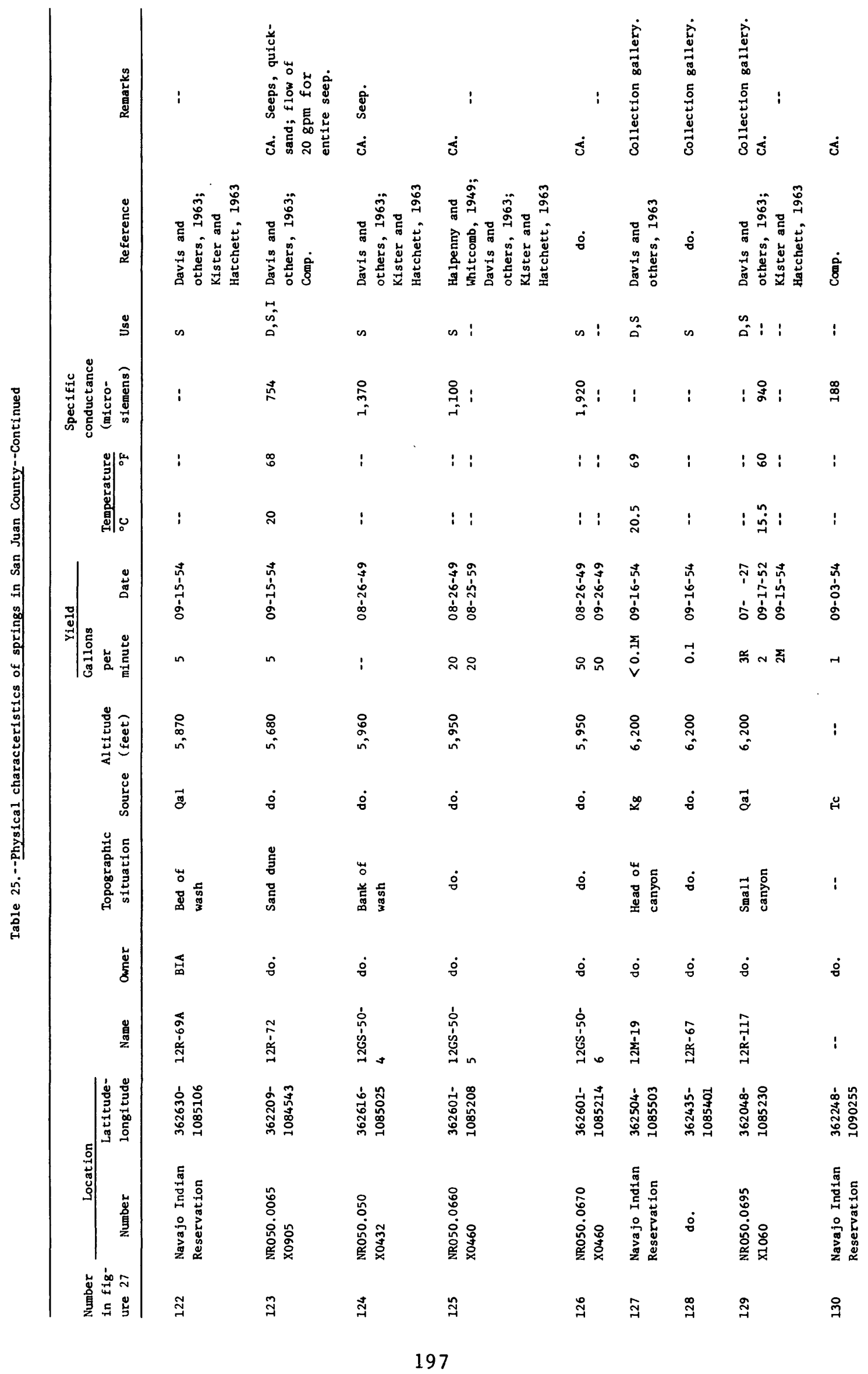




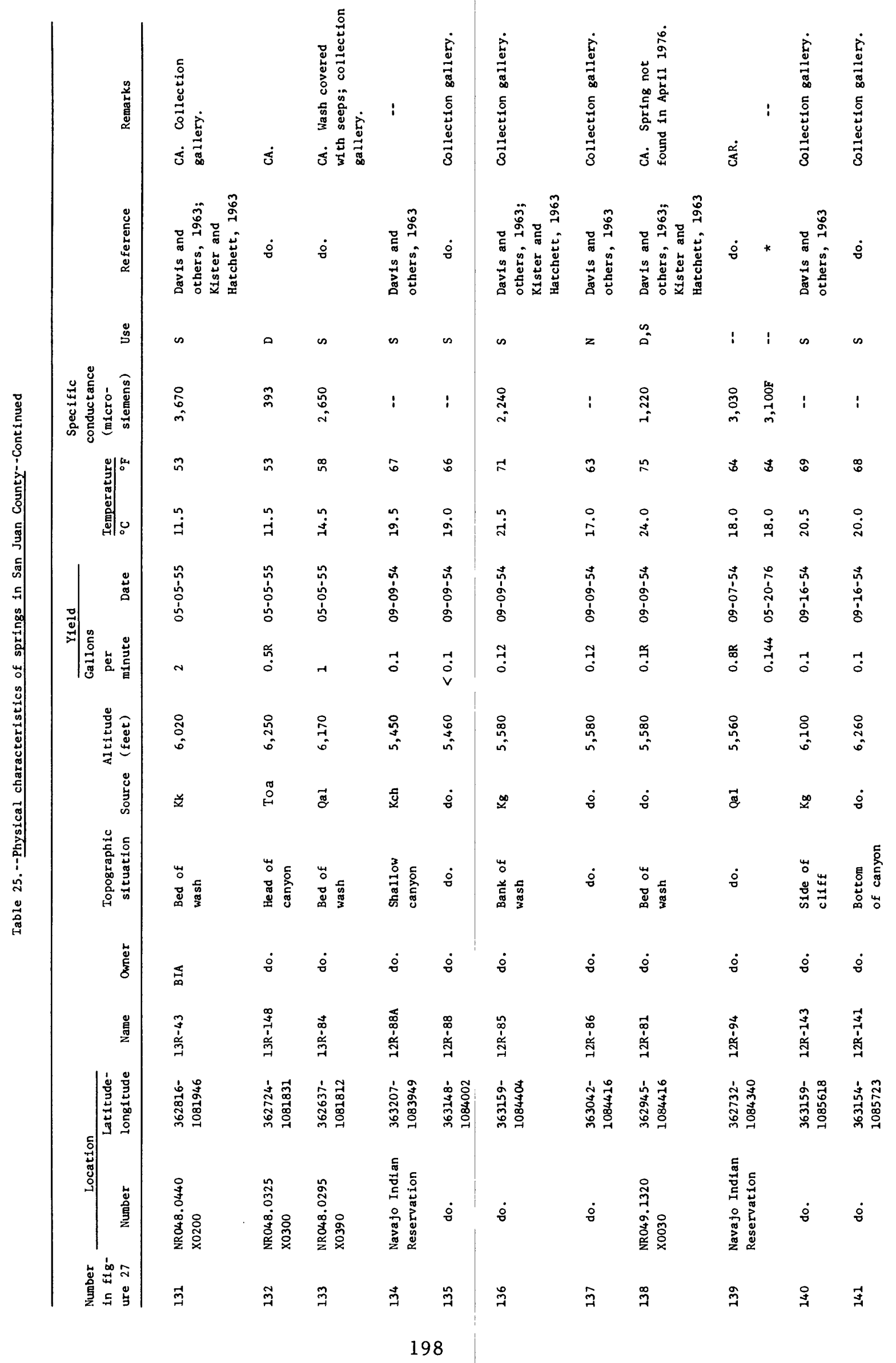




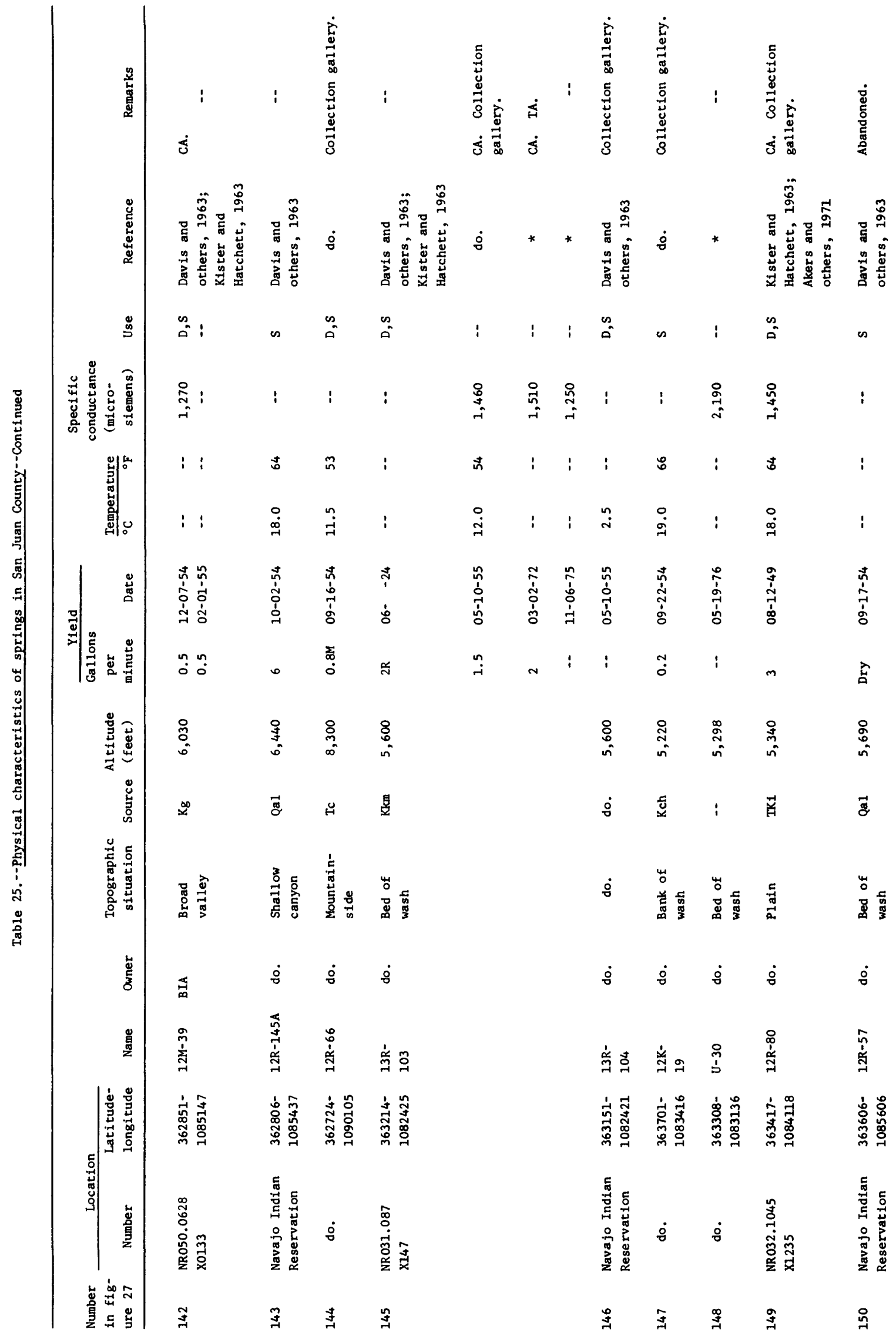




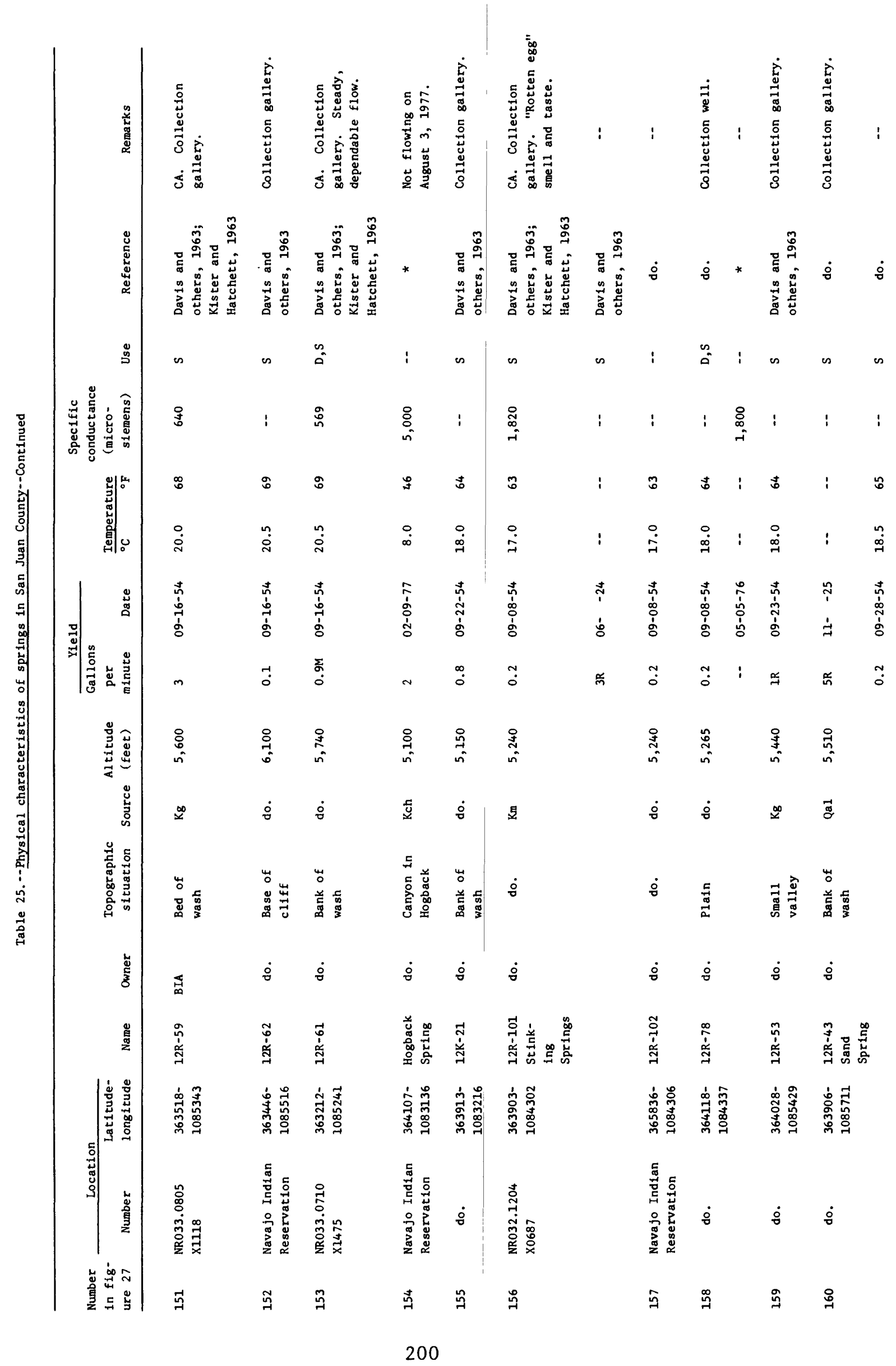




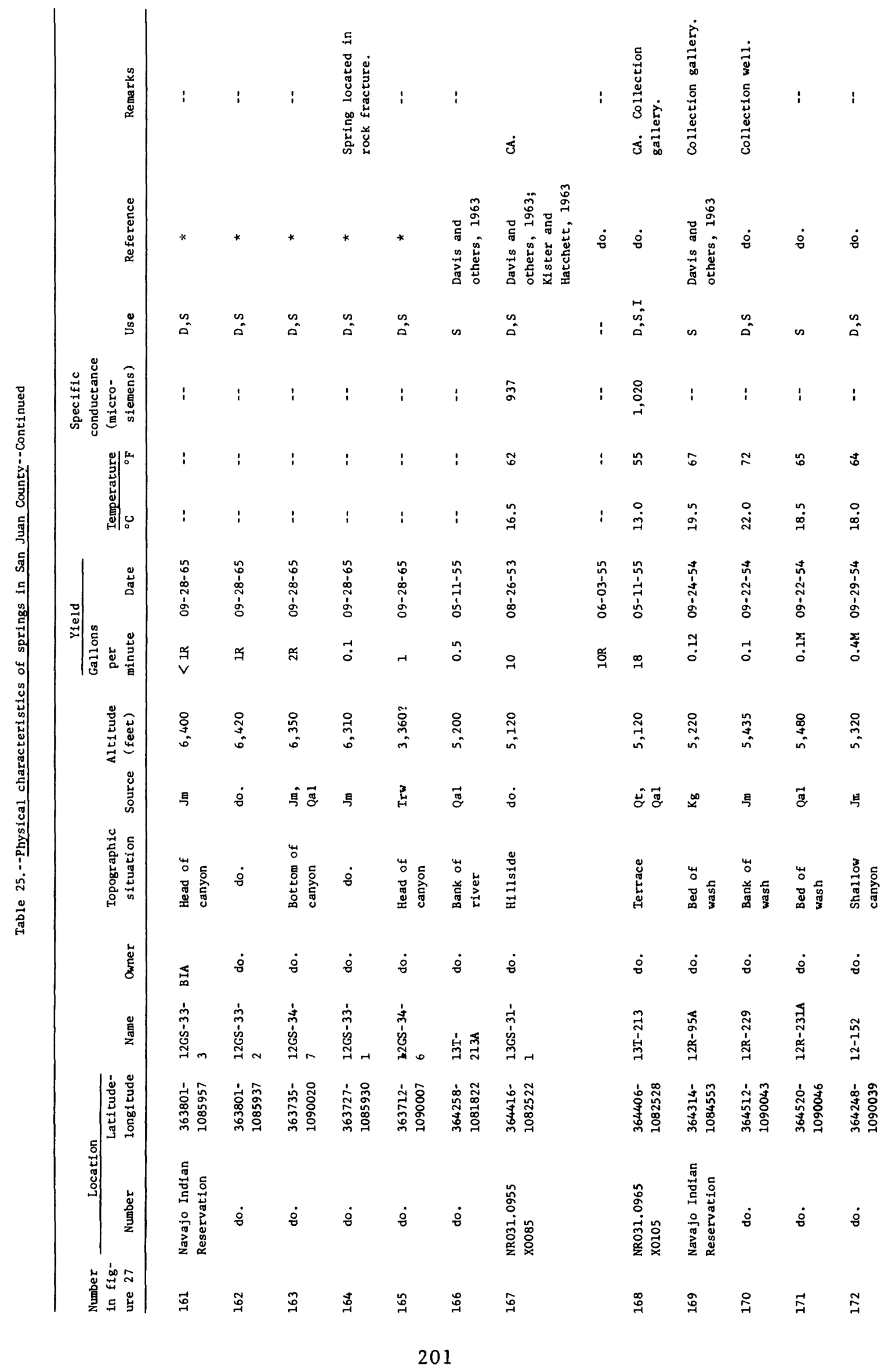




\begin{tabular}{|c|c|c|c|c|c|c|c|c|c|c|c|c|}
\hline 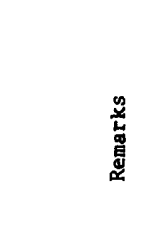 & : & : & $\begin{array}{l}\dot{\mathrm{H}} \\
\dot{8}\end{array}$ & $\begin{array}{l}\dot{घ} \\
\ddot{\emptyset} \\
\ddot{\emptyset}\end{array}$ & $\dot{\jmath}$ & ذ่̇ & 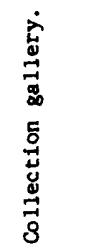 & 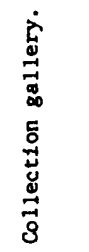 & $i$ & $i$ & $\dot{\delta}$ & : \\
\hline 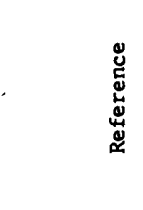 & 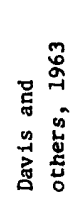 & $\dot{8}$ & * & * & 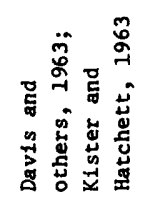 & $\dot{8}$ & 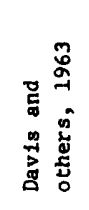 & $\dot{8}$ & $\dot{8}$ & $\dot{8}$ & 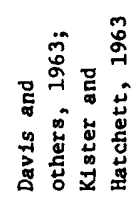 & 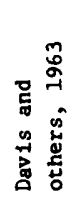 \\
\hline$\stackrel{\Delta}{\Delta}$ & $\stackrel{\infty}{0}$ & is & $:$ & $:$ & $\infty$ & 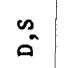 & $\stackrel{s}{a}$ & $D$ & $\stackrel{\infty}{a}$ & $\begin{array}{l}n \\
0 \\
0\end{array}$ & $\stackrel{n}{a}$ & is \\
\hline 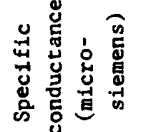 & $:$ & $i$ & 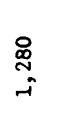 & $\begin{array}{l}8 \\
0 \\
7\end{array}$ & 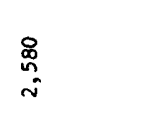 & $\stackrel{\infty}{\infty}$ & $i$ & $:$ & $i$ & ! & 必 & : \\
\hline 氙| & : & $\tilde{0}$ & 3 & in & $\Phi$ & $\stackrel{\circ}{\circ}$ & ธ & 8 & ธิ & $\tilde{\sigma}$ & $\tilde{6}$ & $\tilde{6}$ \\
\hline 。 & ْ̊̀ & $\begin{array}{l}\text { n } \\
\infty \\
\end{array}$ & $\stackrel{n}{7}$ & ஜீ & : & $\stackrel{\circ}{9}$ & 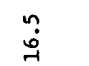 & ֻே & $\stackrel{n}{0}$ & $\stackrel{n}{n}$ & $\stackrel{0}{\stackrel{\leftrightarrow}{H}}$ & $\stackrel{n}{\infty}$ \\
\hline 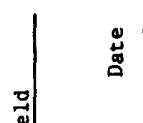 & 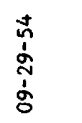 & $\begin{array}{l}\text { प̆t } \\
\text { ஸे } \\
\text { ó }\end{array}$ & 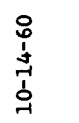 & $\begin{array}{l}\stackrel{\circ}{0} \\
\dot{m} \\
\dot{\sigma}\end{array}$ & 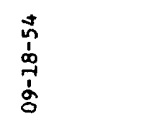 & 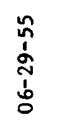 & 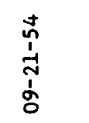 & 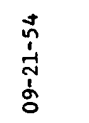 & $\begin{array}{l}\stackrel{t}{0} \\
\stackrel{A}{1} \\
\dot{o}\end{array}$ & 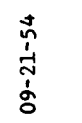 & 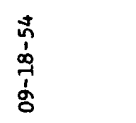 & 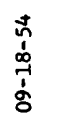 \\
\hline 训㤩 & 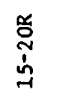 & in & $\stackrel{一}{\circ}$ & $i$ & $\ddot{0}$ & $\stackrel{\infty}{\circ}$ & $\tilde{T}$ & $\stackrel{t}{0}$ & $\stackrel{\mathscr{N}}{*}$ & $\stackrel{n}{0}$ & స̆. & $\stackrel{\text { ? }}{0}$ \\
\hline 总焉 & 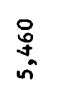 & 兽 & $\begin{array}{l}n \\
\infty \\
\infty \\
j \\
j\end{array}$ & $\stackrel{n}{\alpha}$ & $\begin{array}{l}9 \\
0 \\
\text { n }\end{array}$ & $\begin{array}{c}\stackrel{8}{0} \\
m \\
n\end{array}$ & $\begin{array}{c}0_{0} \\
\text { n. }\end{array}$ & 卓 & $\begin{array}{c}\stackrel{8}{\infty} \\
n_{n}^{n} \\
n^{-1}\end{array}$ & $\begin{array}{c}9 \\
\vdots \\
0 \\
\text { in }\end{array}$ & 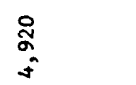 & i̊ \\
\hline 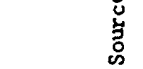 & $\overrightarrow{\mathrm{g}}$ & $\dot{8}$ & 豆 & $\dot{8}$ & $\ddot{\sharp}$ & $\underset{\mathrm{H}}{\mathfrak{Z}}$ & 马 & $\dot{8}$ & 蛋 & $\vec{\Phi}$ & 且 & $\bar{\Psi}$ \\
\hline 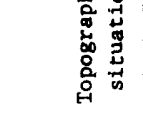 & 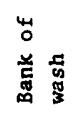 & 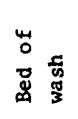 & ! & i & 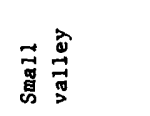 & $\dot{8}$ & 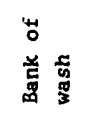 & $\dot{8}$ & 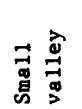 & 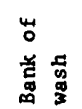 & 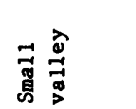 & $\dot{8}$ \\
\hline 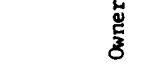 & 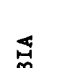 & $\dot{8}$ & $\dot{0}$ & $\dot{8}$ & $\dot{8}$ & : & $\dot{8}$ & $\dot{8}$ & $\dot{8}$ & $\dot{\delta}$ & $\dot{8}$ & $\dot{\circ}$ \\
\hline$\frac{\mathrm{g}}{\mathrm{g}}$ & 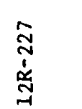 & 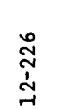 & : & $i$ & 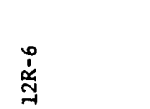 & 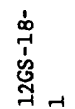 & 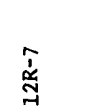 & 产 & 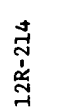 & $\begin{array}{l}\stackrel{\Im}{1} \\
\stackrel{\leftrightarrow}{\pi}\end{array}$ & 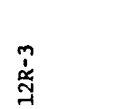 & 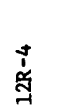 \\
\hline 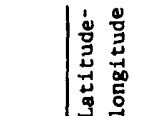 & 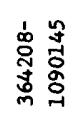 & 苛志 & 㲾忐 & 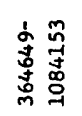 & 突 & 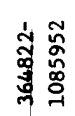 & 宅 & 容号 & 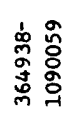 & 灾 & 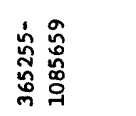 & 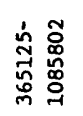 \\
\hline \begin{tabular}{l|l} 
岁 \\
息
\end{tabular} & 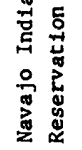 & $\dot{\circ}$ & 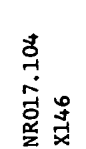 & 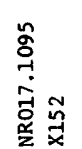 & 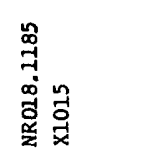 & 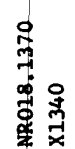 & 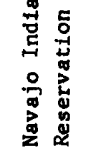 & $\dot{8}$ & $\dot{8}$ & $\dot{8}$ & 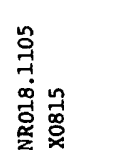 & 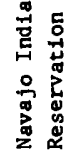 \\
\hline 尊焉焉 & $\stackrel{m}{\sigma}$ & $\stackrel{t}{7}$ & $\stackrel{\varkappa}{\beth}$ & $\stackrel{\circ}{7}$ & $\hat{A}$ & $\underset{\pi}{\infty}$ & $\stackrel{\Omega}{ન}$ & $\underset{\sim}{\stackrel{\Xi}{~}}$ & $\underset{\sim}{-\infty}$ & $\underset{\Im}{\widetilde{J}}$ & 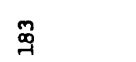 & 㞼 \\
\hline
\end{tabular}




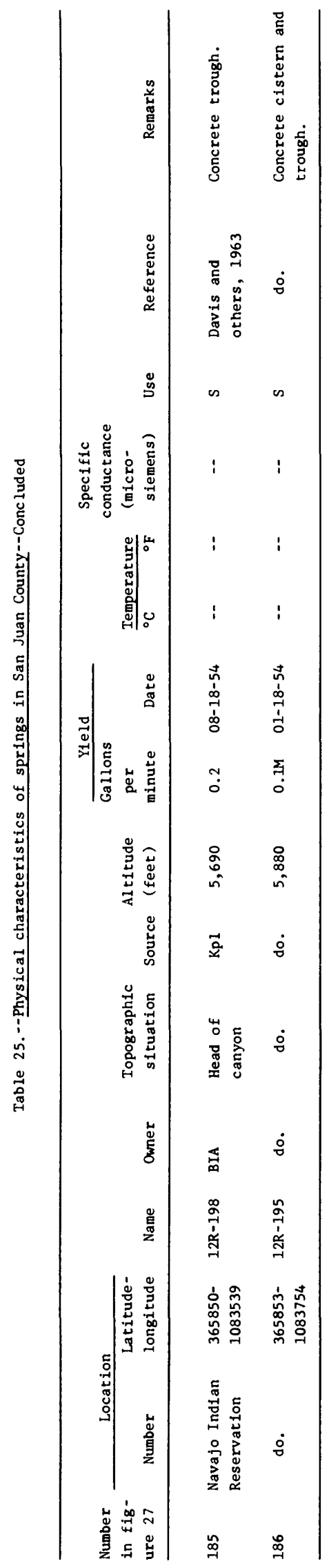



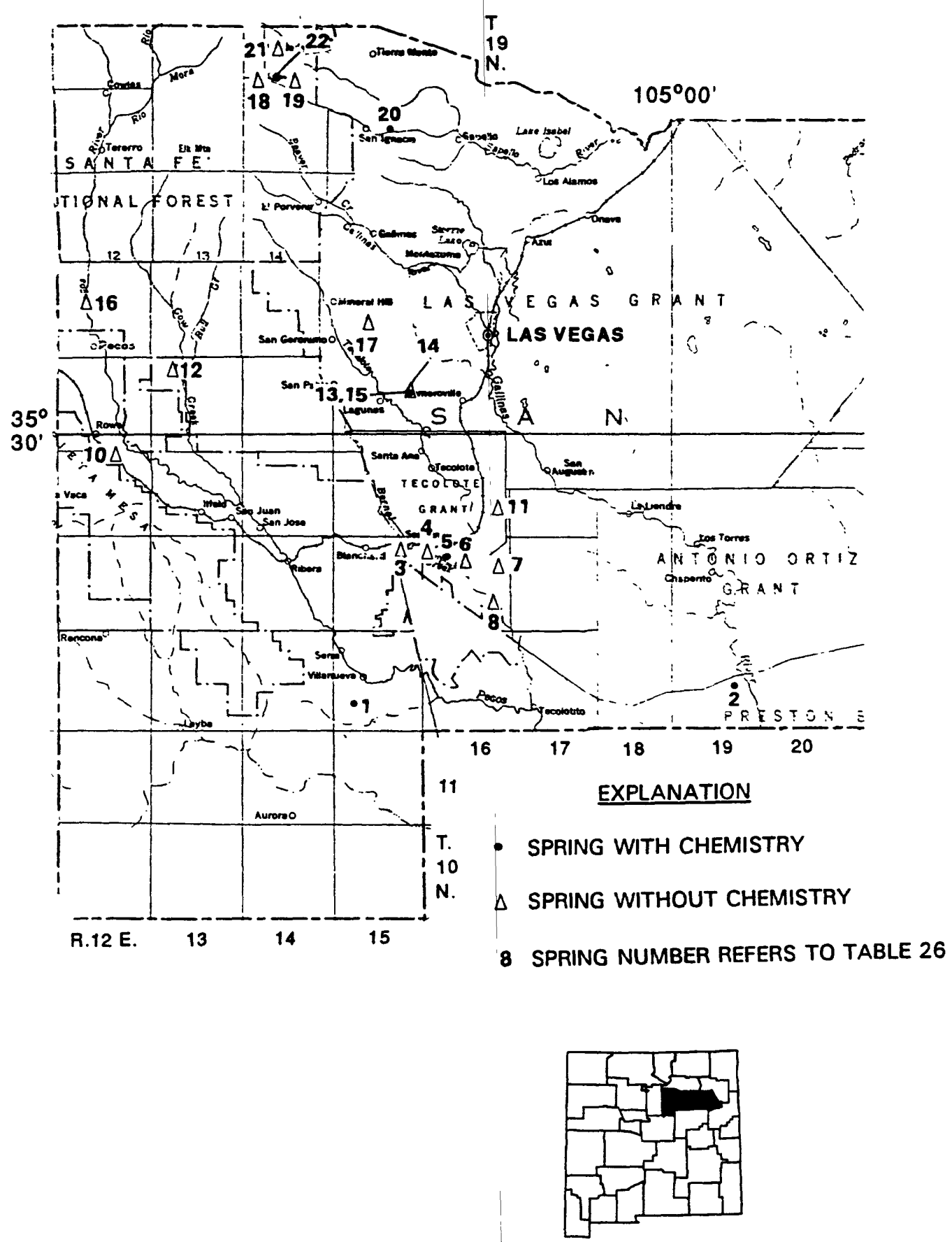


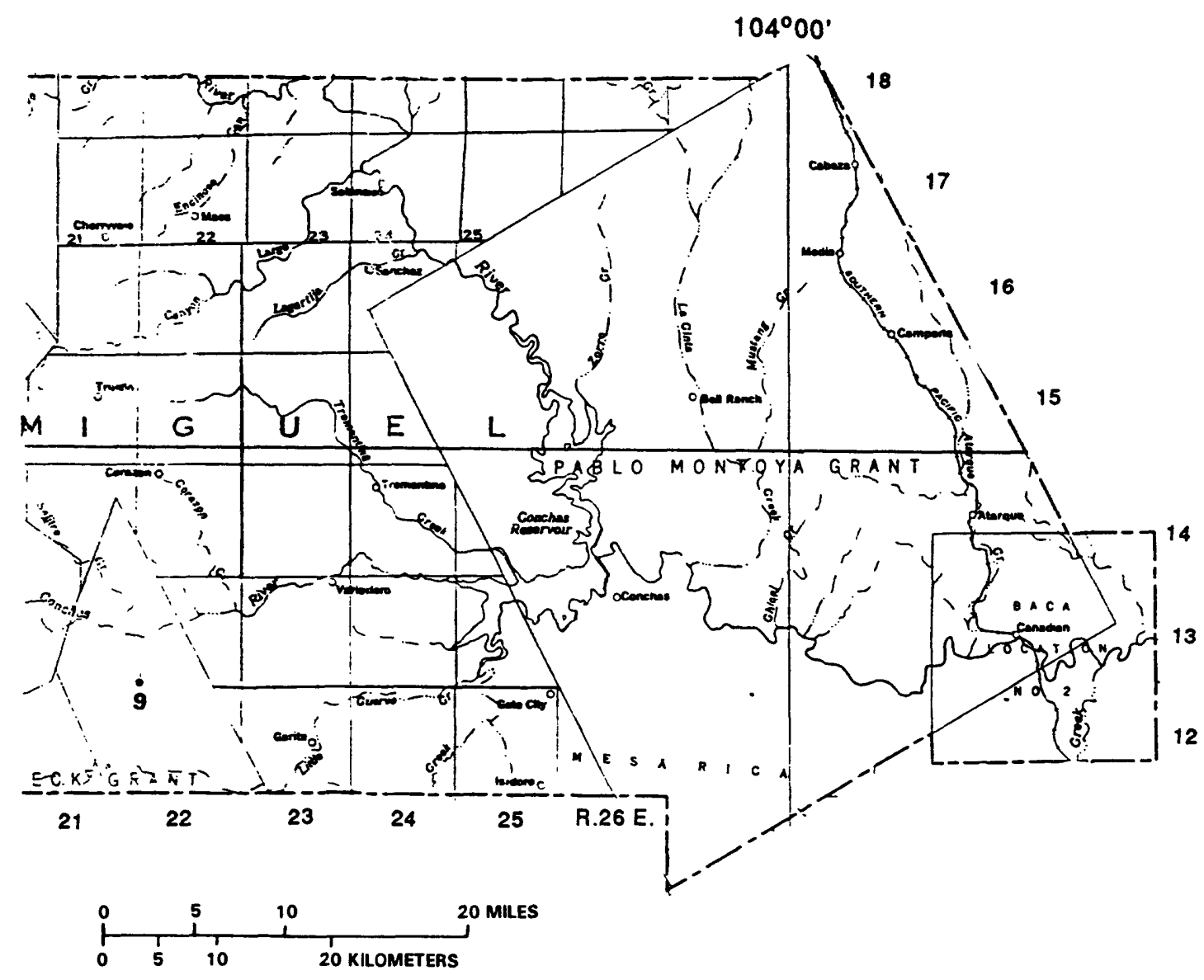

Figure 28.--Location of inventoried springs in San Miguel County. 


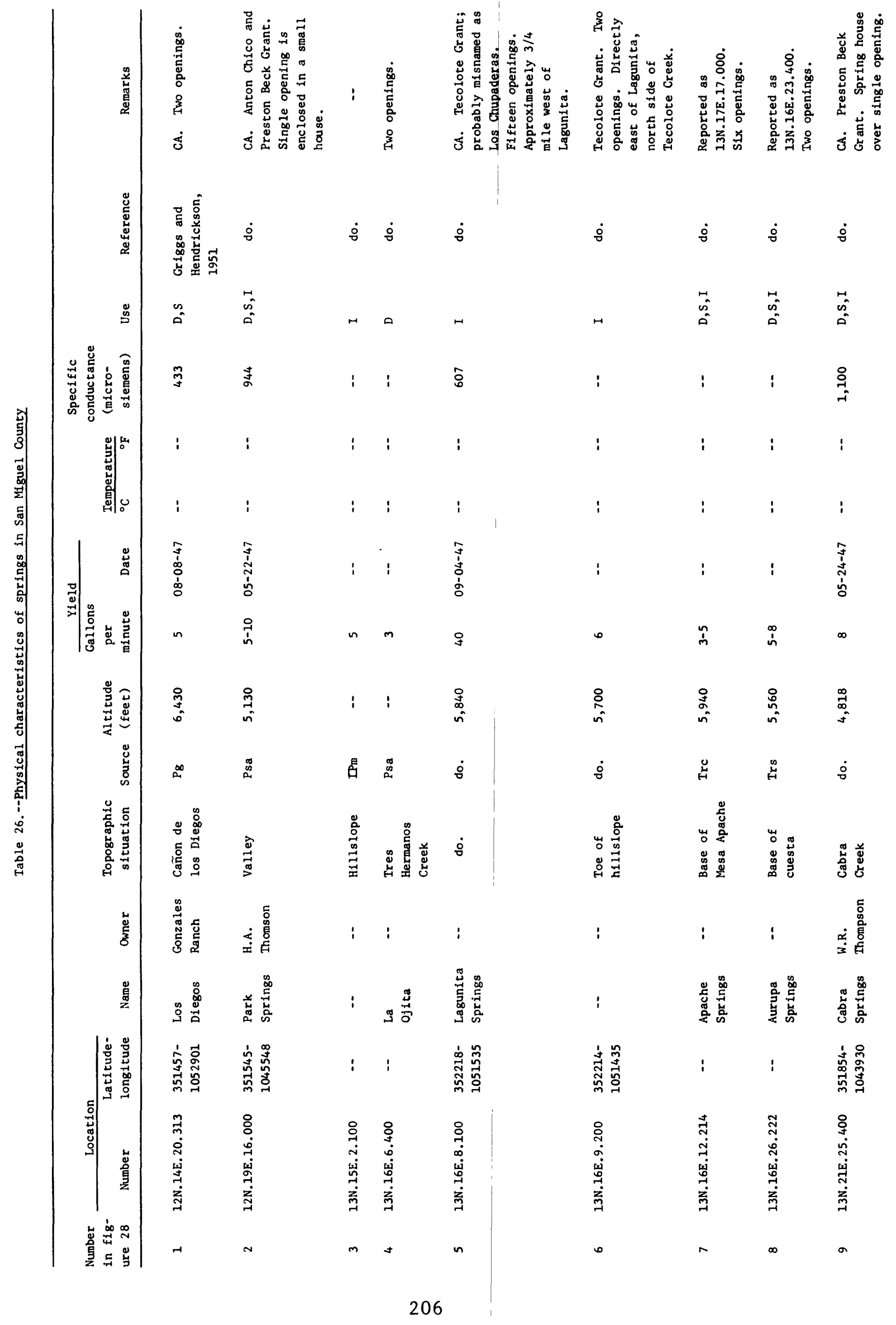




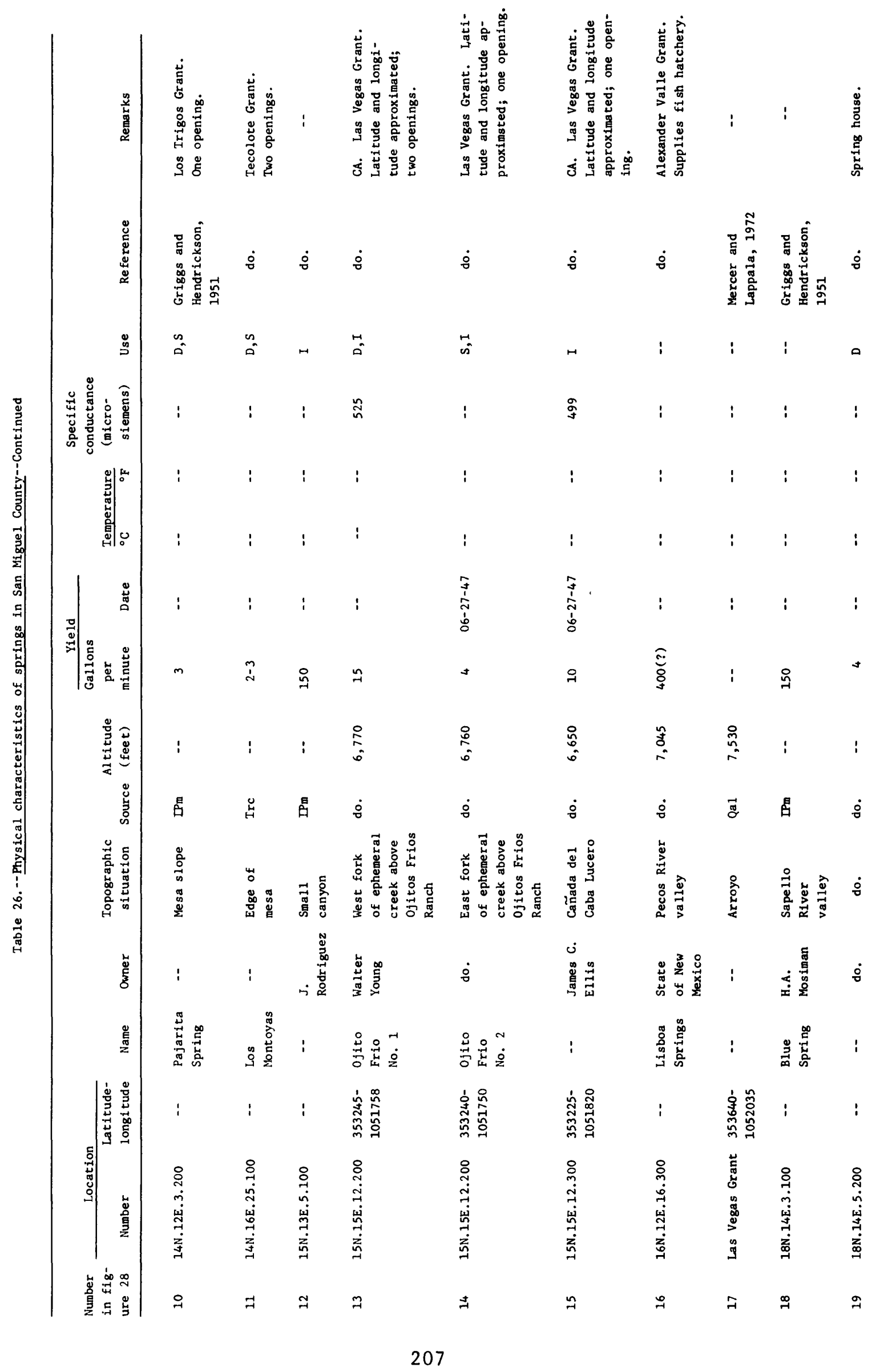




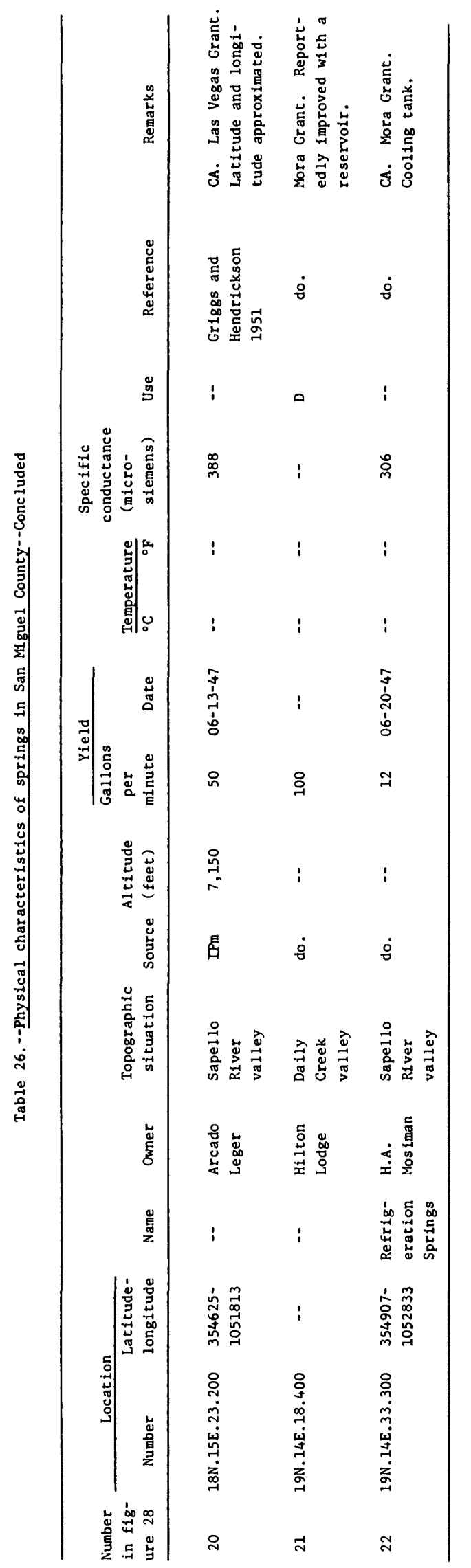



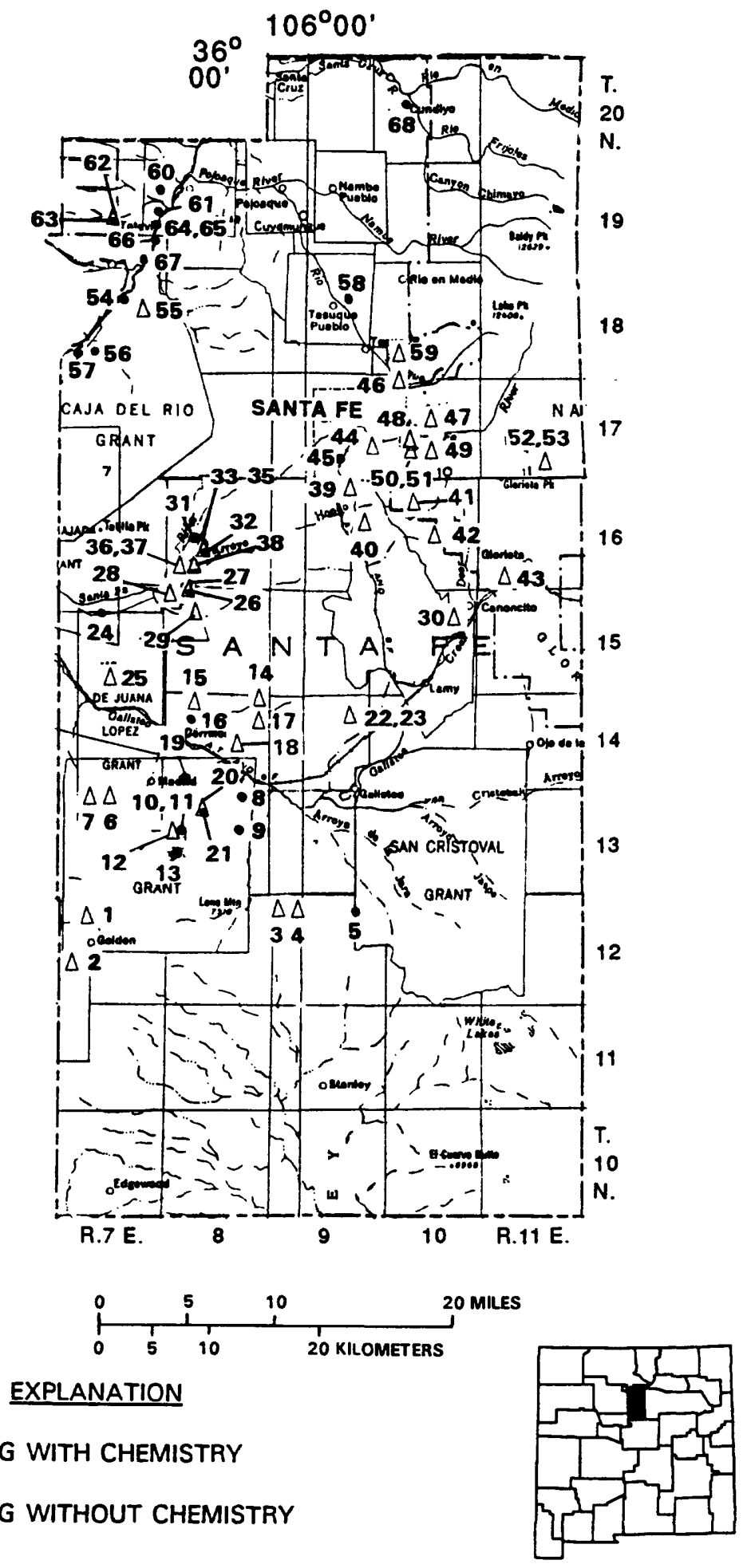

5 SPRING NUMBER REFERS TO TABLE 27

Figure 29.--Location of inventoried springs in Santa Fe County. 


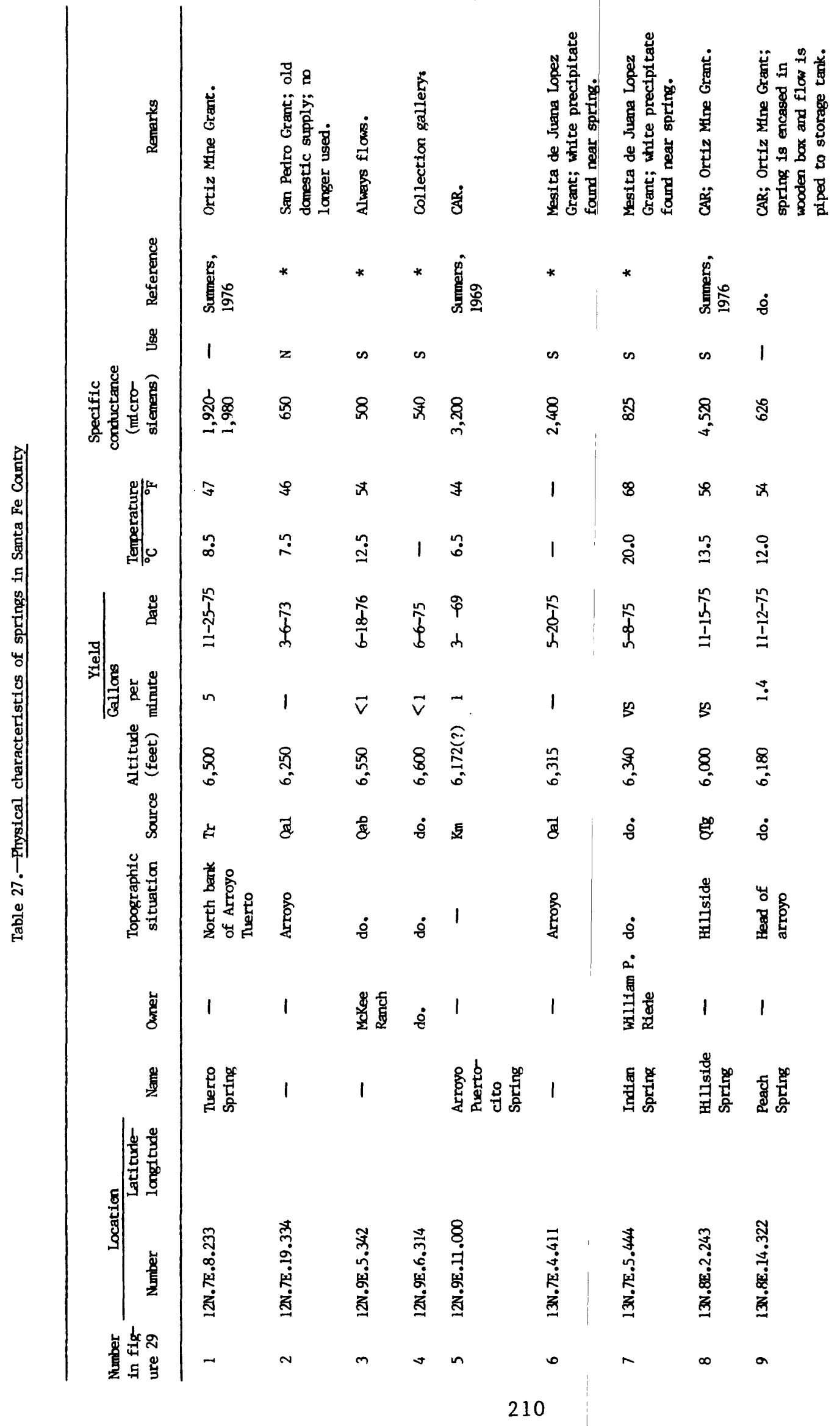




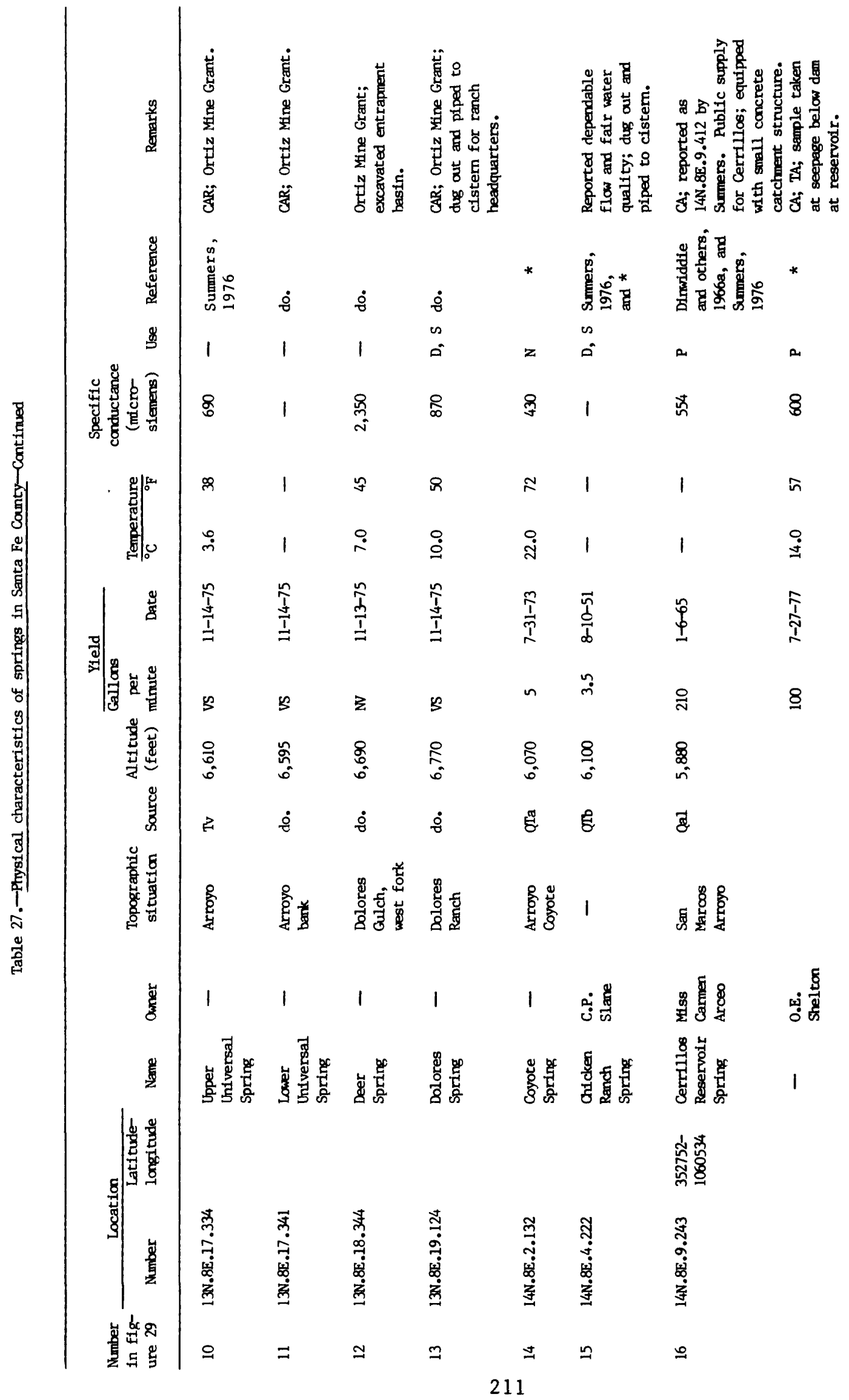




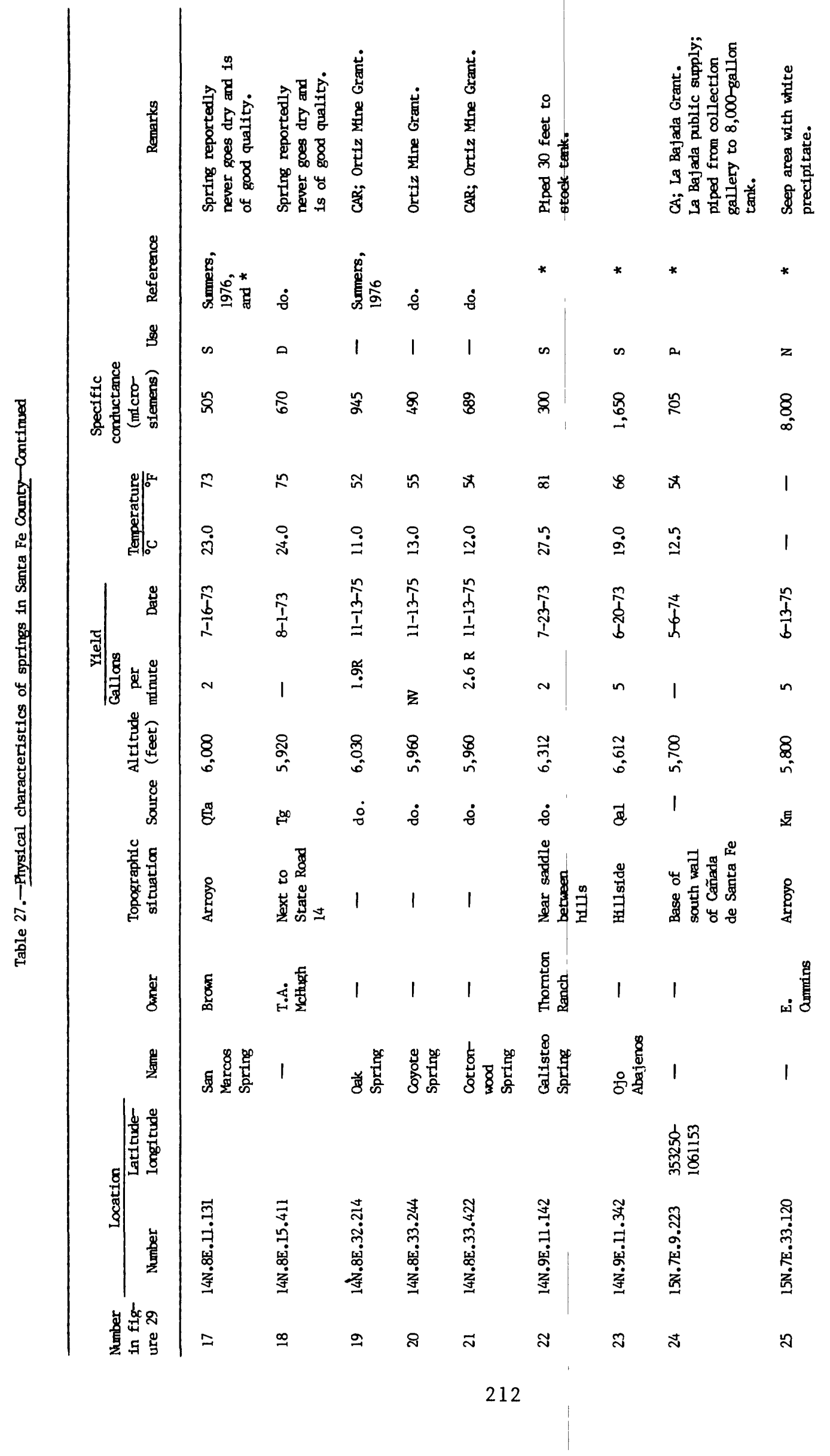




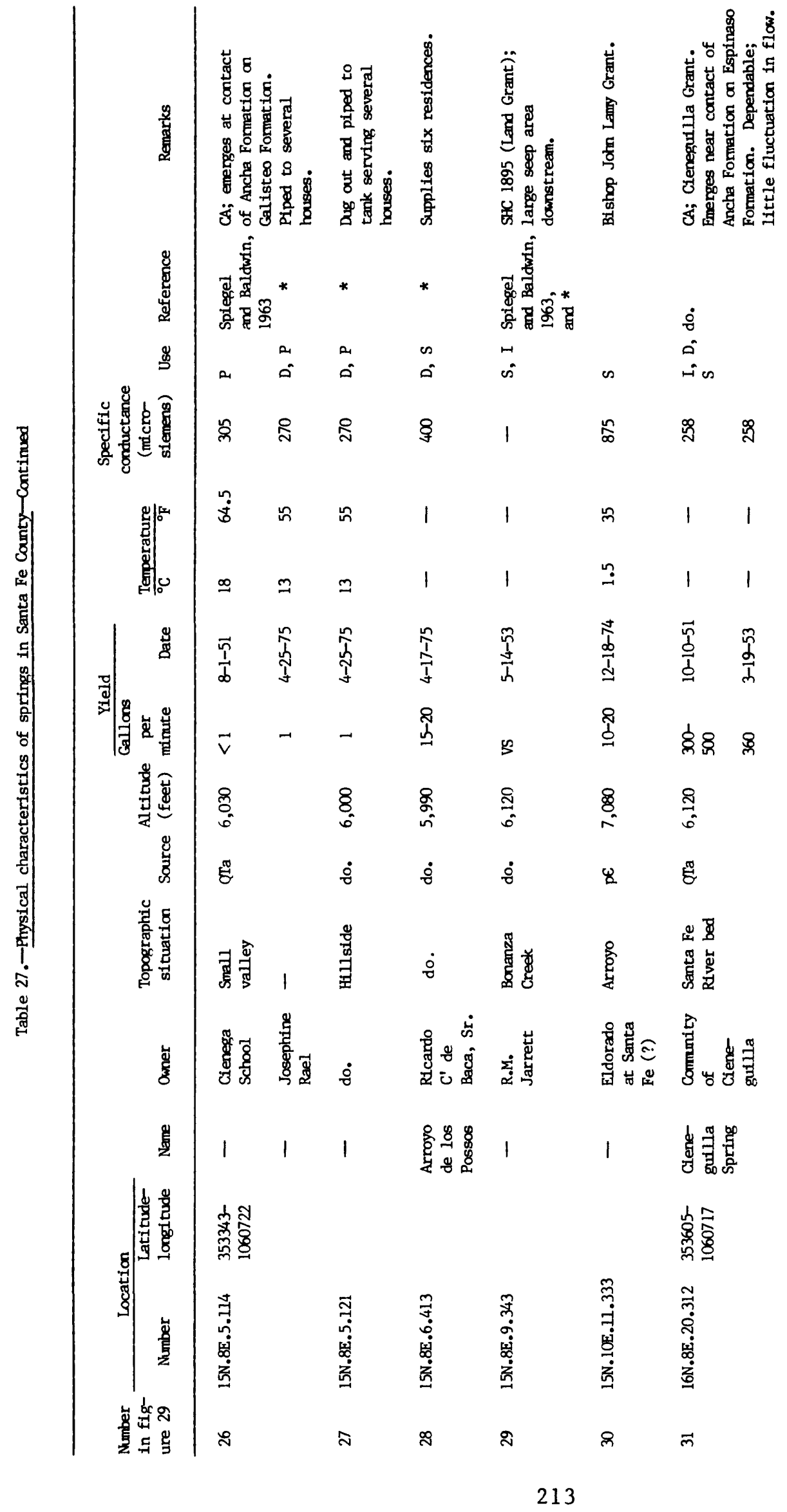




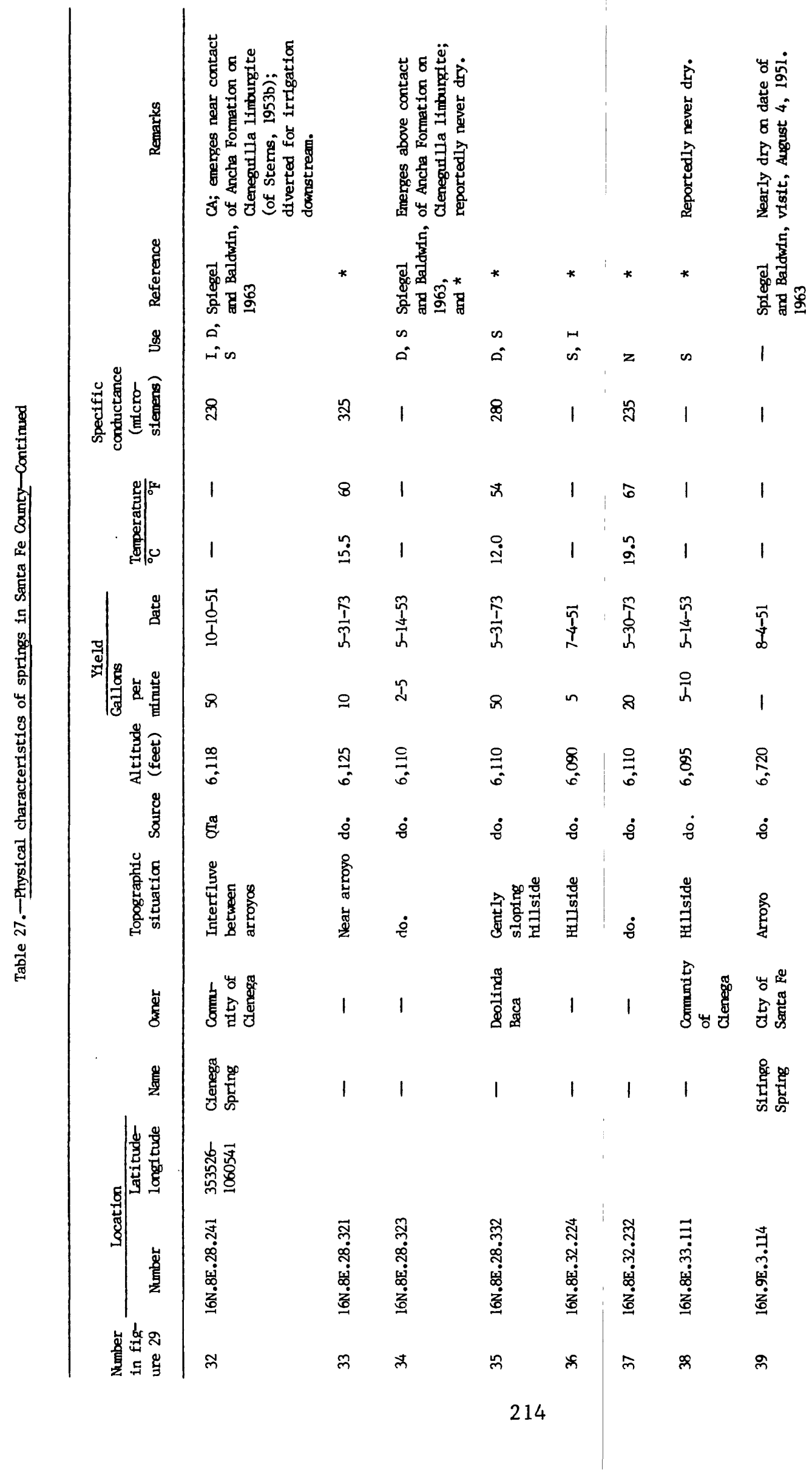




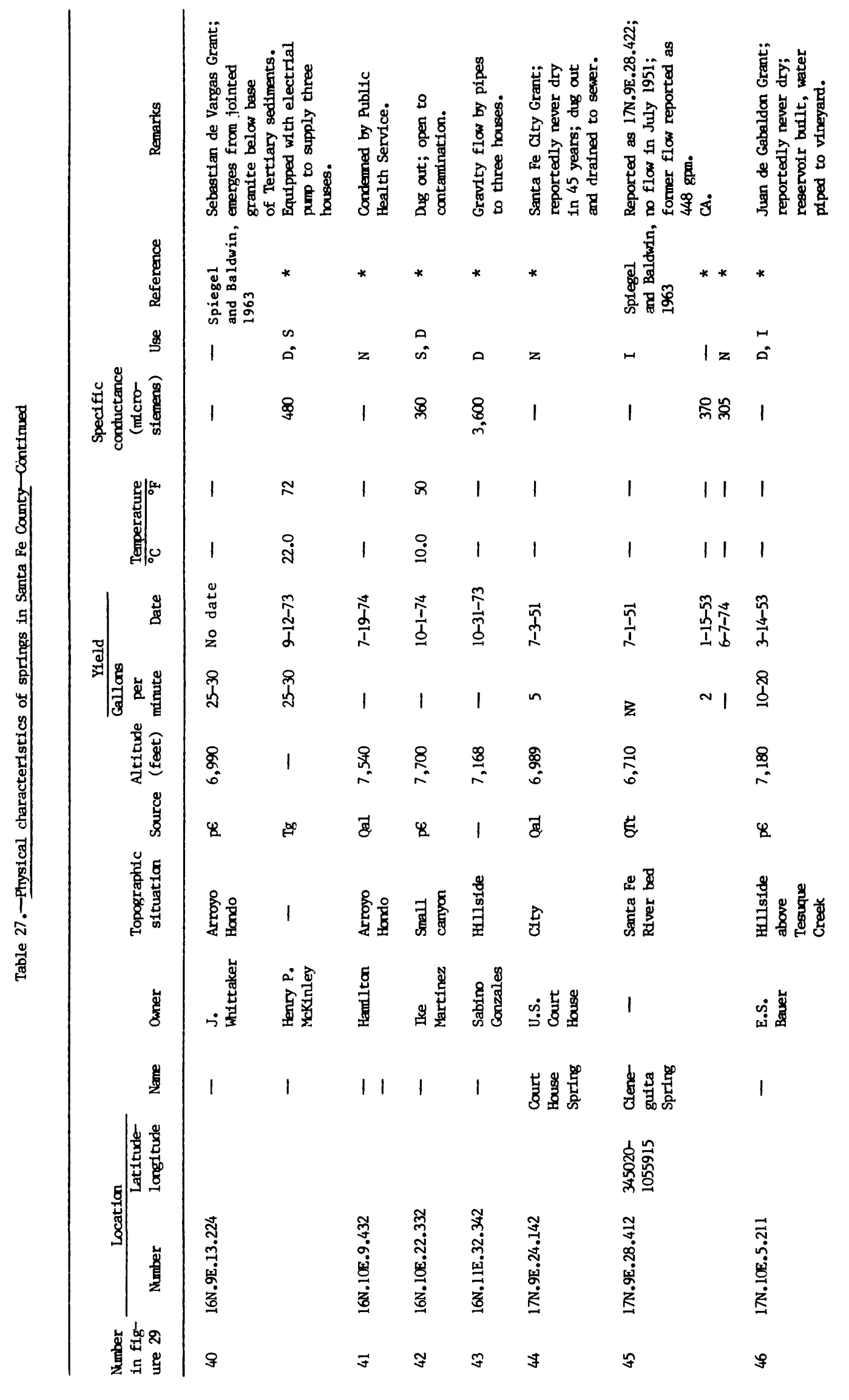




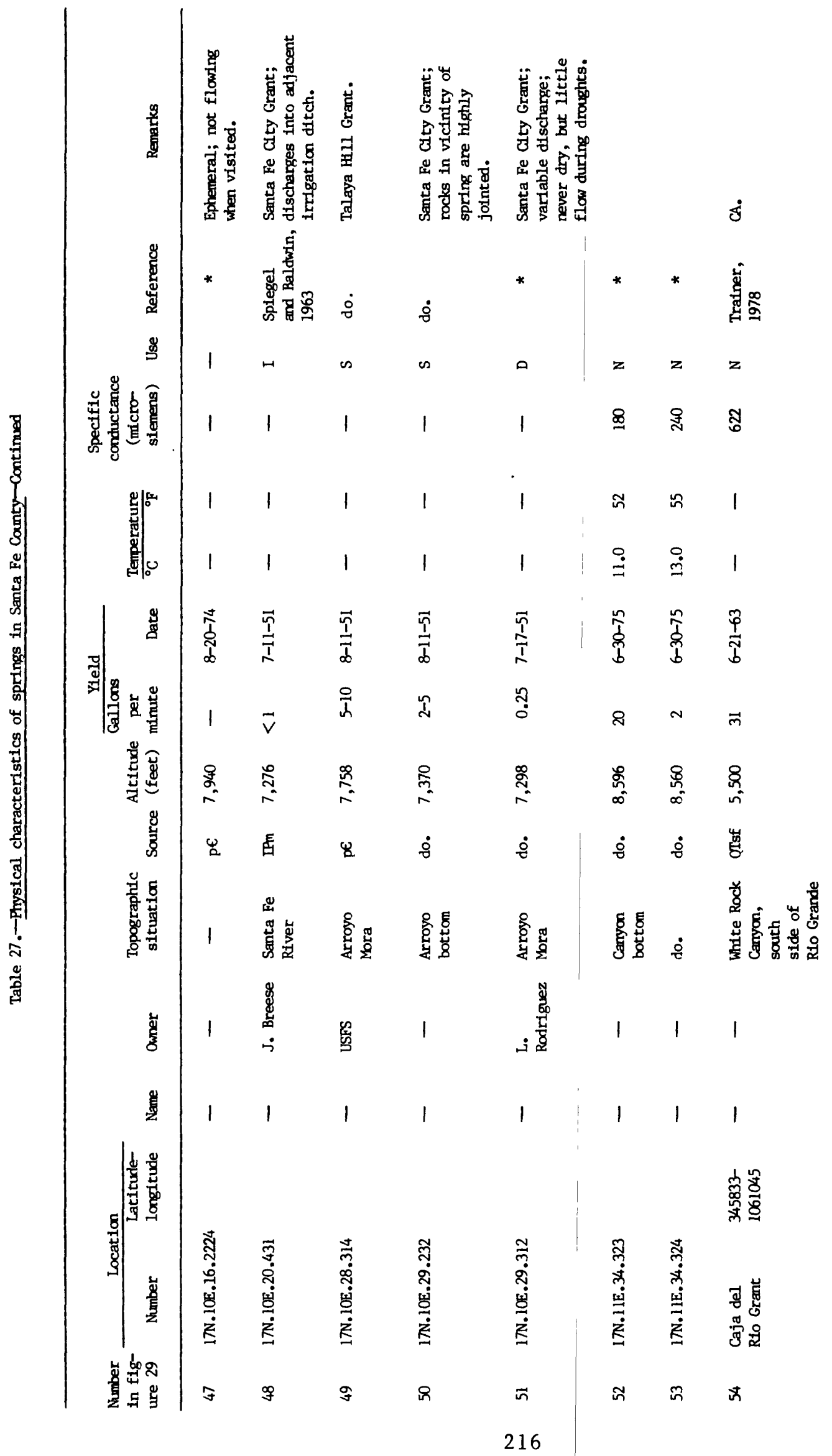




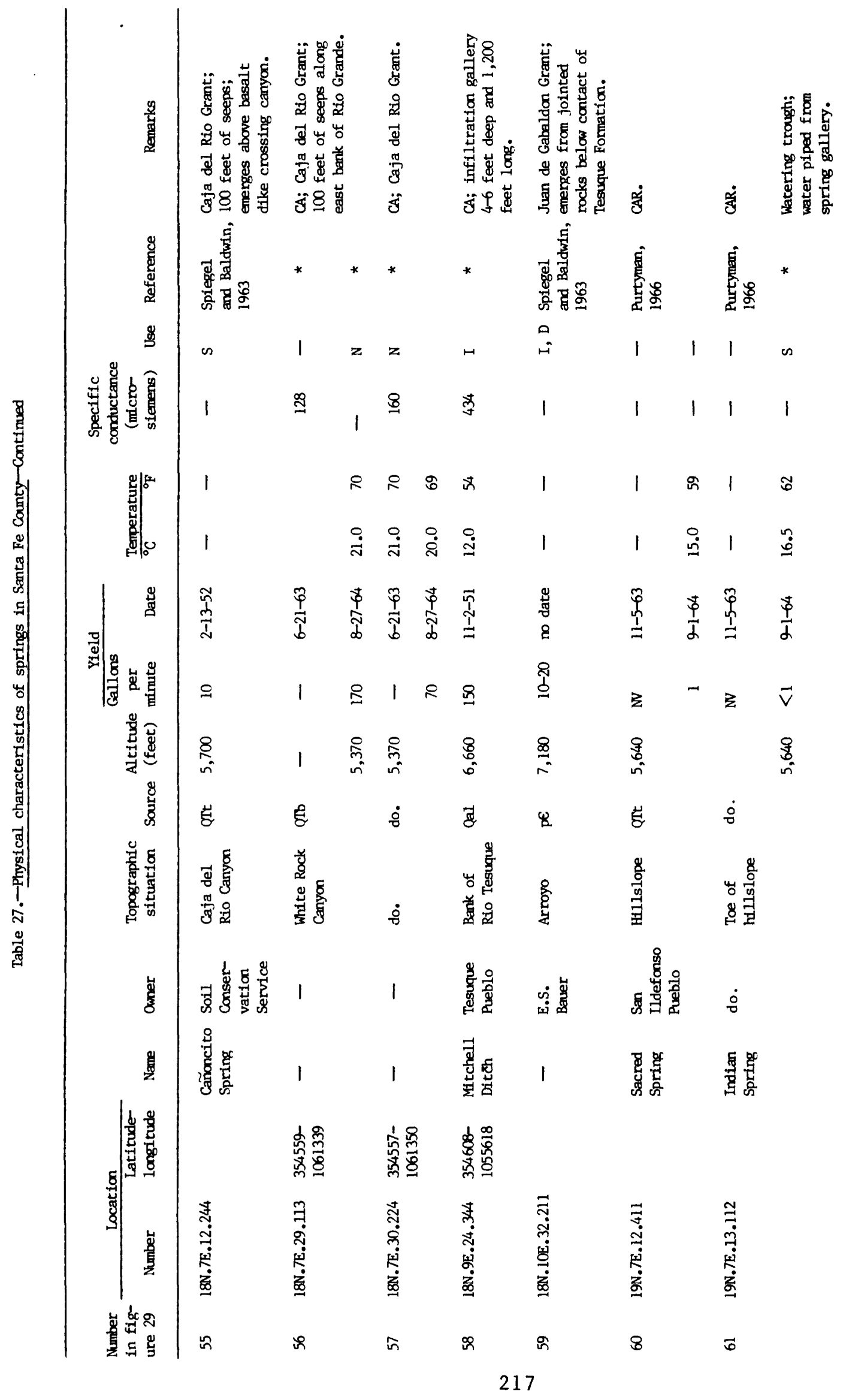




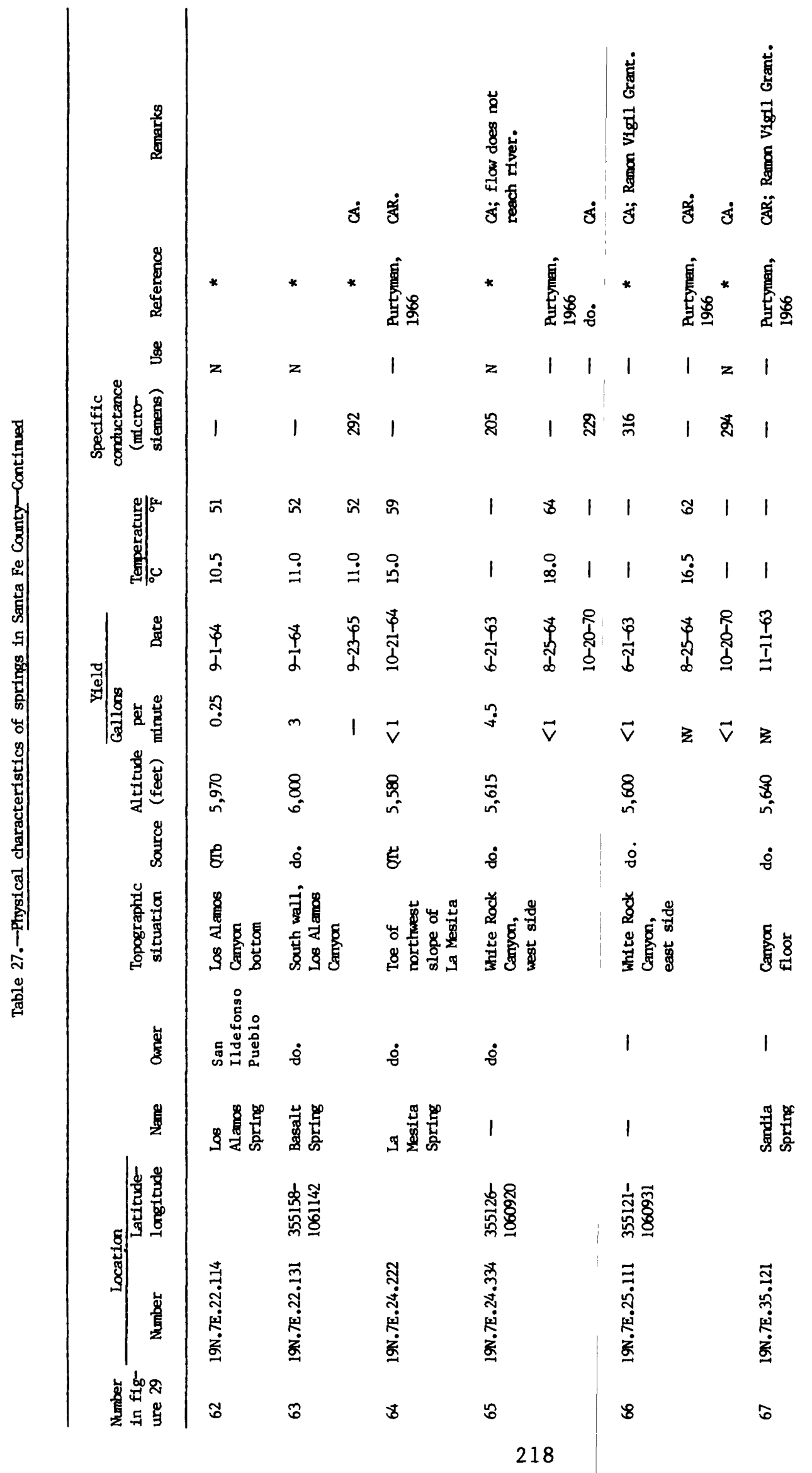




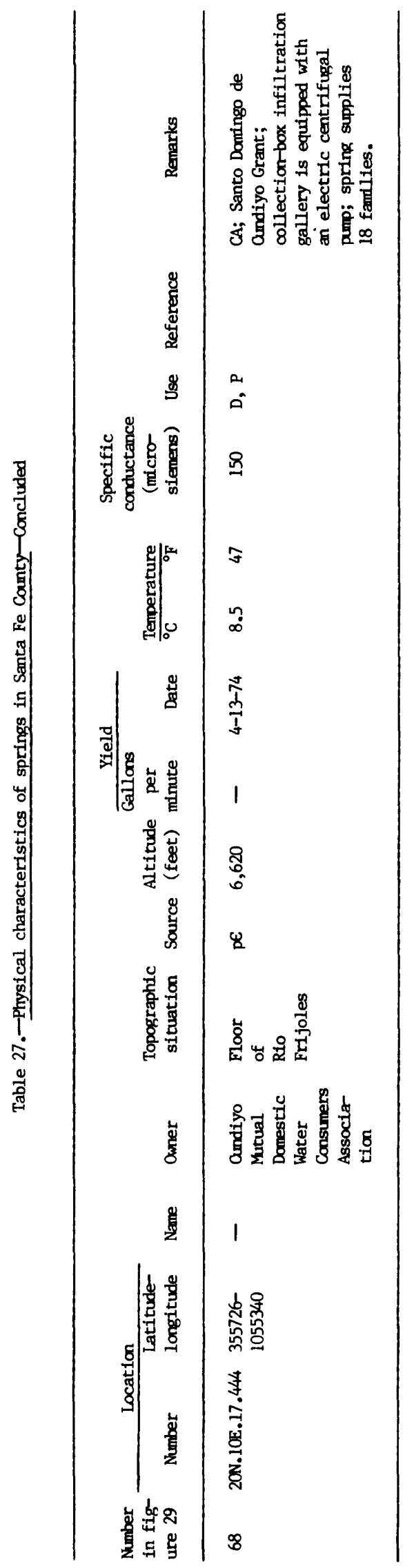




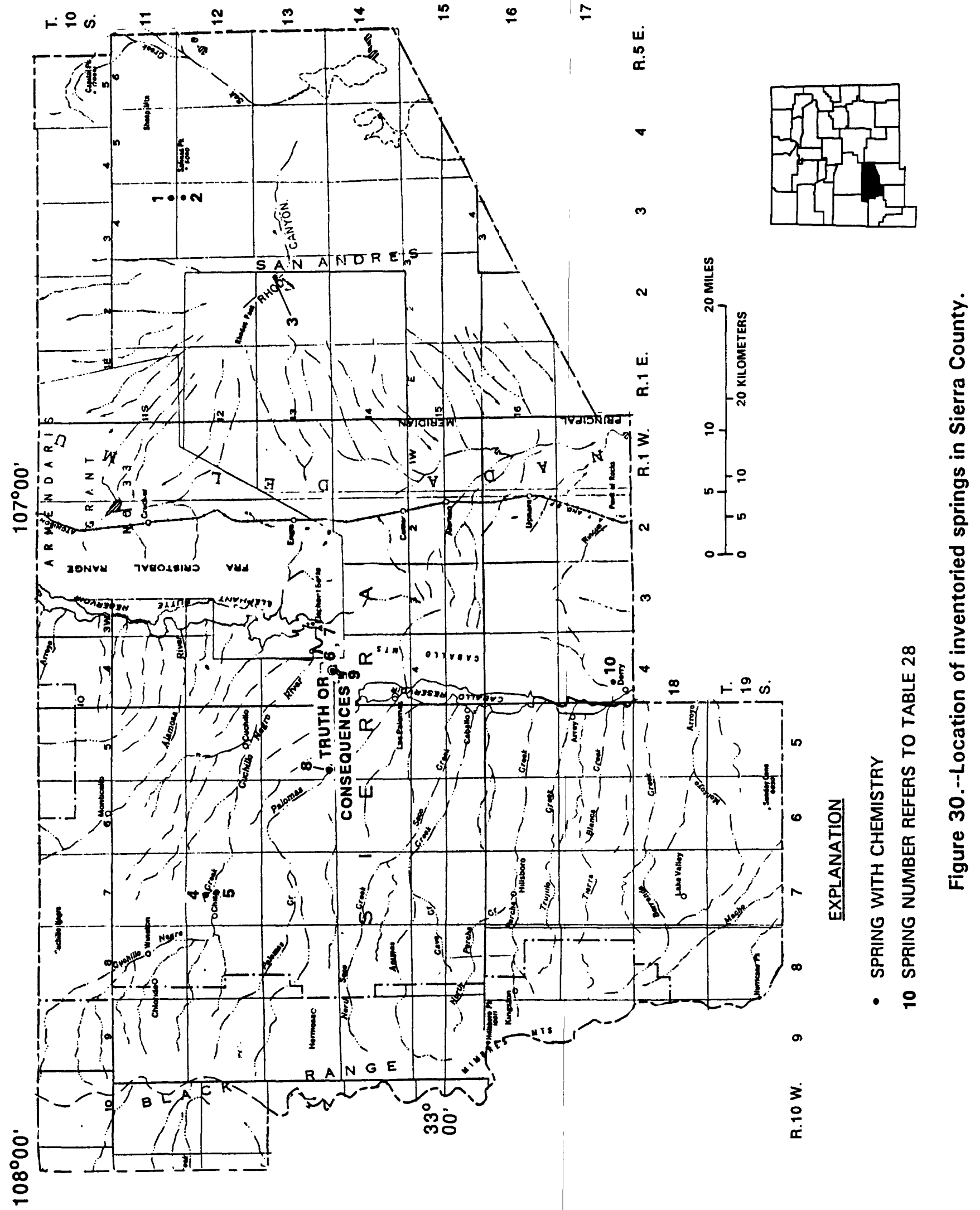




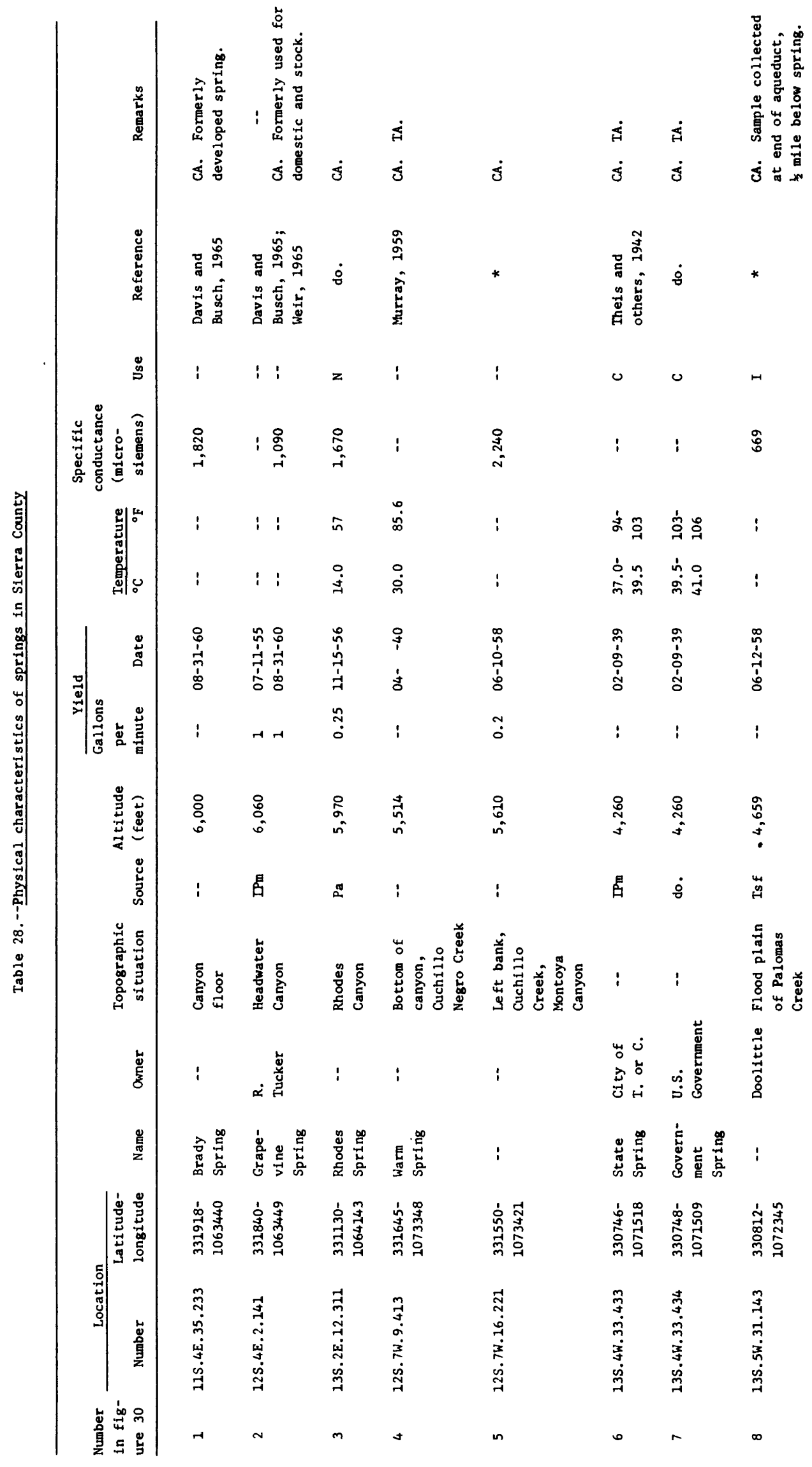




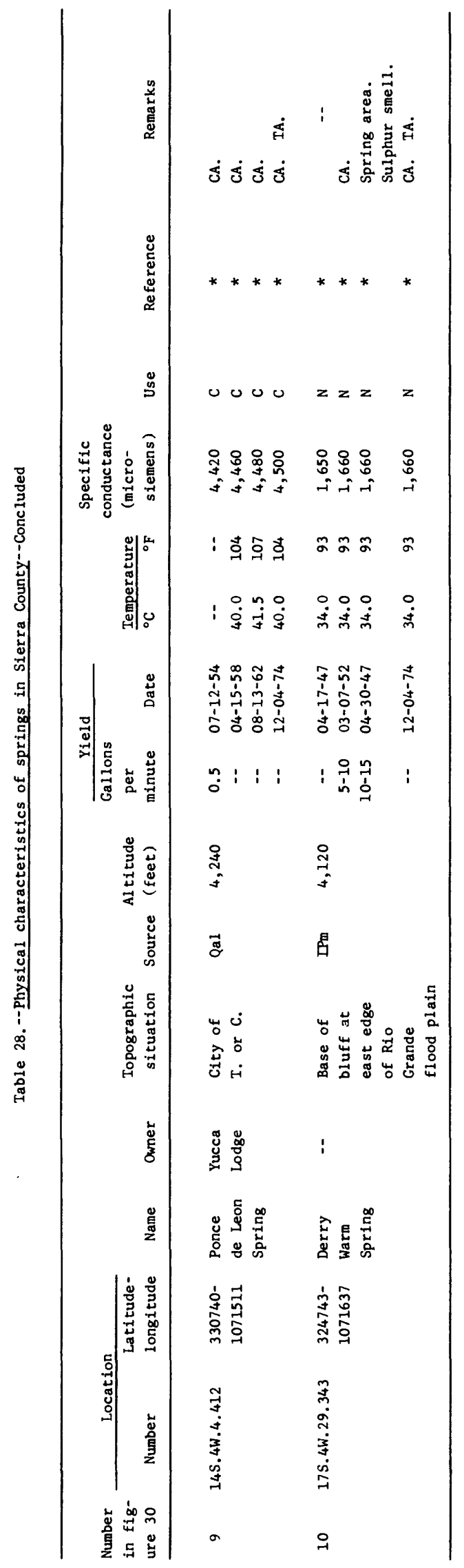




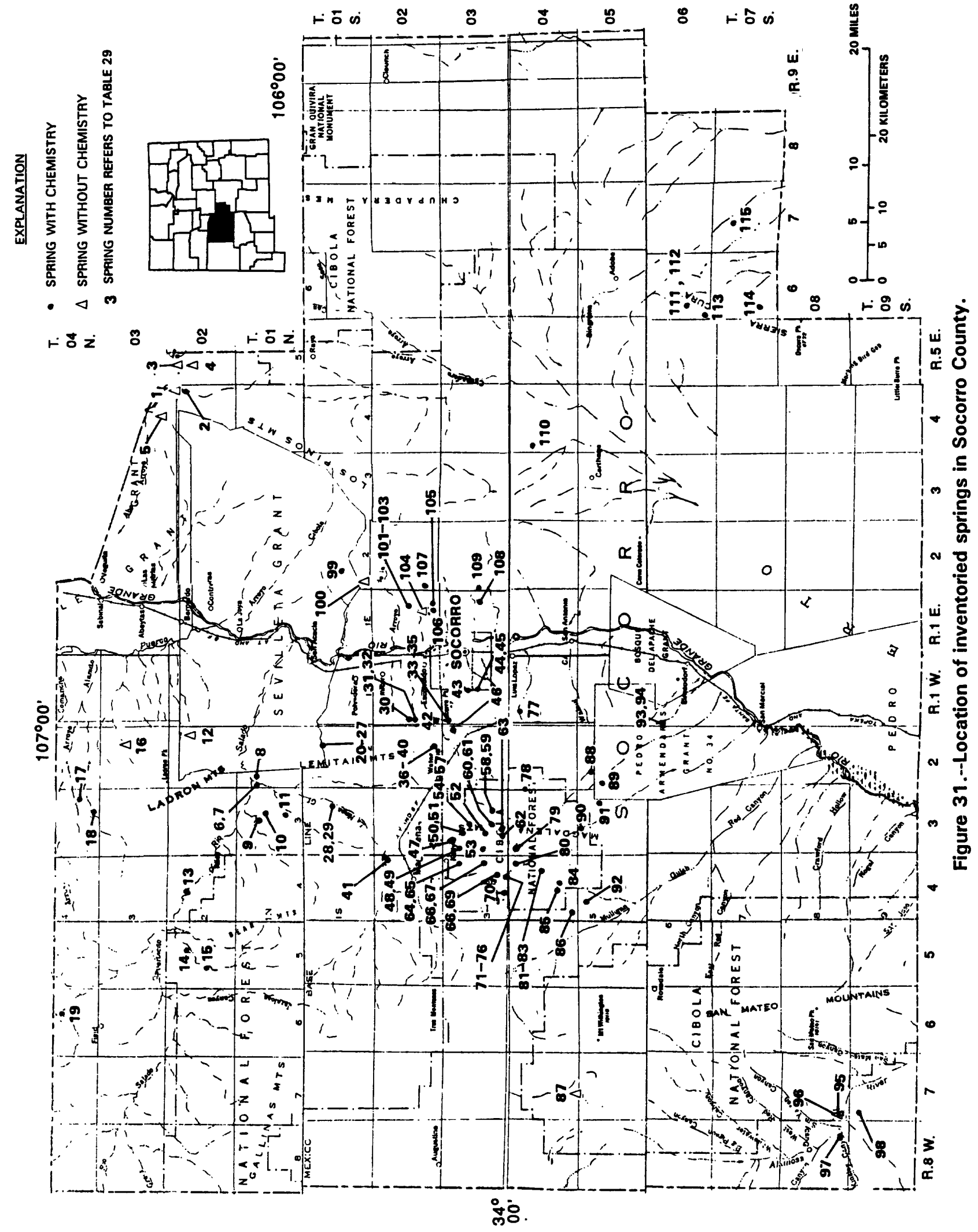




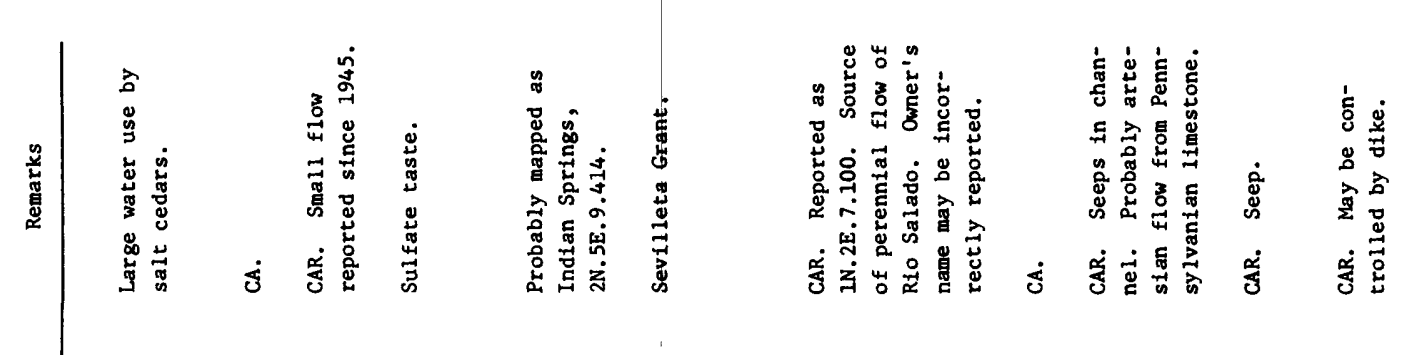

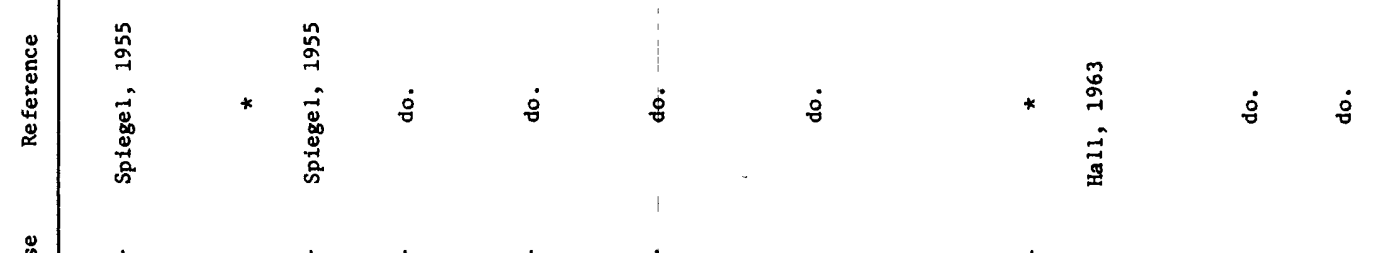

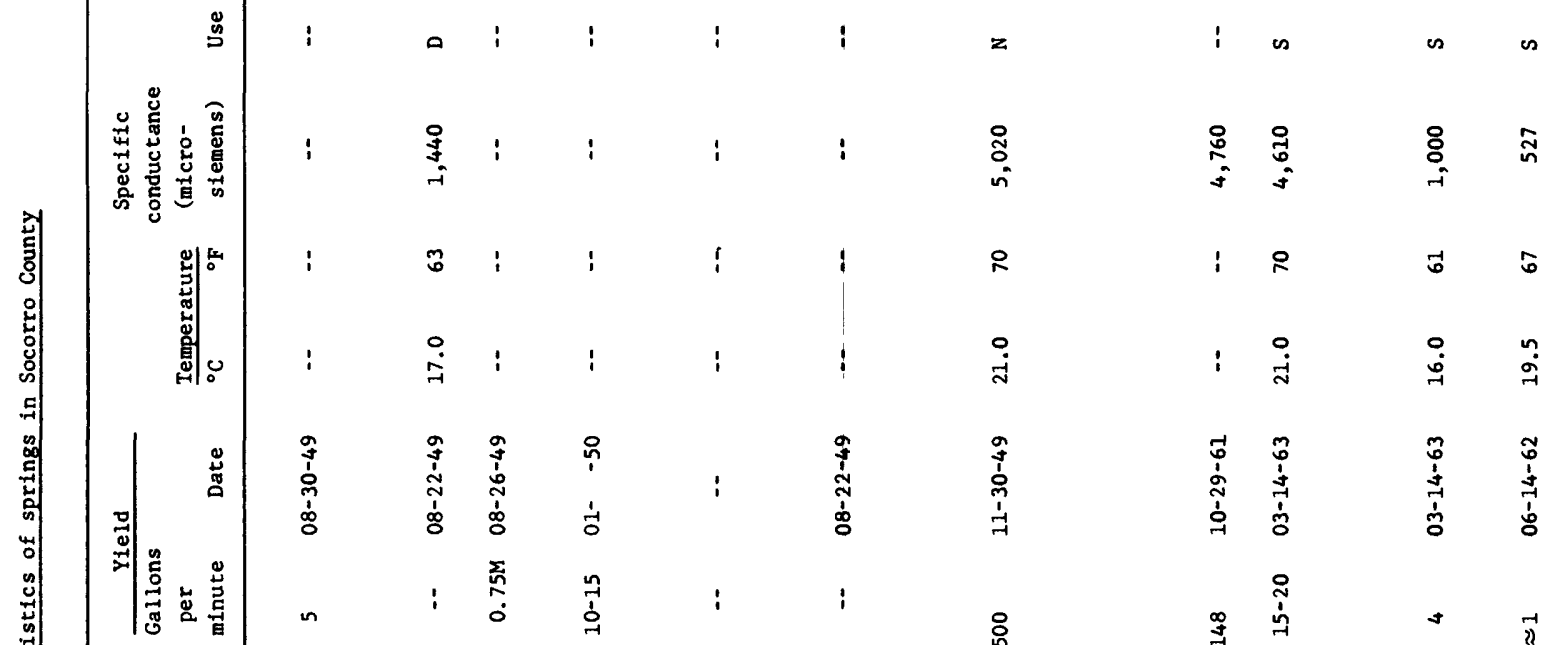

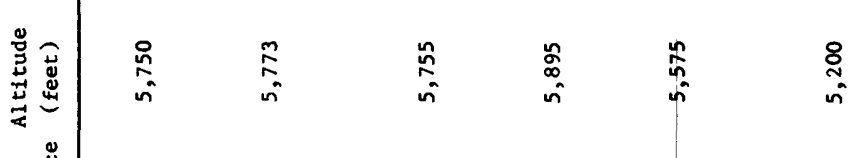

旁

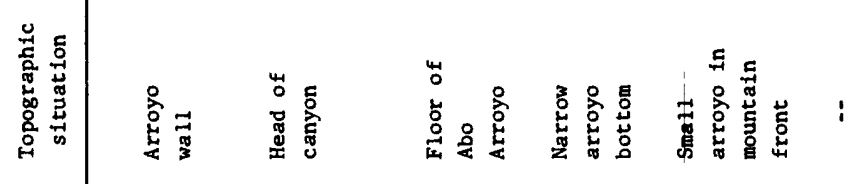

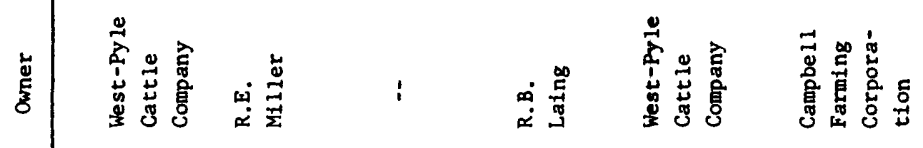

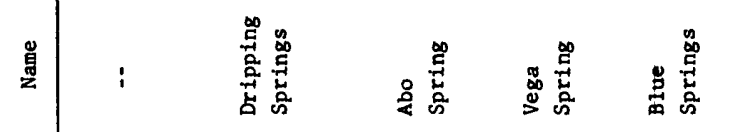

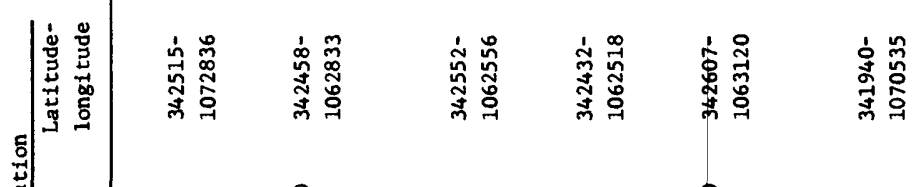

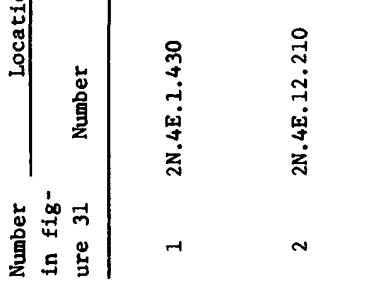

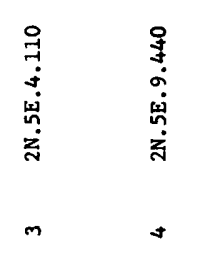

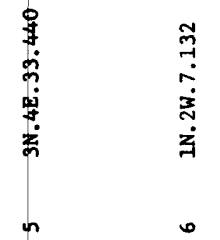

i

ลั $\quad \stackrel{0}{8}$ है

1) 咅

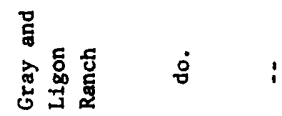

$: \quad: \frac{1}{3}$

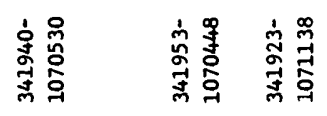

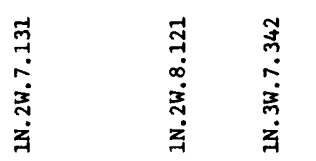




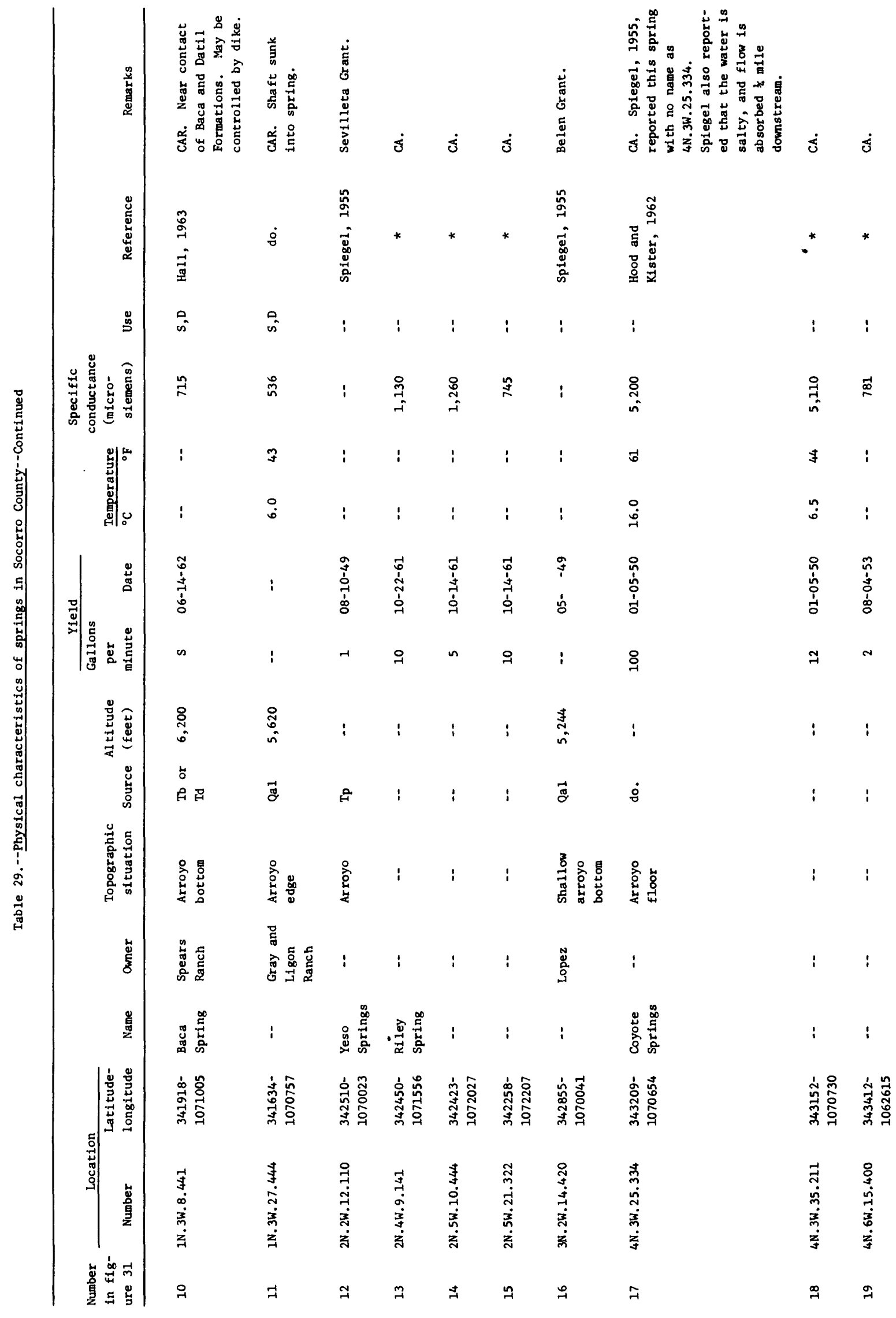




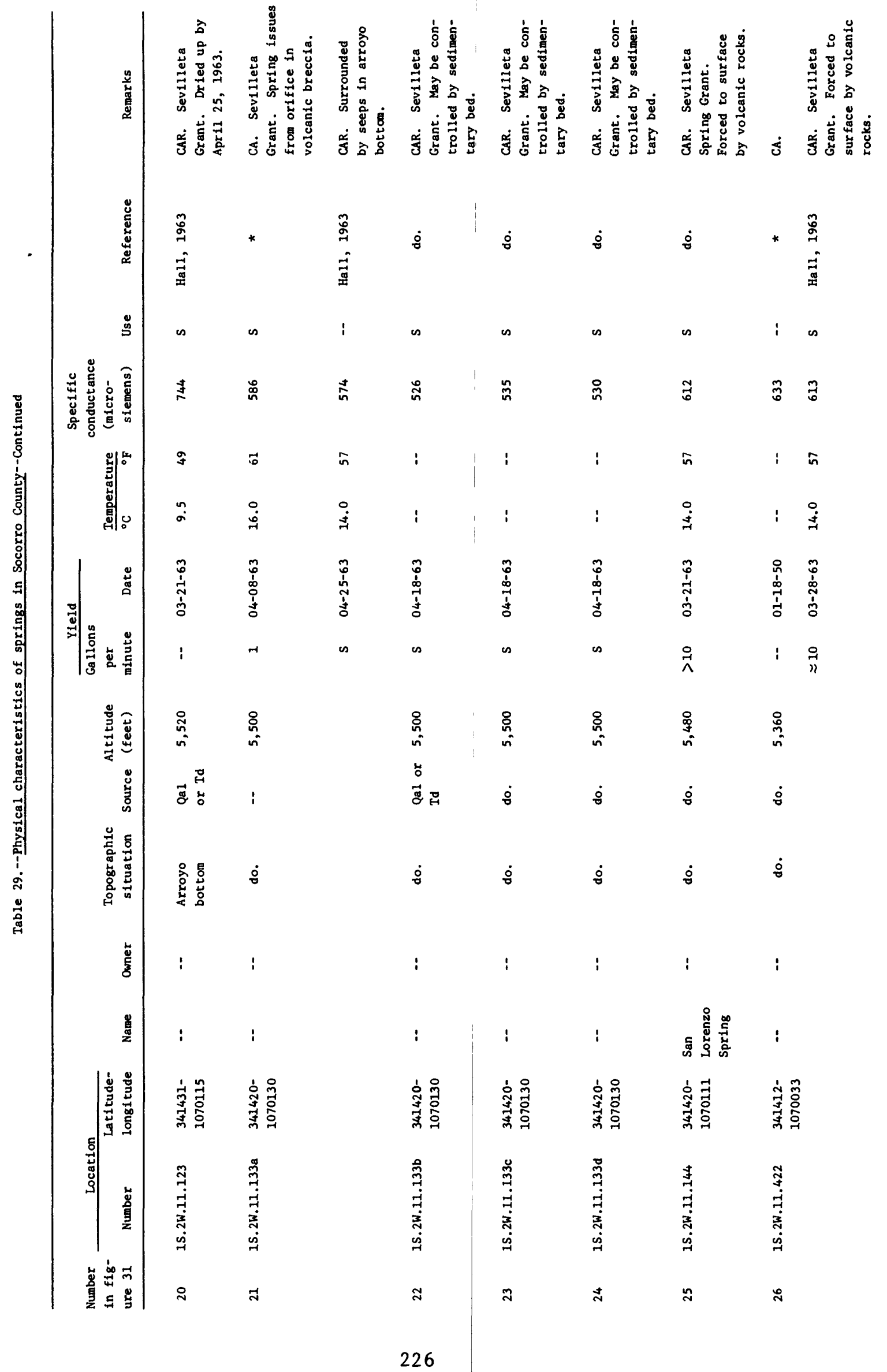




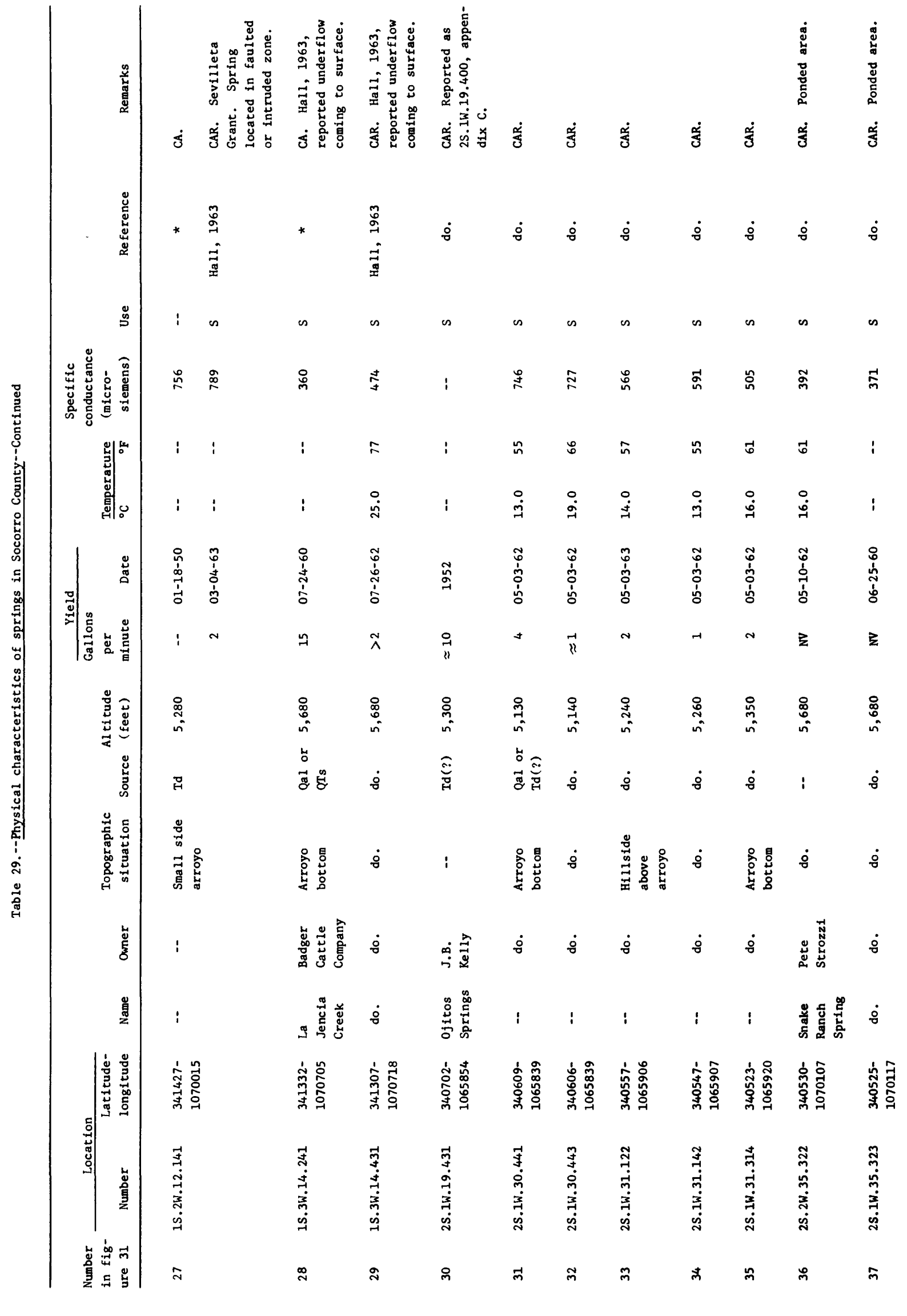




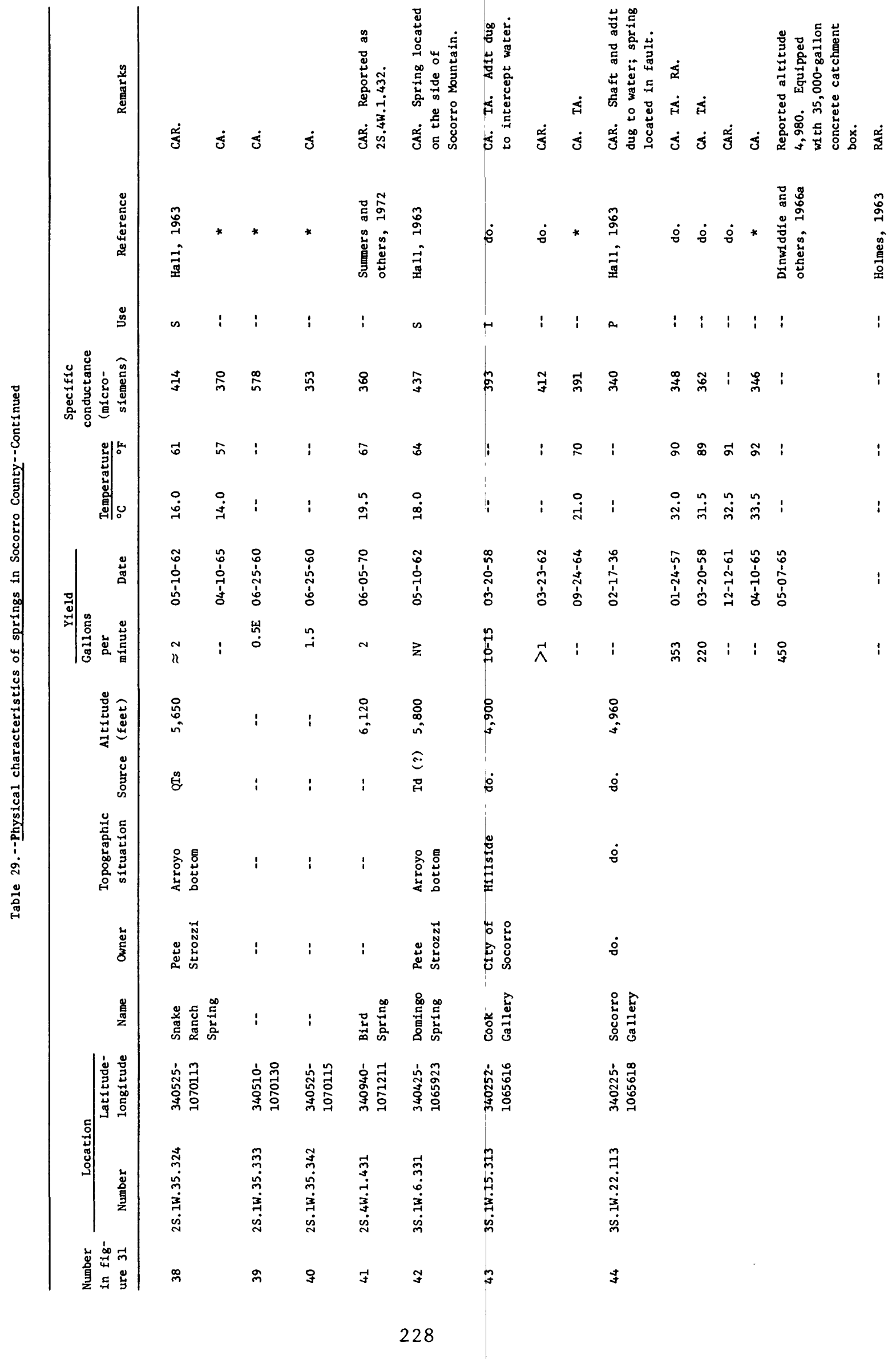




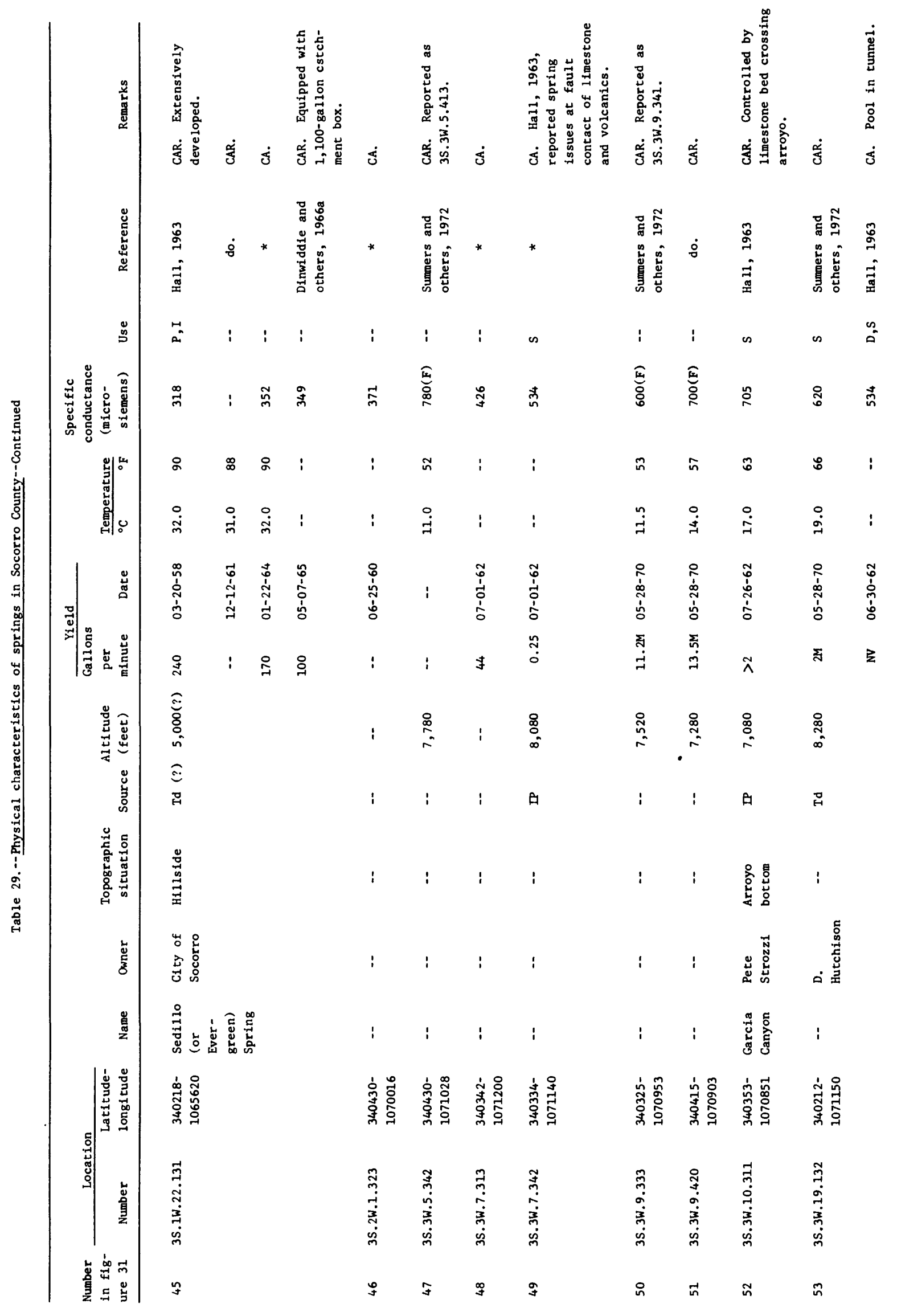




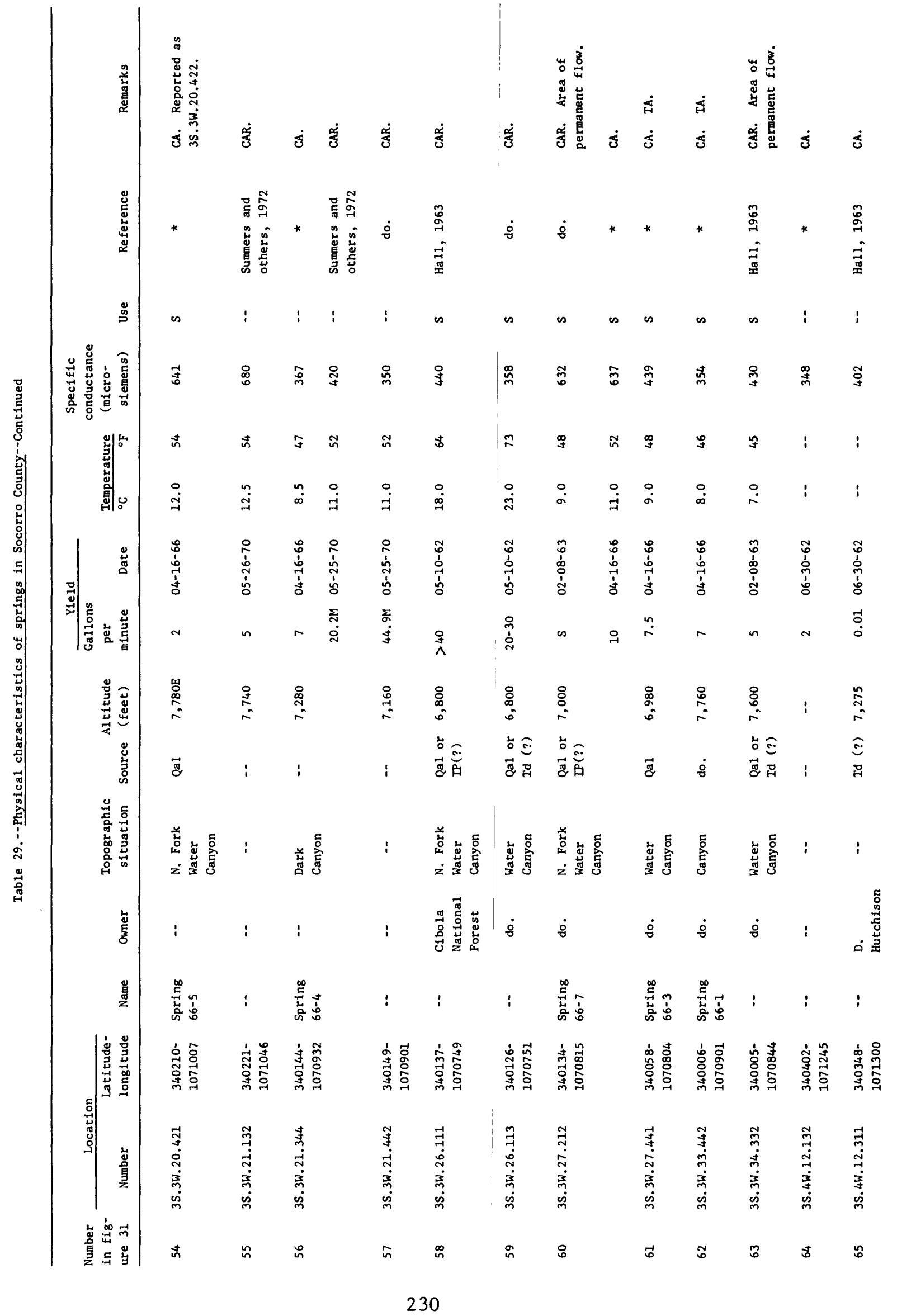




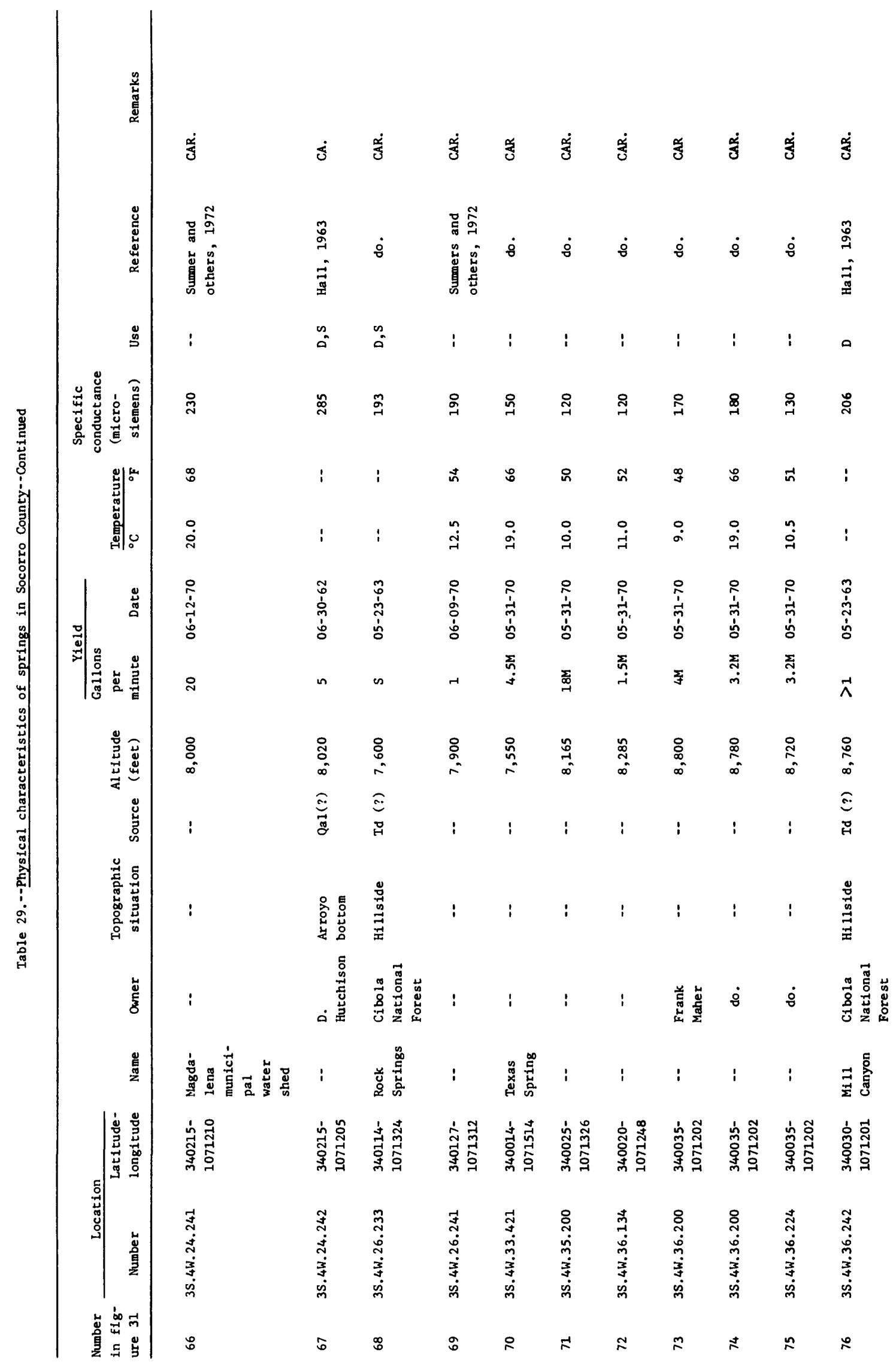




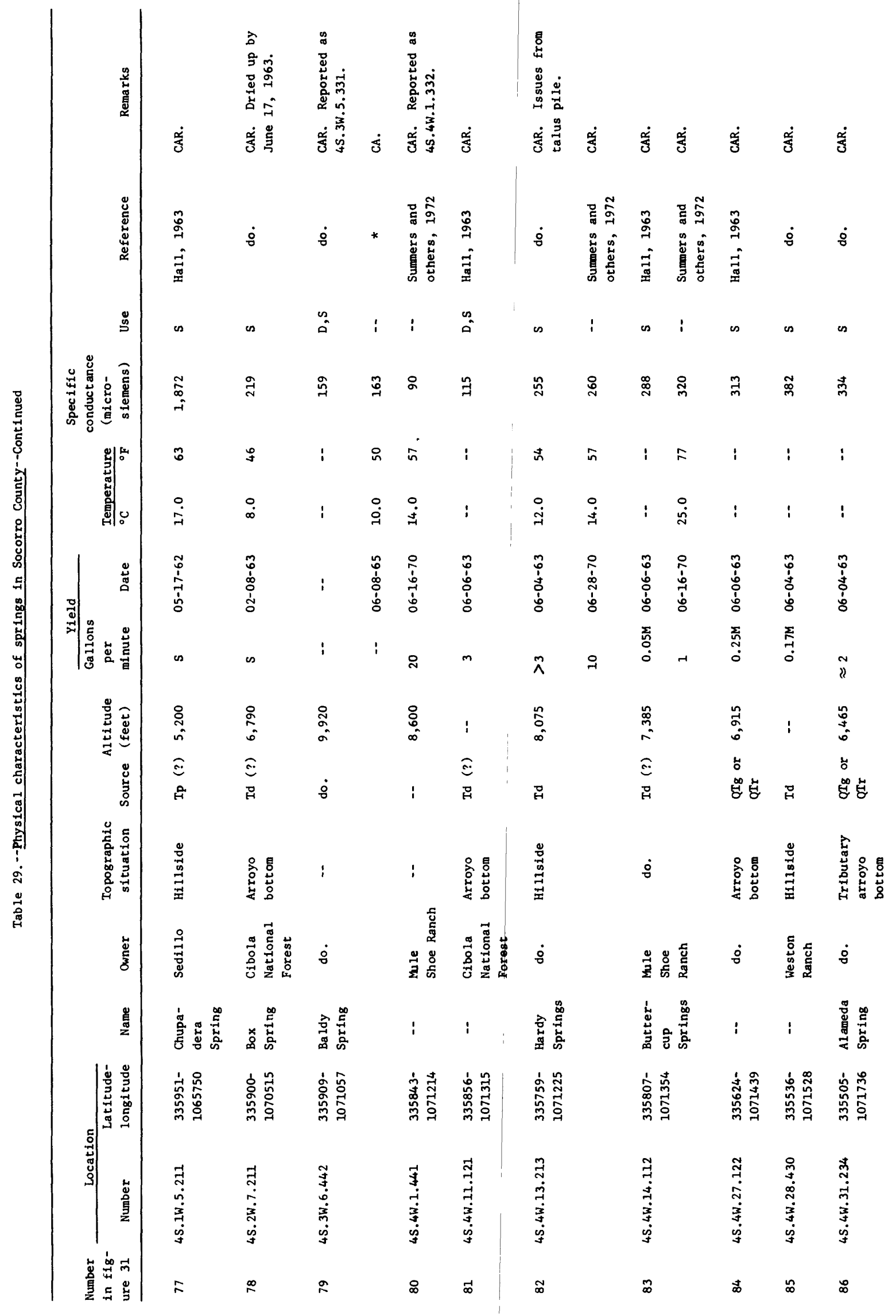




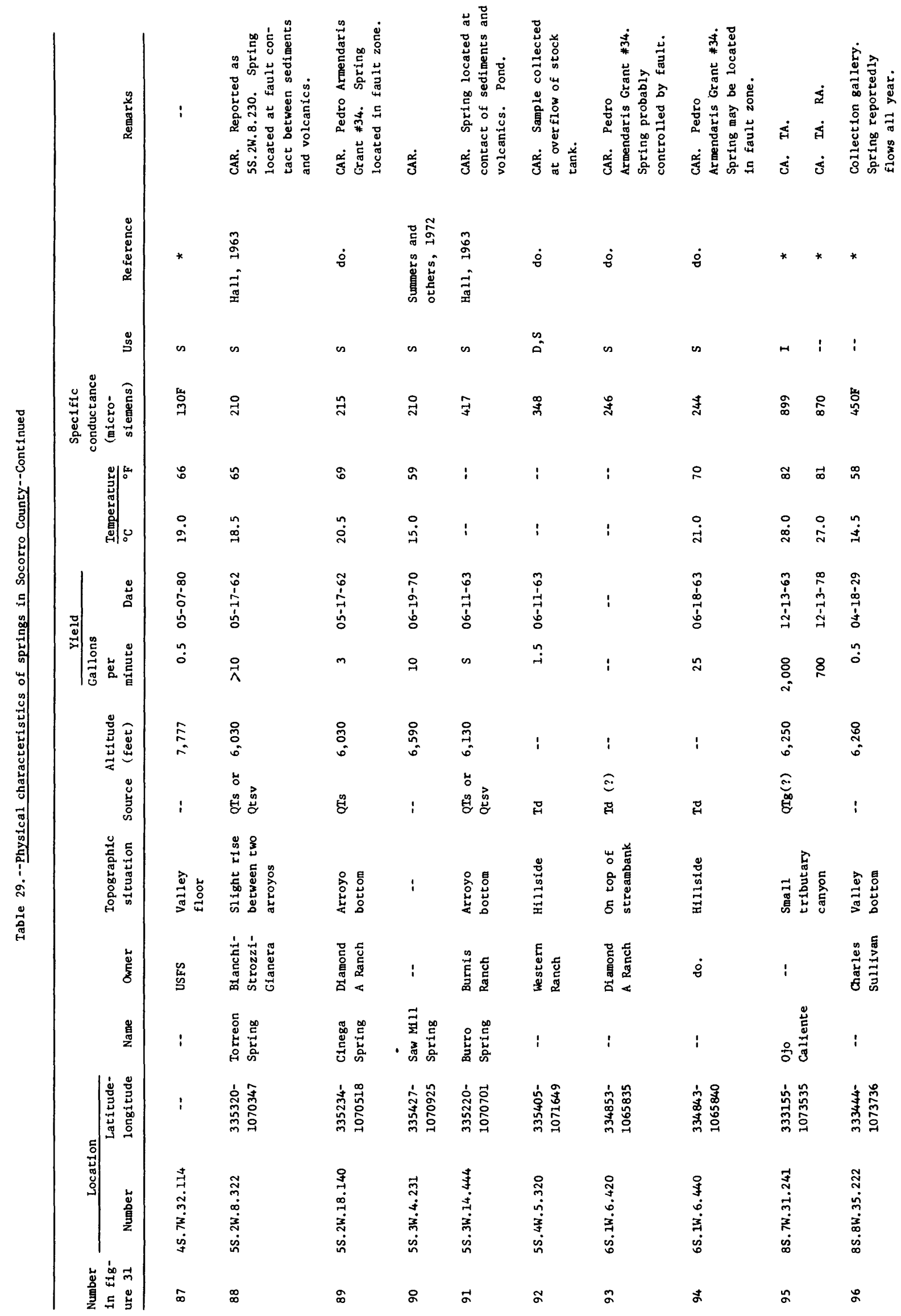




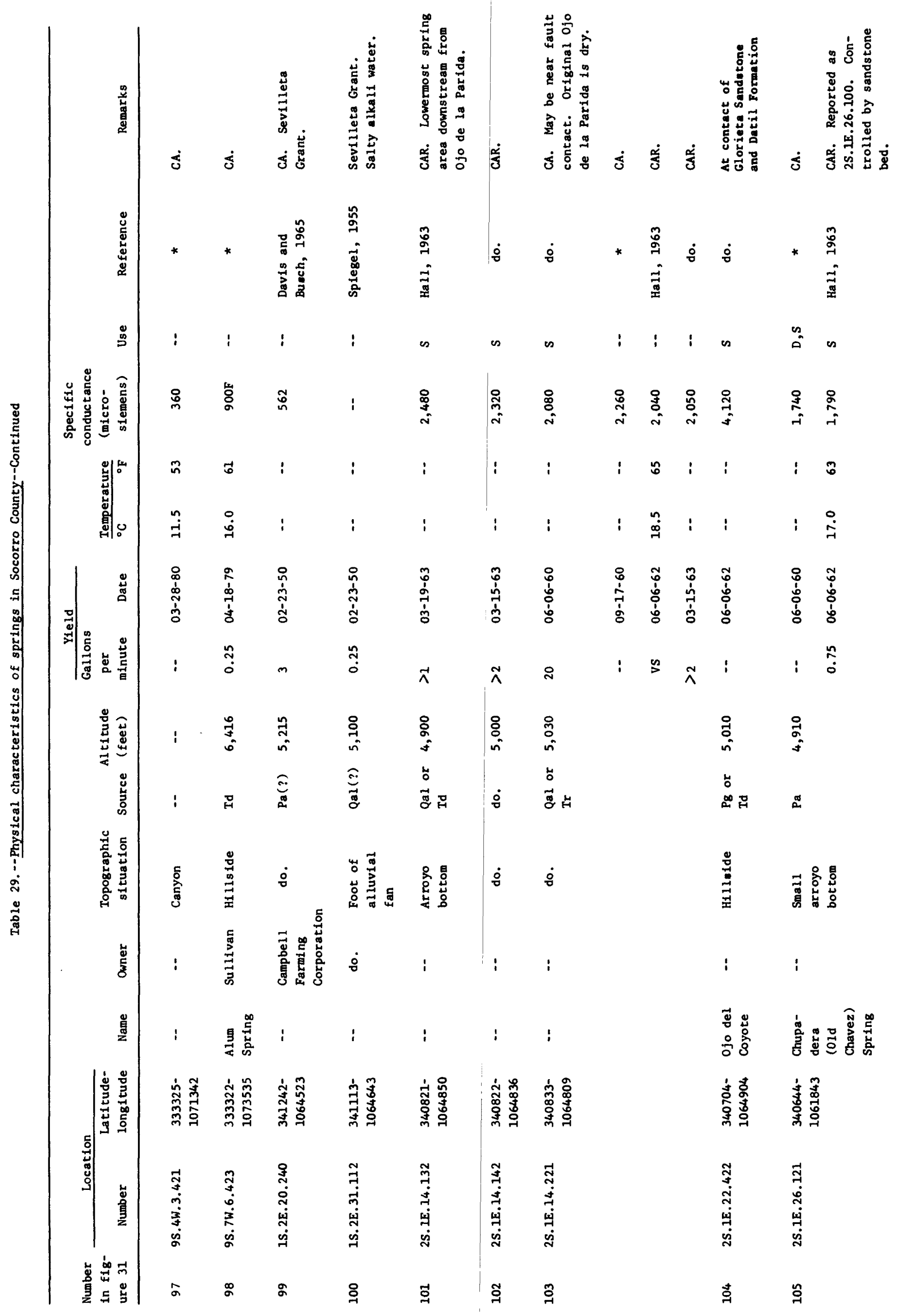




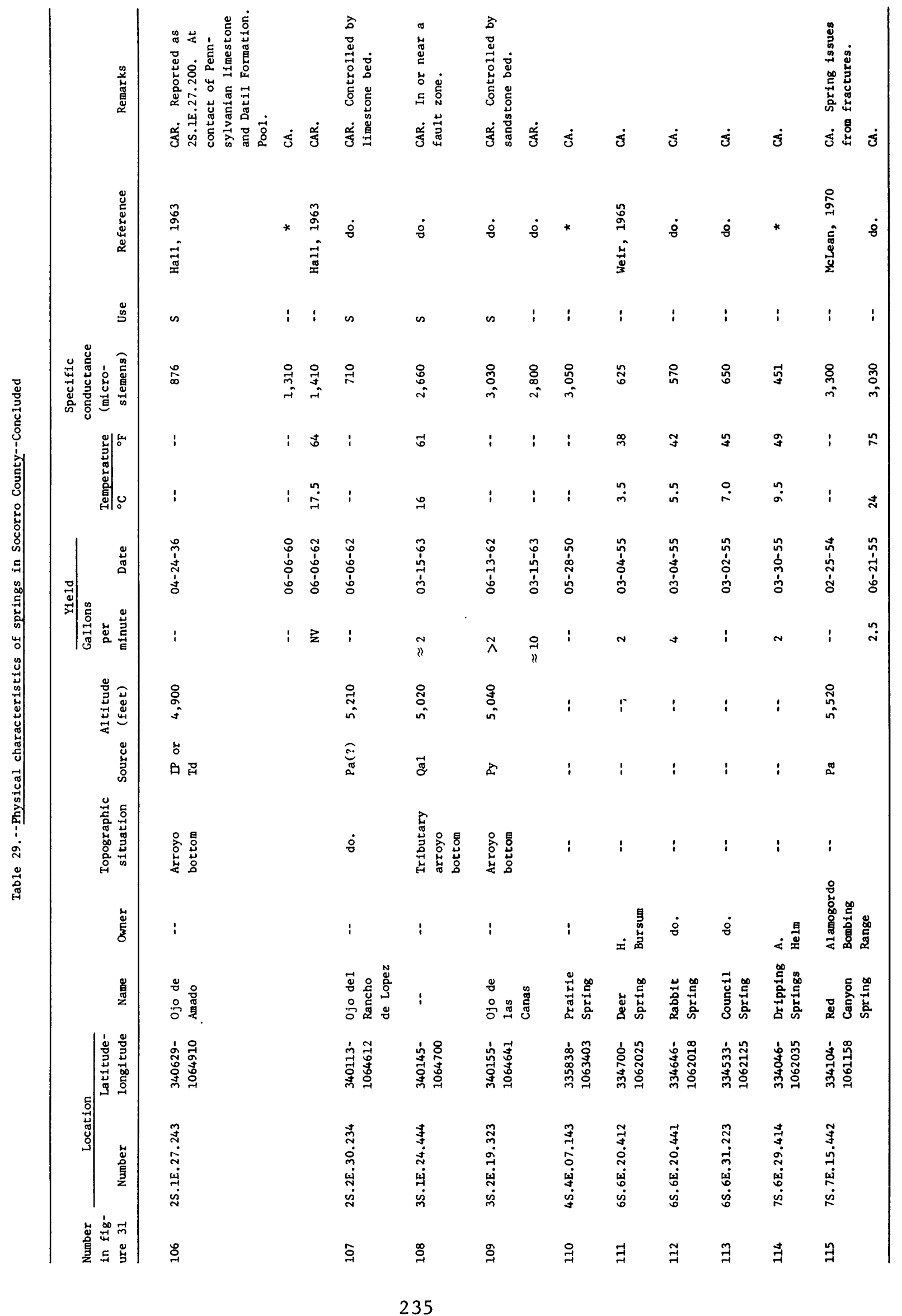




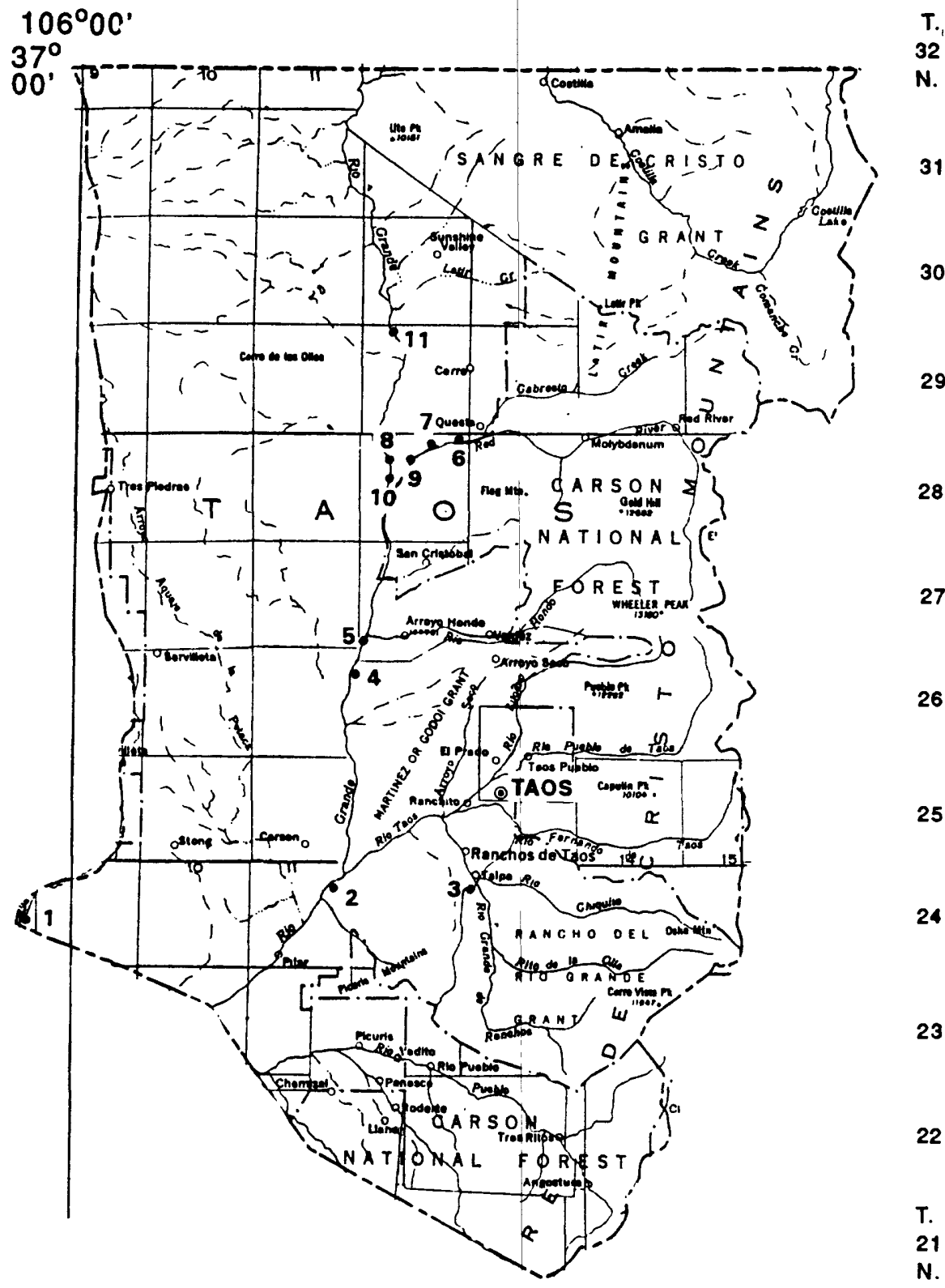

$\begin{array}{lllllll}\text { R.9 E. } & 10 & 11 & 12 & 13 & 14 & \text { R.15 E. }\end{array}$

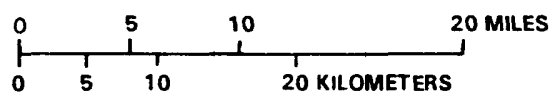

\section{EXPLANATION}

- SPRING WITH CHEMISTRY

2 SPRING NUMBER REFERS TO

TABLE 30

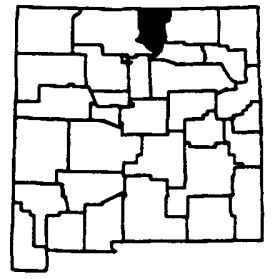

Figure 32.--Location of inventoried springs in Taos County. 


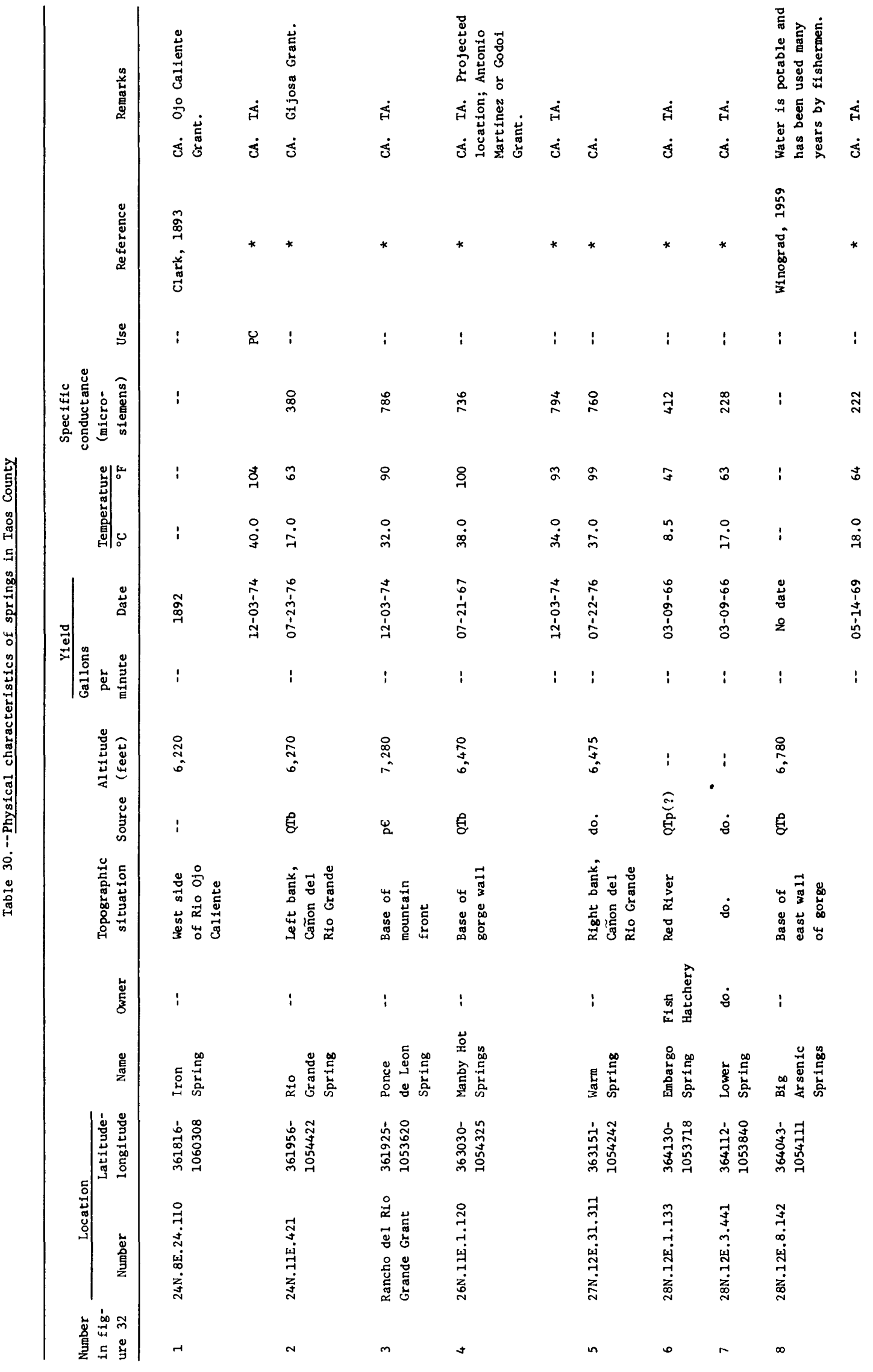




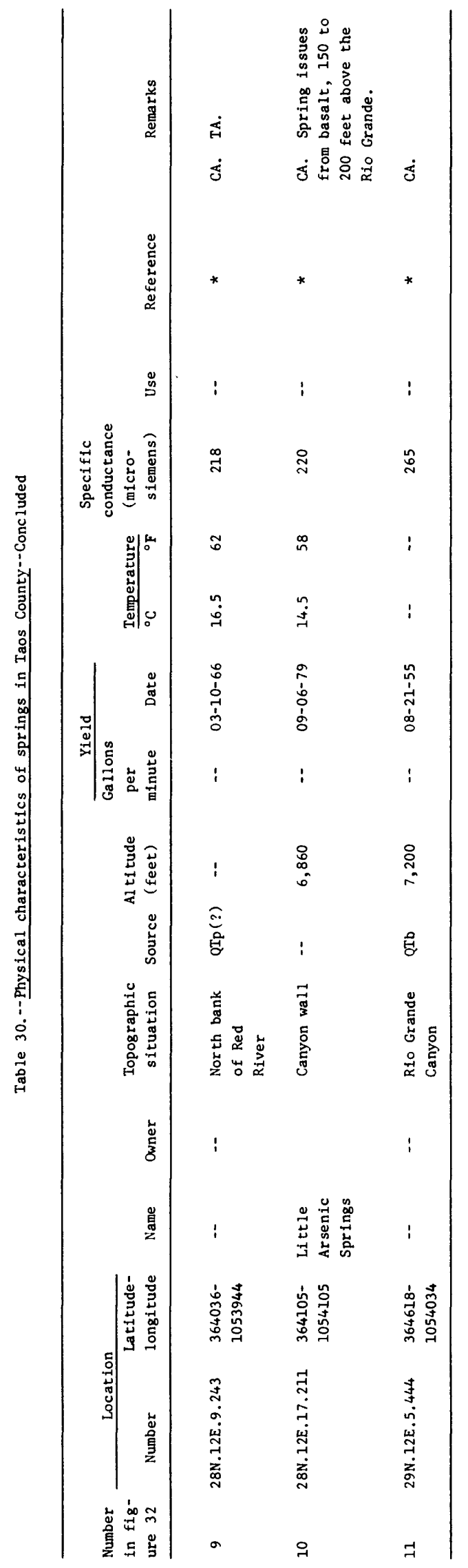




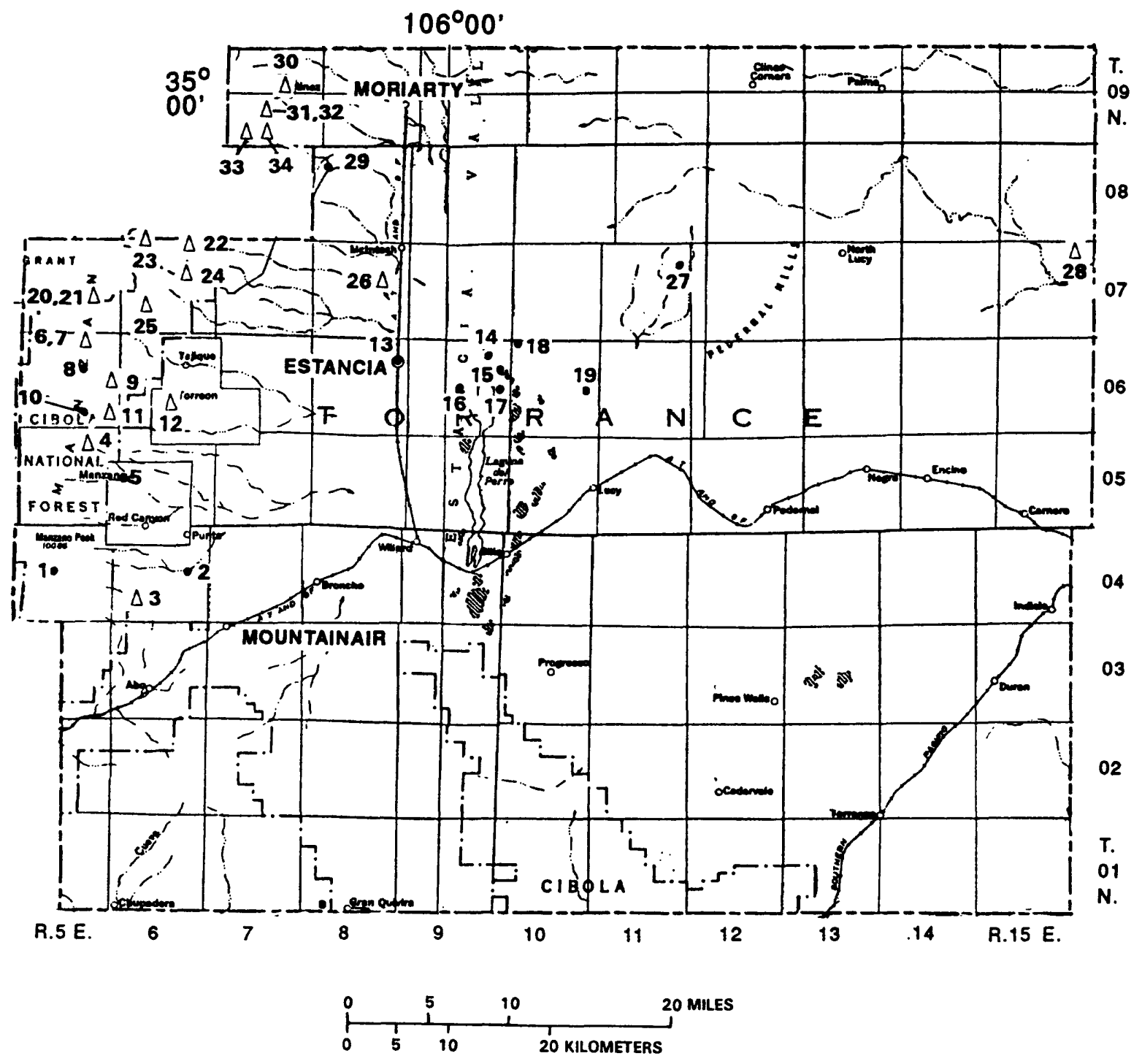

EXPLANATION

- SPRING WITH CHEMISTRY

$\triangle$ SPRING WITHOUT CHEMISTRY

2 SPRING NUMBER REFERS TO TABLE 31

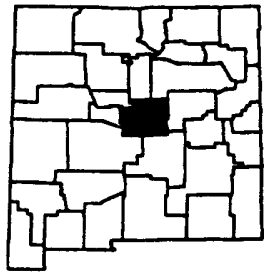

Figure 33.--Location of inventoried springs in Torrance County. 


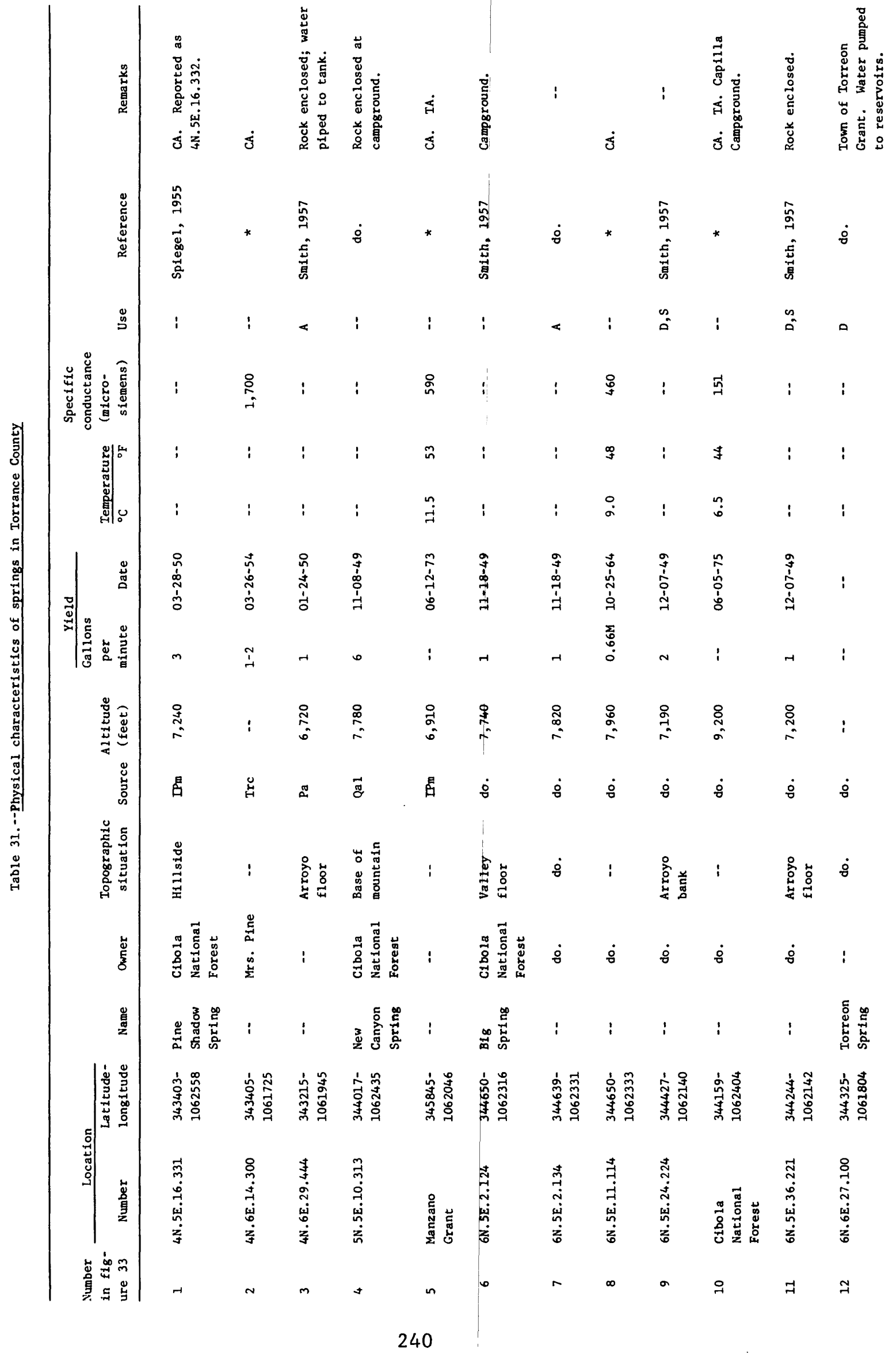




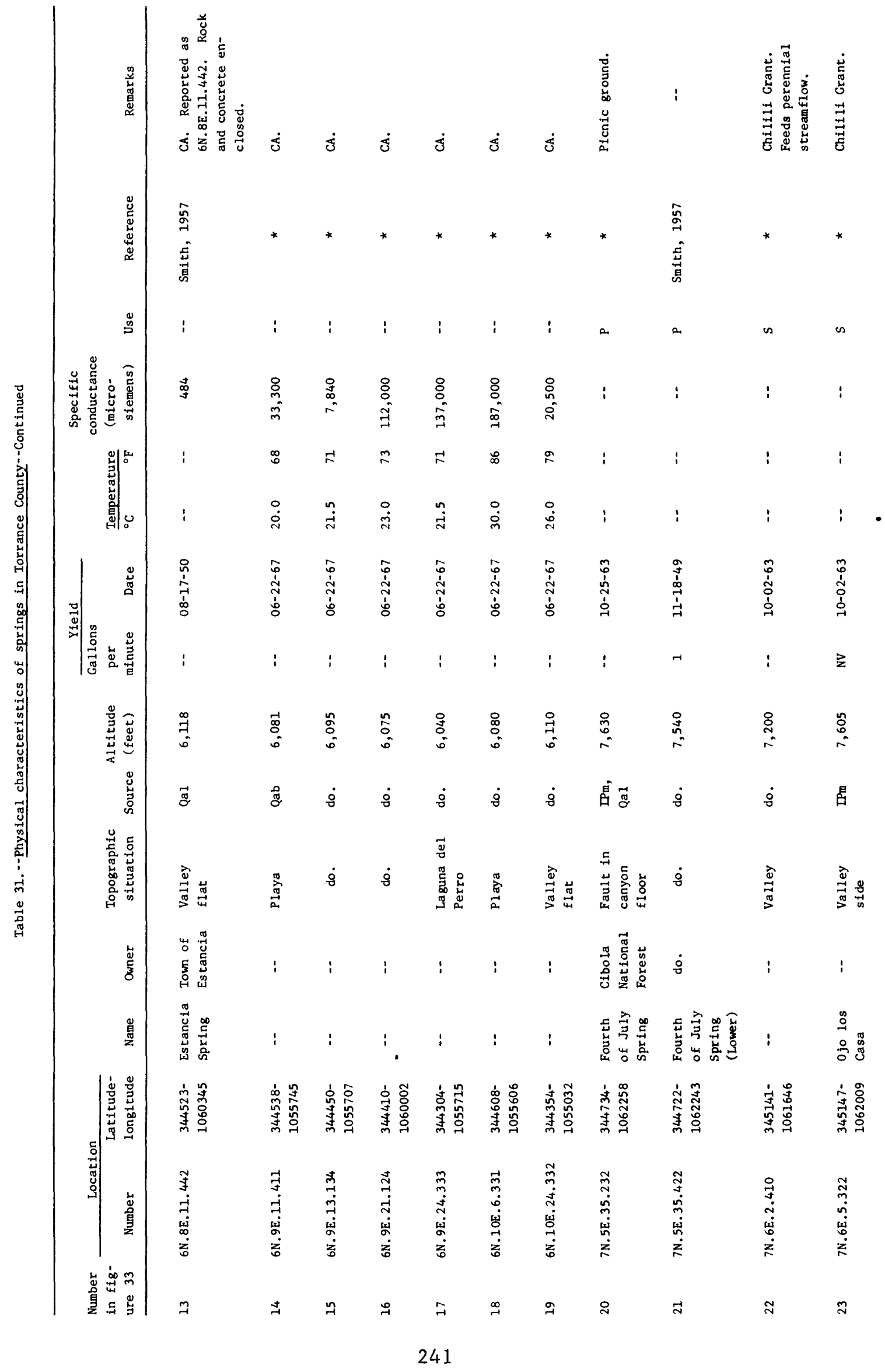




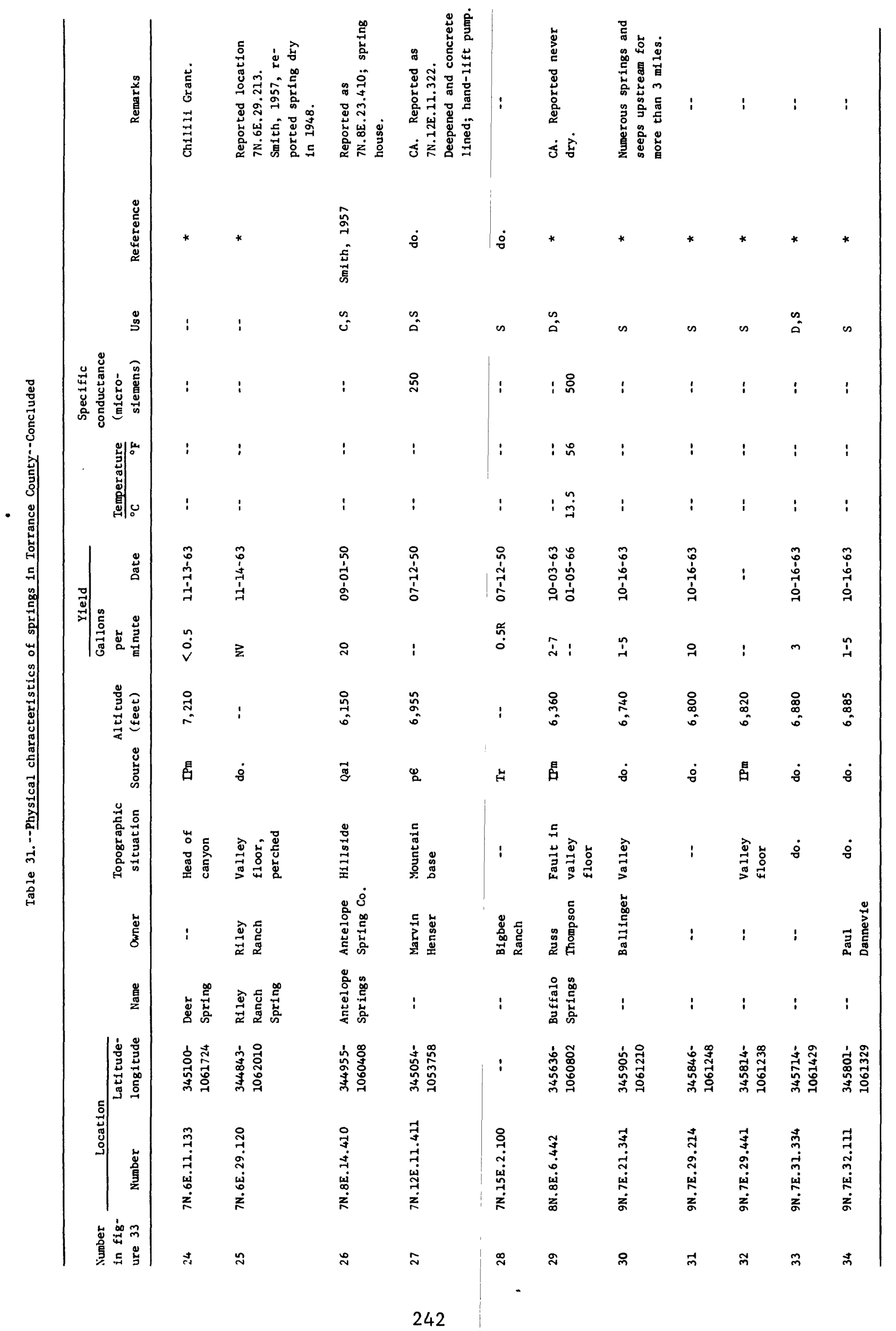




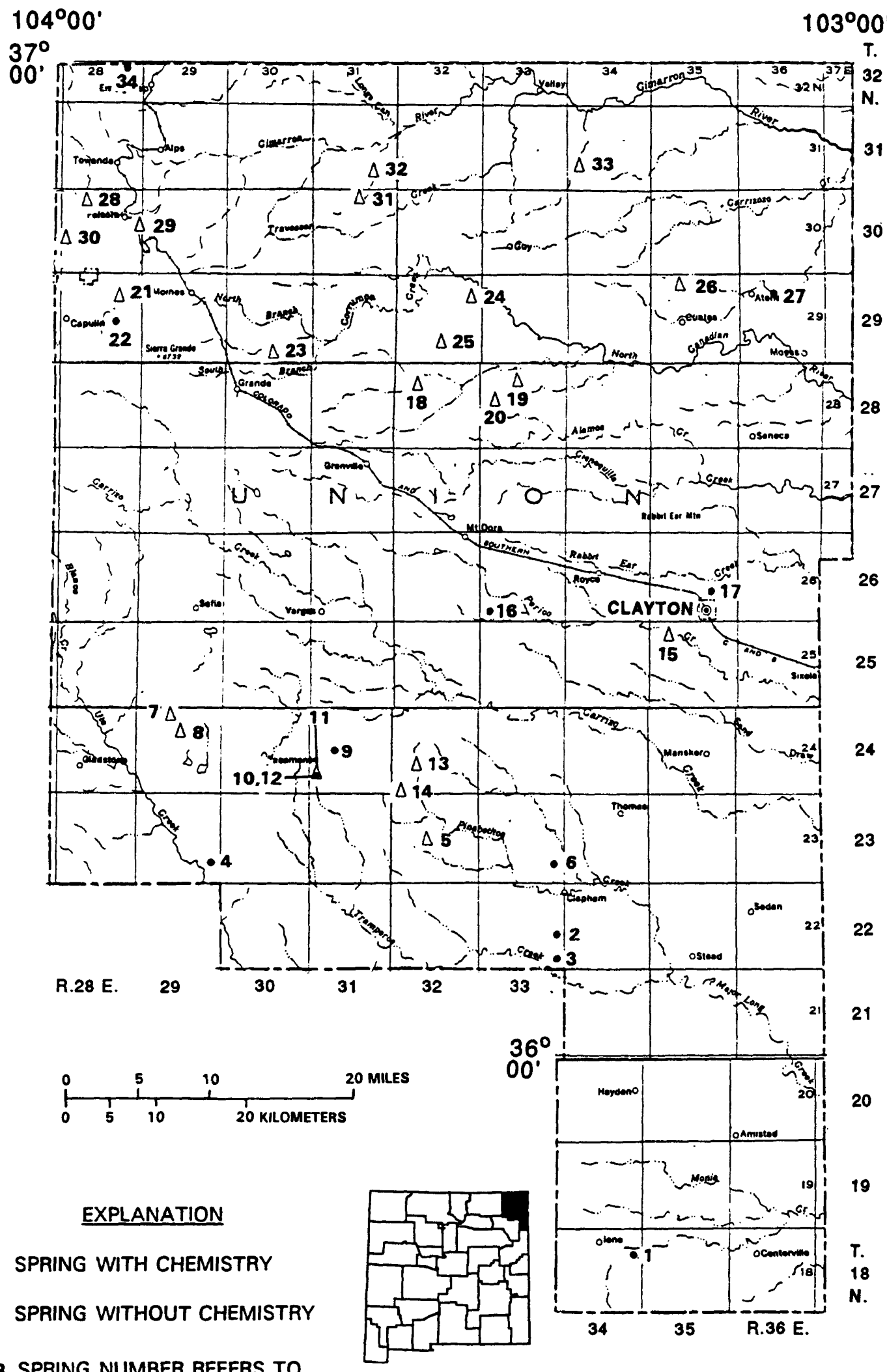

3 SPRING NUMBER REFERS TO

TABLE 32

Figure 34.--Location of inventoried springs in Union County. 


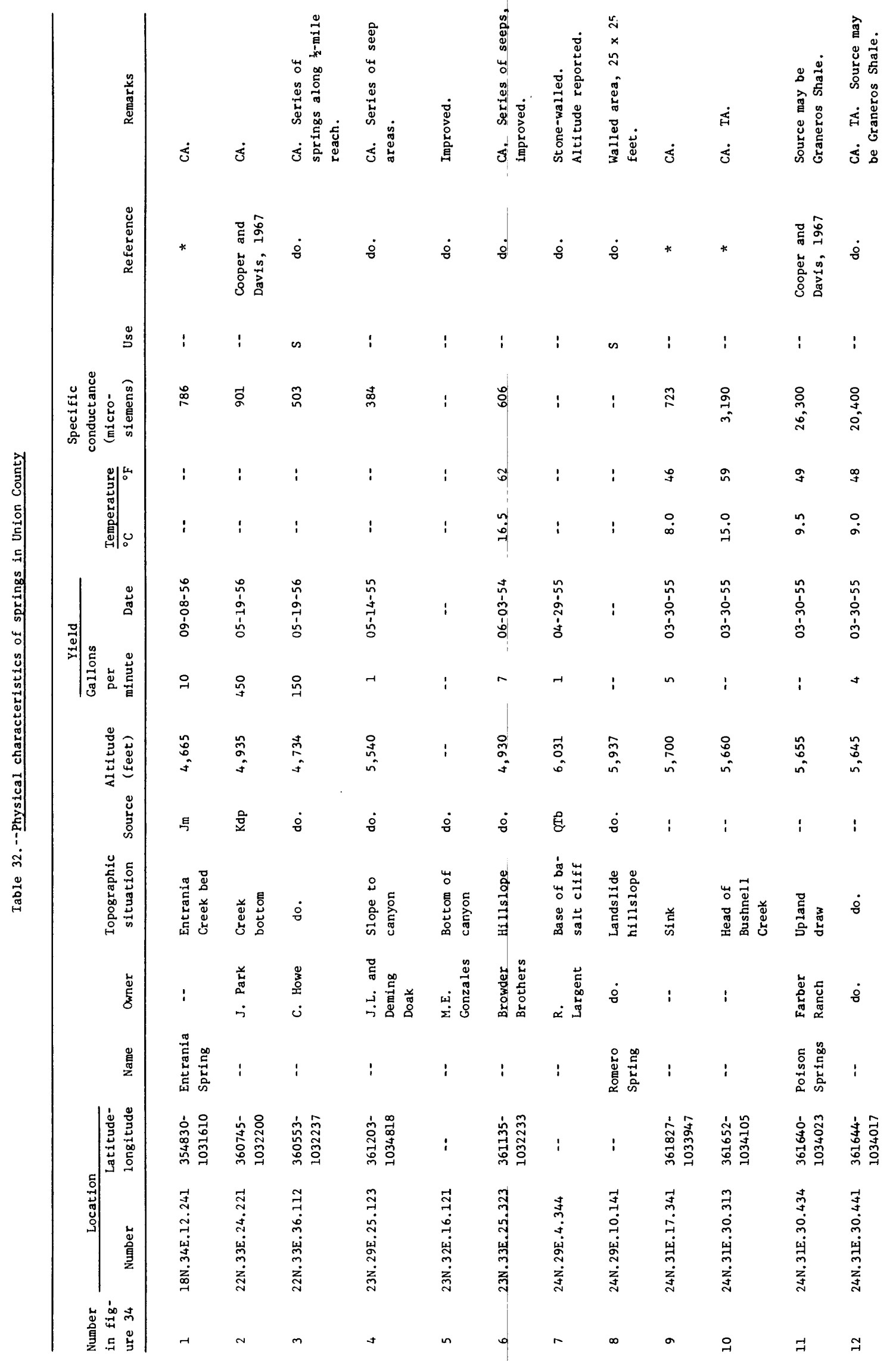




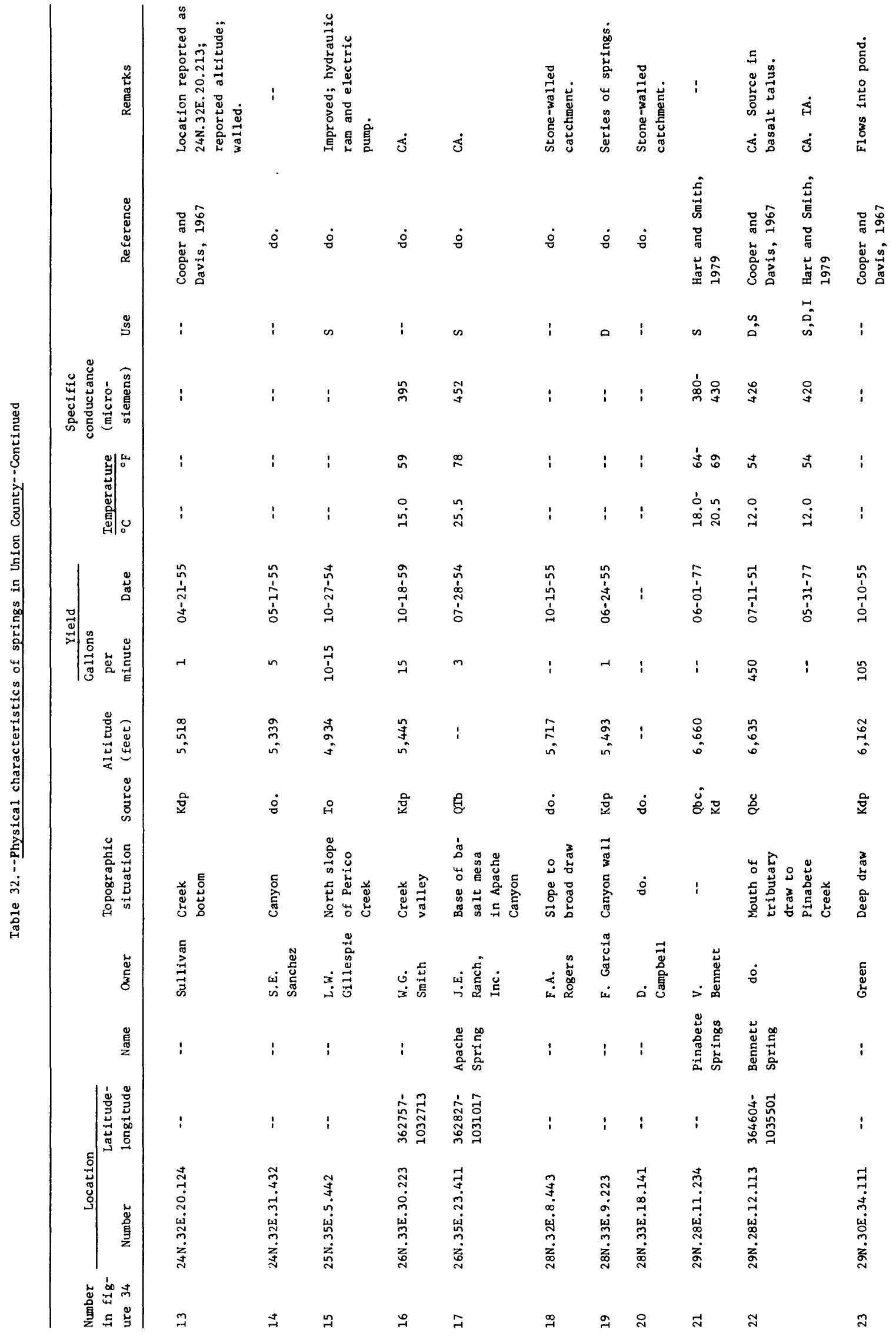




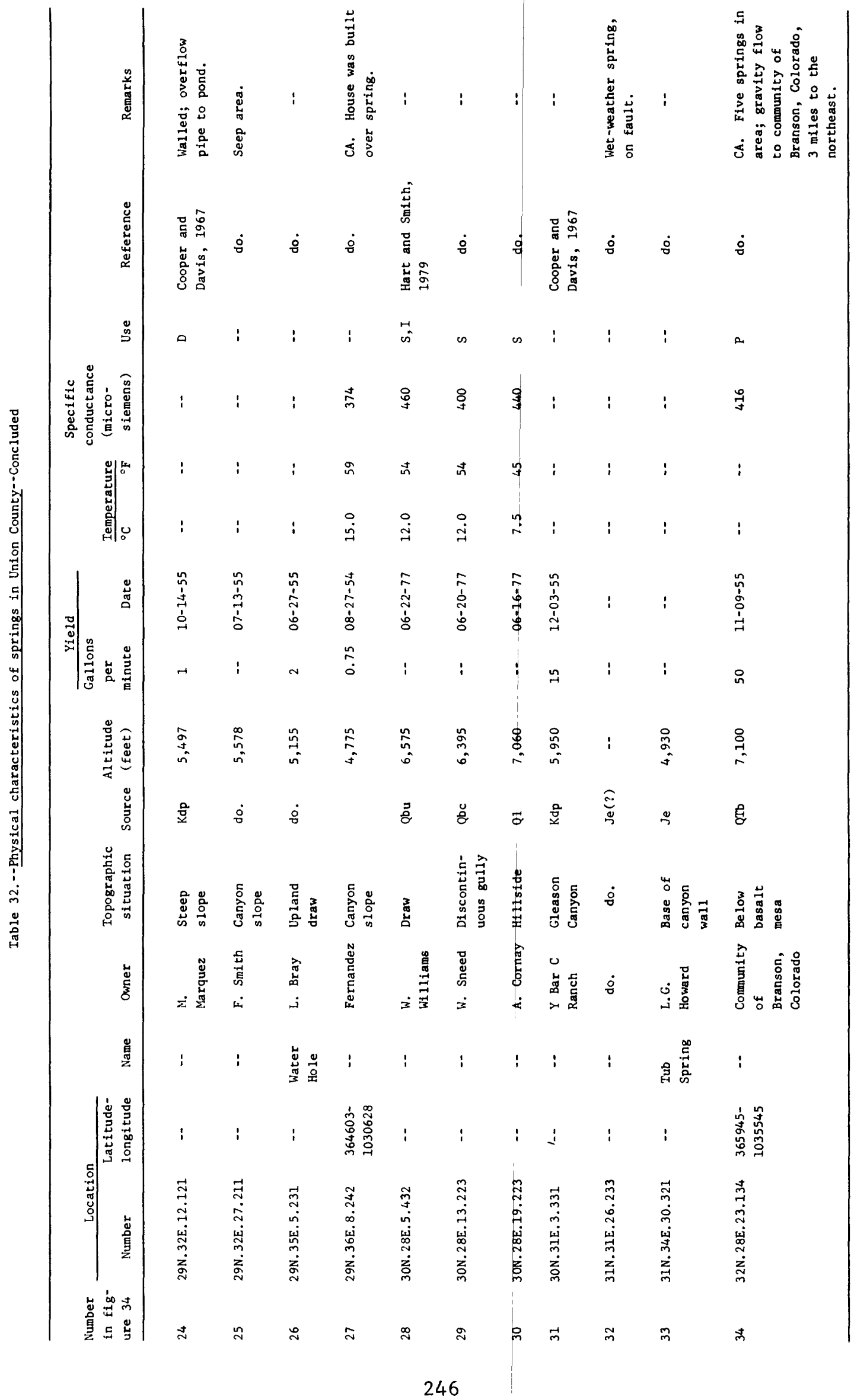



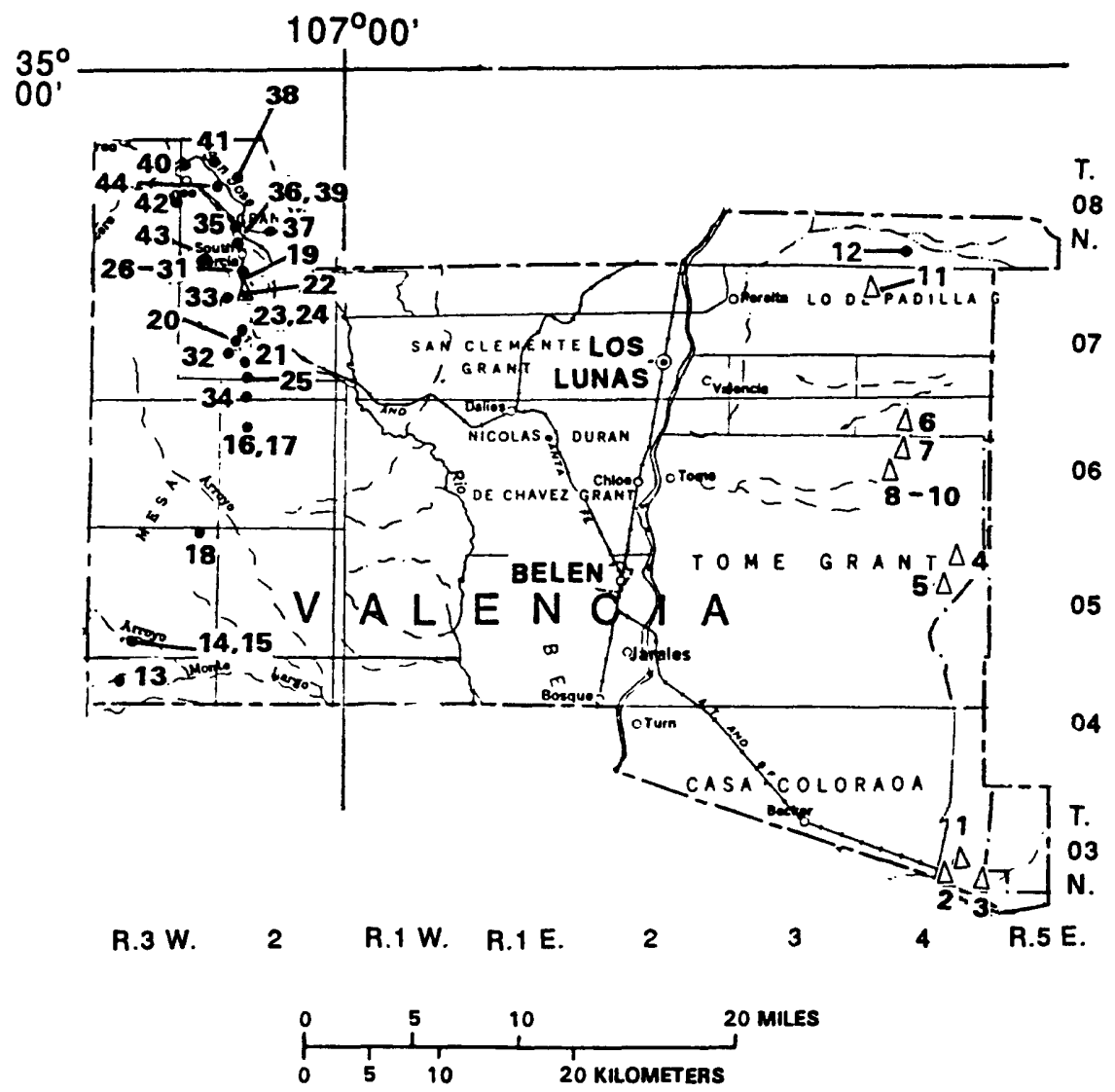

EXPLANATION

- SPRING WITH CHEMISTRY

$\triangle$ SPRING WITHOUT CHEMISTRY

5 SPRING NUMBER REFERS TO TABLE 33

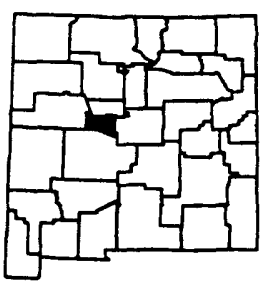

Figure 35.--Location of inventoried springs in Valencia County. 


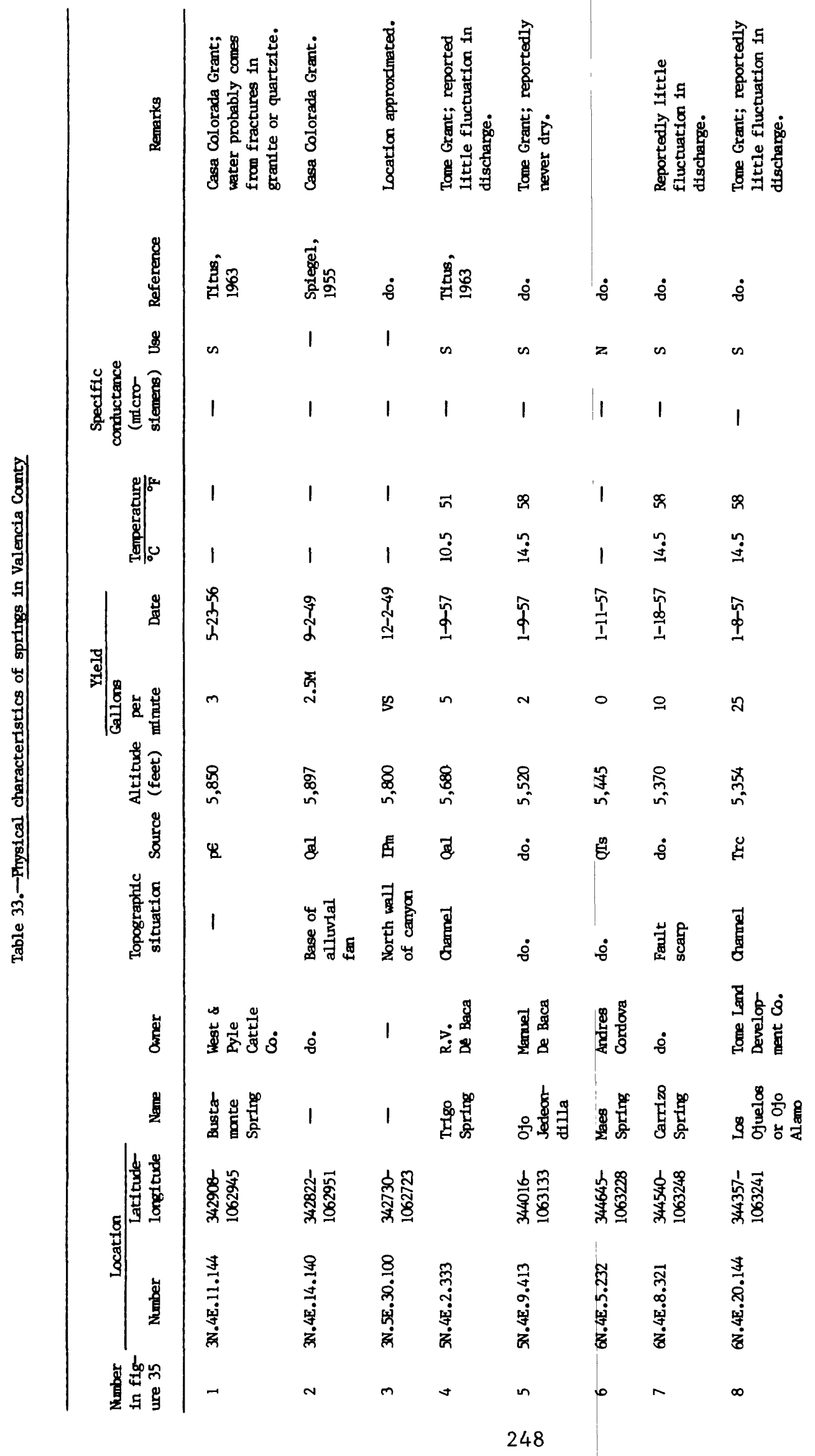




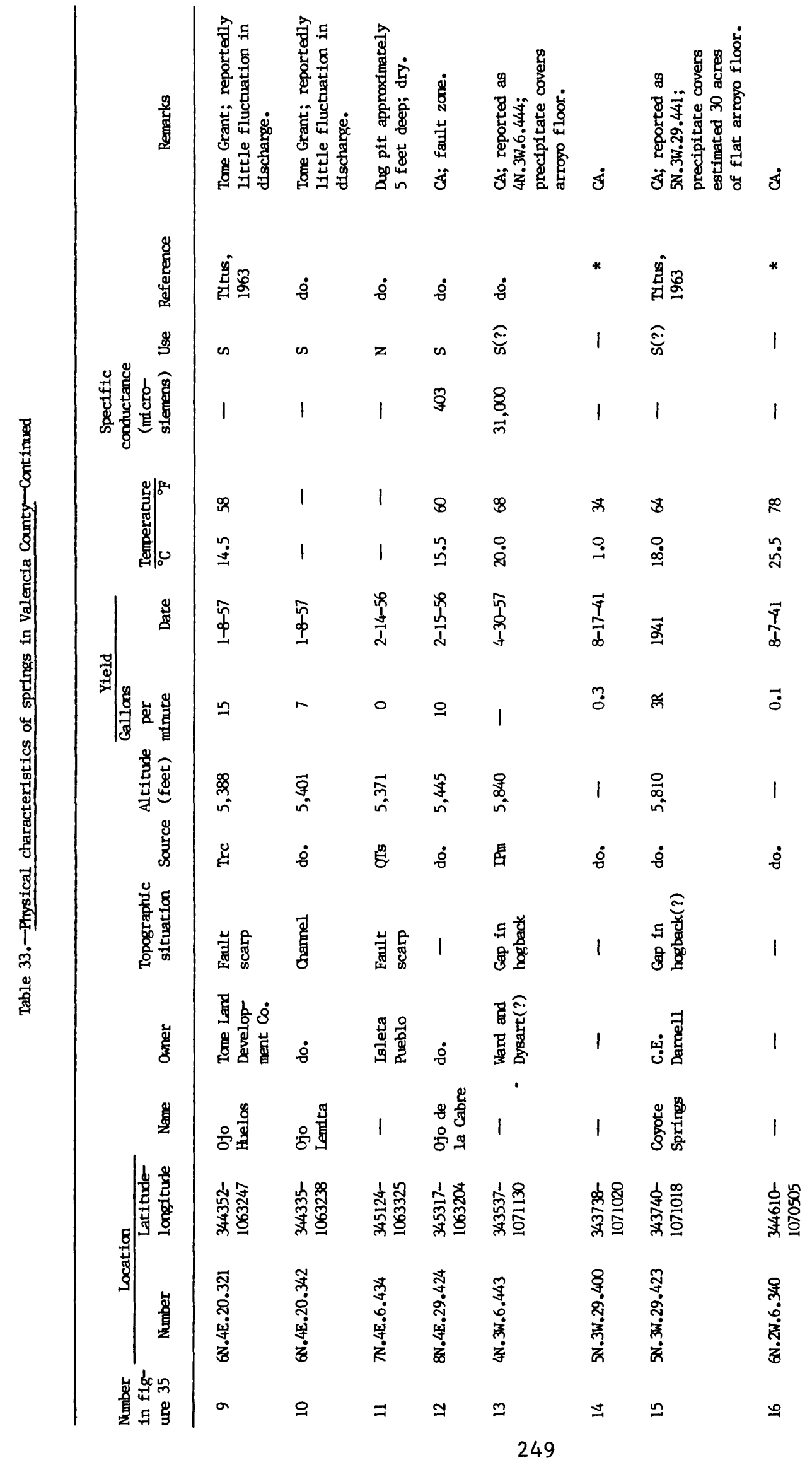




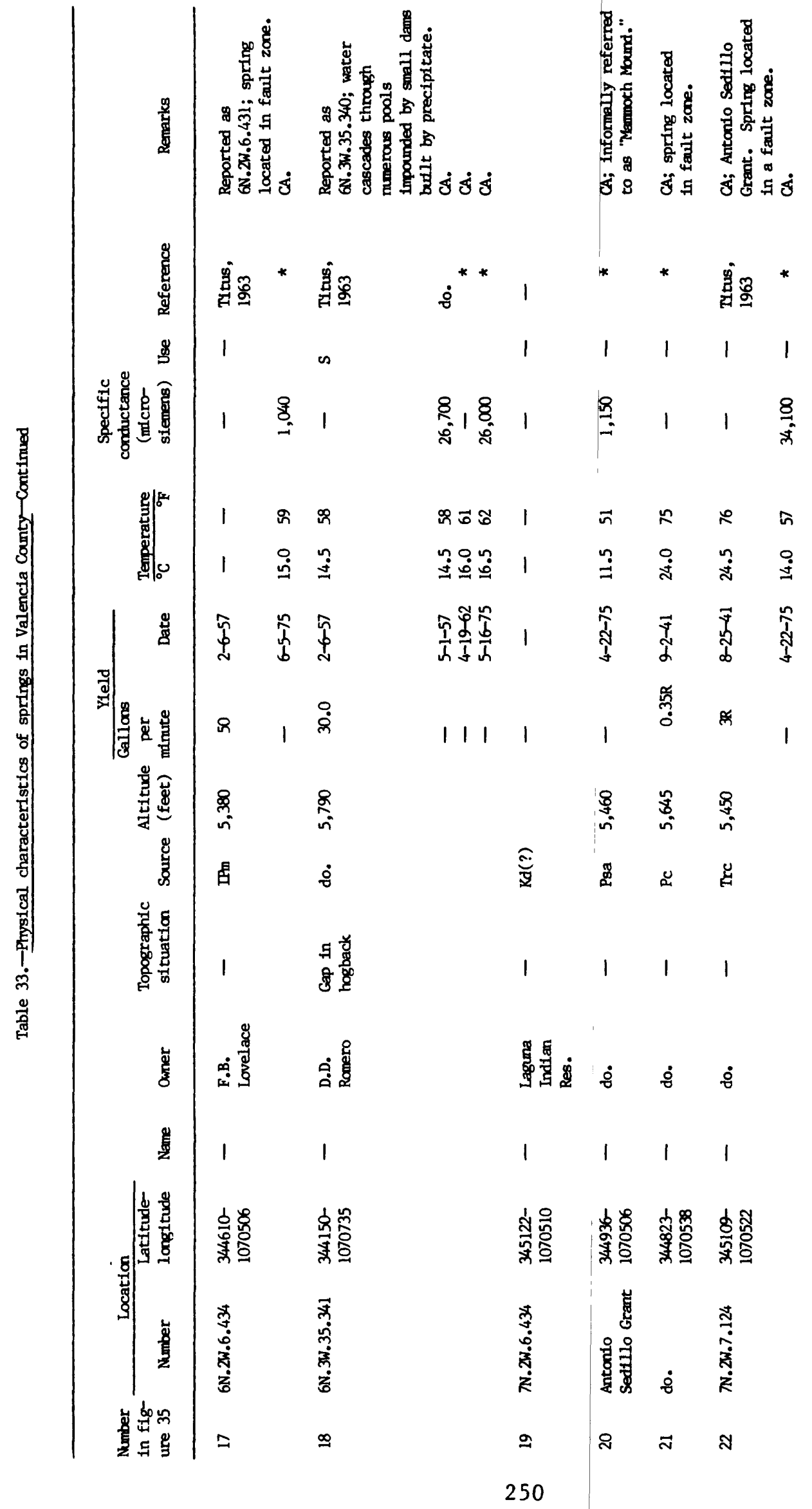




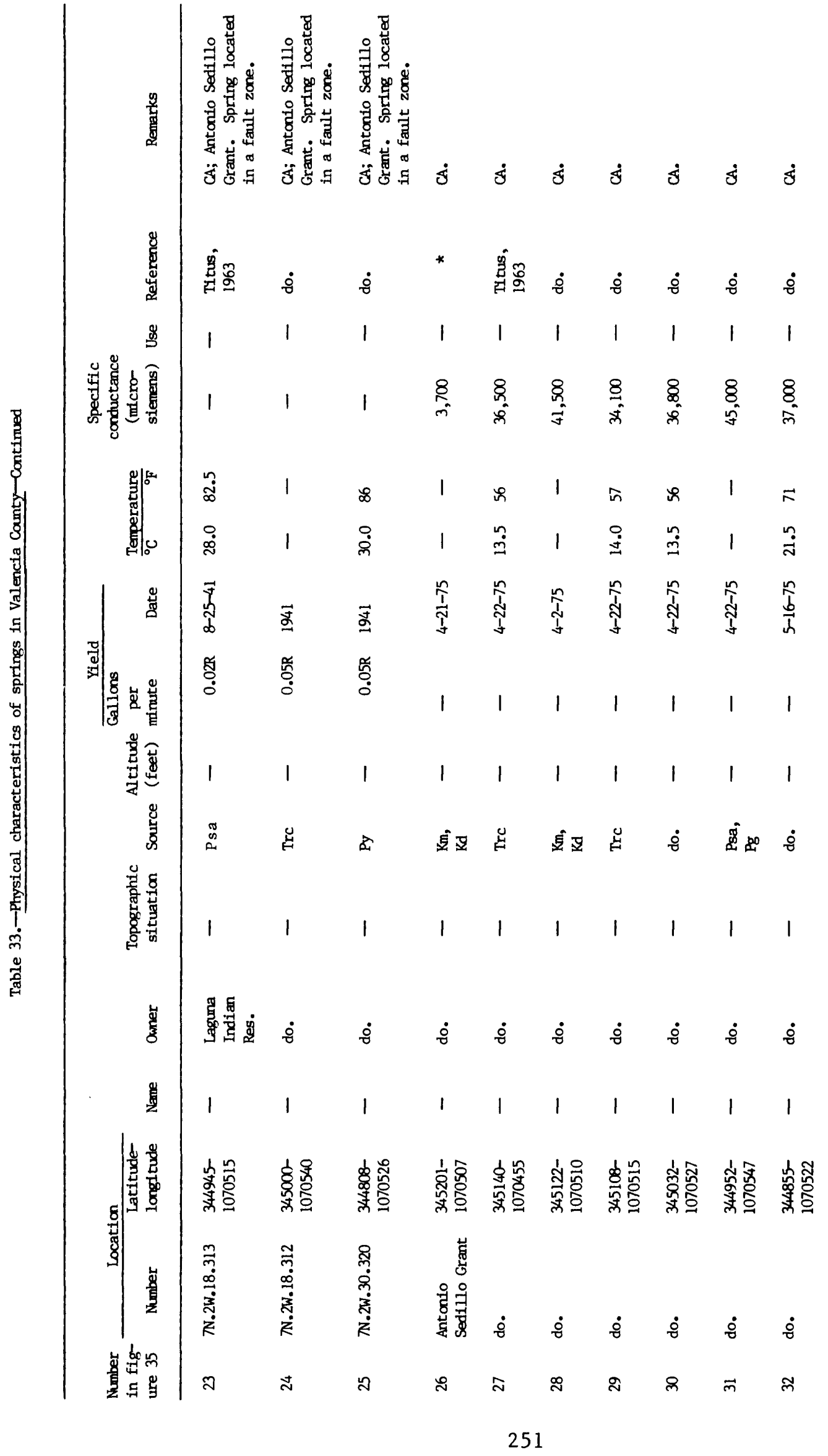




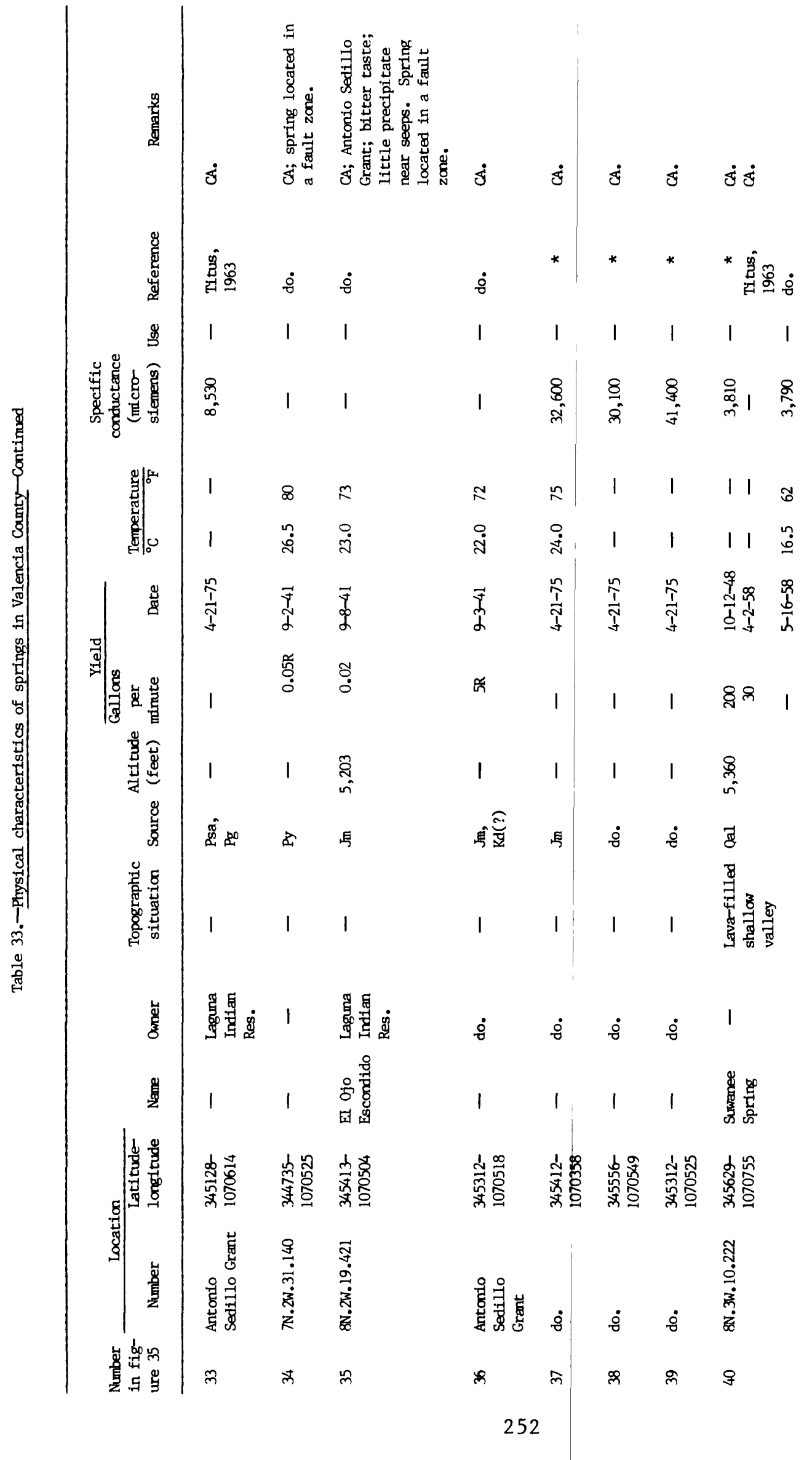




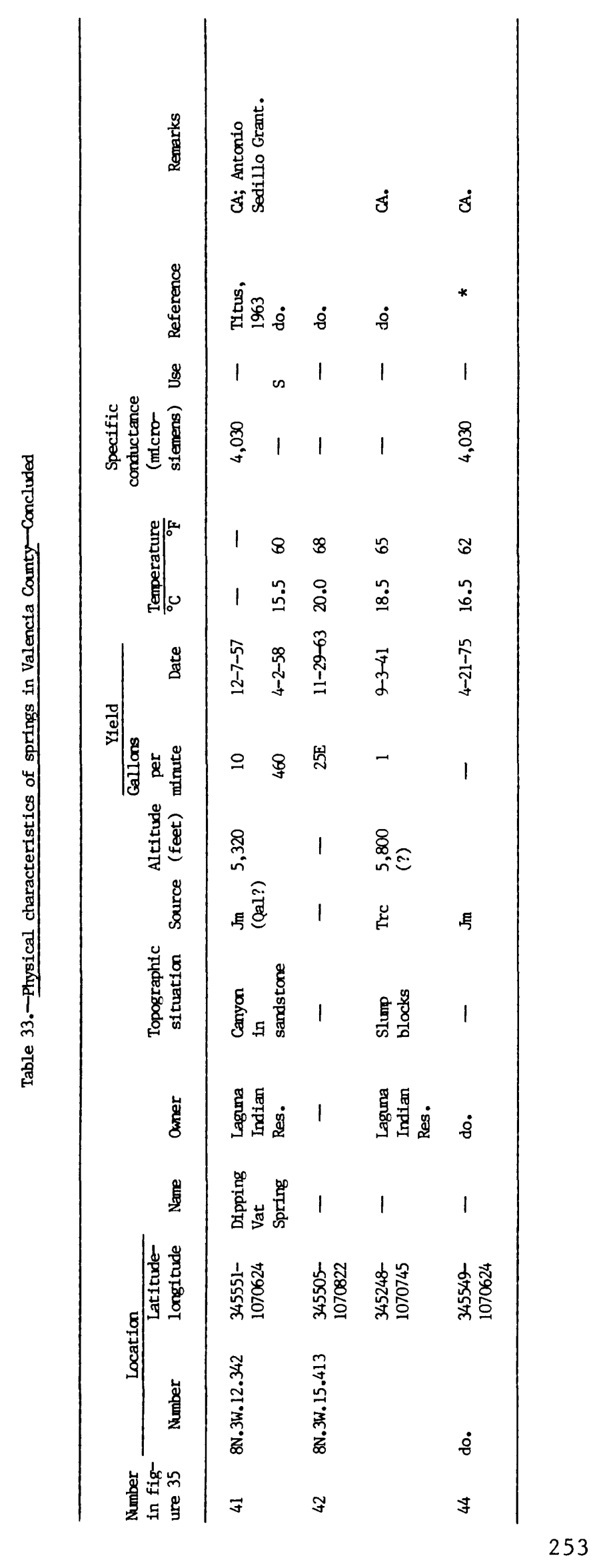

廿U.S. GOVERNMENT PRINTING OFFICE: 1992-836-923 